US Army Corps

of Engineers

Waterways Experiment

Station

\title{
Flow-Induced Structural Response of a 1:5-Scale Olmsted Wicket Model
}

by Mostafiz R. Chowdhury, Robert L. Hall, Winston Glenn Davis 
The contents of this report are not to be used for advertising, publication, or promotional purposes. Citation of trade names does not constitute an official endorsement or approval of the use of such commercial products.

The findings of this report are not to be construed as an official Department of the Army position, unless so designated by other authorized documents. 


\section{Flow-Induced Structural Response of a 1:5-Scale Olmsted Wicket Model}

by Mostafiz R. Chowdhury, Robert L. Hall, Winston Glenn Davis U.S. Army Corps of Engineers

Waterways Experiment Station 3909 Halls Ferry Road

Vicksburg, MS 39180-6199

Final report

Approved for public release; distribution is unlimited

Prepared for U.S. Army Corps of Engineers

Washington, DC 20314-1000

and U.S. Army Engineer District, Louisville Louisville, KY 40201-0059 


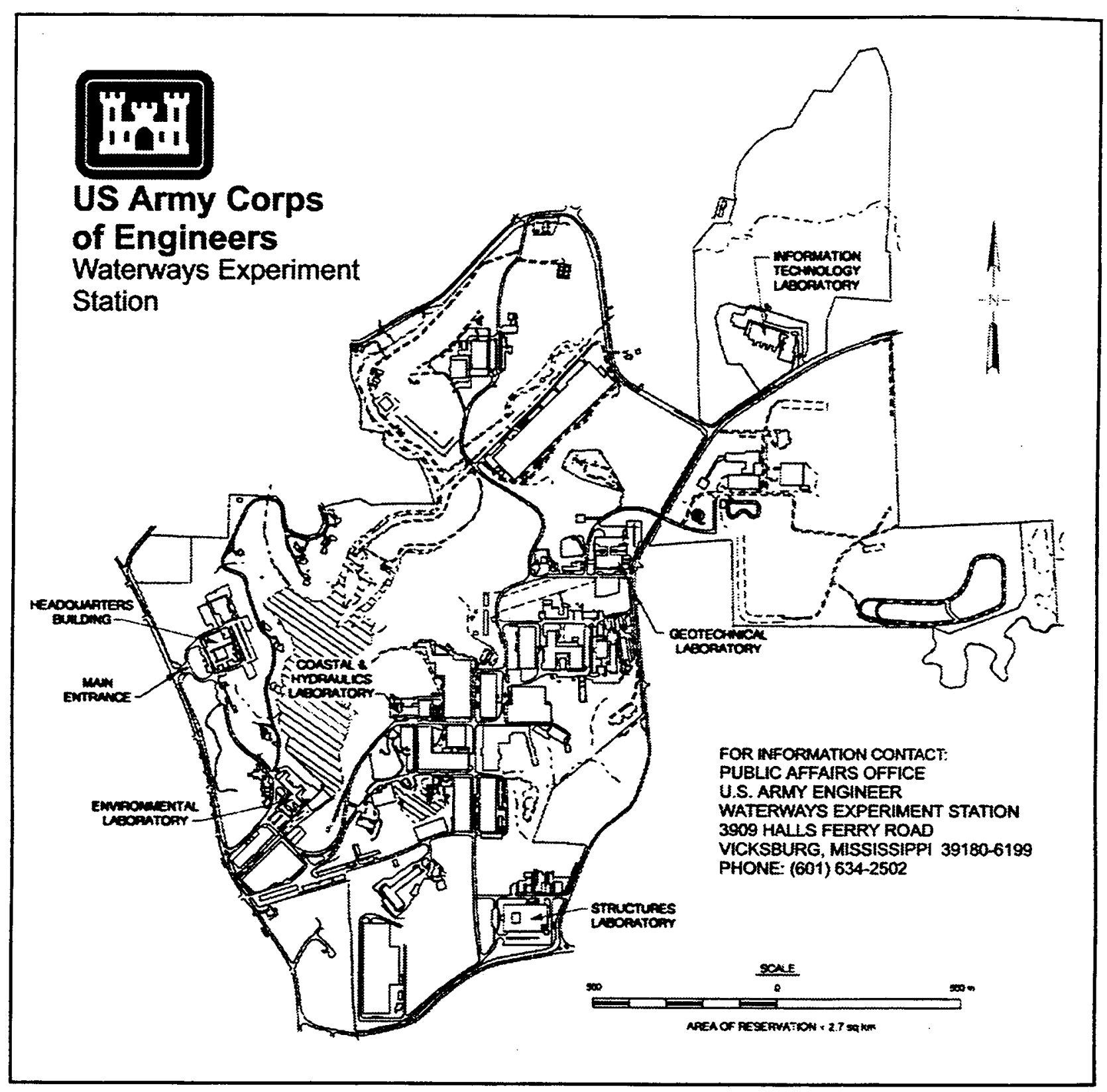

Waterways Experiment Station Cataloging-in-Publication Data

Chowdhury, Mostafiz R.

Flow-induced structural response of a 1:5-scale Olmsted wicket model / by Mostafiz R.

Chowdhury, Robert L. Hall, Winston Glenn Davis ; prepared for Headquarters, U.S. Army

Corps of Engineers and U.S. Army Engineer District, Louisville.

362 p. : ill. ; $28 \mathrm{~cm}$. - (Technical report ; SL-98-2)

Includes bibliographical references.

1. Flow injection analysis - Models - Testing. 2. Structural dynamics - Models

Evaluation. 3. Hydraulic gates - Models - Testing. I. Hall, Robert L. II. Davis, W. Glenn.

III. United States. Army. Corps of Engineers. Louisville District. IV. U.S. Army Engineer

Waterways Experiment Station. V. Structures Laboratory (U.S. Army Engineer Waterways

Experiment Station) VI. Title. VII. Series: Technical report (U.S. Ammy Engineer Waterways

Experiment Station) ; SL-98-2.

TA7 W34 no.SL-98-2 


\section{Contents}

Preface XV

Conversion Factors, Non-SI to SI Units of Measurement ................................xvii

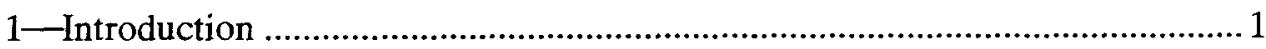

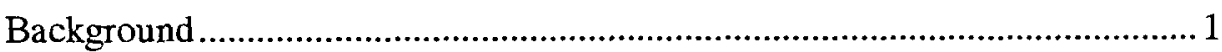

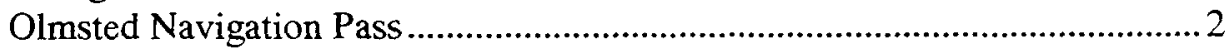

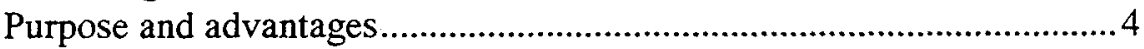

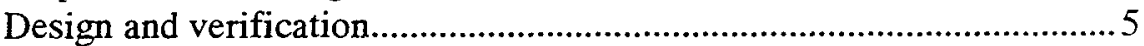

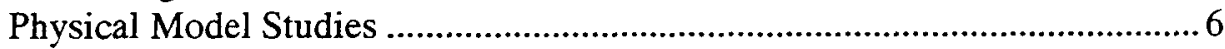

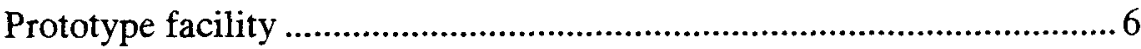

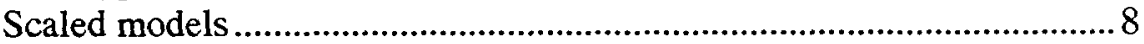

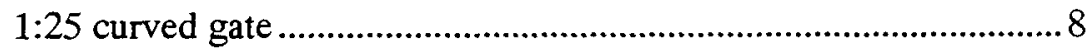

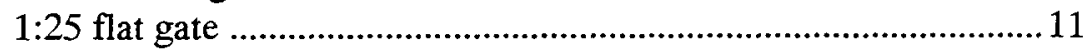

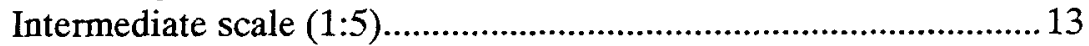

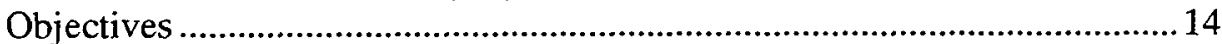

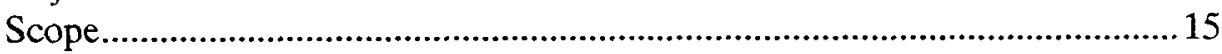

2-Fabrication, Construction, Installation, and Operation ...............................16

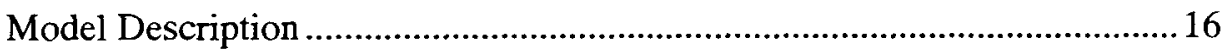

Model appurtenances.......................................................................... 19

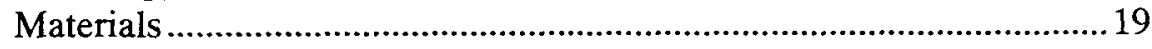

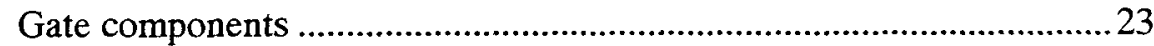

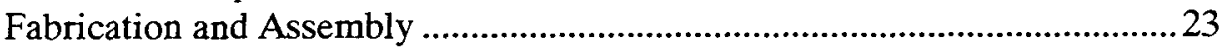

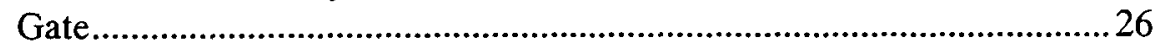

Hinge assembly and sill-bearing frame ............................................2. 27

Prop-rod and hurter mechanism ......................................................28

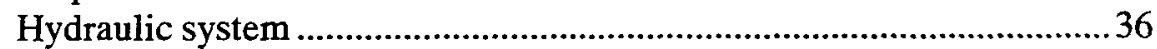

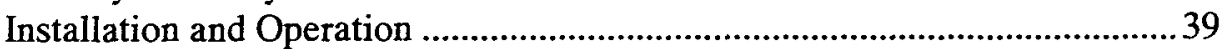

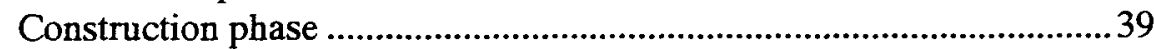

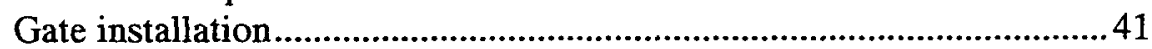

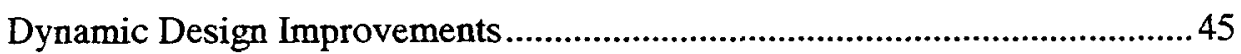

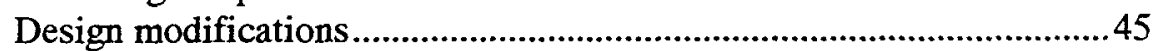

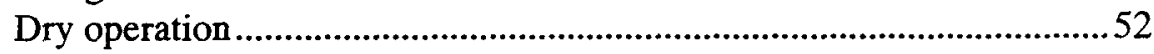

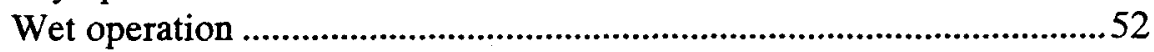

3-Instrumentation, Data Acquisition, and Analysis......................................57

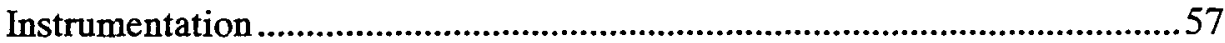

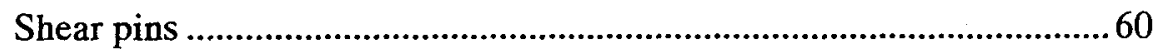

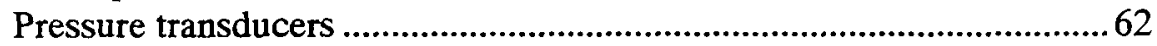

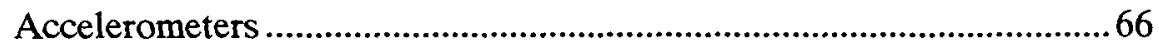

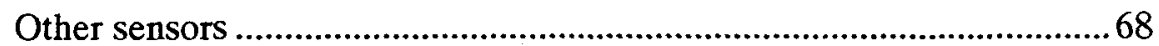


Data Acquisition and Verification......................................................... 70

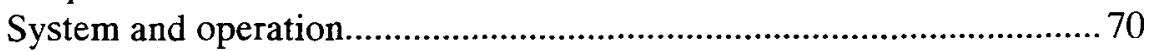

Data Recording and Initialization .............................................................. 71

Pressure transducer output initialization ............................................. 71

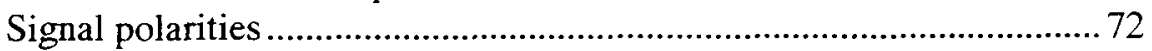

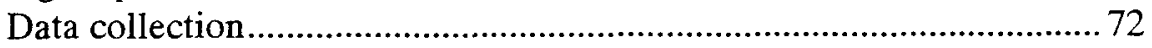

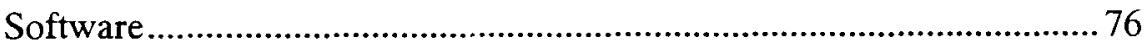

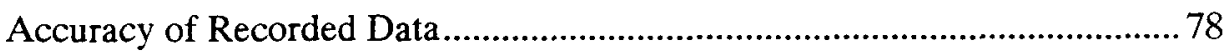

Shear pins ...................................................................................... 78

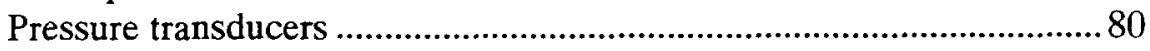

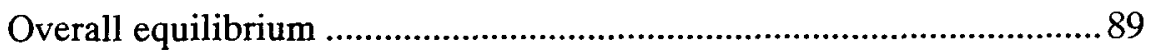

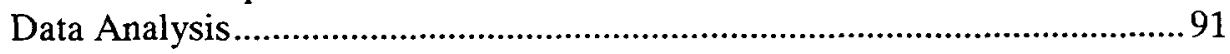

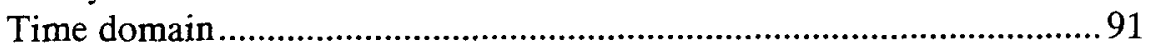

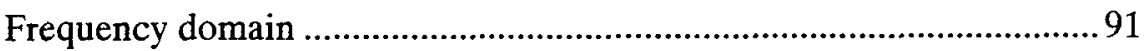

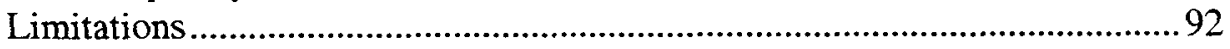

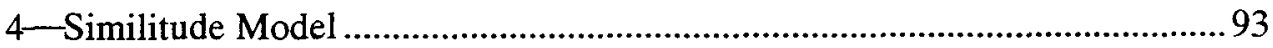

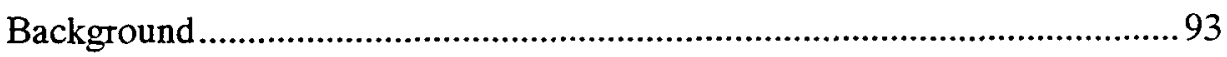

Similitude Relationships for Wicket Gates .............................................. 97

Hydraulic model similitude relations ................................................. 98

Significance of Pi terms............................................................. 100

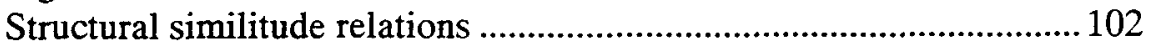

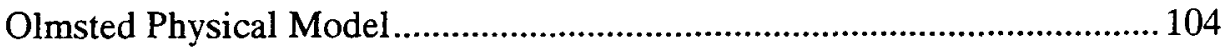

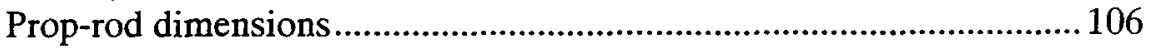

Hydraulic cylinder .............................................................................. 107

Experimental Evaluation of Hydraulic Fluid Bulk Modulus........................ 108

Verification of Similitude Model ............................................................ 109

Comparison of 1:25-scale flat gate and 1:5-scale

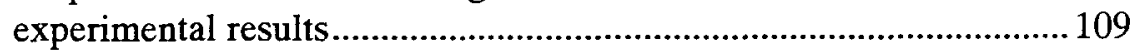

Experimental modal analysis - dry condition ......................................111

Comparison......................................................................................... 112

Experimental modal analysis - wet condition.........................................115

Full-range experiments ...............................................................117

21-ft head difference (Experiment \#173 vs \#2065) ........................118

11 -ft head difference (Experiment \#43 vs \#2072) ........................ 118

Fixed gate at $20 \mathrm{deg}, 11-\mathrm{ft}$ head difference................................... 145

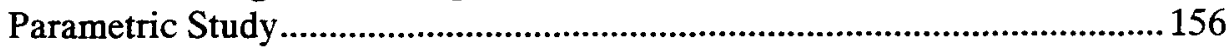

Effects of prop-rod stiffness (Experiments No. 105 and No. 116)...... 156

Effect of oil column volume .............................................................171

Effect of gate-raising rate (144 sec vs $313 \mathrm{sec}$ )................................. 172

Effects of prop-rod dragging (Experiments No. 42 and No. 87) ......... 172

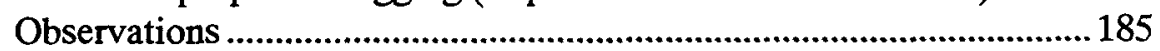

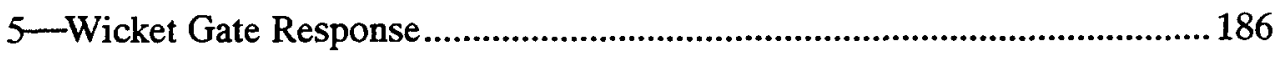

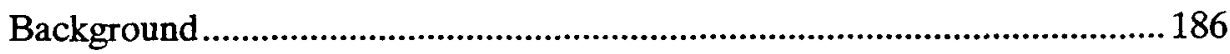

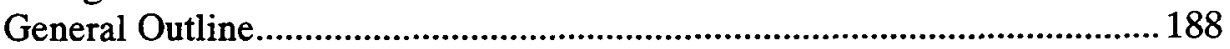

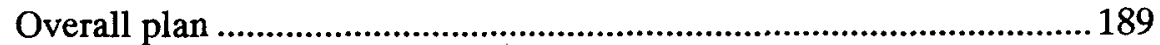

Experimental boundaries and flow configurations.............................. 190 


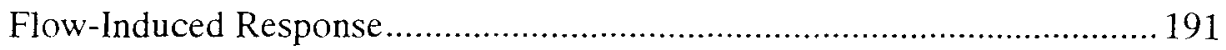

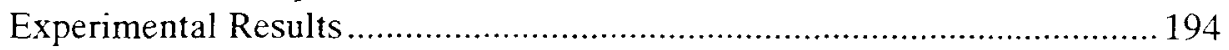

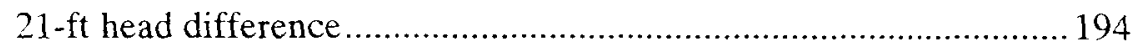

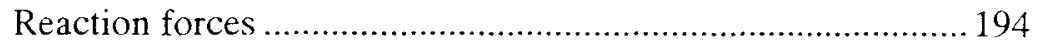

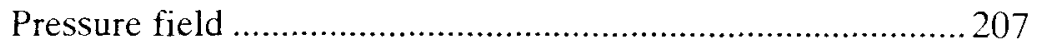

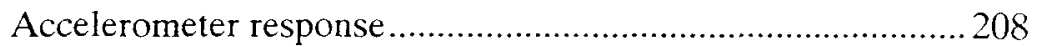

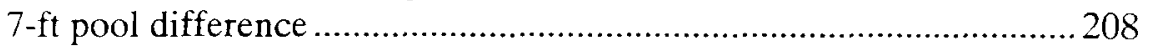

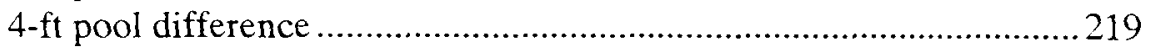

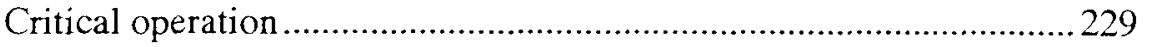

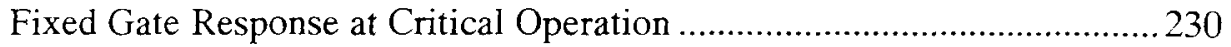

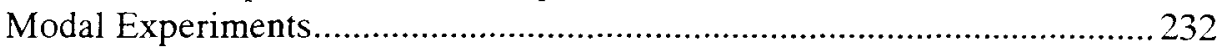

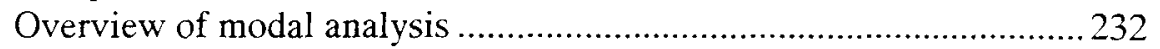

Experimental procedure and data analysis ..........................................238

Experimental setup ................................................................. 241

Data acquisition and modal analysis .............................................. 244

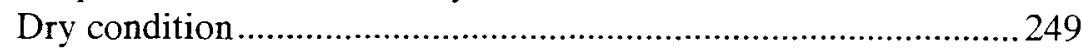

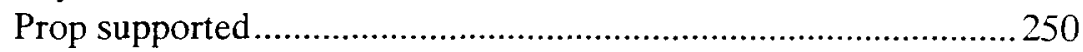

Shaft supported ............................................................... 253

Prop-rod vibrational behavior................................................. 257

Wet Condition ................................................................... 262

Operating Deflected Shape ................................................................. 274

Frequency-domain ODS at critical operation............................2276

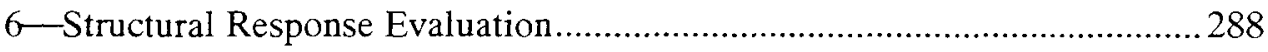

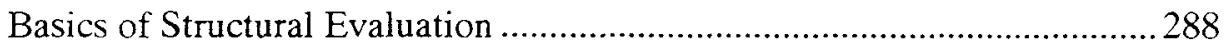

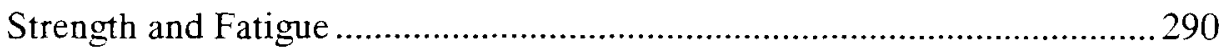

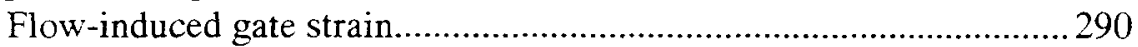

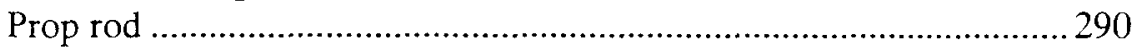

Base hinge assembly ................................................................ 291

7-Physical Parameter Identification .............................................................296

Maximum Measured Responses ...........................................................296

Pressure distributions on a prop-supported gate ............................... 298

Representative pressure history ...........................................................302

Added Mass Effects............................................................................... 302

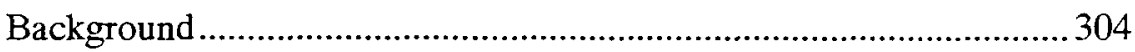

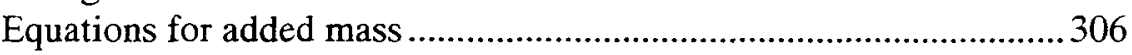

Experimental results for wicket gate model ......................................307

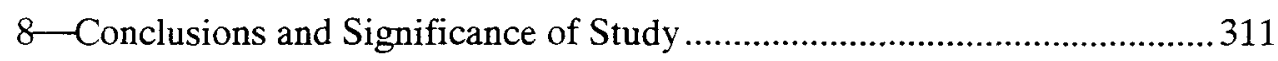

Dynamic Design Development .................................................... 311

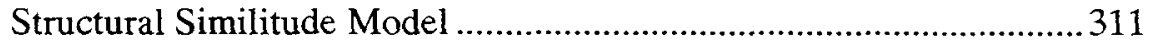

Dynamic Performance Evaluation ...................................................... 312

Design Parameter Identification ..................................................... 313

Analytical Model Development...................................................... 313

Prototype Operational Adjustment and Cost Savings ...........................314 
Appendix A: Selected Prototype Design Sheets ……….............................. A1

Appendix B: Fabrication and Installation Drawings .................................... B1

\section{List of Figures}

Figure 1. Olmsted L\&D project site …........................................................ 2

Figure 2. L\&D 52 across the Ohio River................................................... 3

Figure 3. Olmsted Navigation Pass ............................................................ 4

Figure 4. A five-gate gap flow condition in the $1: 5$ model f...........................5

Figure 5. Prototype facility at Smithland dam........................................... 7

Figure 6. 1:25-scale Olmsted curved gate model .......................................10

Figure 7. 1:25-scale Olmsted flat gate model …........................................12

Figure 8. 1:5-scale Olmsted flat gate model ................................................ 14

Figure 9. Plan and profile view of 1:5-scale Olmsted wicket model .............. 17

Figure 10. A section of Olmsted spillway crest ............................................. 17

Figure 11. Dry bed views of $1: 5$-scale model ................................................ 18

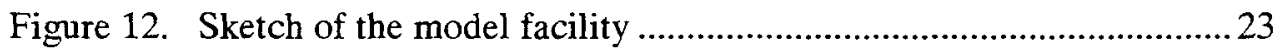

Figure 13. Six water pumps for flume flow regulation ...................................2 24

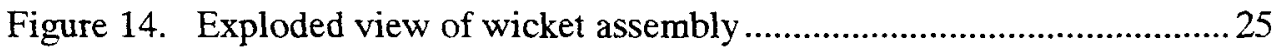

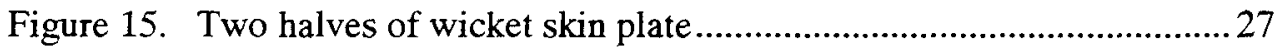

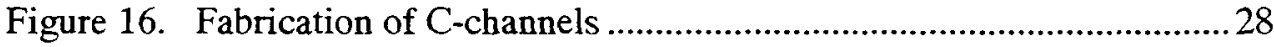

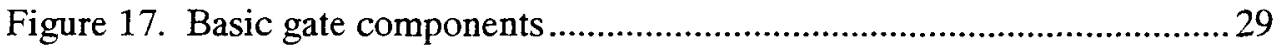

Figure 18. Assembly of stiffener plate, channel, and ribs ..............................30

Figure 19. Mounting conditions of web stiffeners on W-beam.........................31

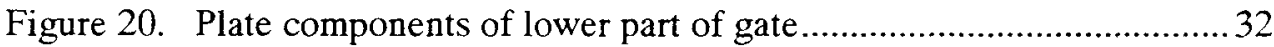

vi 
Figure 21. Interior view showing gate base connection to bearing frame......... 32

Figure 22. Connection details for gate base ….......................................... 33

Figure 23. Closeup view of assembled gate base ....................................... 34

Figure 24. Y-shaped notch to facilitate use of hoist crane hooks..................... 34

Figure 25. Top (front) view of assembled gate ............................................. 35

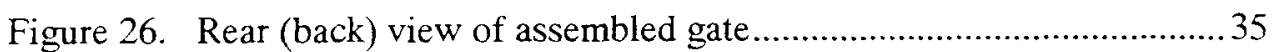

Figure 27. Cutout pieces of ball mounting plate, pillow block .........................36

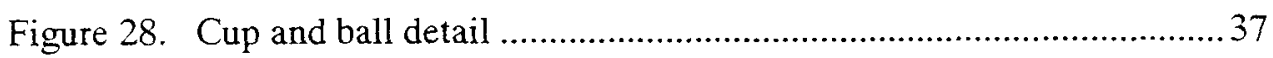

Figure 29. Placement of ball mounting plate on beam web ............................ 38

Figure 30. Overview of 1:5 wicket gate and its accessories............................39

Figure 31. Unfinished assembly of base hinge and bearing frames .................40

Figure 32. Finished gate frame-hinge assembly, cup-ball assembly ................ 40

Figure 33. Relative positioning of embedded frames.................................... 41

Figure 34. Closeup view of cylinder bearing frame assembly .......................42

Figure 35. Closeup view of alignment frame assembly ............................... 43

Figure 36. Hurter bearing frame embedded in the sill....................................4

Figure 37. Schematic diagram of the hydraulic module ..................................45

Figure 38. Connection details of retractable cylinder and support ..................46

Figure 39 . Hydraulic control system with auxiliary cylinder ..........................47

Figure 40. Hydraulic lifting cylinders installed in model..............................4 47

Figure 41. First level of poured concrete................................................... 48

Figure 42. Three views of sill after second level of poured concrete .............. 48

Figure 43. Cable routing pipe outlets and steel hydraulic hoses ......................50

Figure 44. Finished sill with close look at cylinder pit ..................................50

Figure 45. Upstream view of flume ........................................................ 51 
Figure 46. Closeup view of prop rod and cylinder support at 65 deg .51

Figure 47. Prop rod hanging on front edge of hurter due to lateral thrust.........53

Figure 48. Extension of vertical guard plates to avoid lateral obstruction........54

Figure 49. Prop fork breakage during 2GG flow tests .................................54

Figure 50. Twisted guide shoes due to bending of guide bar ..........................55

Figure 51. Completed gate installation in dry operation ................................56

Figure 52. Shear pin locations on instrumented gate ..................................6 60

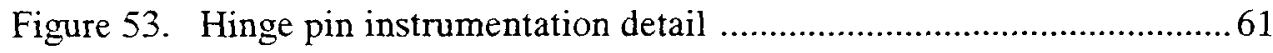

Figure 54. Ball shear pin instrumentation detail ........................................62

Figure 55. Prop shear pin instrumentation detail ..........................................6 63

Figure 56. Pressure gauge calibration device (AMETEK model PKII) ............64

Figure 57. Transducer location on instrumented gate ….................................65

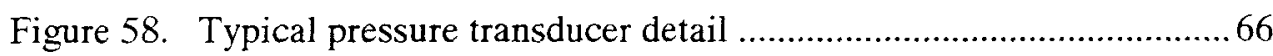

Figure 59. Synthesized FRF plots for measuring goodness of response

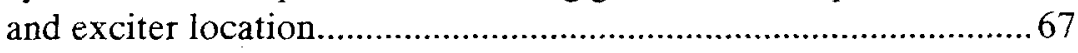

Figure 60. Locations of accelerometers on FE grid .....................................6 68

Figure 61. Transducer installation on gate …..............................................69

Figure 62 . Instrumented gate on flume................................................... 70

Figure 63. Block diagram for data acquisition system ................................ 71

Figure 64. A static load experiment setup ….............................................. 79

Figure 65. Relationship between pressure gauge reading and actual head .......81

Figure 66. Raw pressure gauge readings for a zero-reference ….....................8 82

Figure 67. Relative water depths for overall equilibrium experiment...............90

Figure 68. Spring-mass model for hydraulic system .................................... 110

Figure 69. A schematic diagram of forces on 1:25 scale hydraulic actuator

viii 
Figure 70. Transducer location on 1:25-scale model

Figure 71. Dry gate mode shapes for 1:25-scale wicket.

Figure 72. Dry gate mode shapes for 1:5-scale wicket.

Figure 73. Operating mode shapes for 1:25-scale wicket

Figure 74. Wet mode shapes for 1:5-scale wicket

Figure 75. Gate response comparison for 1:25- and 1:5-scale models, 21 -ft head difference, $3 \mathrm{GG}$ gap configuration

Figure 76. Gate response comparison for 1:25- and 1:5-scale models, 11 -ft head difference, $3 \mathrm{GG}$ gap configuration

Figure 77. Fixed gate response comparison for 1:25- and 1:5-scale models, 20-deg position, 11-ft head difference, and $3 \mathrm{GG}$ configuration ....147

Figure 78. Fixed gate response comparison for the steel and magnesium prop-supported gate, 11-ft head difference, and 3GG configuration.

Figure 79. Driving point FRF comparison for different oil column levels ..... 172

Figure 80. Effect of oil columns on flow-induced dynamic responses for the instrumented gate, $11-\mathrm{ft}$ head difference, $3 \mathrm{GG}$ configuration

Figure 81. Effect of gate-raising rate on flow-induced dynamic responses for instrumented gate, 11-ft head difference, $3 \mathrm{GG}$ configuration 179

Figure 82. A $1 \mathrm{GG}$ flow configuration with instrumented gate at down position (Pool elevations: $300 \mathrm{ft}$ and $279 \mathrm{ft}$ )

Figure 83. A 2GG flow configuration with end gate arrangement, instrumented gate at 24-deg position (Pool elevations: $300 \mathrm{ft}$ and $279 \mathrm{ft}$ ). 193

Figure 84. A $3 \mathrm{GG}$ flow case with lone gate arrangement, instrumented gate at up position (Pool elevations: $299 \mathrm{ft}$, and $279 \mathrm{ft}$ ) 194

Figure 85. Comparison of responses over full-range of operation for various gate configurations, $21-\mathrm{ft}$ pool difference 195

Figure 86. Sign convention for reaction forces 207 
Figure 87. Comparison of responses over full range of operation for various gate configurations, 7 -ft pool difference

Figure 88. Comparison of responses over full range of operation for various gate configurations, 4 -ft pool difference.

Figure 89. Comparisons of fixed gate reaction forces as a function of time

for two gate positions

Figure 90. Comparisons of pressures and accelerations for two gate positions

Figure 91. System block diagram for modal experiment. 238

Figure 92. Modal and FE grid layout 239

Figure 93. Schematic diagram of angular drift error in the SLDV system .....240

Figure 94. Experimental modal test setup 241

Figure 95. Overview of hardware setup for Lazon system. 243

Figure 96. A flowchart for modal analysis using SLDV system. 246

Figure 97. A comparison of SDOF and MDOF curve fitting methods 249

Figure 98. Mobility response functions for downstream and upstream results

Figure 99. Natural dry mode shapes of wicket using SLDV system 252

Figure 100. Verifications of assumptions used in modal experiment 254

Figure 101. Mobility response function comparison for prop- and shaft-supported dry wickets 255

Figure 102. Natural mode shapes from shaft-supported dry wicket. 256

Figure 103. Mobility response function comparison for shaft-supported dry gate at $65-$ and $24-\mathrm{deg}$ positions

Figure 104. Natural mode shapes from downstream side of a

24-deg dry gate.

Figure 105. Mobility response functions of prop-supported wicket for two different reference points 261

Figure 106. Prop-rod modal experiment setup .261 
Figure 107. Directional sensitivity of mobility response functions

for prop rod

Figure 108. Prop-rod mode shapes .263

Figure 109. Superimposed prop-rod FRF responses .264

Figure 110. Comparison of FRF and coherence functions for prop-supported wet and dry gate

Figure 111. Comparison of FRF and coherence functions for shaft-supported dry and wet wickets (3GG), 65-deg position....... 265

Figure 112. Comparison of FRF and coherence functions for shaft-supported dry and wet wicket (3GG), 24-deg position ........266

Figure 113. Natural wet mode shapes of prop-supported wicket in a 3GG condition with $21-\mathrm{ft}$ head difference

Figure 114. Natural wet mode shapes of shaft-supported gate in $3 \mathrm{GG}$ condition with 21 -ft head difference

Figure 115. Natural mode shapes for $24-\mathrm{deg}$ wet gate with $21-\mathrm{ft}$ head difference

Figure 116. Comparison of FRF and coherence plots for no-gap and $3 \mathrm{GG}$ flow configurations for wet gate at $65 \mathrm{deg}$

Figure 117. Mobility response function comparison of wet wicket at 24-deg critical position for 1 GG and $3 G G$ configurations

Figure 118. Mobility response functions for wet wicket at 7-, 11-, and $21-\mathrm{ft}$ head differences.

Figure 119. Transmissibility plot and operating shapes for raising gate during $21-\mathrm{ft}$ head difference, $3 \mathrm{GG}$ flow configuration

Figure 120. Transmissibility plot and operating shapes for prop-supported gate during $21-\mathrm{ft}$ head difference, $3 \mathrm{GG}$ gap flow configuration

Figure 121. Transmissibility plot and operating shapes for fixed gate at $24-\mathrm{deg}$ position during $21-\mathrm{ft}$ head difference, $3 \mathrm{GG}$ flow configuration 281

Figure 122. Strain transducer locations 291

Figure 123. Flow-induced strain response for wicket gate during $21-\mathrm{ft}$ head difference $3 G G$ flow test. 292 
Figure 124. Gate frame hinge assembly

Figure 125. The envelope of fatigue failure using the Goodman line

Figure 126. Safe region for fluctuating shear loads

Figure 127. Finite element model for the base hinge

Figure 128. Pressure gauge locations and column lines for displaying pressure readings

Figure 129. Relative water elevation for pressure measurements

Figure 130. Pressure distributions on prop-supported gate as a function of depth

Figure 131. Pressure envelopes on raising wicket. 303

Figure 132. Wet frequency variation as a function of pool difference. 308

Figure 133. Added mass as a function of water heads 308

\section{List of Tables}

Table 1. Comparison of Material Properties for Olmsted Prototype and 1:5-Scale Wicket Model .22

Table 2. Average Thickness for Major Structural Members Used in 1:5-Scale Wicket Model.

Table 3. Instrumentation List for 1:5 Olmsted Wicket Model .58

Table 4. Typical List of Calibration Factors for 1:5 Olmsted Wicket Model 73

Table 5. Static Experiment Results for Verifying Shear Pin Effectiveness .....80 80

Table 6. Pressure Gauge Verification Table

Experiment 1: Constant Pool 88

Table 7. Pressure Gauge Verification Table Experiment 2: Fixed Gate, Variable Pool

Table 8. Overall Equilibrium of Prop-Supported Instrumented Wicket for 11-ft Head Differential 91

Table 9. Physical Parameters for Hydraulic Modeling. .98 xii 
Table 10. Scale Factors for Dissimilar Materials when Froude Condition is $\mathrm{Met}$

Table 11. Conventional Scaling Relationships.

Table 12. Experimental Natural Frequencies for 1:25- and 1:5-Scale Models at 65-deg Position.

Table 13. Results for 1:25- and 1:5-Scale Flat Gate Models at 20-deg Position (11-ft Head Difference, 3GG Configuration)

Table 14. Results for 1:25- and 1:5-Scale Flat Gate Models at 60-deg Position (11-ft Head Difference, 3GG Configuration).

Table 15. Results for 1:5-Scale Flat Gate Models with and without Prop Rod (11-ft Head Difference, 3GG Configuration)

Table 16. Flow-Induced Vibration Gate Configurations for the Full-Range Experiments

Table 17. Flow-Induced Vibration Gate Cases for Full-Range Experiments

Table 18. A Summary of Test Responses at Critical Configurations 233

Table 19. Lazon System Data Acquisition Setup .245

Table 20. Dry Modal Experiments

Table 21. Correlation Matrix for First 10 Nodes for Upstream and Downstream Laser Scanning

Table 22. Correlation Matrix for Prop- and Shaft-Supported Modes

Table 23. Correlation Matrix for Prop-Supported and 24-deg Position Wicket Modes 260

Table 24. Wet Modal Experiments 263

Table 25. Correlation Matrix for Prop-Supported Dry and Wet Gate (21-ft Head Difference, 3GG Flow Configuration)

Table 26. Correlation Matrix for Dry and Wet Gate Modes at 24-deg Position (21-ft Head Difference, 2GG Flow Configuration)

Table 27. Correlation Matrix of Operating Shapes for Prop-Supported Fixed and Raising Gate (21-ft Head Difference, 3GG Flow Configuration) 
Table 28. Correlation Matrix of Operating Shapes for Fixed Gate at $24 \mathrm{deg}$ and Raising Gate (21-ft Head Difference, 3GG Flow Configuration)

Table 29. Correlation Matrix of Mode Shapes and ODS for Prop-Supported Gate (21-ft Head Difference, 3GG Flow Configuration)

Table 30. Correlation Matrix of Mode Shapes and ODS During Critical Flow Configuration at 24-deg Gate Position (21-ft Head Difference, 3GG Flow Configuration)

Table 31. Correlation Matrix of Mode Shapes for Prop-Supported Gate and ODS for Lifting Wicket (21-ft Head Difference, 3GG Flow Configuration)

Table 32. Estimated Design Responses for Prototype Wicket 297

Table 33. Added Mass Parameters for Wet Gate 309 


\section{Preface}

The research reported herein was sponsored by the U.S. Army Engineer District, Louisville, in support of the Olmsted Navigational Model Study Program. Mr. Rick Schultz was the Program Monitor for the physical model studies, and Mrs. Anjana Chudgar was the technical monitor for the structural performance evaluation of the Olmsted wickets. Mr. Ralph Snowberger provided the structural design guidance, and Mr. Gordon Lance, U.S. Army Engineer Division, Ohio River, contributed to the hydraulic design aspect of the Olmsted project.

All work was carried out by Dr. Mostafiz R. Chowdhury and Robert L. Hall, Structural Mechanics Division (SMD), Structures Laboratory (SL), U.S. Army Engineer Waterways Experiment Station (WES), under the general supervision of Dr. Bryant Mather, Director, SL; Mr. John Ehrgott, Assistant Director; and Dr. Reed Mosher, Chief, SMD; and Mr. Winston Glenn Davis, Hydraulic Structures Division (HSD), Coastal and Hydraulics Laboratory (CHL), WES, under the general supervision of Dr. James Houston, Director, CHL; Mr. Richard Sager, Assistant Director, CHL; and Dr. Phil Combs, Chief, HSD. The work was conducted during May 1993 through January 1996 under the direct supervision of Dr. Chowdhury and Mr. Davis. Mr. Davis was also responsible for design, construction, operation, and maintenance of the hydraulic model facility used in this project.

The contributions of Mr. William Li, graduate student from the Virginia Polytechnic Institute and State University, in acquiring modal data using the laser system, and Ms. Yazmin Seda, SMD, for analyzing recorded data, are gratefully acknowledged. Mr. Billy Benson, SMD, was responsible for purchasing and coordinating the fabrication of the test wickets. Mr. Ken Vitaya-udom, SL, prepared the model shop drawings. Messrs. Bill Tenant and Billy R. Channell, Welding Shop, and Mr. Larry Purvis, Machine Shop, are credited for fabrication of the wicket gates, prop rods, hurter, and flow control gates used in the facility. Messrs. Dan Barnes, Model Construction Section, John E. Gullett, Carpenter Shop, and Mitchel Simmons and Charles Hopkins, Model Shop, constructed the spillway crest and topography. Mr. Joe Ables, Information Technology Laboratory (ITL), was responsible for instrumentation and operation of the model during experiments. Mr. Terry Warren, ITL, wrote the program for data acquisition, and Mr. Homer Greer, ITL, was responsible for coordinating instrumentation and data acquisition tasks. Technical assistance for the preparation of the report by Ms. Vicky Smith, SMD, is gratefully acknowledged. The efforts of the Editorial Section and Visual Production Center, ITL, staff members who participated in the preparation of the report are appreciated. 
At the time of publication of this report, Director of WES was Dr. Robert W. Whalin. Commander was COL Robin R. Cababa, EN.

The contents of this report are not to be used for advertising, publication. or promotional purposes. Citation of trade names does not constitute an official endorsement or approval of the use of such commercial products. 


\section{Conversion Factors, Non-SI to SI Units of Measurement}

Non-SI units of measurement used in this report can be converted to SI units as follows:

\begin{tabular}{|l|l|l|}
\hline Multiply & By & To Obtain \\
\hline cubic feet & 0.02831685 & cubic metres \\
\hline cubic inches & 0.000016387 & cubic metres \\
\hline degrees (angle) & 0.01745329 & radians \\
\hline Fahrenheit degrees & $5 / 9$ & Celsius degrees' \\
\hline feet & 0.3048 & metres \\
\hline G (standard acceleration of free fall) & 9.80665 & metres per second squared \\
\hline inches & 0.0254 & metres \\
\hline ksi (kips per square inch) & 6.894757 & megapascals \\
\hline kips & 4.4484 & kilonewtons \\
\hline miles (U.S. statute) & 1.609347 & kilometres \\
\hline pounds (force) & 4.4484 & newtons \\
\hline pounds (mass) & 0.4535924 & kilograms \\
\hline pounds (force) per square inch & 0.006894757 & megapascals \\
\hline tons & 1,000 & kilograms \\
\hline $\begin{array}{l}\text { To obtain Celsius ( C ) temperature readings from Fahrenheit (F) readings, use the equation: } \\
\text { C = (5/9) - (F - 32). }\end{array}$ & & \\
\hline
\end{tabular}




\section{Introduction}

\section{Background}

Olmsted Locks and Dam (L\&D) is one of the largest civil works projects undertaken by the U. S. Army Corps of Engineers (USACE) to lead the modernization effort of navigational facilities for the twenty-first century. Maintaining a robust navigational infrastructure to facilitate the water-borne transportation and to meet the demand for the ever-increasing traffic flows through our nations waterways is vital to our economy. Transportation of bulk commodities via our vast inland waterways not only provides the most economic mode of conveyance but also helps to conserve energy resources. In this complex world with its global economy, it becomes more demanding to increase productivity by making efficient and effective uses of resources. In this regard, the Corps' continuing effort to improve the navigation facilities using the latest technology is essential.

Considering the complexity of the operating conditions, the uniqueness of the wickets, the economic significance, and a check-and-balance policy on the validation of the hydraulic physical models, an extensive research initiative was undertaken by the USACE Division, Ohio River (ORD). Several research and development phases were initiated and coordinated by the USAE District, Louisville (LRL), to accomplish this monumental navigation project on the Ohio River. The principal focus of this research scheme was to determine the most appropriate type of wicket for the new Olmsted L\&D Project. In January 1990, a wicket model study was initiated to support the design of the prototype structure. In a relentless effort to better understand the performance of these unprecedented hydraulically lifted wickets under variable operating conditions, a series of models was subsequently developed and tested by the U. S. Army Engineer Waterways Experiment Station (WES) (March and Elder 1992; Chowdhury, Hall, and Pesantes 1997). In the final phase of this model study, a 1:5-scale model was studied at WES. Continuous feedback of the experimental results from such physical models was accommodated by the designers at the Louisville District to improve the design of the hydraulically operated Olmsted Wickets. Subsequently, after a series of dynamic design changes, a final version of the prototype structure was adopted for further experimentation and verifications at the Smithland facility. The inability of the prototype facility to simulate broad boundaries of operating conditions demanded major investigations of the 1:5 physical model at WES. This report presents the experimental results for the 1:5-scale model of the prototype wickets. It also describes the experimental and analysis procedures to evaluate the structural dynamic performance of 
operational wickets and provide guidelines for estimating physical parameters for the purpose of design and analytical model development.

\section{Olmsted Navigation Pass}

The Olmsted Navigation Pass is one of the busiest barge traffic routes in the nation. The new $L \& D$ project will be constructed near the community of Olmsted, IL, at Ohio River Mile 964.4. Figure 1 shows the relative location of the Olmsted Dam, the navigation routes, and the old L\&D 52 and 53. As shown in the figure, this reach of the Ohio River is particularly strategic in that it provides a connection between the Ohio, Tennessee, Cumberland, and Mississippi Rivers. The area has been described as the "hub" of the Ohio and Mississippi Rivers waterways system. Barge traffic moving between the Mississippi River system and the Ohio, Tennessee, and Cumberland Rivers must pass through this stretch of river. More tonnage passes this point than any other place in the inland navigation system. For instance, in 1991 alone, $100,000,000$ tons of goods were shipped through this junction of the Ohio River. Clearly, this is a critical reach of water from a commercial navigation perspective.

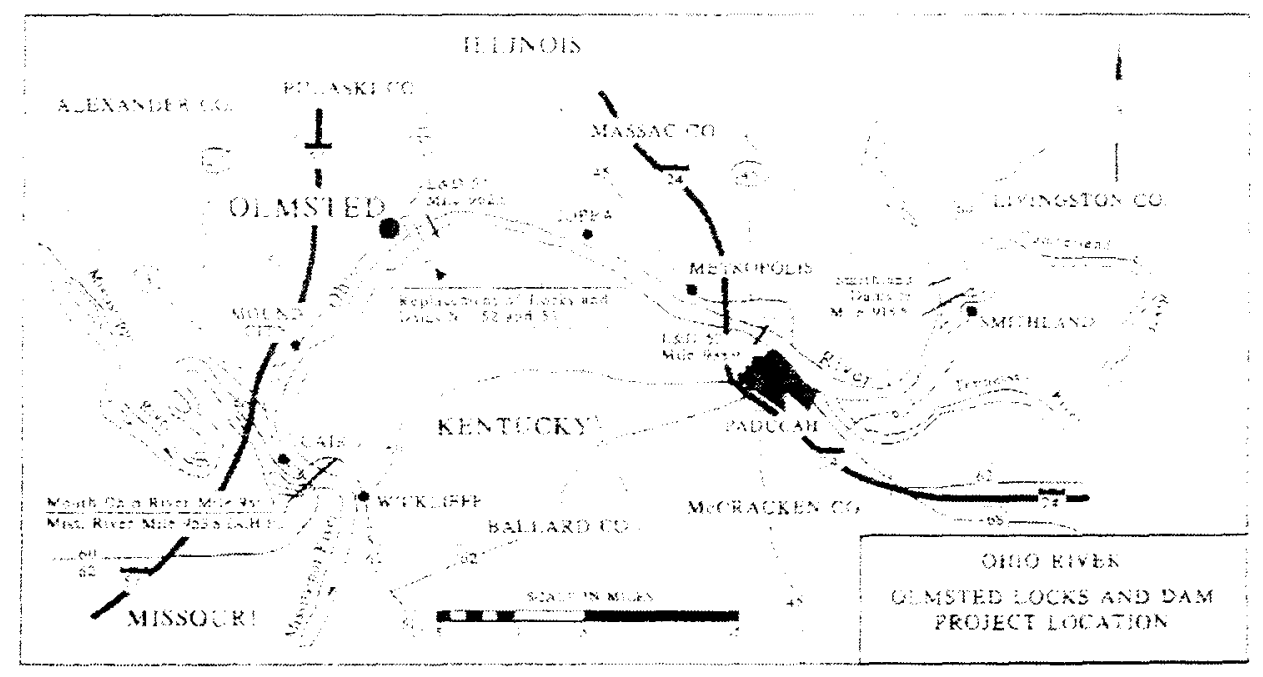

Figure 1. Olmsted L\&D project site

The original dams consisted of wooden wickets that were manually raised to provide navigable depths upstream during periods of low flow and dropped to the river bottom during high water. A view across the Ohio River at L\&D 52, showing the operational condition of the wooden wickets, is displayed in Figure 2. Built in 1929, these aged locks and dam were renovated in the 1970's by adding a temporary 1,200 - $\mathrm{ft}$ lock chamber to meet the traffic demands on the waterway. The present facility, however, is inadequate to handle the current traffic volume without significant delays. The continuing growth in water-borne traffic on the Ohio River and, more importantly, substantial deterioration of 


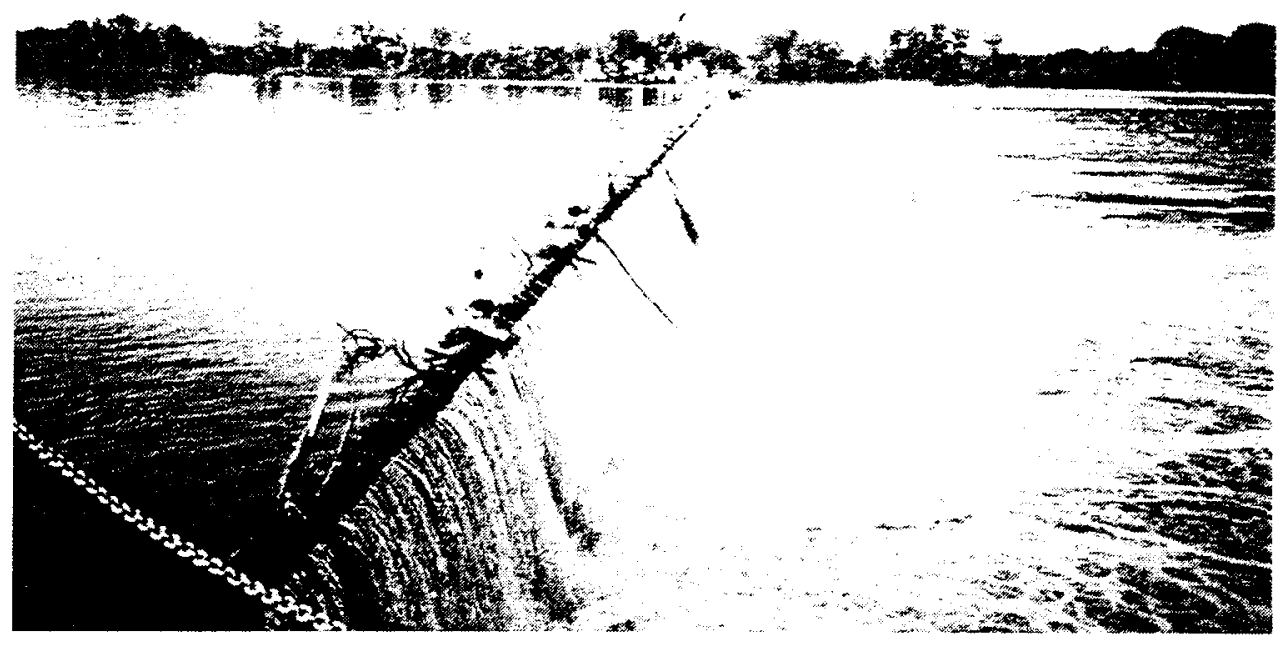

Figure 2. L\&D 52 across the Ohio River

these aged structures demands a replacement of the locks and dam in this strategic hub of the waterways.

The Olmsted L\&D Project is to be constructed 16 miles upstream on the Ohio River from the confluence of the Ohio and Mississippi Rivers. This new L\&D project will replace L\&D No. 52 and 53 on the lower Ohio River. The capacity of this project will be sufficient to meet projected demands of tow traffic through the year 2025. Construction of the Olmsted L\&D Project was authorized by the United States Congress on 17 November 1988, by the passage of the Water Resources Development Act of 1988 (Public Law 100-676). The cost of this project is being equally shared with the navigation industry. Tariffs paid by the navigation traffics on diesel fuel are used to form an Inland Waterways Trust Fund. The trust fund provides 50 percent of the project cost. Estimated total project cost is over $\$ 1$ billion.

Wicket gates have been used as part of L\&D systems by the Corps of Engineers since 1909 (Soast 1994). The Olmsted L\&D, as proposed for this study, will include 220 remotely operated, hydraulically actuated wicket gates. When placed into operation, these hydraulic wickets will be the largest of their kind in the world. Hydraulically operated wickets, 10 by $8.2 \mathrm{ft}$ on a 82 -ft-long dam, are currently in operation at the deNovaul Dam in France (Snowberger 1995). The Olmsted Wickets are about $10 \mathrm{ft}$ wide and $26 \mathrm{ft}$ long with a design lift of $21 \mathrm{ft}$, much larger than those at deNovaul. The project will include twin $1,200-\mathrm{ft}$ by $110-\mathrm{ft}$ lock chambers with a design lift of $21 \mathrm{ft}$ and a $2,200-\mathrm{ft}-$ long navigation pass consisting of wicket gates as shown in Figure 3. In the raised position, the wicket gates will be used to regulate the level of the navigable pool. In the lowered position, the gates will provide a navigable pass (Lance 1992). 
The remaining 426-ft dam will include a fixed weir section which will tie into the Kentucky shore. The LRL gate design team designed the prototype model for the Olmsted wickets that were built at Smithland Dam on the Ohio River.

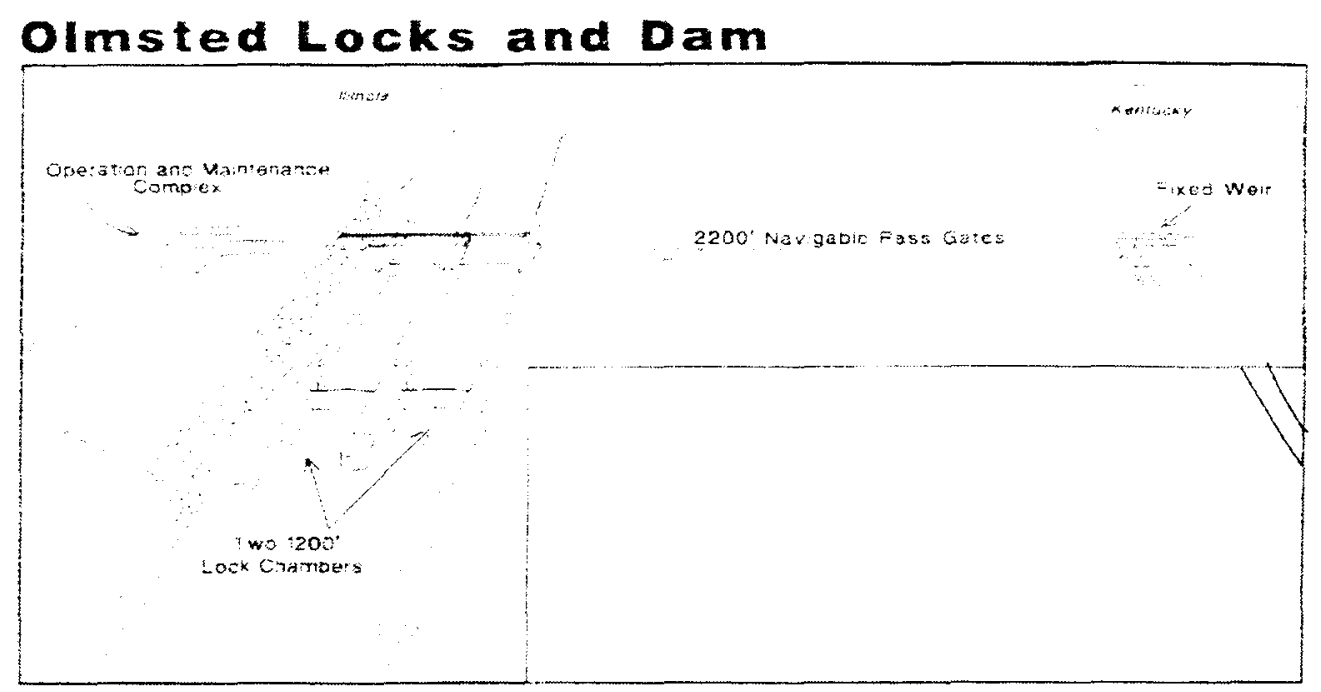

Figure 3. Olmsted Navigation Pass

The current operating proposal calls for the wicket gates to be raised in groups of five with five-gate gaps until 110 gates are raised. Flow over the top of any gate is not anticipated except during its raising or lowering operation. The five-gate gaps will then be closed by raising two gates simultaneously or one gate at a time. In each case, the end gate or gates will be moved. Figure 4 shows a three-gate gap flow configuration after the two end gates have been raised from a five-gate gap and the center gate in the three-gate gap is partially raised. To maintain a uniform flow pattern in the river, as many as 44 gates will be simultaneously and continuously operated in a manner that subjects the gates to hydraulic conditions that will create uneven hydraulic loading and may produce gate vibrations. In addition, during the raising or lowering operations, additional hydraulic conditions will occur that could also induce gate vibrations. A large amount of gate manipulation is expected during the low-flow periods when the gates will be in use to control the major powerhouse-controlled inflows into the Olmsted Reservoir which come from the Tennessee and Cumberland Rivers (Elder 1992).

\section{Purpose and advantages}

The purpose of this L\&D system is to replace the existing L\&D 52 and 53 and regulate navigable depths on the lower Ohio River. In the dry season, the wickets will be raised to maintain the required navigable depths from the Olmsted project upstream to Smithland L\&D. Approximately 60 percent of an average operational year river stages will be sufficient to provide navigable depths without control, and the wickets will be automatically lowered to lay flat 


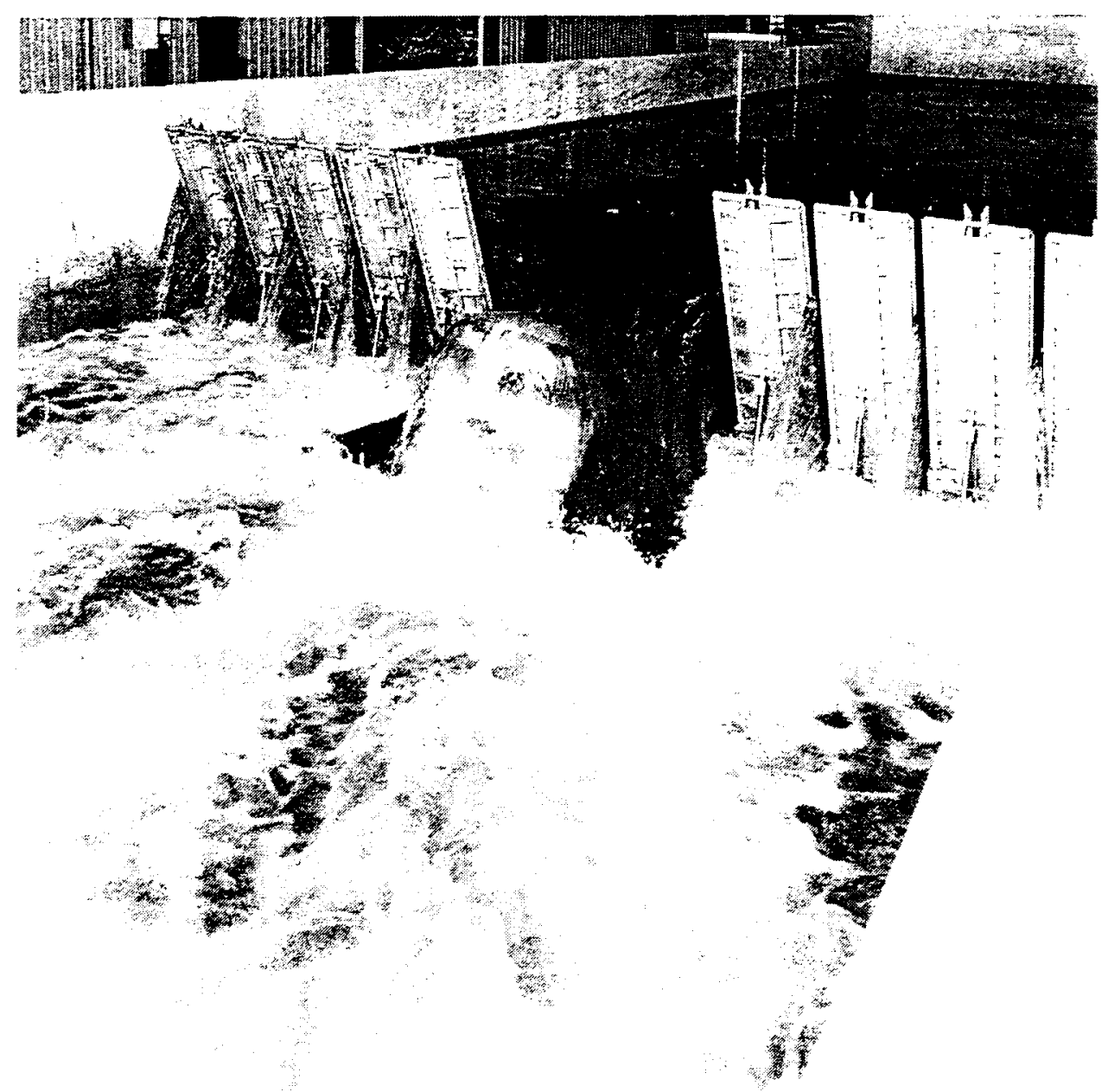

Figure 4. A three-gate gap flow condition in the 1:5 model

on the river bottom, permitting traffic to navigate over the dam without having to pass through the locks. This reduces delays experienced by locking through the system. Delays ultimately raise the price of commodities shipped through the waterways. Such operational advantages and reduction in maintenance and operational costs provided by the new system will substantially outweigh the replacement costs of these second-generation wickets.

The Corps of Engineers estimates that this project will produce average annual economic benefits to the nation of more than $\$ 600$ million. The new locks will operate more efficiently and will pass tows with fewer delays. Total lockage time will be reduced from $5 \mathrm{hr}$ through L\&D No. 52 and 53 to less than $1 \mathrm{hr}$ in the new project.

\section{Design and verification}

On a worldwide basis, the history of dams has shown that they have failed at the rate of about one every year for various reasons (U.S. Department of Interior, Bureau of Reclamation 1992). Recent partial failure of a spillway gate at 
Folsom Dam increased flows into the Lower American River from $6,500 \mathrm{cfs}$ to about $40,000 \mathrm{cfs}$. Lessons from such grievous events must be taken seriously to avoid costly rehabilitation and economic losses. The Olmsted L\&D replacement project cautiously advanced in its multistage design and development phases to ensure a robust design of the dam based on the most advanced engineering practices.

An interactive design practice was coordinated between the designers and the researchers during the prototype model development phase. The wicket design was continually updated based on recommendations provided by the researchers from WES. Experimental results from the physical model studies were used by the LRL to determine the primary size of the structural components (U.S. Army Engineer District (USAED), Louisville 1993). Constructional, operational, and functional difficulties encountered during the fabrication and operation of the physical models were properly taken care of, subsequently, by modifying the design of the gate. Moreover, this design verification process served to identify and address anticipated structural dynamic problems in physical models.

Implementation of corrective measures to modify an installed prototype facility, due to unacceptable performance, would be extremely expensive, time consuming, and damaging to the reputation of the Corps of Engineers (USAED, Louisville 1993). By using a physical model study for early identification of problems that might have potentially disturbing consequences, millions of dollars can be saved by reducing the uncertainty in an untested physical structure.

\section{Physical Model Studies}

The Olmsted Wicket Model studies began in January 1990 when USAED, Louisville, tasked WES with the development of a 1:25-scale curved gate model of the Olmsted L\&D. Since then, two additional models were developed: 1:25and 1:5-scale flat gates. These physical models have been used to investigate the flow-induced vibrational response of Olmsted Wicket Gates and have also helped researchers to better understand the complexities involved in the fluidstructure interaction problem affecting them. The Olmsted Wicket Model studies also includes a prototype test facility in the Smithland Dam on the Ohio River. This facility examined the physical performances of various mechanical/electrical/ hydraulic systems proposed for the Olmsted Dam.

\section{Prototype facility}

The Olmsted prototype hydraulic wickets were installed in an artificial channel constructed in the left bank of the Ohio River at Smithland, KY. An approach channel provided access to the Ohio River on the upstream side of the Smithland Dam, while a retreat channel drained water to the downstream side of the dam. Figure 5 shows a photograph of the prototype facility. Five wickets made of different steels and composite materials with several coatings of different nature were tested in this facility. The purpose of these experiments 


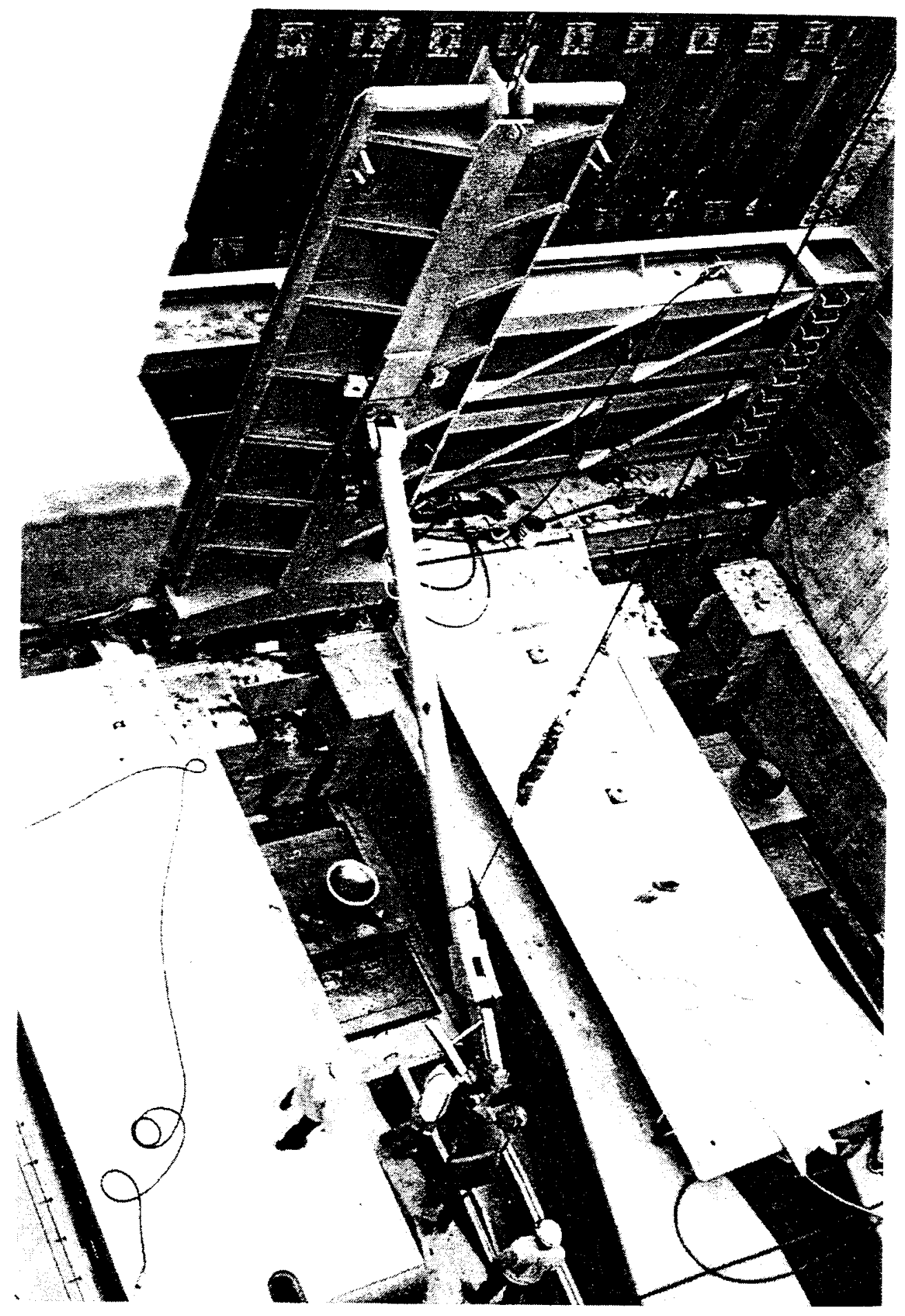

Figure 5. Prototype facility at Smithland Dam 
was to evaluate the long-term operational performance of the wickets under different service conditions. Data provide guidelines to the designers and builders to improve the design of the prototype system for efficient operation. Durability, serviceability, and reliability of various mechanical/electrical/ hydraulic components from several manufacturers were tested to identify the most efficient devices. The facility was also used to develop maintenance procedures for the actual dam by simulating the anticipated courses of action in the prototype facility. Selective structural responses on the prototype gate for available pool conditions were measured to validate the $1: 5$ physical models used by WES to study the flow-induced dynamic performance of the operational wickets (Chowdhury, Ross, and Hall 1997).

Regardless of the capability of the prototype facility, many of the flow conditions anticipated at the Olmsted Dam could not be simulated at the Smithland site. This is because the operational condition of the prototype is dependent upon the available pool elevations of the Smithland Dam. The inability of the prototype facility necessitated the initiation of the Olmsted L\&D physical scale model studies program at WES. A physical model in this regard can efficiently and effectively simulate the likely flow and operational conditions over the life span of the Olmsted Dam. In a regulated environment, the much debated dynamic uncertainty of the operational wickets can be systematically addressed and the design boundaries for mechanical components can be confidently identified.

\section{Scaled models}

The limitations of the prototype facility as mentioned in the previous section and the general concern of the gate design team about the gate performance prompted the gate advisory board to seek assistance of WES in developing physical scaled models of the Olmsted wicket. One of the major concerns of the gate design team was the severity of flow-induced dynamic uncertainty of the hydraulic wickets of unprecedented type (USAED, Louisville 1993). The design team's experience in Tainter gate design could not provide design information for the wickets. Prediction of flow-induced vibrational characters using an accurate model of the wickets was beyond the scope of the Corps design team's ability. A physical scaled model in this regard is ideal to simulate the dynamics of the operating conditions. Several physical models as outlined below were tested and investigated by WES engineers to better understand the gate dynamics in support of the Olmsted L\&D studies.

\section{1:25 curved gate}

A 1:25-scale model of the Olmsted Wicket Dam was constructed by WES, initially for the purpose of conducting scour studies (USAEWES 1989). The model was then modified to include an instrumented gate (USAEWES 1992) to allow the study of forces expected to occur in the lifting rod and on the hinges and to investigate designs developed to provide aeration to the nappe when the 
gate is being raised. In 1994, the gate was instrumented to obtain flow-induced vibration data (March and Elder 1992). In June 1993, Structural Dynamics Research Corporation (SDRC) performed a finite element (FE) analysis on the full-scale model of the gate. In August 1993, SDRC once again performed an FE analysis of the full-scale wicket gate and an additional FE analysis of the 1:25-scale Olmsted Wicket Gate (SDRC 1993a). Since then, WES has developed a numerical model of the Olmsted Wicket. The FE model for the curved gate was validated using results obtained from physical model studies. Detailed information on the numerical aspect of the Olmsted wicket modeling has been described (Seda-Sanabria 1994). The 1:25-scale hydraulic nonstructural curved gate model of the Olmsted wickets is shown in Figure 6.

The objective of the 1:25-scale hydraulic model was to predict the hydraulic response pattern of the prototype gate for various operating conditions. The 1:25-Froude-scaled model of the Olmsted Dam reproduced a 1,250-ft-wide portion of the L\&D. A total of 92 wicket gates were reproduced in the model, and one of them was instrumented.

The instrumented gate was subjected to a variety of dam configurations and flow conditions. The results obtained from these experiments revealed significant transient dynamic loads in the wicket gate hinges and lifting cylinder under certain operating conditions and positions of the gate.

One study identified the source of the transient dynamic loads that was observed during the experiments. This study suggests that an interaction of the first natural mode of vibration of the structure with the forcing frequency may have caused such disturbance (Seda-Sanabria 1994). Modification of the hydraulic conditions is recommended to eliminate any possible link between the pressure oscillations and the gate motion. From a structural point of view, the study suggested that particular attention should be given to the hydraulic device controlling the position of the gate.

The transient dynamic loads which have been observed in the 1:25-scale model have been of great concern throughout the model study. Several previous efforts have investigated the source of dynamic loads and their implications on the design and operation of the Olmsted Wicket Dam (USAEWES 1992; DeGroot 1992; Simpson and Solomon 1992; March and Elder 1992). Actual mode shape and frequency data have been obtained for the 1:25-scale WES model (Chowdhury, Hall, and Pesantes 1997), and computer generated mode shape and frequency data from FE analysis on both full-scale and 1:25-scale models (SDRC 1993a), respectively.

Major DeGroot reviewed data from the 1:25 Olmsted Wicket Dam model during June 1992. This review investigated the role of the hydraulic cylinder and its control system as a source of the observed dynamic loads, including play in the rack and pinion drive for the linear motor, stick-slip friction in the hydraulic cylinder, and sensitivity of the feedback control. DeGroot's review also recognized the role of reduced pressures beneath the gate in increasing the gate loads, emphasized the need for nappe aeration to reduce dynamic loads, and discussed alternatives for providing nappe aeration (DeGroot 1992).

Chapter 1 Introduction 

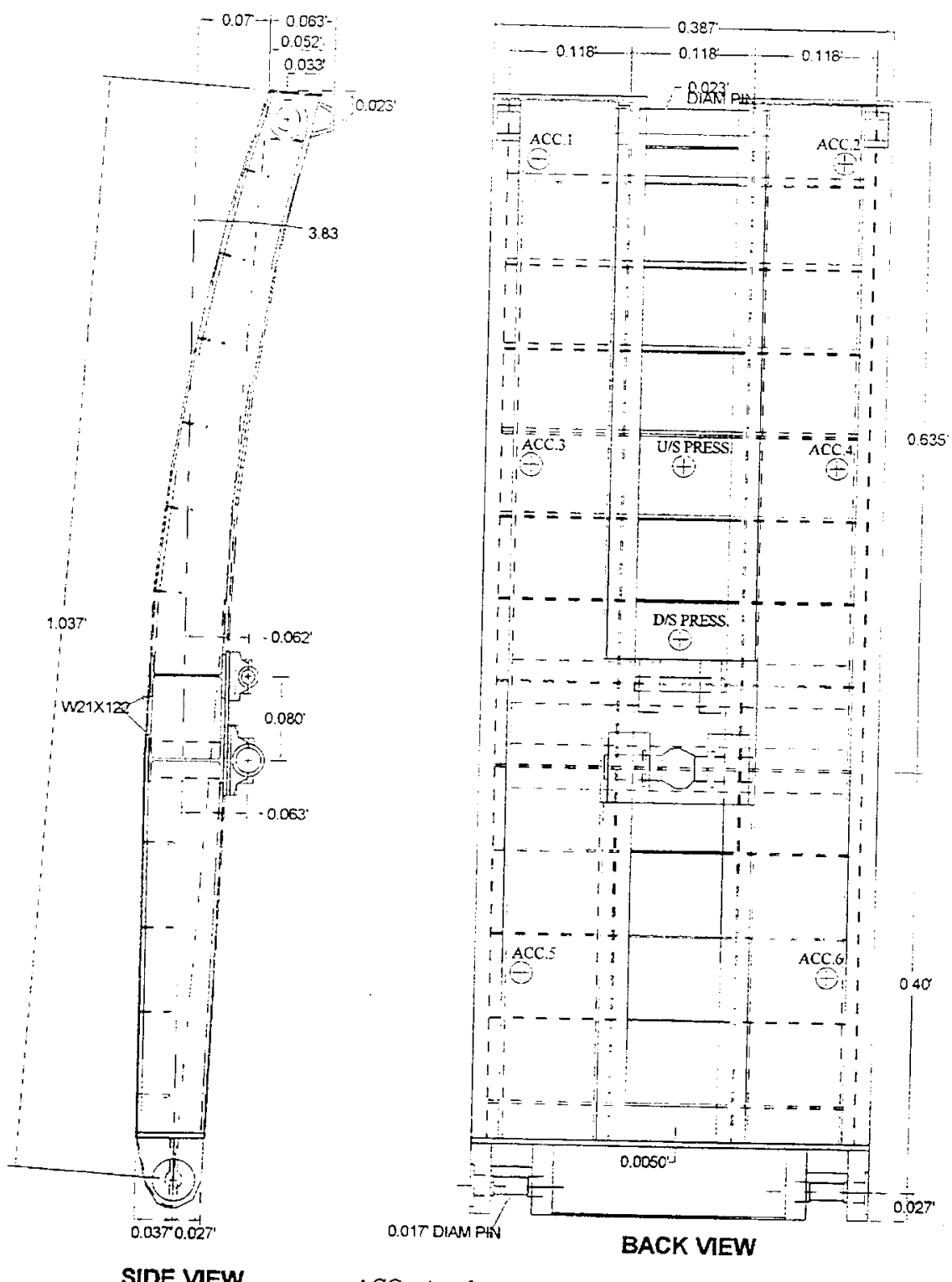

$\mathrm{ACC}=$ Accelerometer

U/S PRESS.=Upstream Side Pressure Transducer

D/S PRESS.=Downstream Side Pressure Transducer

Figure 6. 1:25-scale Olmsted curved gate model

Staff members from SDRC inspected the 1:25-scale Olmsted Wicket Dam model during July 1992, reviewed the model results, and discussed WES testing and data analysis activities. SDRC noted the lack of appropriate structural scaling in the 1:25 model; commented on the cavity beneath model; commented on the low $(40-\mathrm{Hz})$ data sampling rate; suggested an "enhanced" 1:25-scale structural model because the existing 1:25 model was not designed for structural similitude; recommended against a larger hydroelastic model; and recommended 
FE analyses and detailed full-scale prototype testing (Simpson and Solomon 1992).

Consulting Hydraulic Engineers Rex Elder and Patrick March helped in interpreting the hydraulic aspects of the WES work. After analyzing the hydraulic data of the 1:25-scale model at WES, it was determined that both static and dynamic loads in the model appear to be relatively insensitive to gross approach flow patterns. Development of a satisfactory gate design should, in their opinion, proceed in the following manner: upgrade the 1:25-scale WES model to include a more detailed prototype geometry, specifically in the vicinity of the hydraulic cylinder, and continue to develop hydraulic data from the model; develop an intermediate model, of the largest practical size, for vibration and operation studies; develop a full-scale facility; develop FE models of the gate dynamics for use in conjunction with results from the physical models (March and Elder 1992).

\section{1:25 flat gate}

Considering the recommendations provided for the 1:25-scale curved gate model study, a flat-gate geometry of the wicket was selected for further investigation (Figure 7). The geometry of the curved gate was modified to mitigate oscillation and the "bouncing" of the gate upon its hydraulic system, resulting from aeration of the nappe (USAED, Louisville 1993). For this model, a total of 92 ticket gates were reproduced, and one of them was instrumented.

The flow-induced vibrational response of the 1:25 Olmsted Flat Gate was analyzed for three different river models: the Olmsted Dam, the prototype test facility at Smithland Dam, and the 1:25-scale flume configuration. The Olmsted model simulated the open-channel river flow conditions of the Olmsted Navigation Pass (dam) in the Ohio River, whereas the Smithland analysis represented the approach channel conditions of the prototype gate facility. The main objective of the first two studies was to determine any variations in the flow-induced vibrational characteristics of the gate with and without the approach channel for equivalent Smithland Dam navigation pool conditions relative to the Olmsted Dam pool elevations. The 1:25 model of the flume configuration placed in the 1:25 flat gate model was used to determine the effectiveness of partial river modeling for the intermediate scale model. Another purpose of this study was to compare the experimental results of the flat gate with those of the curved gate and determine the effects that geometry changes reflect in the dynamics of the gate.

During the 1:25 flat gate model tests, time-dependent drift of the mechanical and electrical devices introduced an error in the measurement process. This error was particularly noticeable when experiments were conducted for a long period of time. The signal-to-noise ratio for the shaft load was very low compared to the other channels used for the data-acquisition system. As a result, the shaft load record for the 1:25 model was contaminated by harmonic spikes more than any other channels. 

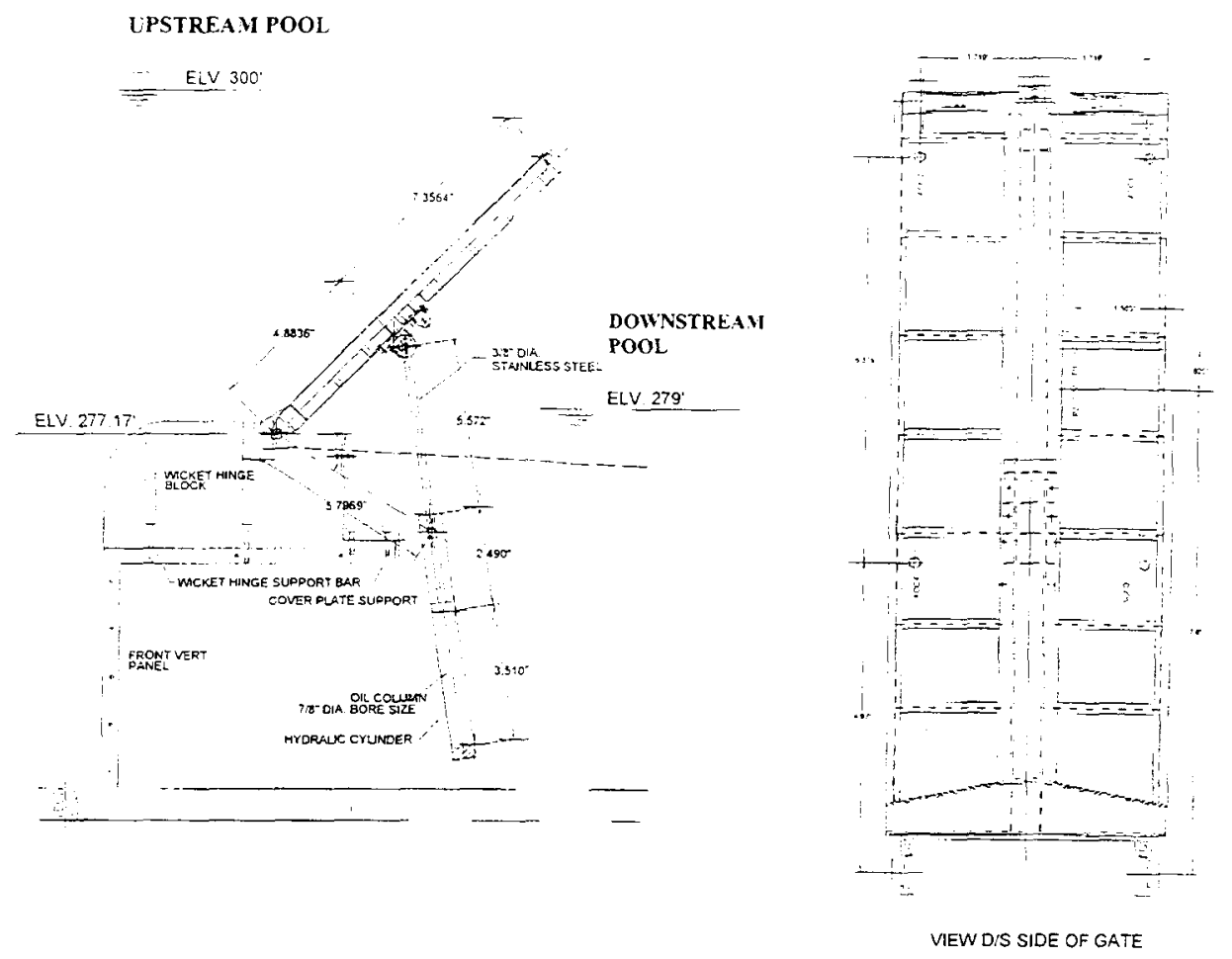

Figure 7. 1:25-scale Olmsted flat gate model

This study found that the pressure, accelerations, and shaft loads for the 1:25scale flat gate, with and without approach channel, did not significantly differ from each other. However, this was not the case for the vertical hinge reactions, which varied significantly. This behavior was believed to be an error introduced by the time-dependent drift discussed above as opposed to a structural response problem. These conclusions were based on observations of the time-domain data of the gate corresponding to four different fixed positions $(-2,30,50$, and $60 \mathrm{deg}$ ). No consistent load variation was observed among the experimental results when the gate was operating around its intermediate position, between the horizontal ( $-2 \mathrm{deg})$ and fully raised $(65 \mathrm{deg})$ positions. This inconsistency was due to the turbulence in the flow while breaking the air gap in the downstream side.

The 1:25 curved gate model consistently provided higher reaction forces than those of the flat gate for identical gate orientations and flow configurations. Curved gate responses were more widely dispersed about their respective means than those of the flat gate. These observations were made based on the flowinduced test results for both 1:25-scale models. As expected, the geometry changes in the flat gate model did change the natural dynamic characteristics of the wicket gate from those of the curved model (Chowdhury, Hall, and Pesantes 1997).

Notice that the 1:25 flat gate was not a fully scaled-down model since its overall dimensions were limited to the availability of the scaled dimensions of 
the material. Although it reproduced the general dimensions of the prototype gate, it was not a true-elastic model since an exact scaling of the thicknesses and construction details was not physically attainable. Brass was used in the 1:25 model instead of steel, which is the prototype material, and the overall dimensions were scaled as practical as possible. Thus, this model should give us an idea of the dynamic behavior of the wickets, but it was not intended to simulate its elastic relationships.

The time- and frequency-domain analysis of the 1:25-scale flat gate data revealed the general dynamic behavior of the operating wickets. Design load boundaries for different flow and gate configurations were identified. The modal parameters for the dry gate were extracted using experimental modal analysis. Dynamic information obtained from this will be compared with the intermediate scale data for verifying the validity of the similitude models as discussed later.

\section{Intermediate scale (1:5)}

Considering the limitations involved in the construction of a prototype facility (i.e., operational inconveniences, potentially excessive modification costs, nature dependency, etc.), and the inadequacy of the 1:25-scale flat gate model in representing the structural similarity of the prototype wicket, it was decided to study the behavior of an intermediate-scale wicket in an easily controlled environment. Thus, a moderate-scale of one-fifth of the prototype geometry was considered for further investigation. The 1:5-scale flat gate model was capable of simulating some of the structural as well as the hydraulic responses of the prototype gate. An intermediate-scale model was selected such that it was neither too small to reproduce the prototype geometric details nor too large to manage and handle as an uneconomical model. For the Olmsted model, selection of a relatively larger-scale model would have tremendously increased the project cost to maintain the simulated (required) flow in the flume. For practical considerations, a 1:5-scale model, therefore, was an effective model of the Olmsted wickets. The 1:5 flat gate model, shown in Figures 4 and 8, was geometrically and dynamically a similitude model. In contrast to the previous 1:25-scale model, its geometrical characteristics simulated those found on the

prototype structure. Since this is a similar-elastic model, all relationships can be scaled up to estimate those expected in the prototype gate.

\section{Objectives}

The objectives of the 1:5-scale flat gate model investigation in support of the Olmsted model studies program are to:

a. Simulate structural and hydraulic behavior of the prototype gate using a physical scaled model.

b. Validate the effectiveness of the similitude model. 


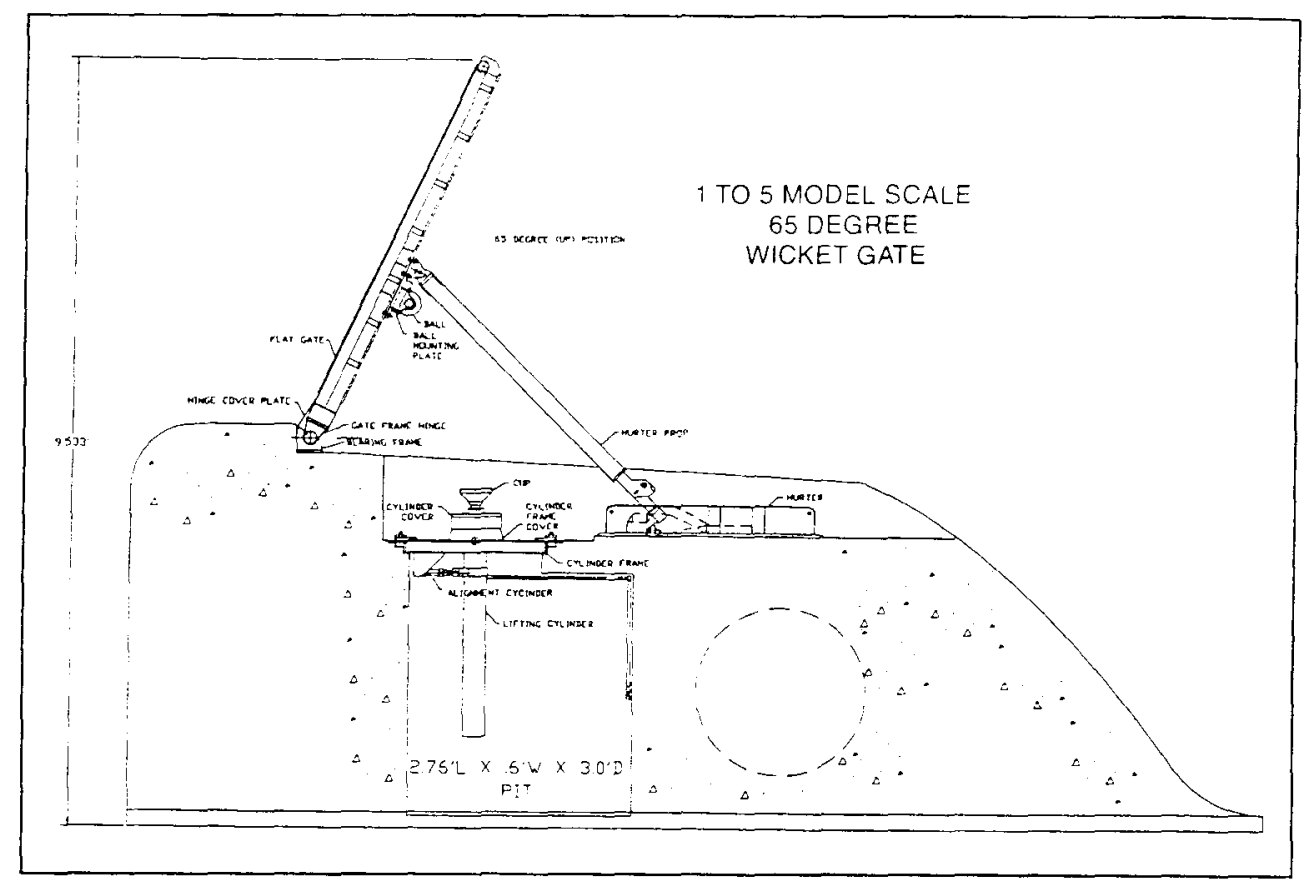

Figure 8. 1:5-scale Olmsted flat gate model

c. Predict and investigate the response pattern for the prototype gate for different flow and operating conditions.

d. Locate sources for any possible vibration-related structural problems and suggest methods to improve the structural performance.

e. Extract the boundaries of design loads for the likely service load conditions.

f. Determine (experimentally) various dynamic physical parameters for the development of an updated FE model of the wicket.

\section{Scope}

The initial portion of the project involved the development of an intermediate 1:5-scale model capable of simulating the structural and hydraulic responses of the prototype wickets. During the construction process, all intricate details of the prototype gate geometry, including hinges, props, cylinders, and hurter (the supporting device for the prop), were reproduced and the materials were kept identical to attain the geometric similarity for the constructed model. Scaling relationships of basic physical parameters between the model and the prototype gate were established using the dynamic similitude laws. Unacceptable performance experienced during the course of the model study program - from 
fabrication to operations - were studied thoroughly to determine the sources and the consequences of the defects. Once the defects were identified, proper modifications were implemented in the system to ensure reliability, increase productivity, and avoid costly adjustments during fabrication, installation, and operation of the prototype wicket.

A fully instrumented gate was installed in the sill to measure the dynamic responses of the model due to various operational sequences. Gate responses to be measured included the pressures on the upstream and downstream faces, accelerations at different locations, and reaction forces at the supports. Several flow-induced experiments were conducted under various flow and operating conditions using the on-board data-acquisition system. Operating conditions were scheduled for conditions expected during normal operation of the Olmsted Dam.

Modal analysis using the state-of-the-art Scanning Laser Doppler Vibrometer (SLDV) was performed for the dry and operating gate. Modal experiments were used to extract the natural vibrational characteristics of the wicket model. Timeand frequency-domain operating deflected shapes (ODS) were extracted for the operating gate for different flow configurations. Wet gate modal parameters were obtained by exciting the operational wicket gate with a shaker and scanning the downstream surface of the gate. Net pressure distribution on the gate surface, the reaction forces, and dynamic responses for different flow and channel configurations were measured. Based on this information, a dynamic evaluation procedure was established to measure the structural dynamic performance of the operational wickets.

Time-domain responses were summarized to estimate the maximum likelihood of design parameters for the gate. Frequency-domain responses were analyzed to obtain information regarding the frequency contents of the flow and the flow-induced structural motion of the wicket. The characteristic mode shapes for the dry and the wet gate (operating condition) were compared to measure the effects of the added mass on the gate dynamics. Parameters needed to develop and update the analytical FE models were identified. 


\section{Fabrication, Construction, Installation, and Operation}

\section{Model Description}

The 1:5-scale model reproduced a 120 -ft-wide section of the spillway crest and stilling basin with topography reproduced approximately $300 \mathrm{ft}$ upstream and downstream from the axis on the dam. The 120 - $\mathrm{ft}$-wide section of the spillway crest included 12 wicket gates with one of the gates (the insrumented gate) reproducing every intricate geometrical detail of the prototype gate (including the materials), the prop rod, the hydraulic lifting cylinder, and hurter. The instrumented gate was positioned in the flume as the sixth gate from the left wall of the flume when looking downstream. Strain gauges, pressure transducers, and accelerometers were installed on the gate and gate components to measure forces exerted on the gate hinges, prop rod, cylinder trunnion and cylinder lift point, pressures on the upstream and downstream face of the gate, and accelerations at selected points on the gate. The instruments used and their functions are presented in detail in Chapter 3 of this report. The other 11 gates, referred to as dummy gates, reproduced the overall geometry of the prototype gates to properly simulate the various flow conditions required for the physical experiments. A general plan and profile of the model and a more detailed sketch of the spillway crest and wicket gate components are provided in Figures 9 and 10 , respectively. Dry bed photographs of the model are shown in Figures 11a through 11 .

The lifting and supporting devices of the instrumented gate are shown in Figures 10 and $11 \mathrm{~d}$. The hydraulic lifting cylinder was used to raise and lower the gate during its operating cycles at constant preset rates. The hydraulic system consisted of a primary and an alignment cylinder. A cup on the end of the primary cylinder piston rod provided a nonrigid bearing connection when coupled with a ball mounted to the back of the gate, therefore the piston rod could be retracted when the gate was being supported by the prop rod. One end of the prop rod is pin connected to the gate and the other end rests on the hurter (Figure 10). There are two one-way tracks in the hurter (Figure 11e) which provide the sliding paths for the prop rod end-blade during raising and lowering cycles. During a raising cycle, the primary cylinder was extended, thereby rotating the gate through an arc from -3 to $68 \mathrm{deg}$, then stopped and retracted and the load from the gate was transferred to the prop rod at the 65-deg position 

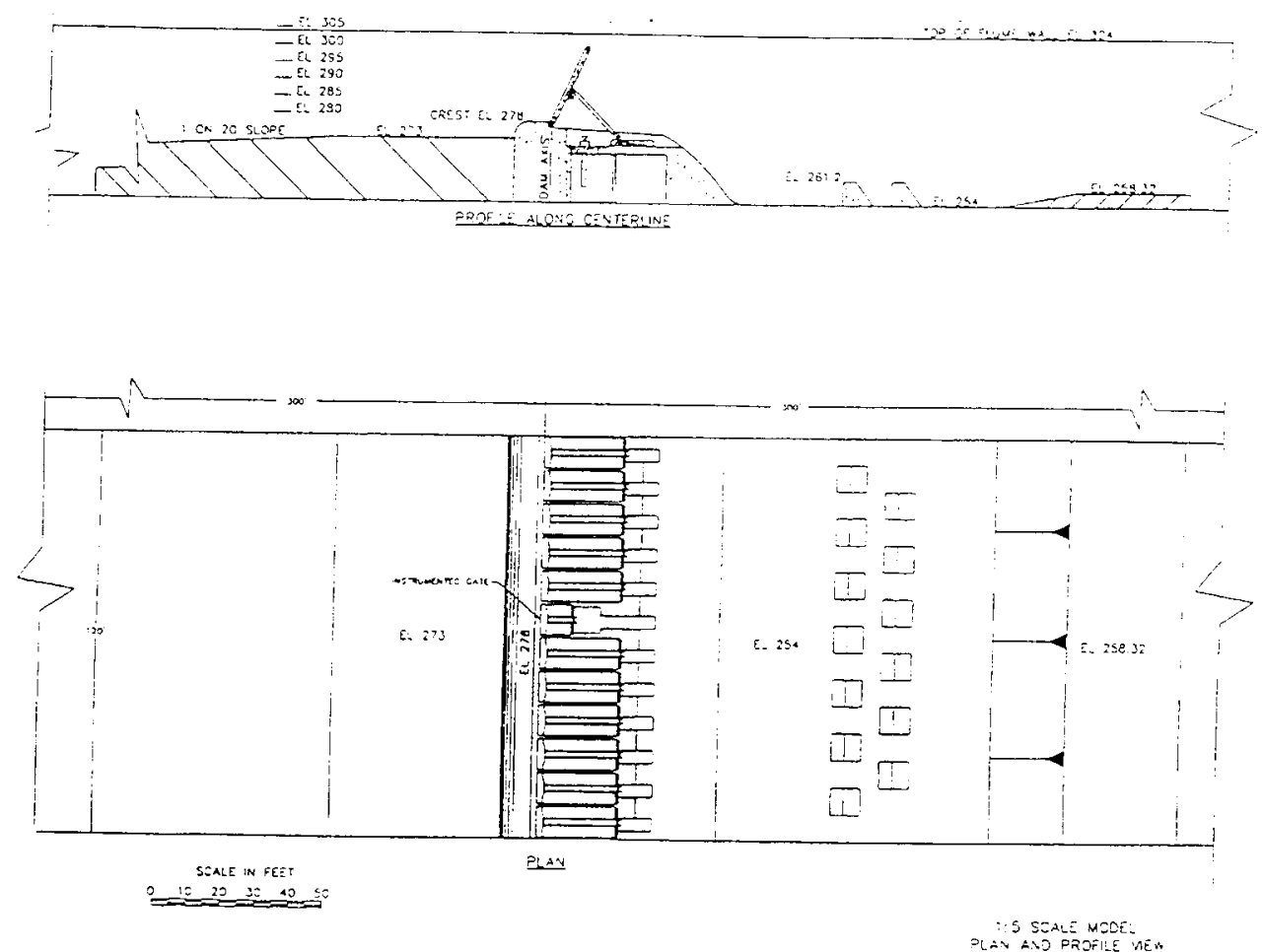

Figure 9. Plan and profile view of 1:5-scale Olmsted wicket model

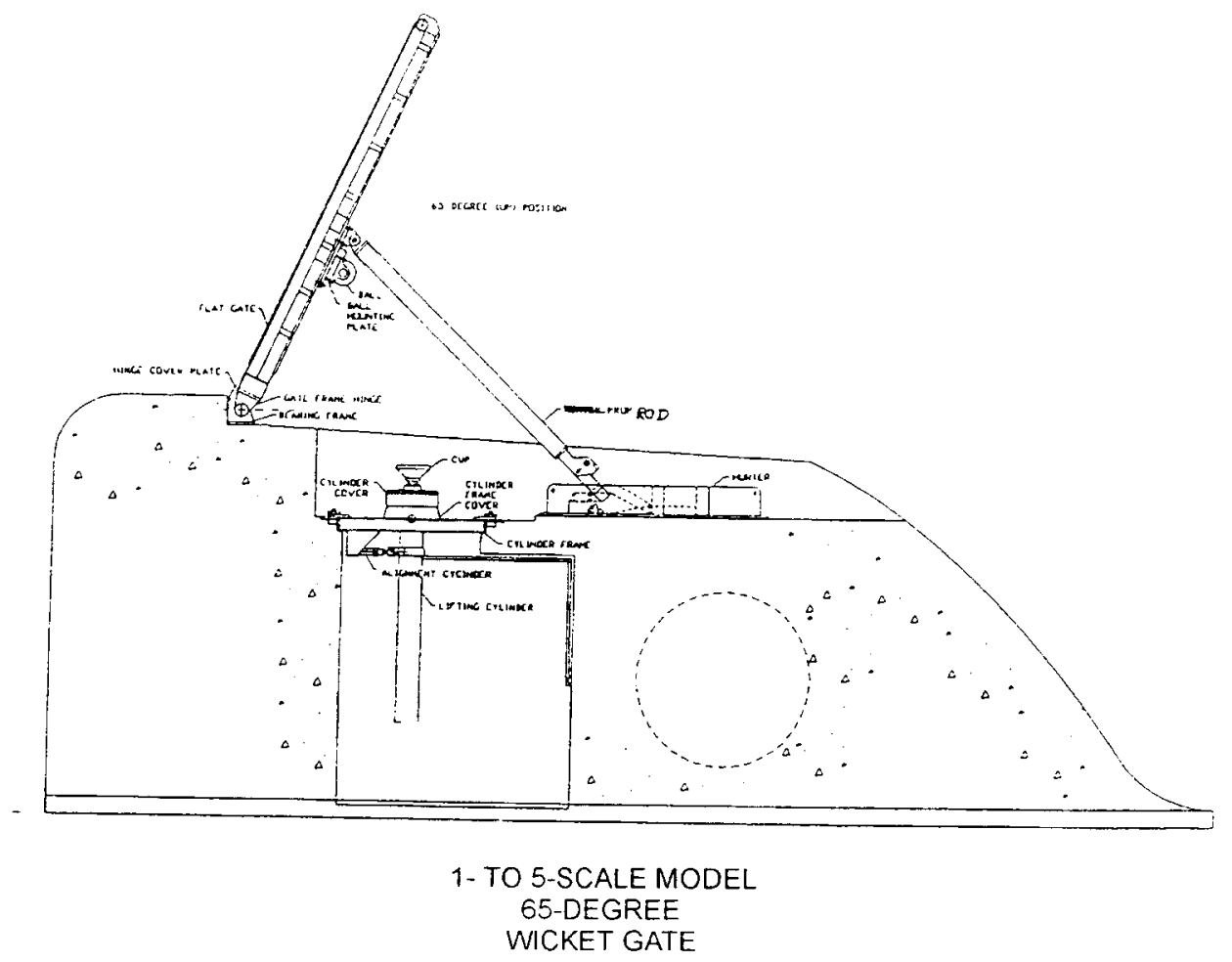

Figure 10. A section of Olmsted spillway crest 


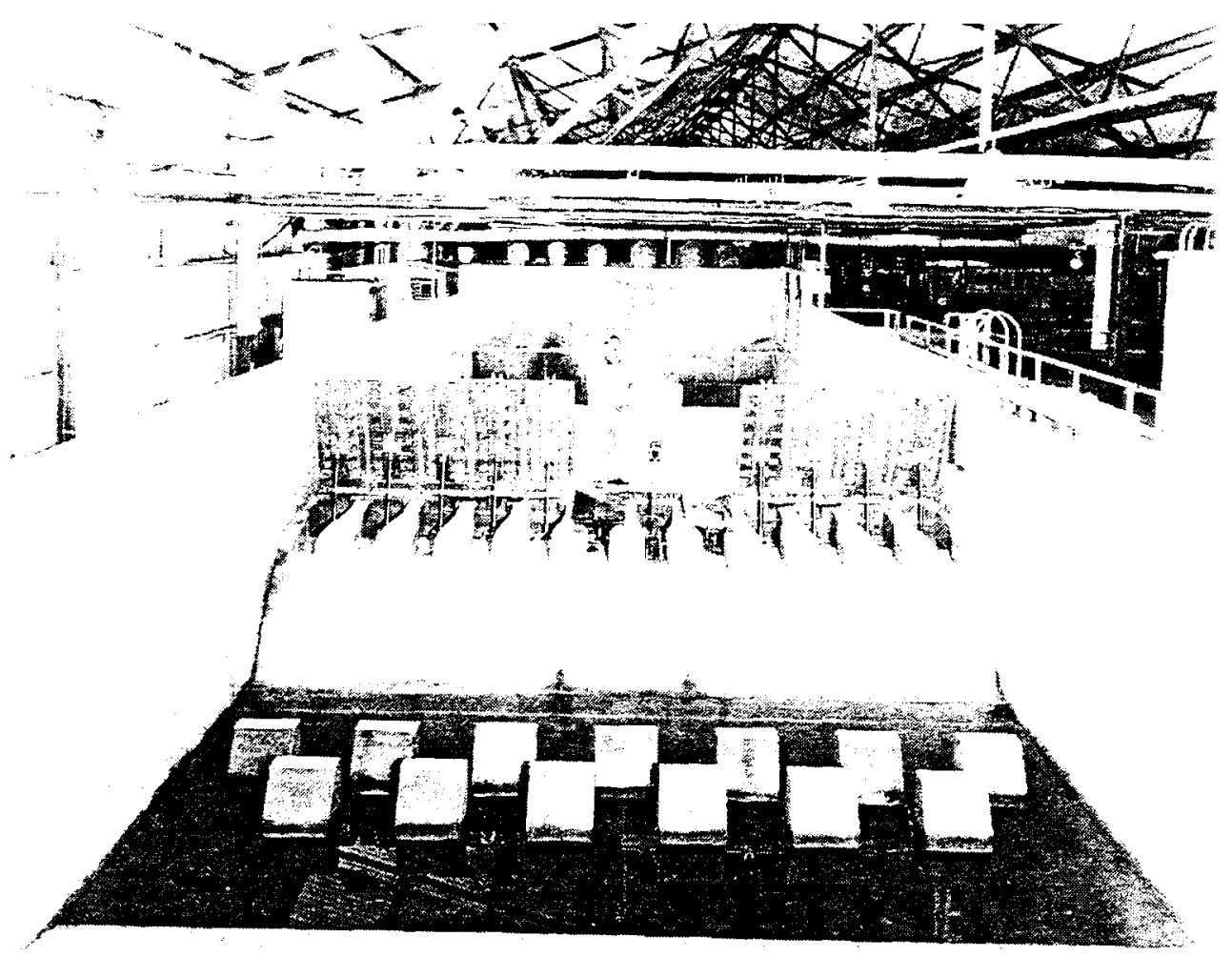

Tax

a. Dry bed looking upstream, general view

Figure 11. Dry bed views of 1:5-scale model (Sheet 1 of 5)

where the prop rod locks in the hurter. During a lowering cycle, the primary cylinder was extended while the alignment cylinder was used to rotate the primary cylinder to match the cylinder cup with the ball mounted to the back of the gate. The gate was then rotated from 65 to $70 \mathrm{deg}$ where the prop rod was disengaged and the primary cylinder was retracted until the gate came to rest at a -3 deg position. 


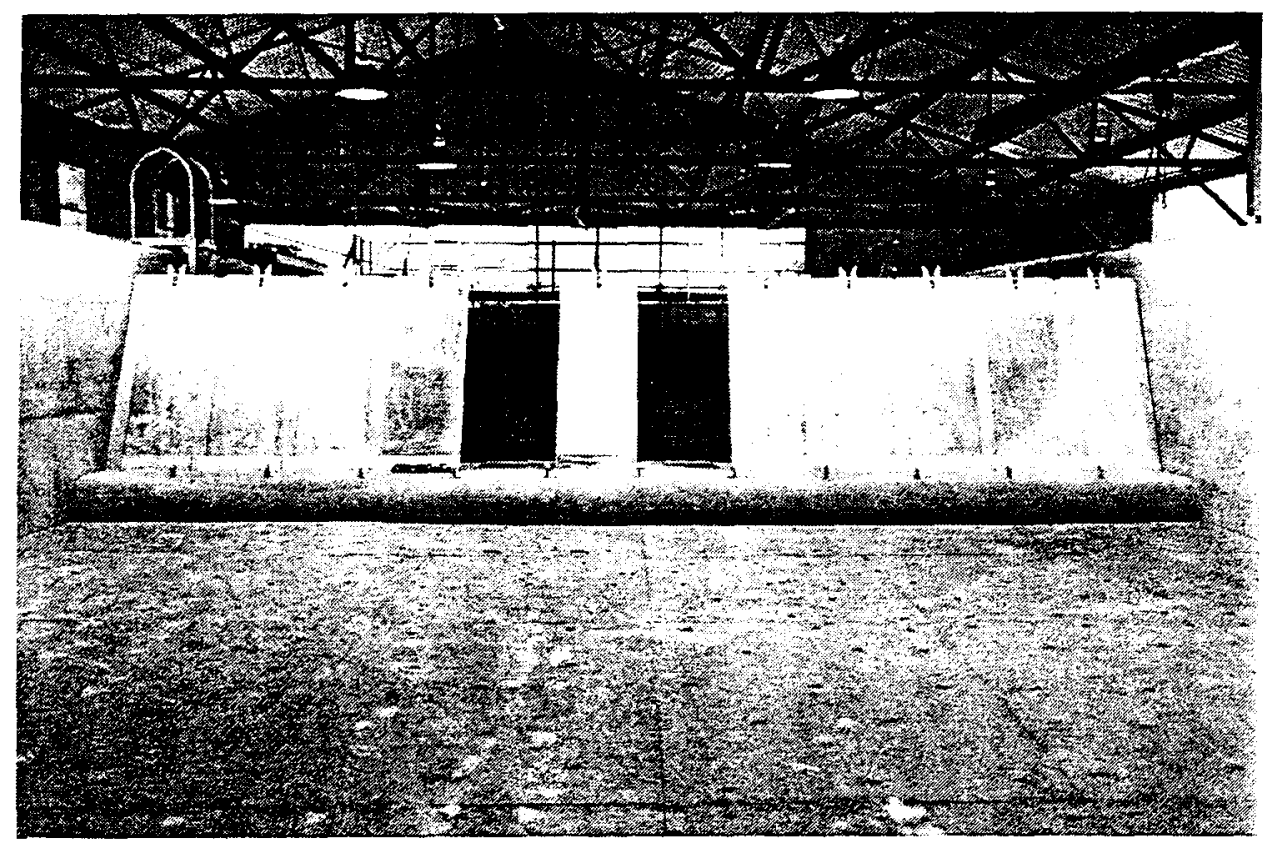

b. Dry bed looking downstream, general veiw

Figure 11. (Sheet 2 of 5 )

\section{Model appurtenances}

Due to the extraordinarily large discharge capacity required to simulate proper river flow conditions, a test facility was constructed for the model. Water used in the operation of the model was supplied by a recirculating system with a discharge capacity of $200 \mathrm{cfs}$ model discharge. Headwater and tailwater elevations were maintained at the desired level by means of an automated sideweir and tailgate, respectively. A detailed sketch of the model test facility is shown in Figure 12. Figure 13 shows the six axial flow pumps and sump used to supply water through the facility. The large pump motors are rated at $300 \mathrm{hp}$ each while the small pump motor are rated at $100 \mathrm{hp} \mathrm{each}$. Commercial pressure transducers were used to monitor pool levels. Hydraulic cylinders were used to raise and lower the dummy gates to achieve the various gate arrangements and flow conditions. Different designs, along with various flow conditions, were recorded photographically.

\section{Materials}

Materials and fabrication details for one of the five prototype wickets are reproducted in the instrumented gate, since each prototype gate possessed a unique material construction. Three types of materials, namely A572 grade 50, A588 grade 50, and fiber-reinforced composite were used for construction of prototype wickets. A588 grade 50 type steel (or its equivalent) was selected for use in the 1:5 model. A588 grade 50 is a high-strength low-alloy structural steel suitable for outdoor structures such as the wicket where savings in weight and added durability 


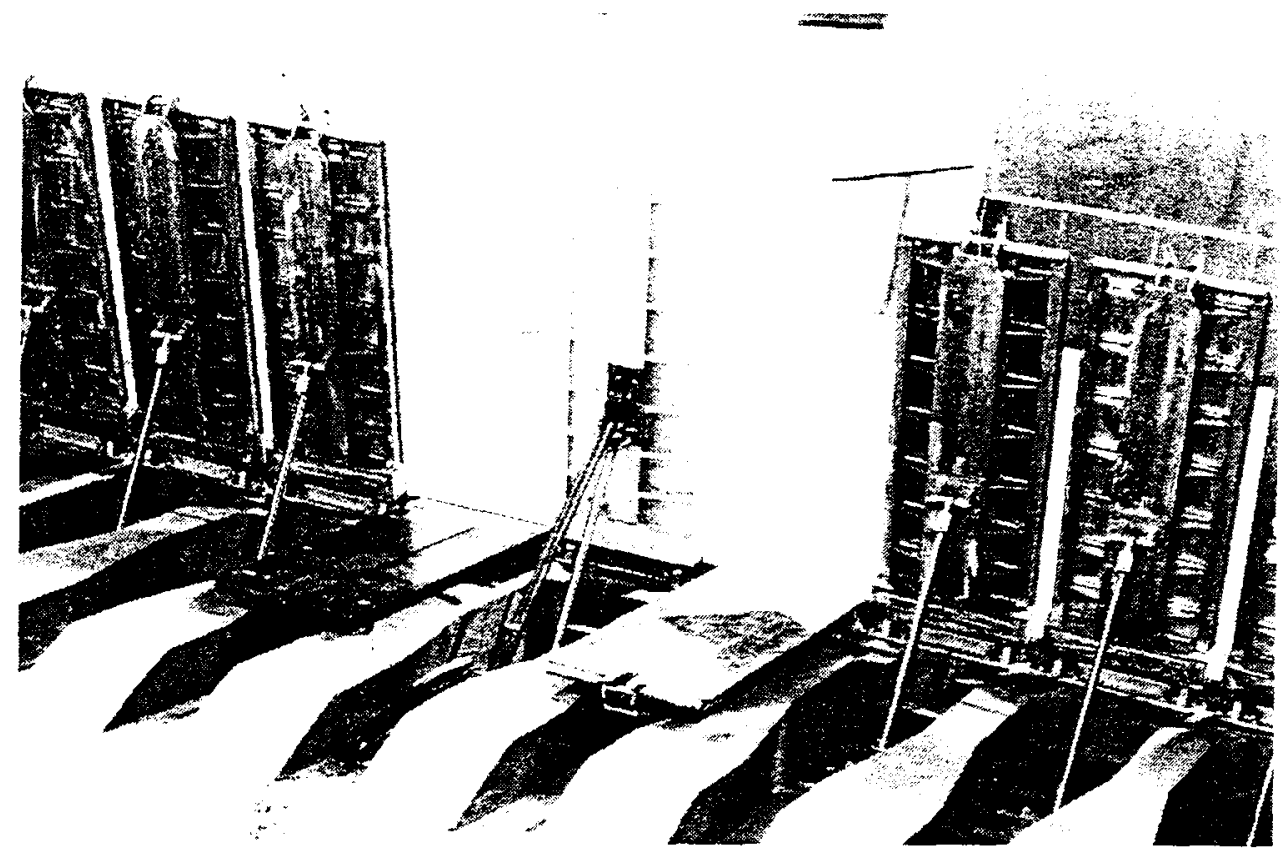

c. Dry bed looking upstream at back of dam with gate at 70 deg

Figure 11. (Sheet 3 of 5 )

were important (American Society of Testing and Materials (ASTM) 1982). Its excellent atmospheric corrosion resistance provides additional protection for the gate during the service condition, and good weldability eased construction of the gate components.

Similar grade steel was used for the corresponding structural components in both prototype and scaled models, unless the specified material could not be located in the national market. In an extensive market search, it was found that the $1 / 5^{\text {th }}$-scale plates for the model with respect to the prototype size were not manufactured by U.S. steel manufacturers. An equivalent grade steel, ASTM A606, thin sheet was used instead of A588 grade 50 steel prescribed for the prototype plate. Manufacturer's mill-certificate and ASTM specifications independently show that ASTM 606 products are rolled to meet the same physical and chemical requirements as ASTM A588. The substitute steel, however, has a higher ductility than that of A588 steel (ASTM 1982). Choice of this different grade steel does not reduce the effectiveness of the similitude model since the elastic properties do not differ between the prototype and the test model.

Moreover, the dynamic stress in the model under operating conditions is so low that the response predicted from the model becomes independent of the limit of yield strength, provided the elastic modulus remains unchanged. As will be discussed in Chapter 4 of this report, the performance of the similitude model in accurately predicting the prototype response depends primarily on the geometric similarity and the establishment of proper similitude relationships and not the elastic material properties of the gates.

Material specifications for structural units including hurter, prop rod, and hydraulic cylinder mounting devices were kept identical as in the prototype. 


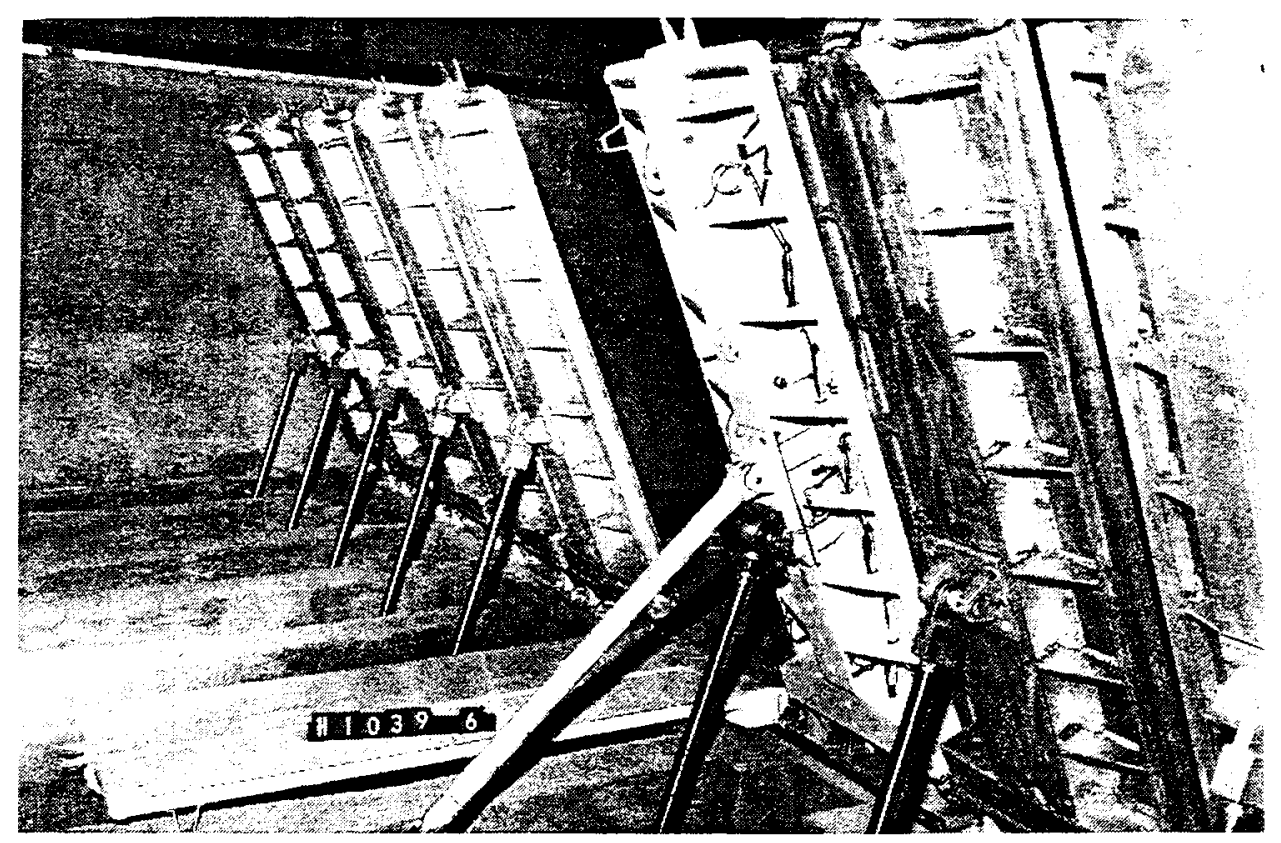

c. Dry bed looking across downstream side of wickets with instrumented gate at $65 \mathrm{deg}$

Figure 11. (Sheet 4 of 5)

Table 1 shows the elastic properties of major structural components used for the prototype and the test model. In this table, the second column presents the design drawing sheet numbers corresponding to each component mentioned in the first column. Prototype design drawings for the Olmsted wicket are enclosed in Appendix A. Elastic properties for the prototype materials presented in the table are obtained from the ASTM specification. Tensile strength tests were conducted for each batch of materials procured for the test model. Tension test results for each component are also shown in the table. All material tests were conducted in the Concrete Materials Division, Structures Laboratory (SL), using ASTM E8 specifications (Derucher and Heins 1981).

Types of stainless steel for major components used for the model had been matched with the prototype requirements. Two different types of stainless steel, namely Types 316 and 410 , were used for the structural components as prescribed in the Olmsted prototype drawings. Type 316 , a molybdenum-bearing, chromium nickel austenitic stainless steel provides better corrosion resistance. Type 410 is a general-purpose martensitic stainless steel with high strength and resistance to erosion and abrasion, although it is less corrosion resistant than the 300 series grade. In the model, the bearing frame was fabricated with A276 Type 316 steel and the embedded frames were made of A36 steel. Stainless steel was used to fabricate the hydralic cylinder system for the model. The material selected for the hydraulic cylinder achieved the similitude relationship for the frequency of the 1:5-scale hydraulic system. 


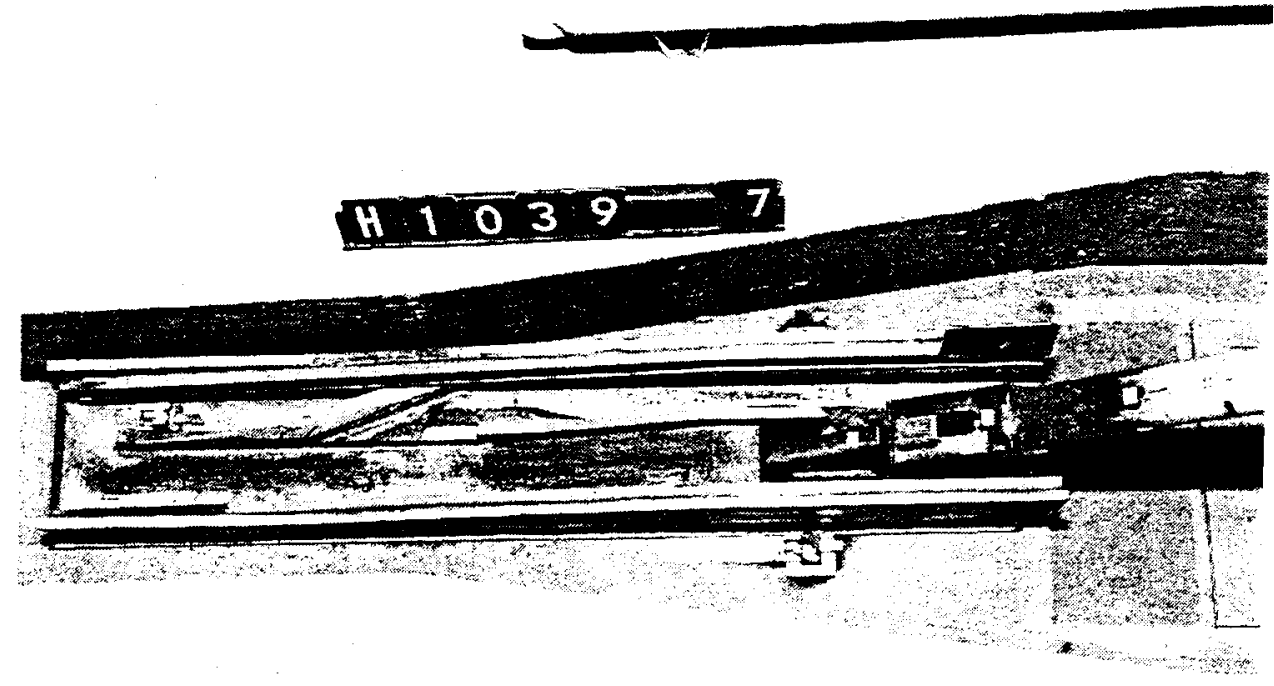

e. Locked-in prop end on hurter recess

Figure 11. (Sheet 5 of 5)

\begin{tabular}{|c|c|c|c|c|c|c|}
\hline \multirow[t]{2}{*}{$\begin{array}{l}\text { Component } \\
\text { Description }\end{array}$} & \multirow[t]{2}{*}{$\begin{array}{l}\text { Reference } \\
\text { Sheet \# }\end{array}$} & \multicolumn{2}{|c|}{ Material Type } & \multirow{2}{*}{$\begin{array}{l}\text { Young's } \\
\text { Modulus, } \\
\text { psi } \\
\text { Model } \\
\end{array}$} & \multicolumn{2}{|c|}{ Yield Strength, psi } \\
\hline & & Prototype & Model & & Prototype & Model \\
\hline Skin plate & S-24, S-25 & A 588 Gr 50 & A 606 & 40,974 & 50,000 & 64,521 \\
\hline Beam & S-24, S-25 & A 588 Gr 50 & A588 Gr 50 & 32.213 & 50,000 & 55,956 \\
\hline Channel & S-24 & A 588 Gr 50 & A588 Gr 50 & 32,213 & 50,000 & 55,956 \\
\hline $\begin{array}{l}\text { Stiffener } \\
\text { plates }\end{array}$ & S-24 & A $514 \mathrm{Gr} 70$ & A514 Gr 70 & 42,083 & 70,000 & 61,216 \\
\hline Web stiffener & S-24 & A $514 \mathrm{Gr} 70$ & A 514 Gr 70 & 32.480 & 70,000 & 65,469 \\
\hline $\begin{array}{l}\text { Gate frame } \\
\text { hinge pins }\end{array}$ & $M-8$ & $\begin{array}{l}\text { A } 276 \\
\text { Stainless } \\
\text { Steel } \\
\end{array}$ & $\begin{array}{l}\text { A } 276 \\
\text { Stainless } \\
\text { Steel } \\
\end{array}$ & 30,850 & 40,000 & 50,676 \\
\hline $\begin{array}{l}\text { Gate frame } \\
\text { hinge }\end{array}$ & $M-8$ & A 588 Gr 50 & A 588 Gr 50 & 35,658 & 50,000 & 52,128 \\
\hline $\begin{array}{l}\text { Ball mounting } \\
\text { plate }\end{array}$ & $M-15$ & $\begin{array}{l}\text { A } 276 \\
\text { Stainiess } \\
\text { Steel }\end{array}$ & $\begin{array}{l}\text { A } 276 \\
\text { Stainless } \\
\text { Steel } \\
\end{array}$ & 30,850 & 40,000 & 50,676 \\
\hline Prop rod & $\mathrm{M}-18$ & A 588 Gr 50 & A588 Gr 50 & 30,737 & 50,000 & 64,940 \\
\hline Hurter & $M-25-A$ & $\begin{array}{l}\text { A } 276 \\
\text { Stainless } \\
\text { Steel } \\
\end{array}$ & $\begin{array}{l}\text { A 276 } \\
\text { Stainless } \\
\text { Steel } \\
\end{array}$ & 30,850 & 40,000 & 50,676 \\
\hline
\end{tabular}



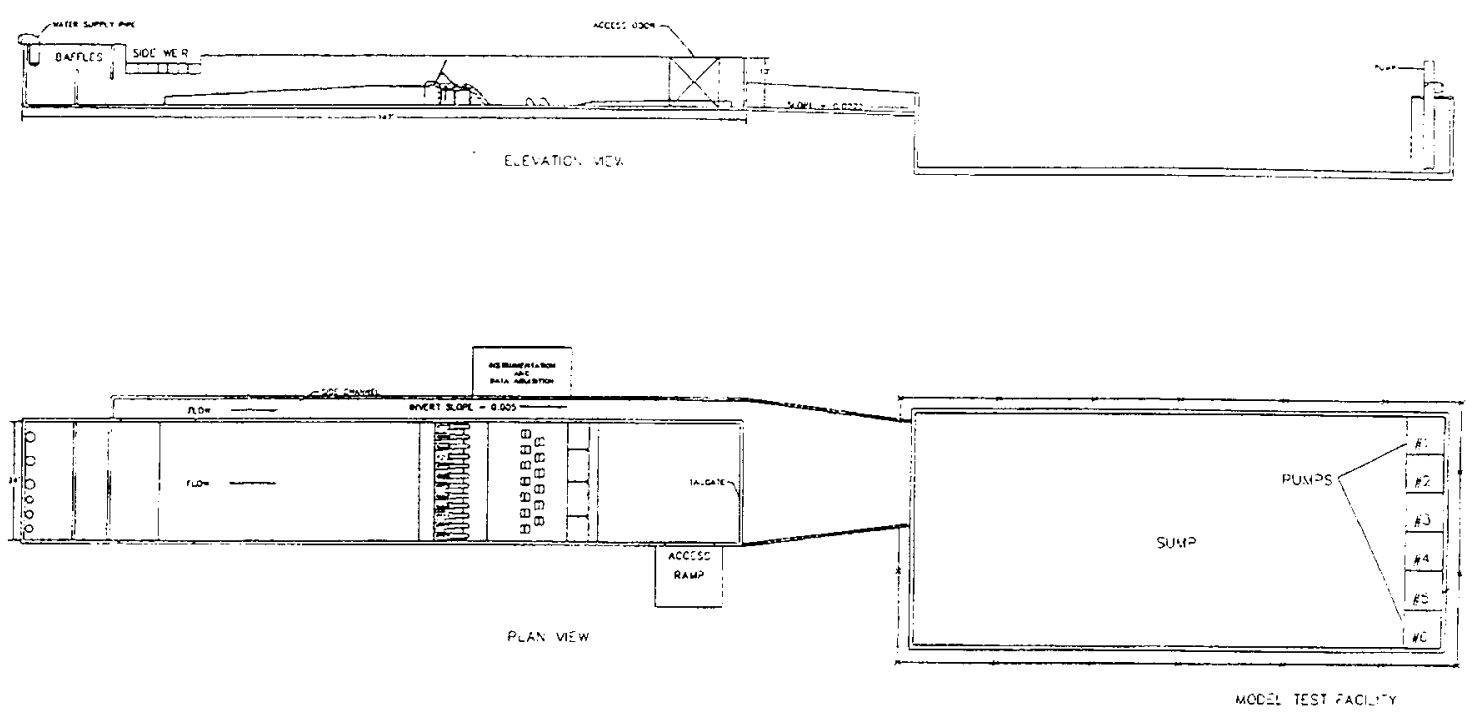

Figure 12. Sketch of the model facility

\section{Gate components}

The wicket system consists of several structural components as shown in Figure 10. These members include the gate, the hoisting devices, bearing frames, and the embedded alignment frames. Figure 10 shows the nomenclature for different components and the supporting devices on the gate. The lifting cylinder is used to raise and lower the gate, and the prop rod supports the gate when the dam is in service. Three bearing frames, one to support the gate hinges, one to support the cylinder assembly, and another to provide track for the prop rod end, were anchored on the sill as shown in the figure. The bearing frame for the prop rod end is called a "Hurter," a term borrowed from wooden wicket terminology, currently in use at L\&D 52 and 53. Embedded frames, not shown in the figure, were installed in the sill prior to pouring concrete to transfer loads, align bearing frames, and provide a strong foundation for the structure. During construction, prototype mounting conditions and framework were duplicated very closely in the test model. An exploded view of the wicket system, including the embedded alignment frames, is shown in Figure 14. Hinge bearing frame and the cylinder frame assemblies were mounted at different levels on the embedded alignment frame, as shown in the figure.

\section{Fabrication and Assembly}

A five-phase work schedule was devised to assemble and erect the wicket system in the model: 


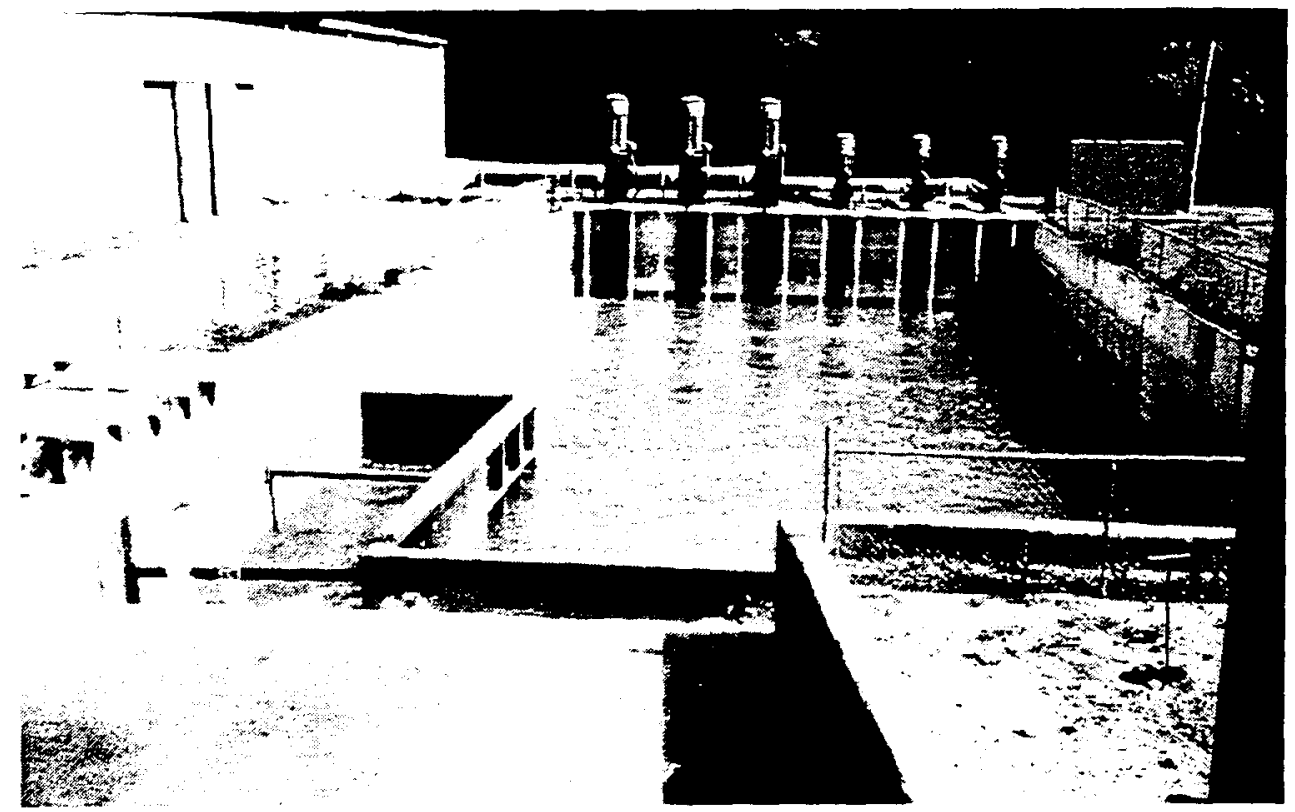

Figure 13. Six water pumps for flume flow regulation

a. Procuring material, preparing shop drawings.

b. Cutting and machining of basic structural units.

c. Welding to form structural components.

d. Assembling machined and welded components, fit testing, sizing adjustments as required.

e. Installing components - embedded, anchored, and mounted.

Once the material was procured, a detailed shop drawing for each part of the wicket was prepared to facilitate the construction process. The shop drawings provided the dimensions of basic structural shapes that formed the structural components of the wicket system. These basic steel shapes consisted of steel sheet, plate, beam, angle, and rods. In the second stage, basic structural units were cut and machined into specified sizes and thicknesses required to construct structural components such as the gate, lifting devices, and bearing frames. Note that during the machining process, linear dimensions of basic shapes were readily attainable. Attaining a uniform thickness, however, depended upon the preexisting curvature, geometric shape, and manufacturing defects of each steel piece. If a steel plate with preexisting curvature, for example, had been machined to a desired thickness, the plate would have been thinner at the warped portion due to leveling of the top surface during the milling process. An average thickness of the major parts is shown in Table 2. Therefore, the steel members were carefully selected and machined to attain an acceptable average thickness of the machined surface. 


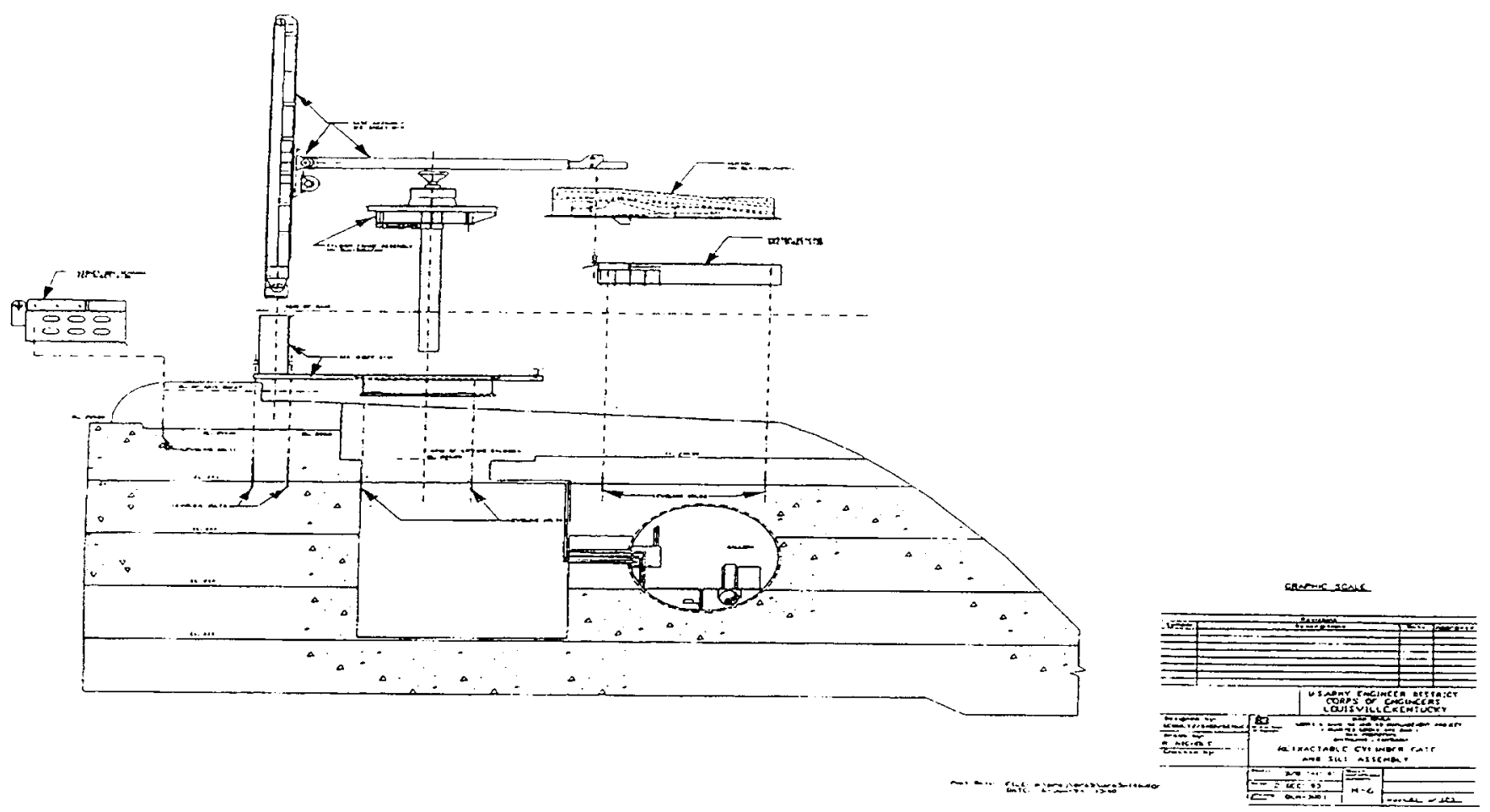

Figure 14. Exploded view of wicket assembly

Once the basic units were prepared, they were then welded together to form the geometry of the structural components in the third stage. During this process, the steel shapes were fastened firmly with clamps to minimize warping due to elongation of welded surfaces. Also, it was necessary to incorporate multiple discontinuous welding across a joint with intermediate breaks to connect members together. A continuous welding across a joint was avoided to reduce warping effects on connected thin steel members. In the fourth stage, the machined and welded components were assembled. During this stage, the adequacy and fit of each part were tested and design drawings were verified. In the case of an unfit or wrong sized part, modifications were made to adjust the size or fitting process. A slight variation in the actual dimensions of the gate components from the required scaled dimensions caused relative misalignment and/or dislocation of parts. In such a case, refined surface finishing or cut-to-fit shape was remade to ensure proper fit of components. In the final phase of the installation process, the assembled parts were sequentially embedded, anchored, and mounted on the sill. 


\begin{tabular}{|c|c|c|c|}
\hline \multicolumn{4}{|c|}{$\begin{array}{l}\text { Table } 2 \\
\text { Average Thickness for Major Structural Members Used in } \\
\text { 1:5-Scale Wicket Model }\end{array}$} \\
\hline $\begin{array}{l}\text { Component } \\
\text { Description }\end{array}$ & $\begin{array}{l}\text { Reference } \\
\text { Sheet No. }\end{array}$ & \multicolumn{2}{|r|}{ Dimensions, in. } \\
\hline & & Prototype & Model \\
\hline Skin plate & S-24, S-25 & $1 / 2$ & 0.1046 \\
\hline Beam & S-24, S-25 & $W 18 \times 175$ & 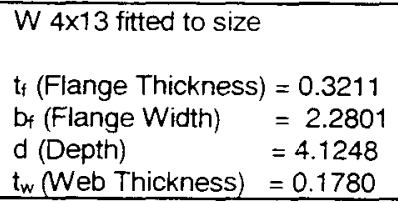 \\
\hline Channel & S-24 & $c 8 \times 18.75$ & $\begin{array}{l}2-\quad L 1 \times 1 \times 1 / 8 \text { were used to } \\
\text { form } C \text { section with } \\
d=1.585, t_{f}=0.77, t_{w}=0.093 \\
\text { and } b_{f}=0.49 \text {. }\end{array}$ \\
\hline $\begin{array}{l}\text { Transverse } \\
\text { stiffener plates }\end{array}$ & S-24 & $3 / 8$ & 0.077 \\
\hline Web stiffener & $\mathrm{S}-24$ & $3 / 4$ & 0.151 \\
\hline Pipe & S-24 & $\begin{array}{l}8 \%: 4 \\
\mathrm{SCH} 80 \text { A53-S }\end{array}$ & $11 / 2 \varphi \mathrm{SCH} 80$ A53-F \\
\hline Prop rod & $\mathrm{M}-18$ & $10 \mathrm{q}$ & 24 \\
\hline
\end{tabular}

\section{Gate}

Twelve-gauge sheet steel was used for wicket gate skin plate sections. Two halves of the skin plate are shown in Figure 15. No surface machining was done to reduce the thickness of the skin plate. An average thickness of $0.1046 \mathrm{in}$. was measured for the skin plate used in the model. The $\mathrm{C}$-channels connected to the outer edges of the skin plate were fabricated by using two $1-\times 1-\times 1 / 8$-in. angles. These angles were milled to the scaled dimensions before welding them together to form the channels shown in Figure 16. Two W $4 \times 13$ (wide-flange, 4-in. nominal depth by 13 -lb per foot length) beams were machined to the required dimensions for the scaled model. Figures $17 \mathrm{a}$ and $17 \mathrm{~b}$ show the basic scaled shapes of the gate components. In these figures, seven transverse stiffener plates, two longitudinal beams, channel sections, and the skin plate are placed in order to show their relative positions in the assembled gate. Figure 18 shows how the stiffener plane, channel, and the ribs were assembled. Web stiffener plates were welded on the W-beam, directly under the ball mounting plate, to support the on-line thrust of the lifting devices during operation. Figure 19 shows the mounting conditions of web stiffeners on the W-beam.

The bottom part of the gate consisted of plates shown in Figure 20. An interior view of the base connection that fastened the gate to the bearing frame are shown in Figure 21. Figures $22 \mathrm{a}$ and $22 \mathrm{~b}$ also show the connection details for the gate base. A $3 / 4$-in. steel pipe was inserted through the base plates to provide routing for the instrumentation cable from the gate to the sill. A closeup view of the assembled gate base is shown in Figure 23. 


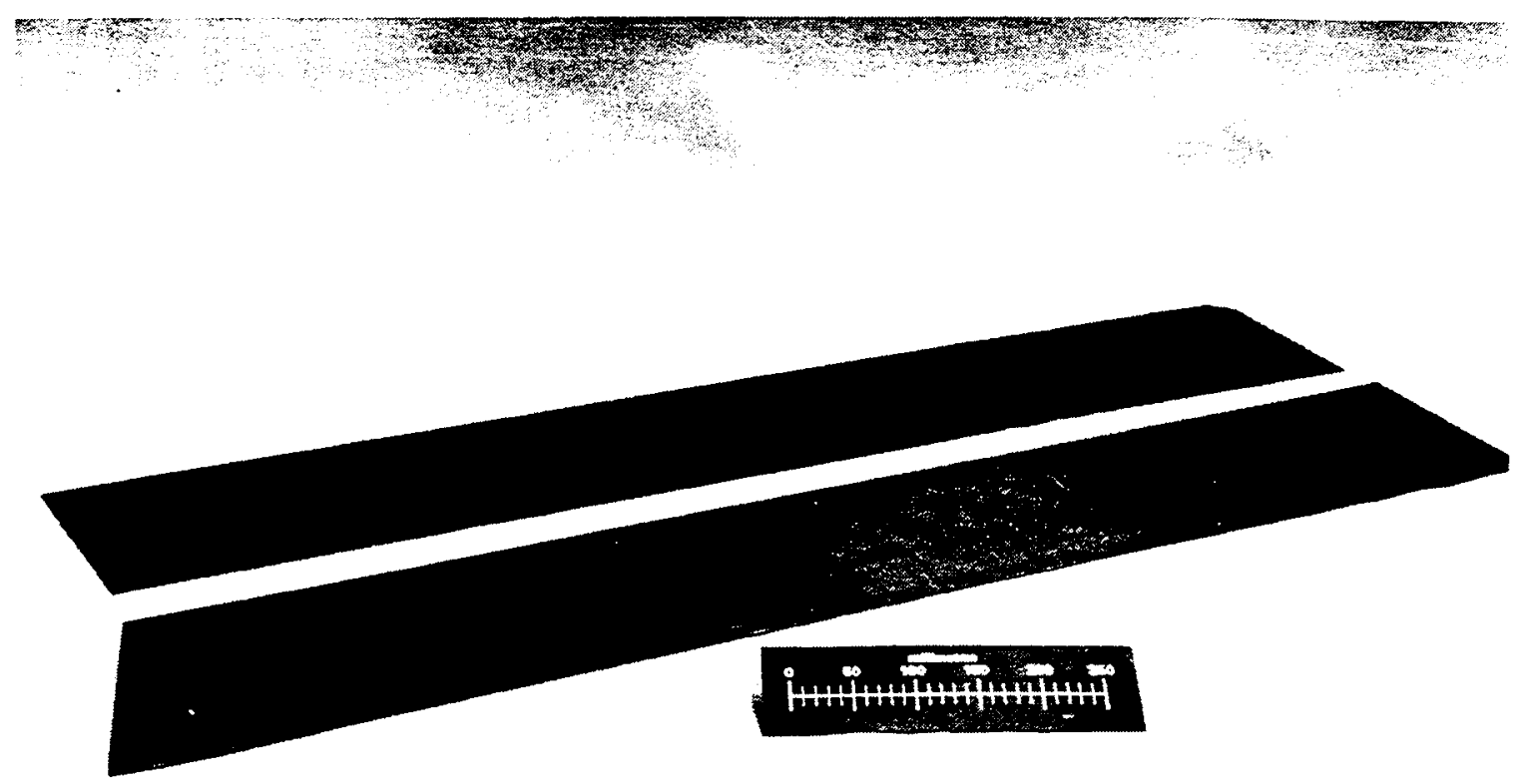

Figure 15. Two halves of wicket skin plate

Figure 24 shows the $Y$-shaped notch and the hoisting rod at the top of the gate. The Y-shaped notch was designed to facilitate access of crane hooks through the slots during manual raising of the gate. A crane hook attached to the hoisting rod could lift the gate when required. Two holes on both ends on the top of the skin plate (not slotted at the time of the photograph) were used to aerate a gate nappe. More discussion relating to the aeration of a bottom-hinged wicket nappe are available in the literature (Stockstill 1992).

Top, front, and rear views of the assembled gate are shown in Figures 25 and 26. As shown in these figures, a 1.7-in.-diam steel pipe was connected to the top edge of the skin plate. Addition of such a pipe provided smooth transition of water flowing over the gate. The cutout pieces of the ball mounting plates and the pillow block for ball connection are shown in Figure 27. Figure 28 shows the cup prior to being connected to the piston rod which raises the gate by pressing against the ball mounted on the pillow block. The placement of ball mounting plates on the beam webs are shown in Figure 29.

\section{Hinge assembly and sill-bearing frame}

An overview of the gate and its accessories is shown in Figure 30. To the left of the gate is shown the sill-bearing frame and the embedded frame assembly in the gate-down configuration. Lugs mounted on the sill-bearing frame provide 

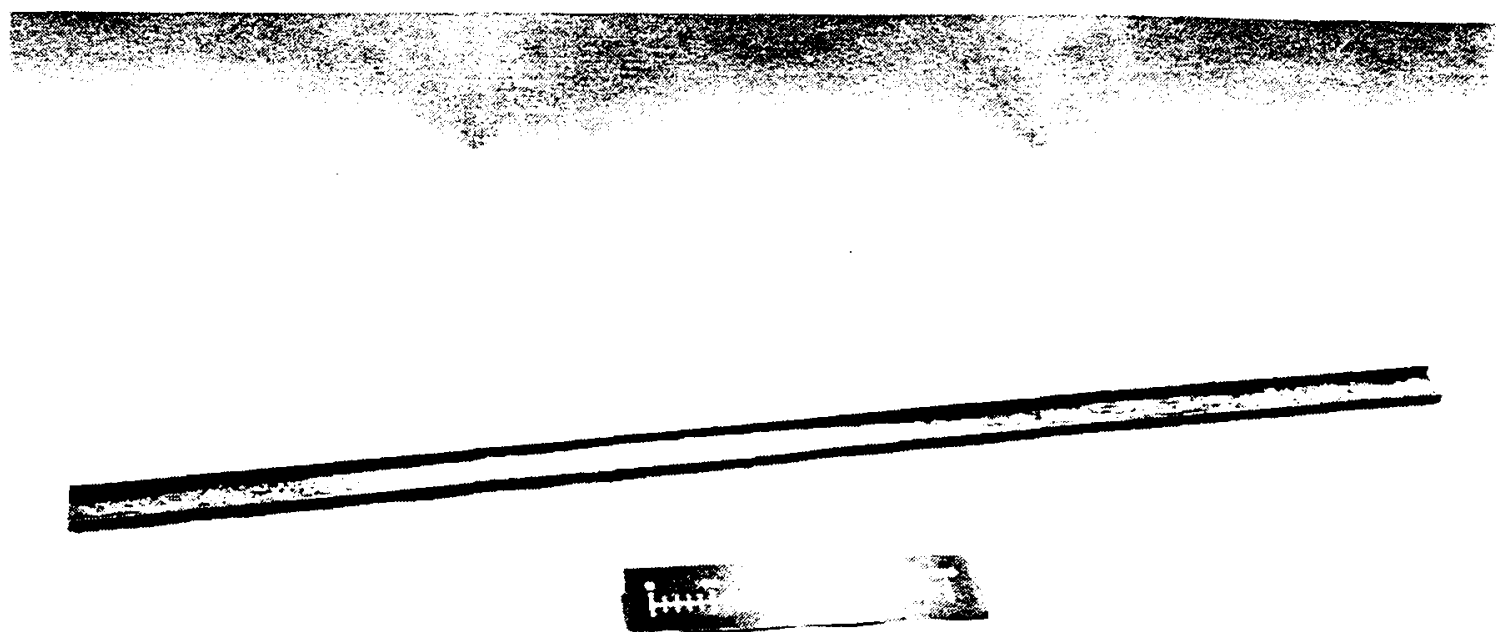

Figure 16. Fabrication of C-channels

base hinge connection for the wicket. The retractable cylinder frame is shown on the top of the figure. An unfinished assembly of the base hinge assembly and the bearing frames are shown in Figure 31. Figure 32 shows the finished gate framehinge assembly and the cup-ball assemblage.

A relative position of embedded frames is shown in Figure 33. The sillbearing frame hinged to the gate base (Figure 32) would be anchored on the top of the alignment embedded frame shown left in Figure 33. The horizontal frame attached to the bottom of the alignment frame would support the cylinderbearing frame shown in Figures $34 \mathrm{a}$ and $34 \mathrm{~b}$. This figure also shows the hurterbearing frame that anchors the hurter assembly. A closeup view of the alignment frame assembly is shown in Figures 35a and 35b. The slotted anchor pins on the top of the alignment frame were used to mount the sill-bearing frame. The alignment pin rod next to the slotted anchor pins facilitated the erection of the gate assembly.

\section{Prop-rod and hurter mechanism}

A locked-in prop end on the hurter recess is shown in Figure 11e. As shown in the figure, the right tracks are used during gate elevation and the left track is used during gate lowering. The retainer pins connecting the hurter with the hurter-bearing frame are also shown in the figure. Note the guide bar that passes 


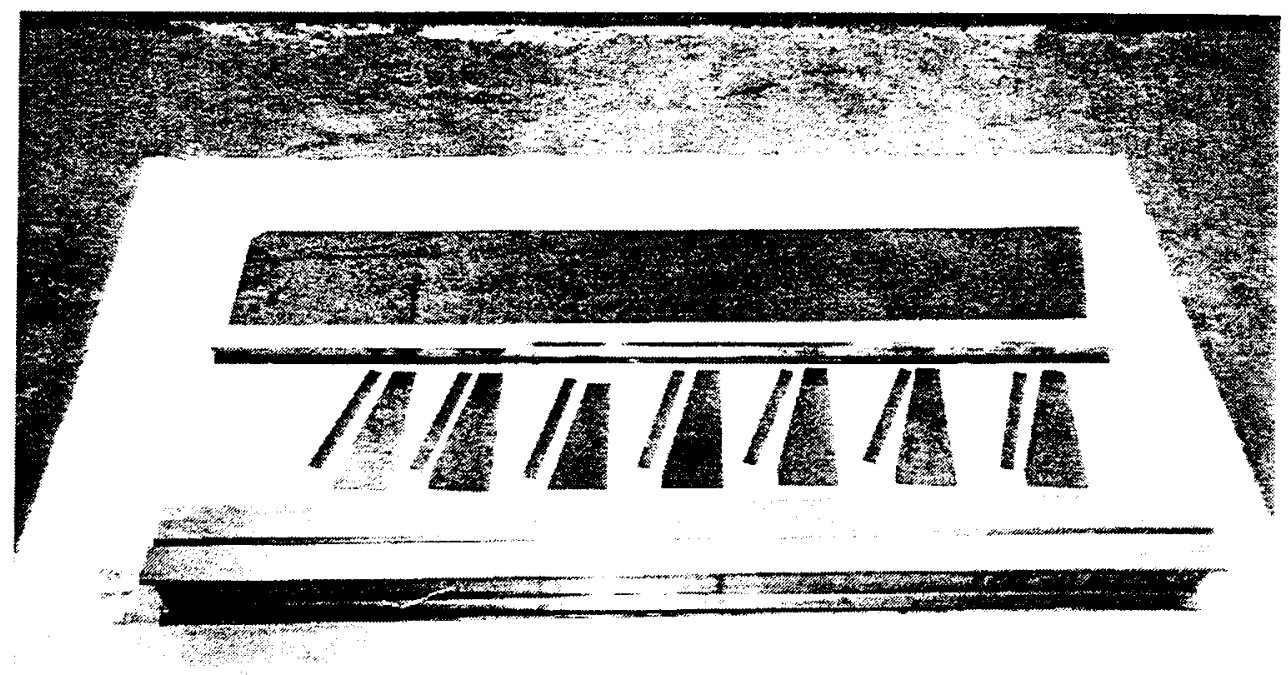

a. Details of transverse stiffeners and W-beam
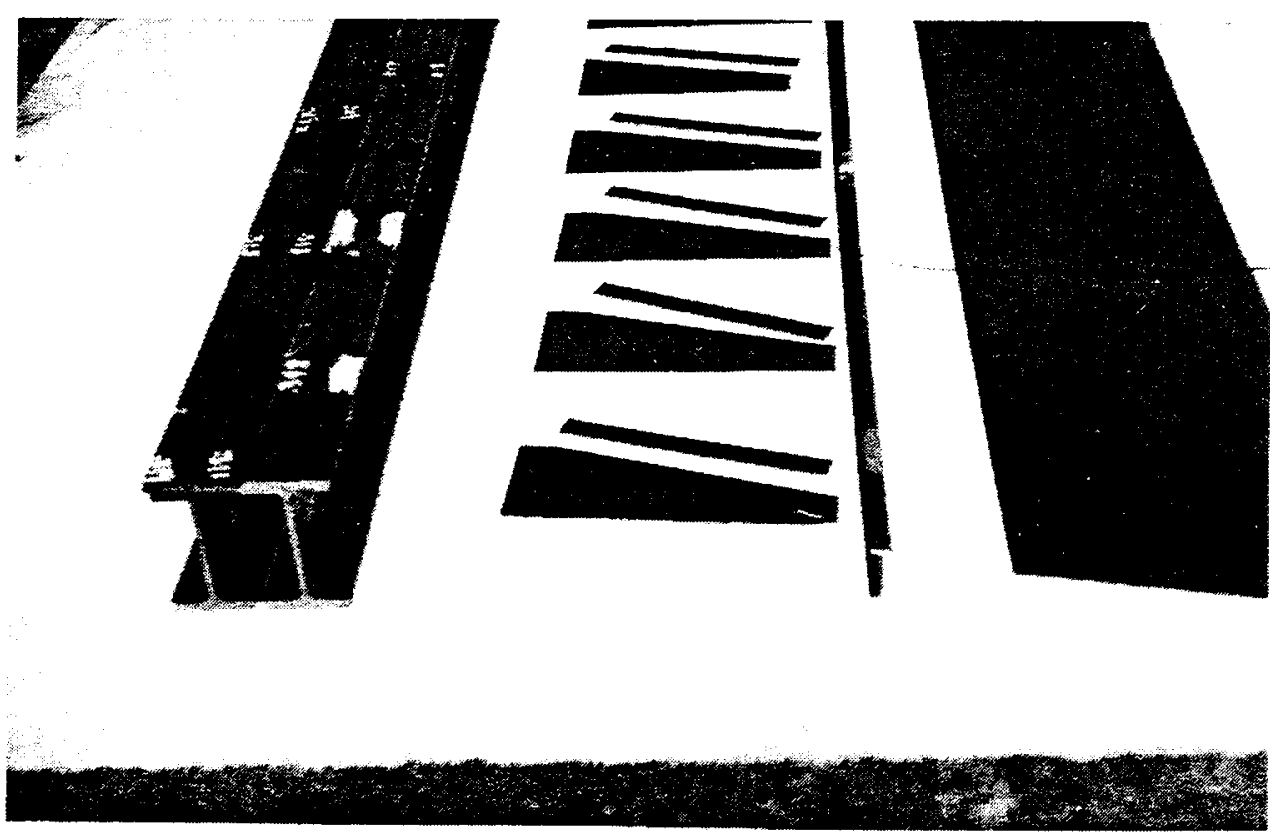

b. Transverse stiffeners, skin plate, and W-beam

Figure 17. Basic gate components

through the slots in the blade connected to the prop end. Both end shoes of the guide bar glide through the slots on either end of the vertical plates. The guide bar restricts motion of the prop blade to prevent the water flow from dislocating the prop end from the hurter track during gate operation. Appendix A presents drawings for the entire assembly. 


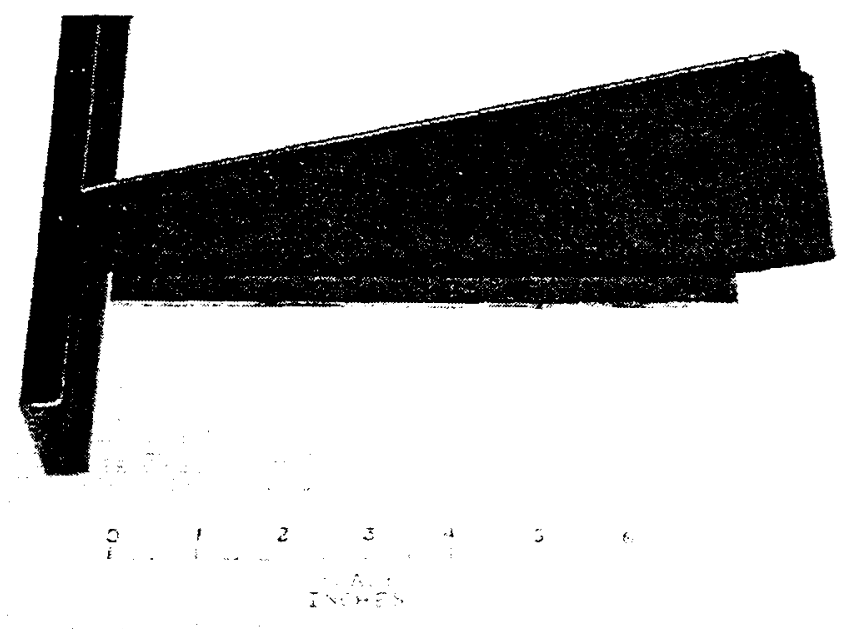

a. Transverse stiffener with channel

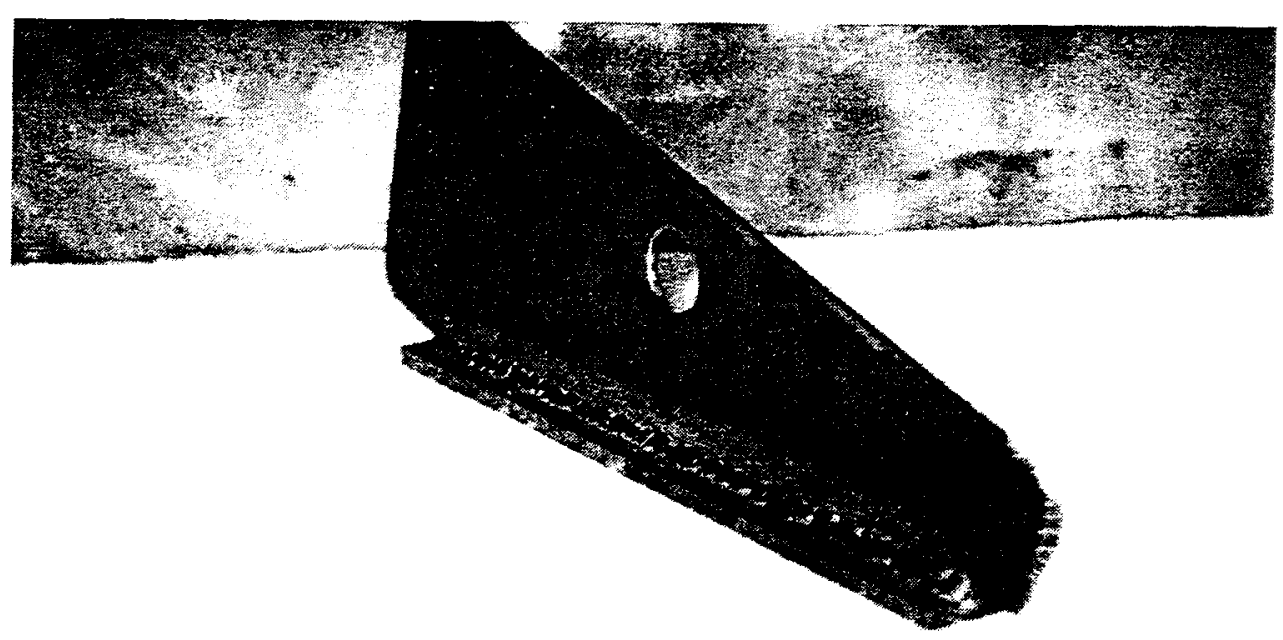

b. Transverse stiffener without channel

Figure 18. Assembly of stiffener plate, channel, and ribs 


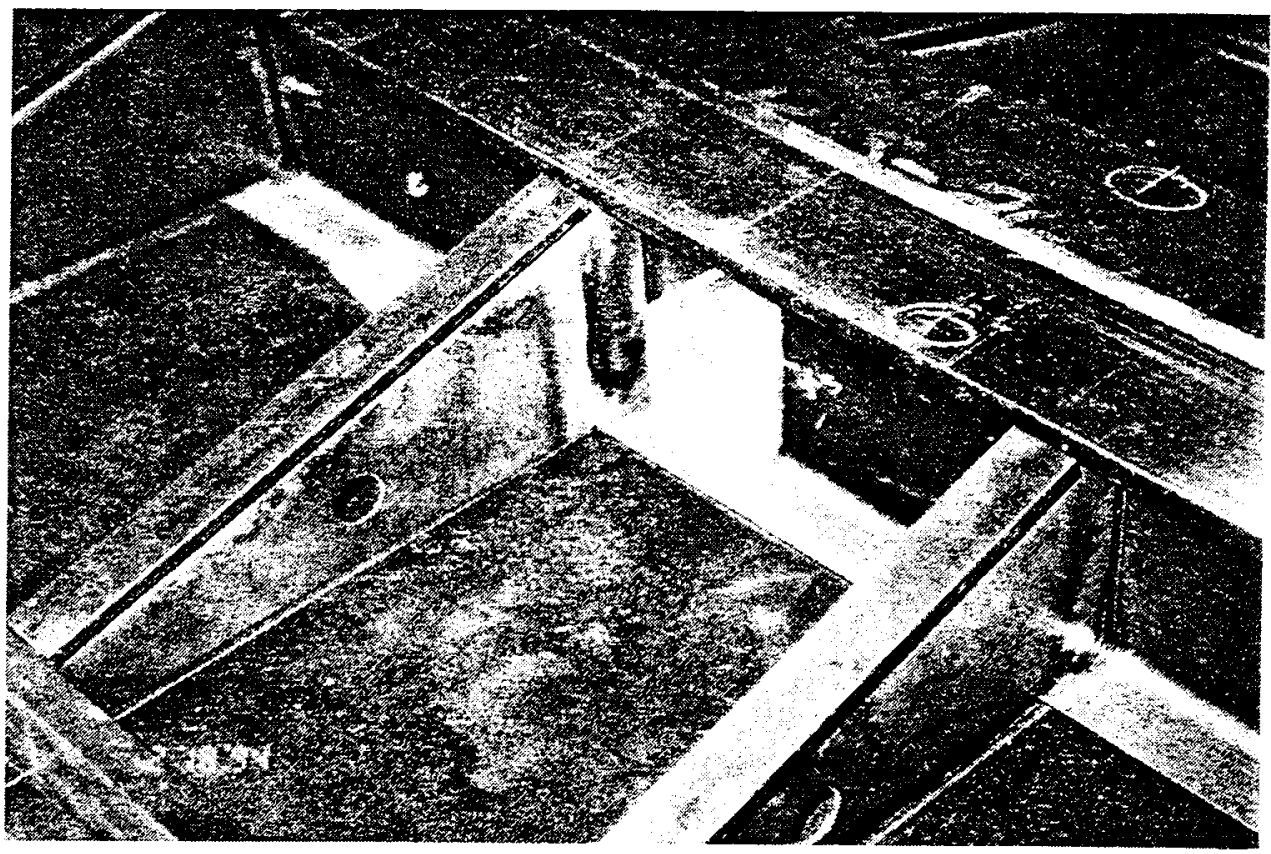

a. Overall assembly of stiffening components

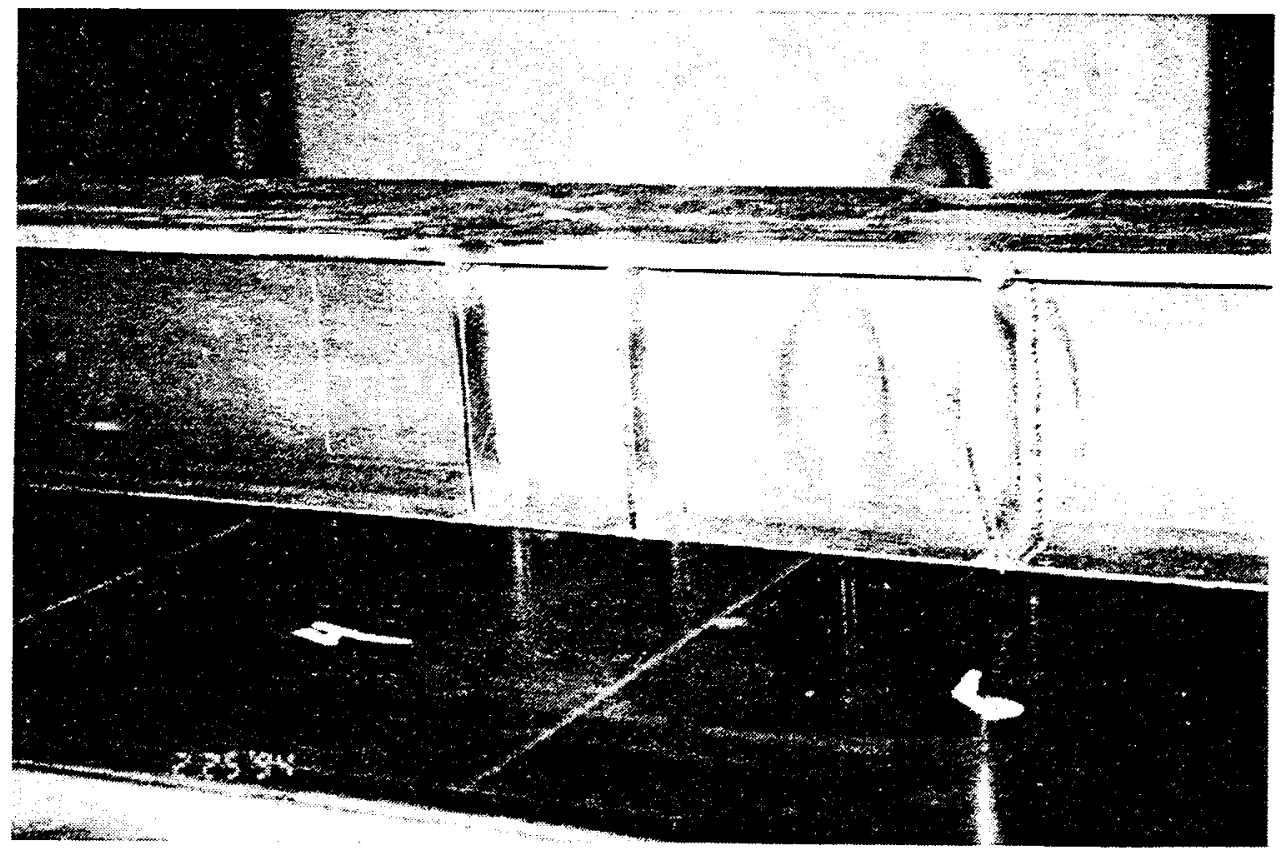

b. W-beam web stiffening at load transfer point

Figure 19. Mounting conditions of web stiffeners on W-beam

The hurter-bearing frame prior to being embedded in the sill is shown in Figures $36 \mathrm{a}$ and $36 \mathrm{~b}$. These figures display the geometric details and positions of structural elements comprising retainer pins, web stiffener plates, the anchor slot, and the anchor bars. 


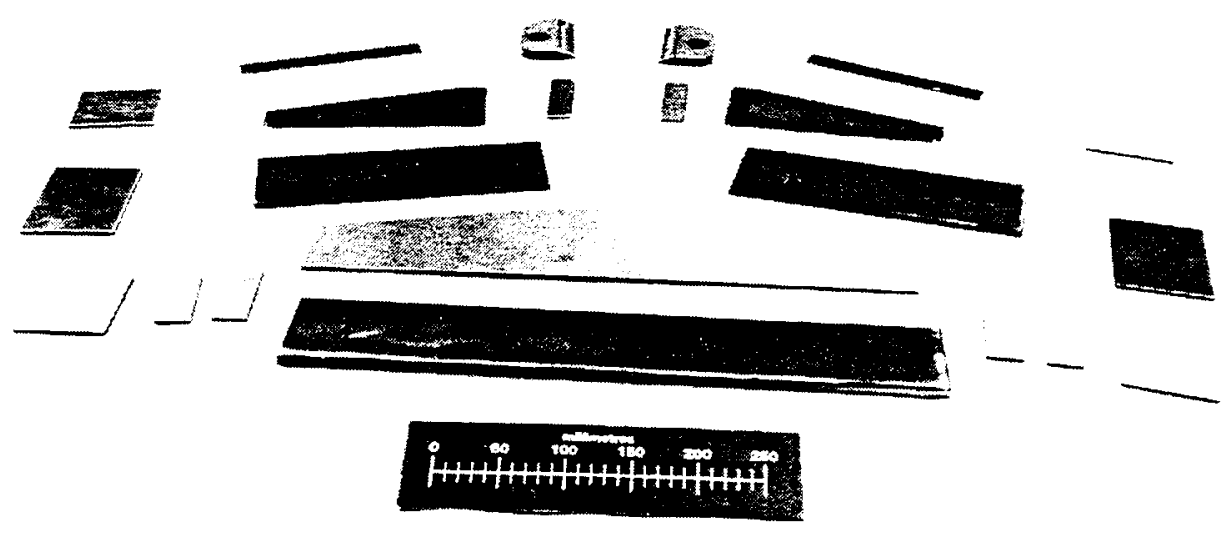

A 15754

Figure 20. Plate components of lower part of gate

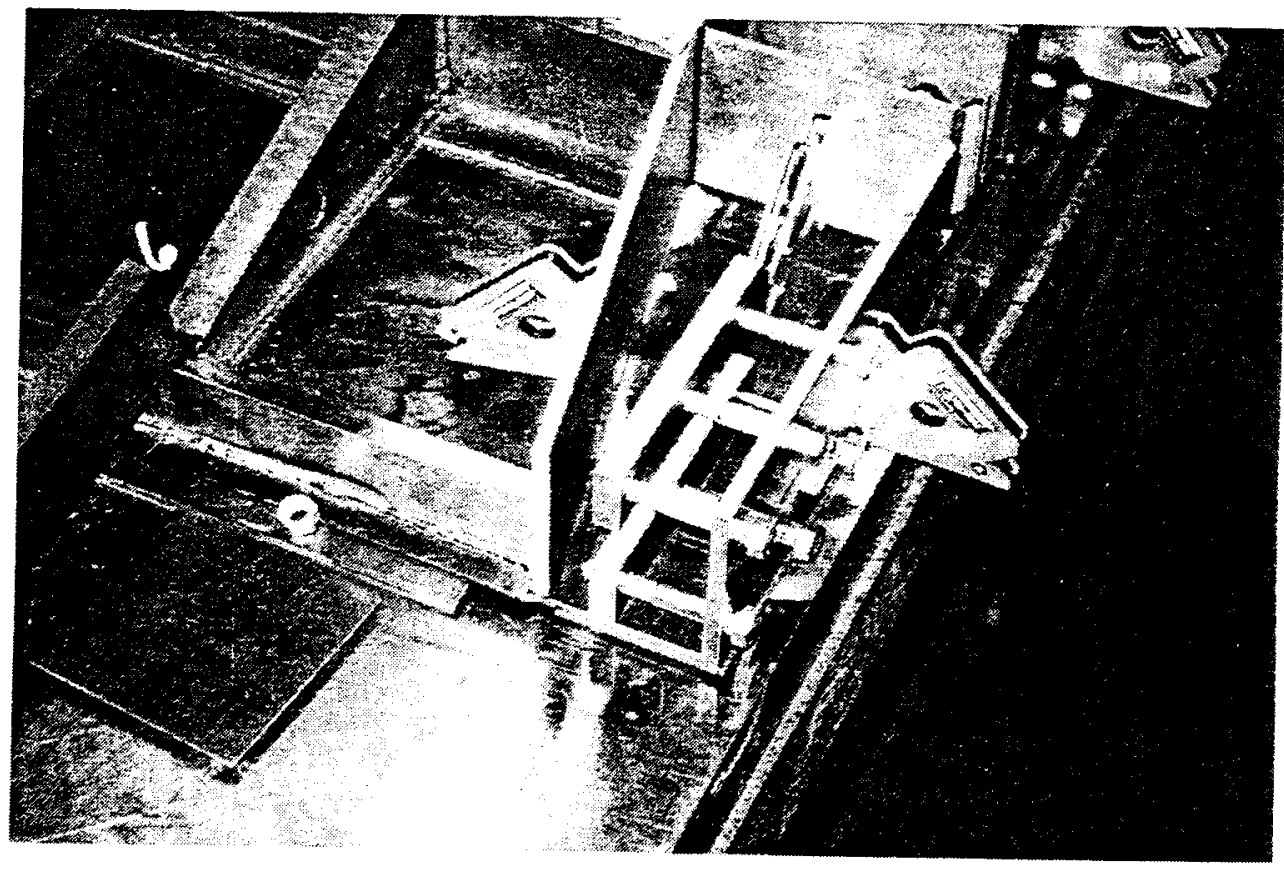

Figure 21. Interior view showing gate base connection to bearing frame 


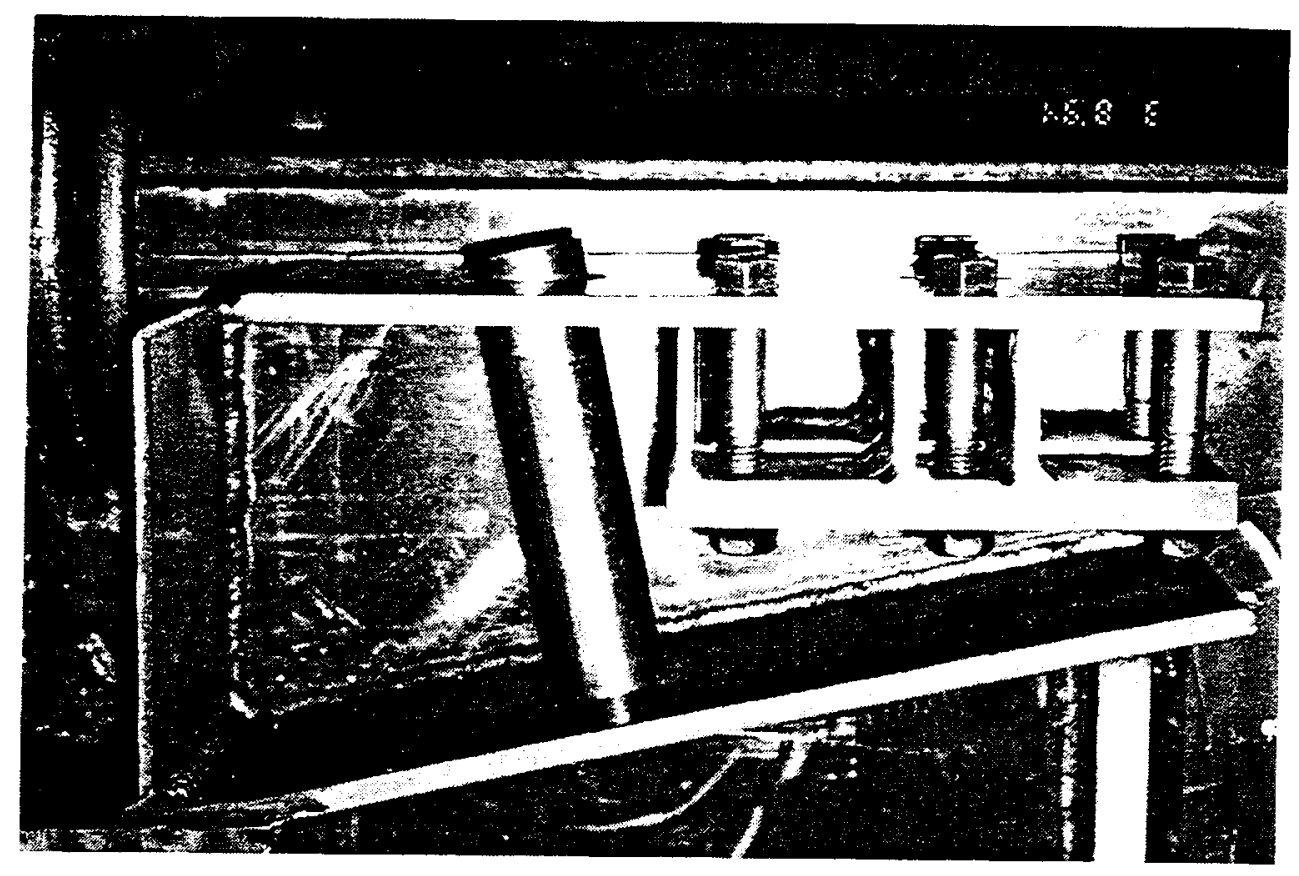

a. Details of box hinge connection

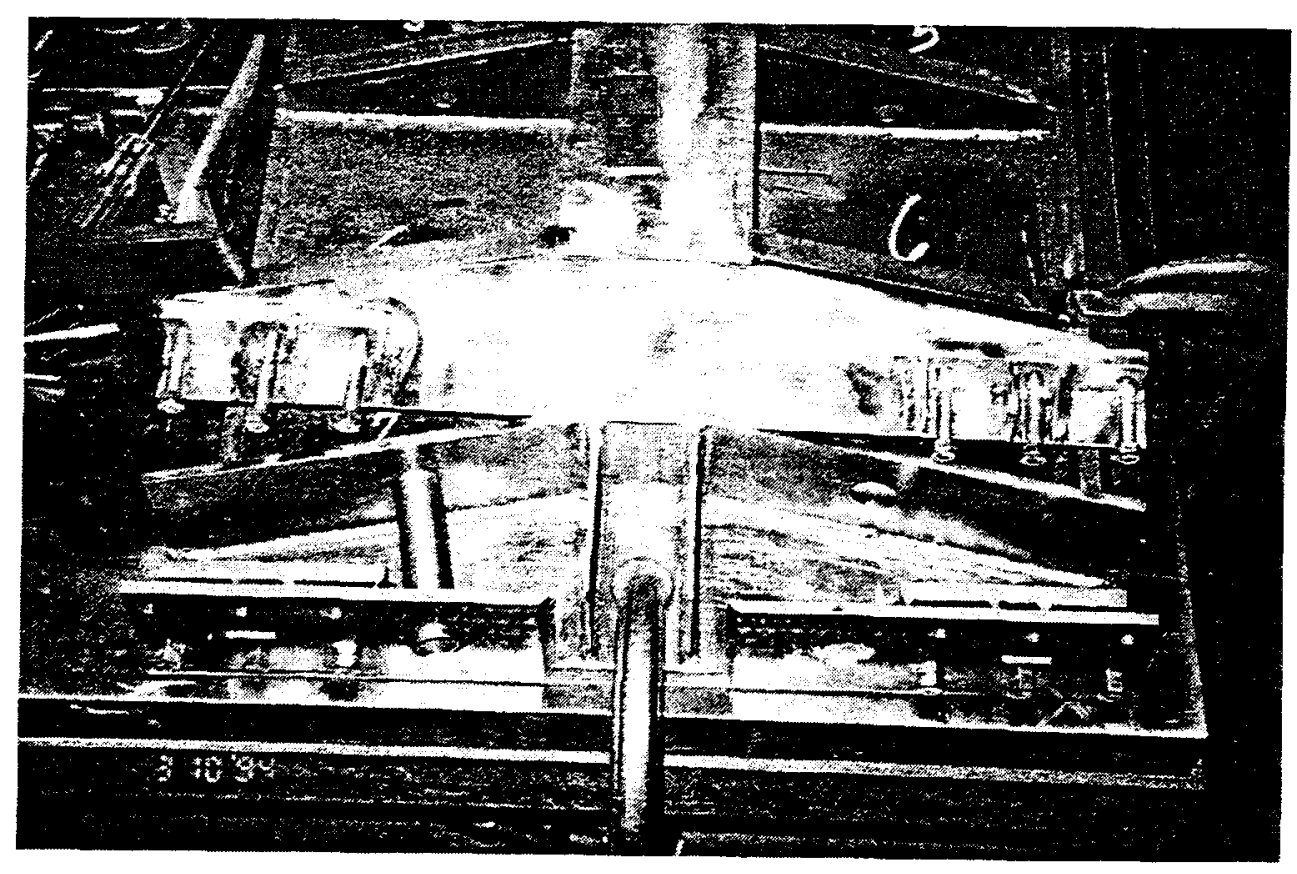

b. Top view of gate base

Figure 22. Connection details for gate base 


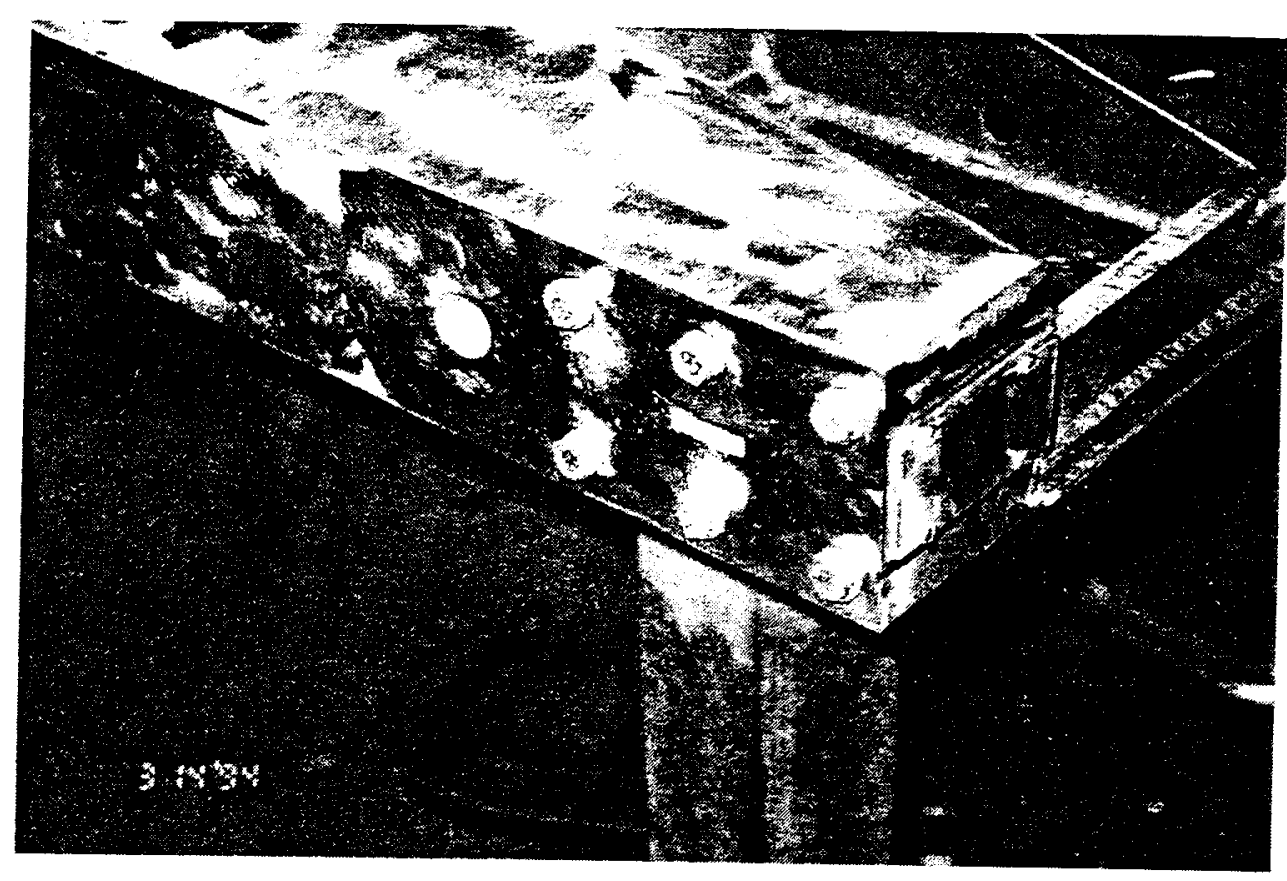

Figure 23. Closeup view of assembled gate base

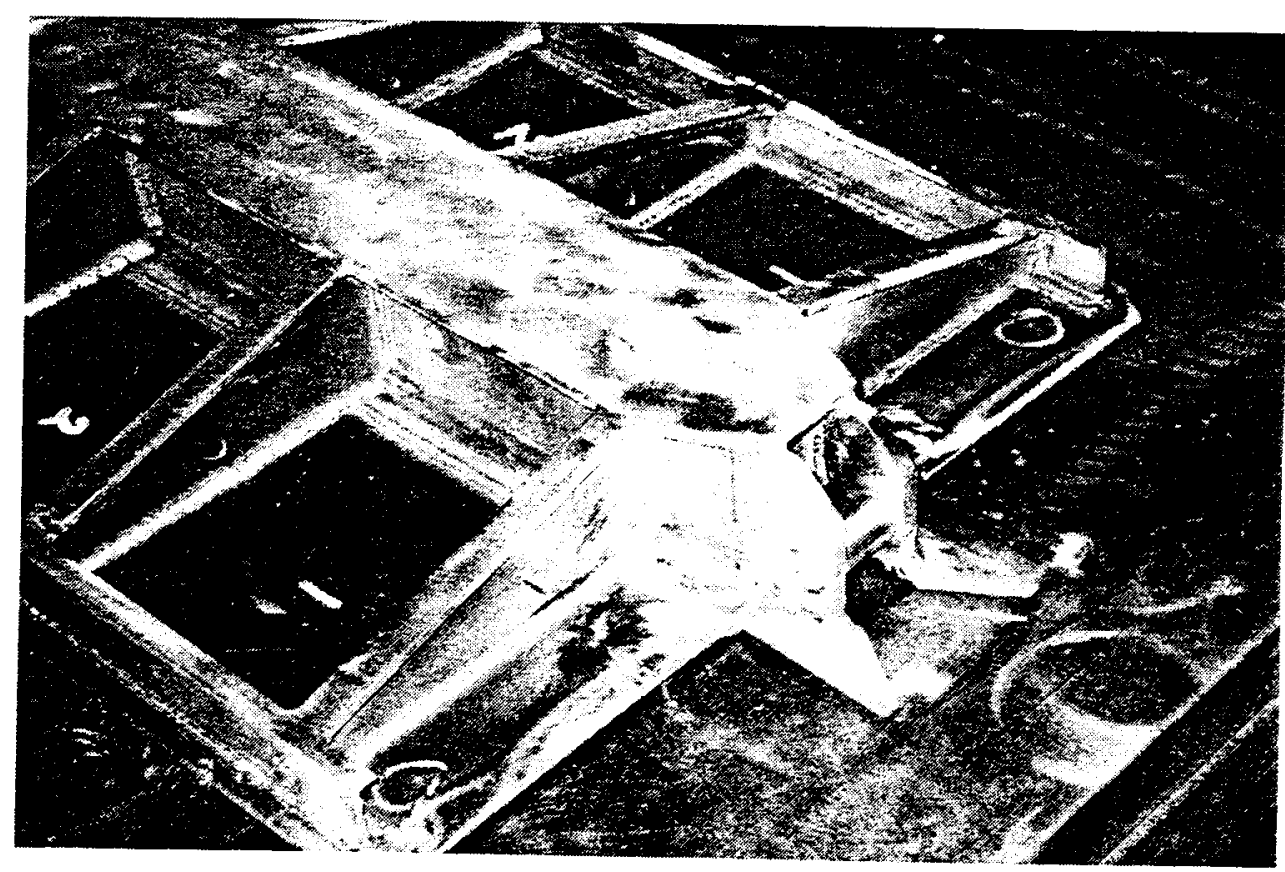

Figure 24. Y-shaped notch to facilitate use of hoist crane hooks 


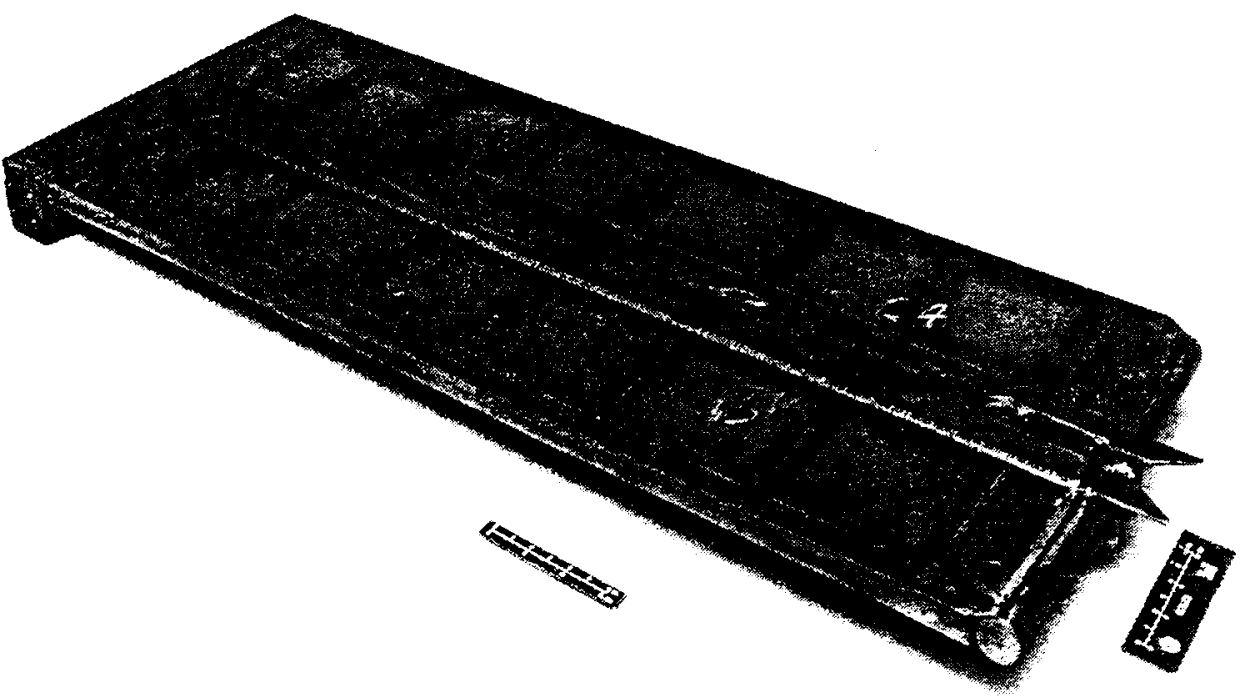

Figure 25. Top (front) view of assembled gate

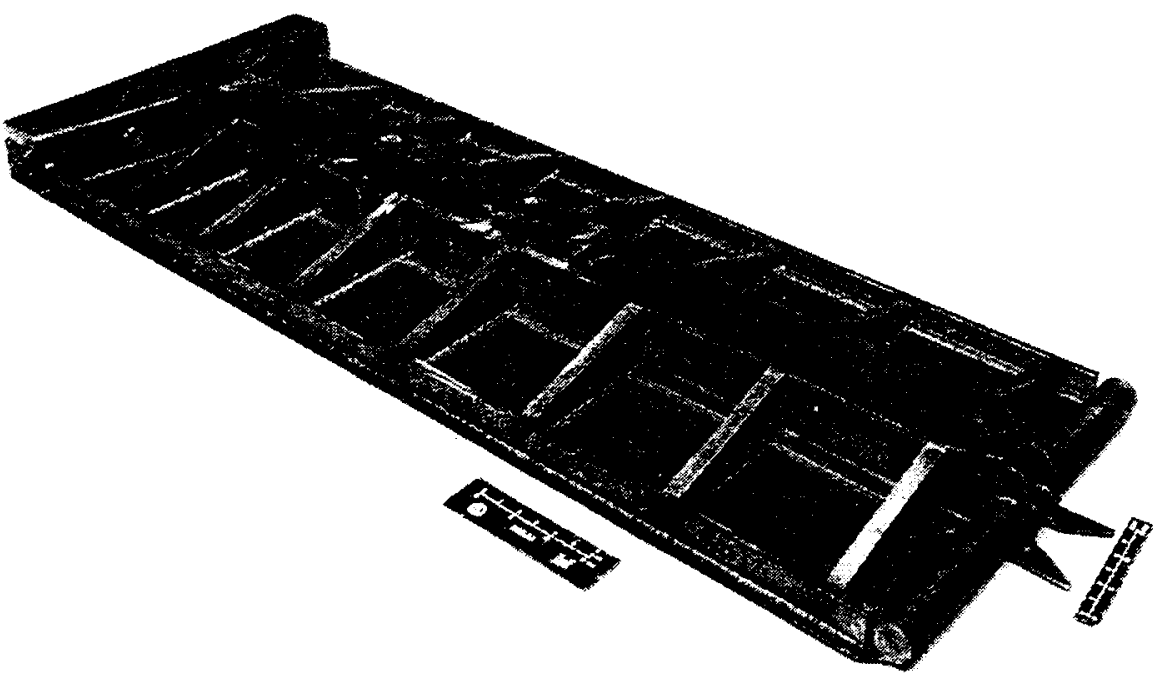

Figure 26. Rear (back) view of assembled gate 


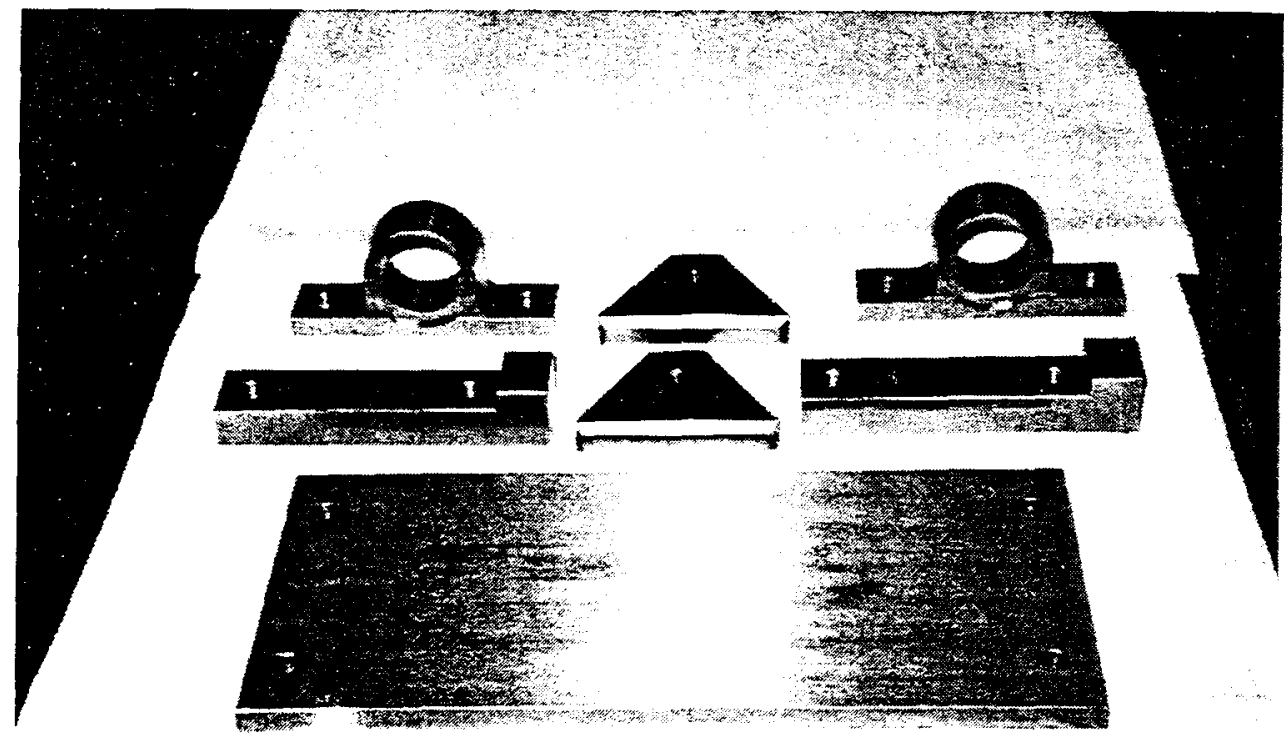

Figure 27. Cutout pieces of ball mounting plate, pillow block

\section{Hydraulic system}

A contract was awarded to Huber, Inc., Jefferson, LA, to furnish a fluid power system to raise and lower wicket gates in the Olmsted 1:5-scale physical models. The system consisted of 1 detachable lifting cylinder, 1 alignment cylinder, 11 direct-connected lifting cylinders, hydraulic power supply, hoses, a combination of directional control valves, popet valves, check valves, solenoid valves, and hydraulic control devices to independently extend, retract, stop, and maintain the positions of each cylinder piston rod.

The hydraulic system used to operate the instrumented gate was a 1:5-scale reproduction of the prototype fluid power system. Model dimensions for the detachable lifting cylinder and alignment cylinder, excluding the steel hydraulic tubing, were scaled down to meet the similitude requirement for the Olmsted model. The hydraulic system was capable of simulating a variable oil-column natural frequency in the model. An adjustable frequency of the hydraulic system was obtained by adding an auxiliary cylinder with a rod-locking mechanism to the system and by maintaining constant oil temperature in the main fluid reservoir. A thermostat-controlled immersion heater was added inside the reservoir to keep fluid temperature at a constant level such that the bulk modulus of the fluid remained invariant. Thus, by adjusting oil volume in the $1: 5$ system, the required oil-column frequency based on the similitude requirement could be attained in the model. In the model, the tubes connected to the lower chamber of the lifting cylinder were connected to the auxiliary cylinder to facilitate frequency adjustment at different gate positions. 


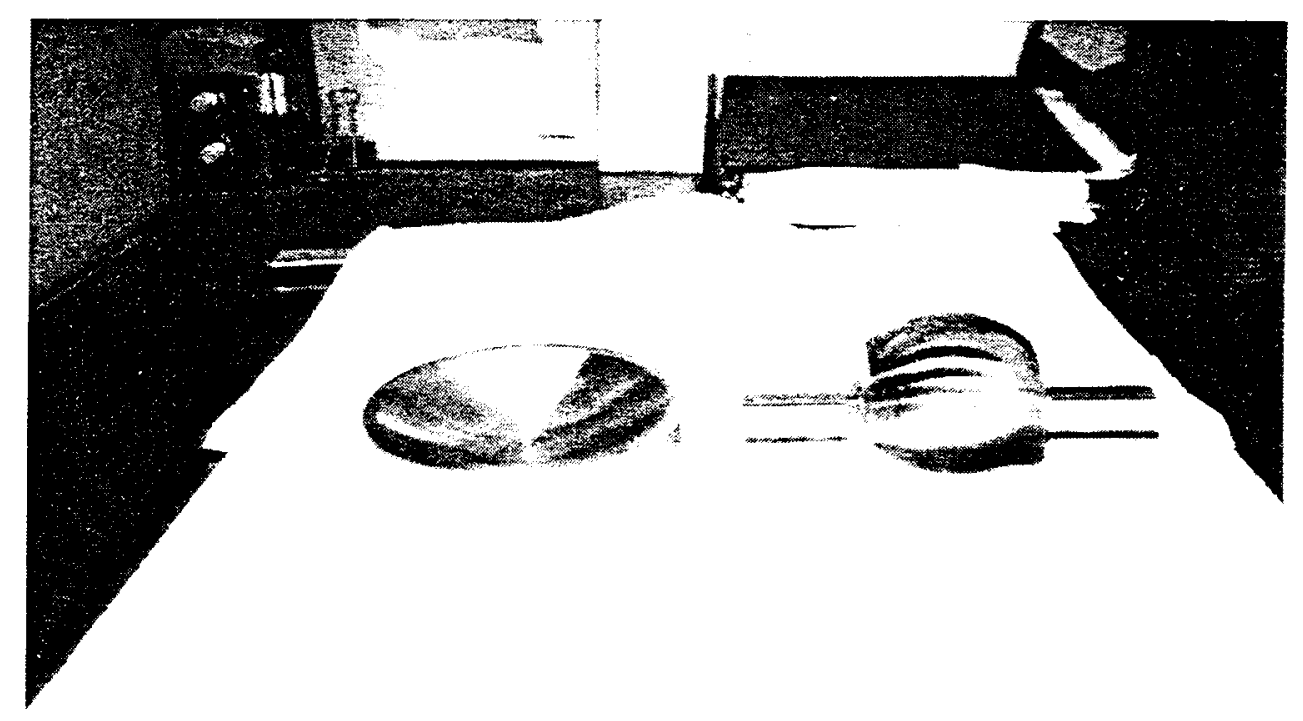

a. Cup and ball

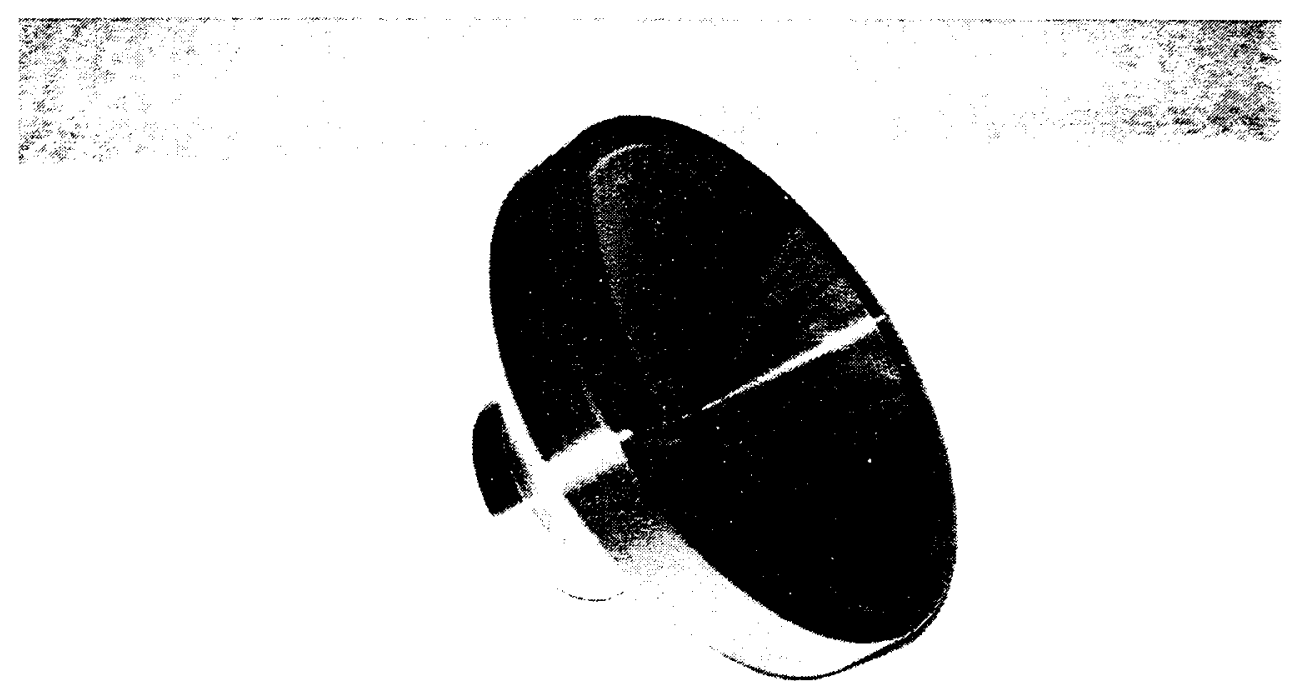

b. Cup

Figure 28. Cup and ball detail

A block diagram showing the schematic of the hydraulic module is presented in Figure 37. As shown in the figure, the cylinder tubing was connected to the hydraulic control system which was driven by a remotely controlled computer. 


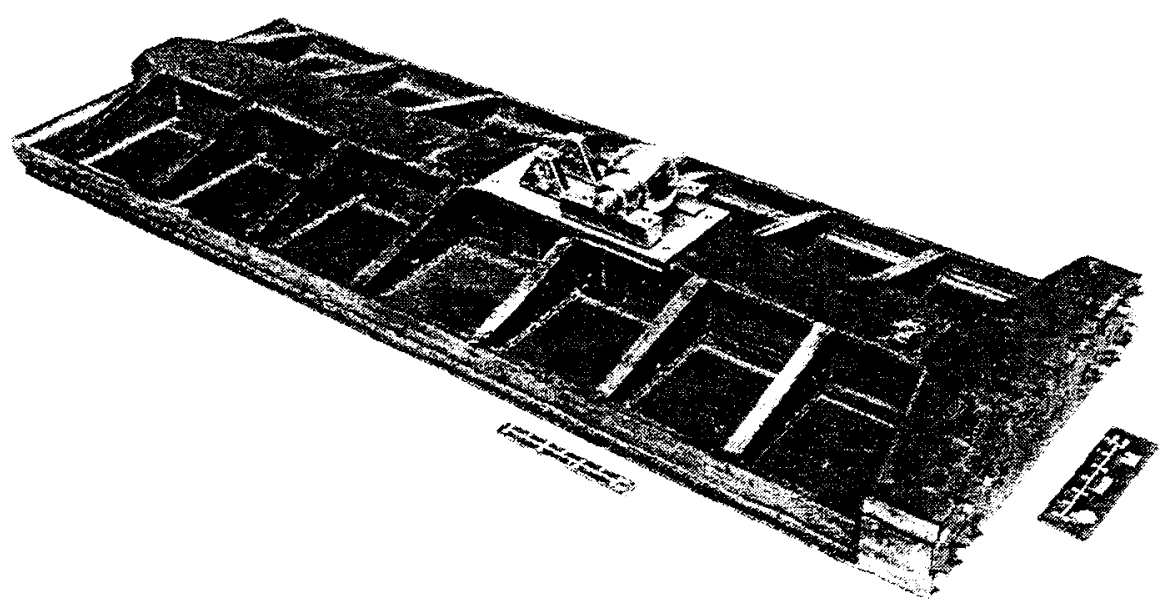

Figure 29. Placement of ball mounting plate on beam web

The computer regulated the motion of the cylinder at a constant speed. Therefore, by setting the desired speed on the hydraulic control panel, the gate could be raised or lowered at that fixed rate. In Figure 37, the cylinder, marked No. 6 , shows one extra hose than the rest in the group. This hydraulic tube provided feedback to the retractable cylinder for adjusting natural frequency of the system.

Figure 38 displays the connection details of the retractable cylinder with its supporting device. As shown in the figure, trunnions were inserted into the pillow blocks and tightly fastened to the cylinder-bearing frame. Also shown is the alignment cylinder that pushes the main cylinder to align the cup with the track of the ball mounted on the gate during operation. Figure 39 shows the hydraulic control system along with the auxiliary cylinder. The auxiliary cylinder had a 6 -in. bore $\times 19$-in. stroke. In addition to the auxiliary cylinder, an accumulator (Figure 39) with a constant volume of 577.27 in. $^{3}$ was added to provide feedback to the main cylinder. A directional control valve regulated the access of these additional oil volumes with the main cylinder fluid. More information is provided in the cylinder design drawings and the hydraulic tubing layout in Appendix B.

Examples of two directly connected and one detachable hydraulic cylinder installed in the model are shown in Figure 40. The main part of the piston rod for the cylinder had a 1.5-in. diam with an overall stroke of 29.6375 in. Also shown is the cylinder mounting mechanism and the position of cylinder bearing frames on the pit. A cylinder frame cover, not shown in the figure, was also constructed based on the prototype design drawings. 


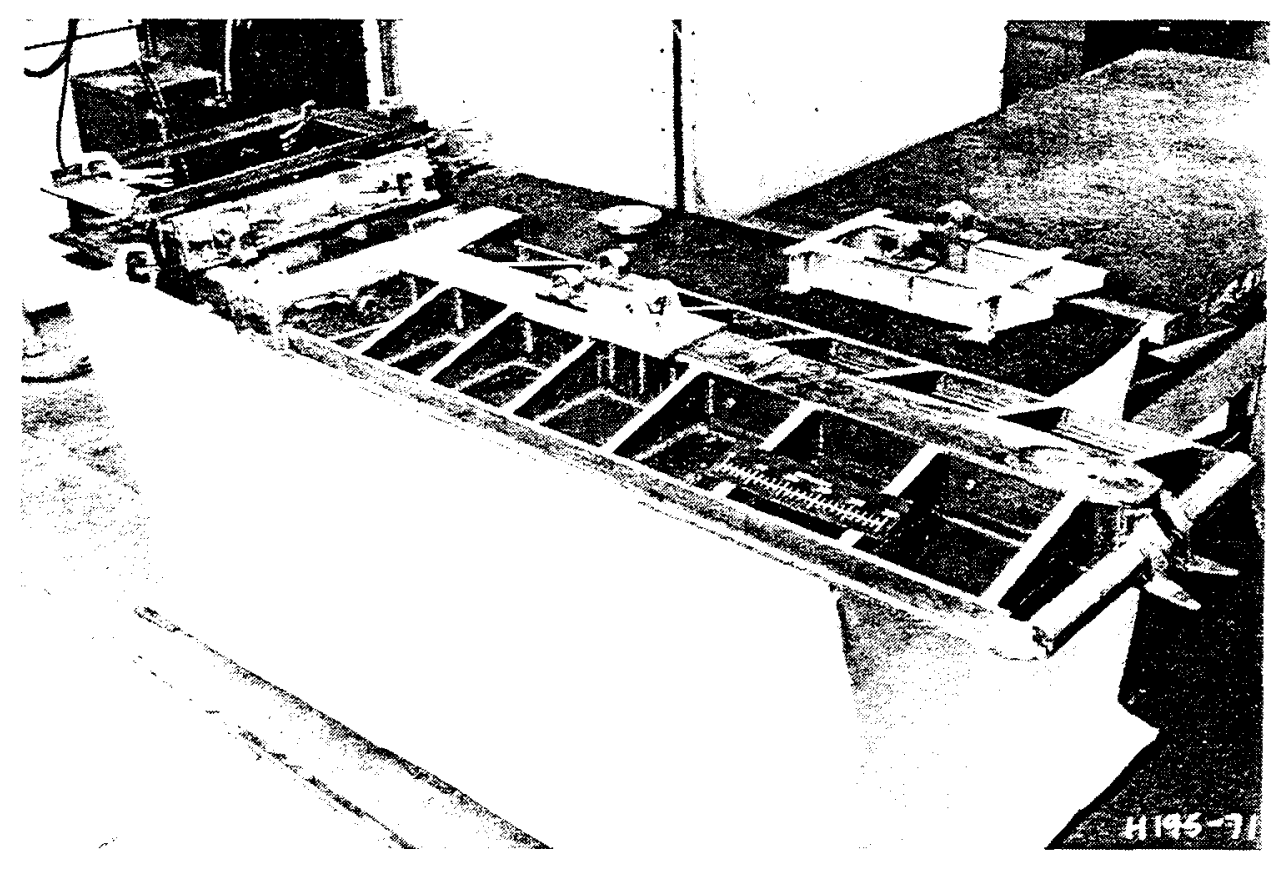

Figure 30. Overview of 1:5 wicket gate and its accessories

\section{Installation and Operation}

\section{Construction phase}

The sill was constructed in phases to accommodate the installation of embedded frames and to retain accurate levels of all components relative to the Olmsted elevations. In the flume, five-gate-width sill sections on both ends of the channels were constructed first to facilitate the proper positioning of test gate accessories in the middle. The remaining two-gate-width sections were constructed to ensure proper elevations of the gate and the supporting devices. Note that the cold joints between the previously constructed sill and the new pouring was adequate enough to resist the lateral thrust of the water current. Lateral frictional resistance for the two-gate-width concrete mass was about $6,400 \mathrm{lbf}$, computed based on a frictional coefficient of 0.2 for concrete.

Three levels of concrete were poured above the floor in lifts. Relative elevations of the concrete bases are shown in Figure 41. The first lift was up to the bottom surface of the embedded frame to form the cylinder pit (Figure 41). As shown in the figure, embedded frames mounted with leveling bolts on a triangular shaped steel base were anchored onto the concrete base. The steel base was required to provide proper elevations of the hinge and retainer pins of the assemblage, since the base of the hurter-bearing frame and the leveling bolts supporting the alignment frame were not at the same elevations. 


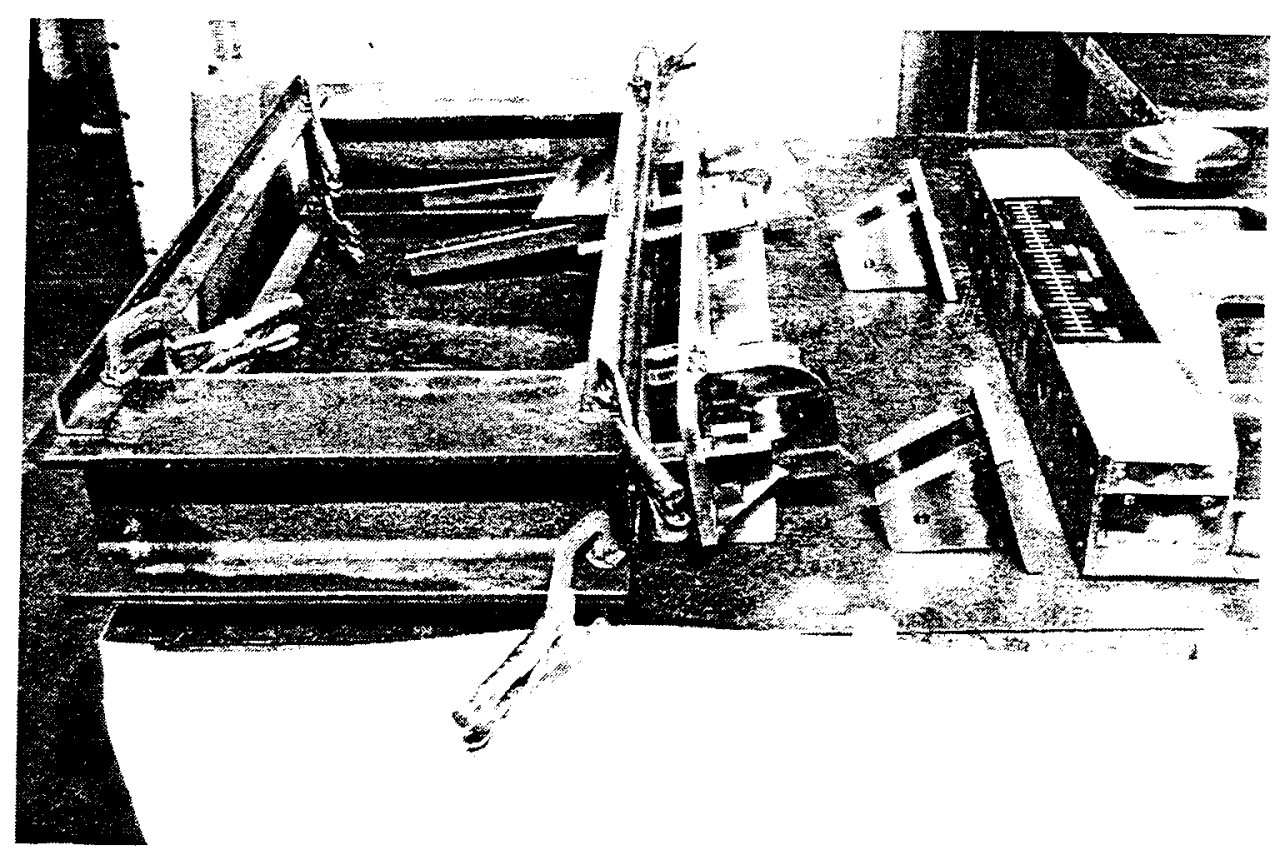

Figure 31. Unfinished assembly of base hinge and bearing frames

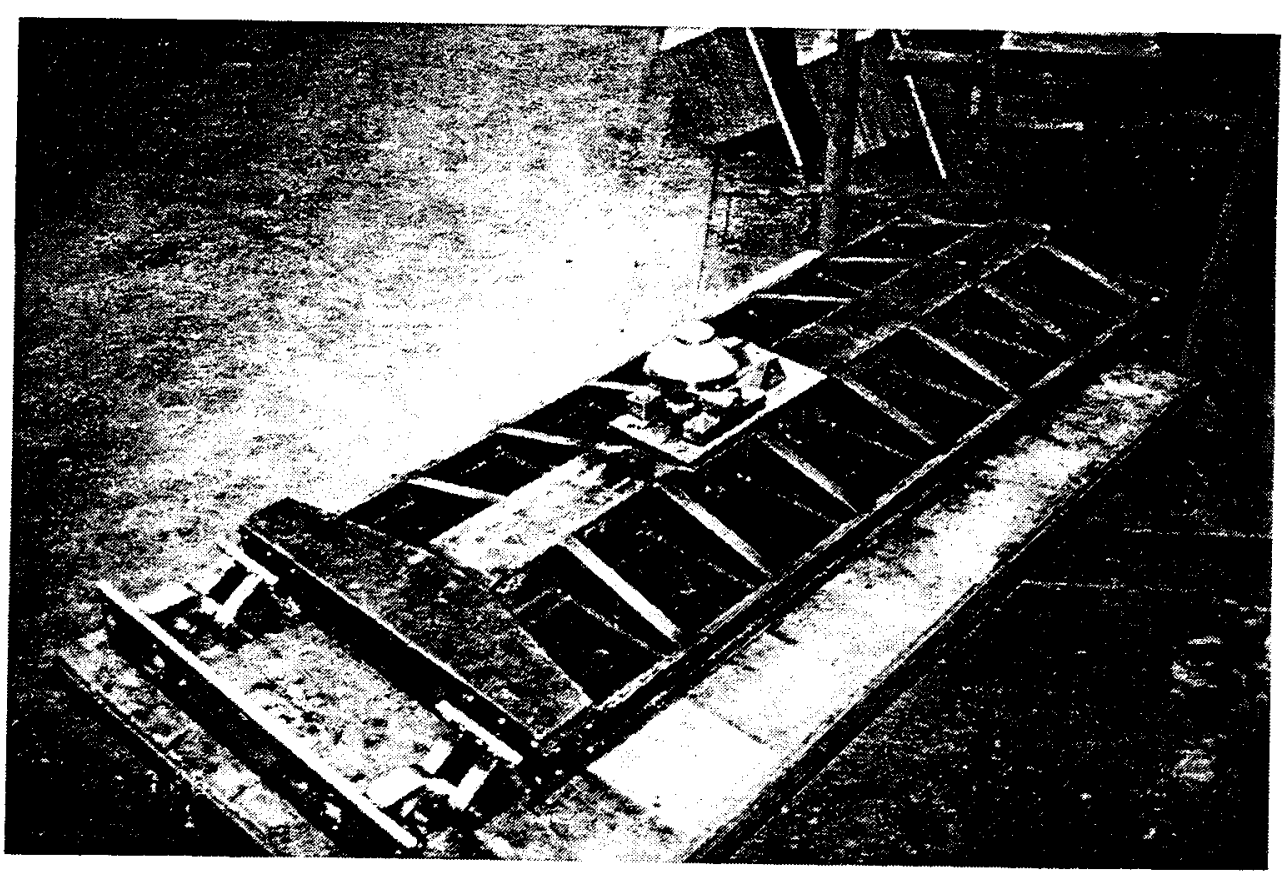

Figure 32. Finished gate frame-hinge assembly, cup-ball assembly

The second level of concrete was poured to cover the hurter-bearing frame such that the base of the hurter and cylinder frame could be installed.

Figures $42 \mathrm{a}$ through $\mathrm{c}$ show three views of the sill upon completion of the second level of concrete pouring. Figure $42 \mathrm{~b}$ shows two inclined plastic pipes inserted into the concrete to route instrumentation cables down to the bottom of the gate 


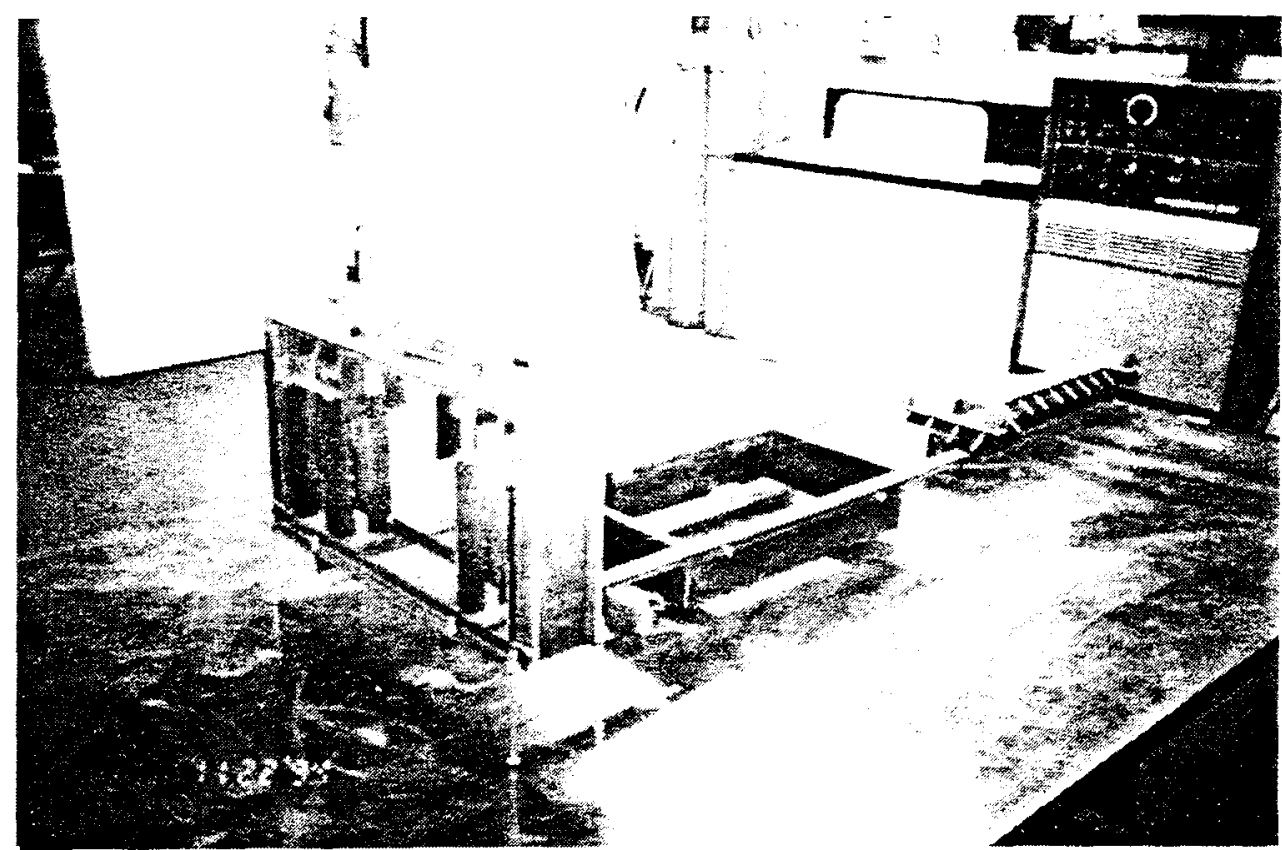

Figure 33. Relative positioning of embedded frames

base through the sill to the instrumentation room. A view shown in Figure 43 displays the steel hoses for the hydraulic lifting cylinders on the upstream side of the sill.

Figure 44 shows a finished sill with a close look at the cylinder pit. Figure 45 displays the upstream view of the flume. On the right in the figure is the upstream water control gate that regulates the water level using servo-controller mechanisms.

\section{Gate installation}

On the completed sill, the protruded anchor and alignment pins were used to anchor the gate-bearing frame. During the installation process, a crane was used to suspend the gate assembly to insert anchor pins through the slots of the bearing frame. On the suspended gate, the bearing frame was connected to the gate frame hinges attached to the base of the gate. Once the bearing frame was aligned with respect to the anchored pins and placed on the sill, the tapered pins were inserted through the holes on the anchor pins to securely lock the gate to the foundation.

The prop, the lifting cylinder, or both in tandem could be used to support the gate in the raised position. A spherical-bearing assembly was mounted in the prop head, and a prop pin was inserted through this spherical bearing to connect the prop rod to the gate as specified in the prototype design drawings. A fully raised gate at $65 \mathrm{deg}$ supported on the prop rod and cylinder is shown in Figure 46. A manual or fully automated computer system could be used to raise, lower, or stop the gate at any position. 


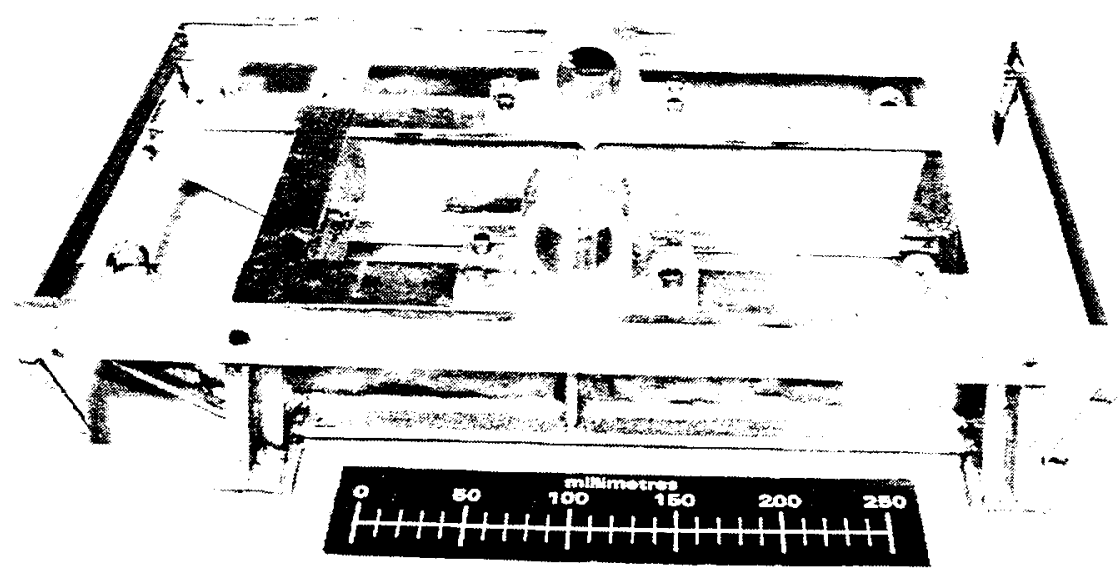

a. Front view

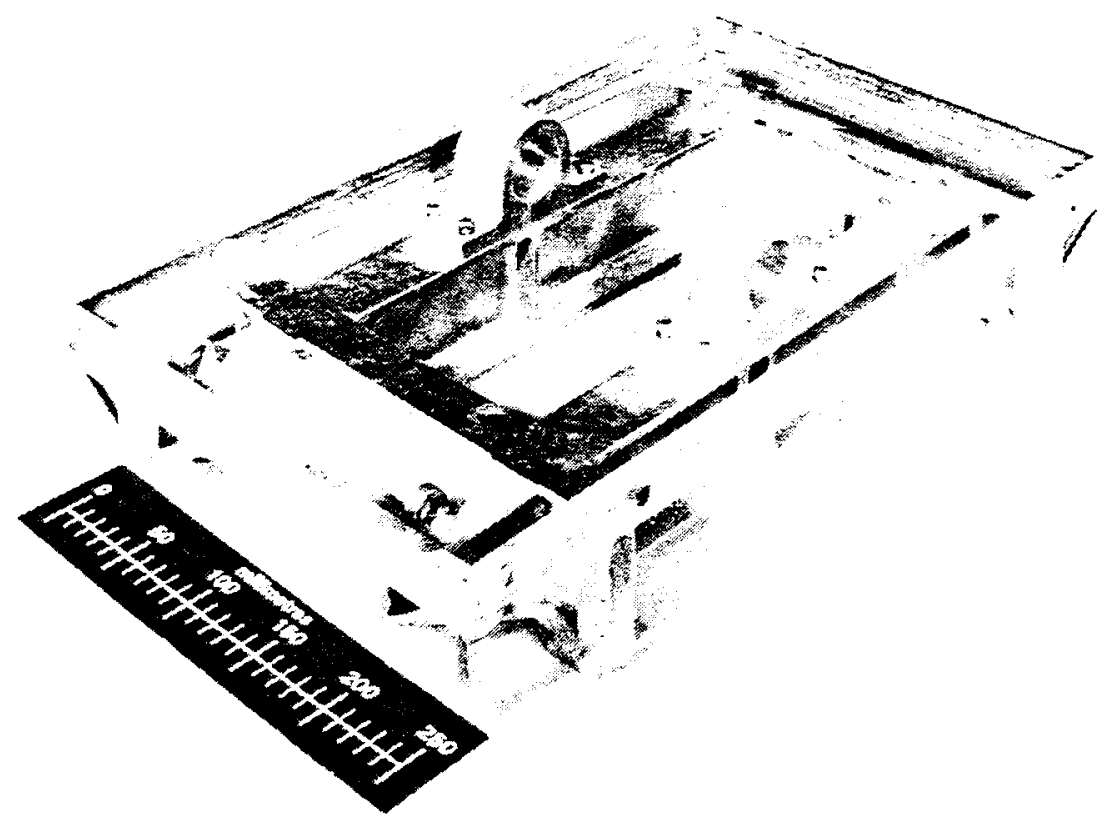

b. Isometric view

Figure 34. Closeup view of cylinder bearing frame assembly 


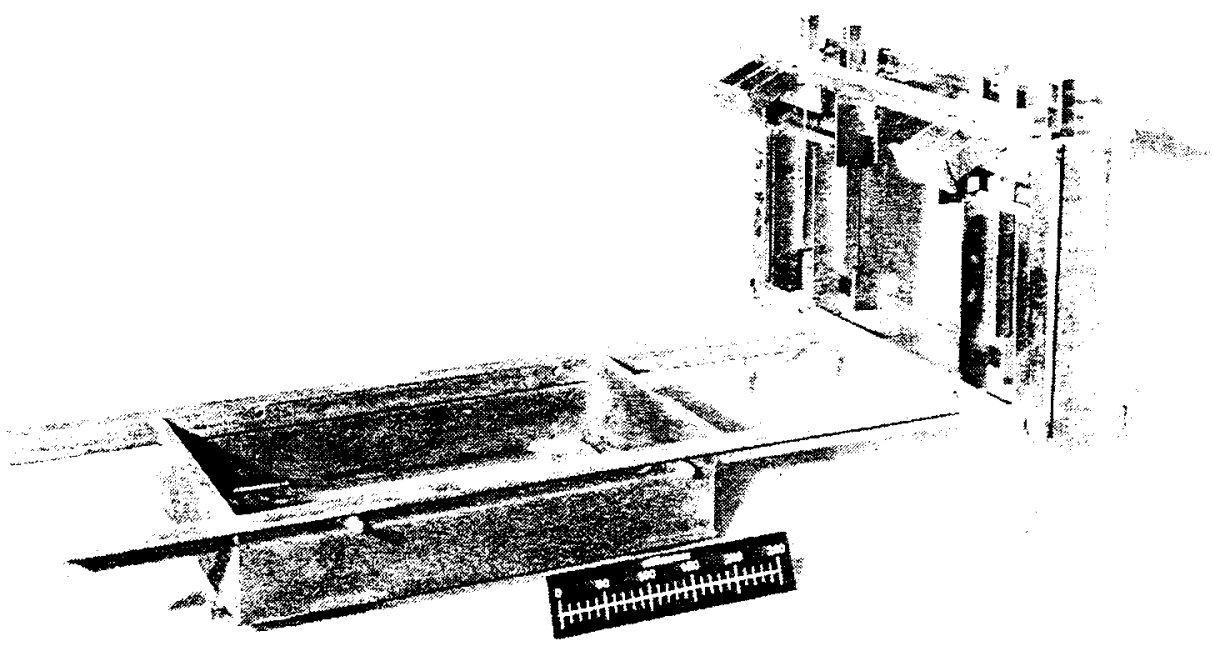

a. Isometric view

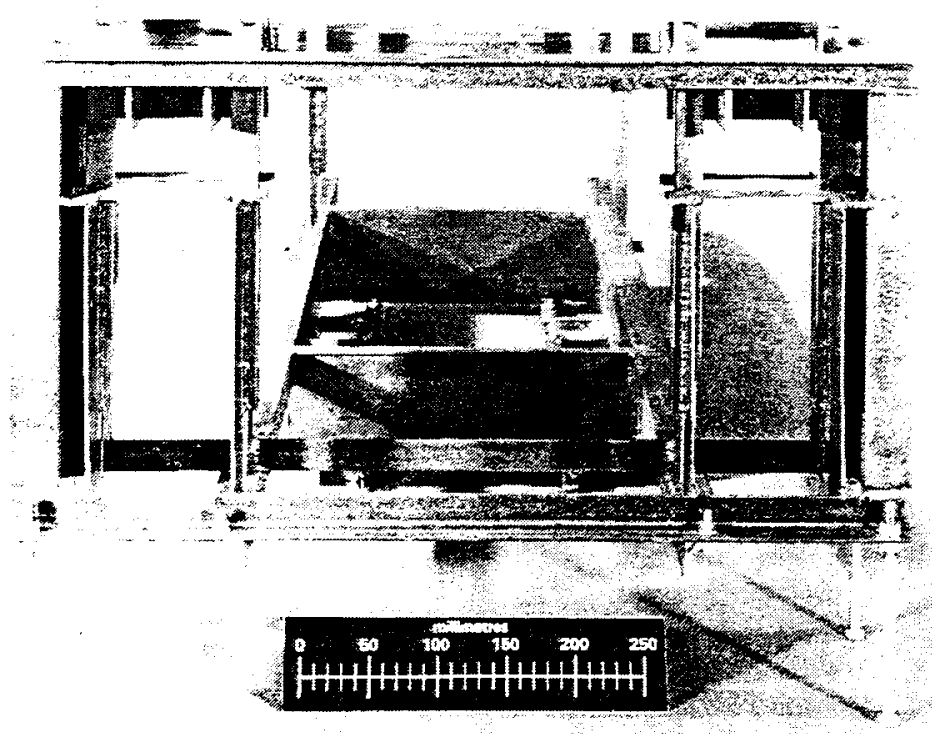

b. Front view

Figure 35. Closeup view of alignment frame assembly 


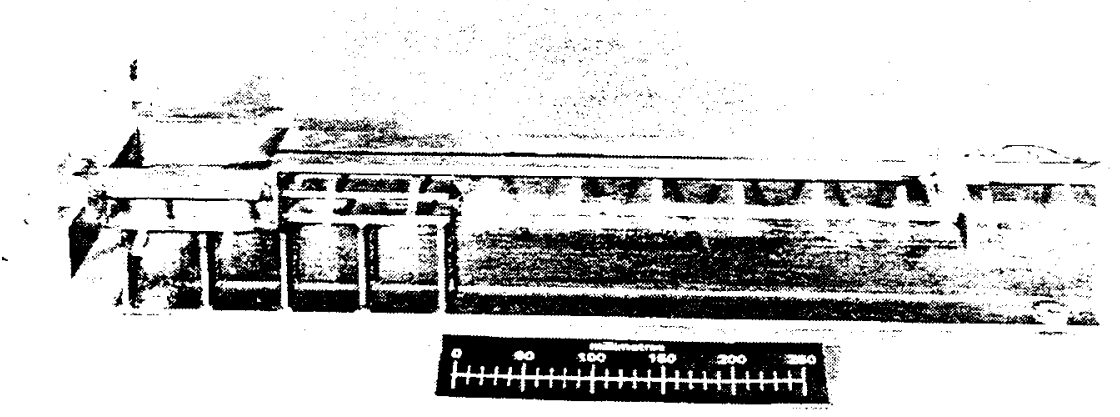

a. Front view

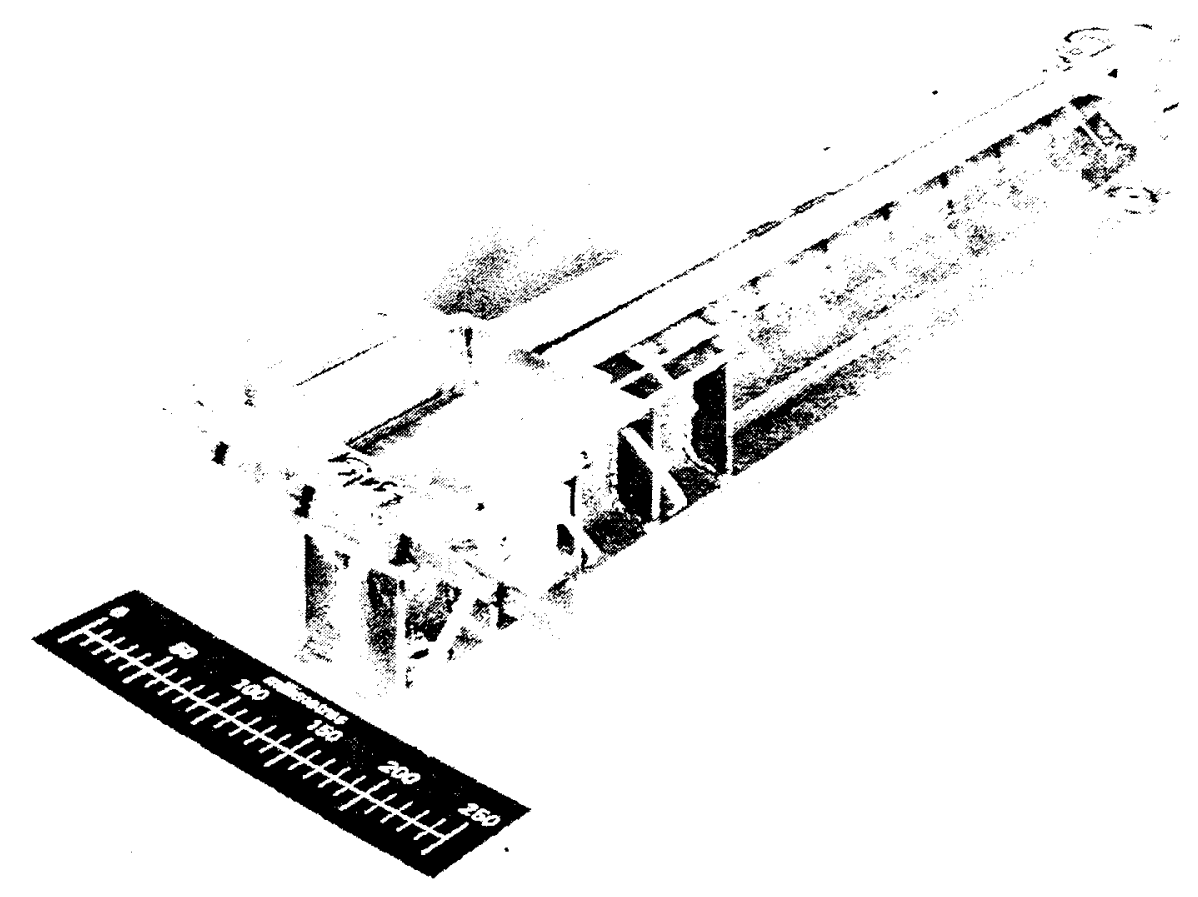

b. Isometric view

Figure 36. Hurter-bearing frame embedded in sill 


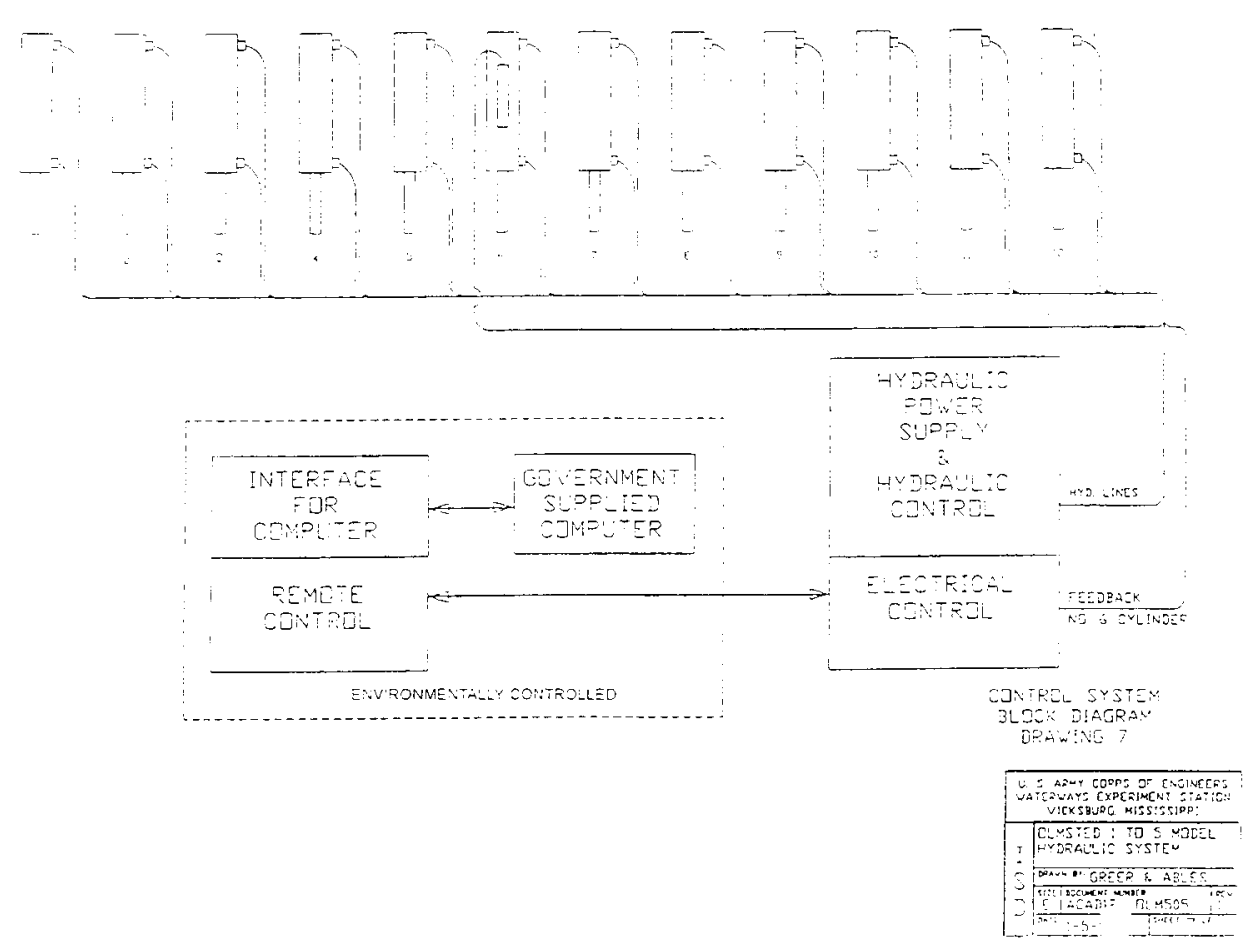

Figure 37. Schematic diagram of hydraulic module

\section{Dynamic Design Improvements}

Since project inception, numerous modifications were made in the design drawings to facilitate fabrication, correct dislocation of fitting parts, adjust design faults, and ensure smooth operation by eliminating operational difficulties. Experience and design data from the 1:25-scale flat gate model were intensively consulted to produce a better wicket design. Moreover, the current wicket design evolved as construction progressed and new ideas were incorporated to resolve detected as well as anticipated construction and operational difficulties. The 1:5-scale model construction was a testing ground for checking the accuracy of the prototype design drawings. Construction details for the current prototype design are distinctly different than the prototype design specifications used to construct the 1:25-scale model. A few of the design changes are listed.

\section{Design modifications}

Construction details of the wicket base were changed to ensure adequate support for the gate frame hinges that transfer loads from the wicket to the foundation. Interior details of supporting brackets for the modified gate frame hinges are shown in Figures 22a and $b$. An additional $0.15-\times 1$-in. webstiffener plate was mounted on the main beams to support the bottom ends of the ball mounting plate on the wide-flange beams. In the final version, altogether 


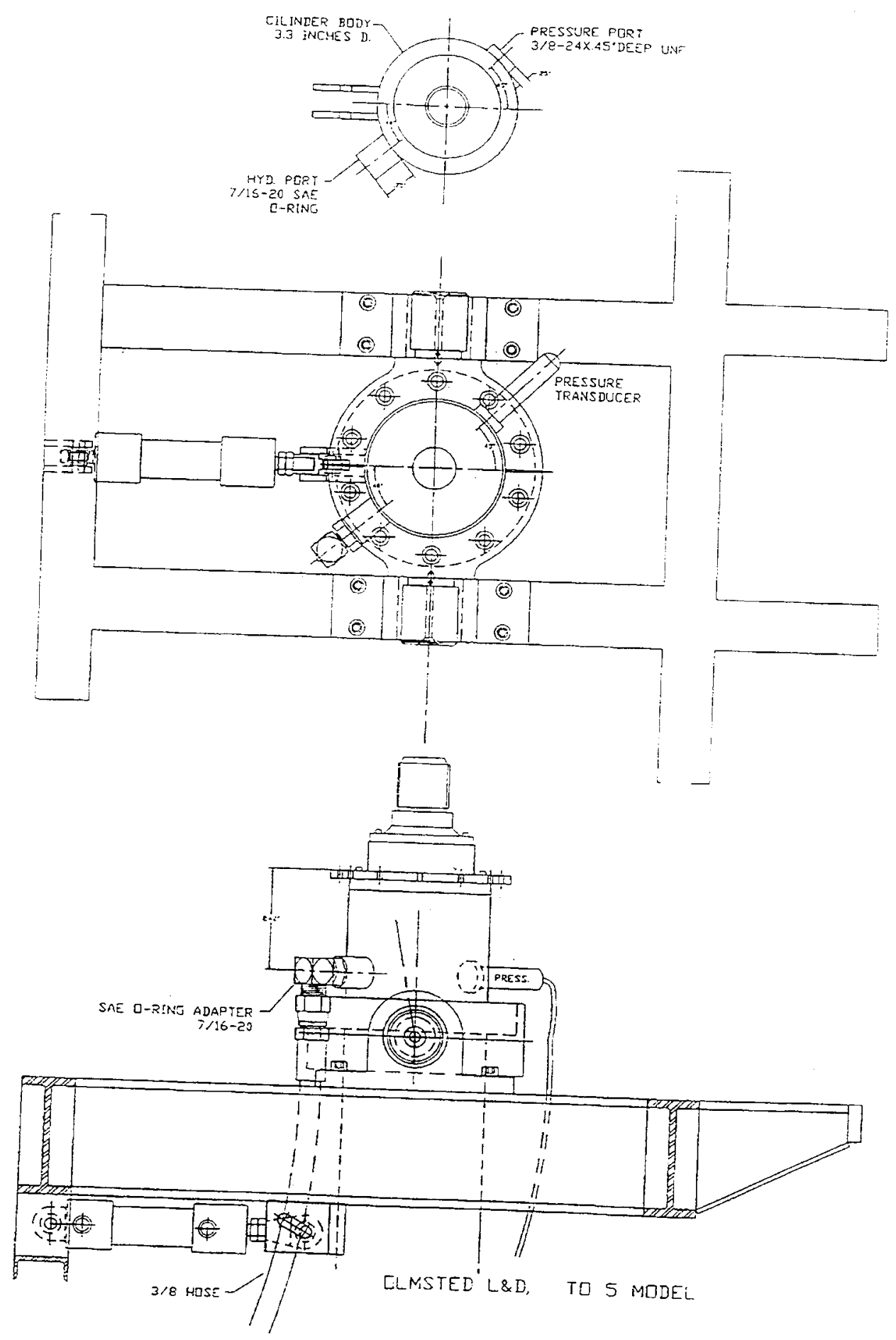

Figure 38. Connection details of retractable cylinder and support 


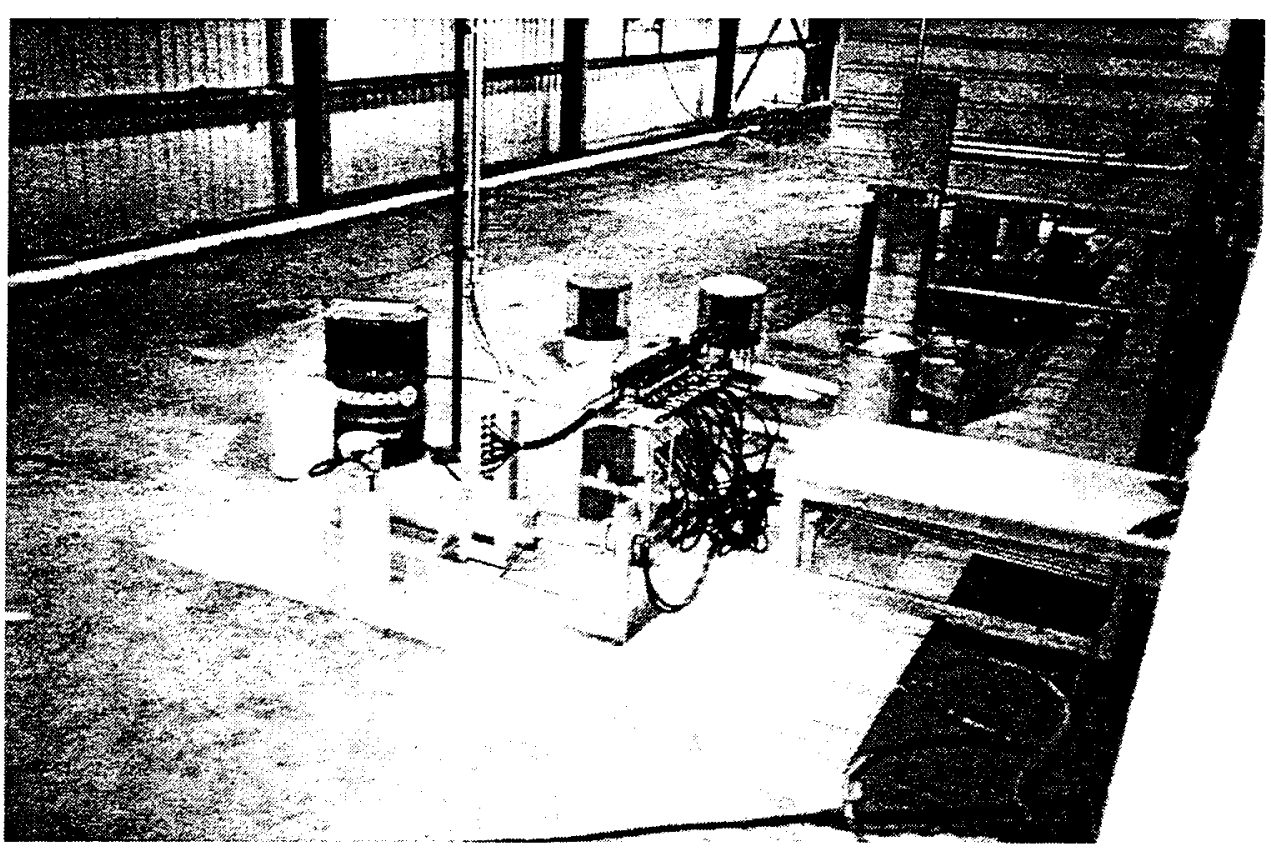

Figure 39. Hydraulic control system with auxiliary cylinder

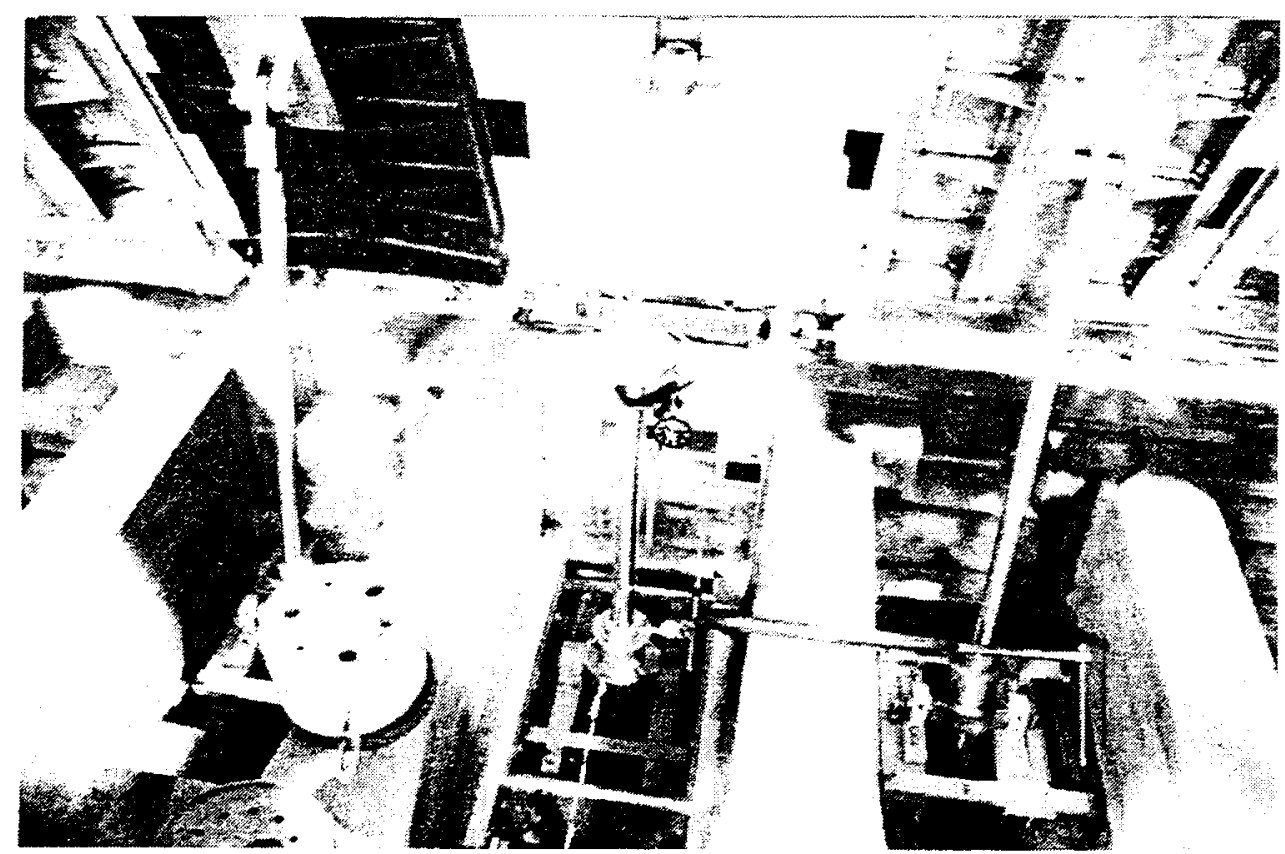

Figure 40. Hydraulic lifting cylinders installed in model 


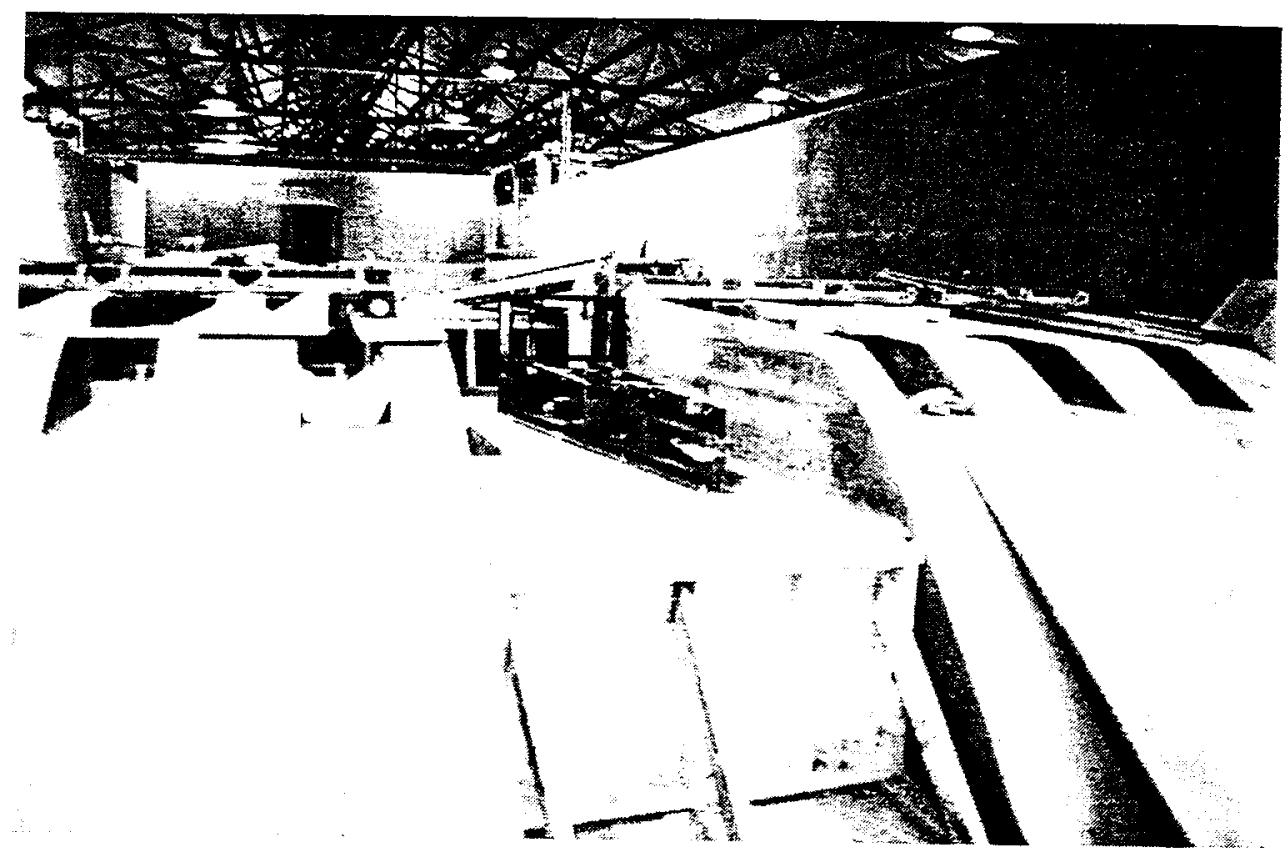

Figure 41. First level of poured concrete

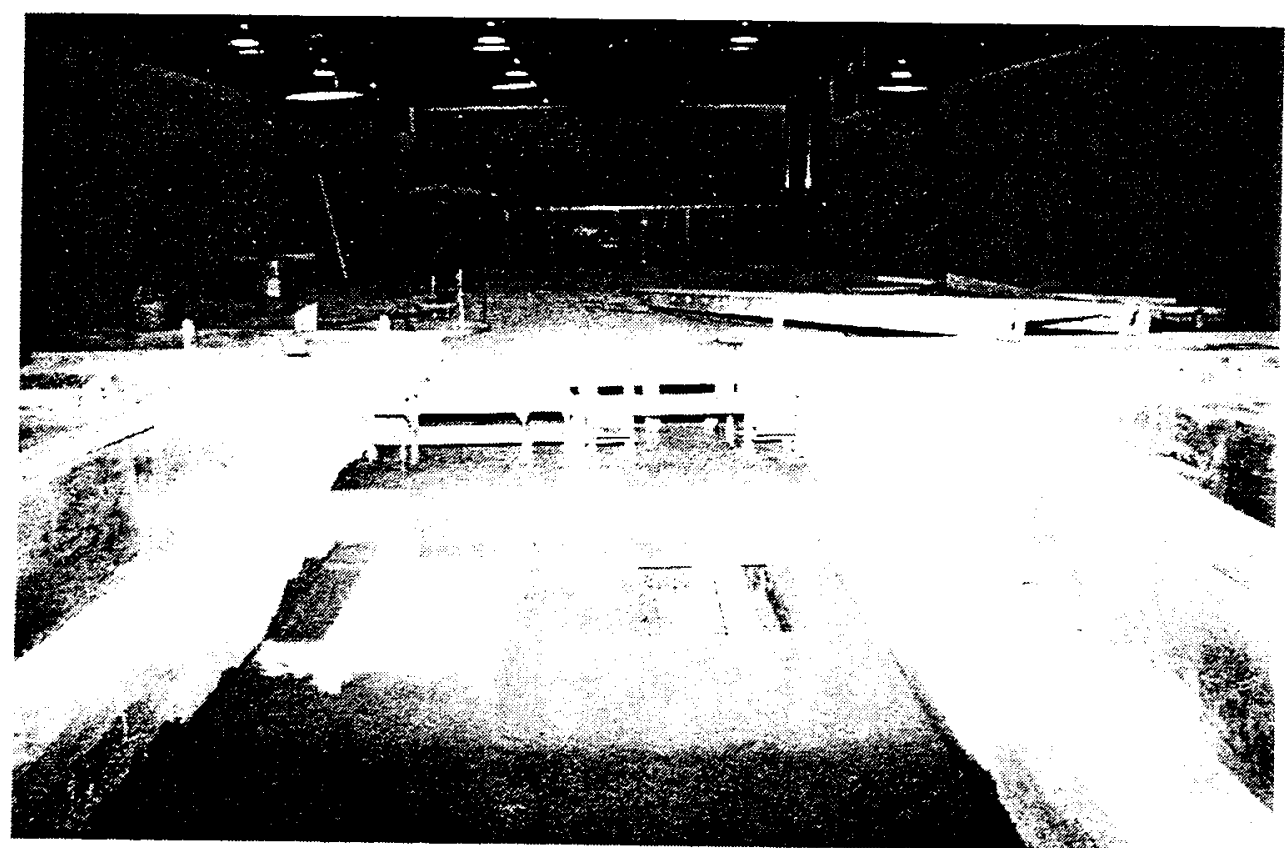

a. Overall view second level of poured concrete

Figure 42. Three views of sill after second level of poured concrete (Continued) 


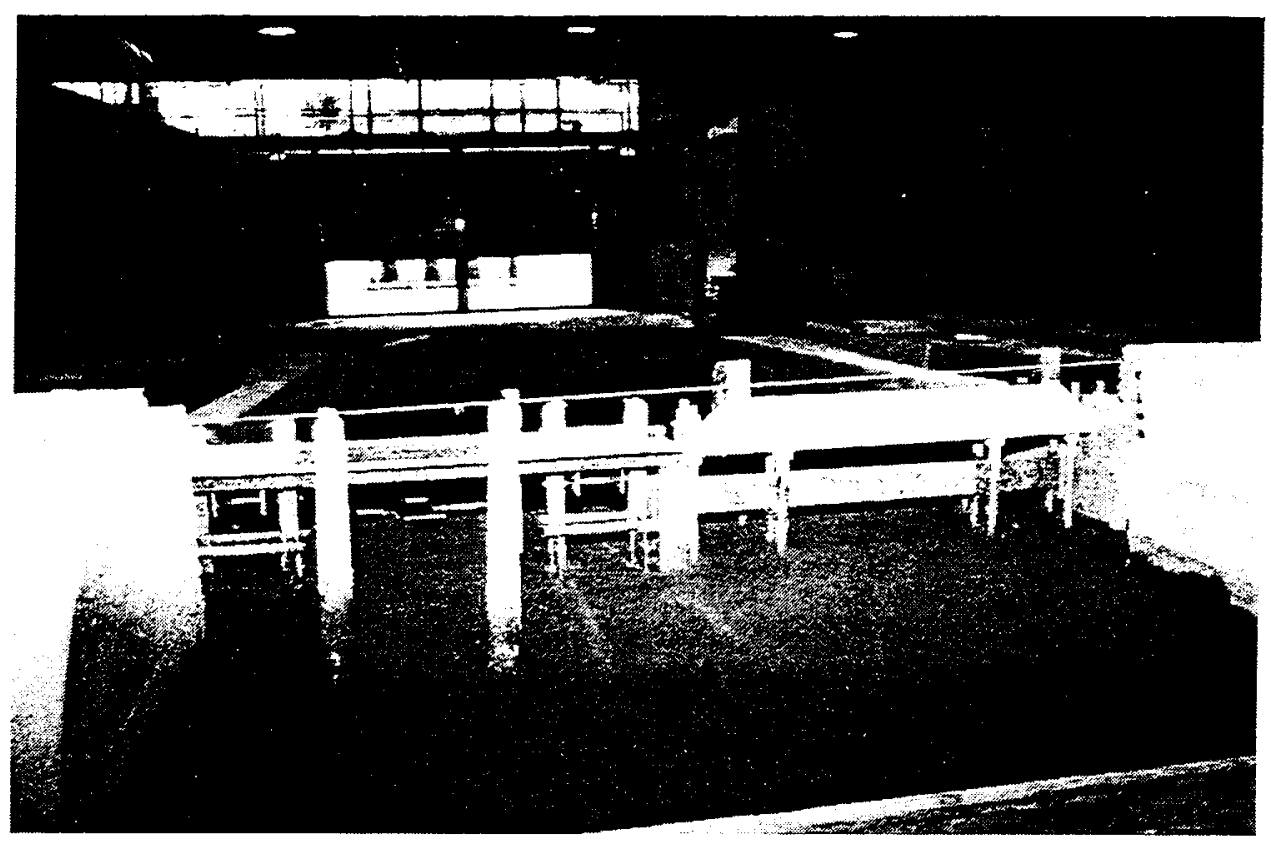

b. Constructed sill for dummy gates

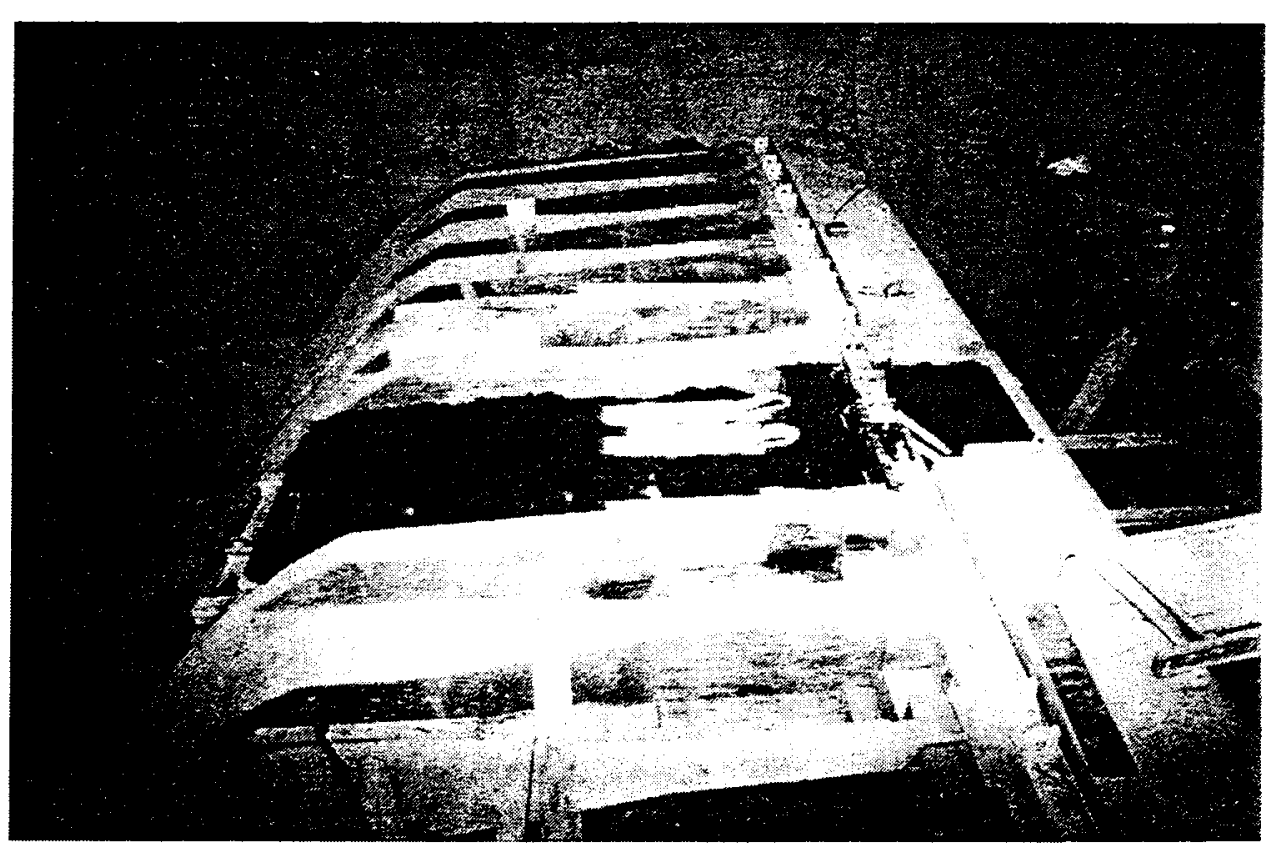

c. Embedded frame placement

Figure 42. (Concluded) 


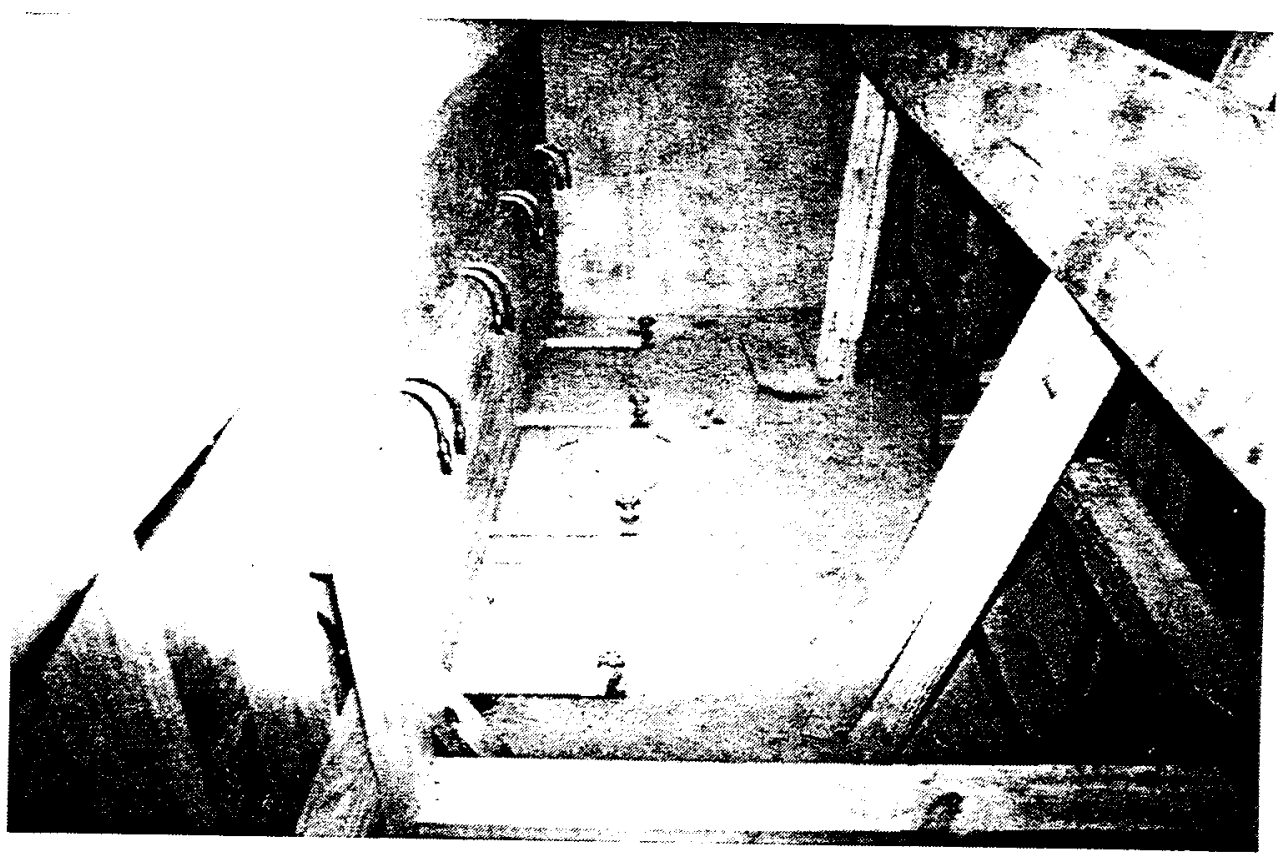

Figure 43. Cable routing pipe outlets and steel hydraulic hoses
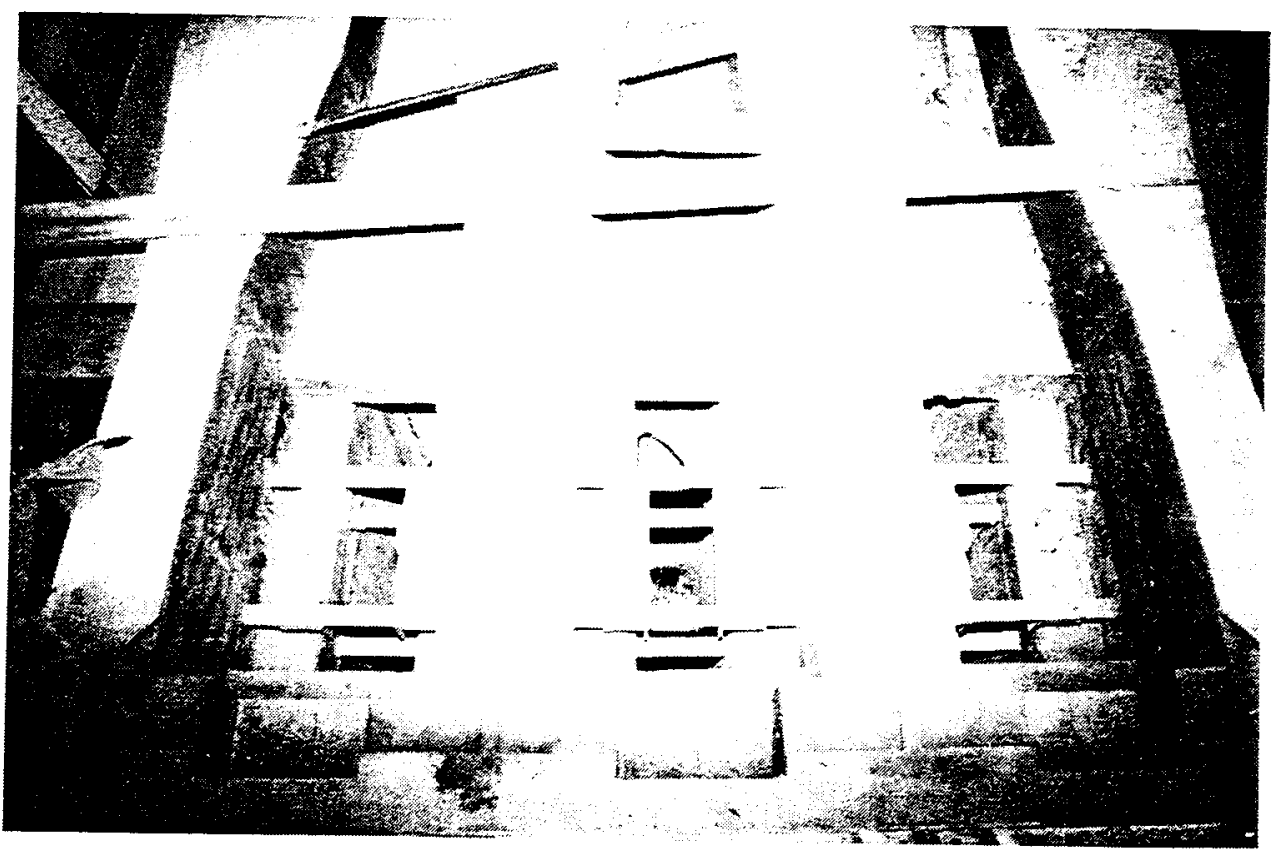

Figure 44. Finished sill with close look at cylinder pit 


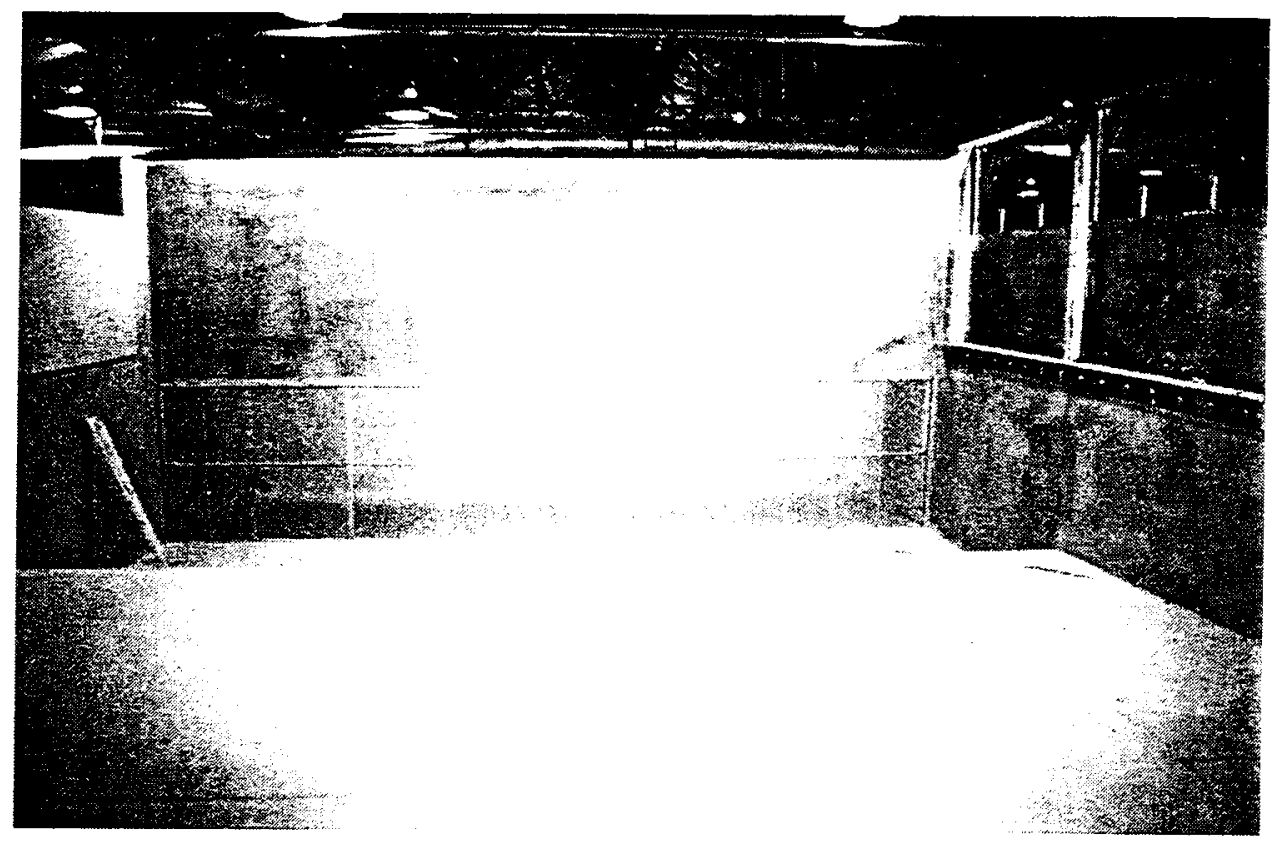

Figure 45. Upstream view of flume

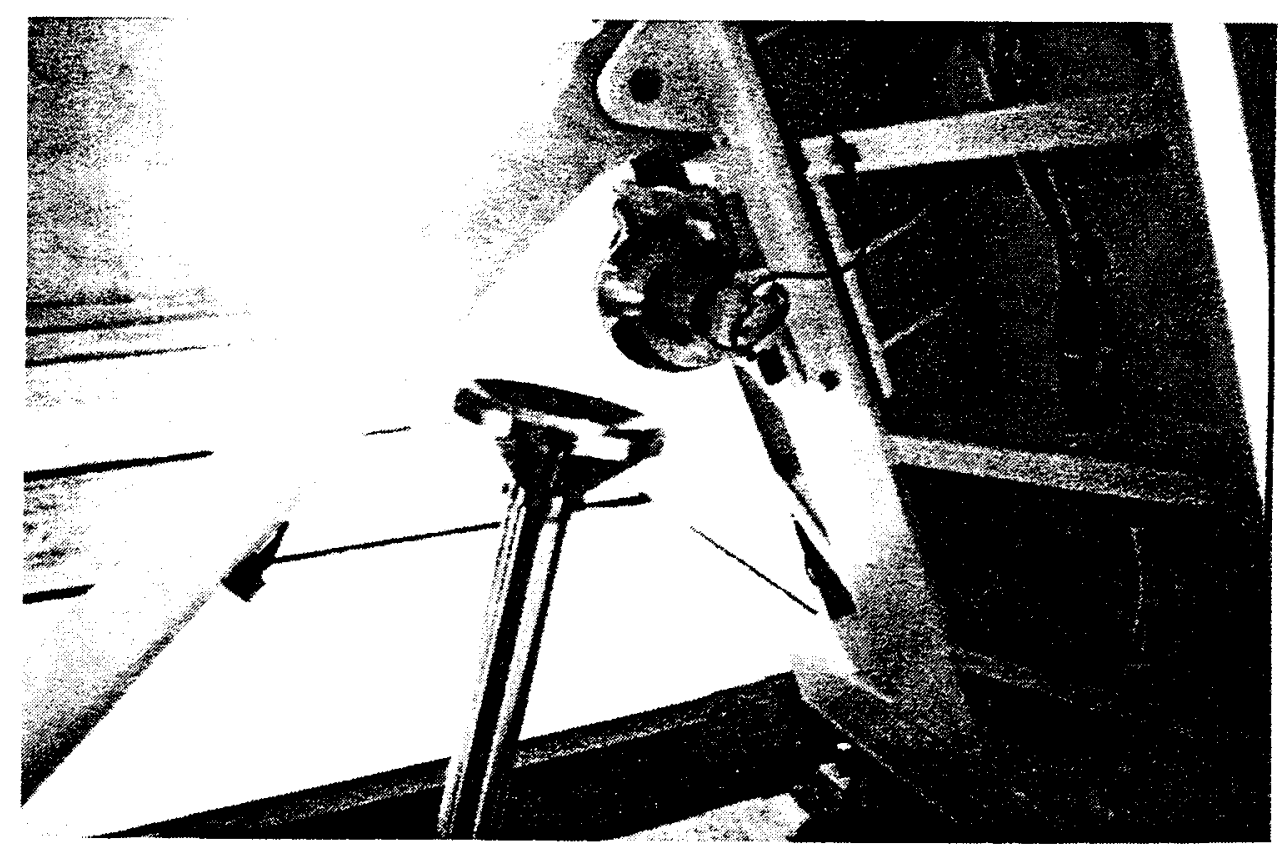

Figure 46. Closeup view of prop rod and cylinder support at $65 \mathrm{deg}$

Five sets of stiffener plates were used instead of the four shown in Figure 19. This web stiffener increased the rigidity of the ball mounting plate. The embedded alignment frame was refabricated to accommodate changes in the bearing frame base-plate thickness from 2 to $4.75 \mathrm{in}$. in the prototype. A thicker bearing plate would uniformly transfer the load from the gate hinge to the foundation. Otherwise, warping of the thin bearing plate could have damaged the sill due to uneven application of very high localized strain. 
Major changes were made in the supporting devices to adjust design faults and to fix operational difficulties. For example, the prop and hurter were completely reconstructed. The prop rod was changed from $12-\times 12-\times 5 / 8$-in. structural tube to 10 -in.-diam pipe, and finally to a 10-in.-diam rod in the latest design. The prop components had been reconstructed from a double-fork pin connection to a single-sided pin connection. The hurter had gone through substantial changes because of difficulties in keeping the prop blade on the track while raising and lowering the gate.

\section{Dry operation}

Several things did not work during the dry test of the wicket. The prop would not drop into the return trough of the hurter. Once placed in the trough, it would bind up when the gate was at about $50 \mathrm{deg}$. It would not shift completely into the raising trough and, as a result, bent the cross rod of the hold-down device. The cup made contact with the bearing bolts when the wicket had been raised above $65 \mathrm{deg}$. These difficulties were resolved by taking the following corrective measures.

The upstream "seat" on the hurter was ground at a 30-deg slope down rearward to the right, and the bearing foot was rounded on the left side. Sufficient grinding allowed the prop to drop off the seat into the return trough as designed. The right-side guide rails were ground back so the prop would not bind at that point. The top of the cam was ground at an angle \pm 30 deg down to the left, and the right side of the prop weak-link fork was ground to a rounded surface. When this was sufficiently done, the prop dropped to the bottom of the trough and stopped bending the cross rod. Bolt heads were ground down, but the cup still made contact. The cup was chamfered off $1 \mathrm{in}$. around the rim which corrected the contacting problem (Figure 46).

The cup was also machined to smoothen the transition from the 18 -in. radius cut to the 9-in. radius arc. This machining was necessary to allow the ball to roll back to the center of the cup during the operation of the gate. The guide bar connected to the bottom of the prop rod was lengthened to prevent the guide shoes from locking on the hurter tracks during the gate lowering. Extending the guide-shoe length minimized the tilting and lateral movement of the shoes that had previously obstructed the movement of the prop rod.

\section{Wet operation}

Adjustment of the hurter component design solved most prop operation problems encountered during the flow experiments. It was noticed, however, that while lowering the gate from a fully raised position, the lateral thrust of the flow caused the prop rod to hang on the front edge of one of the hurter vertical guard plates (Figure 47). At the onset of lowering the gate, no load was transferred to the prop rod. As a result, lateral thrust of the flow caused movement of the unstable prop rod over the side rail. 

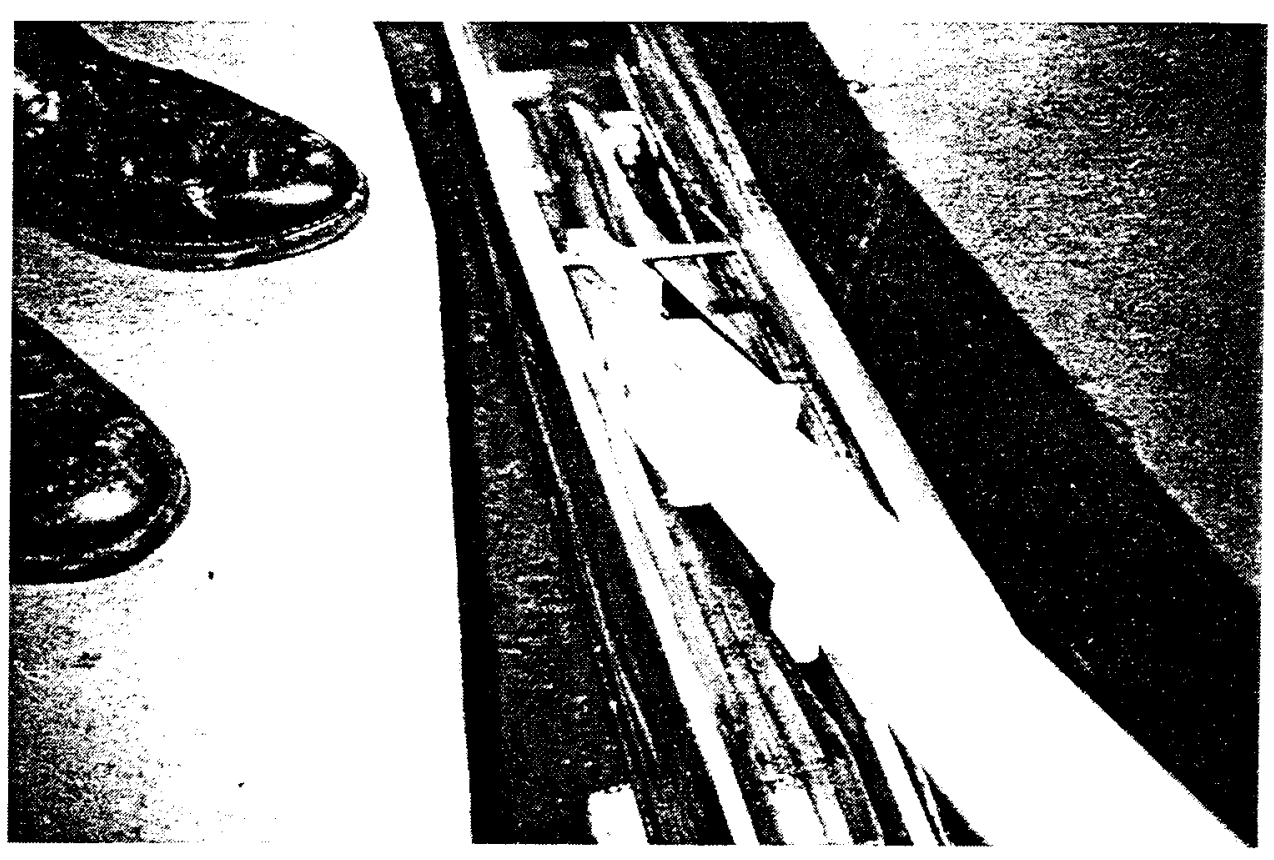

Figure 47. Prop rod hanging on front edge of hurter due to lateral thrust

Derailment of the prop blade during flow tests halted the downward motion of the lowering gate. The uplifting thrust of falling water misguided the prop rod such that its supporting edge would rest on top of the guard plate instead of following the main track. To correct the problem, the vertical guard plates on both sides of the hurter were extended toward the cylinder by 1.5 in. (Figure 48) - Also, an inclined plate was welded on top of the vertical guard plate such that any misguided prop rod will slide back to the track during the lowering of the gate.

WES detected problems that caused the shear pins connecting the prop support blade with the prop support fork to break during the two-gate gap flow tests (Figure 49). In two different instances, these shear pins broke during gate operation. The unsymmetric flow loading from the adjacent gate gap caused the prop blade to ride over the top of the hurter vertical plate which distressed the pins and initiated cracks on the prop support fork. One side of the prop support fork, at the intersection of the two forks and the base, showed a hair-line crack.

A new design of the prop support fork-blade assembly was fabricated and reinstalled in the gate. Design drawings, $M-16$, for the new prop connections are presented in Appendix A. Double-shear pin connections provided adequate strength to operate the gate. For the two-gate gap (2GG) flow conditions, the operating gate jammed several times during the lowering of the gate. Bending of the guide bar caused the guide shoes to twist and consequently block its movement along the hurter tracks (Figure 50). The guide bar was straightened and reinstalled in the model.

A series of experiments with various flow and gap configurations was conducted to demonstrate anticipated problems that could be expected during 


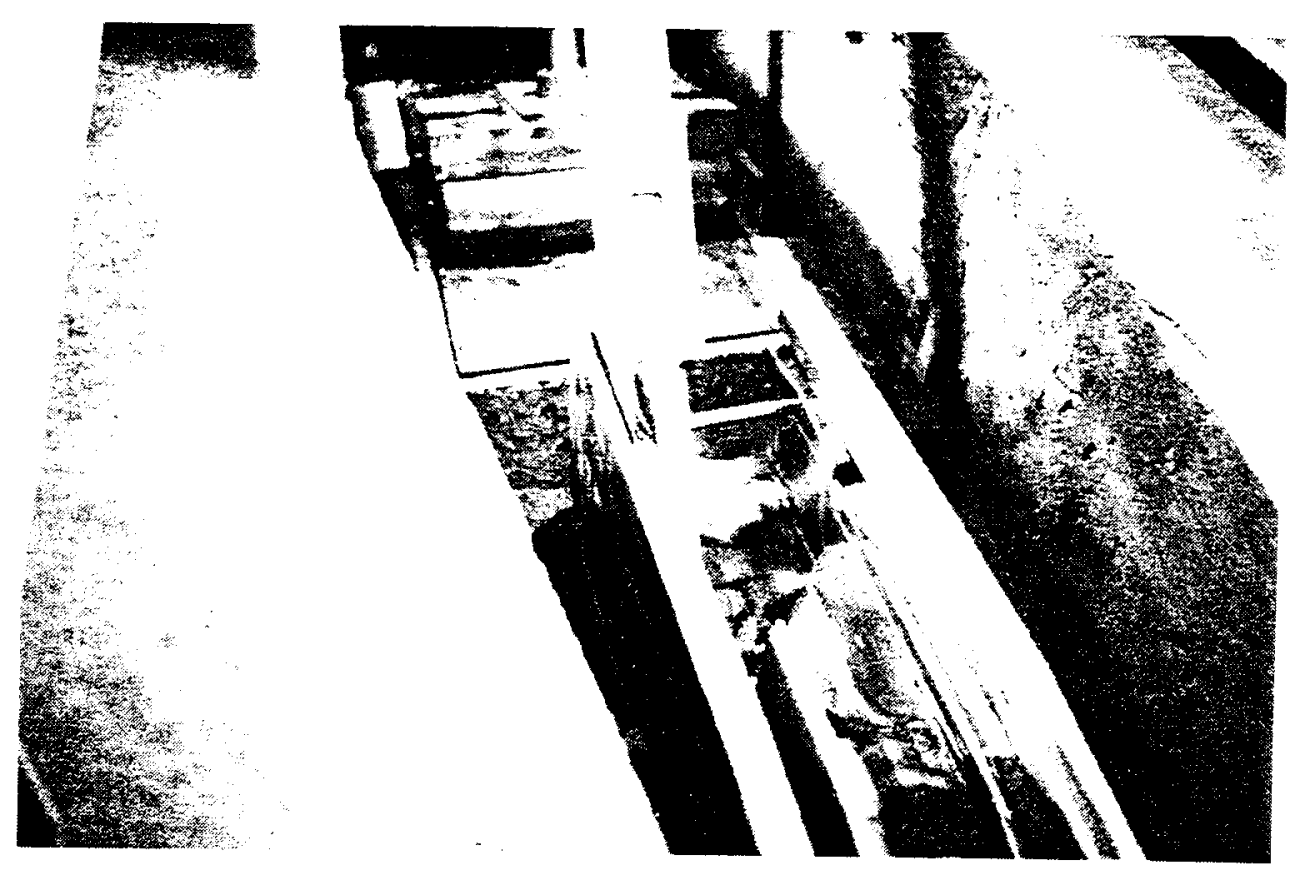

Figure 48. Extension of vertical guard plates to avoid lateral obstruction

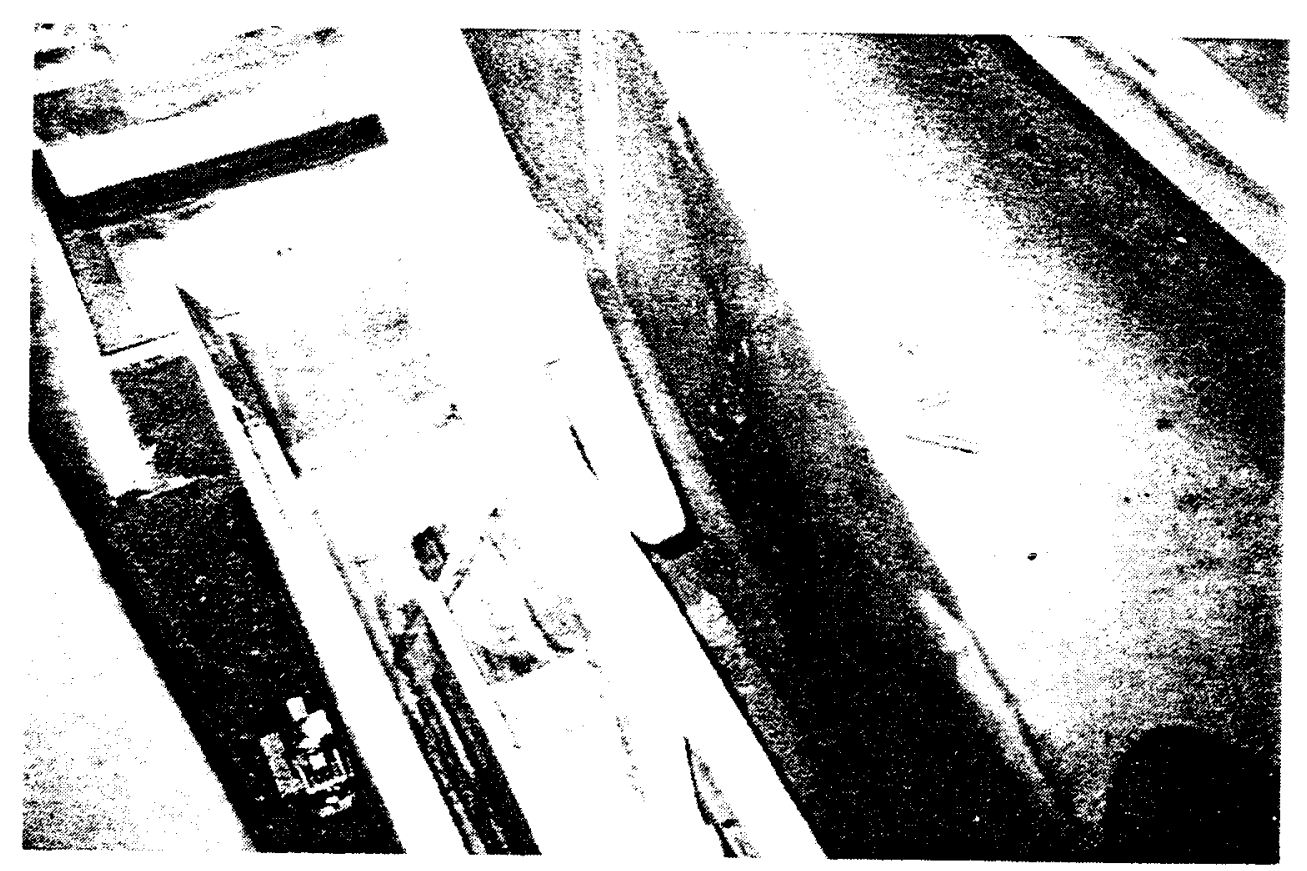

Figure 49. Prop fork breakage during 2GG flow tests 


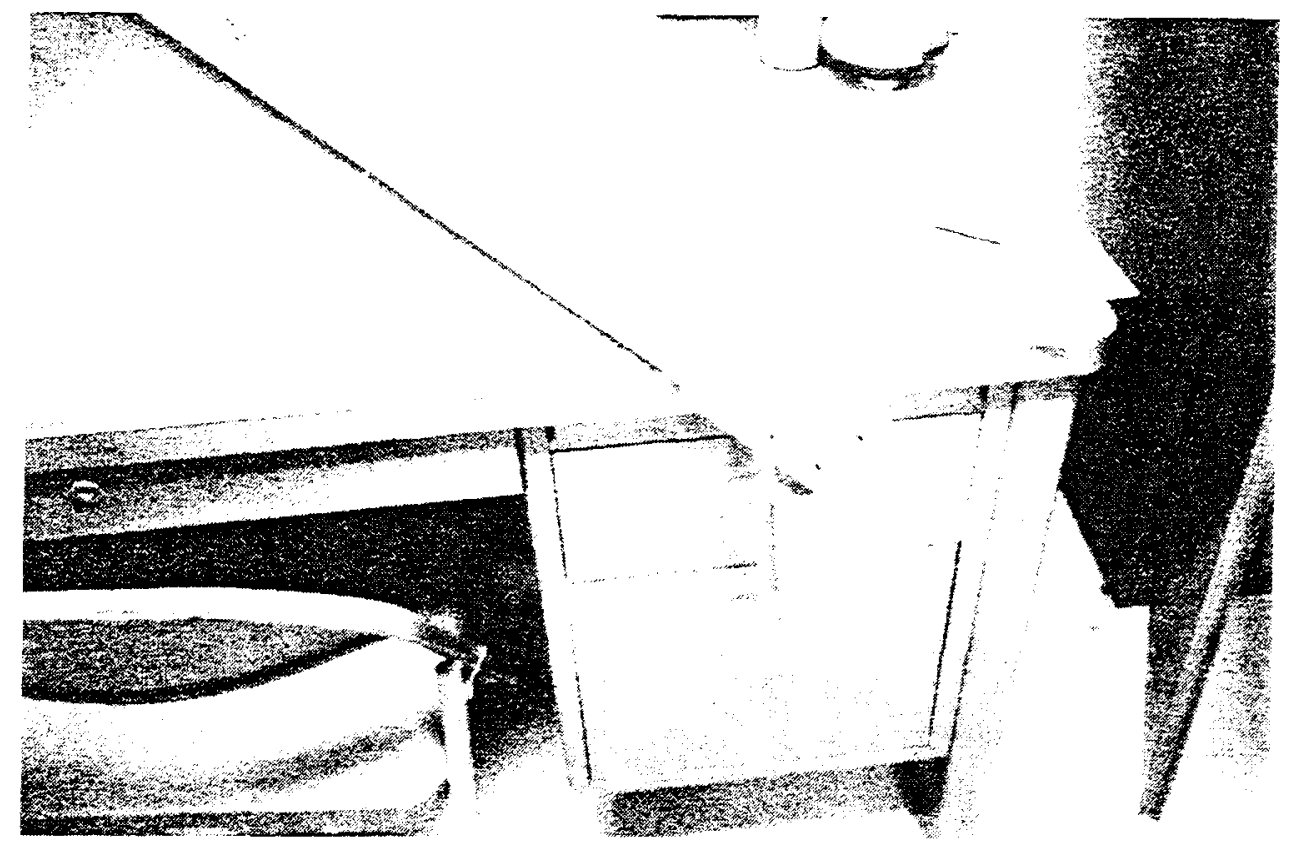

Figure 50. Twisted guide shoes due to bending of guide bar

operation of the prototype gate. It was noticed that a two-gate gap (instrumented gate being the end gate) operation could not engage the prop rod when the head difference was more than $11 \mathrm{ft}$. Two-gate gap flow conditions over $11 \mathrm{ft}$ in head difference resulted in flows causing the prop rod to float and not maintain proper location within the hurter. For this flow condition, the prop blade would not sit on the hurter recess during the retraction of the cylinder from the up position. However, a three-gate gap with the instrumented gate being the center gate could be operated with a minor chance of prop rod disengagement when the head difference was more than $16 \mathrm{ft}$. All tests were conducted without the guide bar on the prop blade. The completed gate installation in the 1:5 model and a dry operational experiment is shown in Figure 51. 

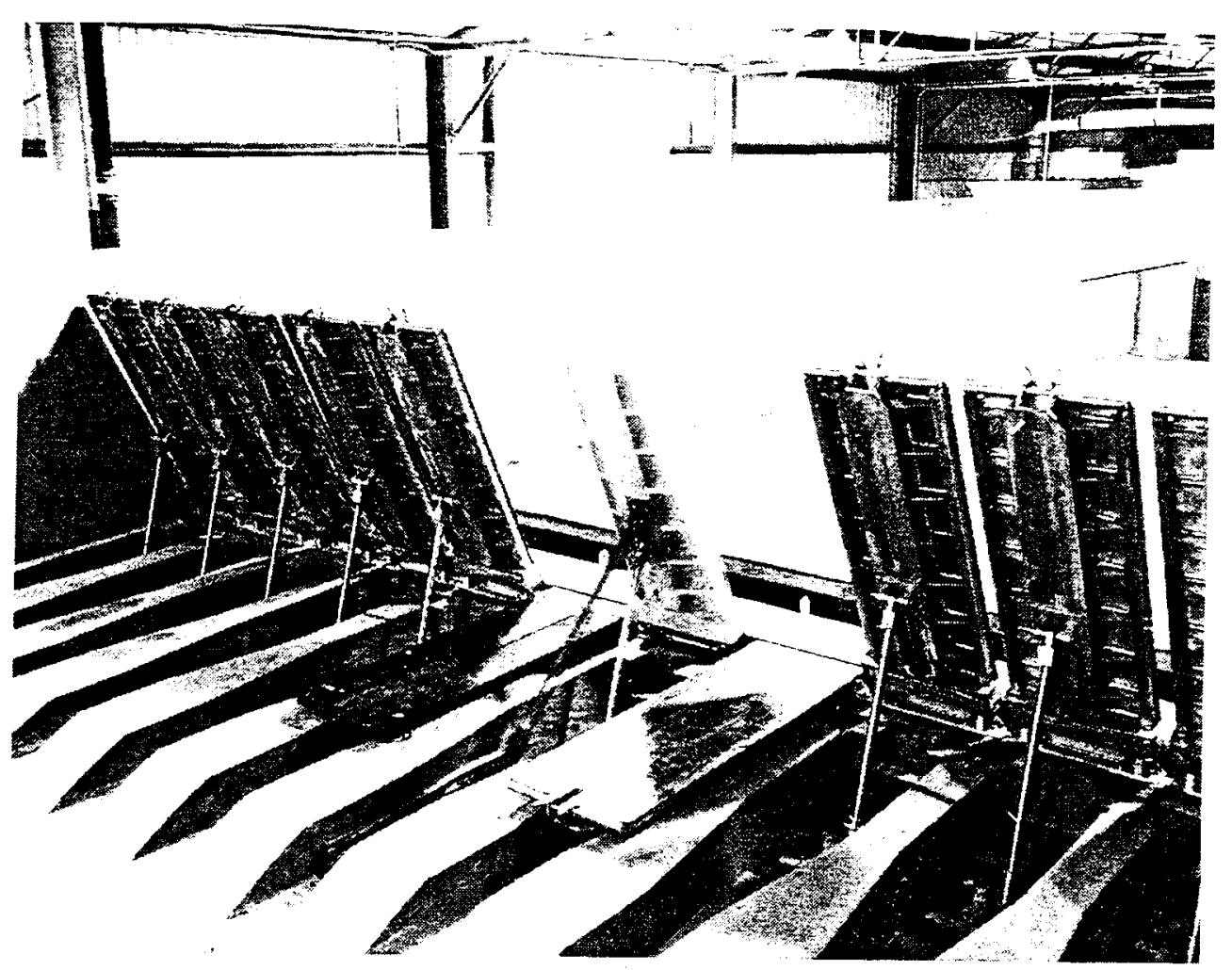

Figure 51. Completed gate installation in dry operation 


\section{Instrumentation, Data Acquisition, and Analysis}

\section{Instrumentation}

A total of 53 different transducers recorded dynamic data for the 1:5-scale Olmsted model. These transducers measured dynamic loading, reaction forces, pressure distributions, rotations, and accelerations of critical gate components. Three major groups of sensors that measured structural dynamic responses included accelerometers, pressure transducers, and shear pins. Accelerometers recorded the dynamic motion of the gate which was used to determine the operating deflected shape of the gate. Accelerometer data were also used to determine the energy content at different frequency levels. The peaks of the acceleration frequency response function (FRF) would indicate the dominant frequency of the flow-induced motion of the gate. Pressure transducers scanned the flow-induced pressure profiles on the upstream and downstream sides of the gate. Net pressure load obtained from the experiment is the total hydrodynamic pressure acting on the gate surface due to the flow-induced operating condition. Shear pins measured the reaction forces at the supports. Reaction forces measured from the experiment would be used to estimate design loads for supporting elements and to examine equilibrium of the system by summing the applied input and the reacting output forces.

The following measurements were made with the 53 transducers:

- Force perpendicular to the face of the gate at the right hinge (r.h.) and the left hinge (1.h.).

- Force parallel to the face of the gate at the r.h. and the I.h..

- Force perpendicular to the face of the gate at the ball.

- Force parallel to the face of the gate at the ball.

- Force on the right side and the left side of the trunnion.

- Pressure on the upstream (U/S) side of the gate at nine locations. 
- Pressure on the downstream $(\mathrm{D} / \mathrm{S})$ side of the gate at nine locations.

- Atmospheric pressure.

- Pressure at the top of the hydraulic cylinder.

- Pressure at the bottom of the hydraulic cylinder.

- U/S pool elevation (measured with a pressure transducer).

- D/S pool elevation (measured with a pressure transducer).

- Accelerations at 15 locations and orientations on the gate.

- Gate rotation.

- Hydraulic cylinder rotation.

- Coupler ball rotation.

- Hydraulic cylinder shaft position.

Table 3 presents the entire sensor list for the instrumented gate. In this table, the second column shows the attributes corresponding to each channel number in

\begin{tabular}{|c|c|c|c|c|c|}
\hline \multicolumn{6}{|c|}{$\begin{array}{l}\text { Table } 3 \\
\text { Instrumentation List for 1:5 Olmsted Wicket Model }\end{array}$} \\
\hline $\begin{array}{l}\text { Channel } \\
\text { Number }\end{array}$ & $\begin{array}{l}\text { Type } \\
\text { gauge }\end{array}$ & Location & Model & $\begin{array}{l}\text { Serial } \\
\text { Number }\end{array}$ & $\begin{array}{l}\text { Full Capacity } \\
\text { in Measuring } \\
\text { Units }\end{array}$ \\
\hline 9 & Force & $\begin{array}{l}\text { Right hinge, } \\
\text { Z-direction }\end{array}$ & Shear pin & 5701 & $1,500 \mathrm{lbf}$ \\
\hline 2 & Force & $\begin{array}{l}\text { Right hinge, } \\
\text { Y-direction }\end{array}$ & Shear pin & 5701 & $1,500 \mathrm{lbf}$ \\
\hline 3 & Force & $\begin{array}{l}\text { Left hinge, } \\
\text { Z-direction }\end{array}$ & Shear pin & 5700 & $1,500 \mathrm{lbf}$ \\
\hline 4 & Force & $\begin{array}{l}\text { Left hinge, } \\
\text { Y-direction }\end{array}$ & Shear pin & 5700 & $1,500 \mathrm{lbf}$ \\
\hline 5 & Force & Ball, Z & Shear pin & 5698 & $2,500 \mathrm{lbf}$ \\
\hline 6 & Force & Ball, $Y$ & Shear pin & 5698 & $2,500 \mathrm{lbf}$ \\
\hline 7 & Force & Trunnion, right & Shear pin & $5933 \mathrm{~A}$ & $2,500 \mathrm{lbf}$ \\
\hline 8 & Force & Trunnion, left & Shear pin & $5933 A$ & $2,500 \mathrm{lbf}$ \\
\hline 9 & Force & Prop rod & Shear pin & 5699 & $2,500 \mathrm{lbf}$ \\
\hline 10 & Pressure & $\begin{array}{l}\text { (P1) U/S top } \\
\text { center (T.C.) }\end{array}$ & Kulite XTM-190-10A & U43-18 & \\
\hline 11 & Pressure & $2 \mathrm{U} / \mathrm{ST}$ T.C. & Kulite XTM-190-10A & $\times 23-31$ & 276.9 in. water \\
\hline 12 & Pressure & $\begin{array}{l}3 \mathrm{U} / \mathrm{S} \text { top left } \\
(\mathrm{T} \text {.L.) }\end{array}$ & Kulite XTM-190-10A & $223-27$ & 276.9 in. water \\
\hline 13 & Pressure & $\begin{array}{l}4 \mathrm{U} / \mathrm{S} \text { middle } \\
\text { right (M.R.) }\end{array}$ & Kulite XTM-190-10A & $R 22-69$ & 276.9 in water \\
\hline
\end{tabular}




\begin{tabular}{|c|c|c|c|c|c|}
\hline \multicolumn{6}{|c|}{ Table 3 (Concluded) } \\
\hline $\begin{array}{l}\text { Channel } \\
\text { Number }\end{array}$ & $\begin{array}{l}\text { Type } \\
\text { gauge }\end{array}$ & Location & Model & $\begin{array}{l}\text { Serial } \\
\text { Number }\end{array}$ & $\begin{array}{l}\text { Full Capacity } \\
\text { in Measuring } \\
\text { Units }\end{array}$ \\
\hline 14 & Pressure & $\begin{array}{l}5 \mathrm{U} / \mathrm{S} \text { middle } \\
\text { center (M.C.) }\end{array}$ & Kulite XTM-190-10A & U23-15 & $\begin{array}{l}276.9 \text { in. } \\
\text { water }\end{array}$ \\
\hline 15 & Pressure & $\begin{array}{l}6 \text { U/S middle left } \\
\text { (M.L.) }\end{array}$ & Kulite XTM-190-10A & U23-17 & $\begin{array}{l}276.9 \text { in. } \\
\text { water }\end{array}$ \\
\hline 16 & Pressure & $\begin{array}{l}7 \mathrm{U} / \mathrm{S} \text { bottom } \\
\text { right (B.R.) } \\
\end{array}$ & Kulite XTM-190-10A & R22-74 & $\begin{array}{l}276.9 \text { in. } \\
\text { water }\end{array}$ \\
\hline 17 & Pressure & $\begin{array}{l}\mathrm{UU} / \mathrm{S} \text { bottom } \\
\text { center (B.C.) } \\
\end{array}$ & Kulite XTM-190-10A & S22-16 & $\begin{array}{l}276.9 \text { in. } \\
\text { water }\end{array}$ \\
\hline 18 & Pressure & $\begin{array}{l}9 \text { U/S bottom left } \\
\text { (B.L.) }\end{array}$ & Kulite XTM-190-10A & R22-78 & $\begin{array}{l}276.9 \text { in. } \\
\text { water }\end{array}$ \\
\hline 19 & Pressure & $\begin{array}{l}10 \mathrm{D} / \mathrm{S} \text { top right } \\
\text { (T.R.) }\end{array}$ & Kulite XTM-190-10A & $\mathrm{G} 24-74$ & $\begin{array}{l}276.9 \mathrm{in} . \\
\text { water }\end{array}$ \\
\hline 20 & Pressure & 11 D/S T.C. & Kulite XTM-190-10A & $\times 23-33$ & $\begin{array}{l}276.9 \text { in. } \\
\text { water }\end{array}$ \\
\hline 21 & Pressure & 12 D/S T.L. & Kulite XTM-190-10A & $\times 23-32$ & $\begin{array}{l}276.9 \text { in. } \\
\text { water }\end{array}$ \\
\hline 22 & Pressure & $13 \mathrm{D} / \mathrm{S}$ M.R. & Kulite XTM-190-10A & S22-19 & $\begin{array}{l}276.9 \text { in. } \\
\text { water }\end{array}$ \\
\hline 23 & Pressure & 14 D/S M.C. & Kulite XTM-190-10A & $\mathrm{S} 22-13$ & $\begin{array}{l}276.9 \text { in. } \\
\text { water }\end{array}$ \\
\hline 24 & Pressure & $15 \mathrm{D} / \mathrm{S} \mathrm{M} . \mathrm{L}$. & Kulite XTM-190-10A & Z23-26 & $\begin{array}{l}276.9 \text { in. } \\
\text { water }\end{array}$ \\
\hline 25 & Pressure & $16 \mathrm{D} / \mathrm{S}$ B.R. & Kulite XTM-190-10A & S22-20 & $\begin{array}{l}276.9 \text { in. } \\
\text { water }\end{array}$ \\
\hline 26 & Pressure & 17 D/S B.C. & Kulite XTM-190-10A & $223-28$ & $\begin{array}{l}276.9 \text { in. } \\
\text { water }\end{array}$ \\
\hline 27 & Pressure & $18 \mathrm{D} / \mathrm{S}$ B.L. & Kulite XTM-190-10A & G24-76 & $\begin{array}{l}276.9 \text { in. } \\
\text { water }\end{array}$ \\
\hline 28 & Pressure & $\begin{array}{l}\text { Cylinder \#6 } \\
\text { (upper) }\end{array}$ & & 409021 & \\
\hline 29 & Pressure & $\begin{array}{l}\text { Cylinder \#6 } \\
\text { (lower) }\end{array}$ & & 409027 & \\
\hline 30 & Accel & 1XT.R. & Triaxial PCB 339A01 & 288 & $\pm 50 \mathrm{gpk}$ \\
\hline 31 & Accel & 1y T.R. & Triaxial PCB 339A01 & 288 & $\pm 50 \mathrm{gpk}$ \\
\hline 32 & Accel & $1 z$ T.R. & Triaxial PCB 339A01 & 288 & $\pm 50 \mathrm{gpk}$ \\
\hline 33 & Accel & 2 T.C. & Uniaxial PCB 353B68 & 7261 & $\pm 50 \mathrm{gpk}$ \\
\hline 34 & Accel & $3 \times$ T.L. & Triaxial PCB 339A01 & 289 & $\pm 50 \mathrm{gpk}$ \\
\hline 35 & Accel & 3y T.L. & Triaxial PCB 339A01 & 289 & $\pm 50 \mathrm{gpk}$ \\
\hline 36 & Accel & $3 z$ T.L. & Triaxial PCB 339A01 & 289 & $\pm 50 \mathrm{gpk}$ \\
\hline 37 & Accel & 4 M.R. & Uniaxial PCB 353868 & 7260 & $\pm 50 \mathrm{gpk}$ \\
\hline 38 & Accel & 5x M.C. & Triaxial PCB 339A01 & 263 & $\pm 50 \mathrm{gpk}$ \\
\hline 39 & Accel & 5y M.C. & Triaxial PCB 339A01 & 263 & $\pm 50 \mathrm{gpk}$ \\
\hline 40 & Accel & $5 z$ M.C. & Triaxial PCB 339A01 & 263 & $\pm 50 \mathrm{gpk}$ \\
\hline 41 & Accel & 6 M.L. & Uniaxial PCB 353B68 & 7258 & $\pm 50 \mathrm{gpk}$ \\
\hline 42 & Accel & 7 B.R. & Uniaxial PCB 353B68 & 7256 & $\pm 50 \mathrm{gpk}$ \\
\hline 43 & Accel & 8 B.C. & Uniaxial PCB 353868 & 7259 & $\pm 50 \mathrm{gpk}$ \\
\hline 44 & Accel & 9 B.L. & Uniaxial PCB 353B68 & 7257 & $\pm 50 \mathrm{gpk}$ \\
\hline 45 & Accel & Prop rod vert. & Triaxial PCB $339 A 01$ & 498 & $\pm 50 \mathrm{gpk}$ \\
\hline 46 & Accel & $\begin{array}{l}\text { Prop rod U/S, } \\
\text { D/S }\end{array}$ & Triaxial PCB $339 \mathrm{A01}$ & 498 & $\pm 50 \mathrm{gpk}$ \\
\hline 47 & Tiltmeter & Gate angle & & 1423 & \\
\hline 48 & Tiltmeter & Cylinder angle & & 1424 & \\
\hline 49 & Tiltmeter & Ball angle & & 1425 & \\
\hline 50 & Position & Actuator \#6 & & & \\
\hline 51 & Elevation & Headwater (ft) & & 94382011 & \\
\hline 52 & Elevation & Tailwater $(\mathrm{ft})$ & & 94382012 & \\
\hline 53 & Pressure & Barometric & & 432290 & \\
\hline
\end{tabular}


the first column. Types of gauges and their respective locations are shown in the third and fourth columns. When available, full-range capacity and model

number are also listed.

\section{Shear pins}

The first nine channels shown in Table 6 measured the reaction forces. Each hinge pin was double-sheared and was locked to the gate to rotate with it. Each pin had two mutually orthogonal sensitive axes, aligned so that one axis was normal to the gate plane (Z-direction) and another was parallel to its longitudinal axis (Y-direction). The general location of the shear pin transducers installed on the test gate is shown in Figure 52. The instrumented shear pins were inserted on-site to connect the gate components together.

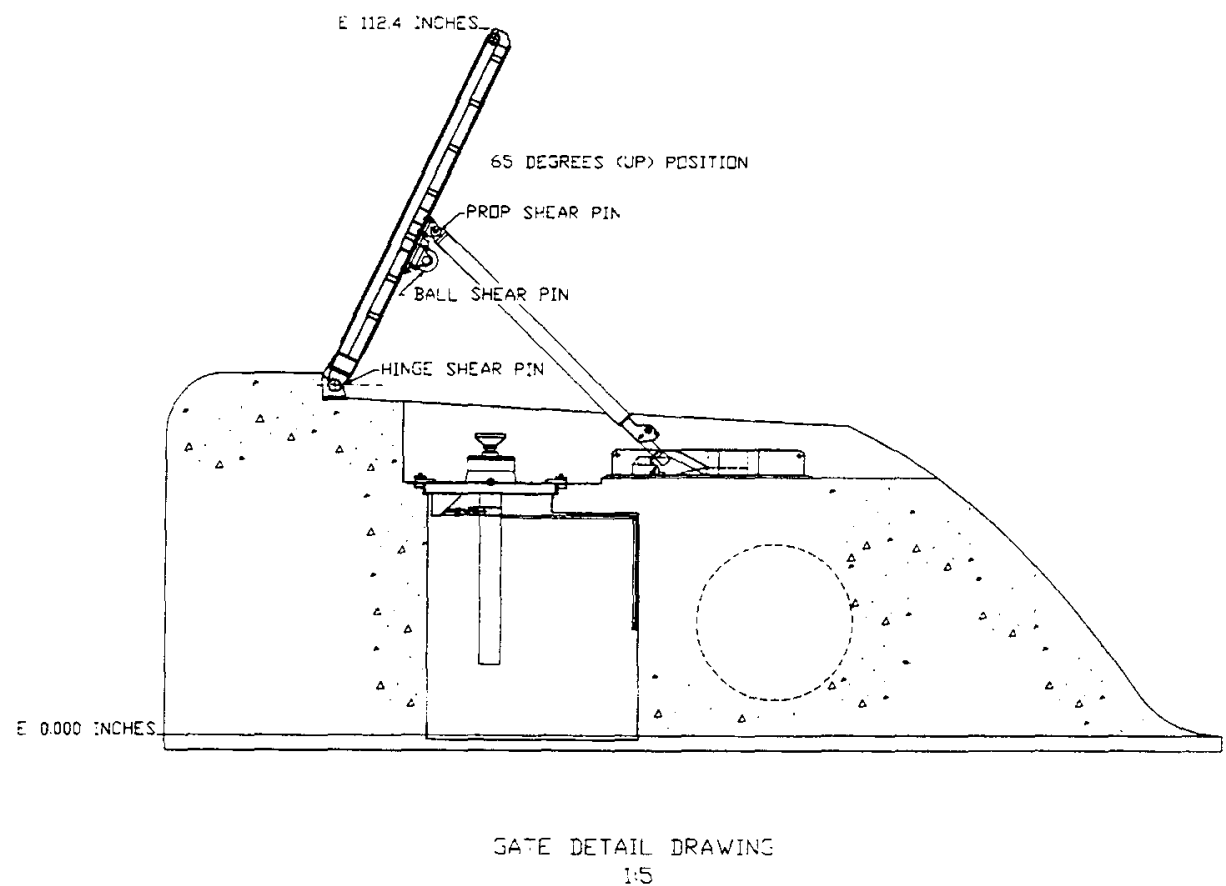

Figure 52. Shear pin locations on instrumented gate

The shear pin transducers installed in the gate measured load using a strain gauge sensing element sensitive to shear load. All shear pins were fabricated and instrumented by the Teledyne Engineering Services ${ }^{\mathrm{TM}}$, a division of Teledyne Brown Engineering. The waterproofed, bonded-resistant strain gauges arranged in full shear bridges were kept in the proper load sensing position by an antirotation plate (Teledyne 1993). Figure 53 shows the placement of the strain gauges on the hinge pins specially made for the Olmsted wicket model. As shown in the figure, gauge locations were aligned with the shear planes (gaps in between the gate hinges and the bearing frame lug) such that the shearing 


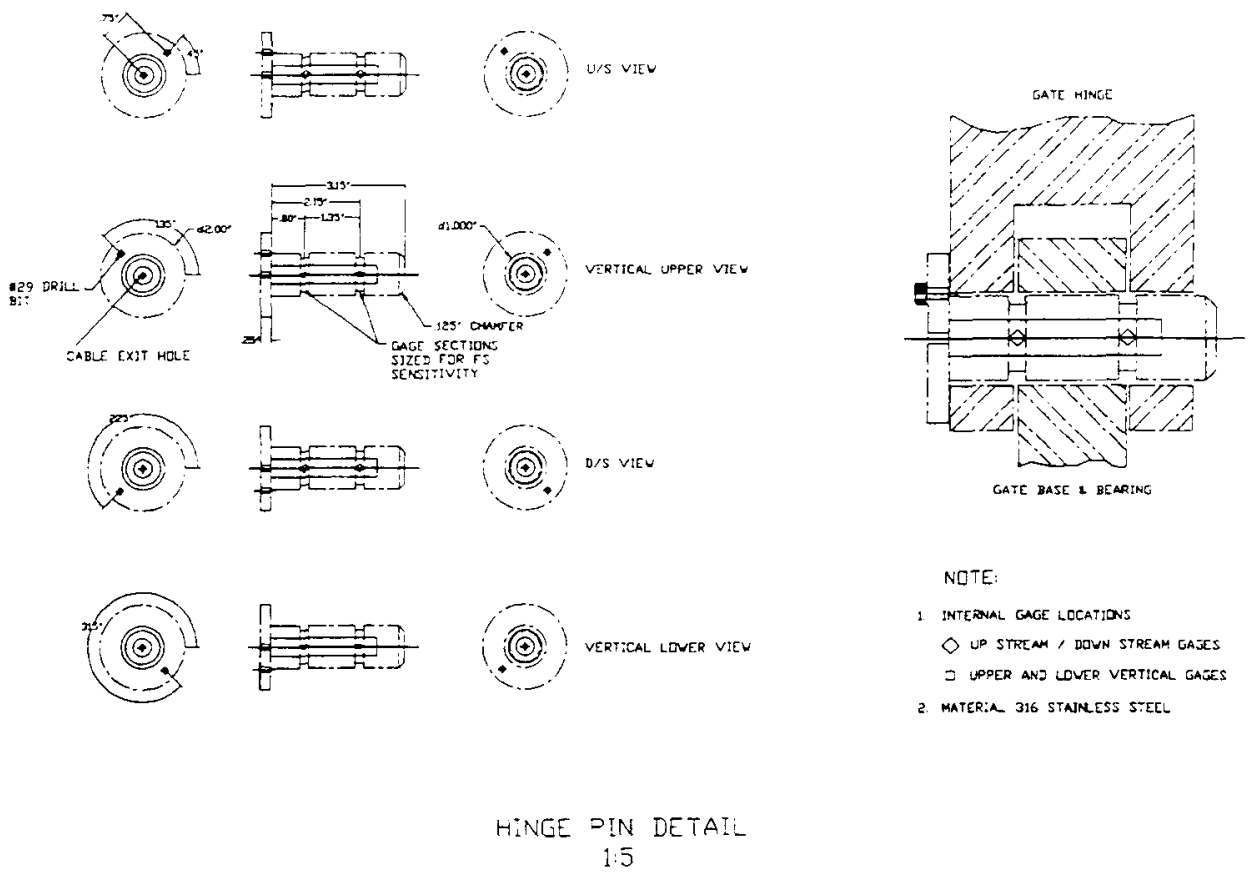

Figure 53. Hinge pin instrumentation detail

deformations were sensed properly by the installed strain gauges. Grooves were made on the pins to increase the sensitivity of the gauged section. The strain gauges mounted on the groves were coated with epoxy to protect them from wear.

Each shear pin transducer was calibrated to its rated capacity in both plus and minus directions by the manufacturer and also independently by WES to measure accuracy, linearity, repeatability, and hysteresis behaviors. A maximum deviation of \pm 0.50 percent of full scale was allowed in the specifications for the accuracy, nonlinearity, and hysteresis of the force transducers used in the test model. A maximum deviation of \pm 0.15 percent was allowed for the nonrepeatability of experiments. The Proving Ring standard loader was used to calibrate the shear pins (Beckwith and Marangoni 1990).

Axial force in the lifting arm (hydraulic cylinder) and in the support arm (prop) was measured using double-shear pin transducers. Two independent measurements were taken to measure the lifting cylinder load. Shear pins installed on the ball measured forces along two fixed mutually perpendicular axes on the ball (channels five and six). Since the ball is free to rotate, an angular position sensor was installed to measure the orientation of the ball corresponding to a fixed normal axis on the gate surface. Channel 48 measured the rotation of the ball angle. Figure 54 shows the positioning of the waterproofed, bondedresistant strain gauges and the angular sensor on the ball-bearing mount for the test wicket. Shear pins installed on the trunnions measured the axial lifting forces transmitted to the cylinder-bearing frame (channels seven and eight). 


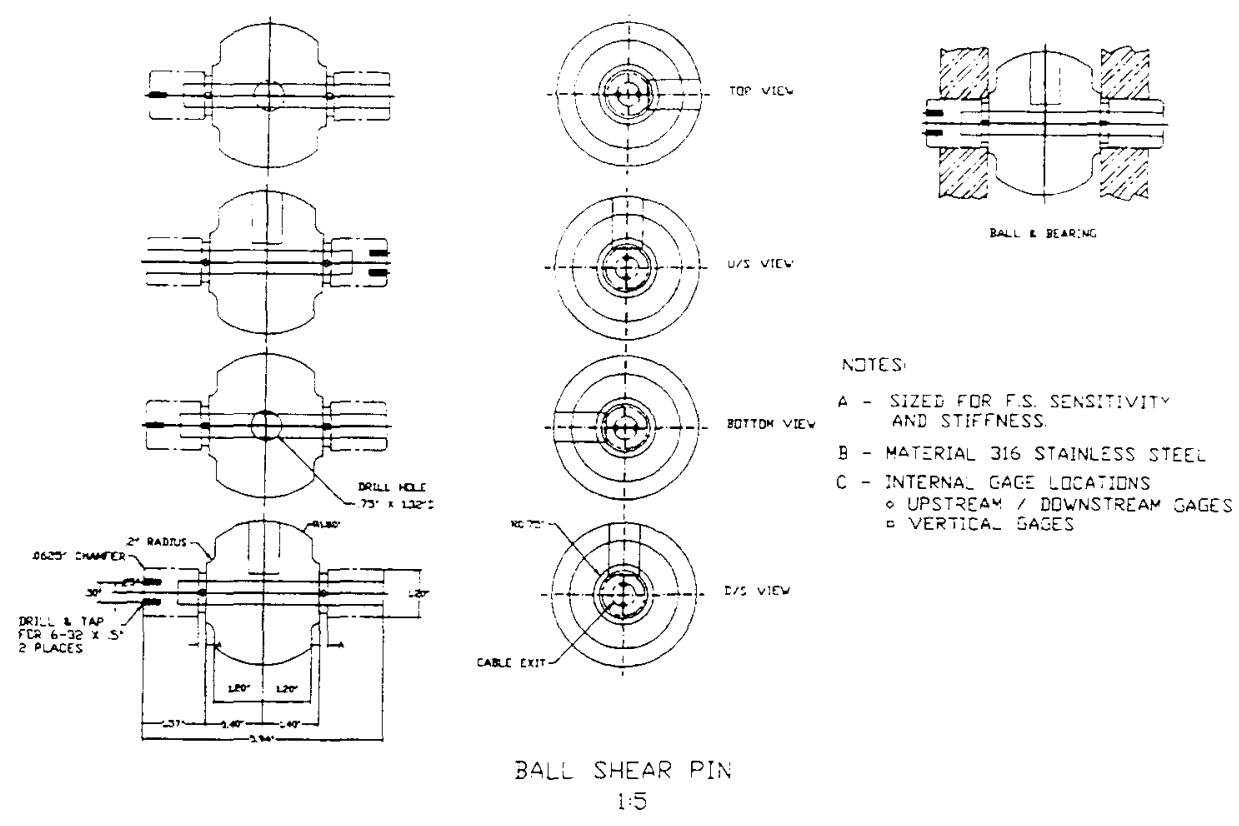

Figure 54. Ball shear pin instrumentation detail

Channel nine measured the prop load when the wicket gate was at the 65-deg position and the prop was in place against the hurter stop. The prop-shear pin was a double-shear, one-strain-gauge bridge to sense the axial force on the prop when it supported the gate in the raised position. Strain-gauge locations for the prop pin are shown in Figure 55.

\section{Pressure transducers}

A total of 18 pressure gauges (on a $3 \times 3$ grid on each face of the gate, upstream and downstream) were installed to measure the pressure distribution on the surface of the gate. As shown in Table 3, kulite ${ }^{\mathrm{TM}}$ XTM-190, 10 psia pressure transducers were used for recording dynamic pressure on the model (Kulite Semiconductor 1994). The XTM-190 utilizes a metal diaphragm and a piezoresistive sensing element, while the 10-32 UNF threaded body permitted easy installation in the model.

Kulite pressure gauges are absolute pressure transducers which were selected as a replacement to the Druck ${ }^{\top M}$ PDCR-200 series used in the 1:25 scale model. In the 1:25 model, humidity buildup inside the reference tube used in the Druck PDCR-200 model caused errors in the pressure measurements, and subsequently damaged the transducers during the 1:25 study. Kulite gauges thus minimized errors in pressure readings and reduced the risk of failure during testing. Also, these gauges are reasonably low in weight, relatively small in size, and adequately fit the anticipated pressure range of the model. 


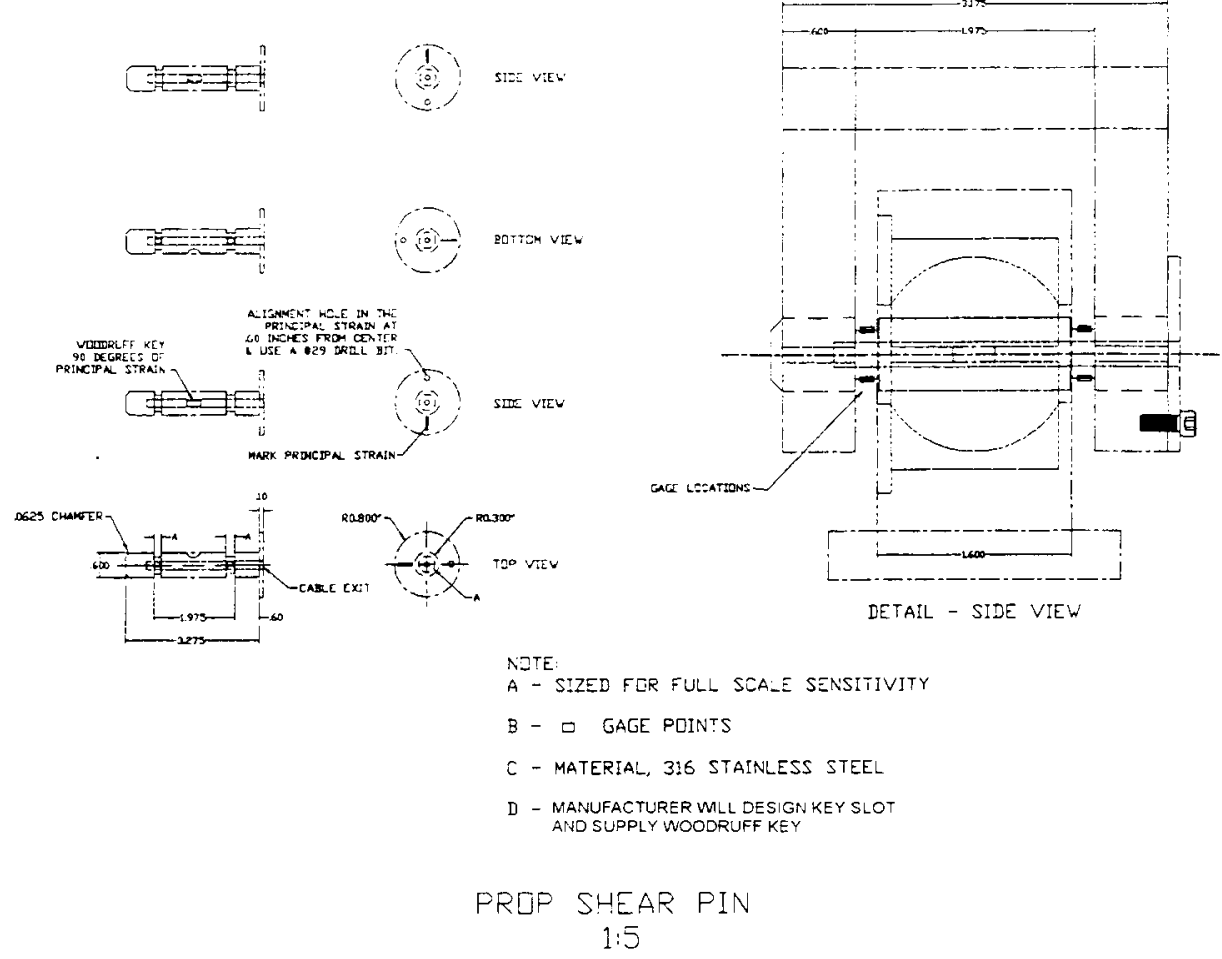

Figure 55. Prop shear pin instrumentation detail

All Kulite pressure transducers were calibrated in the Information Technology Laboratory (TTL), Instrumentation Service Division, using the AMETEK model PK II pneumatic dead-weight tester which had a range of 4- to 250-in. water head (Figure 56). Calibration indicated excellent linearity as the tester sensed pressures as a function of gate rotation. The cable entrance to the pressure cell was waterproofed using standard strain gauge-type procedures. A special RTV silicon compound, type 93205 , was applied to the diaphragm to insulate the diaphragm from thermal transients. Uncoated diaphragm gauges showed considerable errors in the pressure readings during leak checks conducted in the laboratory, a result of heat transfer from the extremely thin diaphragm that caused resistance value changes in the silicon sensor. Uncoated diaphragms often exhibited erroneous results; for example, when a transducer was lowered deeper in the water during a leak check, its output indicated transient peaks as if the gauge had been raised. Successive tests of the RTV coated diaphragm showed remarkable improvement in eliminating such thermal errors without significantly altering the frequency response of the transducer. The natural frequency for the RTV coated transducer was $48 \mathrm{kHz}$.

Figure 57 shows the location and orientation of the pressure transducers on the instrumented gate. Table 3 shows which channel numbers were associated with the pressure gauges in Figure 57. In this figure, transducer numbers are prefixed with ' $P$ ' such that $P x$ stands for the ' $x$ ' numbered transducer. Detailed positioning of pressure gauges and cable routing is shown in Figure 58. 


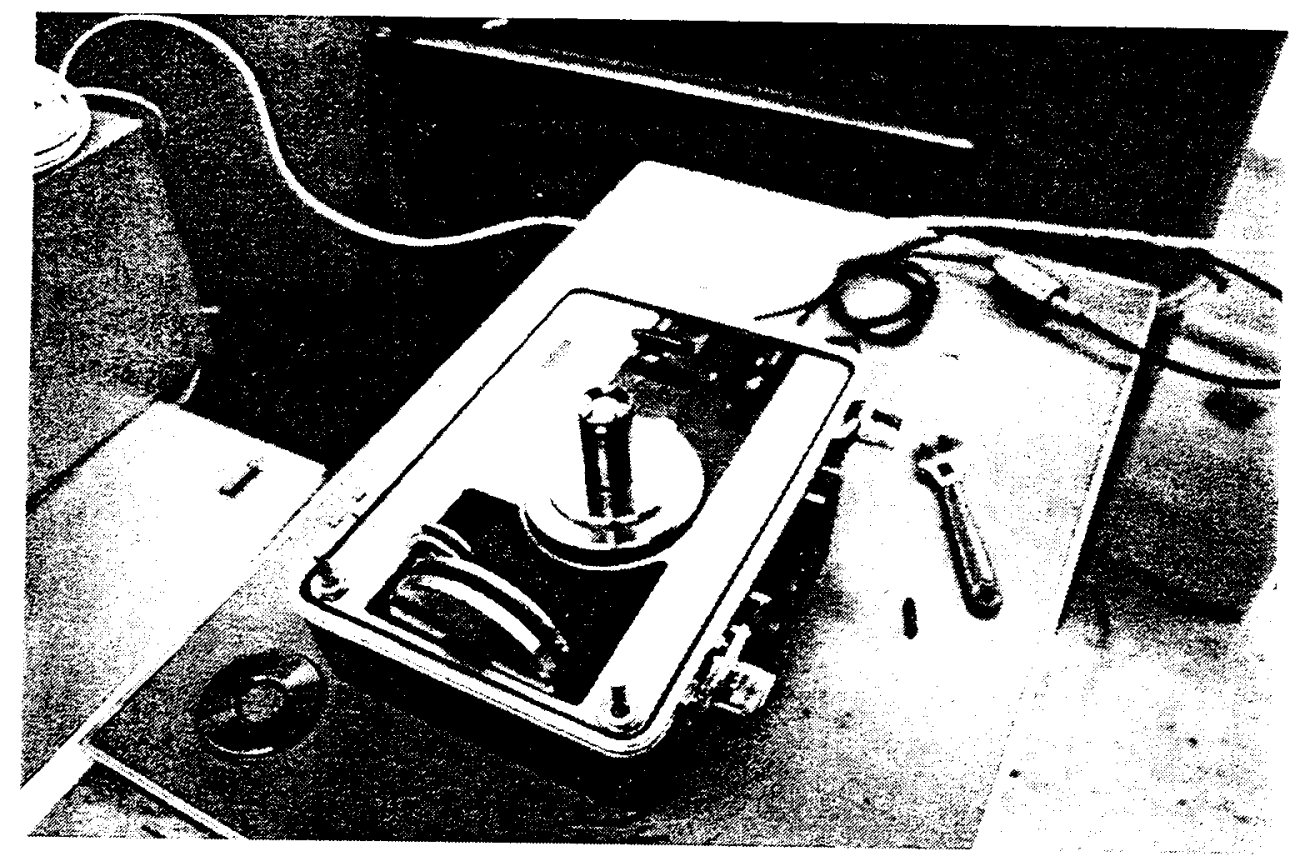

a. PKII pneumatic dead-weight tester

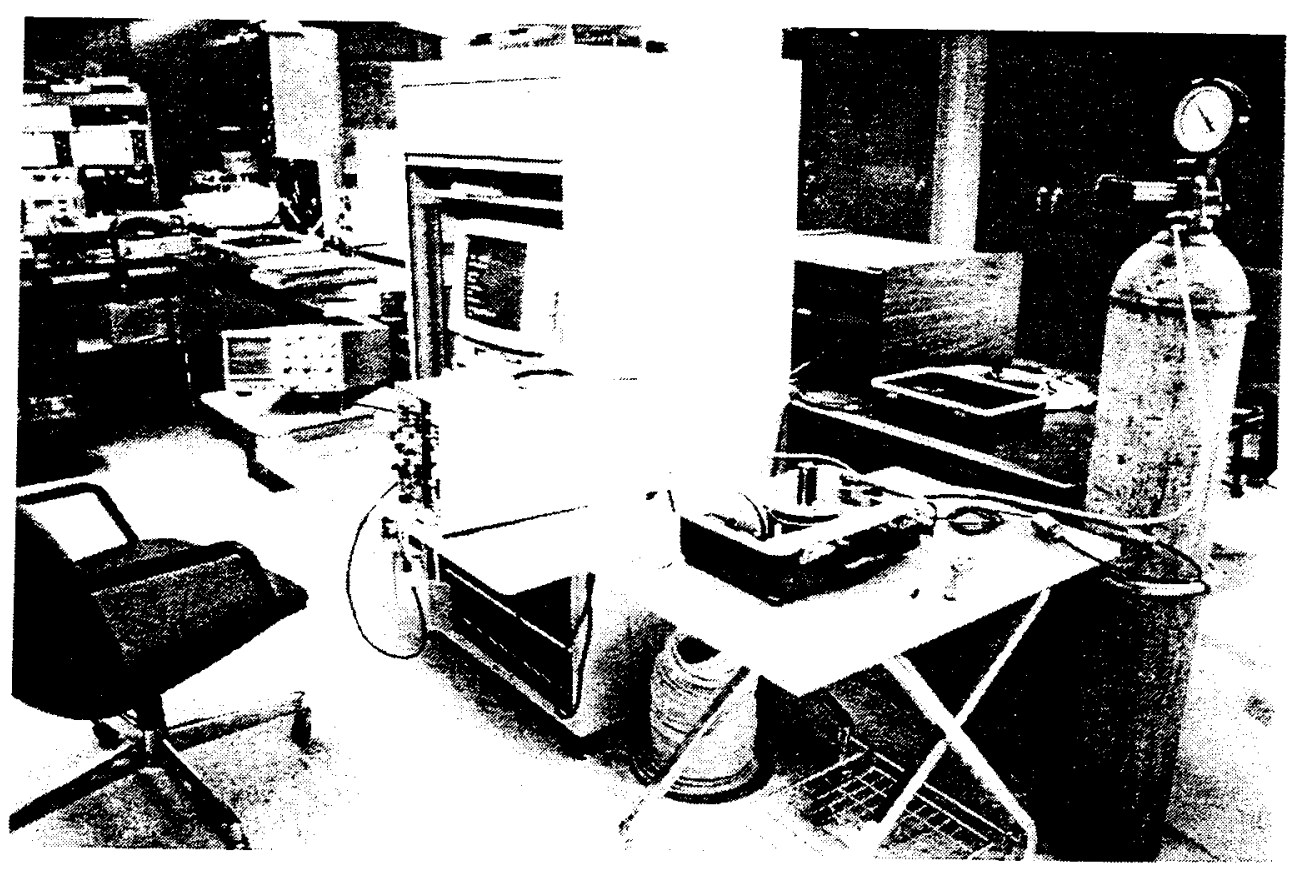

b. Overall test setup

Figure 56. Pressure gauge calibration device (AMETEK model PKII) 

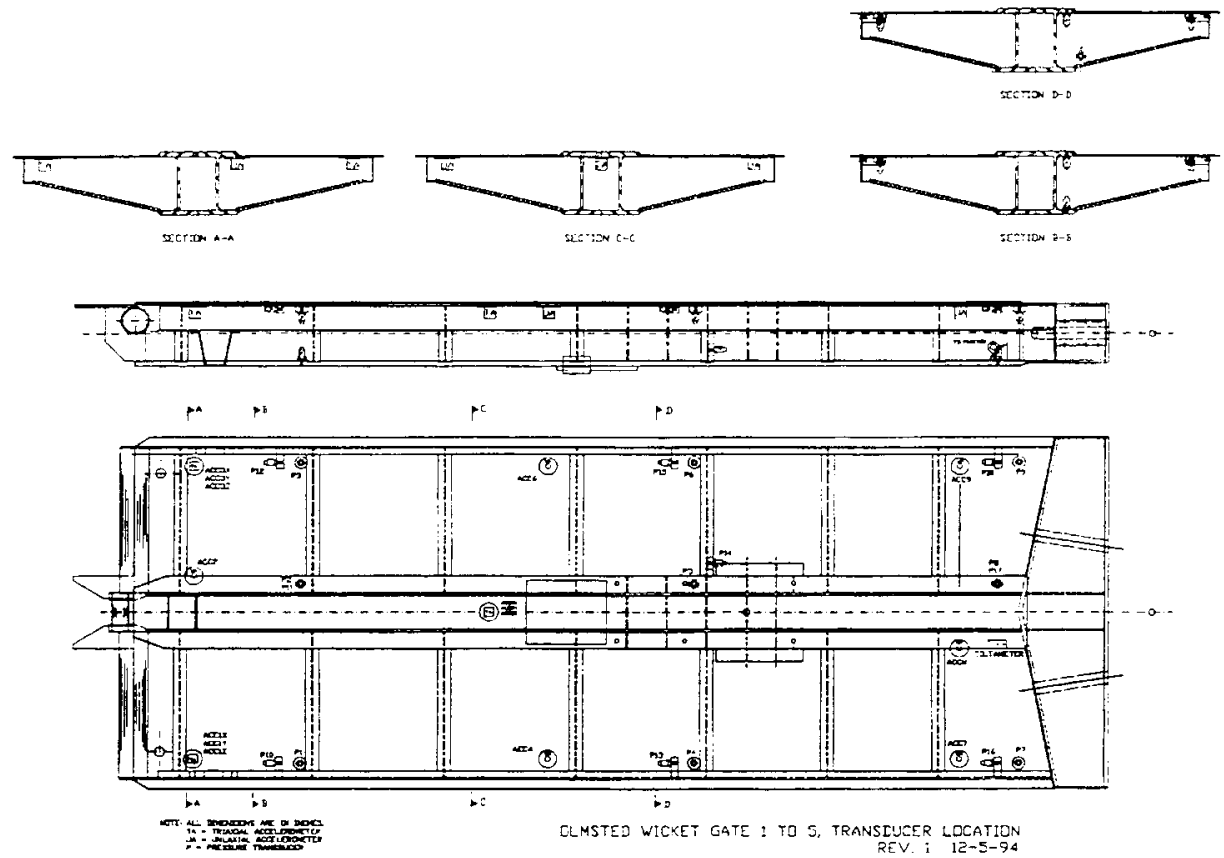

Figure 57. Transducer location on instrumented gate

Upstream pressure gauges, inserted from the backside of the wicket, had tips flashed to the flat skin plate. Downstream transducers on both edges of the wicket were mounted on additional steel plates which were fastened to the channel webs. Interior pressure gauges, excepting the middle one, were installed on the bottom beam flanges as shown in the figure. Pressure gauge P14 was installed on the transverse stiffener plate because the beam flange directly beneath the corresponding top pressure gauge was inaccessible due to the positioning of the ball mounting plate.

A nonuniform pressure distribution is common for the subject gate, and the unbalanced nature of the flow is evident during an end gate flow configuration. Such a nonuniform pressure distribution requires a minimum of three pressure transducers along a line in each direction to adequately measure the pressure distribution. Thus, three pressure transducers were mounted linearly in each direction on the surface of both sides of the gate to meet these criteria. A spatial distribution of the measured pressure would allow accurate modeling of the load pattern on the wicket gate. The downstream pressure distributions would provide information to check system equilibrium while considering the reaction forces and net pressure loads on both faces. This would also be used to correlate the flow conditions for the prototype wicket gate.

Channels 26 and 27 measured dynamic pressures in the upper and lower sections of the hydraulic lifting cylinder. These measurements permitted monitoring of the pressure buildup in the cylinder during shaft operation. 


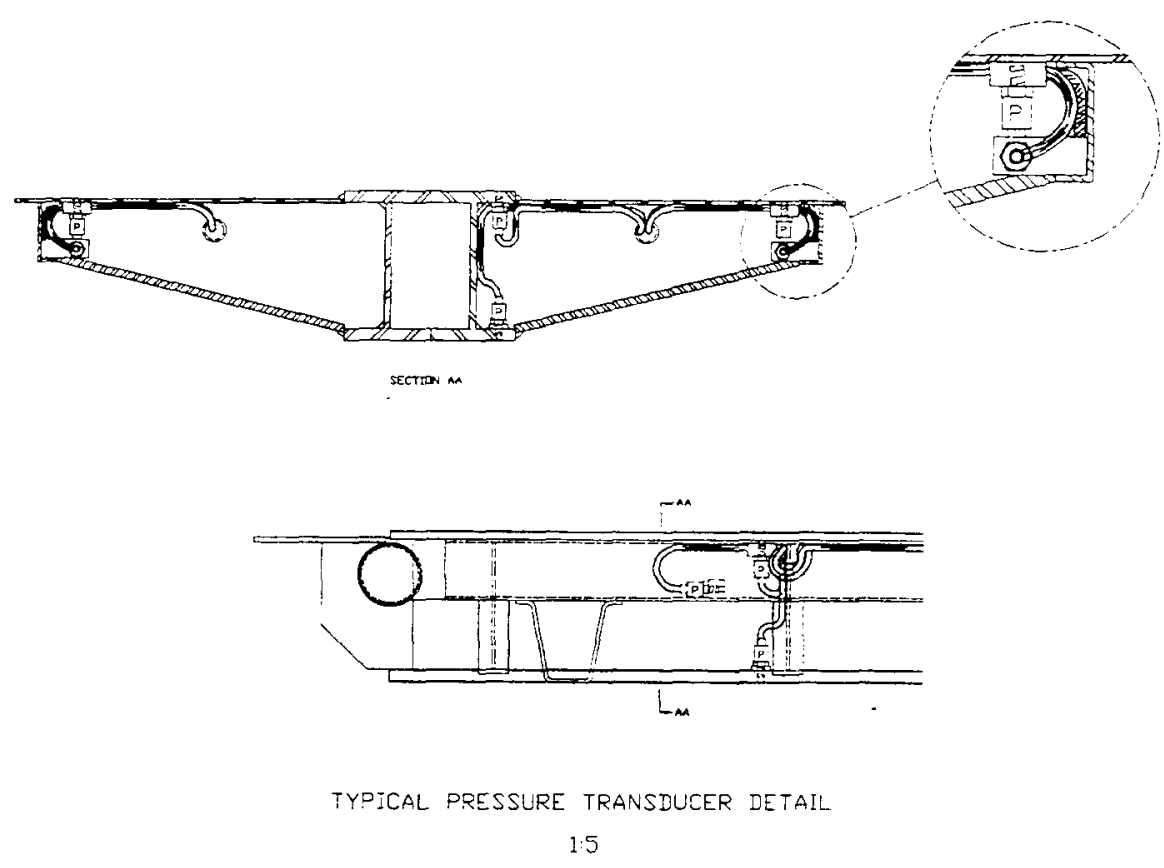

Figure 58. Typical pressure transducer detail

\section{Accelerometers}

A total of nine accelerometers in three columns along the length of the gate were installed to measure gate acceleration at predetermined locations (see Figure 57 for accelerometer locations). The accelerometers were positioned to capture the first two pure bending modes and the first and second torsional modes of the gate. These gauge locations were determined using an FE preanalysis of the wicket.

Synthesized FRF were used to determine optimal accelerometer locations for the test gate using the I-DEAS Master series-Test Module (SDRC 1993b). A preliminary $\mathrm{FE}$ model of the gate geometry, excluding the support mechanisms, was developed to extract the modal dynamic characteristics of the wicket using ABAQUS FE general-purpose computer code (HKS 1992). The extracted modal parameters were transported to the I-DEAS Master series platform for synthesizing FRF corresponding to excitor and response locations. Two criteria were used to select each accelerometer position by examining the FRF plots for a various combination of response and excitation points. The first was to verify the response points were not at the nodal points for the first few fundamental mode shapes, while the second ensured the energy level of determining modes was high at the response location. These criteria were met by observing the FRF for different response points and ensuring: (a) the accelerometers were not placed on nodal lines and (b) the peaks of the FRF were dominant in the synthesized plots. Figure 59 shows the synthesized FRF plots for a few response points on the gate surface. In this figure, the node numbers from the FE model 


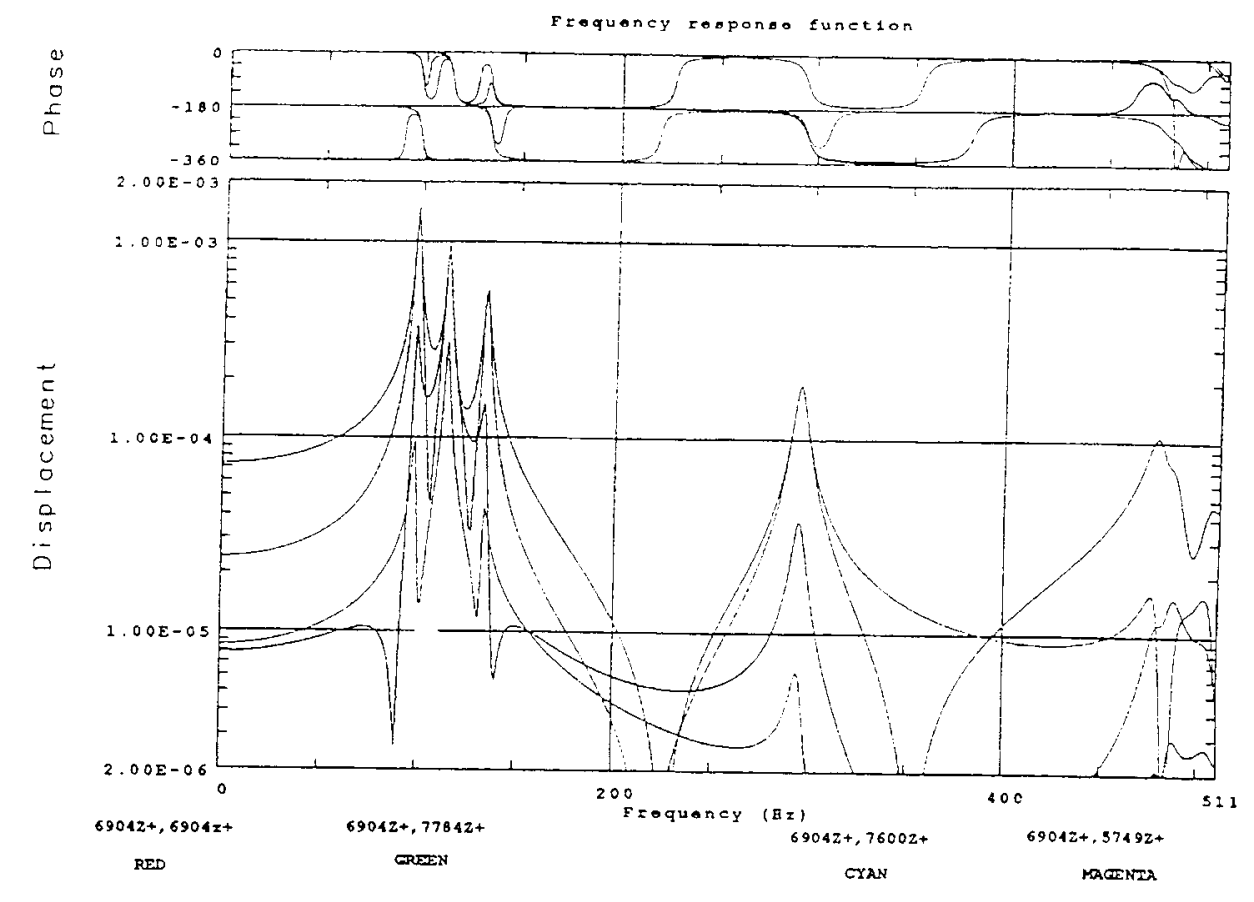

Figure 59. Synthesized FRF plots for measuring goodness of response and exciter location

which were used for extracting the FRF are also shown. Figure 60 shows the location of the nodes used to synthesize the FRF plots. Note that the top three accelerometer locations shown in Figure 60 were moved upward an additional 1.65 -in. during the actual instrumentation process to more fully capture the upper region of gate motion. The upper accelerometer locations were raised 8.25 in. on the prototype gate to better capture its upper gate motion, and hence the model accelerometers were raised to corresponding locations to ensure similitude. Although the accelerometers were placed higher than the positions recommended by the FE analysis, these new positions still satisfied the criteria used for selecting the accelerometer location.

Three high-sensitivity triaxial accelerometers, model PCB 339A01, were installed on the gate to sense its three-directional motion. PCB 339A01 uses a quartz flexural-beam sensing element to provide long-term measurement stability required for permanent mount applications. It is a 15 -gm, stud mount, 0.62 -in. cube, with a sensitivity of $100 \mathrm{mV} / \mathrm{gm}$, and adequate for a frequency range of 1 to $2,000 \mathrm{~Hz}, \pm 5$ percent (PCB Piezotronics (PCB) 1993). Six high-frequency, miniature uniaxial accelerometers, model PCB 353B68, were also installed on the gate as shown in Figure 57. PCB 353B68 uses a quartz shear mode sensing element to provide repeatable and reliable vibration measurements. Insensitivity to transverse motion is also an inherent feature of this type of shear mode design. The miniquartz shear accelerometers weigh 1.8 gm each, 10-32 top connected, 


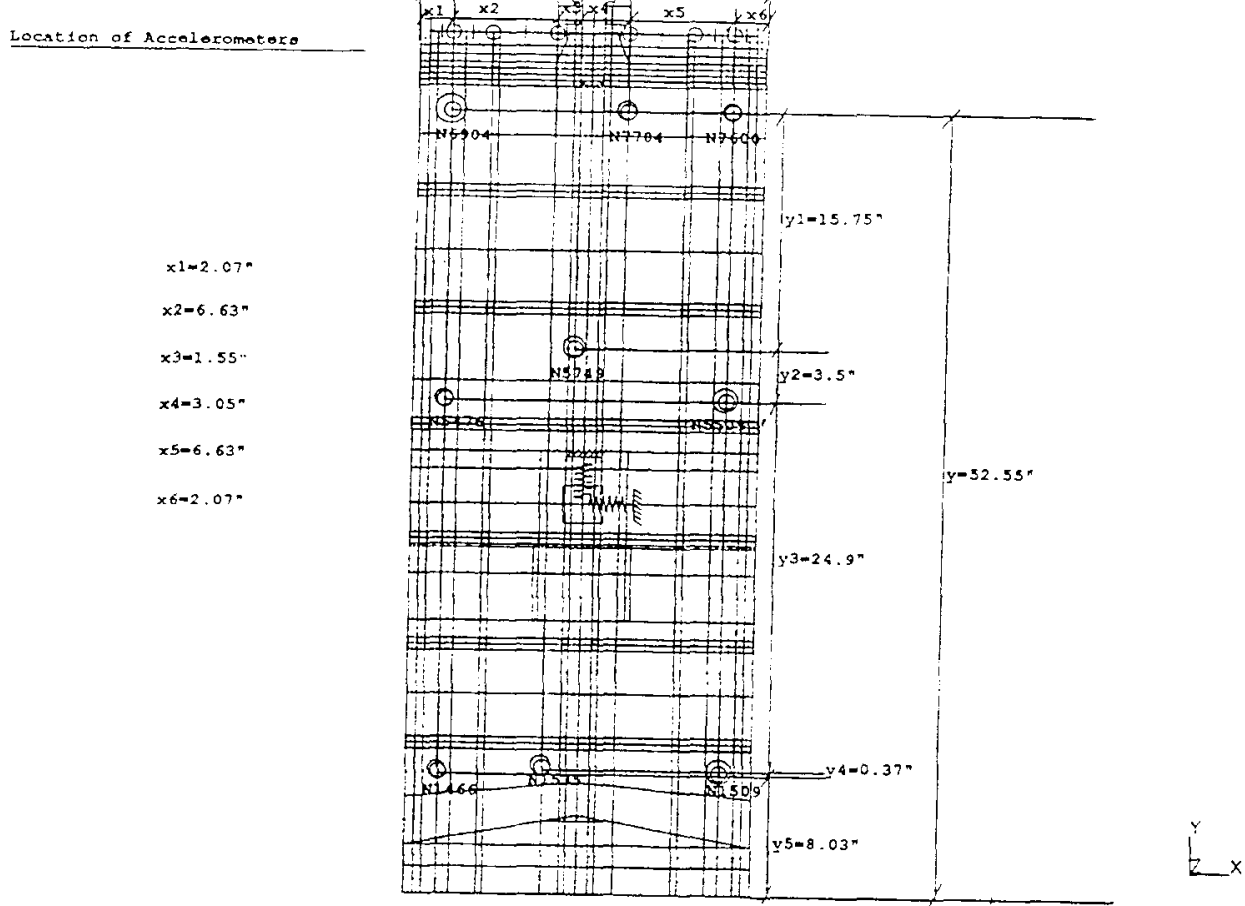

Figure 60. Locations of accelerometers on FE grid

with a frequency range of 1 to $10,000 \mathrm{~Hz}, \pm 5$ percent, and a sensitivity of $100 \mathrm{mV} / \mathrm{gm}$ (PCB 1993). All accelerometers were calibrated in TTL to verify the calibration certificate provided by the manufacturer, Piezotronics, Inc. The calibration procedure was in compliance with MIL-STD-45662A and traceable to NIST (National Institute of Standard and Technology).

Prop dynamics were also measured using a triaxial PCB accelerometer, installed on the prop about one-fourth the length up from the bottom of the prop rod. As shown in Table 3, motion in two mutually orthogonal directions was recorded for the prop. One sensing direction was normal to the prop and the other was along the length of the prop.

\section{Others sensors}

Gate orientation was measured by a tiltmeter attached to the base of the gate. Positive gate angles are represented by rotation of the gate about its base from its horizontal to its upright position. Channel 48 measured cylinder angle from a vertical axis on its mount going clockwise. Channel 49 measured rotation of the ball normal to the gate surface. Headwater and tailwater elevations were also recorded on Channels 51 and 52 using pressure sensors. Barometric pressure was recorded on Channel 53. 
Figure 61 shows a partial view of the transducer installation method for the gate. In this figure, the ball and prop shear pins are displayed along with the location of the tiltmeter that measured the ball angle. Figure 62 shows an overall view of the instrumented gate placed in the model. Also displayed in this figure are the cable routing and the positioning of pressure gauges and ball shear pins.

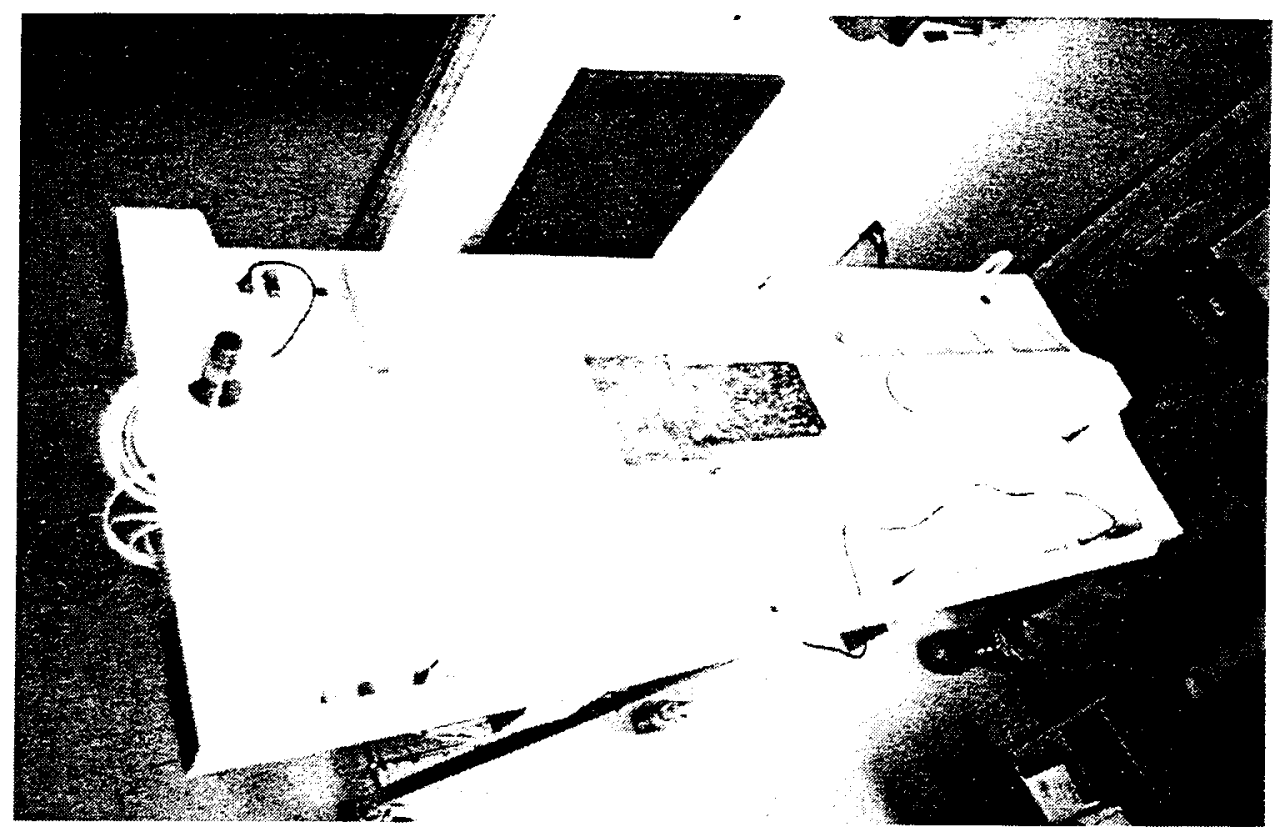

\section{a. Transducers mounted on skin plate}

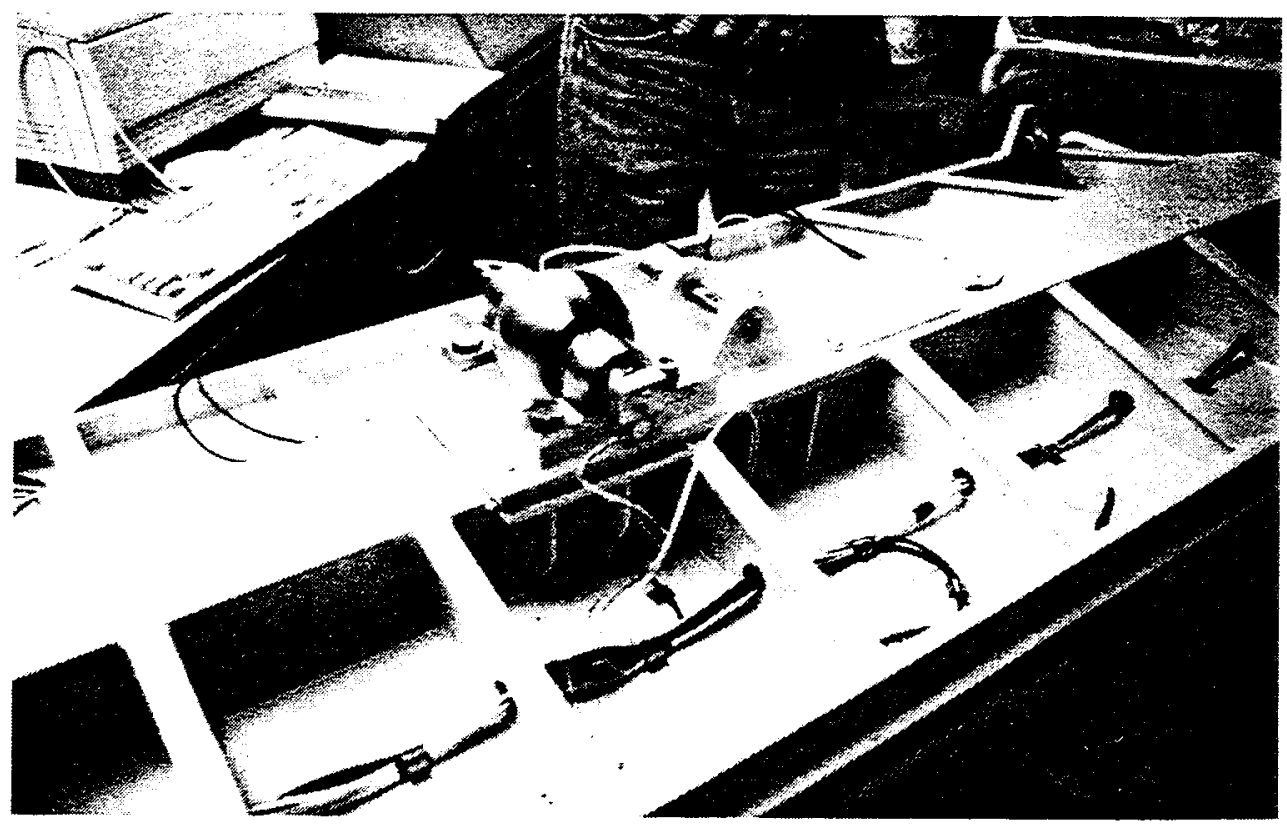

b. Transducer cable routing

Figure 61. Transducer installation on gate 


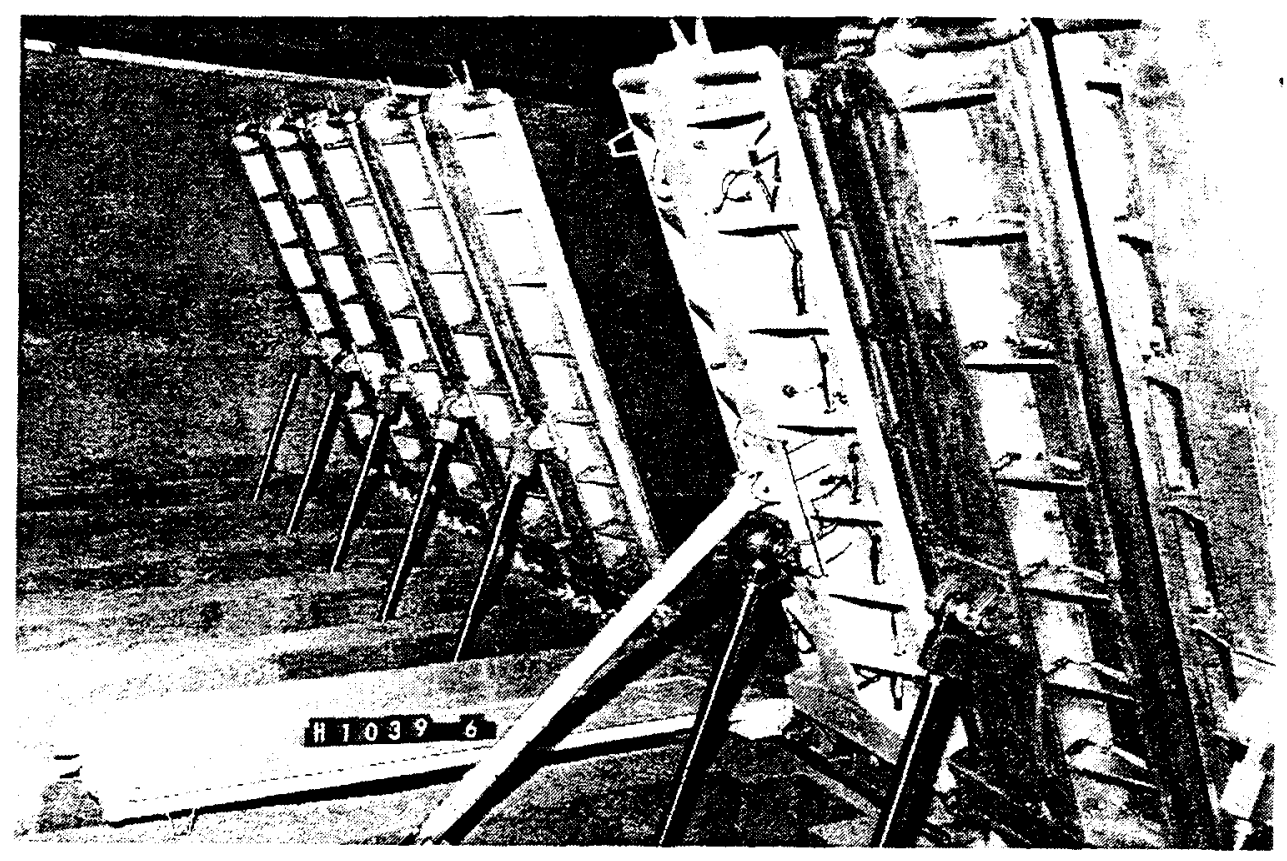

Figure 62. Instrumented gate on flume

\section{Data Acquisition and Verification}

\section{System and operation}

The data acquisition system for the Olmsted 1:5 Model Study consisted of two personal computers (PC) for data storage, data processing, and process control, an analog-to-digital converter (ADC), signal conditioning amplifiers, and a printer. Signal conditioning included continuous variable gain amplifiers, tracking filters, and antialias filters. The signal conditioning amplifiers were manufactured by Vishay and could supply gains up to 10,000. Acquisition of additional data was provided by Zonic standalone data acquisition system (Zonic 1991).

Two PCs were used to facilitate simultaneous data collection and data processing. Both PC were PC compatibles with $90 \mathrm{MHz}$ Pentium CPU, 32 megabytes of RAM, 1 gigabit hard disk drives, 150 megabyte Bernoulli removable hard disk drives, quad-speed CD-ROM drives, Simply LANtastic network adapters, and 15-in. Super VGA monitors. Custom made programs were installed in the $\mathrm{PC}$ to regulate data acquisition and control gate position. The PC used to record data also contained a National Instruments AT-MIO-16F$5 \mathrm{ADC}$ board and a Real Time Devices digital input/output (DIO) board. The DIO were used to activate the desired hydraulic system function for the experiment to be run. The ADC had a 12-bit resolution and was configured for 5 to +5 volt input range. The $A D C$ was a printed circuit board that plugged into a $\mathrm{PC}$ expansion slot. A block diagram showing the data acquisition system for the 1:5 Olmsted Wicket model is shown in Figure 63. 


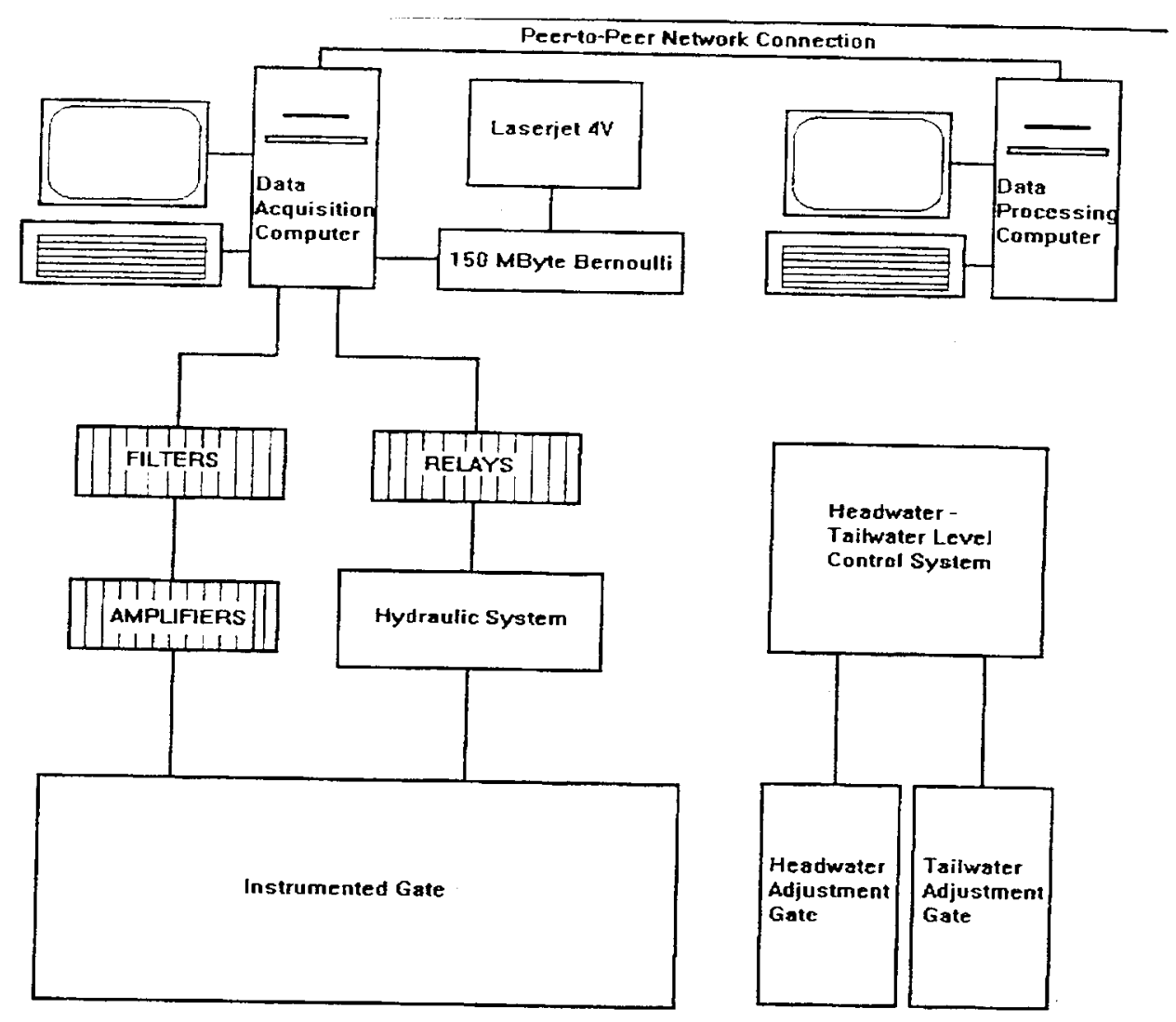

Figure 63. Block diagram for data acquisition system

Custom software was written to take calibration measurements, record data during an experiment, and make time-history plots of the data recorded. Custom software was also written to perform processing, such as subtracting the atmospheric pressure data from the absolute pressure data that had been recorded. MATLAB matrix analysis software was used for much of the data analysis; it contains many powerful numerical and graphical tools to manipulate matrices, perform frequency analyses, plot graphs as well as perform other crucial mathematical functions (The MathWorks 1992). Frequency plots were made with several short custom m-files written for MATLAB, and MATLAB was used to process the stored time-domain data as well.

\section{Data Recording and Initialization}

\section{Pressure transducer output initialization}

During these experiments, it was desirable to have the pressure transducers indicate $0 \mathrm{ft}$ of water when the transducers were dry. As water was applied, their 
output would then increase in proportion to the water column above the transducer. This turned out to be impractical with these transducers. They could be set to indicate $0 \mathrm{ft}$ of water when they were dry. but when water was applied the resulting temperature change in the diaphragm caused a shift in the outputs. thereby yielding erronenus measurements.

To circumvent this problem. the model was filled with water to a level of $302 \mathrm{ft}$ Olmsted elevation and the instrumented gate was lowered to its rest position of $-3 \mathrm{deg}$. Note that all pool elevations mentioned in this report are reported in Olmsted elevations. The transducer outputs were set to indicate $0 \mathrm{ft}$ of water under this condition. From knowledge of the geometry of the model, the proper pressures could be computed after completion of each experiment.

\section{Signal polarities}

Force signals were configured to give a positive indication for forces acting downstream in the horizontal plane and forces acting with gravity in the vertical plane. Pressure signals were configured to indicate increasing positive pressures as the water column increased. Acceleration signals were configured such that accelerations acting upstream were positive.

\section{Data collection}

At the beginning of a day's testing, measurements were taken to determine transducer outputs with zero excitation and correlated against transducer outputs of known excitation to establish calibrations. Typical transducer calibration factors are listed in Table 4. After calibration, the model channel was filled to prototype $302 \mathrm{ft}$. The gate was then lowered to its rest position of $-3 \mathrm{deg}$. All transducer outputs were set to zero at this no-flow condition. All shear pins and pressure transducers measured responses with reference to the static pool head. Actual responses for these transducers, therefore, consisted of the measured dynamic response (negative values, since head pressure decreases as gate angle increases) plus the initial static response.

$$
P_{\text {flow induced }}=P_{\text {static }}+P_{\text {gauge (measured) }}
$$

The flow-induced pressure readings, for example, can be obtained by adding the pressure gauge reading with the static pressure head corresponding to a $302-\mathrm{ft}$ pool elevation. The recorded accelerations represented the actual accelerations that the gate experienced due to the flow. 


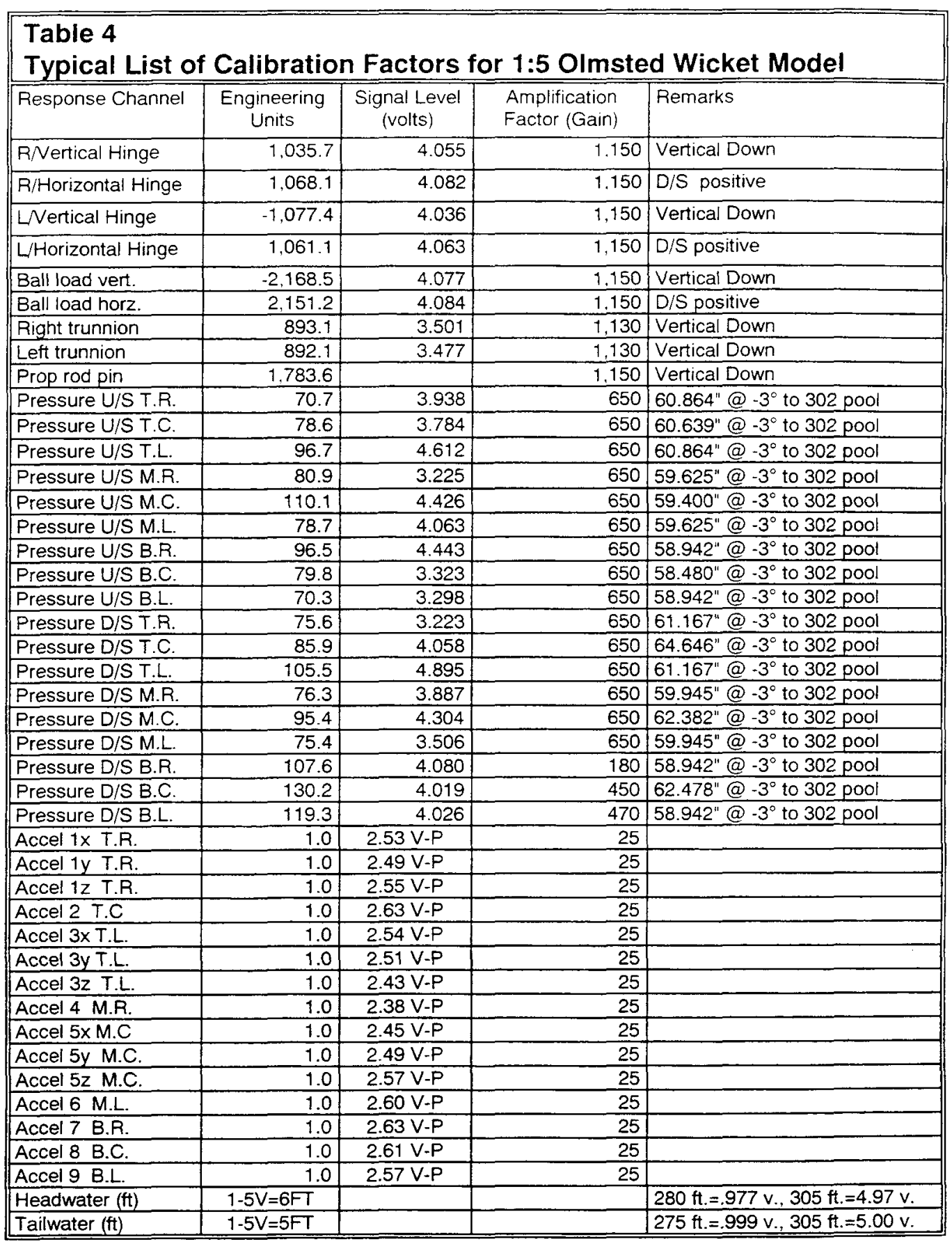

The gate was rotated from -3 to $65 \mathrm{deg}$ with the still water depth of $302 \mathrm{ft}$ prototype in the model channel. Data were recorded during this rotation to subtract the forces due to the weight of the gate out of data recorded with water in the channel. Only the forces the water exerted on the gate (hydrodynamic) were of interest in this study. 
With the headwater and tailwater levels in the test channel set to desired levels, each test was run. After the data for each test were recorded, timehistories were examined to ensure conducting subsequent tests would be sensible. The file could then be moved to the data processing computer for further analysis, such as examining frequency plots and making comparisons of data obtained from the Olmsted Prototype or 1:25 Model studies.

Depending upon the operating instructions inserted into the input list, the water level would be arranged, the gate would be positioned, and the transducer data would be sampled at the prescribed rate for the registered channel numbers. A typical input list used to drive the gate at different position was:

\section{Typical input list}

Experiment Number:

147

Channel List:

$1-8,10-53$

Sampling Rate:

Length of Time Sampled:

500.000 samples/sec/channel

Gate Rotation Measurement Channel:

$410.0 \mathrm{sec}$

Headwater Level:

Tailwater Level:

$300.0 \mathrm{ft}$

$279.0 \mathrm{ft}$

Gate Operation

( 0 for fixed, and 1 for rotation): 1

Gate Rotation Angle, Start of Experiment: $\quad-3.0 \mathrm{deg}$

Gate Rotation Angle, End of Experiment: $\quad 65.0 \mathrm{deg}$

NOTE: Filter at $250.0 \mathrm{~Hz}, \mathrm{~W} /$ PROP ROD TYPE 2

1 GATE GAP \#6 GATE DOWN, NOTE PROP ROD LOAD CELL BAD. USED 2 SMALL AND 2 BIG PUMPS

Date: 6-22-1995 Time: 10:51:04

Three types of operating cycles could be attained in the model: a raising cycle, a lowering cycle, and a fixed gate operating position.

Typical sampling rates for all three types of experiments were 500 samples per model sec per measurement (times given in the following descriptions of the three types are in 1:5 model sec). Experiments typically yielded 21,746,384-byte data files (binary format). Whenever hard disk space became short, files were transferred to a 150 -megabyte Bernoulli removable hard disk.

The gate-raising cycle experiments lasted $410 \mathrm{sec}$. This was the time required to rotate the gate from a lowered position of $-3 \mathrm{deg}$ to a raised position of $65 \mathrm{deg}$, disengage the hydraulic lifting arm, and allow the hydraulic lifting arm to assume a rest position at the top of the hydraulic cylinder. This type of experiment was primarily used to measure the changing forces on the gate during upward rotation. 
The gate-lowering cycle experiments lasted $212 \mathrm{sec}$. This was the time required for the hydraulic lifting arm to engage the gate and rotate the gate from a raised position of $65 \mathrm{deg}$ to a lowered position of $-3 \mathrm{deg}$. This type of experiment was primarily used to measure the changing forces on the gate during downward rotation.

The fixed-gate experiments generally lasted approximately $30 \mathrm{sec}$. For these experiments the gate would be adjusted to a critical position and remain in that position for the duration of the experiment. These experiments were primarily used to determine the vibrational characteristics of the gate.

Upon experiment completion, an output status file was generated to summarize the input and output information for the experiment conducted. A typical status file showing the recorded information of the experiment run is listed below. This file also provides a general statistical description of each recorded data.

\section{$\underline{\text { Output list }}$}

\begin{tabular}{|c|c|c|c|c|c|c|c|}
\hline $\begin{array}{l}\text { Channel } \\
\text { Number }\end{array}$ & $\begin{array}{l}\text { Gauge } \\
\text { Type }\end{array}$ & $\begin{array}{l}\text { Units Of } \\
\text { Measure- } \\
\text { ment }\end{array}$ & $\begin{array}{l}\text { Minimum } \\
\text { Reading }\end{array}$ & $\begin{array}{l}\text { Average } \\
\text { Reading }\end{array}$ & $\begin{array}{l}\text { Maximum } \\
\text { Reading }\end{array}$ & $\begin{array}{l}\text { Root Mean } \\
\text { Square }\end{array}$ & $\begin{array}{l}\text { Standard } \\
\text { Deviation }\end{array}$ \\
\hline 1 & Force, R. Hinge, $z$ & lbf & -164.054 & 74.682 & 246.082 & 128.53 & 104.606 \\
\hline 2 & Force, R. Hinge, y & $\mathrm{lbf}$ & -152.013 & 190.788 & 530.469 & 262.578 & 180.408 \\
\hline 3 & Force, L. Hinge, z & $\mathrm{Ibf}$ & -170.965 & -4.176 & 259.225 & 83.422 & 83.318 \\
\hline 4 & Force, L. Hinge, y & Ibf & -30.645 & 233.051 & 506.379 & 280.222 & 155.601 \\
\hline 5 & Force, ball, z & $\mathrm{lbf}$ & -11.733 & $1,020.226$ & $1,576.108$ & $1,175.2$ & 583.296 \\
\hline 6 & Force, ball, y & $\mathrm{Ibf}$ & -1.299 & 582.01 & 951.031 & 669.832 & 331.572 \\
\hline 7 & Force, R. Trunnion & $\mathrm{lbf}$ & -41.893 & 618.664 & $1,029.283$ & 714.18 & 356.801 \\
\hline 8 & Force, L. Trunnion & $\mathrm{lbf}$ & -87.407 & 550.67 & 869.028 & 642.529 & 331.067 \\
\hline 10 & Pressure, U/S T.R. & in. wc & -54.681 & -48.289 & -38.489 & 48.406 & 3.356 \\
\hline 11 & Pressure, U/S T.C. & in. $w c$ & -56.767 & -43.304 & -30.285 & 43.914 & 7.292 \\
\hline 12 & Pressure, U/S T.L. & in. $w c$ & -54.419 & -48.507 & -39.117 & 48.612 & 3.186 \\
\hline 13 & Pressure, U/S M.R. & in. wc & -45.94 & -36.368 & -27.689 & 36.573 & 3.866 \\
\hline 14 & Pressure, U/S M.C. & in. $w c$ & -34.654 & -27.157 & -22.64 & 27.261 & 2.382 \\
\hline 15 & Pressure, U/S M.L. & in. wc & -47.343 & -35.875 & -27.161 & 36.151 & 4.462 \\
\hline 16 & Pressure, U/S B.R. & in. wc & -44.268 & -21.711 & -10.273 & 23.95 & 10.111 \\
\hline 17 & Pressure, U/S B.C. & in. wc & -32.322 & -16.967 & -10.471 & 17.902 & 5.711 \\
\hline 18 & Pressure, UIS B.L. & in. wc & -40.559 & -20.186 & -10.15 & 22.054 & 8.882 \\
\hline 19 & Pressure, D/S T.R. & in. wc & -71.4 & -62.445 & -51.484 & 62.57 & 3.956 \\
\hline 20 & Pressure, D/S T.C. & in. wc & -71.38 & -64.229 & -54.17 & 64.382 & 4.427 \\
\hline 21 & Pressure, D/S T.L. & in. wc & -70.166 & .60 .653 & -50.864 & 60.69 & 2.134 \\
\hline 22 & Pressure, D/S M.R. & in. wc & -75.712 & -59.63 & -53.971 & 59.691 & 2.711 \\
\hline 23 & Pressure, D/S M.C. & in. wc & -75.945 & -61.688 & -52.621 & 61.625 & 0 \\
\hline 24 & Pressure, D/S M.L. & in. wc & -74.143 & -58.961 & -49.369 & 58.929 & 0 \\
\hline 25 & Pressure, D/S B.R. & in. $w c$ & -81.4 & -60.187 & -55.39 & 60.511 & 6.254 \\
\hline 26 & Pressure, D/S B.C. & in. $w c$ & -68.498 & -62.493 & -55.61 & 62.36 & 0 \\
\hline 27 & Pressure, D/S B.L. & in. wc & -71.652 & -59.333 & -55.673 & 59.442 & 3.591 \\
\hline 28 & Pressure, upper cy & psi & -13.941 & 28.999 & 163.872 & 49.389 & 39.979 \\
\hline
\end{tabular}




$\begin{array}{lcccrrrr}29 & \text { Pressure, lower cy } & \text { psi } & 1.704 & 223.103 & 332.824 & 243.915 & 98.587 \\ 30 & \text { Accel., 1xT.R. } & \text { g. } & -0.389 & 0 & 0.471 & 0.014 & 0.014 \\ 31 & \text { Accel., 1y T.R. } & \text { g. } & -0.36 & 0.003 & 0.311 & 0.006 & 0.006 \\ 32 & \text { Accel., 1z T.R. } & \text { g. } & -1.065 & 0.008 & 0.976 & 0.032 & 0.031 \\ 33 & \text { Accel., 2 T.C. } & \text { g. } & -1.007 & 0.008 & 1.073 & 0.026 & 0.025 \\ 34 & \text { Accel., 3x T.L. } & \text { g. } & -0.384 & -0.001 & 0.492 & 0.013 & 0.013 \\ 35 & \text { Accel., 3y T.L. } & \text { g. } & -0.257 & 0.009 & 0.285 & 0.011 & 0.005 \\ 36 & \text { Accel., 3z T.L. } & \text { g. } & -1.076 & 0.01 & 1.43 & 0.036 & 0.035 \\ 37 & \text { Accel., 4 M.R. } & \text { g. } & -0.47 & -0.002 & 0.447 & 0.019 & 0.019 \\ 38 & \text { Accel., 5x M.C. } & \text { g. } & -0.153 & 0.004 & 0.251 & 0.009 & 0.008 \\ 39 & \text { Accel., 5y M.C } & \text { g. } & -0.26 & 0.002 & 0.281 & 0.004 & 0.004 \\ 40 & \text { Accel., 5z M.C. } & \text { g. } & -0.399 & 0.002 & 0.346 & 0.015 & 0.015 \\ 41 & \text { Accel., 6 M.L. } & \text { g. } & -0.453 & -0.009 & 0.603 & 0.02 & 0.019 \\ 42 & \text { Accel., 7 B.R. } & \text { g. } & -0.367 & 0.006 & 0.422 & 0.013 & 0.011 \\ 43 & \text { Accel., 8 B.C. } & \text { g. } & -0.294 & 0.013 & 0.37 & 0.015 & 0.007 \\ 44 & \text { Accel., 9 B.L. } & \text { g. } & -1.059 & 0.008 & 0.642 & 0.029 & 0.028 \\ 45 & \text { Accel., Prop rod z } & \text { g. } & -1.954 & -0.009 & 1.953 & 0.034 & 0.033 \\ 46 & \text { Accel., Prop rod y } & \text { g. } & -1.356 & 0.006 & 1.887 & 0.015 & 0.014 \\ 47 & \text { Gate angle } & \text { DEG } & -2.48 & 37.574 & 67.472 & 44.21 & 23.295 \\ 48 & \text { Cylinder angle } & \text { DEG } & 1.269 & 7.857 & 16.402 & 9.63 & 5.568 \\ 49 & \text { Ball angle } & \text { DEG } & -29.822 & -12.242 & 11.182 & 19.892 & 15.679 \\ 50 & \text { Shaft position } & \text { INCHES } & -0.019 & 14.041 & 28.811 & 16.425 & 8.522 \\ 51 & \text { Elevation, U/S pool } & \text { FT. WC } & 299.694 & 299.983 & 300.336 & 300.111 & 8.744 \\ 52 & \text { Elevation, D/S pool } & \text { FT. WC } & 278.439 & 279.475 & 279.811 & 279.049 & 0 \\ 53 & \text { Barometric pressure } & \text { IN. WC } & -0.318 & 0.054 & 0.44 & 0.058 & 0.02\end{array}$

\section{Software}

Commercial software used at the model included DOS 6.2, Windows for Workgroups 3.11, Matlab 4.x, and Simply LANtastic. DPLOT, a Windowsbased plot analysis program written at WES, was used for time-domain data analysis.

The software listed below was custom written to collect and analyze data at the model. All were written to run under MS-DOS on a PC compatible computer.

- NICAL3 - Program to read transducer outputs in two different states: excited and unexcited. These outputs were used to determine slope and offset values for converting transducer outputs into engineering units.

- NIDA3 - Program to accept the desired sampling rate, date record time, experiment number, and other information pertinent to a experiment run such as water levels, gate configurations, etc. This program then accepted measurements from the ADC and put them in RAM. Data were moved from RAM to the hard disk at the end of the experiment.

- EGATERM - Program displayed the raw data on the PC screen. 
- PROTPLOT - This program generated plot files from the raw data recorded. The plot files could then be output to the Laserjet printer. Also, the data could be displayed in model or prototype units.

- NOFLOW - Program that reads a raw data file recorded with a still water pool of 302-ft prototype. The forces are saved as a function of the gate rotation. Later these noflow forces would be subtracted from experiments run with flowing water to determine the forces due to the flowing water.

- SUBNFPRE - Program that subtracts noflow force data from an experiment and subtracts the atmospheric pressure readings from the data obtained from the pressure transducers. The noflow forces were subtracted to obtain the forces due to the flow of water over the gate. The atmospheric pressure was subtracted from the absolute pressure readings recorded to obtain differential pressure readings.

- TRANSFRM - This program manipulated the data recorded to obtain data that could be compared to the Olmsted 1:25 model data. The gate hinge forces on the 1:5 model were measured horizontal and vertical to the face of the gate. These forces were rotated to give forces horizontal and vertical to the channel bottom which was equivalent to forces measured on the 1:25 model. The forces measured on each side of the trunnion (two of these forces) were summed to give the total force on the shaft. There were only two pressures measured on the 1:25 model, one on the upstream side and one on the downstream side of the gate. To make a comparison to the $1: 25$ upstream pressure, the upstream top center and upstream middle center pressure readings from the 1:5 model were averaged. Likewise, the downstream top center and downstream middle center pressure readings were averaged to compare to the 1:25 model downstream pressure measurement.

- BINMATCH - This program extracts one or more channels of data from a raw data file and puts the channel(s) of data in a binary file compatible with Matlab.

- BINDPLCH - This program extracts one or more channels of data from a raw data file and puts the channel(s) of data in an unformatted data file compatible with DPLOT.

- IDAP2 \& IDAP525 - These were interactive programs that displayed data on-screen corresponding to different scale model experiments. IDAP2 was used to compare data from experiments run on the same model. IDAP525 was used to compare data when some of the data came from the 1:5 model and some from the 1:25 model. This program had zoom-in and zoom-out capabilities and the ability to perform screen dumps to the Laserjet printer.

To examine the frequency content of the data recorded, some custom Matlab m-files were written. 
A piece of software was written to ease importation of the binary data from the model into the IDEAS software package on the Structural Mechanics Division's Silicon Graphics computer. Without this software, the data from the model would have required conversion to ASCII format, greatly increasing the disk space required for intermediate data storage and the time required for data importation.

\section{Accuracy of Recorded Data}

Tests were conducted to determine the accuracy, sensitivity, and connectivity of the transducers installed on the gate. A static load experiment was used to check the effectiveness of the shear pins by equilibrating the input and measured output forces recorded in the shear pins. The pressure readings were calibrated with static water head tests to measure the accuracy of the pressure transducers. Also, overall system equilibrium was determined by equating the pressure field to the reaction forces measured with the installed transducers.

\section{Shear pins}

Three static dry load experiments were conducted to measure the accuracy of the shear pin transducers. A 500-lb load was applied at three positions over the gate surface and the experiment data were recorded for $30 \mathrm{sec}$. All experiments were conducted with the gate at the 0 -deg position. A 500-lb mass was applied:

a. On the tip of gate at 57.60-in. from base hinge axis (Experiment No. 37).

b. Above the hydraulic lifting cylinder, at 24.60 in. from base hinges (Experiment No. 38).

c. At or near hinges, at 5.3 in. from base hinges (Experiment No. 39).

Figure 64 displays the second experiment case in which the 500-lb mass was applied above the hydraulic lifting cylinder. The output responses recorded for the experiments are tabulated in Table 5. The second column in this table presents the transducer response for the 'zero reference test' (Experiment No. 36). A zero reference experiment was conducted prior to the static experiments to determine the residual magnitudes of forces for each transducer at the beginning of the static experiment. These residual forces were the initial readings for the sensors which nullify the effect of static dead load and the physical condition of the lowered gate. 


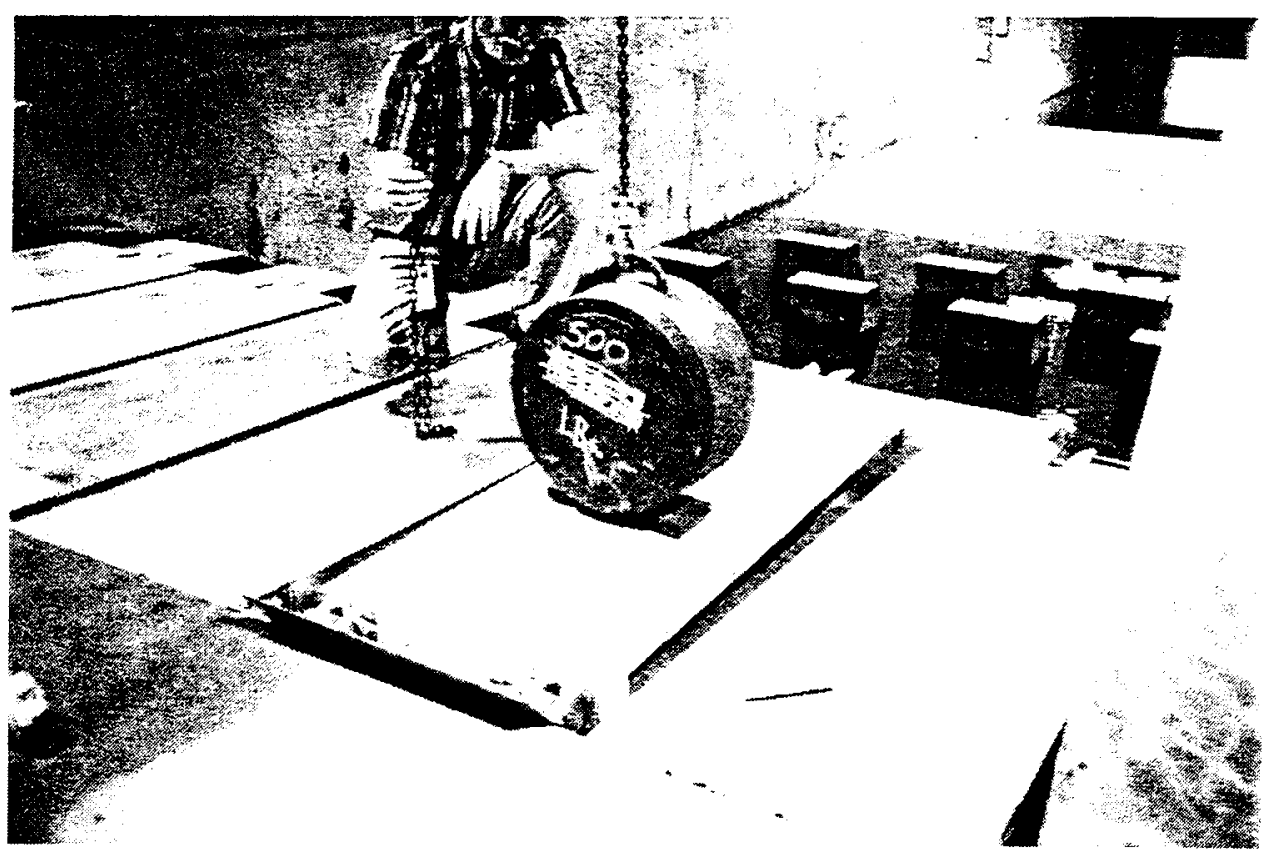

a. Positioning of load above lifting cylinder

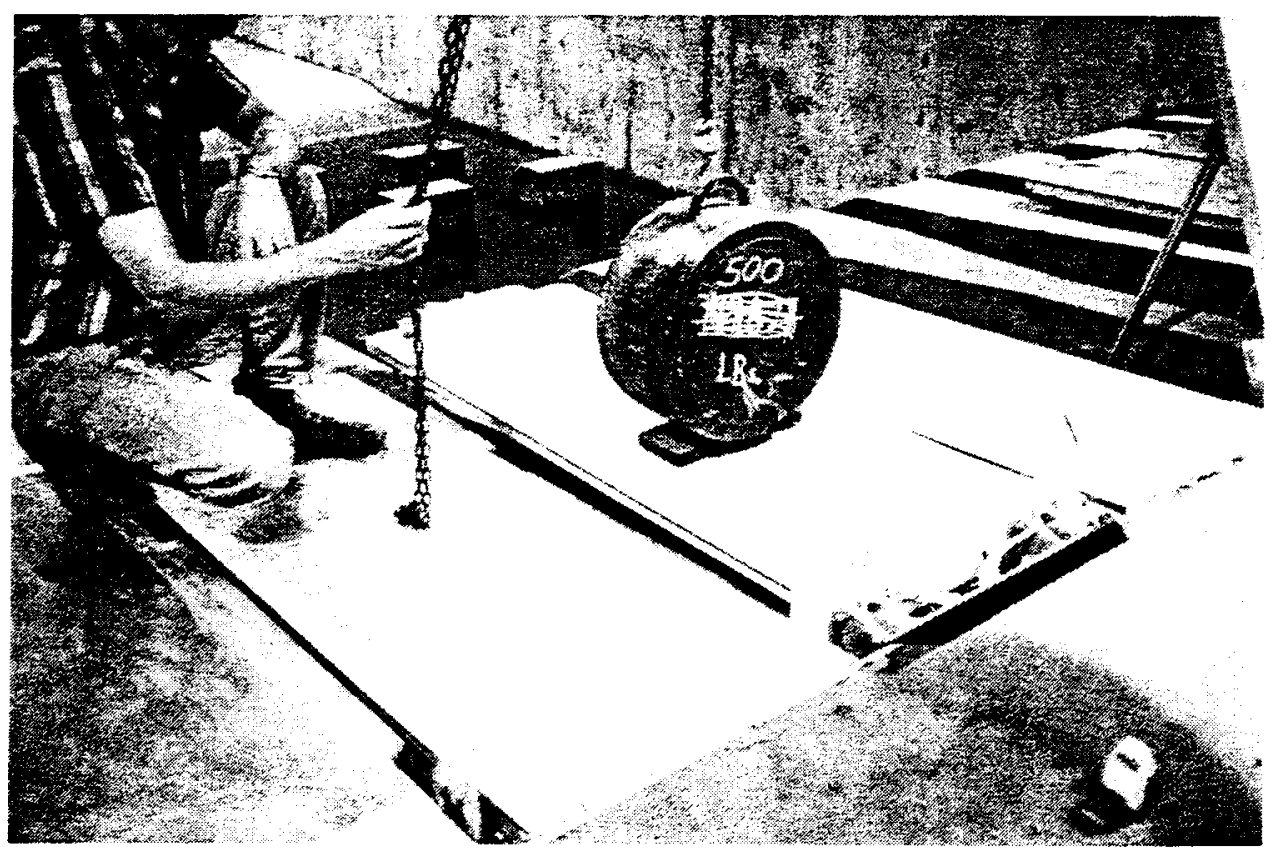

b. Isometric view

Figure 64. A static load experiment setup 


\begin{tabular}{|c|c|c|c|c|}
\hline \multicolumn{5}{|c|}{$\begin{array}{l}\text { Table } 5 \\
\text { Static Experiment Results for Verifying Shear Pin } \\
\text { Effectiveness }\end{array}$} \\
\hline Gauge Type & $\begin{array}{l}\text { Zero Reference } \\
\text { Experiment }\end{array}$ & $\begin{array}{c}\text { Load Case } \\
1 \\
\end{array}$ & $\begin{array}{l}\text { Load Case } \\
2\end{array}$ & $\begin{array}{c}\text { Load Case } \\
3\end{array}$ \\
\hline R. hinge, $z$. lbf $+v e \uparrow$ & -0.55 & -350.43 & -2.82 & 189.48 \\
\hline R. hinge, $y$, Ibf, $+v e \rightarrow$ & -1.44 & -8.59 & -31.54 & -29.41 \\
\hline L. hin $z$, lbf, +ve $\uparrow$ & -0.82 & -324.52 & -20.08 & 194.07 \\
\hline L. hir,$y$, lbf, $+v e \rightarrow$ & -0.80 & -22.75 & -3.96 & 25.95 \\
\hline R. trunnion, lbf, + ve $\uparrow$ & -0.81 & 599.57 & 265.00 & 58.22 \\
\hline L. trunnion, Ibf + ve $\uparrow$ & -0.30 & 584.20 & 241.67 & 40.82 \\
\hline Prop rod, Ibf & -1.00 & -0.67 & -1.74 & -0.98 \\
\hline Gate angle, deg & -0.35 & -0.38 & -0.35 & -0.36 \\
\hline Cylinder angle, deg & +4.13 & 4.17 & 4.15 & 4.13 \\
\hline $\begin{array}{l}\text { Sum of hinge forces, } z \text { - } \\
\text { direction, +ve } \uparrow\end{array}$ & -1.37 & -674.95 & -22.90 & 383.55 \\
\hline $\begin{array}{l}\text { Sum of trunnion forces, } z \text { - } \\
\text { direction. }+ \text { ve } \uparrow\end{array}$ & -1.02 & $1,180.43$ & 505.34 & 98.78 \\
\hline $\begin{array}{l}\text { Sum of reactions in } z- \\
\text { direction, }+v e \uparrow\end{array}$ & -2.39 & 505.48 & 482.44 & 482.33 \\
\hline
\end{tabular}

In this table the sum of hinge reactions in the z-direction provides the total vertical reaction forces at the gate hinges. Sum of the trunnion forces in the $z$-direction is the component of the total trunnion forces acting in the $z$-direction. Cylinder angle was considered in computing this directional force for the trunnion. From the equilibrium of the system, the sum of reactions row provides the weight of the applied load on the gate for each load case. A maximum error of 3.5 percent of the full load was observed in the experiments. This error may be partially attributed to the load transfer on the prop rod that had not been considered in the above calculations. Note that closer the shear pin readings were to the full range value (see Table 3 for full capacity of shear pins), the better the estimation. Deviation in the results was due to nonlinearity of the cause-effect relationship when the reading magnitudes were small compared to the full-range capacity. This experiment verified that the shear pins could measure loads with sufficient accuracy.

\section{Pressure transducers}

Pressure readings from two noflow experiments were used to test the validity of the pressure gauges. For static pool conditions, gauge readings for transducers were related to the actual depth of water column at their respective locations. Thus, by comparing the gauge readings and the static pressure heads, the sensitivity of the pressure transducers could be verified.

As mentioned earlier in the initialization of pressure gauges, pressure readings were recorded with respect to the initialization datum, gate at -3-deg position with a static $302-\mathrm{ft}$ pool. Therefore, the pressure reading at any position of the gate would indicate a negative reading equal to the vertical path traversed by the gauge above the datum (-3-deg position). Figure 65 shows the relationship between the recorded value and the actual pressure head at the 


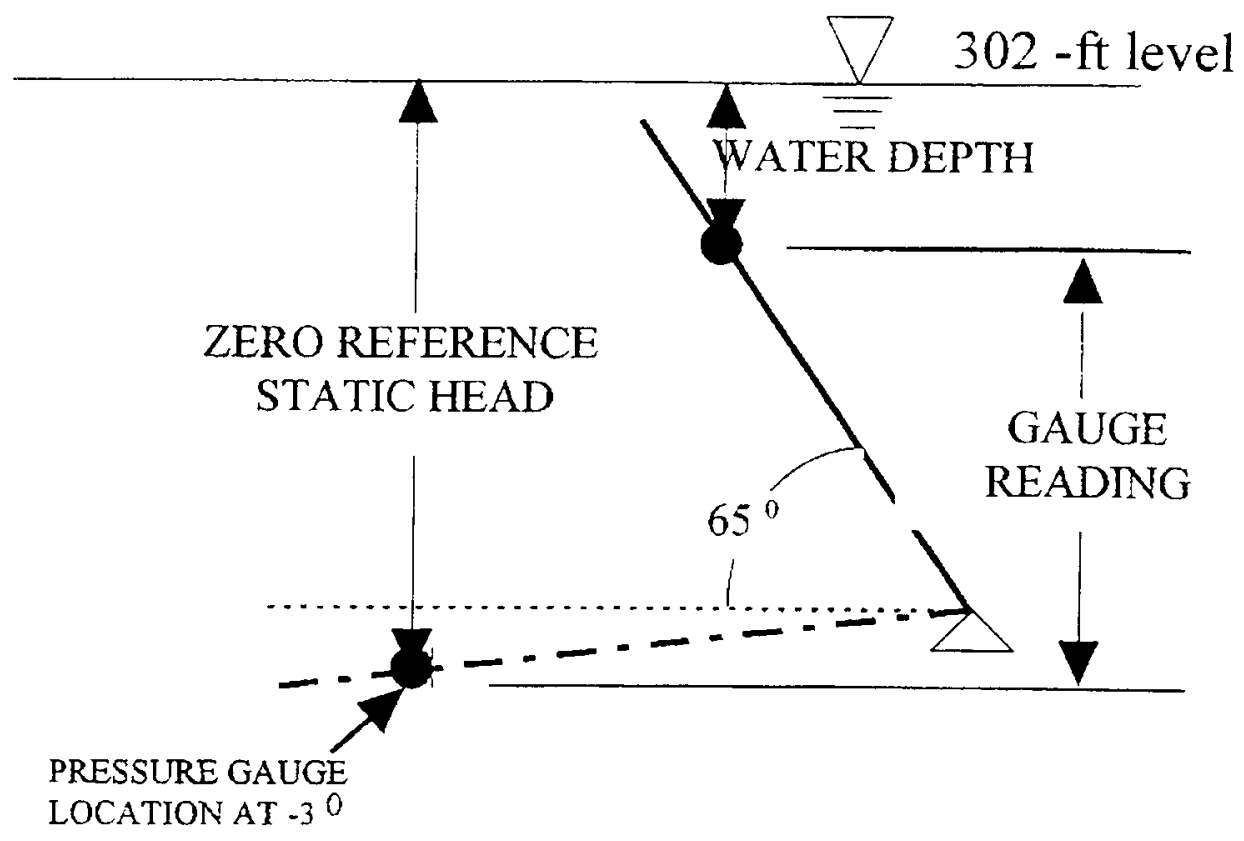

Figure 65. Relationship between pressure gauge reading and actual head

transducer location. As shown in the figure, the actual pressure on the gate at the gauge location (65-deg position) could be obtained by subtracting the gauge reading from the zero reference head.

After initializing the gauges, two experiments were conducted to verify pressure gauge validity. These were:

a. Constant Pool Elevation (302-ft) - pressure readings were taken when the submerged gate was raised from -3 to 65 deg (Experiment No. 41) at constant upstream and downstream pool elevations (noflow).

b. Fixed Gate, Variable Pool - conducted for the closed dam condition (all gates in the up position with their gaps closed by needles) with differing upstream and downstream pool elevations (Experiment No. 111).

Upstream and downstream pool elevations were constant at $296.5 \mathrm{ft}$ and $285.5 \mathrm{ft}$, respectively.

Pressure gauge readings corresponding to the initial datum are shown in Figure 66. As shown in this figure, head progressively decreased from the initial reference level $(302 \mathrm{ft}$ ) as the gate was raised from -3 to $65 \mathrm{deg}$. In the physical structure, the vertical distance traversed by each sensor thus represented the respective pressure drops recorded during the experiment. At any given gate angle, the distance traversed by each sensor could be calculated from the trigonometric relationship of the pressure gauge locations (Figure 60) and the gate angle. Actual pressure head, however, is the difference between the zero reference depth and the recorded water column height. Table 6 summarizes the 

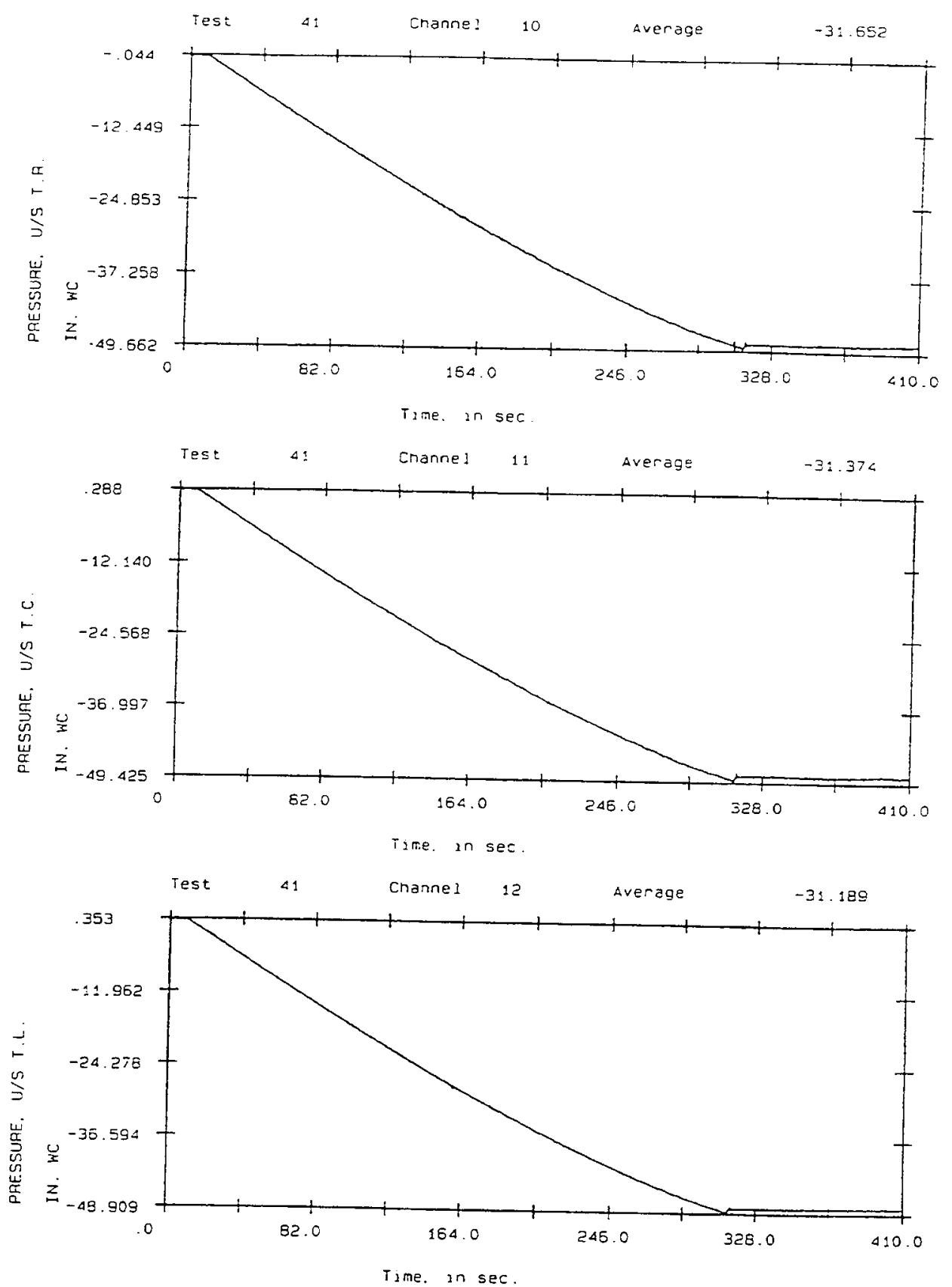

Figure 66. Raw pressure gauge readings for a zero-reference (Sheet 1 of 6)

effectiveness of the gauge readings by comparing the actual sensor movement from the datum (column 3 ) with the corrected gauge readings (column 6) obtained from the experiment.

In the second experiment, the pressure records and the physical static heads of each sensor were compared for a fixed gate-dam configuration. Note that due 

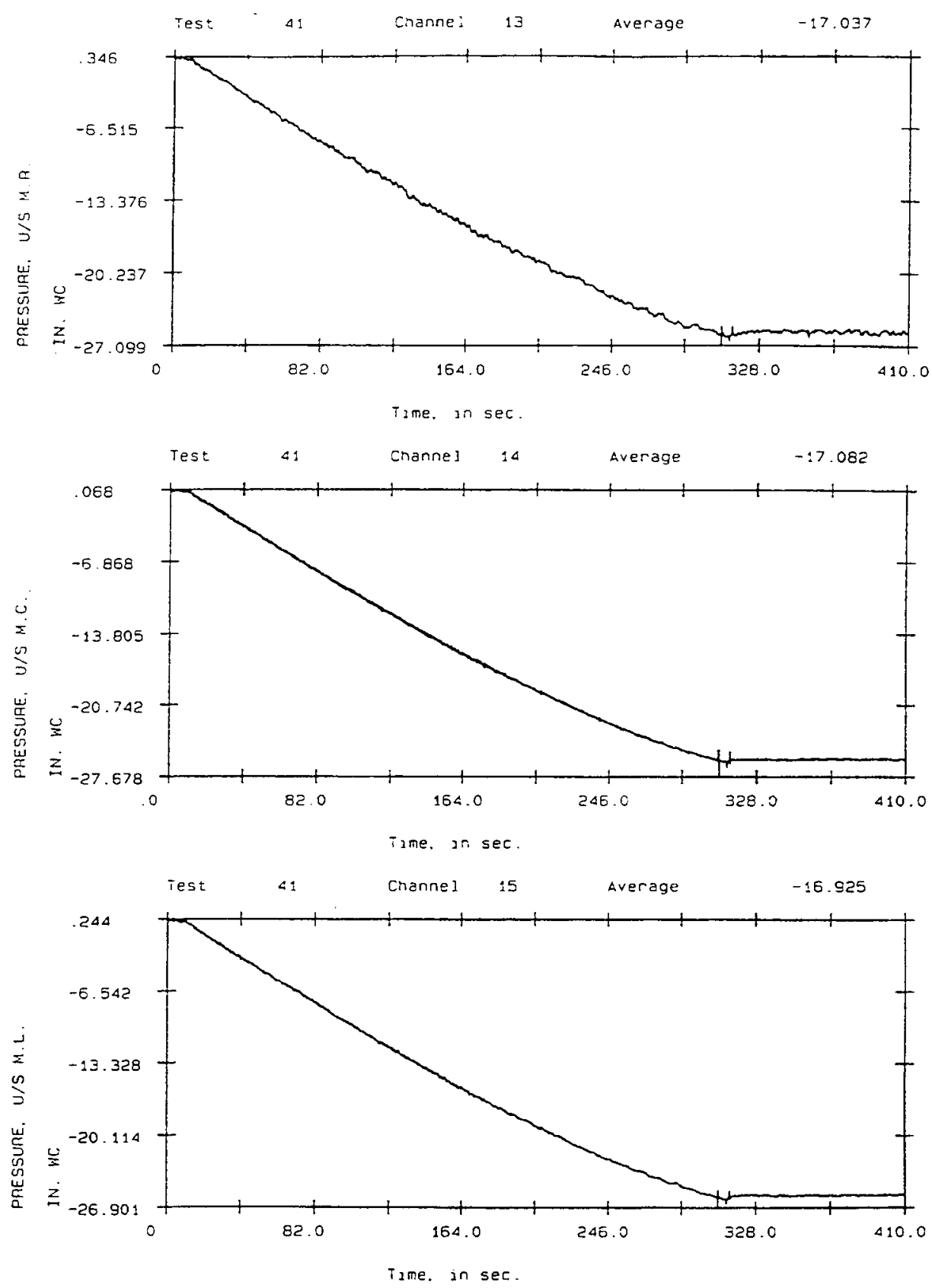

Figure 66. (Sheet 2 of 6 )

to difference in pool levels on upstream and downstream sides of the gates, all upstream gauges and the bottom three downstream gauges were always submerged, while the rest of the downstream gauges were exposed to the air. A negative head on estimated reading indicated that the gauge was being exposed to the atmosphere. The results for this experiment are summarized in Table 7. 

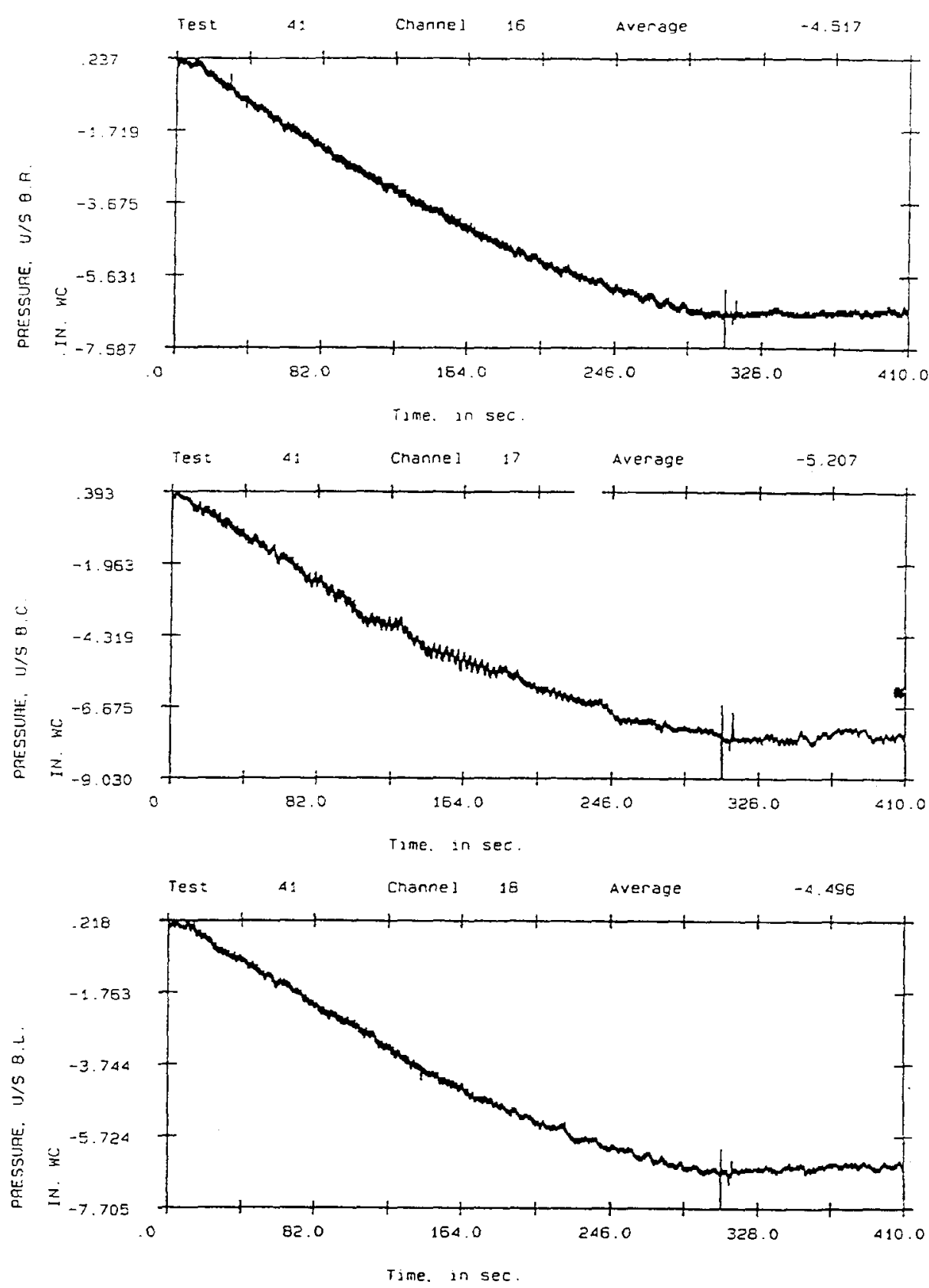

Figure 66. (Sheet 3 of 6 )

In this table, the third column represents the actual static head of the water column above the sensor. This water head could be obtained from the physical dimensions of the structure as described earlier in this section. The measured head is shown in the sixth column. Measured water head was computed by subtracting the corrected gauge reading (the fifth column) from the reference (static) head presented in the second column. As seen in the table, the estimated 

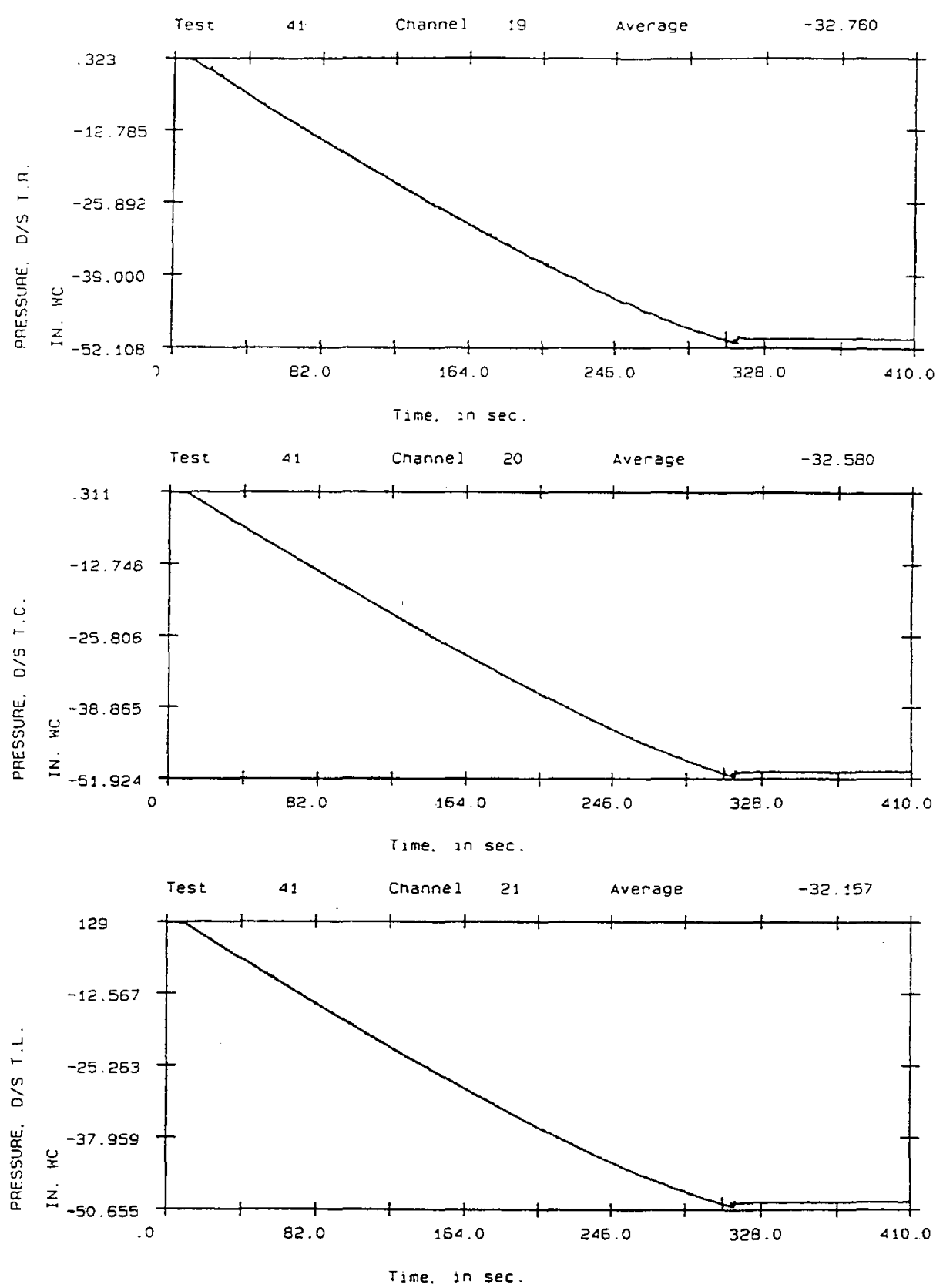

Figure 66. (Sheet 4 of 6 )

readings for all but the downstream bottom right gauge matched reasonably well with the corresponding static heads. An unaccounted increase in the velocity head due to flow through the gaps between the gate could decrease the pressure head at the downstream bottom right gauge location. 

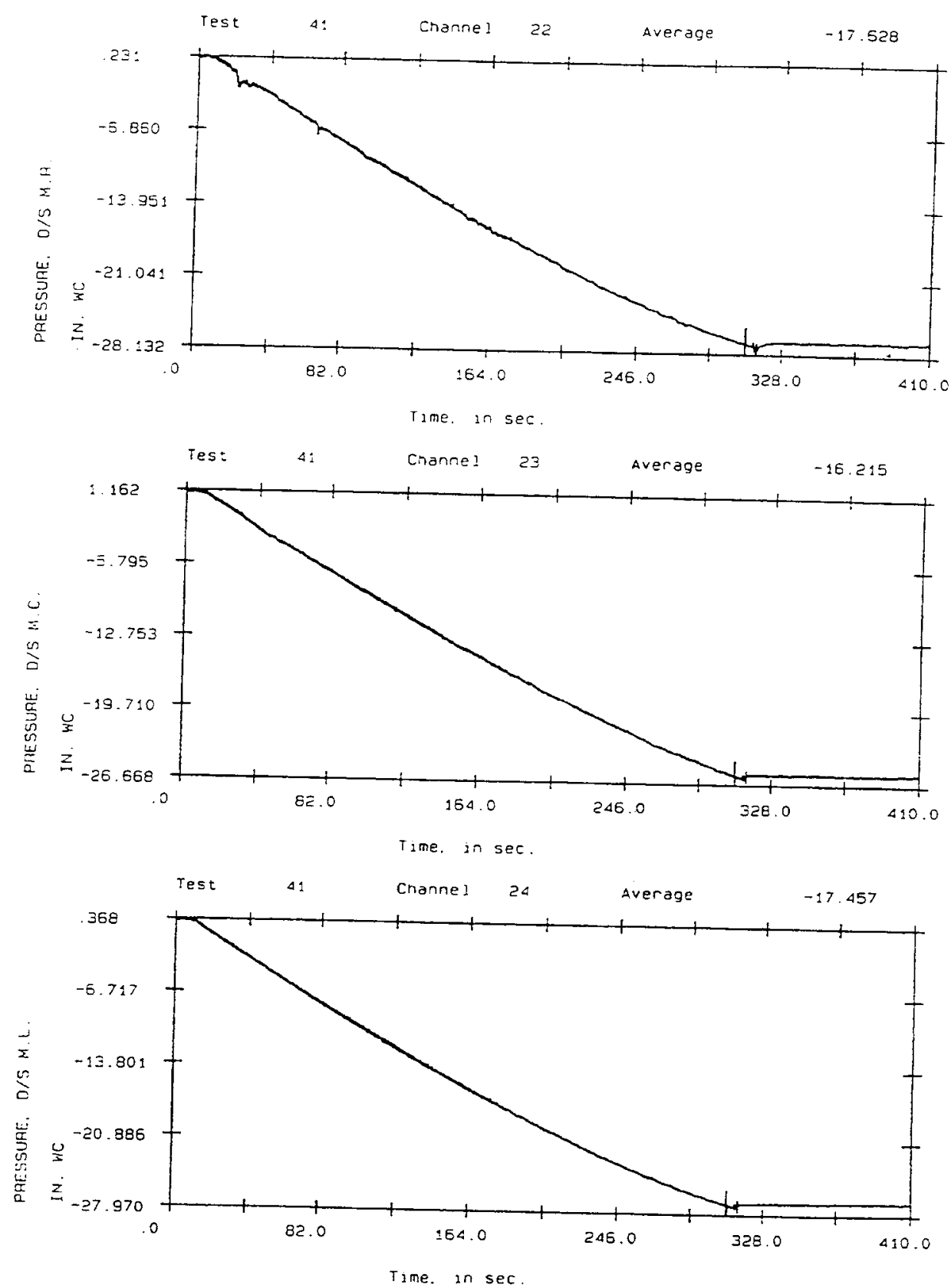

Figure 66. (Sheet 5 of 6 ) 

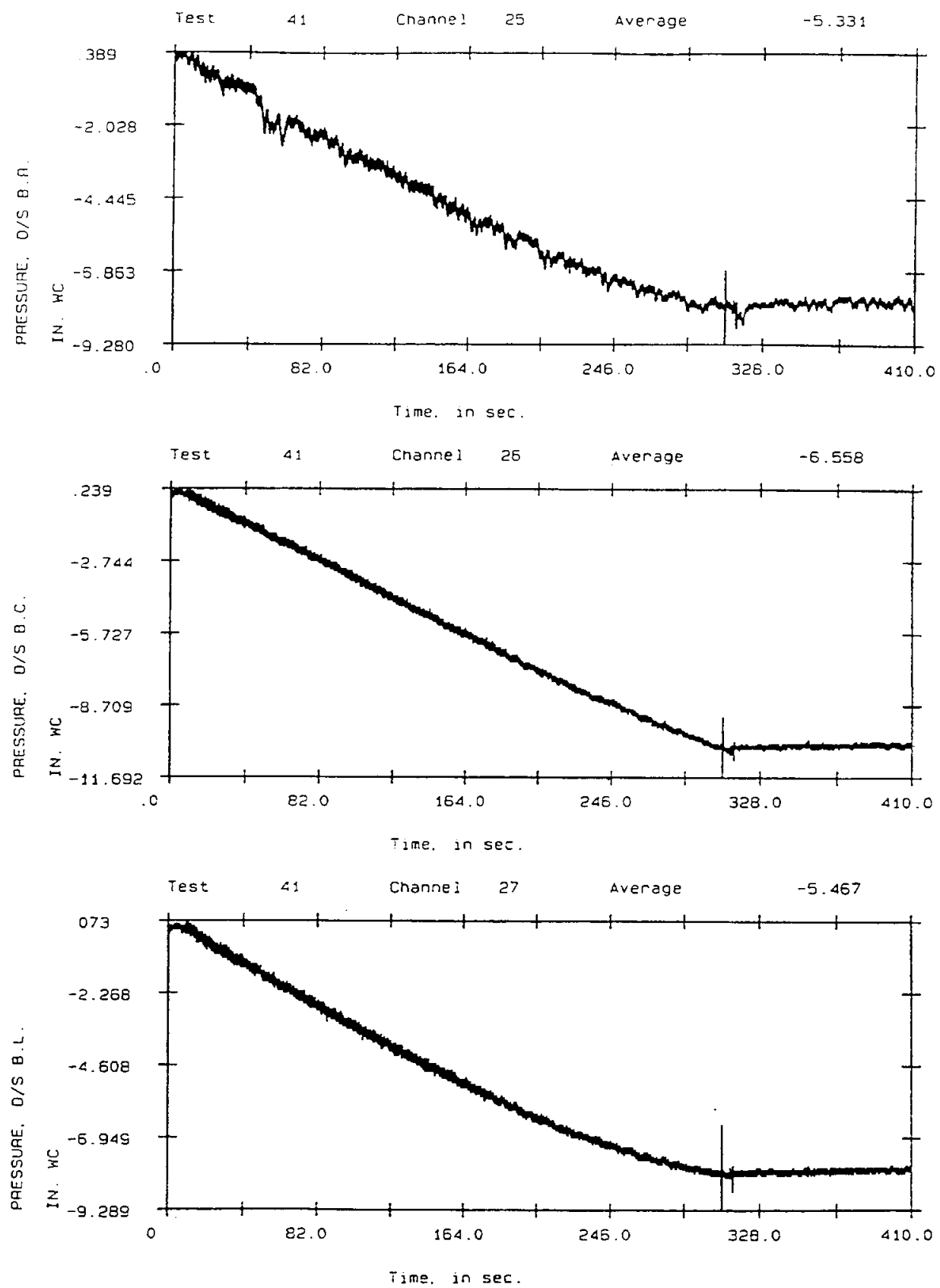

Figure 66. (Sheet 6 of 6 ) 


\begin{tabular}{|c|c|c|c|c|c|}
\hline \multicolumn{6}{|c|}{$\begin{array}{l}\text { Table } 6 \\
\text { Pressure Gauge Verification Table - Experiment 1: Constant Pool }\end{array}$} \\
\hline & \multicolumn{2}{|c|}{ Physical Condition } & \multicolumn{2}{|c|}{ Recorded Data } & \multirow{2}{*}{ Estimation } \\
\hline (1) & (2) & (3) & (4) & (5) & \\
\hline Position & $\begin{array}{l}\text { Static Head } \\
@-3^{\circ} \text { to } 302 \\
\text { Pool, in. of }\end{array}$ & $\begin{array}{c}\text { Traversed } \\
\text { Depth (from }-3^{\circ} \\
\text { to } 65^{\circ} \text { position). }\end{array}$ & $\begin{array}{c}\text { Zero Reference } \\
\text { Test Gauge } \\
\text { Readinq. in. of }\end{array}$ & $\begin{array}{l}\text { Difference in } \\
\text { Gauge Readings } \\
\text { at }-3^{\circ} \text { and } 65^{\circ} \text { in. }\end{array}$ & $\begin{array}{l}\text { Corrected Head, } \\
\text { in. of Water, } \\
\text { Column (5) - }\end{array}$ \\
\hline $\begin{array}{c}\text { Pressure, U/S } \\
\text { T.R. }\end{array}$ & 60.864 & -48.45 & -0.147 & -48.068 & -47.92 \\
\hline $\begin{array}{c}\text { Pressure, U/S } \\
\text { T.C. }\end{array}$ & 60.639 & -48.45 & 0.285 & -48.160 & -48.45 \\
\hline $\begin{array}{c}\text { Pressure, U/S } \\
\text { T.L. }\end{array}$ & 60.864 & -48.45 & 0.305 & -47.954 & -48.26 \\
\hline $\begin{array}{c}\text { Pressure, U/S } \\
\text { M.R. }\end{array}$ & 59.625 & -26.094 & 0.051 & -26.158 & -26.21 \\
\hline $\begin{array}{c}\text { Pressure, U/S } \\
\text { M.C. }\end{array}$ & 59.400 & -26.094 & 0.013 & -26.012 & -26.03 \\
\hline $\begin{array}{c}\text { Pressure, U/S } \\
\text { M.L. }\end{array}$ & 59.625 & -26.094 & 0.089 & -25.872 & -25.96 \\
\hline $\begin{array}{c}\text { Pressure, U/S } \\
\text { B.R. }\end{array}$ & 58.942 & -7.794 & 0.164 & -6.846 & -7.01 \\
\hline $\begin{array}{c}\text { Pressure, U/S } \\
\text { B.C. }\end{array}$ & 58.480 & -8.93 & 0.149 & -7.951 & -8.10 \\
\hline $\begin{array}{c}\text { Pressure, U/S } \\
\text { B.L. }\end{array}$ & 58.942 & -7.794 & 0.190 & -6.685 & -6.88 \\
\hline $\begin{array}{l}\text { Pressure, D/S } \\
\text { T.R. }\end{array}$ & 61.167 & -49.395 & 0.239 & -50.793 & -51.03 \\
\hline $\begin{array}{c}\text { Pressure, D/S } \\
\text { T.C. }\end{array}$ & 64.646 & -48.449 & 0.208 & -50.603 & -50.81 \\
\hline $\begin{array}{c}\text { Pressure, D/S } \\
\text { T.L. }\end{array}$ & 61.167 & -49.395 & -0.001 & -49.197 & -49.20 \\
\hline $\begin{array}{c}\text { Pressure, D/S } \\
\text { M.R. }\end{array}$ & 59.945 & -27.03 & 0.181 & -27.033 & -27.21 \\
\hline $\begin{array}{l}\text { Pressure, D/S } \\
\text { M.C. }\end{array}$ & 62.382 & -26.07 & 1.049 & -26.960 & -28.01 \\
\hline $\begin{array}{l}\text { Pressure, D/S } \\
\text { M.L. }\end{array}$ & 59.945 & -27.03 & 0.285 & -27.010 & -27.30 \\
\hline $\begin{array}{c}\text { Pressure, D/S } \\
\text { B.R. }\end{array}$ & 58.942 & -8.66 & 0.330 & -8.007 & -8.33 \\
\hline $\begin{array}{l}\text { Pressure, D/S } \\
\text { B.C. }\end{array}$ & 62.478 & -8.98 & 0.097 & -10.440 & -10.54 \\
\hline $\begin{array}{c}\text { Pressure, D/S } \\
\text { B.L. }\end{array}$ & 58.942 & -8.66 & -0.059 & -7.900 & -7.96 \\
\hline
\end{tabular}




\begin{tabular}{|c|c|c|c|c|c|}
\hline \multicolumn{6}{|c|}{$\begin{array}{l}\text { Table } 7 \\
\text { Pressure Gauge Verification Table - Experiment 2: Fixed Gate, Variable } \\
\text { Pool }\end{array}$} \\
\hline \multirow[b]{2}{*}{ (1) } & \multicolumn{2}{|c|}{ Physical Condition } & \multicolumn{2}{|c|}{ Recorded Data } & \multirow{2}{*}{$\begin{array}{c}\text { Estimation } \\
(6) \\
\end{array}$} \\
\hline & (2) & (3) & (4) & (5) & \\
\hline Position & $\begin{array}{l}\text { Static Head } \\
@-3{ }^{\circ} \text { to } 302 \\
\text { Pool, in. of } \\
\text { Water }\end{array}$ & $\begin{array}{l}\text { Water Head @ } \\
\text { Sensor } \\
\text { Position, } \\
\text { in. }\end{array}$ & $\begin{array}{l}\text { Zero Reference } \\
\text { Test Gauge } \\
\text { Reading, in. of } \\
\text { Water at 3-in. } \\
\text { position } \\
\end{array}$ & $\begin{array}{l}\text { gauge } \\
\text { Readings } \\
\text { at } 65^{\circ}, \text { in. } \\
\text { of Water }\end{array}$ & $\begin{array}{l}\text { Measured Head, } \\
\text { in. of Water, } \\
\text { Column (5)- } \\
\text { Column (4) }\end{array}$ \\
\hline $\begin{array}{c}\text { Pressure, U/S } \\
\text { T.R. }\end{array}$ & 60.864 & 0.5872 & -0.147 & -60.112 & 0.783 \\
\hline $\begin{array}{l}\text { Pressure, U/S } \\
\text { T.C. } \\
\end{array}$ & 60.639 & 0.5872 & 0.285 & -62.414 & -1.619 \\
\hline $\begin{array}{c}\text { Pressure, U/S } \\
\text { T.L. }\end{array}$ & 60.864 & 0.5872 & 0.305 & -60.490 & 0.500 \\
\hline $\begin{array}{c}\text { Pressure, U/S } \\
\text { M.R. }\end{array}$ & 59.625 & 21.7187 & 0.051 & -39.275 & 20.452 \\
\hline $\begin{array}{l}\text { Pressure, U/S } \\
\text { M.C. }\end{array}$ & 59.400 & 21.7187 & 0.013 & -39.435 & 20.215 \\
\hline $\begin{array}{c}\text { Pressure, U/S } \\
\text { M.L. }\end{array}$ & 59.625 & 21.7187 & 0.089 & -38.711 & 21.191 \\
\hline $\begin{array}{c}\text { Pressure, U/S } \\
\text { B.R. }\end{array}$ & 58.942 & 39.0237 & 0.164 & -20.587 & 38.431 \\
\hline $\begin{array}{l}\text { Pressure, U/S } \\
\text { B.C. }\end{array}$ & 58.480 & 37.9543 & 0.149 & -21.912 & 36.866 \\
\hline $\begin{array}{c}\text { Pressure, U/S } \\
\text { B.L. }\end{array}$ & 58.942 & 39.0237 & 0.190 & -20.534 & 38.512 \\
\hline $\begin{array}{c}\text { Pressure, D/S } \\
\text { T.R. } \\
\end{array}$ & 61.167 & AMBIENT & 0.239 & -62.930 & -1.965 \\
\hline $\begin{array}{c}\text { Pressure, D/S } \\
\text { T.C. }\end{array}$ & 64.646 & AMBIENT & 0.208 & -63.991 & 1.015 \\
\hline $\begin{array}{c}\text { Pressure, D/S } \\
\text { T.L. }\end{array}$ & 61.167 & AMBIENT & -0.001 & -60.378 & 0.917 \\
\hline $\begin{array}{c}\text { Pressure, D/S } \\
\text { M.R. } \\
\end{array}$ & 59.945 & AMBIENT & 0.181 & -58.774 & 1.461 \\
\hline $\begin{array}{c}\text { Pressure, D/S } \\
\text { M.C. }\end{array}$ & 62.382 & AMBIENT & 1.049 & -63.197 & -0.690 \\
\hline $\begin{array}{c}\text { Pressure, D/S } \\
\text { M.L. }\end{array}$ & 59.945 & AMBIENT & 0.285 & -60.554 & -0.382 \\
\hline $\begin{array}{l}\text { Pressure, D/S } \\
\text { B.R. }\end{array}$ & 58.942 & 11.808 & 0.330 & -54.528 & 4.214 \\
\hline $\begin{array}{c}\text { Pressure, D/S } \\
\text { B.C. }\end{array}$ & 62.478 & 11.5452 & 0.097 & -51.478 & 11.042 \\
\hline $\begin{array}{c}\text { Pressure, D/S } \\
\text { B.L. }\end{array}$ & 58.942 & 11.808 & -0.059 & -48.319 & 10.729 \\
\hline
\end{tabular}

\section{Overall equilibrium}

Equilibrium of the entire system was measured by equating the input and output forces for the prop supported gate under differential pool elevations, as shown in Figure 67. Figure 67 is a cross-sectional view of the dam showing the relative water depths and the orientations of the structural elements. In this figure, all pool elevations are shown in terms of Olmsted reduced level. The water depths, however, correspond to the 1:5-scale model. Note that the top longitudinal edge of the hinge cover plate rests on the gate skin plate to close the gap between the gate base and the bottom of the sill-bearing plate. Therefore, the 


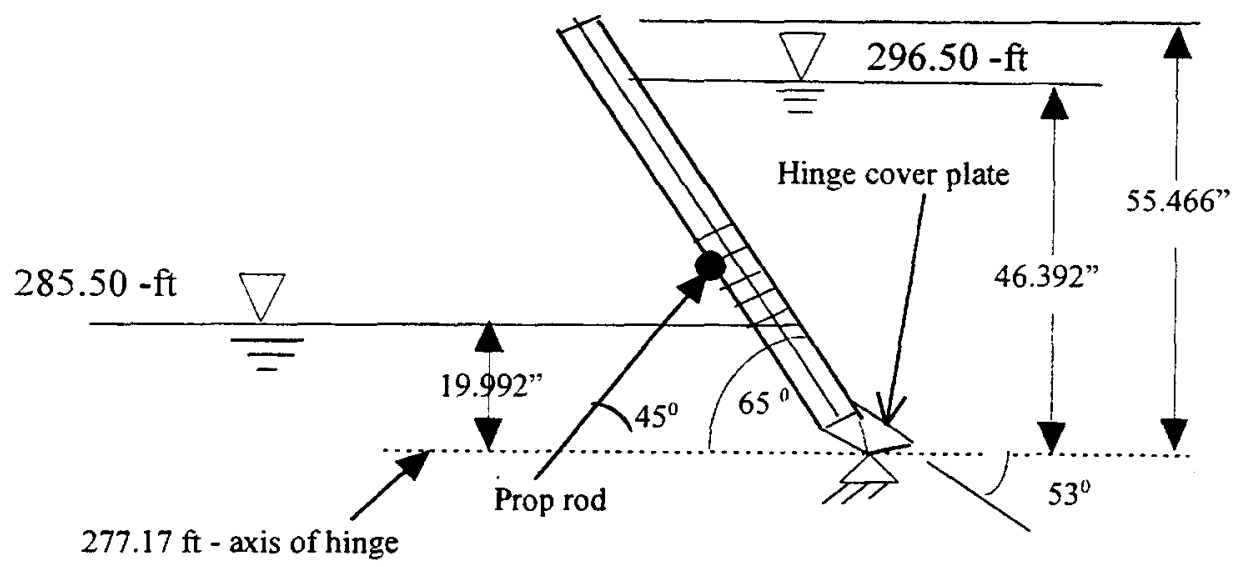

Figure 67. Relative water depths for overall equilibrium experiment

reaction forces transmitted from the hinge cover plate are acting on the gate along the line of their intersection.

The input forces consisted of pressure loads from the upstream and downstream sides of the gate. The upstream forces included the pressure on the skin plate and the reaction force transmitted from the hinge cover plate to the base of the gate. The backward water pressure on the submerged downstream side of the gate provided the downstream load. The output reactions included the prop and hinge reactions. Two sources of input forces caused the reactions on the gate. One due to the hydraulic pressure and another due to the dead weight of the gate.

Two experiments were conducted to determine the reaction forces which were due only to the hydraulic load. In the first experiment, Experiment No. 1000 , the reaction forces were measured for the prop-supported dry gate. In the second experiment, Experiment No. 111, all gates were raised, the gaps in between the gates were closed by needles, and the data were recorded for a $11-\mathrm{ft}$ differential head difference. A difference of the second test from the first one provided the net reaction forces which were solely due to the hydraulic pressure loads:

$$
F_{\text {hydraulic }}=F_{\text {wet total }}-F_{\text {dry }}
$$

These net reaction forces due only to hydraulic load are compared with the analytical results in Table 8 . The analytical reactions were computed by using the static equations of equilibrium of the system. Three such equations, one by summing the moment of all forces about the base hinge and two others by equating the normal and tangential forces, were used to compute the analytical results presented in the table. The measured and actual pressure heads for the 11-ft differential head difference experiment are compared in Table 7 . Note that the total hinge reactions along the axes are computed from the analytical results. This verifies that the transducers used in the model are functioning satisfactorily. 


\begin{tabular}{|c|c|c|c|c|}
\hline \multicolumn{5}{|c|}{$\begin{array}{l}\text { Table } 8 \\
\text { Overall Equilibrium of the Prop-Supported Instrumented Wicket } \\
\text { for } 11-\mathrm{ft} \text { Head Differential }\end{array}$} \\
\hline Gauge Type & $\begin{array}{c}\text { Dry } \\
\text { Condition }\end{array}$ & $\begin{array}{c}\text { 11-ft Head } \\
\text { Difference Test }\end{array}$ & $\begin{array}{c}\text { Net } \\
\text { Reactions }\end{array}$ & $\begin{array}{c}\text { Analytical } \\
\text { Results }\end{array}$ \\
\hline R. hinge, $z, \mathrm{lbf},+v e \uparrow$ & 0.989 & 128.959 & 127.97 & \\
\hline L. hinge, $z$, lbf, +ve $\uparrow$ & -7.590 & 32.456 & 40.046 & $\sum 176.618$ \\
\hline R. hinge, y. lbf, +ve $\rightarrow$ & -20.091 & 16.924 & 37.015 & \\
\hline L. hinge, $y$, lbf, +ve $\rightarrow$ & -44.401 & 46.424 & 90.825 & $\sum 139.7$ \\
\hline Prop Rod, Ibf, +ve 1 & 93.433 & 699.819 & 606.386 & 616.70 \\
\hline
\end{tabular}

\section{Data Analysis}

\section{Time domain}

The measured input was originally an analog signal. The front end converted the analog signal to a series of digital values. The digitized input signal as a set of $\mathrm{N}$ discrete values, evenly spaced in the period $\mathrm{T}$, could be displayed as a function of time using real-time.

The real-time scope displays a number of input channels in a variety of formats. MATLAB (The Mathworks 1992), DPLOT (WES 1995) and I-DEAS Master Series 1.2 (SDRC 1993b) were used to generate the time-domain data presented in this report. Time-domain signals could be displayed as function of time or as a function of gate rotation angle. All packages allow mathematical operations of the recorded channel such that the units of measurement could be converted, the analytical operations could be performed, and data could be displayed in model or prototype units. As mentioned earlier, the IDAP2 program was used during the experiment to check the consistency and quality of the recorded data by observing each recorded channel on site. The PROTPLOT program generated plot files from the raw data recorded. The plot files could then be output on the Laserjet printer.

\section{Frequency domain}

Signal processing operations on time-history records are based on the Fast Fourier Transform for converting time variant data to frequency data. Digital Fast Fourier transform capabilities of both MATLAB and I-DEAS were used to obtain the entire frequency spectrum of a time signal in a selected bandwidth. Details of mathematical formulation for Fourier Transform or Spectral Densities of signals can be found in several references (Ewins 1984, Bendat and Piersol 1980, Paz 1985, Stearns 1975, or Hewlett Packard 1986).

In I-DEAS, the Signal Processing task was used to process data using standard signal processing methods to obtain the desired results. These results included time averaging, auto correlation, correlation matrix, autospectra, 
spectral matrix, frequency response functions, order functions, and composite power functions, etc. It is the simplest method but has limitations when dealing with data that change rapidly with time. With the FFT technique, the frame size and number of spectral lines are interdependant. The number of spectral lines is equal to half the number of time elements plus one. Furthermore, the FFT technique assumes stationary and periodic data within each time slice. Consequently, when performing time variant analysis, one could shorten the time slice to improve stationarity, but this would also lower the spectral resolution and may increase leakage error. Use of a Hanning window helps but does not eliminate the smearing caused by low spectral resolution. Hanning windows with a 50 percent overlap were used to generate spectra from time variant data.

\section{Limitations}

Although extensive precautionary efforts were undertaken to minimize the sources of error that could affect data recording, there were still a number of nature-dependent external sources in the model. Errors in the data could result from environmental uncertainty, human tolerance, and/or machine or instrument tolerance. One such error was due to pool level fluctuation resulting from the unsteady flow pattern that could not be maintained within a 1\2-ft of prototype head in the model. Temperature variations could easily affect the performance of the mechanical and electrical devices used for recording the sensor responses. A time-dependent drift of the mechanical and electrical systems may also add to deviation of the recorded response from the true one.

As outlined in the sensor verification section in this chapter, a low signal-tonoise ratio could also affect the measured res . nses. The higher the response, the better the prediction. Thus, when the measured response is closer to the calibrated value, it is more accurate. In the model study, however, this is not always the case. A random variation of responses from experiment to experiment existed in the study presented in this report. Thus, an error is expected due to low sensor sensitivity, primarily for lower order responses.

A slight misalignment of the entire gate assembly, a random lateral tilting of the clevis attached to the prop rod, and repositioning of the ball in the cup caused nonuniform distributions of reaction forces at the bottom hinges. Table 8 confirms such phenomena of uneven hinge reactions distributions due to the symmetric load application on the gate. 


\section{Similitude Model}

\section{Background}

Historically, controlled field experiments and/or laboratory experiments have provided the bulk of experimental data for the investigation of many engineering problems. Controlled field experiments have the advantage of modeling the complexity of the full-scale condition. However, these experiments are sometimes prohibitively expensive, difficult to perform, and usually offer little direct control over boundary conditions. On the other hand, reduced-scale laboratory experiments, which are often inexpensive and relatively easy to perform, are frequently limited by their inability to model realistic boundary conditions in many of the engineering problems. Establishment of scaling laws therefore requires an in-depth understanding of the physical behaviors or existing theories of the prototype system (Batista, Carvalho, and Roitman 1991, and Tanaka 1990).

The scaling laws are established such that the scale model replicates the behavior of the actual system (prototype). The accuracy of the predicted response for the prototype (from the experimental results of the scale model) depends upon the relationship between the corresponding variables and parameters of the model and its prototype. Parameters that describe the physical behavior include geometry, material behavior, dynamic response, and energy characteristic of the system. System similarity requires that the relevant system parameters are identical in model and prototype and these systems are governed by a unique set of characteristics equations. Similitude laws establish scale factors $(\lambda)$ for each important physical variable in a system. Ratios of physical parameters are such that they represent the analytical formulation of system behavior.

Two procedures are widely used to establish similarity conditions between the model and prototype. In modeling a relatively unknown phenomena, for which mathematical models are unknown or uncertain, dimensional analysis are used to establish similarity conditions between variables in model and prototype. By using a dimensional analysis, an incomplete form of the characteristics equations can be formulated in terms of nondimensional products of system variables and physical parameters. The direct use of governing equations, however, can be used to establish similitude relations for a system in which the effects of all system variables and parameters are known. More detailed 
information on establishing similitude scale factors using these methods can be found in the literature (Novak and Cabelka 1981, Baker. Westine. and Dodge 1973, and Simitses and Razaeepazhand 1992).

Scaling laws are the most fundamental aspect of every physical theory and led to the explanation of many complex problems, which are too large to be tested systematically (Caccese and Harris 1990. Farrar, Baker, and Dove 1994), or too complicated to model numerically (Roitman, Andrade, and Batista 1992), or too uncertain in their behavioral relationships (Schofield 1981). Primarily, scaling laws are used to validate analytical formulation or extract system parameters for analytical model, to develop empirical relationship, to aid in developing improved conceptual models of fundamental process, and to understand the nature of the problem from reduced-scale laboratory experiments (Bazant 1993, Boyce and Kana 1993, Tanaka 1990, Key 1989, Tao 1990, and Solignac, Pagan, and Molton 1989).

Bazant (1993) has examined scaling laws to study the deterministic role of size effect in various types of failure theories in structural mechanics. He has shown very effectively that the understanding of the physical phenomena is the core of the scaling law. Bazant's investigation was strictly theoretical with an attempt to synthesize the experiences from numerical and experimental studies.

In modeling the gravitational effects on engineering structures, centrifuge modeling has played a critical role in developing improved conceptual models of fundamental processes (Schofield 1981). In a gravity-dominant engineering scale model, such as an earthquake model, offshore gravity platforms, soil contaminant transport processes, and soil liquefaction studies, scaling of the gravitational field is essential for obtaining stress conditions that are homologous in the soil model and the prototype (Schofield 1981, Culligan-Hensley and Savvidou 1995). Centrifuge modeling provides the most versatile technique for reproducing prototype stress conditions, self-weight and buoyancy-induced forces, and accelerations of certain time effects such that the governing constitutive equations are valid in both the model and prototype. This is achieved by subjecting a geometrically scaled model to a centrifugal acceleration of $n$ gravities (ng). In a steady centrifuge flight, the parameters of centrifuge motion can be adjusted such that the three components of acceleration can simulate three types of motion in a prototype. In such a simulation, horizontal shaking, vertical shaking, and the earth's gravity are simulated by the three components of angular acceleration of the flight motion (Schofield 1981). Centrifuge model experimentation, however, is expensive and limited to the size of the centrifuge facility.

Another important use of similitude models in understanding physical phenomena can be found in wind tunnel investigations of bridge structures. The similitude study of wind-bridge interaction enables researchers to identify causes of wind-induced catastrophic disasters (Tanaka 1990). Consideration of windinduced dynamic behavior was not a serious topic for bridge engineers until the discovery of self-excited oscillations through wind-tunnel investigation of bridges. Wind-tunnel modeling requires: aeroelastic similarity, similarity of wind-induced dynamics, and consistent matching of dimensional scale and 
geometrical shape for the structure. In reality, however, all aeroelastic models are so-called equivalent models, which maintain only the geometric shape and dynamic characteristics (modal parameters) of the prototype (Tanaka 1990 and Solignac. Pagan, and Molton 1989).

Adjustment of scaling factors to minimize scaling-factor distortion errors is normally required in similitude studies. Almost invariably, a complete duplication of system behavior by satisfying all governing equations in a model or satisfying nondimensional relations established by dimensional analysis is impractical or impossible ( Tanaka 1990, Boyce and Kana 1993, Caccese and Harris 1990, Batista, Carvalho, and Roitman 1991, and Chakrabarti 1992). In reality, the requirements for exact similitude can be met only when model and prototype are identically scaled. Hence, it becomes a critical decision for the modeler to determine which parameters should be relaxed for each simulation without neglecting the major phenomena of the system. Therefore, the decision must be based an understanding of the physical phenomena and a knowledge of dominant parameters. The current trends in addressing such modeling distortion in different branches of engineering are summarized below.

Boyce and Kana (1993) have developed a rational relationship between soil strain levels in laboratory compression tests and pile response tests to account for the distortion in soil properties due to geometric effects. They used a scale model for predicting the earthquake response of pile foundations embedded in over-consolidated soil. Gravity effects were simulated in the models by scaling pile and soil material properties. Similitude requirements were used to provide the correctly scaled stiffness and mass properties for the model piles. Using a trial-and-error procedure, a model soil was synthesized such that its stiffness and damping properties were correctly modeled.

Caccese and Harris (1990) have addressed the limitations of small-scale modeling in predicting the earthquake response of reinforced concrete structures. They have accounted for the mass-density distortion (by using the same material for prototype and model, the scaling factor for mass density, $1 / S_{l}$, was not met in the study, where $S_{l}$ is the scaling factor for length) by using artificial mass in the structure such that the added mass would not appreciably change the stiffness. Considering the limitations of the small-scale models, they suggested that the large-scale simulations and/or mathematical analyses should support the adequacy of a small-scale model if prototype behavior is to be predicted. In earthquake simulation, lack of large-capacity facilities severely limits the scale (size) of models that may be used.

Farrar, Baker, and Dove (1994) examined the similarity between the elastic dynamic parameters of reinforced concrete replica models and their prototypes. Similitude relations between variables influencing the dynamic response of concrete structures for different damping mechanisms were also developed. The study showed that the viscous damping forces were distorted in the model and a proper scaling of damping forces requires consideration of both material properties and geometric scaling factors for the model. Experimental results 
showed that the scale mo: I could accurately predict the modal dynamic parameters (frequency and mode shapes) of a prototype structure. This study again emphasized that any distortion in the scaling factors due to modeling constraints must be accounted for during the subsequent data reduction and comparison process. By examining the overall deviations in the dynamic response due to various damping mechanisms. they pointed out the necessity of error quantification for measuring the effectiveness of a scale model. In their study, they used a normalized scaling factor, $\omega_{n}=\omega_{m} \sqrt{E_{n} / E_{m}}$ (where $\omega_{n}$ and $\omega_{m}=$ normalized and measured resonant frequencies and $E_{m}$ and $E_{n}=$ modulus of elasticity for the test structure to which the resonant frequency is being normalized) to adjust the measured resonant frequency to account for the variations in the modulus of elasticity and mass density between the model and prototype.

Simitses and Razaeepazhand (1992) used a similitude model to study the cylindrical bending of orthotropic laminated beam-plates. They verified, with reasonable accuracy, that the scale model could effectively predict the prototype behavior. They also demonstrated that the sensitivity of size-effect increases as the model size decreases. As a result, the difference in behavior between the model and prototype increases as the size decreases. In their investigation, both dimensional analysis and direct use of governing equations were considered to derive the similarity relationships between the model and prototype parameters. In case of a new phenomena for which the mathematical model is not known, dimensional analysis could be used to formulate an incomplete form of characteristic equations for the system.

A hydroelastic small-scale model was investigated by Roitman, Andrade, and Batista (1992) to understand the mechanical behavior of a deep-water tension leg platform subjected to wave action. In this study, steady-state time response was analyzed to identify the general dynamic parameters to verify a simplified numerical model and to conceptualize the effects of fluid-structure interaction due to simulated impacts and wave action. This experiment testifies that the global dynamic parameters could be simulated favorably with a very small-scale model (1:169) without going through elaborate reproduction of an exact replica of its prototype.

Batista, Carvalho, and Roitman (1991) have demonstrated that even a geometrically distorted, very reduced scale model (1:169), when carefully constructed by adjusting the physical geometry within the similitude conditions, could favorably assess the dynamic characteristics of large offshore structures. An experimental model was built to simulate the behavior of an offshore compliant tower that behaves mainly as an inverted pendulum immersed in water, oscillating under action of sea waves. In that experiment, the physical parameters of the model were adjusted by considering the geometric, elastic modulus, and fluid density scale factors in the general similitude relations. Note that one of the basic elastic requirements $\left(\lambda_{E}=\lambda_{\rho} \lambda_{l}\right)$ for the Froude model was not met in this experiment by not using a properly scaled elastic modulus for the model. Steel was used in the prototype while a combination of steel, aluminum, 
polypropylene, and plywood were used in the model. The major feature of this physical model was that both rigid body and elastic motions were observed in tests performed using plain water as the modeling fluid. It was reported that for such a reduced scale-model hydroelastic structural similitude conditions could be approximated best by performing the experiments in plain water.

Tao (1990) has experimented with a prototype dam and five small-scale models to derive the similitude relationship for flow pressure pulsation. He showed that the scale factor for pressure conformed to the similarity relations of gravity, $\lambda_{p}=\lambda_{1}$ ), but the corresponding frequency scale did not.

Distortion of scaling laws in modeling the hydro-elastic behavior of an offshore oil storage tank was systematically examined by Chakrabarti (1992). Two modeling criteria based on the structural and hydraulic similitude relationships were considered to study the overall dynamics of the model. In the experiment, the elastic forces provided by the tank wall stiffness were the dominant factor for correctly simulating the structural response, while the Froude law was used to simulate the flow dynamics. By using a different material (modulus of elasticity directly scaled by the geometric scale) in the Froude model, both elastic and hydraulic relationships were considered in one experiment. The experiment showed that the internal waves generated by a rigid (nonelastic) and a Froude model were different. A variation in the wave amplitude was related to the movement of the tank at its foundation. Note that the weak supporting condition (model base was placed on a $1 / 2$-in.-thick aluminum slab) in the rigid model may have contributed to the differences in the wave height. Elastic deformation of the tank due to the use of a different material (different modulus of elasticity) did not significantly affect the sloshing behavior of the fluid. No study was conducted to determine the effects of geometric scaling of the tank stiffness on the dynamics of the tank fluid without changing the prototype tank material.

\section{Similitude Relationships for Wicket Gates}

Flow-induced structural motion of a wicket gate is the result of interaction between the hydraulic and structural behavior of the system. A similitude model thus requires simulation of these two dominant physical phenomena by reproducing the hydraulic phenomena caused by the flow and gate structural motion. Hydraulic similarity is usually based on empirical relationships established by dimensional analysis. Structural similitude relations can be obtained directly from the governing dynamic equation of motion. As mentioned in the previous discussions on the use of similitude models, it is impossible to attain a model that satisfies exactly the hydro-elastic similitude requirements for both physical phenomena unless the model and prototype are identical.

Considering the limitations of a similitude model, similar behavior for the flow-induced vibration of both prototype and scale-model wicket gates was achieved by using the hydraulic and structural behavior in the modeling technique. A hydraulic relationship was used to ensure similar flow-induced forces for both systems by equating the Froude and Strouhl law relationships. 
An equality in Froude numbers, together with geometrical similarity for both gates, ensured dynamic similarity for the system.

Using the similitude scaling laws, as described below, scale factors (ratios of a certain quantity in model and corresponding quantity in the prototype) for important variables, such as mass density, frequency, modulus of elasticity and time were established between the model and prototype system. These scaling relationships included geometric, kinematic, and dynamic similarities.

A geometrically similar model should have the same shape as that of the prototype, and all dimensions are reduced in the same scale. Kinematic similarity signifies similarity in motion of homologous points (corresponding points but not necessarily same points). Two systems are dynamically similar if homologous parts of systems experience homologous net forces.

\section{Hydraulic model similitude relations}

Basic physical parameters and system variables that influence the gate and fluid motion are presented in Table 9. The dimensions in terms of the fundamental quantities, $F, L$, and $T$, are also shown in this table.

\begin{tabular}{|c|l|c|}
\hline \multicolumn{2}{|l|}{$\begin{array}{l}\text { Table } 9 \\
\text { Physical Parameters for Hydraulic Modeling }\end{array}$} \\
\hline \hline Symbol & Parameter & Basic Dimensions \\
\hline $\mathbf{E}$ & Length & $\mathrm{L}$ \\
\hline $\boldsymbol{E}$ & Material modulus & $\mathrm{FL}^{-2}$ \\
\hline $\boldsymbol{v}$ & Mass density & $\mathrm{FL}^{-4} \mathrm{~T}^{2}$ \\
\hline $\mathbf{F}$ & Poission's Ratio & - \\
\hline $\mathbf{T}$ & Force & $\mathrm{F}$ \\
\hline $\mathbf{a}$ & Time & $\mathrm{T}$ \\
\hline $\mathbf{V}$ & Acceleration & $\mathrm{LT}^{-2}$ \\
\hline $\boldsymbol{\delta}$ & Velocity & $\mathrm{LT}^{-1}$ \\
\hline $\mathbf{f}$ & Fisplacement & $\mathrm{L}$ \\
\hline $\mathbf{P}$ & Pressure & $\mathrm{T}^{-1}$ \\
\hline $\boldsymbol{\varepsilon}$ & Strain & $\mathrm{FL}^{-2}$ \\
\hline $\boldsymbol{\sigma}$ & Stress & -- \\
\hline
\end{tabular}

The functional relationship between these variables can be established in terms of $\mathrm{N}$ dimensionless quantities called "Pi $(\pi)$ terms" by the Buckingham theorem, where $\mathrm{N}$ is the number of significant variables minus the number of primary 
dimensions (Novak and Cabelka 1981). In this case, there are three primary dimensions $(F, L, T)$, so there are 10 Pi-terms which could be obtained from the functional relations of the relevant variables. In functional format the above parameters can be written as:

$\phi(L, E, \rho, v, F, T, a, V, \delta, f, P, \varepsilon, \sigma)=02$

Writing the functional equation in terms of dimensional homogeneity (Baker, Westine, and Dodge 1973) and using the matrix approach, the equation of dimensional homogeneity can be established. Equating the terms of like exponents in the equation of dimensional homogeneity, the dimensionless $\pi$-terms could be obtained. The nondimensional Pi terms are:

$$
\begin{gathered}
\pi_{1}=v, \pi_{2}=\varepsilon, \pi_{3}=\frac{F}{E L^{2}}, \pi_{4}=\frac{T \sqrt{E}}{L \sqrt{\rho}}, \pi_{s}=\frac{L \rho a}{E}, \\
\pi_{6}=\frac{\sqrt{\rho V} V}{\sqrt{E}}, \pi_{7}=\frac{\delta}{L}, \pi_{8}=\frac{L \sqrt{\rho} f}{\sqrt{E}}, \pi_{9}=\frac{p}{E}, \pi_{10}=\frac{\sigma}{E}
\end{gathered}
$$

It can be shown that the above Pi terms could satisfy the Newton's second law of motion as well, thus ensuring that dynamic similarity for the model and prototype are attained. A dynamic similarity of the homologous parts of the model and prototype are obtained from Newton's law, force = mass $\mathrm{x}$ acceleration, such that the scaling factors are identical on both sides of the equation. For a Froude model, the scale factor for force is $\lambda_{F}=\lambda_{E} \lambda_{L}^{2}$, and the right-hand side scale factor can be obtained from $\left(\lambda_{m} \lambda_{a}=\lambda_{\rho} \lambda_{L}^{3} \lambda_{E} / \lambda_{L} \lambda_{\rho}\right)$. The scaling factor $(\lambda)$ for a variable or parameter is obtained when the ratio of the Pi terms for model and its prototype are set to unity. For example, the scale factor for forcing function can be obtained from the third Pi term, which is, $\lambda_{F}=\lambda_{E} \lambda_{L}^{-2}$. For geometric similarity (scaled model $\mathrm{m}$ and prototype $\mathrm{p}$ ), the 1:5 Olmsted model was such that its shape corresponded exactly to the prototype, and all dimensions were reduced by the same scale, $\lambda_{L}=L_{m} / L_{p}$.

Thus the similitude relationship, which includes geometric, kinematic and dynamic similarity between model and prototype, is obtained by equating corresponding Pi terms for the model and prototype.

A close relationship of these Pi terms with the physical phenomena of the hydraulic model is well established. These nondimensional quantities, commonly known as Reynolds number, Froude number, Cauchy mumber, etc., are extensively used to define the characteristics of hydraulic flows. A brief discussion of these significant quantities is summarized. 


\section{Significance of Pi terms}

The dynamic equilibrium of the hydraulic model includes four types of forces. These are: (a) inertial, (b) damping, (c) gravitational, and (d) elastic. The first two force systems represent the state of movement, and the last two represent the reaction to movement.

In the above equations, the Cauchy and Strouhl conditions are met. These can be verified by substituting the general similitude relationships stated above into the following:

Cauchy Number $(\mathbf{C a})=C a=\frac{\text { Inertial Force }}{\text { Gravitional Force }}=\frac{\rho V^{2}}{E}$

$$
\left(\frac{\rho V^{2}}{E}\right)_{m}=\left(\frac{\lambda_{p} \rho_{p} \lambda_{E} \lambda_{p}^{-1} V^{2}}{\lambda_{E} E}\right)_{p}=\left(\frac{\rho V^{2}}{E}\right)_{p} \equiv \pi_{6}
$$

Strouhl Number $($ Sh $)=S h=\frac{\text { Vortex Shedding Frequency }}{\text { Flow Velocity }}=\frac{f L}{V}$

$$
\left(\frac{f L}{V}\right)_{m}=\left(\frac{\sqrt{\lambda_{E}} \lambda_{L}^{-1} \sqrt{\lambda_{\rho}} f \lambda_{L} L}{\sqrt{\lambda_{E} \sqrt{\lambda_{\rho}}} V}\right)_{p}=\left(\frac{f L}{V}\right)_{p} \equiv \pi_{8}
$$

Froude Condition $\left(\mathbf{N}_{\mathrm{F}}\right)=N_{F}=\frac{\text { Inertial effect }}{\text { Gravity effect }}=\frac{V^{2}}{g L}$

Using the general similitude relations and defining, $\lambda_{g}=\frac{g_{m}}{g_{p}}$ the Froude equation becomes

$$
\left(\frac{V^{2}}{g L}\right)_{m} \cong\left(\frac{V^{2}}{g L}\right)_{p}=\frac{\left(\sqrt{\lambda_{E}} \lambda_{\rho}^{-1 / 2}\right)^{2}}{\lambda_{L} \lambda_{g}} \frac{V^{2}}{L g}
$$

Thus, for the Froude condition to be met, the following relation must exist: $\lambda_{E}=\lambda_{\rho} \lambda_{L} \lambda_{g}$. In general, $\lambda_{g}=1$. Thus, $\lambda_{E}=\lambda_{p} \lambda_{L}$, which suggests that the material for the model should have a low elastic modulus and a high density.

The Froude condition, however, can only be satisfied by using dissimilar materials for model and prototype. The Froude and Strouhl conditions were considered in the hydraulic modeling technique to ensure similar flow pattern for both systems. These similarities guarantee that the ratios of inertial and gravity 
effects (Froude) and the vortex shedding frequency and flow velocity (Strouhl) will be identical for both systems. The Froude law is widely used for modeling open channels in which the flow is exclusively governed by gravity (Novak and Cabelka 1981, and Baker, Westine, and Dodge 1973).

As shown in the previous calculation, the Strouhl condition is readily attainable for both the model and the prototype. The Froude condition, however, requires that the model material be such that the scale factor for modulus of elasticity (E) equals the product of scale factors for mass density $(\rho)$ and length (L). This condition can only be satisfied by using dissimilar materials for the model and the prototype gates. An investigation of materials for the model suggests that commonly available materials, including metals and plastics, cannot provide a geometrical scale factor of over one-half of the prototype dimension. As shown in Table 10, other available materials could not be used for producing a moderate size scale model. A hypothetical synthesized material would, however, be ideal for satisfying the elastic similitude relationship. Considering the excessive expense and uncertainties inherent in modeling the various natural aspects of the river, development of a synthesized material was deemed impractical and beyond the scope of the investigation.

Plastics were deliberately eliminated from the selection process due to skepticism regarding their long-term mechanical performance and the uncertainty in replicating conditions such as welding and bolting and the fabrication process of the prototype. A half-size model of the prototype was not a good choice for the scale model either, since a prototype of the Olmsted wicket existed for full-scale study. A half-size model would tremendously increase the simulation costs and would not serve the purpose of the scale-model study program. Therefore, a reduced scale model of materials identical to those of the prototype was used in this study, while keeping in mind that such a material selection would obviously violate the similitude relations for the elastic modulus established by the Froude model.

Another, dimensional quantity that works well with pumps, fans, and turbine modeling is the Reynolds number, which can be defined as:

Reynolds Number $\left(\mathbf{N}_{\mathrm{R}}\right)=\frac{\text { Inertial Force }}{\text { Viscous Force }}=\frac{V L}{v}$, where $v$ is the kinematic viscosity of the fluid, and viscosity is the property of a liquid which retards flow. In the Olmsted model, it was impractical to satisfy Reynolds number similitude relations, since the viscous forces are usually at least an order of magnitude smaller and relatively unimportant compared to the fluid inertia forces. A Froude model requires a fluid with a kinematic viscosity of $1.08 \mathrm{E}-06 \mathrm{ft}^{2} / \mathrm{sec}\left(\lambda_{v}=\lambda_{Z}^{3 / 2}\right)$ to simulate the viscous damping effect of the prototype water at $60^{\circ} \mathrm{F}$ (water has a kinematic viscosity of $1.217 \mathrm{E}-05 \mathrm{ft}^{2} / \mathrm{sec}$ at $60^{\circ} \mathrm{F}$ ). A low viscosity of such order can be available if mercury is used as a model fluid (see fluid properties, Lindeburg 1986). Therefore, a distortion of viscous effect will exist in a Froude model when plain water is used in the model. This distortion results in measured viscous damping forces in a reduced scale model that would overpredict the 


\begin{tabular}{|c|c|c|c|c|}
\hline Model & Prototype & $\lambda_{\mathrm{E}}$ & $\lambda_{\rho}$ & $\lambda_{L}=\frac{\lambda_{E}}{\lambda_{\rho}}$ \\
\hline AL380 & $\begin{array}{l}\text { A588 / } \\
\text { A572 Grade } 50\end{array}$ & $\frac{10.3}{29.1}=.354$ & $\frac{.097}{.286}=.339$ & $1.04 " 1: 1$ \\
\hline Copper & $\begin{array}{l}\text { A588 / } \\
\text { A572 Grade } 50\end{array}$ & $\frac{14.5}{29.1}=.498$ & $\frac{.285}{.286}=.996$ & $0.50 \geqslant 1: 2$ \\
\hline Magnesium & $\begin{array}{l}\text { A588 / } \\
\text { A572 Grade } 50\end{array}$ & $\frac{6.5}{29.1}=.223$ & $\frac{.065}{.286}=.227$ & $0.98 \# 1: 1$ \\
\hline Titanium & $\begin{array}{l}\text { A588 / } \\
\text { A572 Grade } 50\end{array}$ & $\frac{6.50}{29.1}=.2239$ & $\frac{.065}{.286}=.227$ & $0.98 \gg 1: 1$ \\
\hline
\end{tabular}

viscous damping forces in the prototype. In a Froude model, the scaling factor for Reynolds number is:

$$
\lambda_{N_{R}}=\left(\lambda_{v} \lambda_{L}\right) / \lambda_{v}=\lambda_{L}^{3 / 2}
$$

The drag coefficients, as functions of Reynolds number, will differ between the systems. No significant change in the dynamics of the flow is anticipated due to such a Reynolds number difference between the prototype and the model. This is true since the drag coefficients are low for the turbulent open channel flow with high Reynolds numbers for the prototype and the model (Lindeburg 1986).

Ignoring the elastic behavior of the wicket, a similitude hydraulic model was obtained by using similar materials for both the model and the prototype. As discussed in the latter part of this chapter, a method to minimize the effect of distortion in similitude scales was developed for the Olmsted model to simulate the flow dynamics of the river on a rigid gate. This model will provide correct hydraulic loads on the gate but the response will not be a true elastic simulation of the prototype response. A true elastic similitude model requires a different set of scale factors that are discussed below.

\section{Structural similitude relations}

The equation of a structural system with proper boundary and initial conditions characterizes the behavior of the system in terms of its variables and parameters. The response of the system is a function of its independent 
variables. In the case of a true elastically similar model, the equations of motion of the scale-model and its prototype must be invariant under transformation.

This transformation defines the scaling factors between all parameters, inputs, and responses for both systems. Similitude ensures similar responses between a model and a prototype system. The equations of motion for the gate dynamics can be written as:

$$
M_{n} \ddot{z}_{n}(t)+\omega^{2} M_{n} z_{n}(t)=F_{n}(t)
$$

where

$$
\begin{aligned}
& M_{n}=n t h \text { modal mass } \\
& z_{n} \quad=n t h \text { modal displacement } \\
& F_{n}(t)=n t h \text { modal forcing function }
\end{aligned}
$$

Similitude model laws could be developed by defining the nondimensional relationships for variables associated with the above equations. By equating nondimensional quantities between the model and the prototype, the scale factors are established; conversely, transformation of the above equation from prototype to model using these scale factors would lead to the identical equation (Baker, Westine, and Dodge 1973). Using similar materials for both the model and the prototype, the scale factors for force $(F)$, time $(T)$, and frequency $(f)$ become:

$$
\lambda_{F}=\lambda_{E} \lambda_{L}^{2}, \lambda_{T}=\lambda_{L} \sqrt{\lambda_{\rho}} / \sqrt{\lambda_{E}} \text {, and } \lambda_{f}=\sqrt{\lambda_{E}} /\left(\lambda_{L} \sqrt{\lambda_{\rho}}\right)
$$

Note that the frequency for the model will be scaled by the inverse of $\lambda_{\mathrm{L}}$, if the dimensions are scaled by $\lambda_{\mathrm{L}}$. A structural similitude model for similar materials thus requires different scaling relationship than the one presented for the hydraulic case. Note that the representation of prototype damping behavior in a model is not considered in the above scaling formulation.

Damping could be included in a similitude analysis based upon assumptions regarding the energy dissipation formulation for the structure of interest. Viscous damping could be incorporated in a model by using the nondimensional terms $\xi, v$, where $\xi$ is the damping ratio and $v$ is the fluid viscosity. These constants are a function of both material properties and system geometry. It has been shown earlier that the fluid viscosity for the prototype can not be easily replicated in the model. However, the concept of structural damping could be retained in a geometrically scaled model. This is because the structural damping forces scale the same as elastic and inertial forces; hence, the structural damping would scale without distortion. Not considered here, though, is the coulomb formulation of damping due to friction between the interfacing surface. Farrar, Baker, and Dove (1994) have shown that coulomb could produce a different set of scaling terms for reproducing prototype frictional behavior in a model. A comparison of damping factors would be used in the latter part of this chapter to determine the effectiveness of the model in reproducing prototype damping mechanism. 
A comparison of the scaling relationships using the hydraulic and structural similitude laws is presented in Table 11. As mentioned previously, the hydraulic model ensures that the flow-induced dynamic forces are identical for the model and the prototype. The structural model resembles the structural response of the prototype system.

\section{Olmsted Physical Model}

An extensive experimental evaluation of the Olmsted wicket was needed to formulate the mathematical model of the system and to understand the dynamic performance of this hydraulically lifted gate of unprecedented size. General similitude relations for the Olmsted model were based on principles for major physical system attributes.

The structural modeling criteria noted above were not directly used in modeling the wicket gates presented in this study. A direct structural model could not properly model the hydraulic behavior of the flow, and more importantly, a model of this type would tremendously increase the cost of the project to attain an equal velocity relationship (1:1). Maintaining a velocity corresponding to a prototype discharge of $11,700 \mathrm{cfs}$ in the model would require higher-capacity pumps or pressurization of the channel flow in a closed conduit. Considering these disadvantages of a direct structural model, the Froude model was adopted with an adjustment to the elastic behavior of the system.

It appears that the hydraulic scaling relationship presented above would only be valid for simulating the rigid body motion of the gate since the effects of modulus of elasticity were not scaled down properly. An adjustment to the performance of the hydraulically operated gate was done in such a way that the low-frequency rigid body interaction of the gate dynamics with the flow motion was preserved for both models.

The Froude scaling law is most widely used in hydraulic problems involving turbulent free-surface flow (U. S. Department of the Interior 1994). Note that the scaling factors based on the Froude model satisfy the same equation of motion describing the structural response of the gate. This identity can be verified by substituting the Froude scaling relationships into the modal equation of motion for the gate. As shown below, the transformed equation for the prototype (with subscript $p$ ) is identical to the model equation.

$$
\begin{aligned}
& M_{n} \ddot{z}_{n}(t)+\omega_{n}{ }^{2} M_{n} z_{n}(t)=F_{n}(t) \\
& {\left[\lambda_{\rho} \lambda_{L}^{3}\left(M_{n p} \ddot{z}_{n p}(t)\right)+1 / \lambda_{L} \omega_{n p}{ }^{2} \lambda_{\rho} \lambda_{L}^{3} M_{n p} \lambda_{L} z_{n p}(t)=\lambda_{\rho} \lambda_{L}^{3} F_{n p}(t)\right]}
\end{aligned}
$$

Although the Froude model satisfies the structural equation of motion, an adjustment to the similitude model was still needed to determine the effects of modeling distortion due to noncompliance with the elastic-inertial relationship. 


\begin{tabular}{|c|c|c|c|c|}
\hline \multicolumn{5}{|c|}{$\begin{array}{l}\text { Table } 11 \\
\text { Conventional Scaling Relationships }\end{array}$} \\
\hline Parameter & Symbol & $\begin{array}{l}\text { Basic } \\
\text { Dimensions }\end{array}$ & $\begin{array}{l}\text { Scale Factor } \\
\left(\frac{\text { model }}{\text { prototype }}\right) \\
\text { (Froude) }\end{array}$ & $\begin{array}{l}\text { Scale Factor } \\
\text { (STRUCTURAL) } \\
\text { when, } \lambda_{E}=1\end{array}$ \\
\hline Length & $\bar{L}$ & $\bar{L}$ & $\overline{\lambda_{L}}$ & $\lambda_{L}$ \\
\hline Material Modulus & $E$ & $\mathrm{FL}^{-2}$ & $\lambda_{E}=\lambda_{\rho} \lambda_{L}$ & 1 \\
\hline Mass Density & $\cdot$ & $\mathrm{FL}^{-1} \mathrm{~T}^{2}$ & $\lambda_{\rho}$ & 1 \\
\hline Poisson's Ratio & $\cdot$ & -- & 1 & 1 \\
\hline Force & $F$ & $F$ & $\lambda_{\rho} \lambda_{L}^{3}$ & $\lambda_{L}^{2}$ \\
\hline Time & $T$ & $T$ & $\sqrt{\lambda_{L}}$ & $\overline{\lambda_{L}}$ \\
\hline Acceleration & $\bar{a}$ & $\mathrm{LT}^{-2}$ & 1 & $\frac{1}{\lambda_{L}}$ \\
\hline Velocity & 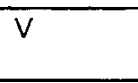 & $L T^{-1}$ & $\sqrt{\lambda_{L}}$ & 1 \\
\hline Displacement & $d$ & $L$ & $\lambda_{L}$ & $\lambda_{L}$ \\
\hline Frequency & $\bar{f}$ & $T^{-1}$ & $\frac{1}{\sqrt{\lambda_{L}}}$ & $\frac{1}{\lambda_{L}}$ \\
\hline Pressure & $P$ & $\mathrm{FL}^{-2}$ & $\lambda_{\rho} \lambda_{L}$ & 1 \\
\hline Strain & $\cdot$ & - & 1 & 1 \\
\hline Stress & $\cdot$ & $\mathrm{FL}^{-2}$ & $\lambda_{\rho} \lambda_{L}$ & 1 \\
\hline Discharge & $Q$ & $\mathrm{~L}^{3} \mathrm{~T}^{-1}$ & $\lambda_{L}^{\frac{5}{2}}$ & $\lambda_{L}^{2}$ \\
\hline Mass & $M$ & $F L^{-1} T^{2}$ & $\lambda_{\rho} \lambda_{L}^{3}$ & $\lambda_{L}^{3}$ \\
\hline Prop Stiffness & $\mathrm{k}$ & $\mathrm{FL}^{-1}$ & $\lambda_{\rho} \lambda_{L}^{2}$ & $\lambda_{L}^{3}$ \\
\hline
\end{tabular}

A Froude model is representative of a rigid prototype gate in which the deformable mode contributions are not considered in the scaling relationships. Froude relationships are valid as long as the rigid-body motions control the dynamics of the gate (i.e. modulus of elasticity is not an important factor). This assumption was verified for the 1:25 scale model (Chowdhury, Hall, and Pesantes 1997). It was found that the operating deflected shapes (ODS) of the 1:25 gate were predominantly low-frequency modes. An ODS (or vibration pattern) displays the actual vibrational behavior during operations. The ratio of maximum deviation of frequency responses for the flexible gate from that of the assumed rigid gate would depend on the spectral density ratio of the excitation and the fundamental modes of vibration. 
The above assumption for rigid body motion is valid for the gate only. The prop or the hydraulic system stiffness, however, cannot be neglected in modeling the rigid-body motion of the gate. This is because a rigid prop stiffness (rigid motion) will not allow the rigid gate to move at all. Thus, a dissimilar material must be used for the prop that retains the Froude law relations discussed above.

As shown below, a magnesium prop rod satisfies the elastic requirement for the model system. For magnesium, the scale factor for modulus of elasticity $\lambda_{E}$, is about one-fifth of the prototype material. Thus, for a valid 1:5 geometrically scaled Froude model, one needs a mass density for the prop rod similar to the prototype material, such that $\lambda_{\rho}=1$. This model neglects the contribution of prop inertia so that the inertial effects $(\rho)$ for the gate are more important and the elastic effects $(E)$ of the prop are dominant. Considering the prop rod inertia as insignificant compared to the gate inertia, it was assumed that a difference in the inertial effects of the prop resulting from a density unequal to that of the prototype would not considerably change the dynamics of the system.

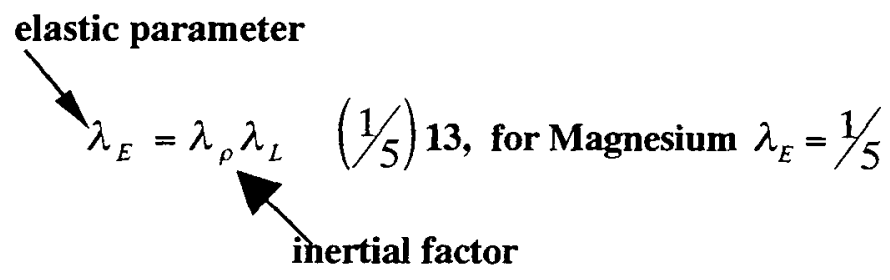

For the rigid gate, the inertial effects are more important than the elastic effects. Therefore, a combination of materials for the model (magnesium for the prop and steel for the gate) approximately validates the scaling-law relationships by maintaining the fundamental physical properties of the system.

\section{Prop-rod dimensions}

The prototype prop-rod diameter was 10 in. A scaled 2-in.-diam magnesium rod was selected for the proposed model to simulate the axial frequency of the supporting mechanism. The fundamental axial frequency, $\sqrt{\frac{k}{m}}$, of the selected rod itself is approximately scaled down by a factor of $\lambda_{L}^{-1}$, which is the scale factor for the frequency using the structural law. In this computation, standard values for mass density and modulus of elasticity for the steel prototype and the magnesium model were considered. With such dimensional adjustments, the prop rod satisfied the Froude condition $\lambda_{E}=\lambda_{\rho} \lambda_{L}$ as well as the proposed geometric scale factor of $1: 5$.

A 2-in.-diam magnesium rod, however, did not model the buckling capacity and the mass of the prototype properly. A scaled magnesium rod of about $2.9 \mathrm{in}$. and 4 in. in diameter would be required to model the buckling capacity and mass of the model, respectively. Considering the high design factor of safety of about 4, a 2-in.-diam rod would not be distressed due to the anticipated flow-induced load. Therefore, to maintain the proper flow pattern at the downstream side of the gate a 2-in.-diam rod appeared appropriate. 


\section{Hydraulic cylinder}

An equivalent hydraulic model was designed to simulate the approximate natural frequency of the prototype system using the Froude scaling relationship $\left(\lambda_{f}=1 / \sqrt{\lambda_{z}}\right)$. An approximate spring-mass model of the hydraulic system is shown in Figure 68. In this figure, the mass (M) is the effective mass applied on the tip of the piston rod resulting from the flow-induced vibration of the instrumented gate. Results from the 1:25 flat gate model were used to estimate the magnitude of this total mass for the 1:5 model. The spring components in a series represent the resistance provided by the piston rod in compression, the oil compressed in steel pipe and tubing, and the auxiliary cylinder.

The undamped natural frequency of the hydraulic system can be obtained by using, $\omega_{n}=\sqrt{\kappa_{e} / M}, \mathrm{rad} / \mathrm{sec}$, where $\kappa_{\mathrm{e}}$ is the equivalent spring stiffness for the axial resistance provided by the piston rod and the oil column. The adjustable volume of additional oil $\left(\mathrm{V}_{\mathrm{Req}}\right)$ can be related to the oil column spring stiffness $\left(\kappa_{\mathrm{o}}\right)$ of the hydraulic system using the equation shown below:

$$
\begin{aligned}
& V_{\mathrm{Req}}=a_{t} l+A_{a u x} l_{a u x} \\
& V_{\mathrm{Req}}=\frac{\beta A_{c}^{2}}{\kappa_{o}}-A_{c} L \\
& \text { and, } \\
& \kappa_{o}=\frac{\beta A_{c}^{2}}{A_{c} L+a_{t} l}
\end{aligned}
$$

where

$$
\mathrm{V}_{\mathrm{Req}}=\text { required additional volume of hydraulic fluid }
$$

$a_{t}$ and $1=$ area of cross section and the length for the tubing, respectively

$$
\begin{aligned}
A_{\text {aux }} \text { and } l_{\text {aux }}= & \text { cross-sectional area and the length of the auxiliary cylinder, } \\
& \text { respectively } \\
\beta= & \text { bulk modulus of elasticity of the hydraulic fluid } \\
\mathrm{A}_{c} \text { and } \mathrm{L}_{c}= & \text { cross-sectional area and the associated length for the cylinder, } \\
& \text { respectively } \\
\kappa_{\mathrm{o}}= & \text { equivalent spring stiffness of the series oil column and tubing }
\end{aligned}
$$


By adjusting the volume of the oil column, the natural frequency of the spring-mass model was attained to model the scaled frequency of the equivalent prototype system. In this regard, an auxiliary cylinder with a rod locking mechanism and a small accumulator was used to adjust the required volume of the oil in the transmission line of the system. Addition of the auxiliary cylinder provided a relatively easy adjustment for the model to operate the test gate through a wide range of angular positions ( -2 to $65 \mathrm{deg}$ ) by moving and locking the rod at desired locations. It also allowed for some "tuning" of the system to compensate for tolerance in the estimate used for the computations.

Computation of oil column in the auxiliary cylinder could be performed using the steps listed below:

a. Compute the natural frequency of the prototype system using the estimated equivalent stiffness of the piston rod and oil column

$$
\left(\omega_{p}^{2}=\kappa_{e \cdot p} / M_{p}\right)
$$

b. Compute equivalent stiffness for the simulated model based on the required model frequency, $\omega_{\mathrm{n}, \mathrm{m}},\left(\kappa_{e, m}=M_{m} \omega_{n, m}^{2}\right)$

c. Determine the required oil column stiffness for the model

$$
\left(\frac{1}{\kappa_{o}}=\frac{1}{\kappa_{e}}-\frac{1}{\kappa_{r}}\right)_{m} \text {, from the known values of } k_{e} \text { and } k_{r}
$$

d. Compute additional oil volume $\left(V_{\mathrm{Req}}=\frac{\beta A_{C}^{2}}{\kappa_{o}}-A_{c} L\right)_{m}$

Note that the secant bulk modulus of oil $(\beta)$ is a very sensitive physical property that depends on pressure, temperature, and entrained air in the oil chamber. For a constant temperature and pressure, secant bulk modulus as a function of oil volume was experimentally determined for the Olmsted Hydraulic System. Thus, by representing the assumed experimental value of $\beta$ as a function of oil volume, the required oil volume could be computed from this equation.

\section{Experimental Evaluation of Hydraulic Fluid Bulk Modulus}

This test measured the stiffness of the hydraulic system to calibrate the bulk modulus of oil with the oil volume in the tube and the cylinder. The relationship of physical stiffness to the oil property presented above could be used to determine the bulk modulus of oil. A 500-lb load was applied on the cup and the displacements of the cup and the platform were monitored using two dial gauge as shown in Figure 40. Experiments were repeated for various oil columns by changing the volume of oil column in the tube and cylinder (auxiliary stroke 
length was set at different positions). Note that during the experiment, a relaxation time of $5 \mathrm{~min}$ was allowed to minimize the time-dependent deformation due to compressibility of the air pockets inside the tube. The measured stiffness is an approximation of the actual behavior of the operating system since the preload for the hydraulic system will be different during the raising of the gate, and the data will be recorded for a finite time period.

Using a linear curve-fitting algorithm, the bulk modulus was related to the oil volume by the equation, $\beta=74,116+181 \mathrm{~V}$, when $\mathrm{V}$ is less than $700 \mathrm{in}^{3}$. The hydraulic fluid used in the prototype is an ISO standard VG 32-46. The cylinder casing is made up of steel that is compatible with water and the hydraulic fluid used in the system. A realistic magnitude for bulk modulus of 100,000 psi could be assumed for the hydraulic fluid (ISO Standard VG 32-46) for a well-managed hydraulic system with minimum practical amount of entrained air (Merritt 1967). However, the experimentally determined value of $\beta$, as mentioned above, is a better approximation for the model in absence of an elaborate laboratory result. The same type of fluid will be used for the prototype and the scaled model. It is assumed that the compliance of the steel hydraulic tubing is negligible. Also, the model dimensions were geometrically scaled by a factor of 1:5 of the prototype except for the steel hydraulic tubing, where the required volume $\left(A_{t} l+A_{\text {aux }} L\right)$ would be adjusted to achieve the desired natural frequency of the system. The spring effect of the piston rod in the series system shown in Figure 68 is almost negligible. As a spring, the piston rod is about 17 times stiffer than that of the oil column between the piston and the closed directional-control valve.

\section{Verification of Similitude Model}

\section{Comparison of 1:25-scale flat gate and 1:5-scale experimental results}

Experimental results from two similitude models of Olmsted wickets were compared to investigate the effects of modeling distortions and to verify the effectiveness of the 1:5-scale model in representing prototype conditions. Similar experiments for identical flow configuration and gate position were conducted for a 1:25-scale hydraulic and a 1:5-scale pseudo-elastic model. Although these models reproduced the general dimensions of the prototype wicket, the fabrication distortion due to size effect was more dominant in the 1:25-scale model. Note that the 1:5-scale model was geometrically and dynamically a similitude model. However, the 1:25 model was a partially scaled-down model with a directly connected cylinder.

As explained in the 1:25-scale model report, the random lateral shift of the clevis due to its freedom to rotate about the longitudinal axis of the gate caused nonuniform distribution of reaction forces at the bottom hinges (Chowdhury, Hall, and Pesantes 1997). In the 1:25 model, the coupling reaction force at the clevis attachment between the gate and the cylinder rod introduced a biased 


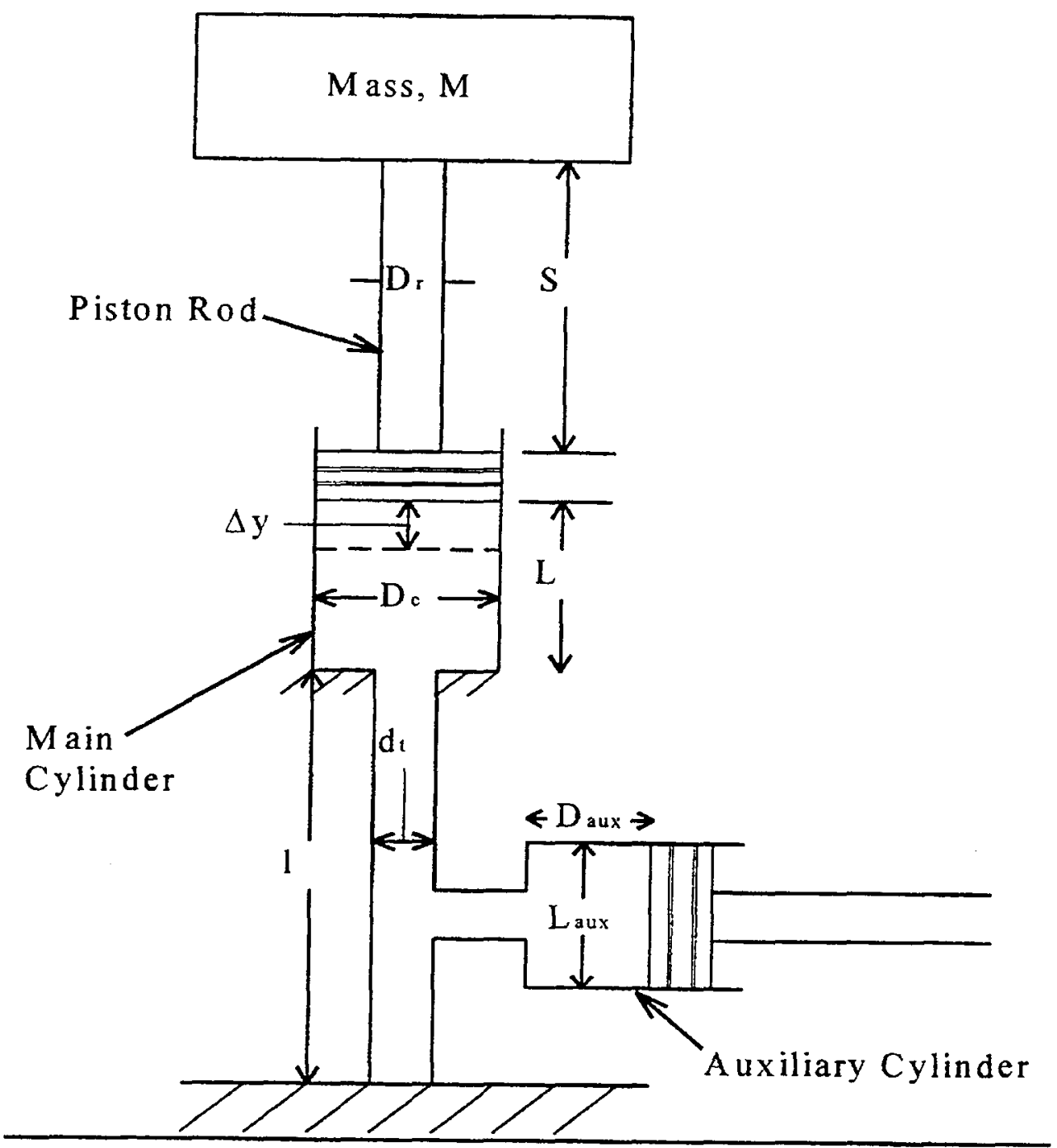

Figure 68. Spring-mass model for hydraulic system

reaction on the bottom hinges. Figure 69 shows the cylinder load transfer mechanism on the 1:25-scale model. These biased forces are not expected to occur in the 1:5-scale model since the alignment cylinder provides the lateral support to hold the cylinder at its position. In the 1:5 model, the axial loads from the cylinder are transmitted to the gate through the roller mechanism of the cup and ball connection. Therefore, a biased error in the measured bottom hinge reaction forces needs to be accounted for during the comparison of responses between the $1: 25$ and 1:5 models.

In the 1:25-scale model, the overall dimensions of the gate were limited to the availability of material to meet the required scaled dimensions. For instance, the geometric details of using $1 / 25^{\text {th }}$ of the 0.5 -in. skin plate (prototype thickness) were practically impossible to reproduce. Brass was used in the 1:25 model instead of steel, the prototype material, and the overall dimensions were scaled as practically as possible. The mass per unit length $(\bar{m})$ was $0.0964 \mathrm{lb}-\mathrm{sec}^{2} / \mathrm{ft}^{2}$ and $0.0045163 \mathrm{lb}-\mathrm{sec}^{2} / \mathrm{ft}^{2}$, for the 1:5-scale and 1:25-scale models, respectively. 


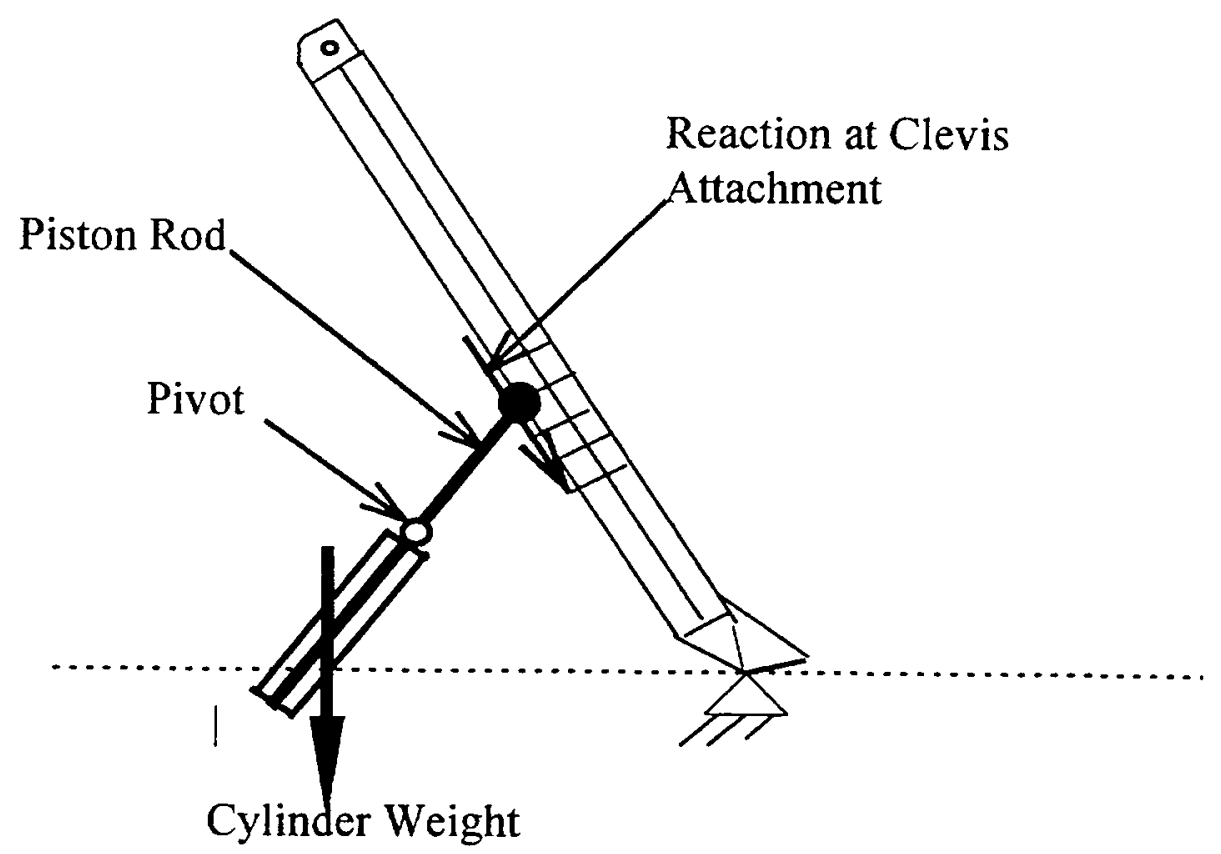

Figure 69. A schematic diagram of forces on 1:25 scale hydraulic actuator

Thus, this latter model should give us an idea of the dynamic behavior of the wickets, but it was never intended to simulate the elastic behavior of the prototype. The 1:5 flat gate, however, is geometrically similar with respect to the prototype structure. Since this is a similar elastic model, all relationships can be scaled up to estimate those expected in the prototype.

Four accelerometers (PCB Model 353B68), five strain-gauge load cells (two for each of the hinges and one for the shaft), and two pressure transducers (DRUCK Model PDCR-200) recorded data for the 1:25 model which were compared to corresponding measurements from the 1:5 model. The gauge locations for the 1:25-scale model and 1:5-scale model are shown in Figures 70 and 57. Since the pressure gauges in the 1:25 model were not mounted at the same locations as in the 1:5 model, an average of the top and middle center pressure gauges on the intermediate-scale model were used to compare the corresponding (upstream and downstream) measured pressures from the 1:25-scale model.

\section{Experimental modal analysis - dry condition}

Table 12 shows a comparison of the results obtained from the experimental modal analysis conducted on the 1:25- and 1:5-scale models. Modal information for the dry 1:25 flat gate at various fixed positions was extracted using a hammer-impacted modal experiment. For the 65-deg position, the first bouncing mode was found at $154 \mathrm{~Hz}$, first torsion at $239 \mathrm{~Hz}$, second bending at $369 \mathrm{~Hz}$, 


\section{UPSTREAM POOL}
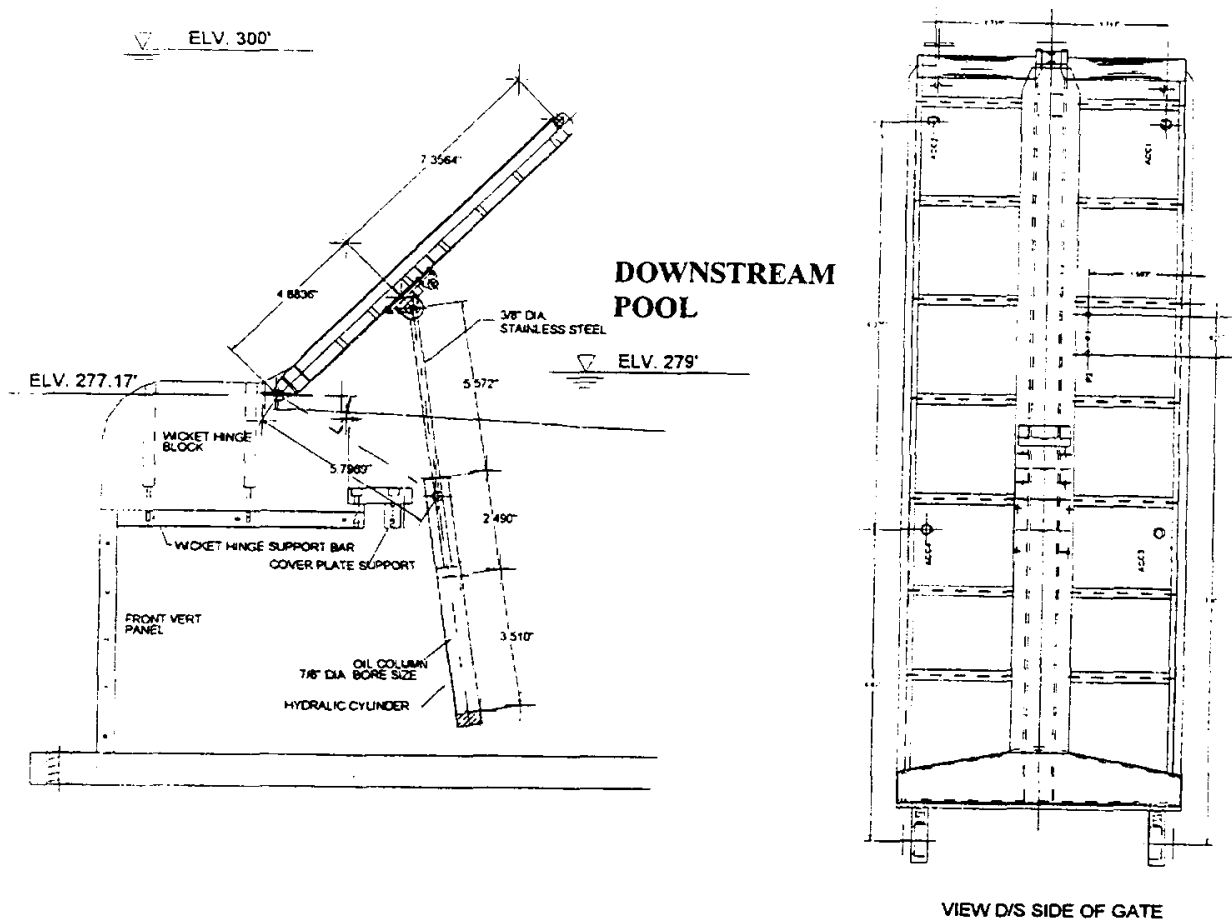

Figure 70. Transducer location on 1:25-scale model

and second torsion at $649 \mathrm{~Hz}$. A visual description of these modes is shown in Figure 71 .

The experimental modal experiment for the cylinder supported 1:5 flat gate at the 65-deg position was conducted using a scanning doppler laser vibrometer system. The IDEAS Master Series-TEST module was used to extract the modal parameters for the gate under dry conditions. Figure 72 presents the dominant modes of vibration of the 1:5-scale wicket at the 65-deg position. As shown in this figure, the first mode was found at about $60 \mathrm{~Hz}$, first torsion at $95 \mathrm{~Hz}$, second bending at $142 \mathrm{~Hz}$, and second torsion at $223 \mathrm{~Hz}$.

\section{Comparison}

A comparison of the mode shapes indicates that the qualitative dynamic characteristics for both gates are similar. As displayed in Figures 71 and 72, there is an overall similitude in the mode shape trends of both models. For a properly scaled structural similitude model, the frequency scale factor for the model (1:25) of a model (1:5) should be $1 / \lambda_{1}$ or 5 (see Table 11 ). Experimental results presented in Table 12 indicates that the average frequency scaling factor for the 1:25- and 1:5-scale model is 2.643. An average value of 2.643 for the frequency scaling factor agrees well with the estimated scaling factor for the true geometry obtained using the known values of the parameters for both models. 
Mode 1

Frequency: $179.1 \mathrm{~Hz}$

Damping: $4.19 \%$

Mode 2

Frequency: $242.7 \mathrm{~Hz}$

Damping: $1.26 \%$
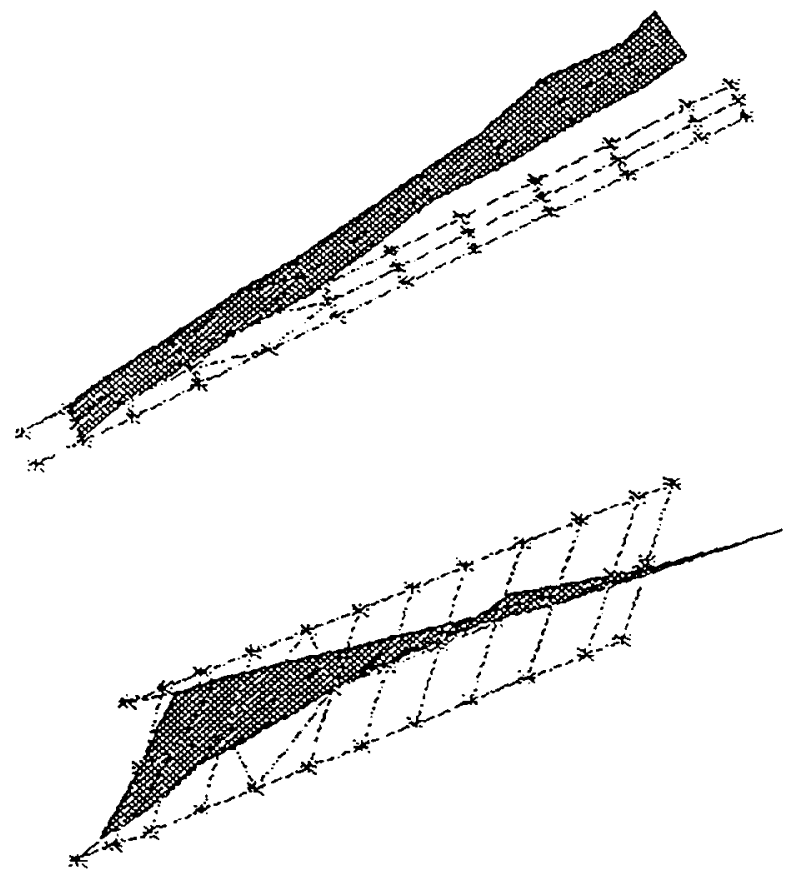

Mode 3

Frequency: $375.1 \mathrm{~Hz}$

Damping: $2.83 \%$

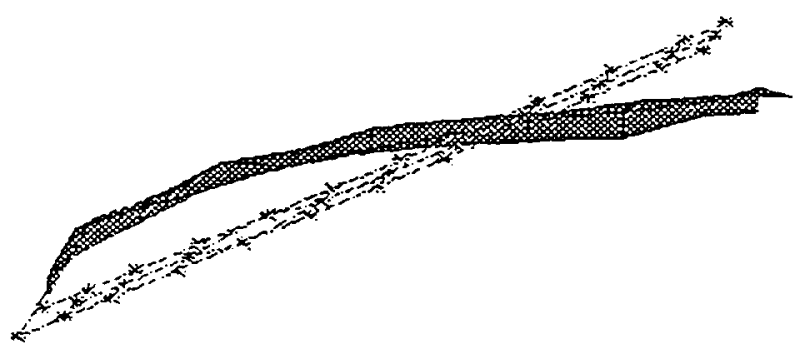

Mode 4

Frequency: $605.7 \mathrm{~Hz}$

Damping: $1.44 \%$

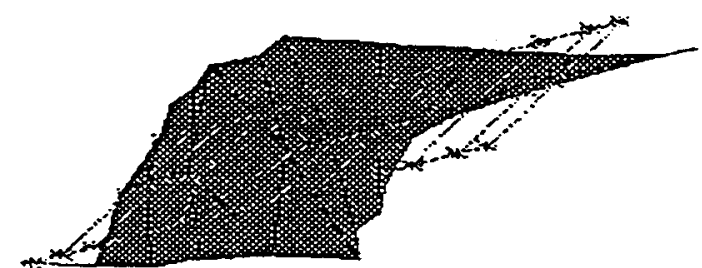

Figure 71. Dry gate mode shapes for 1:25-scale wicket

The equation below shows that the 1:25-scale model provides a frequency scale factor of 2.633 .

$\omega_{1: 5}=\omega_{1: 25} \sqrt{\left.\left[(E I)_{1: 5}\left(\bar{m} L^{4}\right)_{1: 25}\right] /(E I)_{1: 25}\left(\bar{m} L^{4}\right)_{1: 5}\right]}=\omega_{1: 25} / 2.633$ 


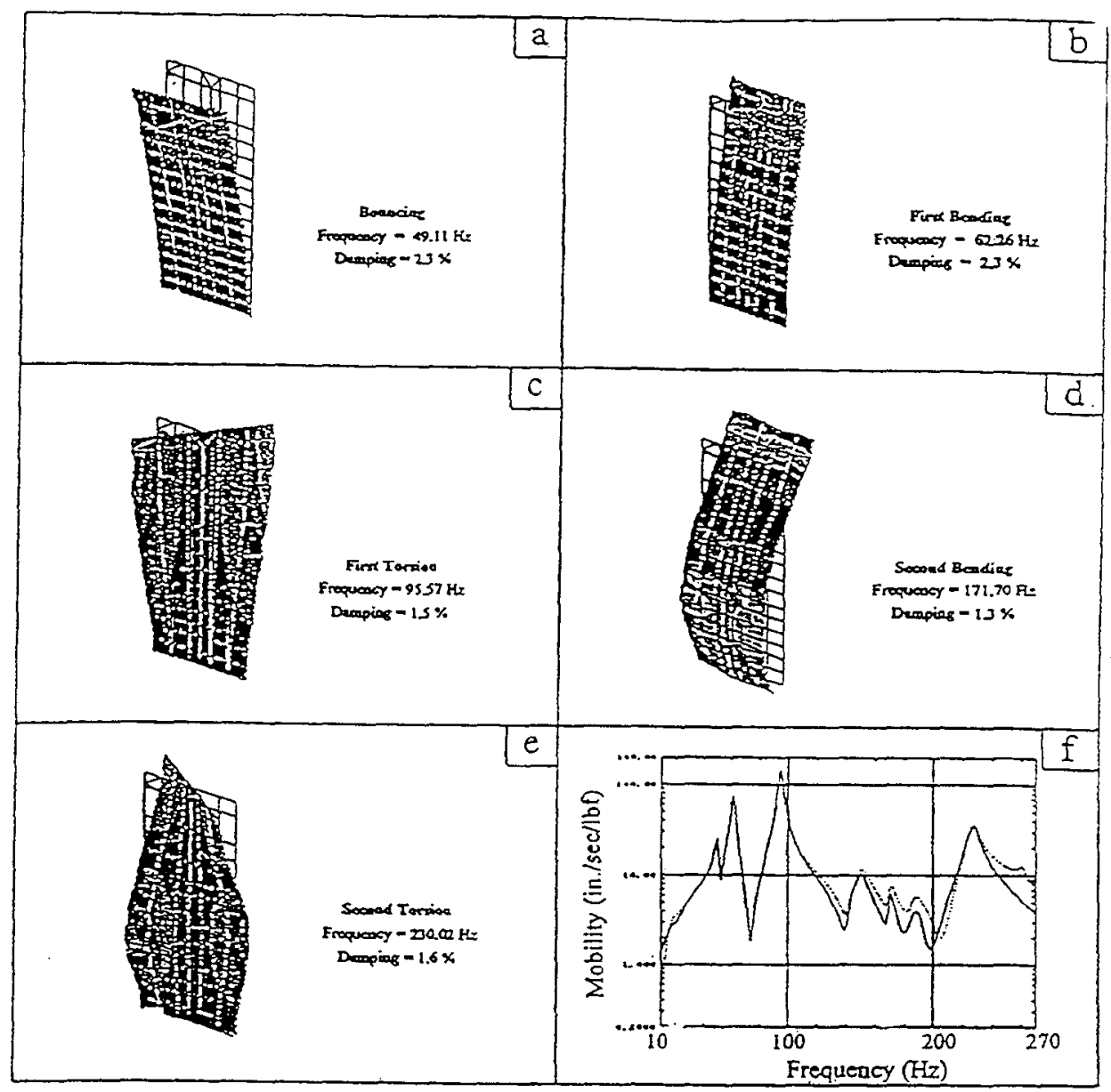

Figure 72. Dry gate mode shapes for 1:5-scale wicket

where

$\bar{m}=$ mass per unit length

$\mathrm{E}$ and $\mathrm{I}=$ the modulus of elasticity and moment of inertia of the wicket.

A scaling factor of 2 was assumed for the modulus of elasticity, and scaling factors of 21.3449 and $15396 \times 5^{4}$ were used for the mass per unit length and moment of inertia, respectively, for the model.

A deviation in the actual frequency scaling factor verifies that the 1:25-scale model was not an exact structural replica of the 1:5-scale model. This is due to the fact that the mass and stiffness properties were not intended to be reproduced in the 1:25 model, and thus its natural frequencies do not scale up properly to those obtained in the 1:5 model. The natural frequencies of the 1:5 model, however, should accurately predict those expected on the prototype. Although deviation was observed, the dynamic behavior of the intermediate model were was preserved in the 1:25-scale model. 


\begin{tabular}{|c|c|c|c|c|c|c|}
\hline \multicolumn{7}{|c|}{$\begin{array}{l}\text { Table } 12 \\
\text { Experimental Natural Frequencies for 1:25- and 1:5-Scale Models at } \\
\text { 65-deg Position }\end{array}$} \\
\hline \multirow[b]{3}{*}{$\begin{array}{l}\text { Mode } \\
\text { Shape }\end{array}$} & \multicolumn{4}{|c|}{ Dry Condition } & \multirow{2}{*}{\multicolumn{2}{|c|}{$\begin{array}{c}\text { Wet Condition } \\
1: 5-S c a l e \\
\text { (11-ft HD, } 3 G G)\end{array}$}} \\
\hline & \multicolumn{2}{|c|}{ 1:25-Scale } & \multicolumn{2}{|c|}{$\begin{array}{c}1: 5-\text { Scale } \\
\text { Cylinder Supported }\end{array}$} & & \\
\hline & $\begin{array}{c}\text { Frequency } \\
\mathrm{Hz}\end{array}$ & $\begin{array}{c}\text { Damping } \\
\%\end{array}$ & $\begin{array}{c}\text { Frequency } \\
\mathrm{Hz}\end{array}$ & $\begin{array}{c}\text { Damping } \\
\%\end{array}$ & $\begin{array}{c}\text { Frequency } \\
\mathrm{Hz}\end{array}$ & $\begin{array}{c}\text { Damping } \\
\% \\
\end{array}$ \\
\hline $\begin{array}{l}\text { Mode } 1 \\
\text { (Bending) }\end{array}$ & 154.07 & 1.65 & 59.61 & 3.57 & 61.87 & 1.9 \\
\hline $\begin{array}{l}\text { Mode } 2 \\
\text { (Torsion) }\end{array}$ & 234.24 & 1.20 & 94.69 & 1.96 & 70.96 & 2.35 \\
\hline $\begin{array}{l}\text { Mode } 3 \\
\text { (Bending) }\end{array}$ & 369.81 & 2.34 & 141.98 & 3.23 & 94.94 & 2.84 \\
\hline $\begin{array}{l}\text { Mode } 4 \\
\text { (Torsion) }\end{array}$ & 649.12 & 2.69 & 223.09 & 4.01 & 144.39 & 2.9 \\
\hline
\end{tabular}

\section{Experimental modal analysis - wet condition}

Table 12 also presents the associated deflected shape of the wicket corresponding to the dominant peaks in the wet response power spectral density (PSD). These shapes are commonly referred to as ODS, which could be analyzed to understand the actual response pattern of the gate during operation. These shapes were animated using the IDEAS-TEST module by generating a frame of deflected shapes for each instance of time response. Four accelerometer readings from the 1:25-scale model were used to generate the frames of accelerometer responses. Then, the frames were animated and displayed sequentially as if the structure were vibrating in real-time. A scanning of the ODS indicated that the gate vibrated with four distinct mode shapes similar to the natural mode shapes identified using the experimental modal analysis. However, they occurred in different frequency ranges than the dry modal frequencies for the gate. Figure 73 shows the ODS for the 1:25-scale model.

The characteristic mode shapes for the 1:5-scale gate under wet conditions were also extracted for a fixed operating position using the scanning laser vibrometer system. The ODS for the operating gate were generated using the transmissibility functions for each response accelerometer with respect to a reference accelerometer. These transmissibility functions were consequently used to display the ODS in the frequency domain. A scanning of the ODS indicated that the gate vibrated with distinct operating shapes similar to the wet natural mode shapes identified previously in the experimental modal analysis. 
Mode 1

Frequency: $72.3 \mathrm{~Hz}$

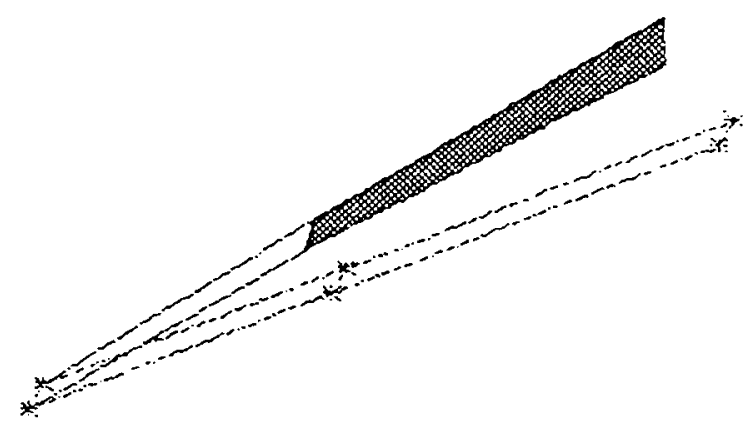

Mode 2

Frequency: $162.1 \mathrm{~Hz}$

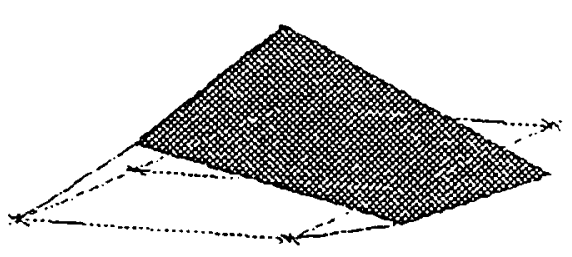

Mode 3

Frequency: $185.5 \mathrm{~Hz}$

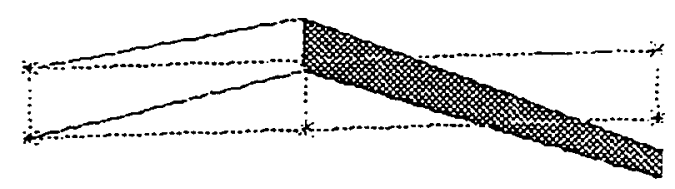

Mode 4

Frequency: $357.4 \mathrm{~Hz}$

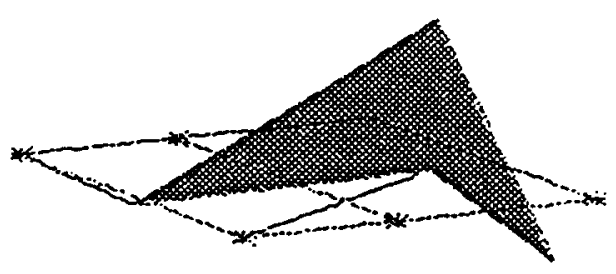

Figure 73. Operating mode shapes for 1:25-scale wicket

Though the amplification at the resonance point is marginal, similarity in the wet mode shapes and the ODS suggests that the energy content of the random flow excites the natural wet modes of the gate. As seen in time-history plots, the fluctuation of the responses about the mean is very low. Figure 74 shows the wet mode shapes for the 1:5-scale operating gate.

A similarity in the operating deflected shapes for both models again shows the capability of the reduced model to retain the dynamic characteristics of the prototype system. This is so because the scale factors established using the 


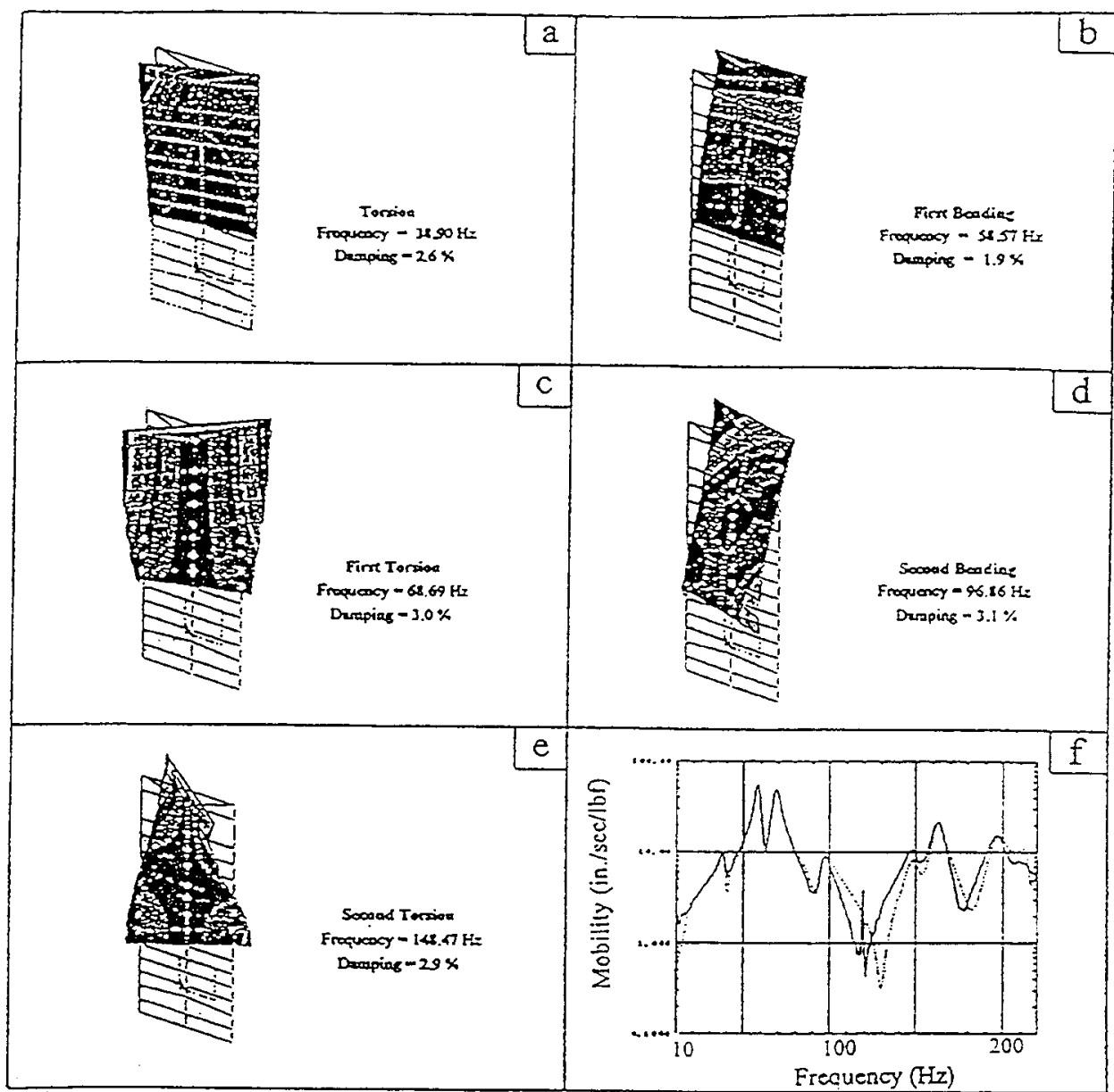

Figure 74. Wet mode shapes for 1:5-scale wicket

hydraulic model would ensure structural similitude identity defined by the dynamic equations of motion. The effect of neglecting the Froude criteria $\left(\lambda_{E}=\lambda_{\rho} \lambda_{L}\right)$ in the similitude model could not be substantiated without analyzing the flow-induced responses for both models. A systematic flowinduced response comparison for both models is presented.

\section{Full-range experiments}

A comparison of the responses for both scaled models is shown in Figure 75 through 76. In these figures, the predicted responses from the 1:25 model were obtained by using the similitude scaling relationships. All magnitudes in the time-domain correspond to the 1:5-scale model. The predicted forces obtained from the 1:25 model were generated by multiplying the actual forces by a scale factor of $\lambda^{3}(\lambda=5)$, the pressure by $\lambda$, and the time by a factor of $\sqrt{\lambda}$, where $\lambda$ corresponds to the ratio between the prototype and the model lengths. The net pressures obtained from the top center and middle center transducers of the 1:5 
model are compared to the net pressure readings calculated from the 1:25 model readings. All 1:25-scale model responses were adjusted to correspond to the 1:5-scale model. All full-range experiments for the 1:5-scale model were sampled at a rate of $500 \mathrm{samples} / \mathrm{sec} / \mathrm{channel}$ and filtered at $250 \mathrm{~Hz}$. The sampling rate for the 1:25-scale model was 100 samples/sec/channel and filtering rate was $50 \mathrm{~Hz}$.

\section{1-ft head difference (Experiment No. 173 vs No. 2065)}

Data corresponding to a three-gate gap configuration, 21-ft head difference (prototype elevation), full-range operation of the gate were recorded during this test. The measured responses for the 1:25- and 1:5-scale model were filtered at $250 \mathrm{~Hz}$ and $50 \mathrm{~Hz}$, respectively. Figures $75 \mathrm{a}$ and b present time- and frequencydomain comparisons for the shaft and net pressures. Autospectra density (ASD) functions for the shaft and net pressures are also presented in Figure 75. As shown in the ASD plots, the low-frequency peak for the 1:5-scale model differs from the 1:25-scale model. This dominant low-frequency peak is associated with a rigid body mode in the 1:5-scale model. Time- and frequency-domain comparisons of the total horizontal and vertical hinge reactions are presented in Figures $75 \mathrm{c}$ and $\mathrm{d}$. Although not presented in Figure 75, the PSD functions for the acceleration (system response) were also calculated. They showed those frequencies of the gate which were excited by the flowing water. The PSD for the pressure are a good representation of the frequency content of the actual flow. These functions were computed using the Structural Dynamics Research Corporation's (1993b) IDEAS-TEST module (a frame size of $0.98 \mathrm{sec}$, Hanning narrow window with 50 percent overlapping).

As discussed previously, a biased error is introduced in the 1:25-scale model hinge and cylinder rod reactions due to the direct connected cylinder. This error can be omitted by displaying only the forces resulting from loadings caused by flow over the gate. This is achieved by subtracting the forces required to raise the gate under submerged quiescent conditions from values recorded under flow conditions for both models. A comparison of flow-induced loads between the 1:25-scale and 1:5-scale models for a 21-ft head differential and a three-gate gap configuration is provided in Figures $75 \mathrm{e}$ through $\mathrm{i}$. A comparison of flow induced loads between the 1:25-scale and 1:5-scale models for a 21-ft head differential and a one-gate gap configuration is provided in Figures $75 \mathrm{j}$ through n. These data have been scaled to prototype equivalents and do not reflect the weight of the gate, they are the results of flow induced forces only. The left and right hinge reactions are displayed as horizontal and vertical components. All measured responses, including hinge reactions, agree very well for both models.

\section{1-ft head difference (Experiment No. 43 vs No. 2072)}

Using the same procedures as in the $21-\mathrm{ft}$ head difference data analysis, the time-and frequency-domain data for the 1:25- and 1:5-scale models corresponding to a three-gate gap configuration, 11-ft head difference (prototype 

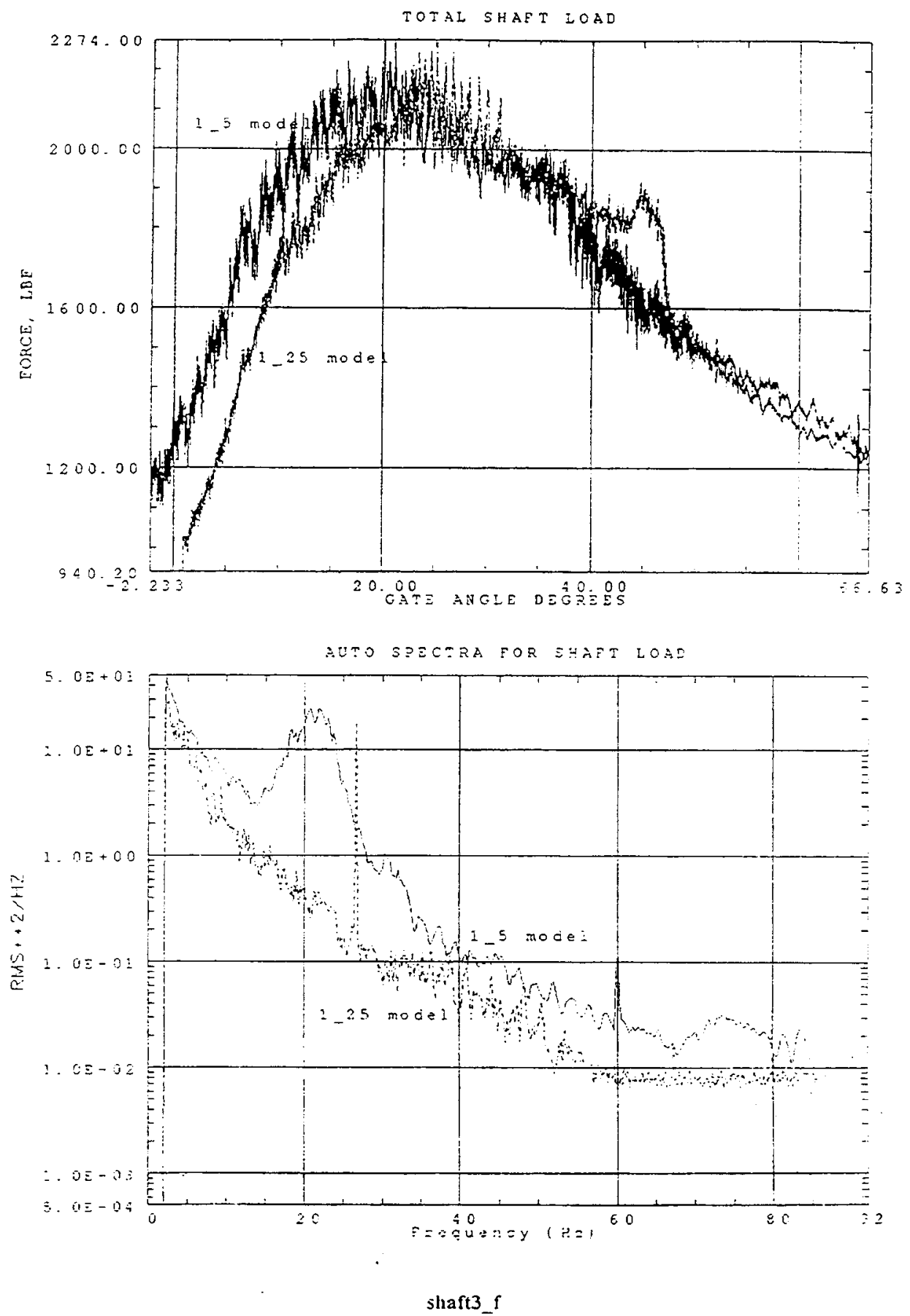

a. Shaft load

Figure 75. Gate response comparison for 1:25- and 1:5-scale models, 21-ft head difference, 3GG configuration (Sheet 1 of 14)

elevation), and full-range operation of the gate are compared in Figure 76. Considering the low magnitude of the reaction forces and the lower sensitivity of the transducers used in the 1:25 model, the overall trend in the responses for 

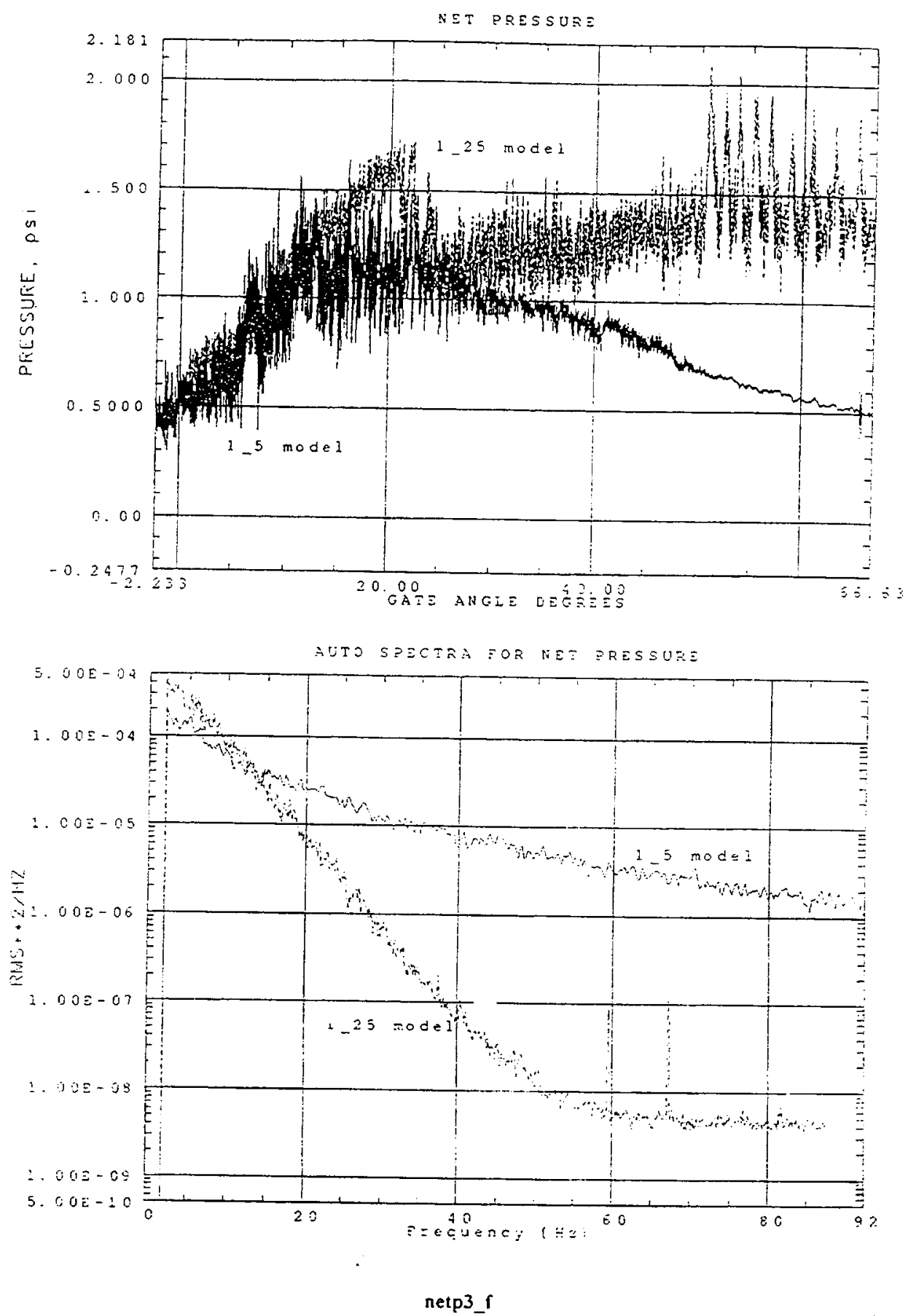

b. Net pressure

Figure 75. (Sheet 2 of 14)

both models agrees well. As shown in the figures, except for the hinge reactions, all measured responses agreed very well for both models. 

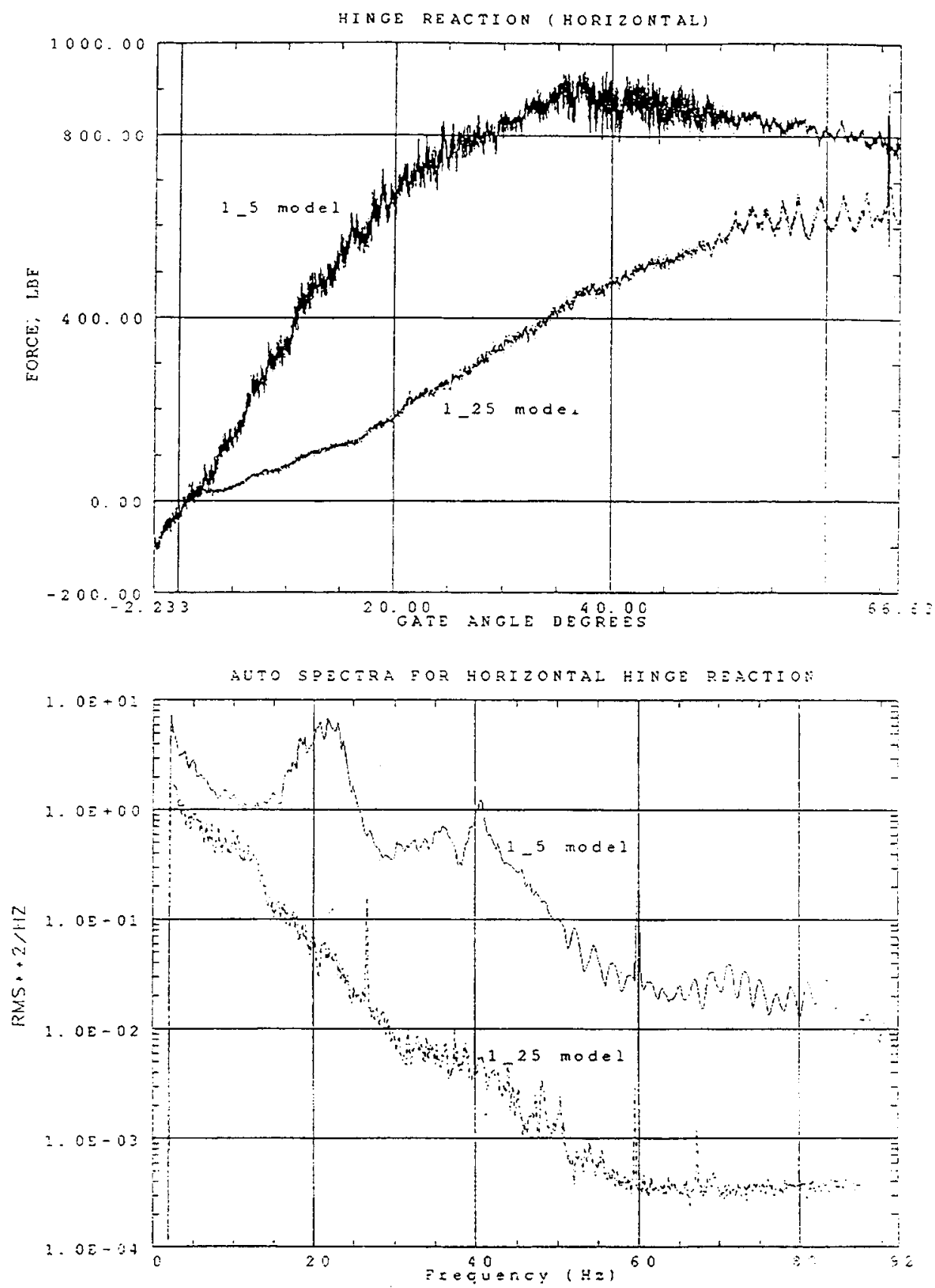

top source: hingehn

bottom source: hingehf

c. Horizontal reaction

Figure 45. (Sheet 3 of 14) 

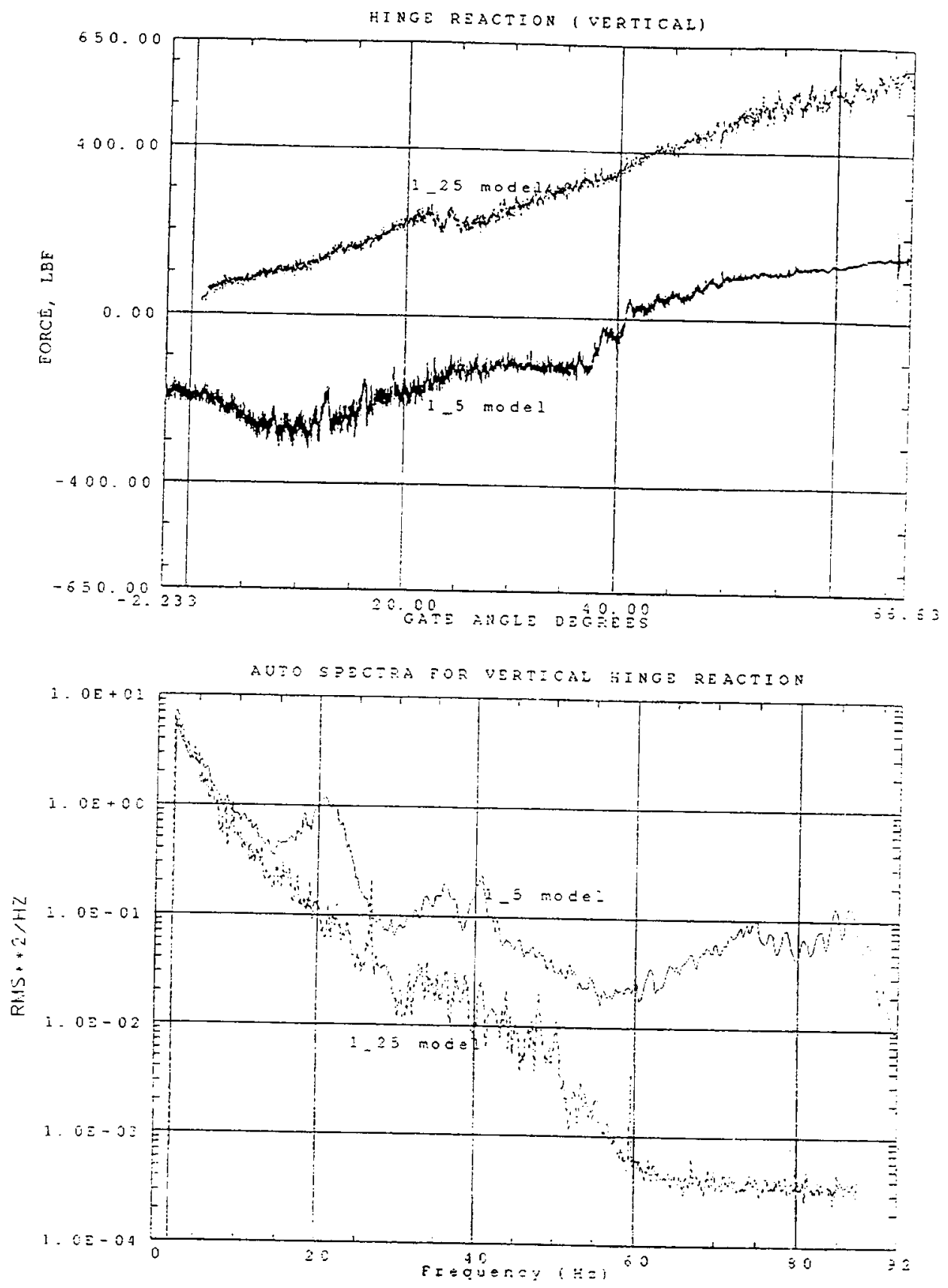

hingev and hingevf

\section{d. Vertical reaction}

Figure 75 . (Sheet 4 of 14) 


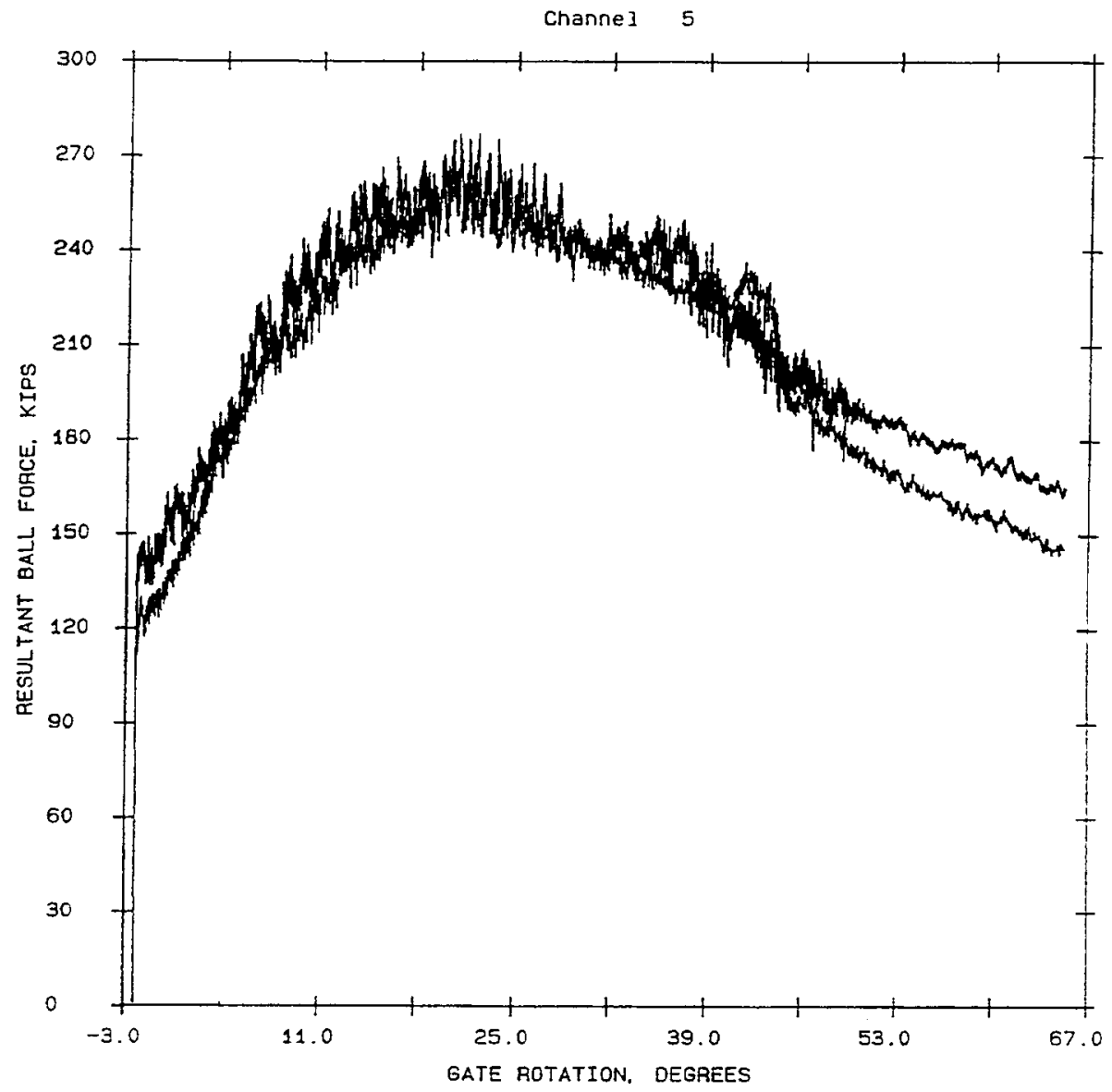

\author{
BALL FORCE VS GATE ROTATION \\ COMPARISON OF 1:5 SCALE MODEL \\ WTH 1:25 SCALE MODEL \\ POOL ELEVATION 300.0 \\ TAILWATER ELEVATION 279.0 \\ 3-GATE GAP - CENTERED
}

e. Prototype equivalent ball force

Figure 75. (Sheet 5 of 14) 

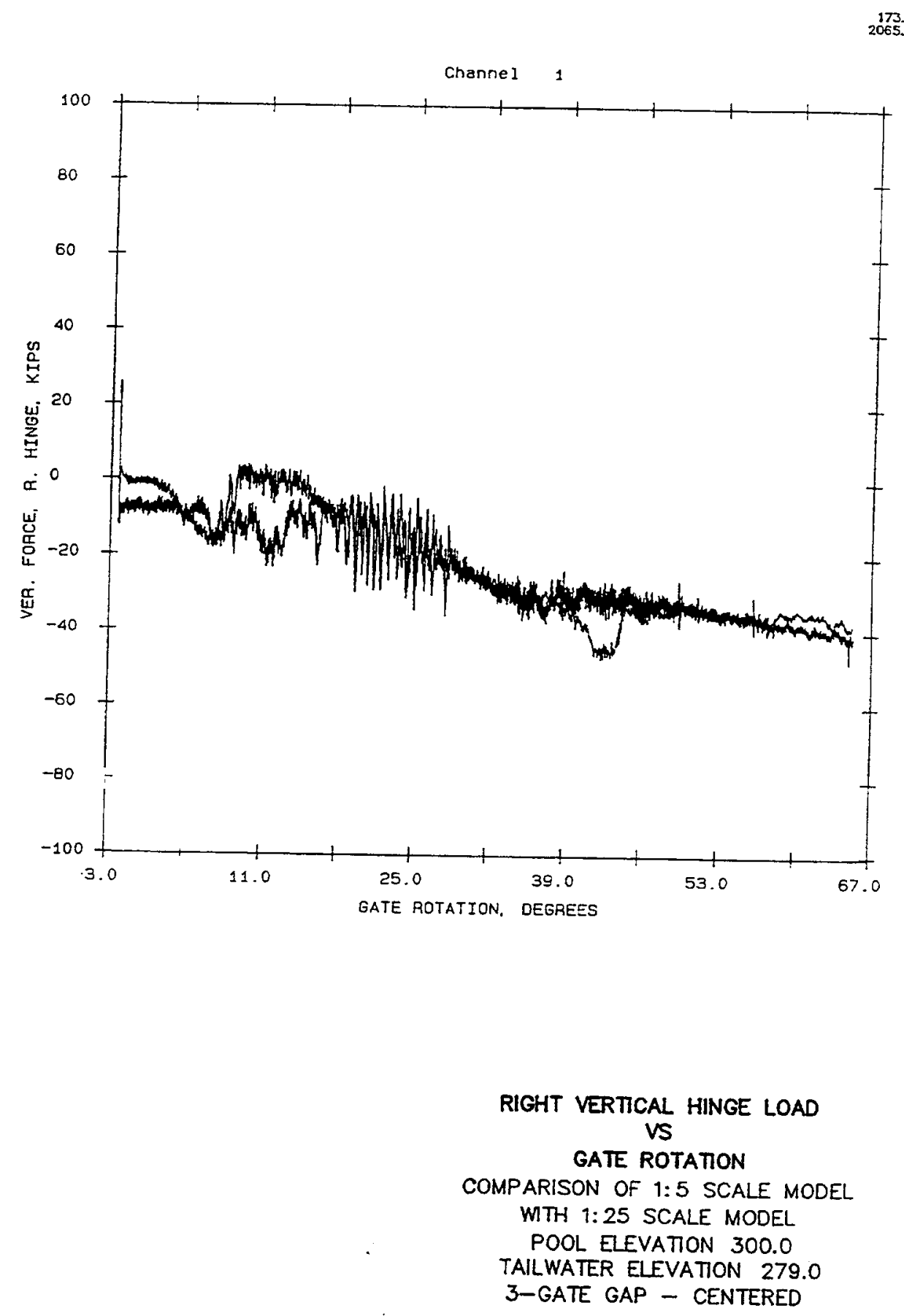

f. Prototype equivalent right vertical hinge load

Figure 75. (Sheet 6 of 14) 

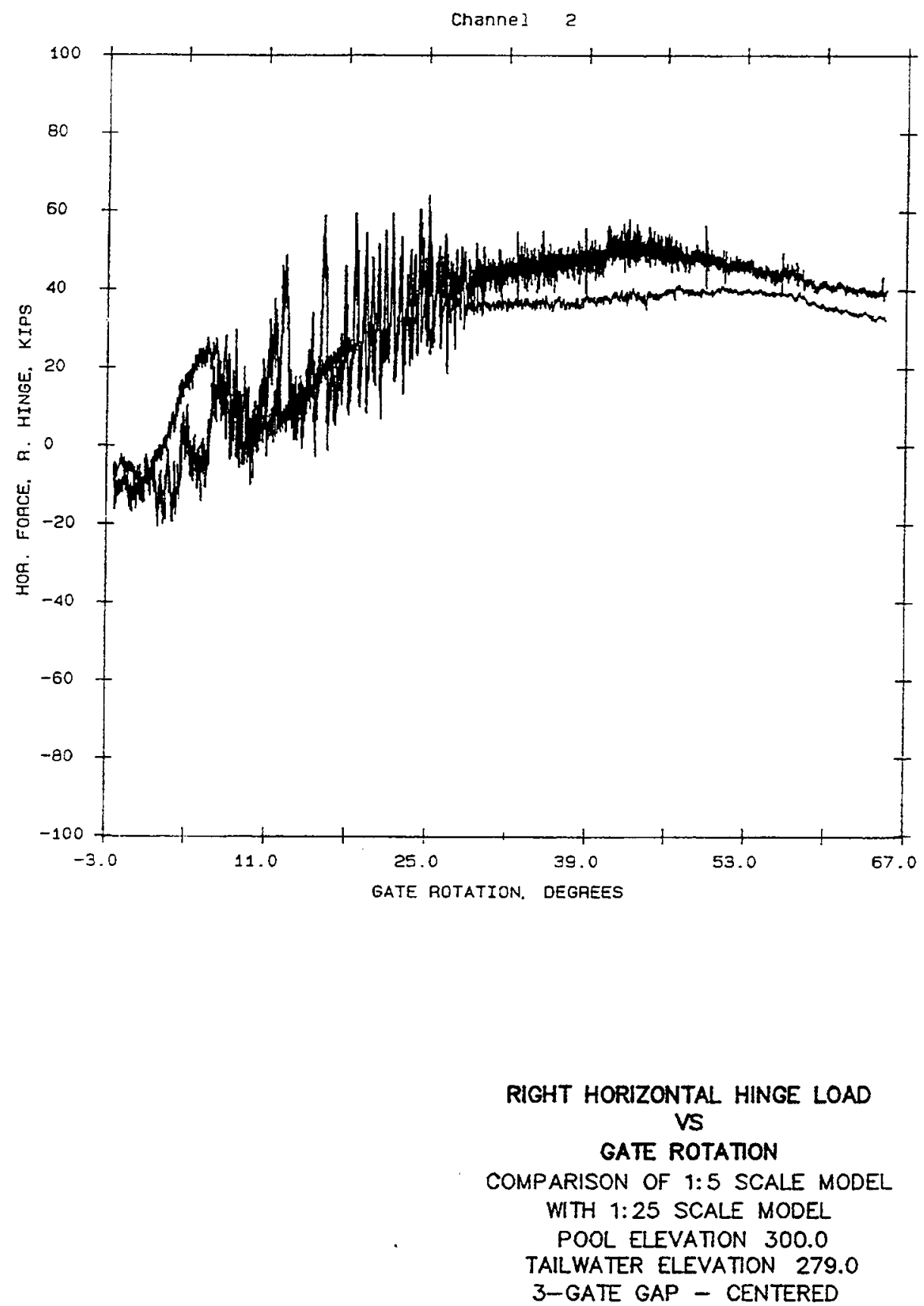

g. Prototype equivalent right horizontal hinge load

Figure 75. (Sheet 7 of 14) 

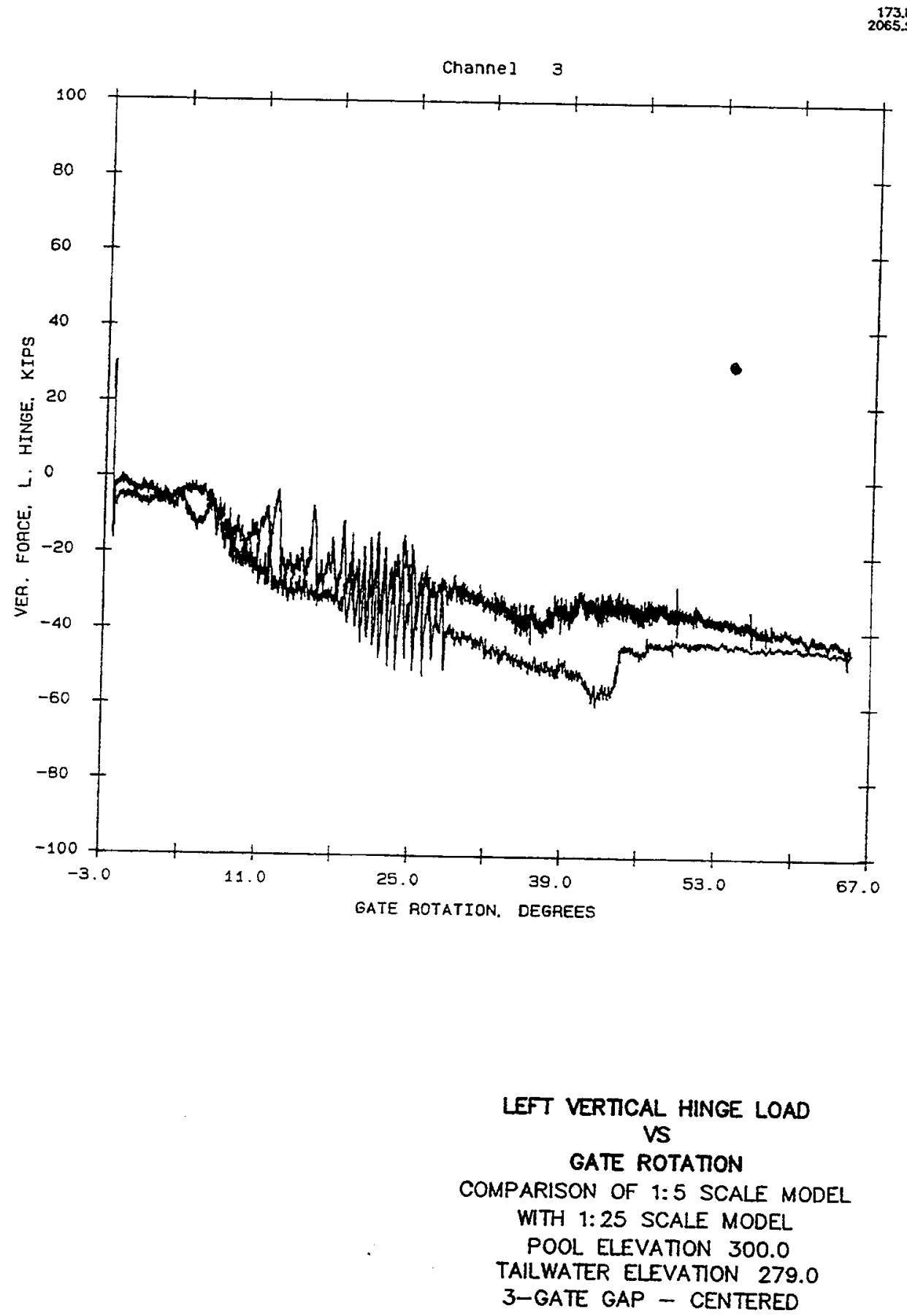

h. Prototype equivalent left vertical hinge load

Figure 75. (Sheet 8 of 14) 

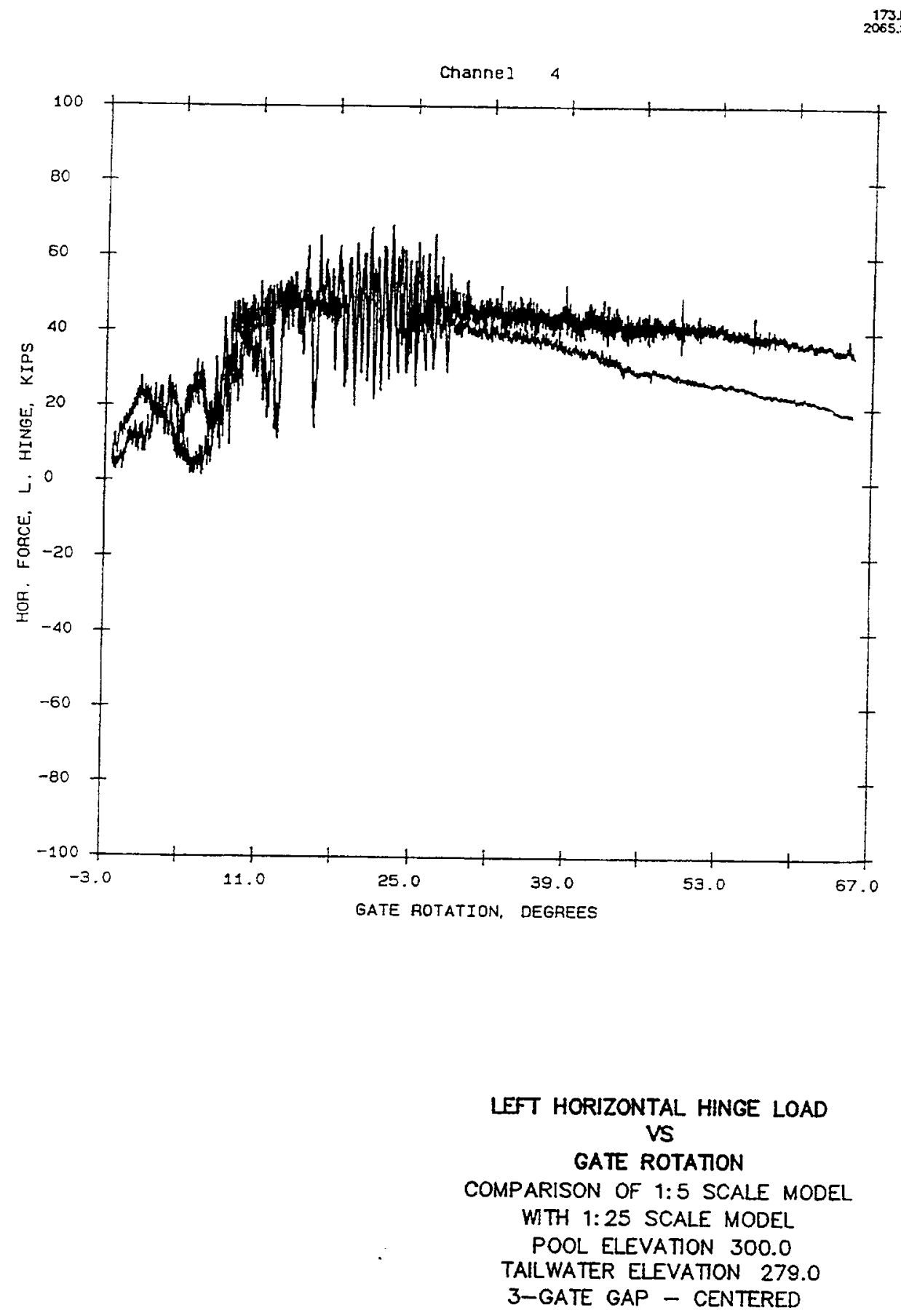

i. Prototype equivalent left horizontal hinge load

Figure 75. (Sheet 9 of 14) 


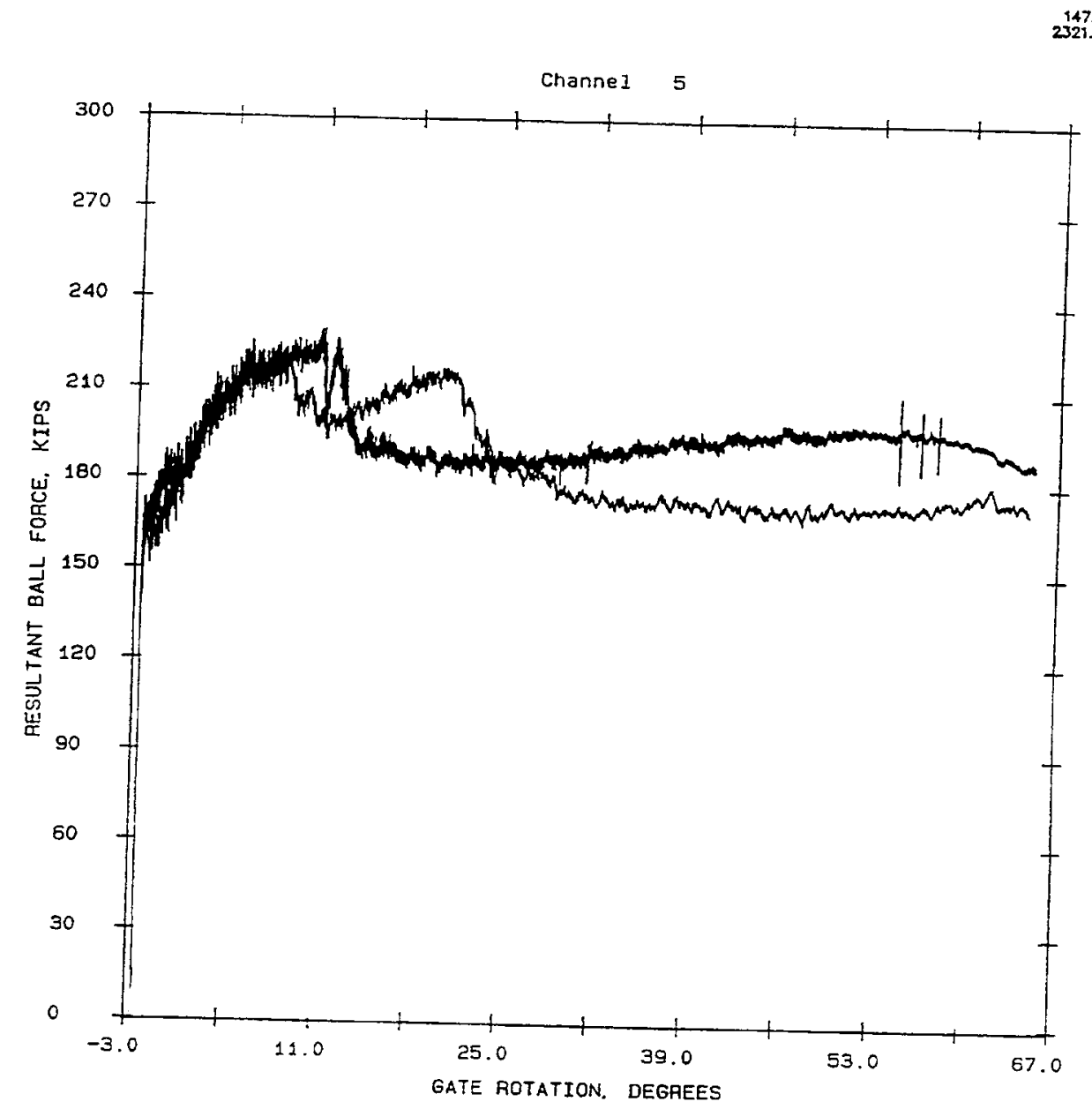

BALL FORCE VS GATE ROTATION

COMPARISON OF 1:5 SCALE MODEL WTH 1:25 SCALE MODEL POOL ELEVATION 300.0

TAILWATER ELEVATION 279.0 1-GATE GAP

j. Prototype equivalent ball force, 1GG

Figure 75. (Sheet 10 of 14) 

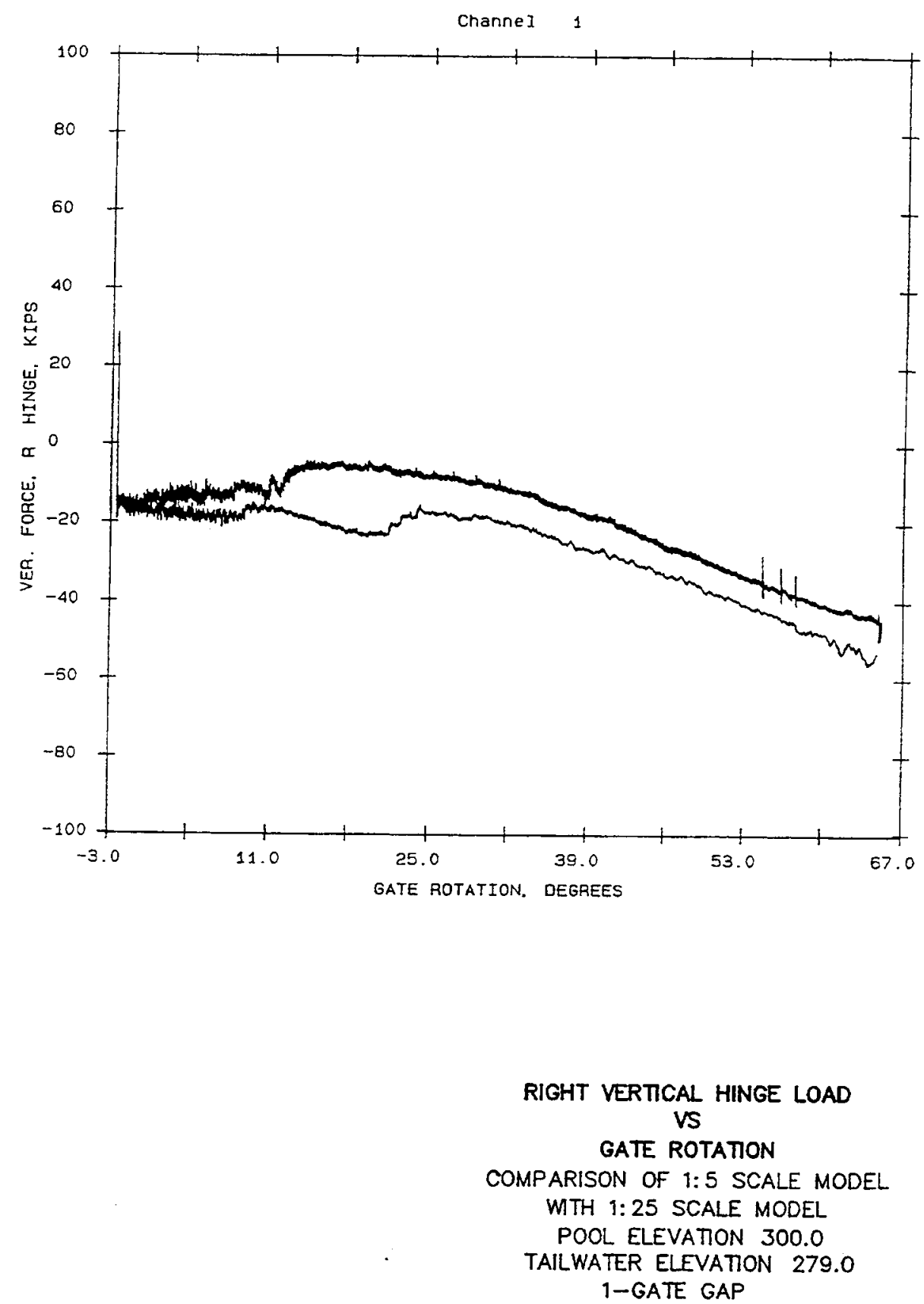

k. Prototype equivalent right vertical hinge load, 1GG

Figure 75. (Sheet 11 of 14) 


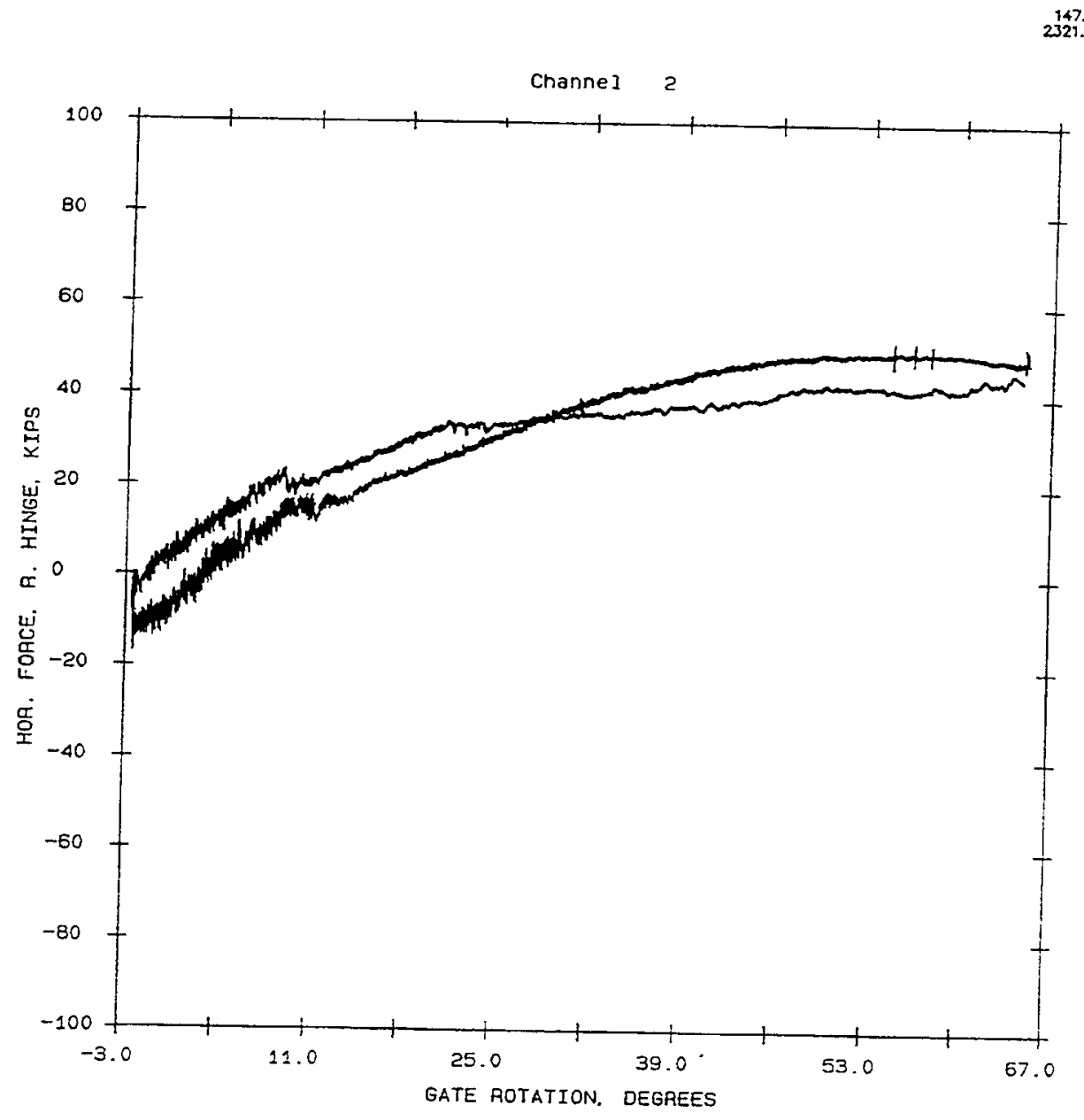

\section{RIGHT HORIZONTAL HINGE LOAD VS GATE ROTATION \\ COMPARISON OF 1:5 SCALE MODEL WITH 1:25 SCALE MODEL POOL ELEVATION 300.0 TAILWATER ELEVATION 279.0 1-GATE GAP}

I. Prototype equivalent right horizontal hinge load, 1GG

Figure 75. (Sheet 12 of 14) 

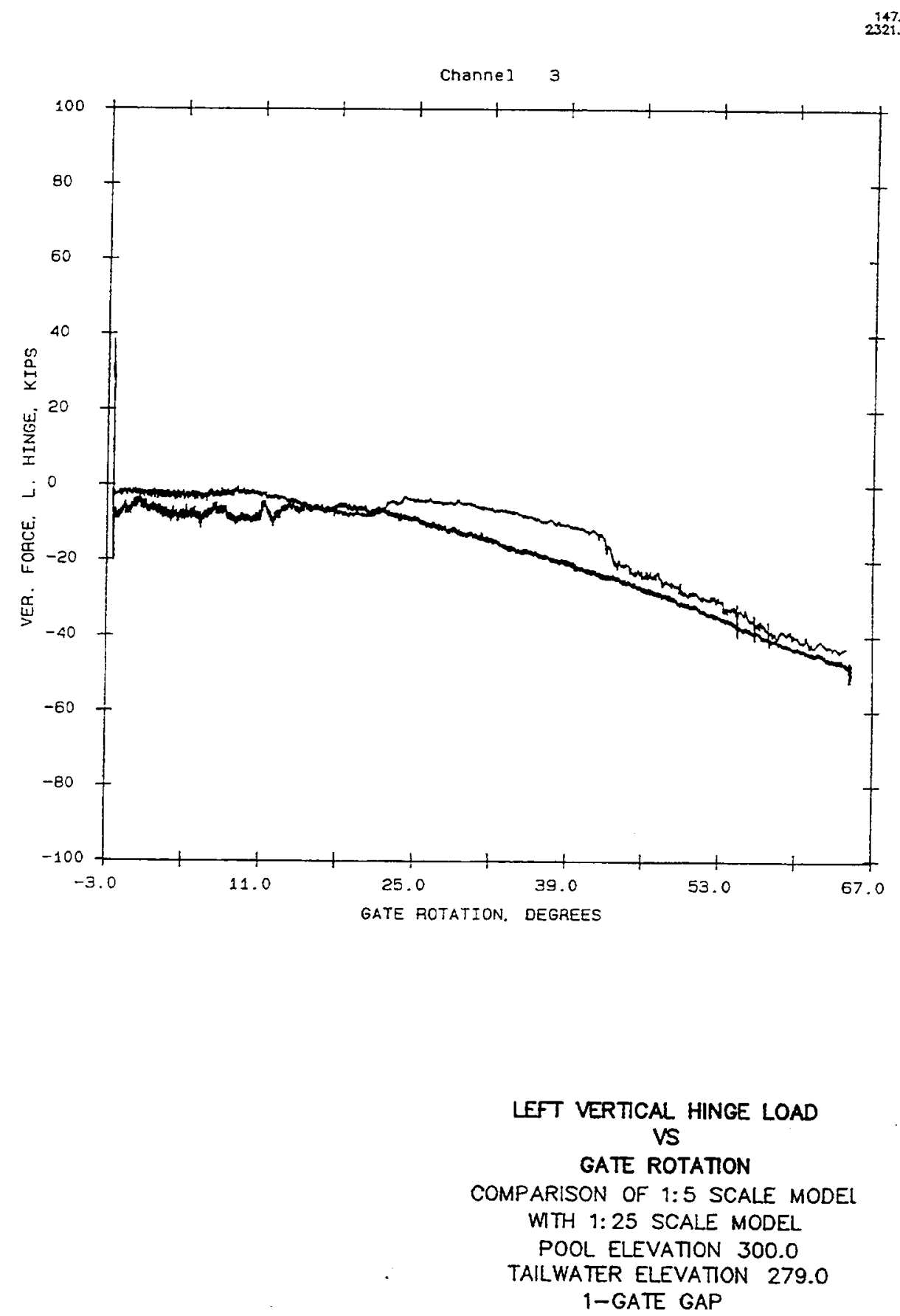

m. Prototype equivalent left vertical hinge load, 1GG

Figure 75 . (Sheet 13 of 14) 


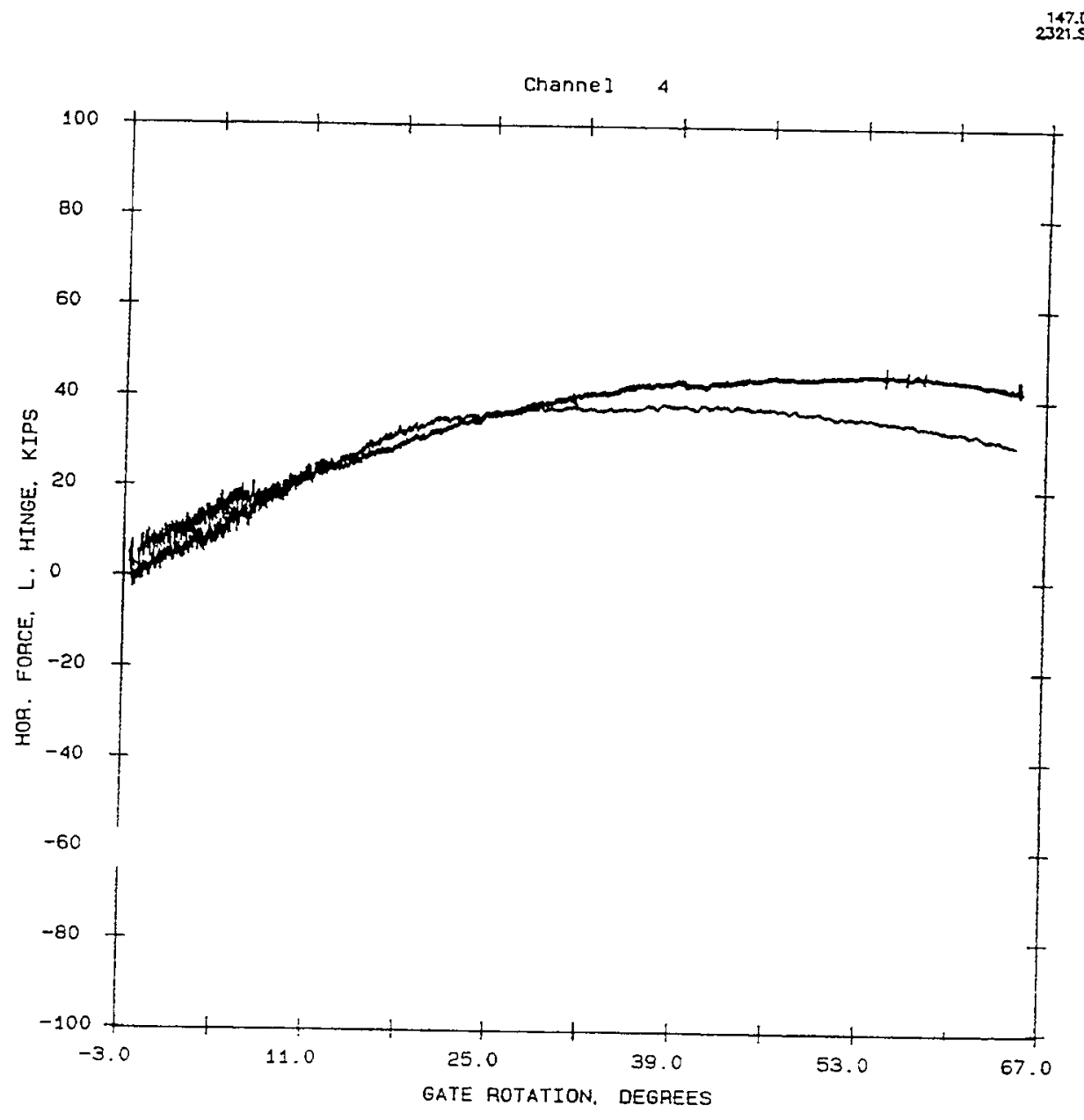

\author{
LEFT HORIZONTAL HINGE LOAD \\ VS \\ GATE ROTATION \\ COMPARISON OF 1:5 SCALE MODEL \\ WITH 1:25 SCALE MODEL \\ POOL ELEVATION 300.0 \\ TAILWATER ELEVATION 279.0 \\ 1-GATE GAP
}

n. Prototype equivalent left horizontal hinge load, 1GG

Figure 75. (Sheet 14 of 14) 


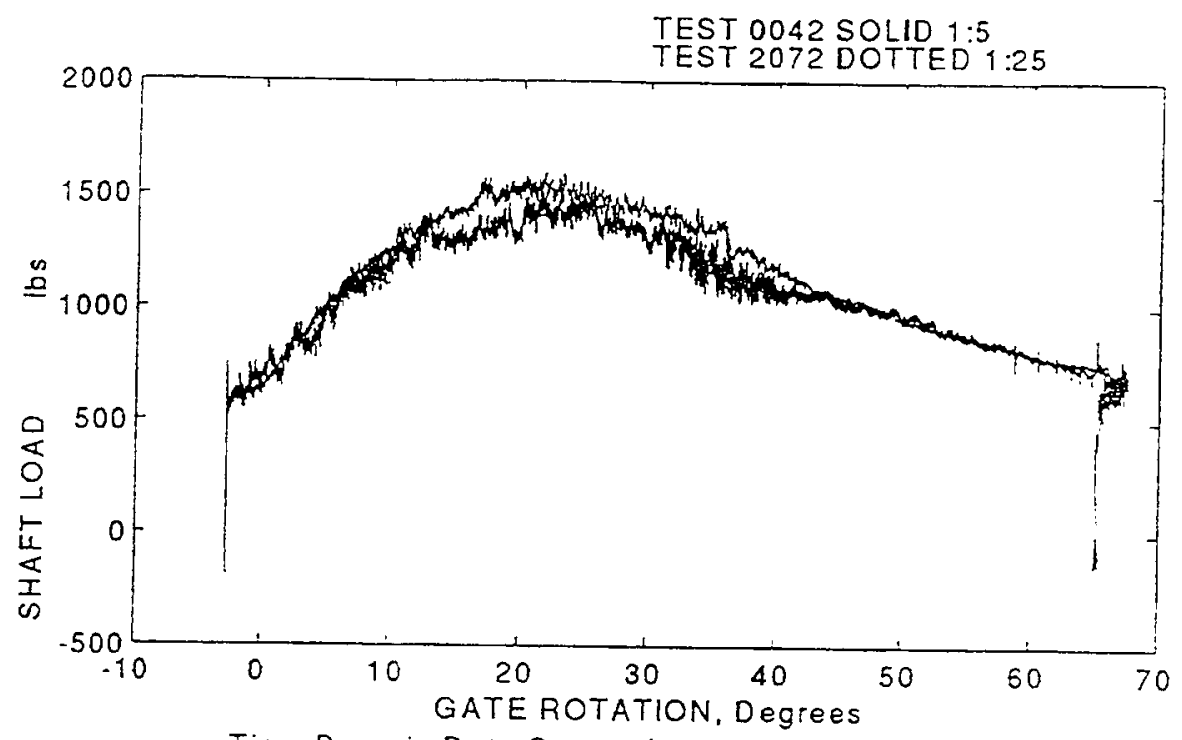

Time-Domain Data Comparison for $1: 5$ and $1: 25$ Olmsted Flatgate Models.

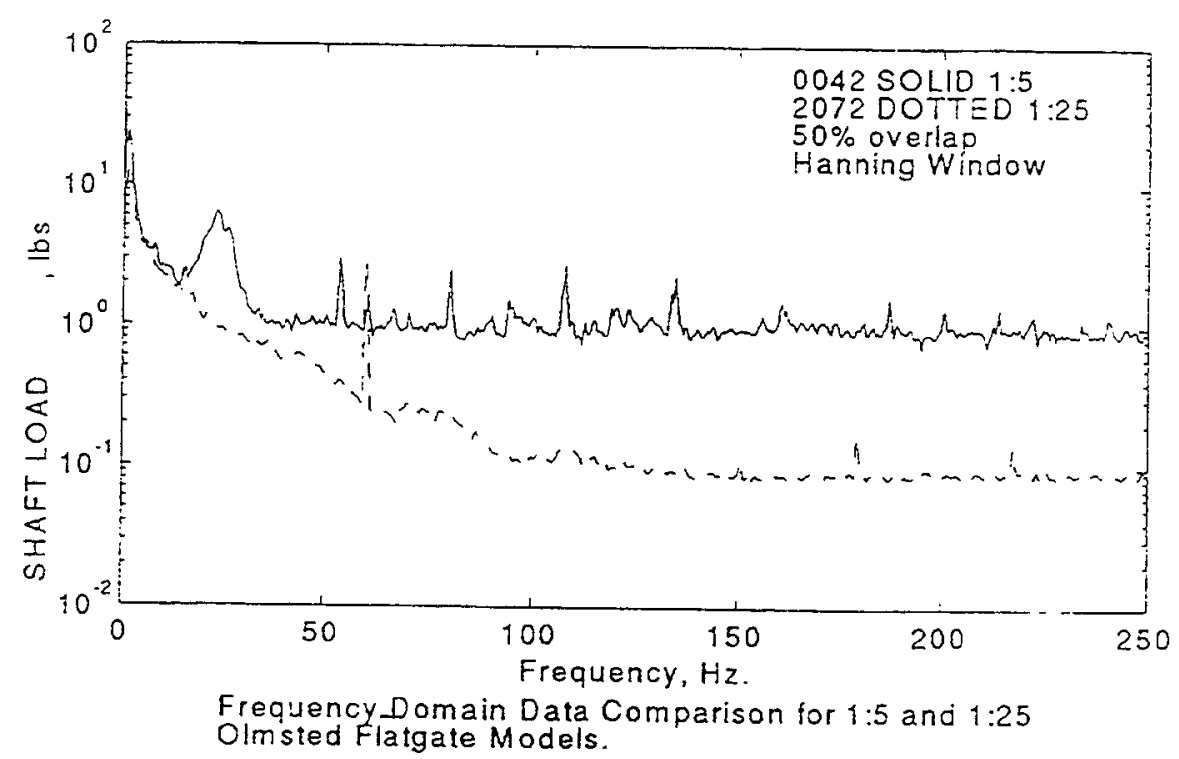

\section{a. Shaft load}

Figure 76. Gate response comparison for 1:25- and 1:5-scale models, 11-ft head difference, $3 G G$ configuration (Sheet 1 of 12) 


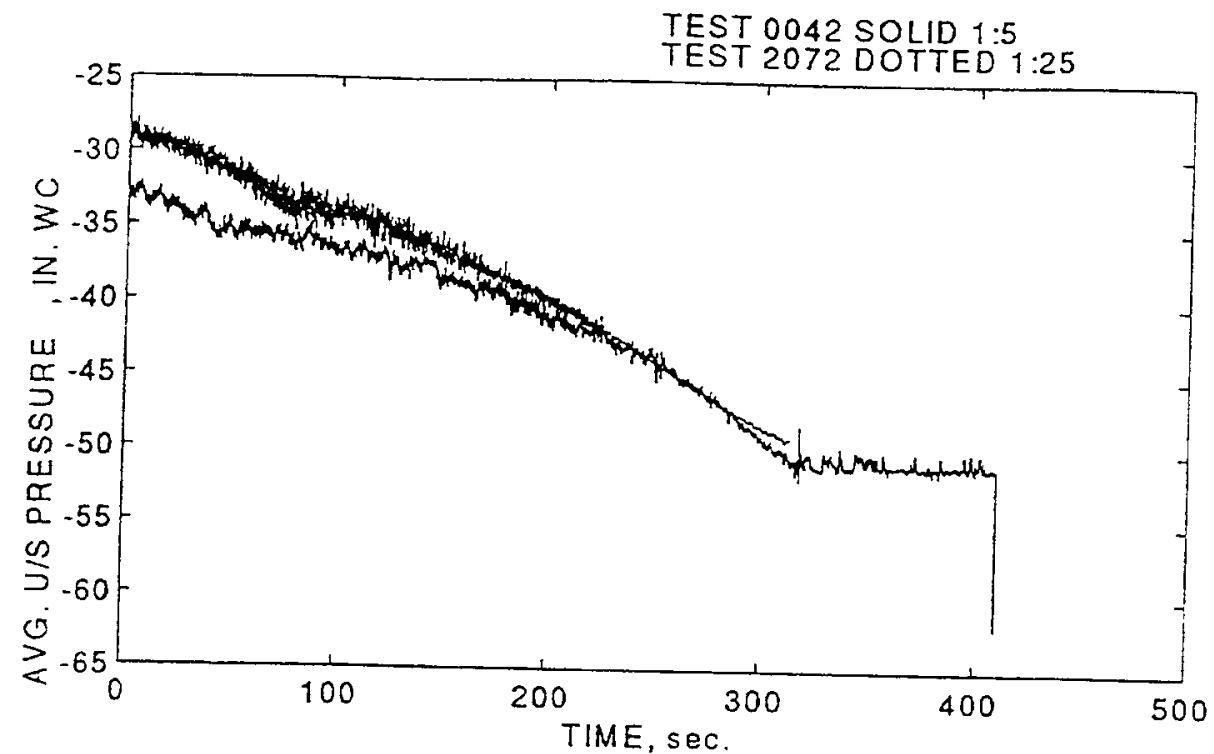

Time Domain Data Comparison for $1: 5$ and $1: 25$ Olmsted Flatgate Models

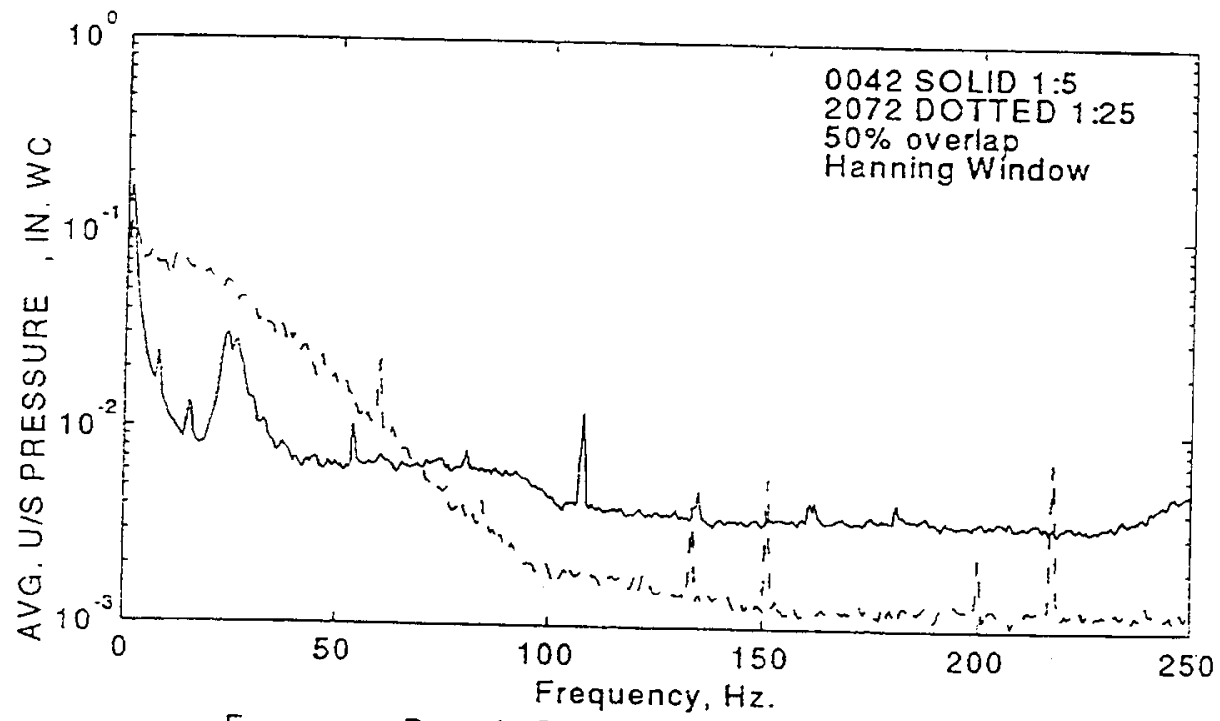

Frequency-Domain Data Comparison for $1: 5$ and $1: 25$

Olmsted Flatgate Models.

b. Upstream pressure

Figure 76. (Sheet 2 of 12) 


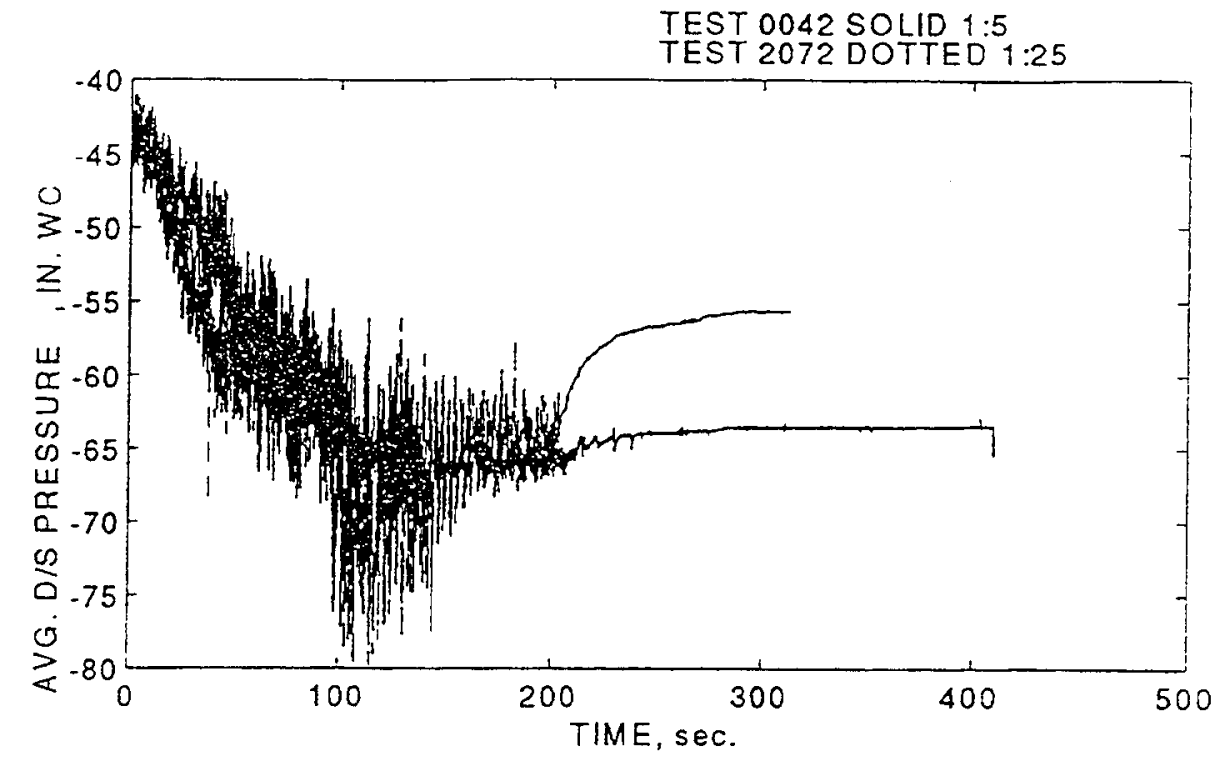

Time.Domain Data Comparison for $1: 5$ and $1: 25$ Olmsted Flatgate Models.

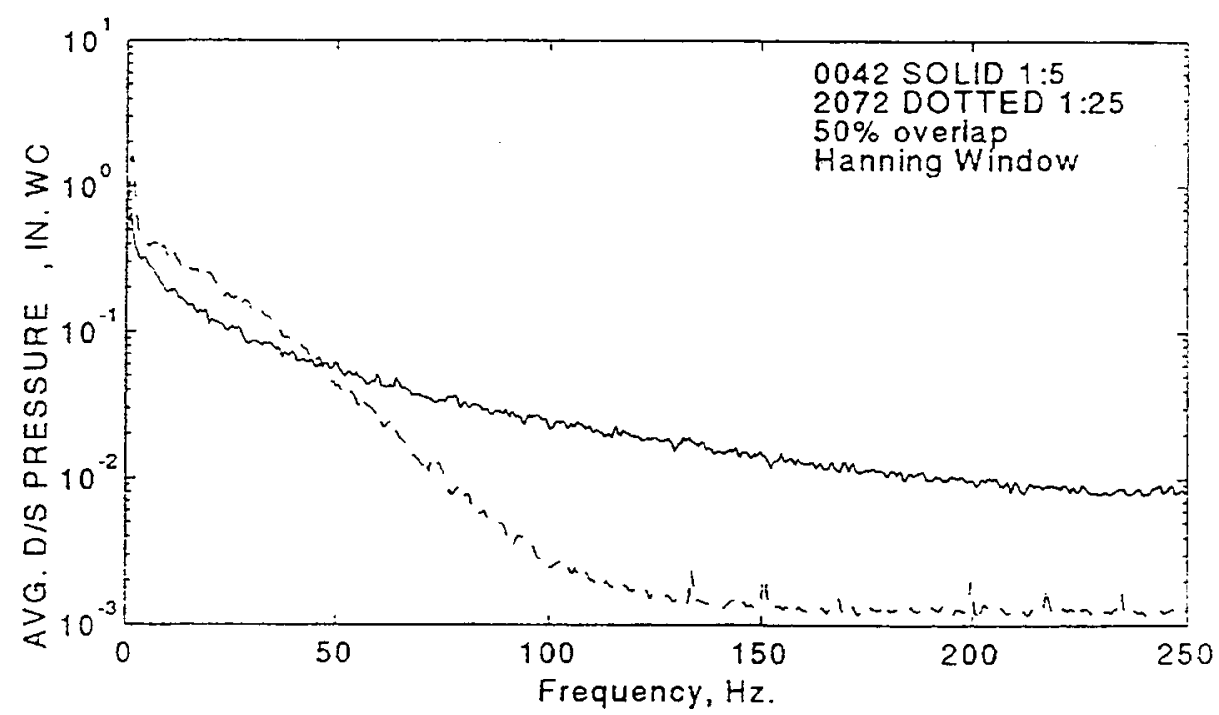

Frequency-Domain Data Comparison for $1: 5$ and $1: 25$ Olmsted Flatgate Models.

\section{c. Downstream pressure}

Figure 76. (Sheet 3 of 12) 


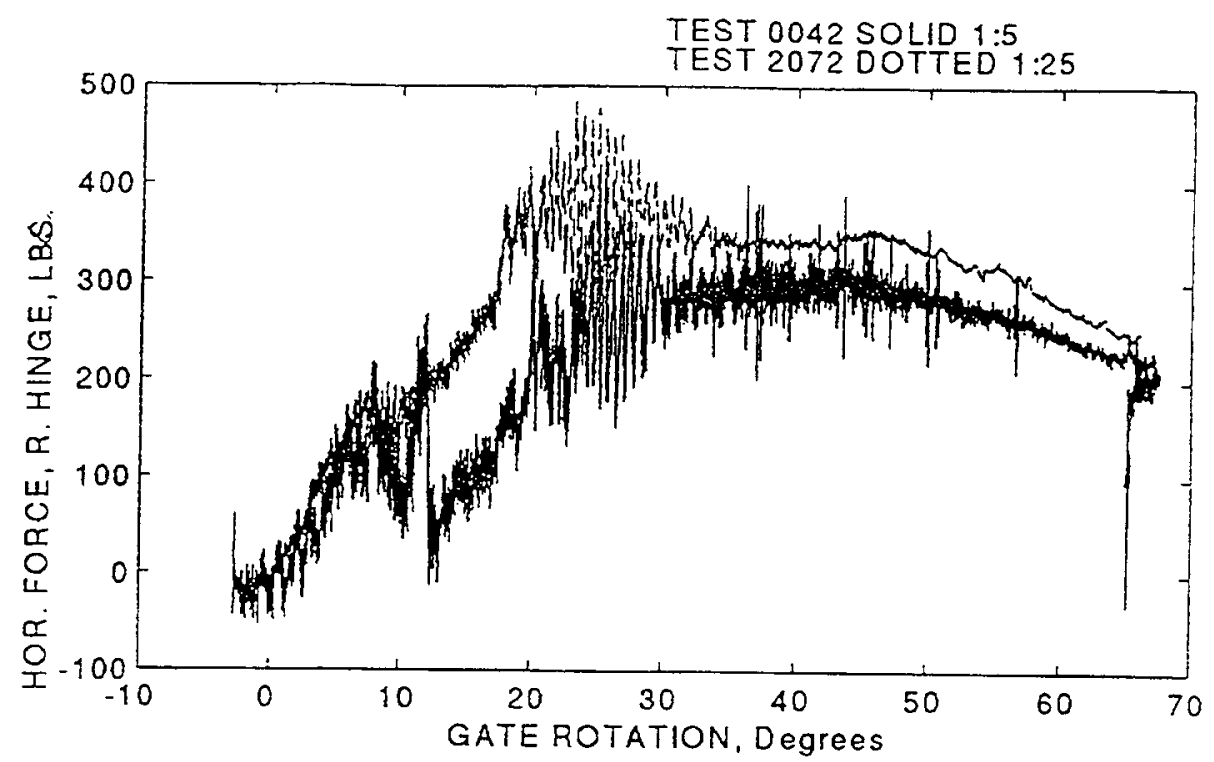

Time Domain Data Comparison for $1: 5$ and $1: 25$ Olmsted Flatgate Models.

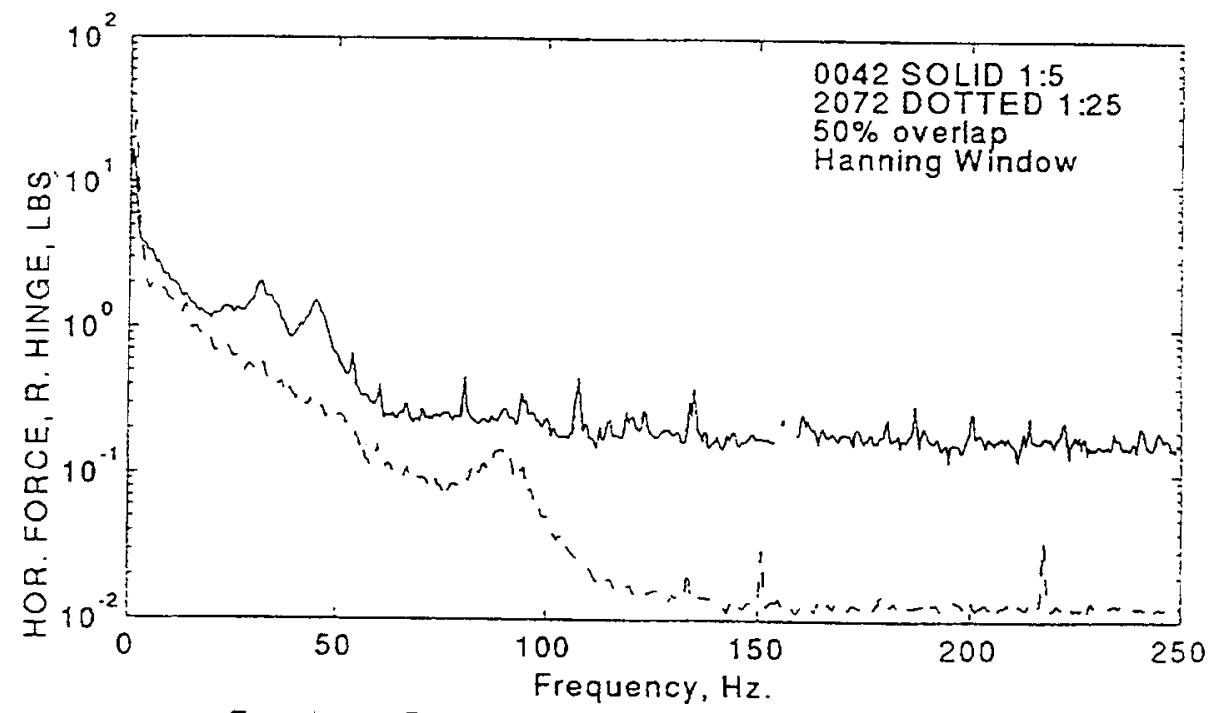

Frequency-Domain Data Comparison for $1: 5$ and $1: 25$ Olmsted Flatgate Models.

d. Horizontal right hinge reaction

Figure 76. (Sheet 4 of 12) 


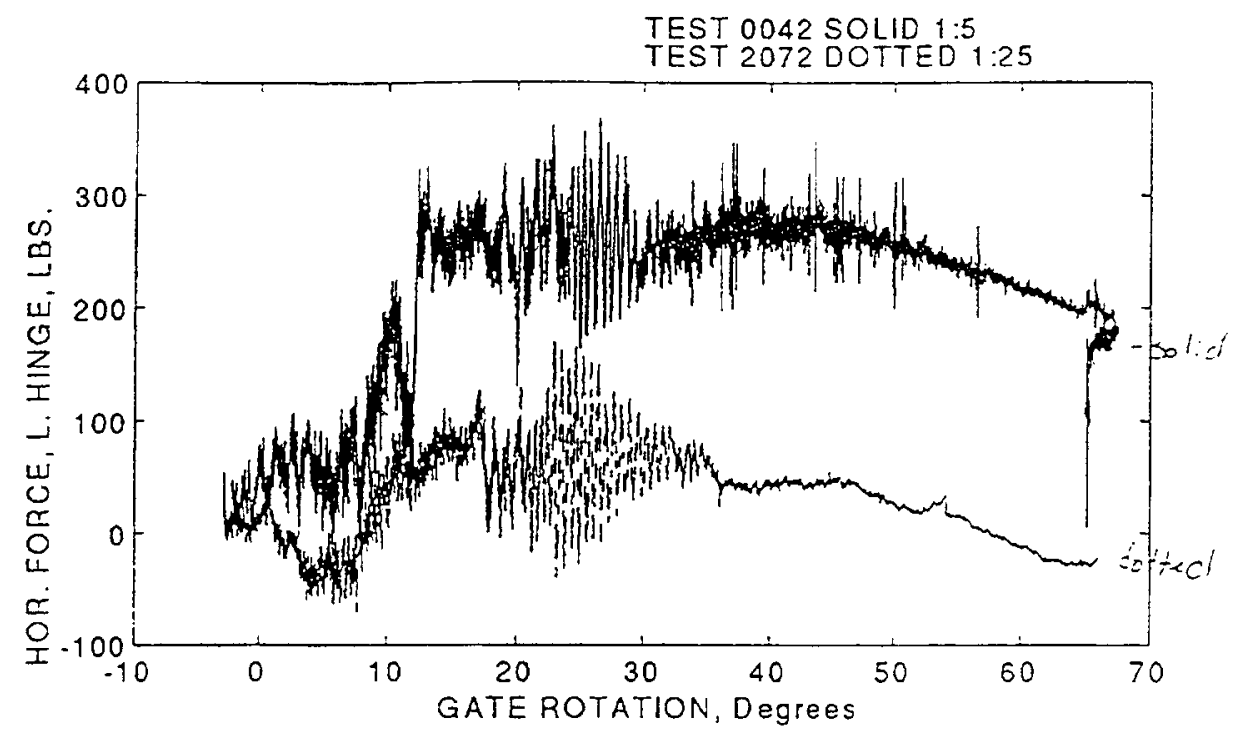

Time-Domain Data Comparison for $1: 5$ and $1: 25$

Oimsted Flatgate Models.

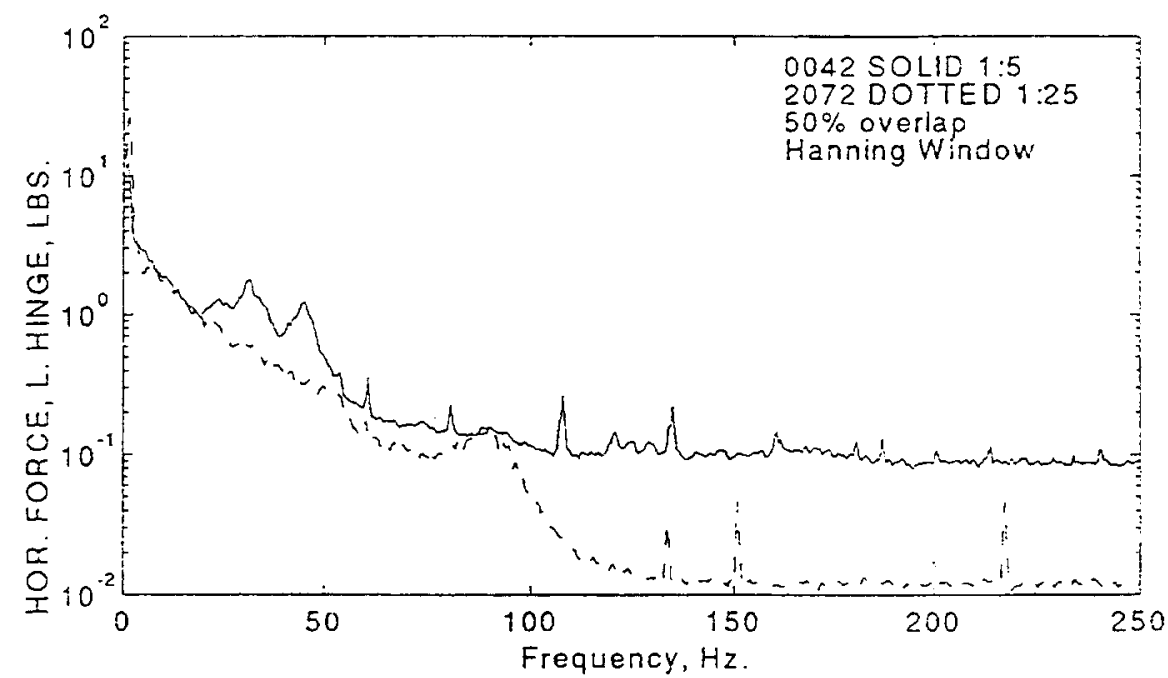

Frequency_Domain Data Comparison for $1: 5$ and $1: 25$ Olmsted Flatgate Modeis.

e. Horizontal left hinge reaction

Figure 76. (Sheet 5 of 12) 


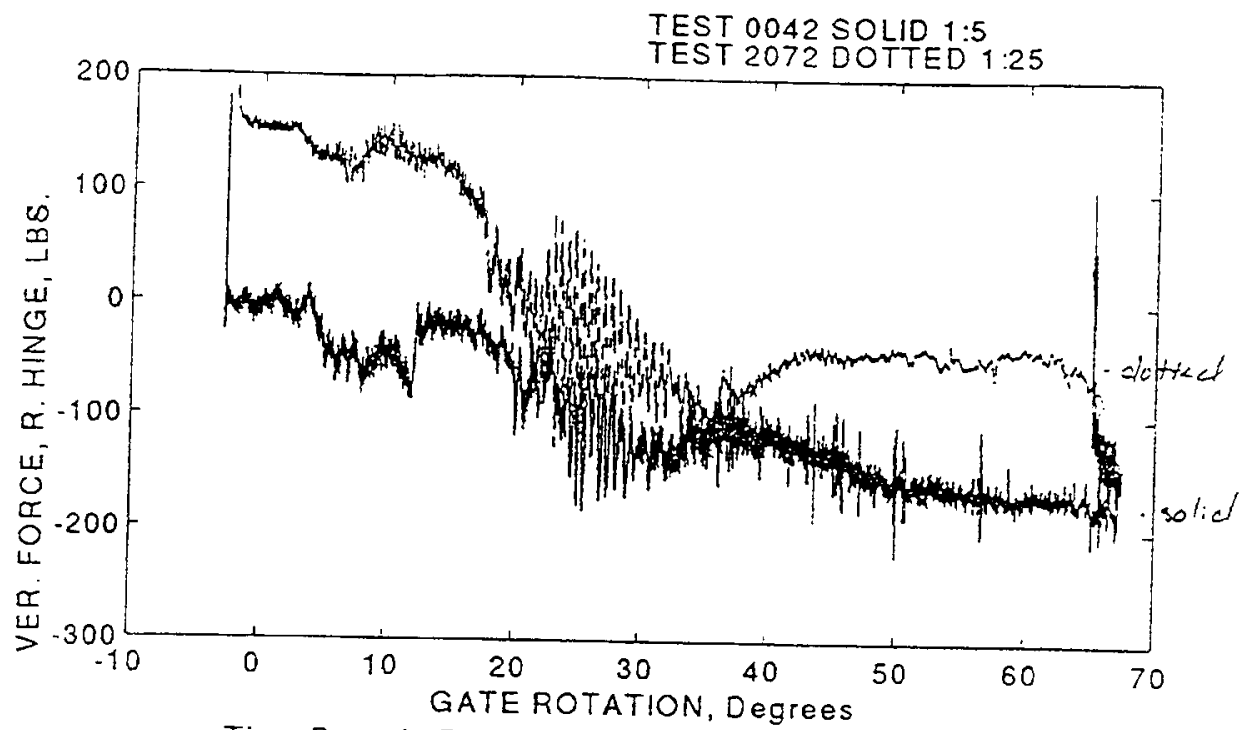

Time-Domain Data Comparison tor $1: 5$ and $1: 25$

Olmsted Flatgate Models.

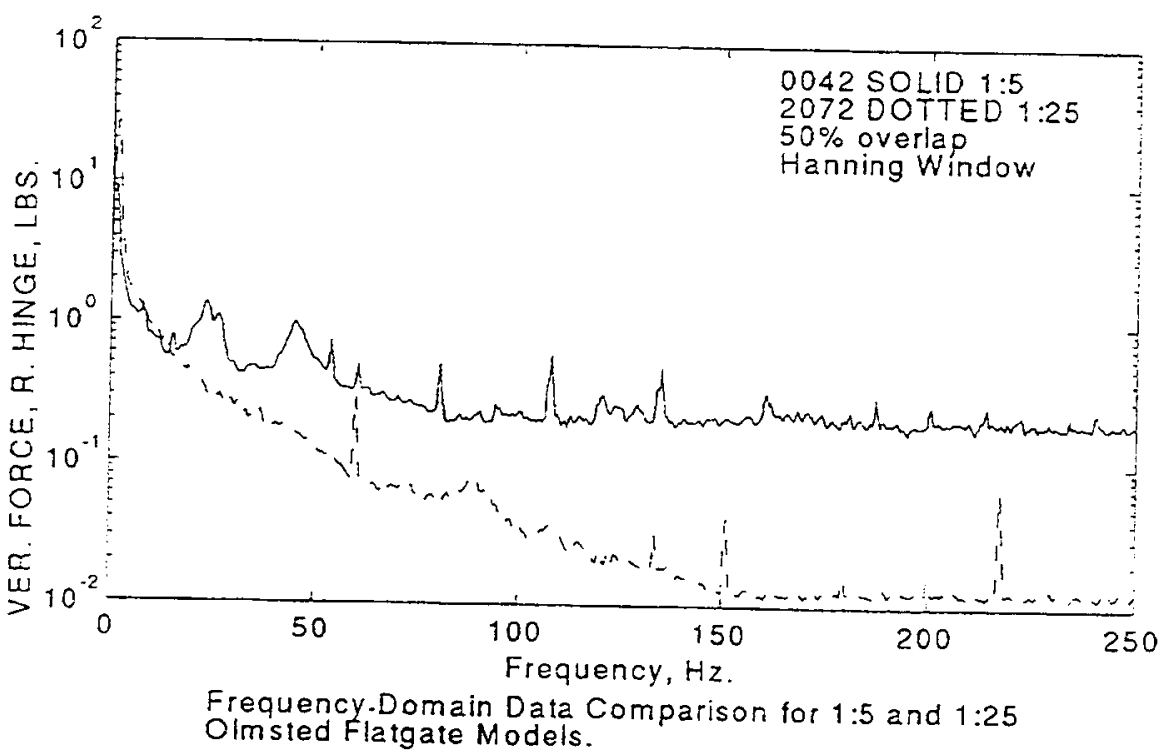

f. Vertical right hinge reaction

Figure 76. (Sheet 6 of 12) 


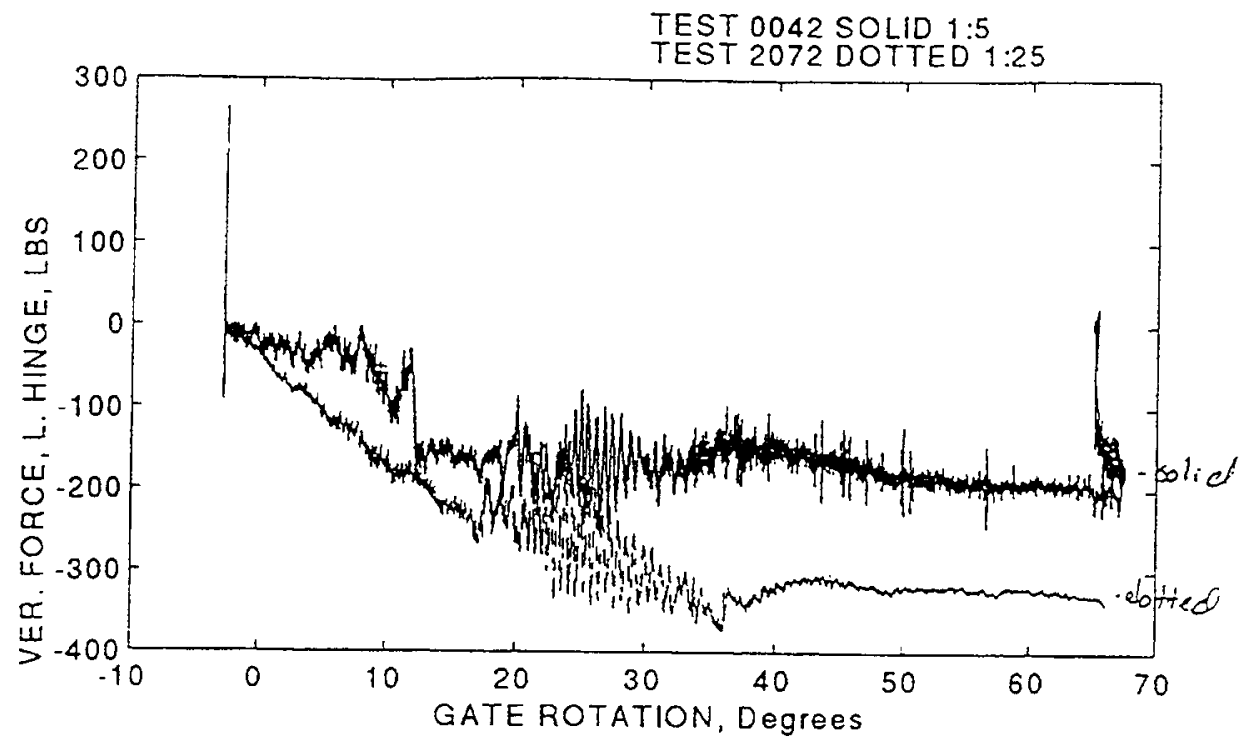

Time Domain Data Comparison for $1: 5$ and $1: 25$

Olmsted Flatgate Models.

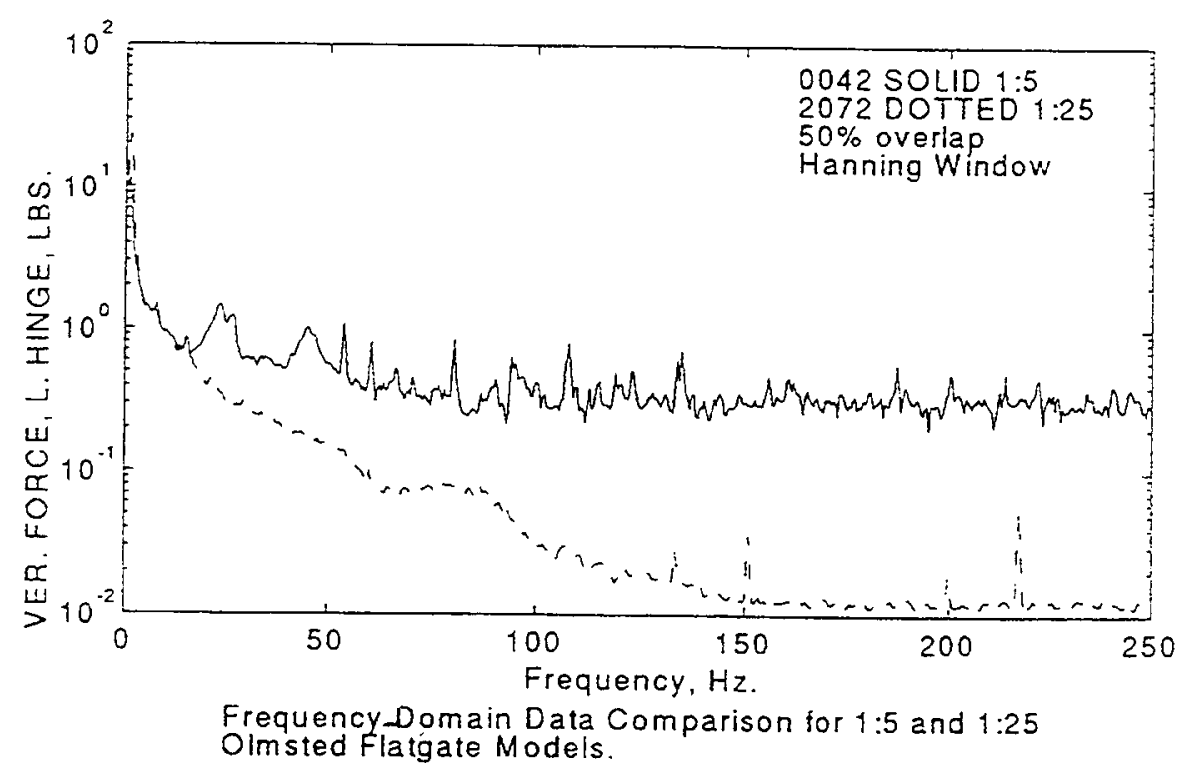

g. Vertical left hinge reaction

Figure 76. (Sheet 7 of 12) 


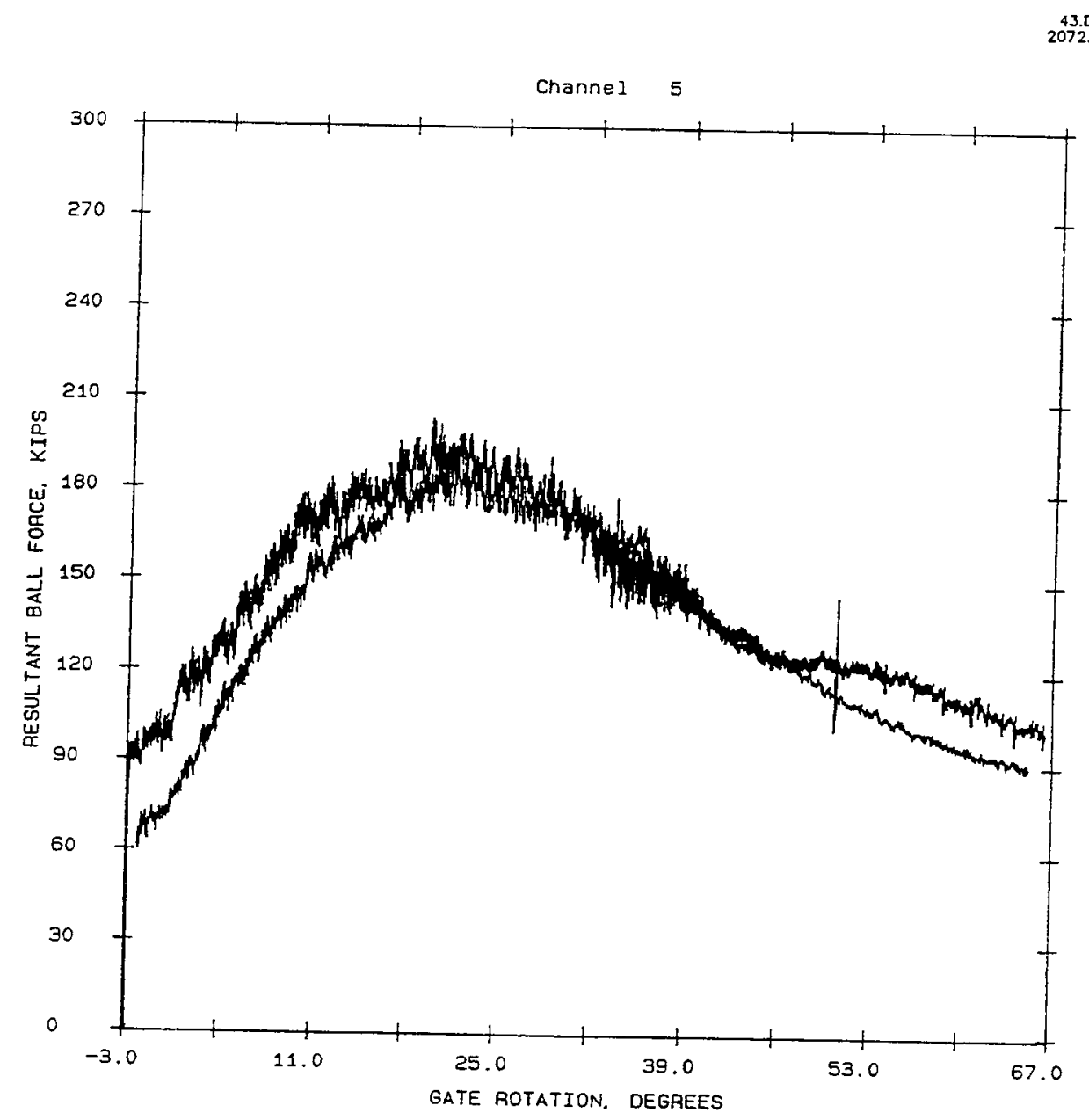

BALL FORCE VS GATE ROTATION

COMPARISON OF 1:5 SCALE MODEL WTH 1:25 SCALE MODEL POOL ELEVATION 296.5

TAILWATER ELEVATION 285.5

3-GATE GAP - CENTERED

h. Prototype equivalent ball force

Figure 76. (Sheet 8 of 12) 

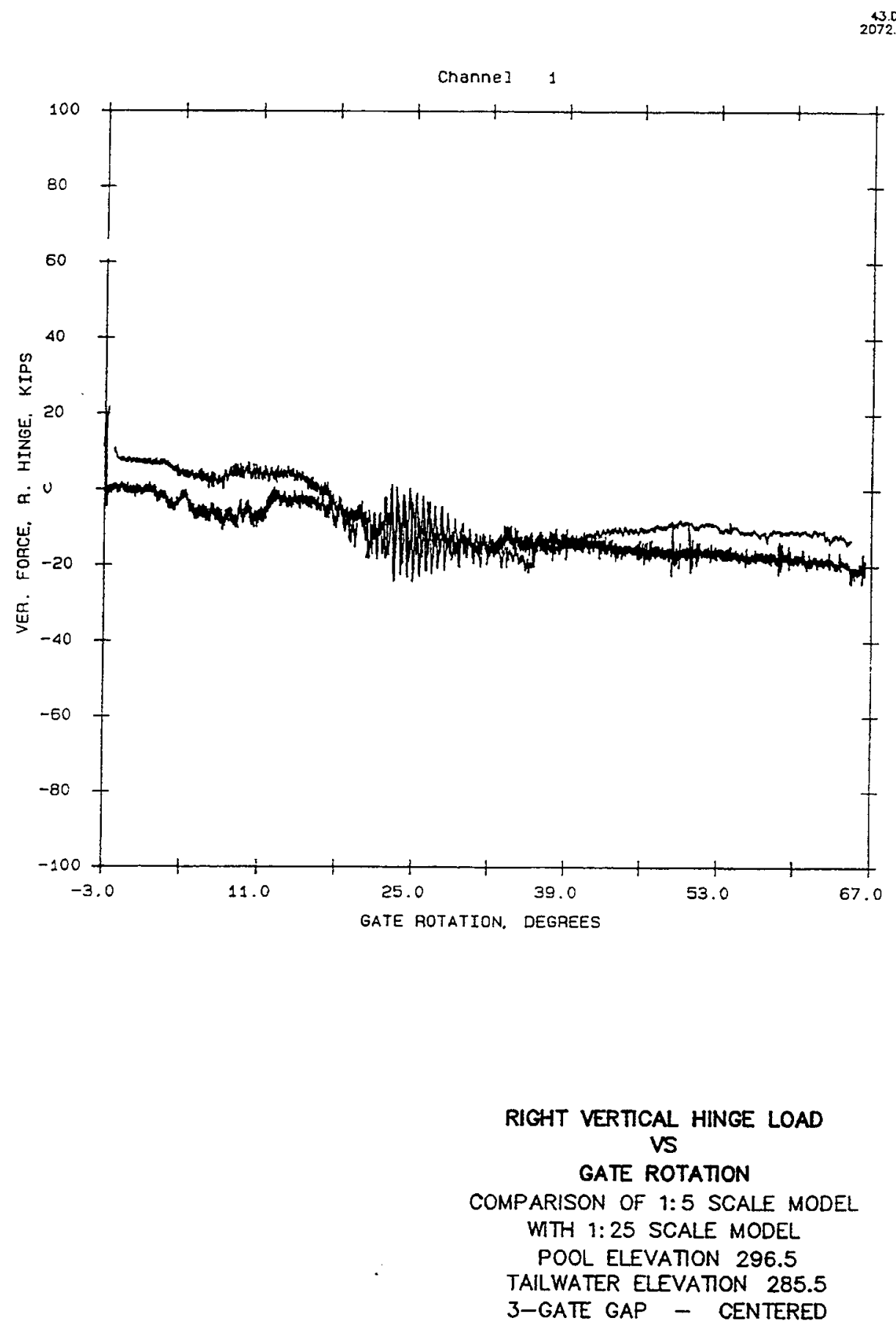

i. Prototype equivalent right vertical hinge load

Figure 76. (Sheet 9 of 12) 

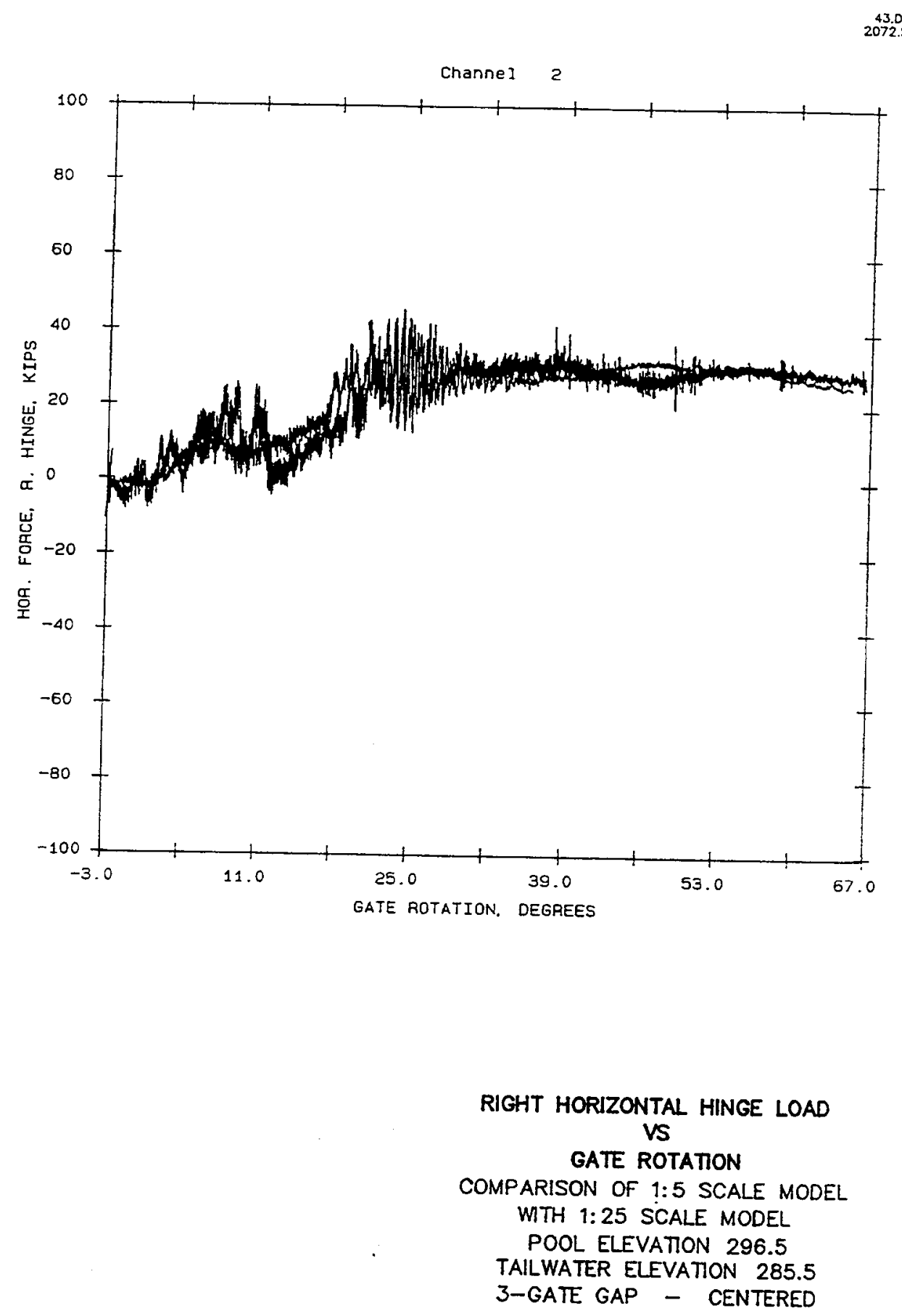

j. Prototype equivalent right horizontal hinge load

Figure 76. (Sheet 10 of 12) 

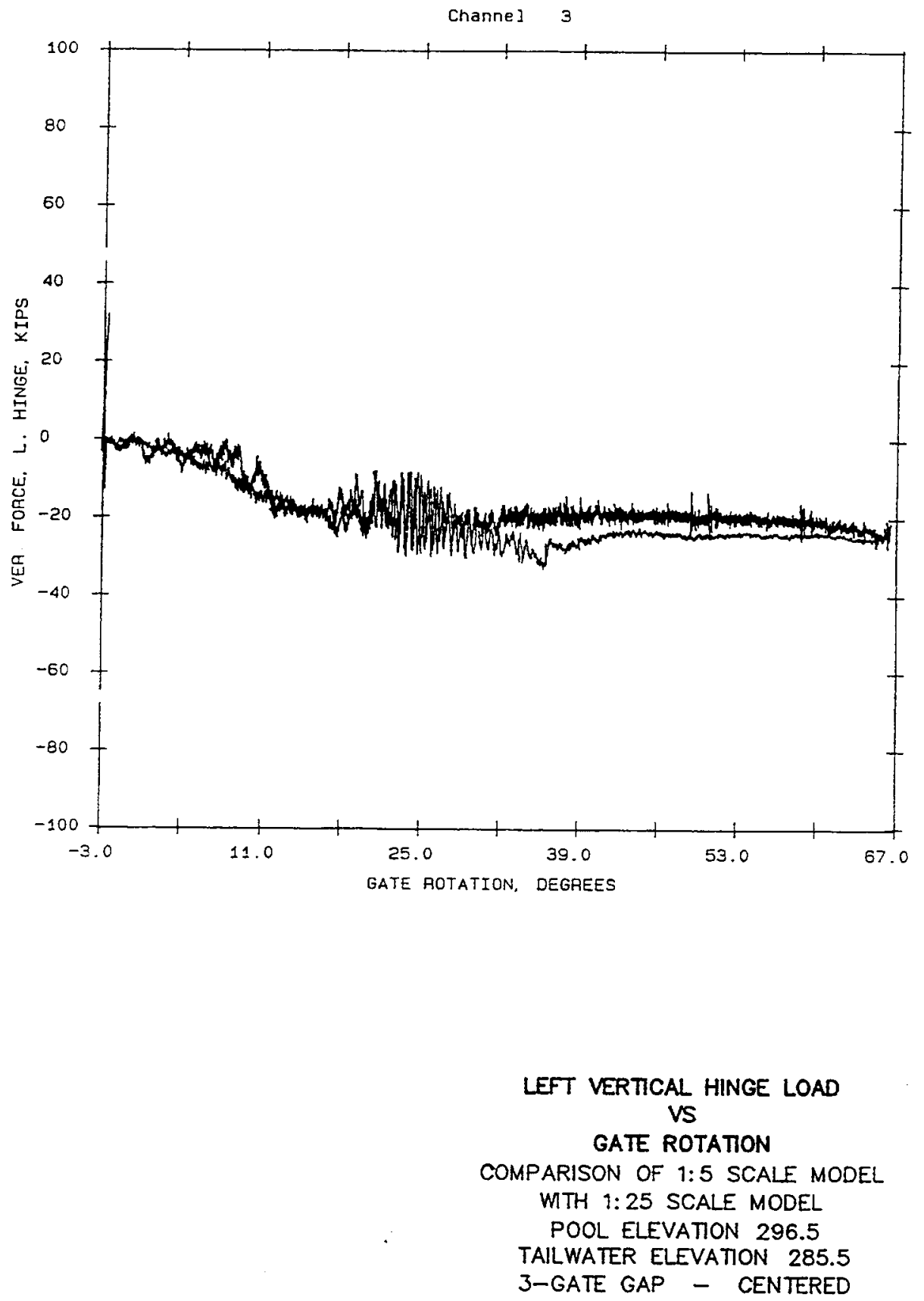

k. Prototype equivalent left vertical hinge load

Figure 76. (Sheet 11 of 12) 

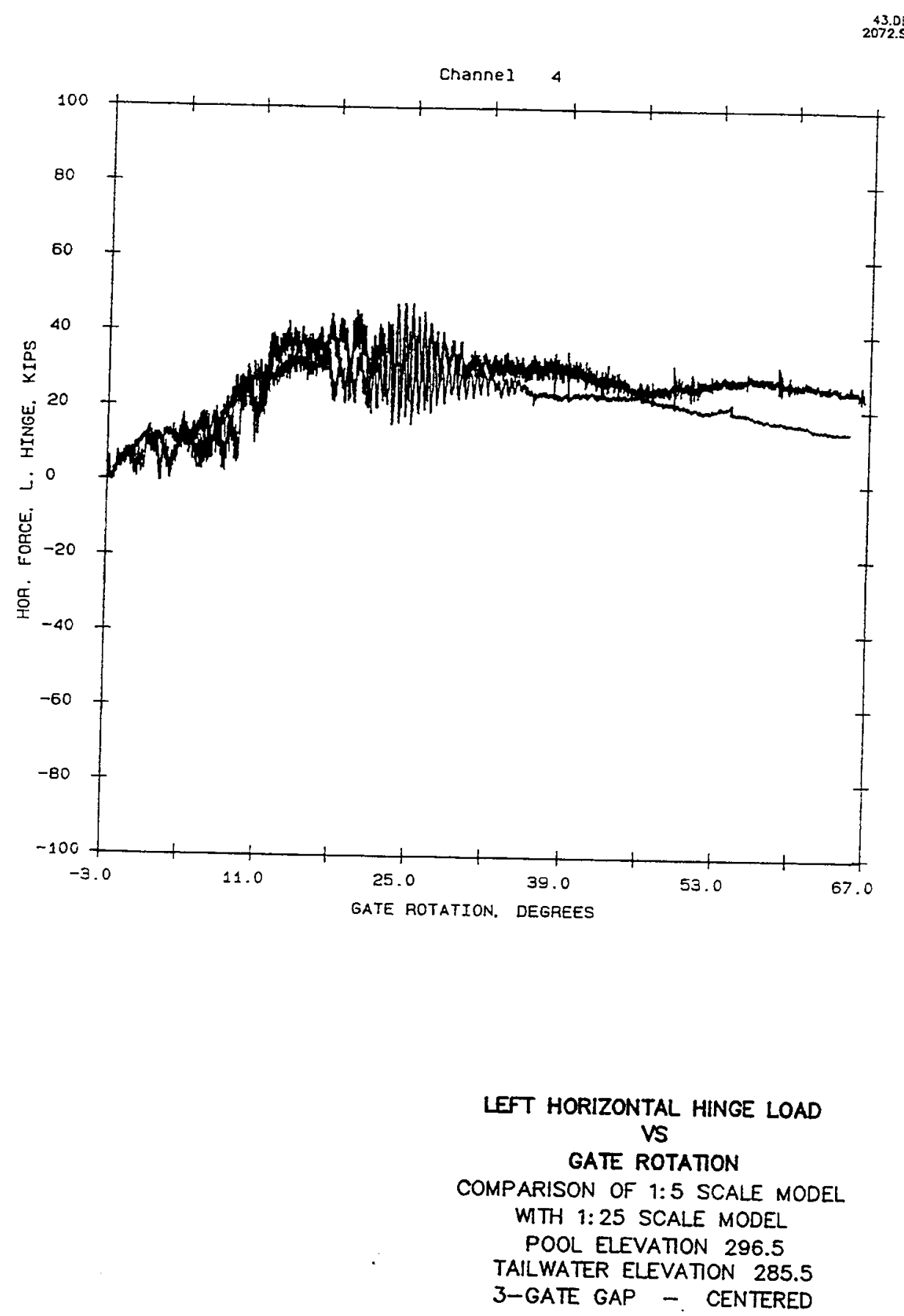

I. Prototype equivalent left horizontal hinge load

Figure 76. (Sheet 12 of 12)

A comparison of flow-induced loads between the 1:25- and 1:5-scale models for an 11-ft head differential and a three-gate gap configuration is provided in Figures $76 \mathrm{~h}$ through 1 . It should be noted that these data are the results of flowinduced forces only. 
Noticeable dynamic disturbances at or near the nappe break were identical for both models. The fluctuation of responses during the burst of air gap beneath the gate on the downstream side indicate a major turbulence in the response for both models. A fixed-gate dynamic response analysis at this location would adequately identify the structural response pattern for both models. A closeup view of the detailed responses at or near the nappe break was obtained by measuring the test gate responses for a fixed-gate position as presented below.

\section{Fixed gate at 20-deg, 11-ft head difference}

Results corresponding to a 20-deg position, 11-ft head difference, and threegate gap configuration for the 1:25- and 1:5-scale flat gate models are compared in Table 13. In this table, the ratio for each of the parameters corresponding to their respective mean plus one and mean plus two standard deviations is presented. A one plus $(+\sigma)$ and two plus $(+2 \sigma)$ standard deviation about the mean indicate that about 68 and 96 percent of the total samples in a normally distributed variable would lie within these ranges, respectively (Wirsching and Ortiz 1993). Thus, by comparing the ratios of $(\mu \pm \sigma)$ and $(\mu \pm 2 \sigma)$ for each random variable, the deviation of frequency distribution between the models are measured. As shown in the table, except for the quantitative differences in the net vertical hinge reaction force and the two acceleration records, the remaining responses agreed reasonably well. The elastic response, as evidenced in the accelerometer responses, differed between the two models. The results that showed variations are printed in bold. Net reaction forces were obtained by summing the corresponding reactions for both hinges. Also, the net pressure represents the actual pressure that was exerted on the gate due to the flow and was obtained by subtracting the downstream pressure from the upstream pressure. The standard deviation of the newly formed random variable $Y$, which is a linear function of uncorrelated random variables $X_{1}, \ldots . X_{n}$, such that

$\left[Y=a_{0}+\sqrt{\sum_{1}^{n}\left(a_{i} X_{i}\right)}\right]$, was computed with the generalized

equation, $\sigma_{\gamma}=\sqrt{\sum_{1}^{n}\left(a_{i}^{2} \sigma_{X_{i}}{ }^{2}\right)}$ (Thoft-Christensen and Baker 1982).

Time- and frequency-domain comparisons of responses from these experiements are also presented in Figure 77. The measured responses for the 1:25- and 1:5-scale model were filtered at $1,000 \mathrm{~Hz}$ and $500 \mathrm{~Hz}$, respectively. Using the Hanning window and 50 percent overlapping, the PSD for the respective channel responses were computed with MATLAB. A brief comparison of the mode shapes, as explained above, would be necessary at this point to understand the role of modal participation in the flow-induced dynamics of both models. A detailed procedure for evaluating the modal parameters of the wicket gate is explained later in this report. 


\begin{tabular}{|c|c|c|c|c|c|c|}
\hline \multicolumn{7}{|c|}{$\begin{array}{l}\text { Table } 13 \\
\text { Results for 1:25- and 1:5-Scale Flat Gate Models at 20-deg Position } \\
\text { (11-ft Head Difference, 3GG Configuration) }\end{array}$} \\
\hline \multirow[b]{2}{*}{ Gauge Type } & \multicolumn{2}{|c|}{$\begin{array}{c}1: 5-S c a l e \\
\text { (Experiment No. 47) }\end{array}$} & \multicolumn{2}{|c|}{$\begin{array}{c}\text { 1:25-Scale } \\
\text { (Experiment } \\
\text { No. 7085) } \\
\end{array}$} & \multicolumn{2}{|c|}{ Ratio } \\
\hline & $\begin{array}{c}\text { Avg. } \\
\text { Reading } \\
(\bar{\mu})\end{array}$ & $\begin{array}{c}\text { Std. } \\
\text { Deviation } \\
(\sigma)\end{array}$ & $\begin{array}{c}\text { Avg. } \\
\text { Reading } \\
(\bar{\mu})\end{array}$ & $\begin{array}{c}\text { Std. } \\
\text { Deviation } \\
(\sigma)\end{array}$ & $\frac{(\bar{\mu}+\sigma)_{1: 5}}{(\bar{\mu}+\sigma)_{1: 25}}$ & $\frac{(\bar{\mu}+2 \sigma)_{1: 5}}{(\bar{\mu}+2 \sigma)_{1: 25}}$ \\
\hline Shaft Load, Ibf & $1,566.18$ & 22.72 & $1,596.38$ & 39.93 & 0.971 & 0.962 \\
\hline $\begin{array}{l}\text { Net Vertical } \\
\text { Force at Hinges, Ibf }\end{array}$ & 269.78 & 30.65 & 384.00 & 80.43 & 0.647 & 0.608 \\
\hline $\begin{array}{l}\text { Net Horizontal } \\
\text { Force at Hinges, lbf }\end{array}$ & 349.12 & 67.33 & 324.37 & 110.72 & 0.957 & 0.886 \\
\hline $\begin{array}{l}\text { Upstream Pressure, } \\
\text { in. of water }\end{array}$ & -37.725 & 0.385 & -33.355 & 0.139 & 1.137 & 1.144 \\
\hline $\begin{array}{l}\text { Downstream } \\
\text { Pressure, in. of water }\end{array}$ & -65.886 & 3.218 & -65.885 & 0.944 & 1.0340 & 1.067 \\
\hline $\begin{array}{l}\text { Net Pressure, in. of } \\
\text { water }\end{array}$ & -28.161 & 3.241 & -32.53 & 0.955 & 0.938 & 1.00 \\
\hline Accel. T.R., g's & 0.008 & 0.017 & -0.027 & 0.020 & 0.531 & 0.625 \\
\hline Accel. T.L., g's & 0.010 & 0.016 & -0.018 & 0.017 & 0.754 & 0.824 \\
\hline Accel. M.L., g's & -0.009 & 0.013 & -0.002 & 0.016 & 1.229 & 1.036 \\
\hline
\end{tabular}

PSD for the reaction forces show that the first peak (mode 1) is the dominant mode of vibration for the operating wicket. The energy level for the first peak (mode) is at least one order of magnitude higher than that of the remaining frequency spectra. A slightly higher frequency for the operating dominant modes is noticeable in the 1:25-scale model. The distribution of peaks in the 1:25-scale model is different than those of the 1:5-scale model. The cumulative contributions of these differences, however, does not significantly affect the time-domain responses as shown in the time-domain reaction plots.

Effectiveness of the higher modes decreases since the higher-order energy content in the flow asymptotically decreases for higher orders as shown in the pressure PSD.

The acceleration PSD show the dominant modes of vibration for both models. This PSD comparison shows that the peaks in the 1:25-scale model were exited at higher frequencies than those of the 1:5-scale model. A difference in the distribution of peaks is noticeable; however, for the same reason as reaction forces, the difference in the time scale is very marginal.

A similar trend in the response pattern was also noticeable when both models were tested at a 6-deg position, three-gate gap configuration with a pool difference of $11 \mathrm{ft}$. Note that at the 60 -deg gate position both pressure gauges were exposed to the air; thus, in this case the net pressure is the same as the 


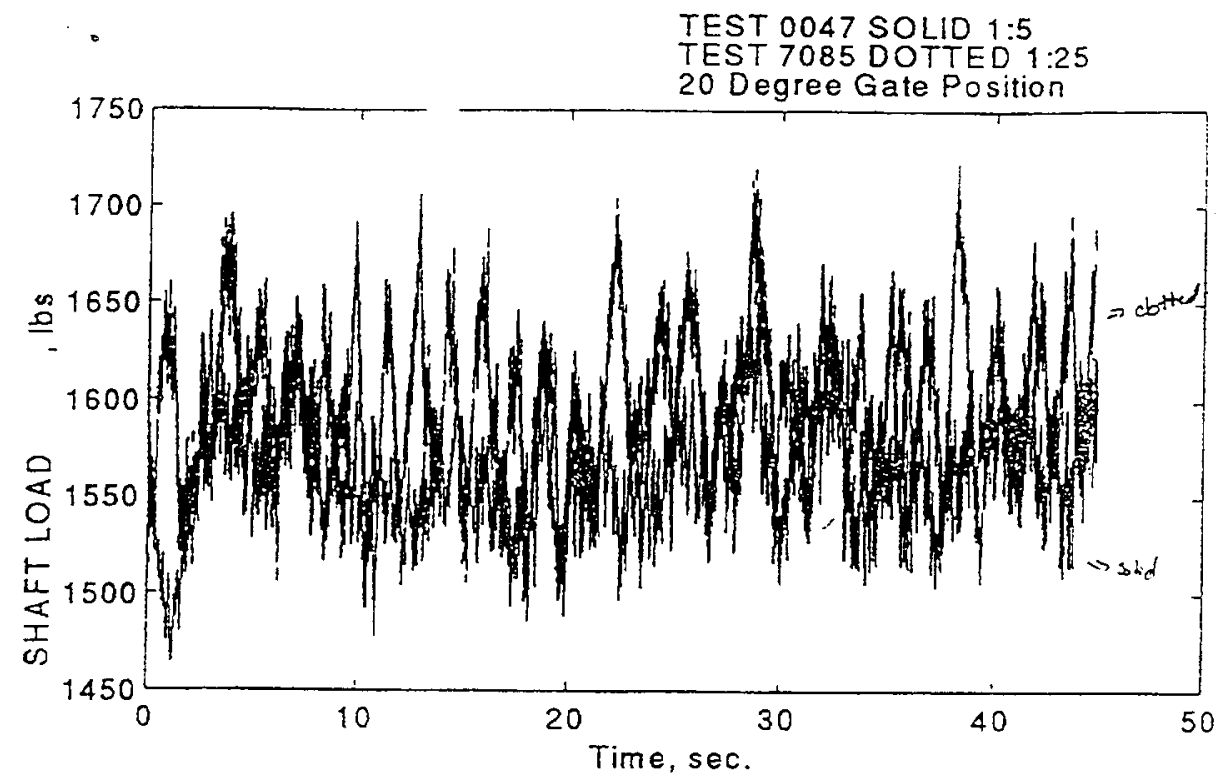

Time Domain Data Comparison for $1: 5$ and $1: 25$

Olmsted Flatgate Models.

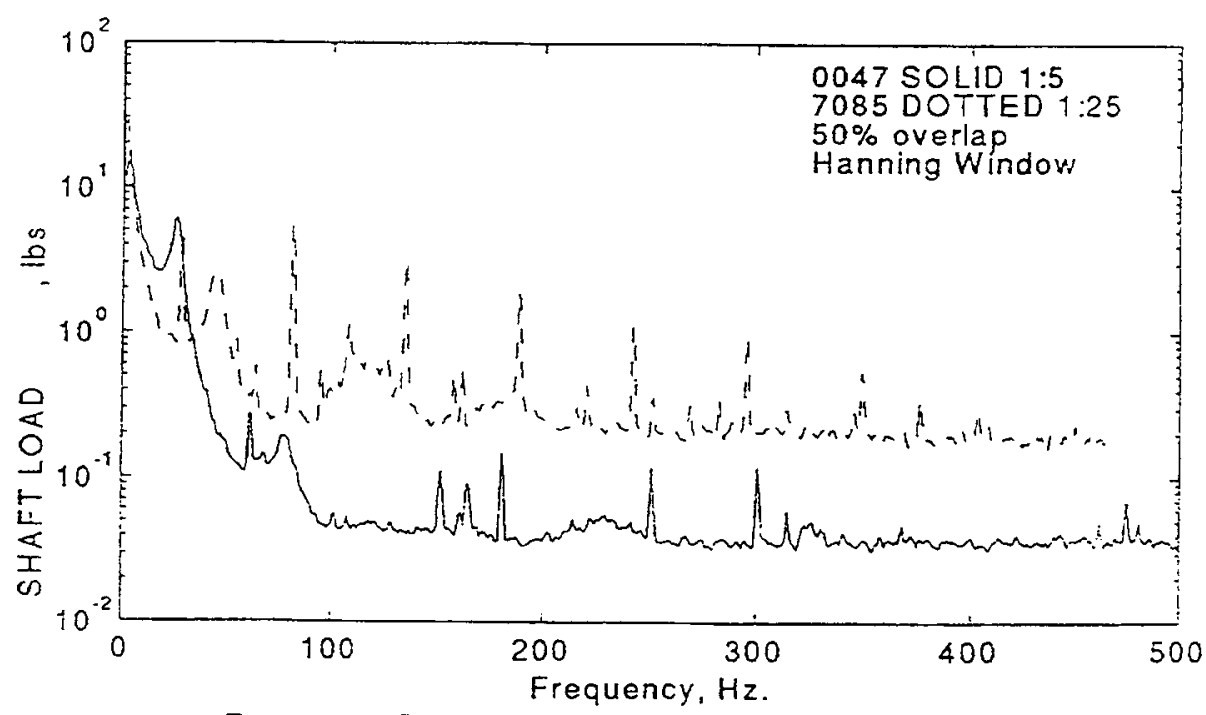

Frequency Domain Data Comparison for $1: 5$ and $1: 25$ Olmsted Flatgate Models.

a. Shaft load

Figure 77. Fixed gate response comparison for 1:25- and 1:5-scale models, 20-deg position, 11-ft head difference, and $3 G G$ configuration (Sheet 1 of 8 ) 


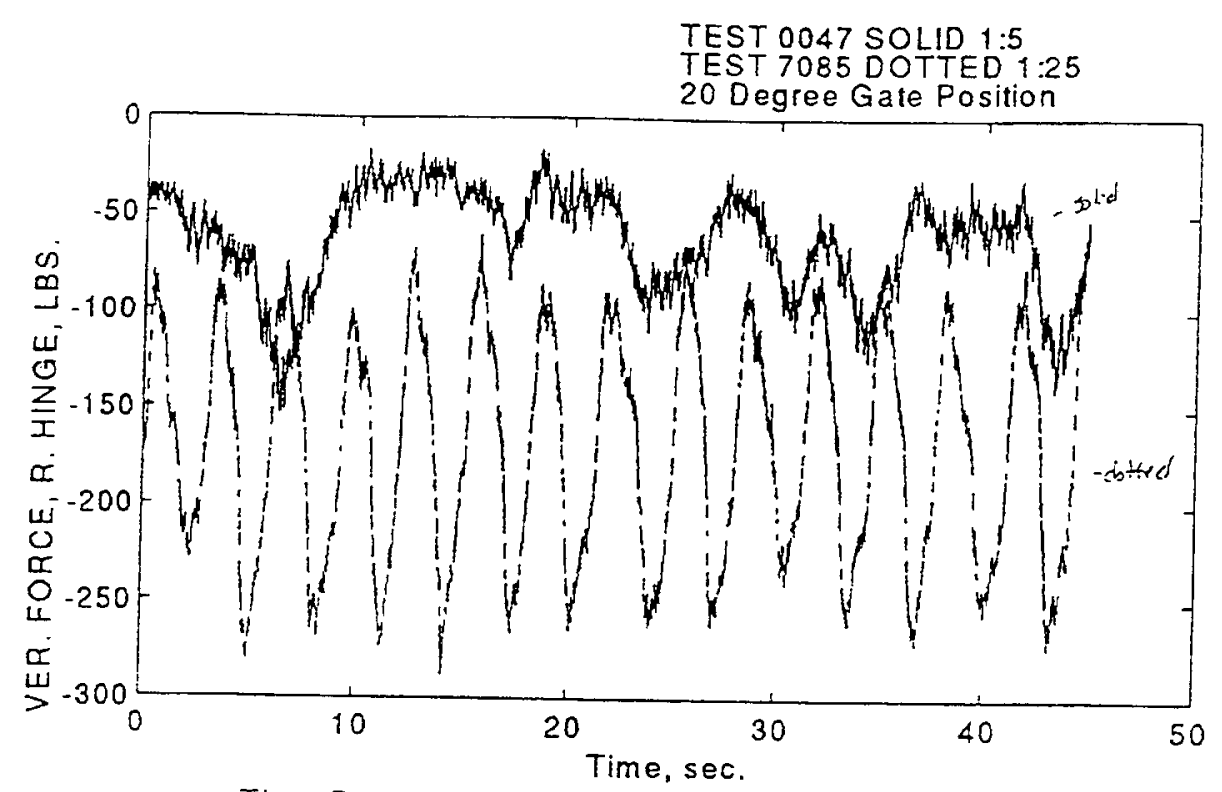

Time Domain Data Comparison for $1: 5$ and $1: 25$ Oimsted Flatgate Models.

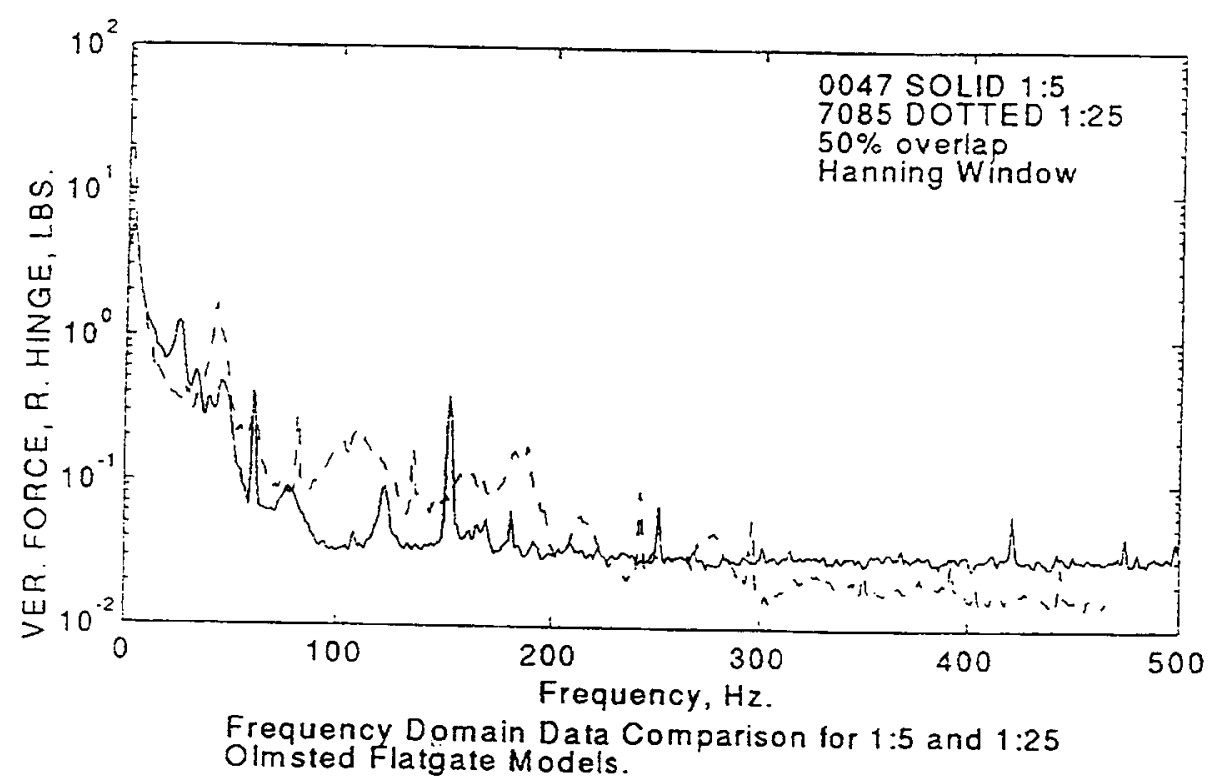

b. Right vertical hinge load

Figure 77. (Sheet 2 of 8 ) 
TEST 0047 SOLID $1: 5$

TEST 7085 DOTTED 1:25

20 Degree Gate Position

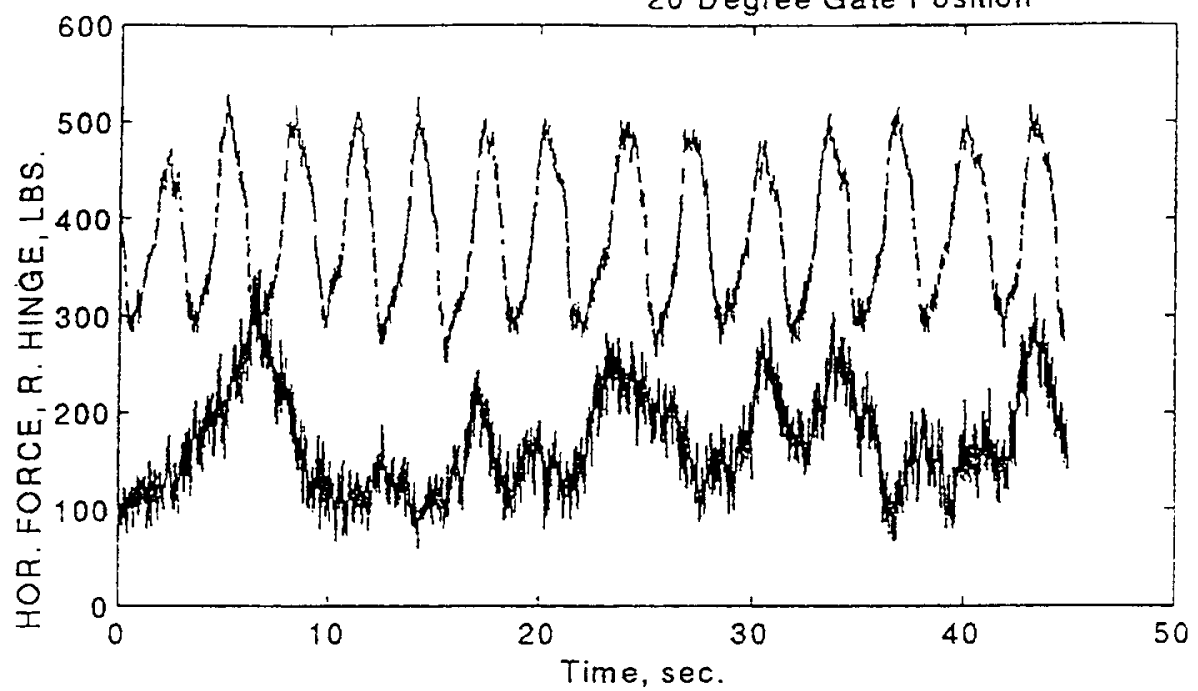

Time Domain Data Comparison for $1: 5$ and $1: 25$

Olmsted Flatgate Models.

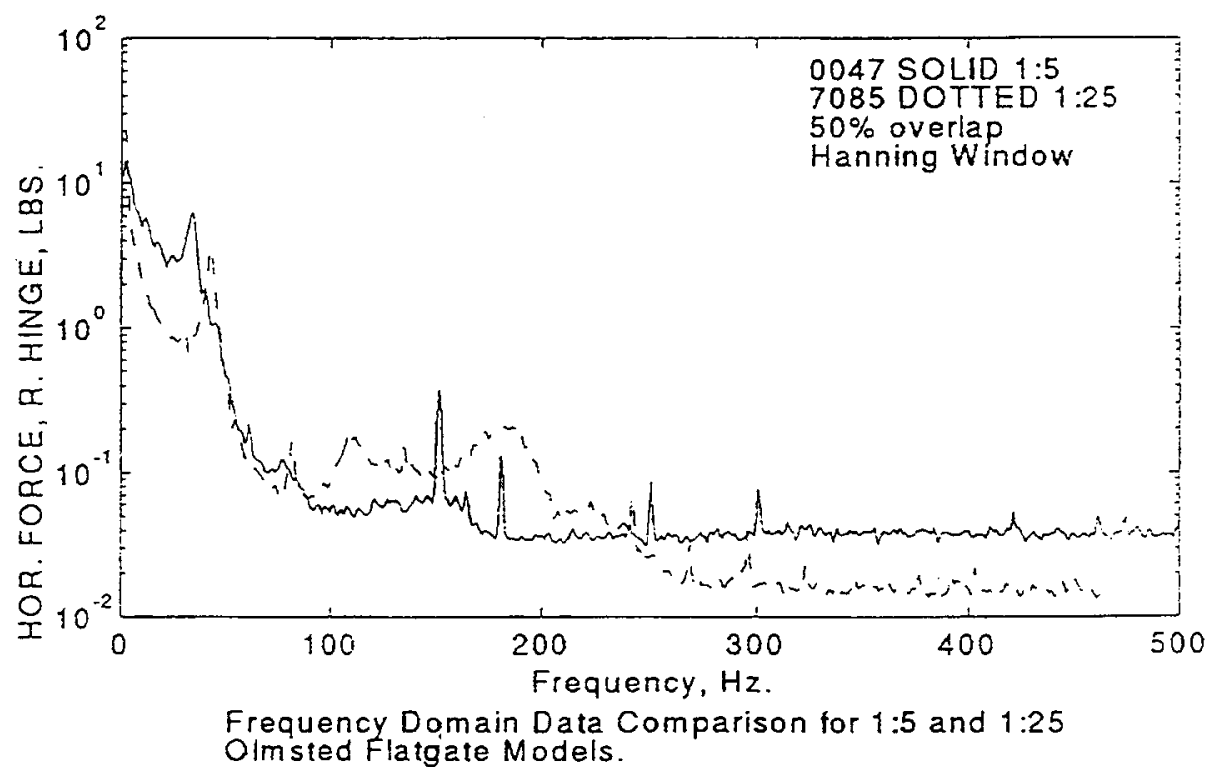

c. Right horizontal hinge load .

Figure 77. (Sheet 3 of 8 ) 


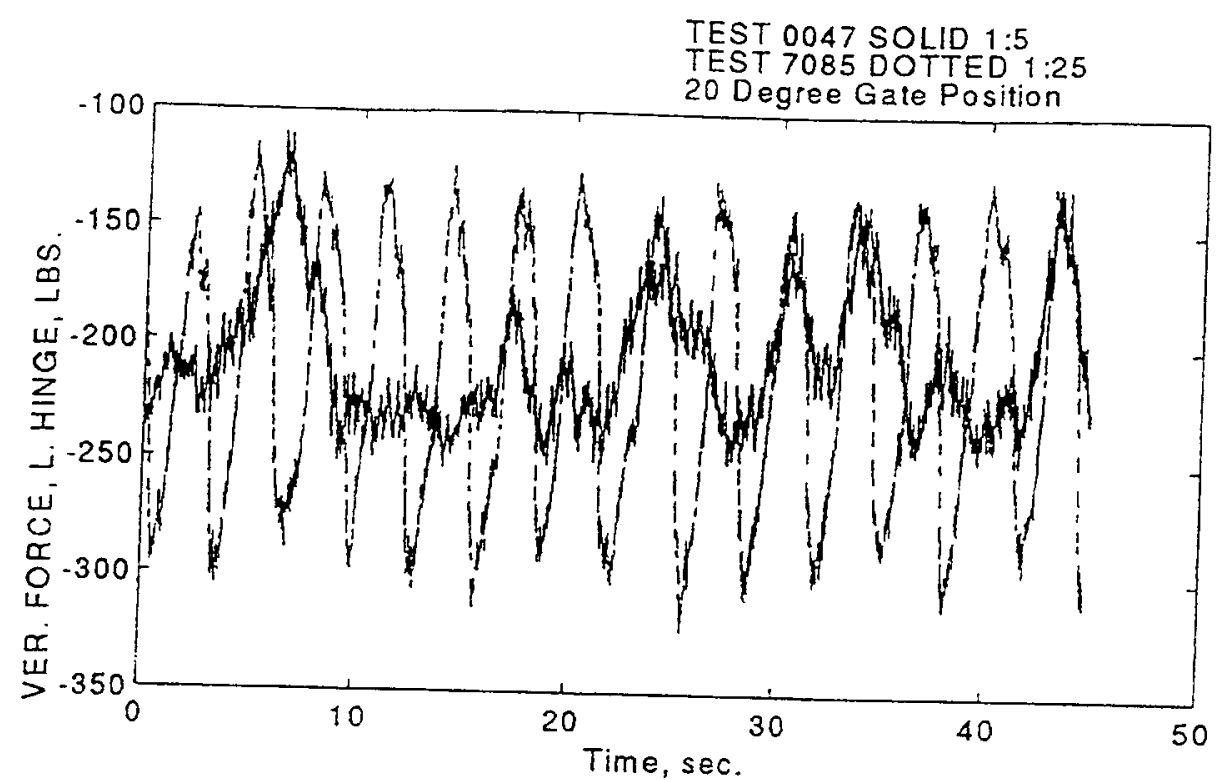

Time Domain Data Comparison for $1: 5$ and $1: 25$

Olmsted Flatgate Models.

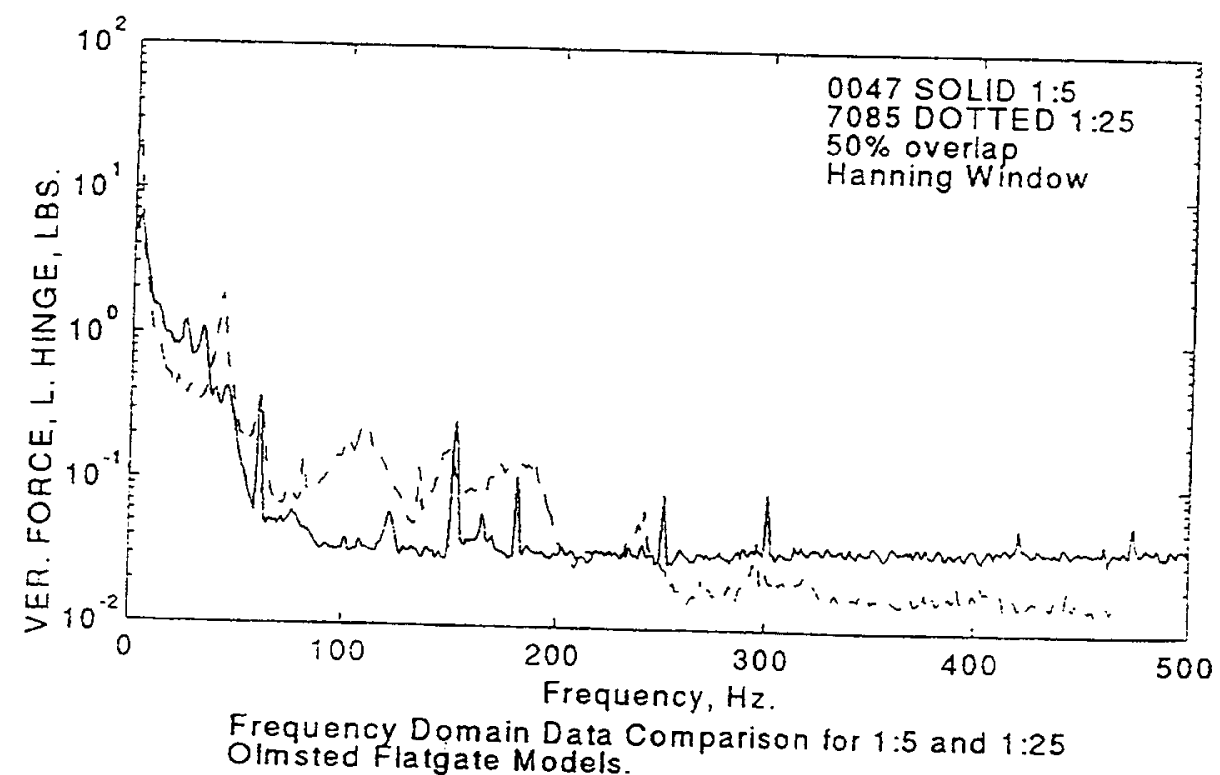

d. Left vertical hinge load

Figure 77. (Sheet 4 of 8 ) 


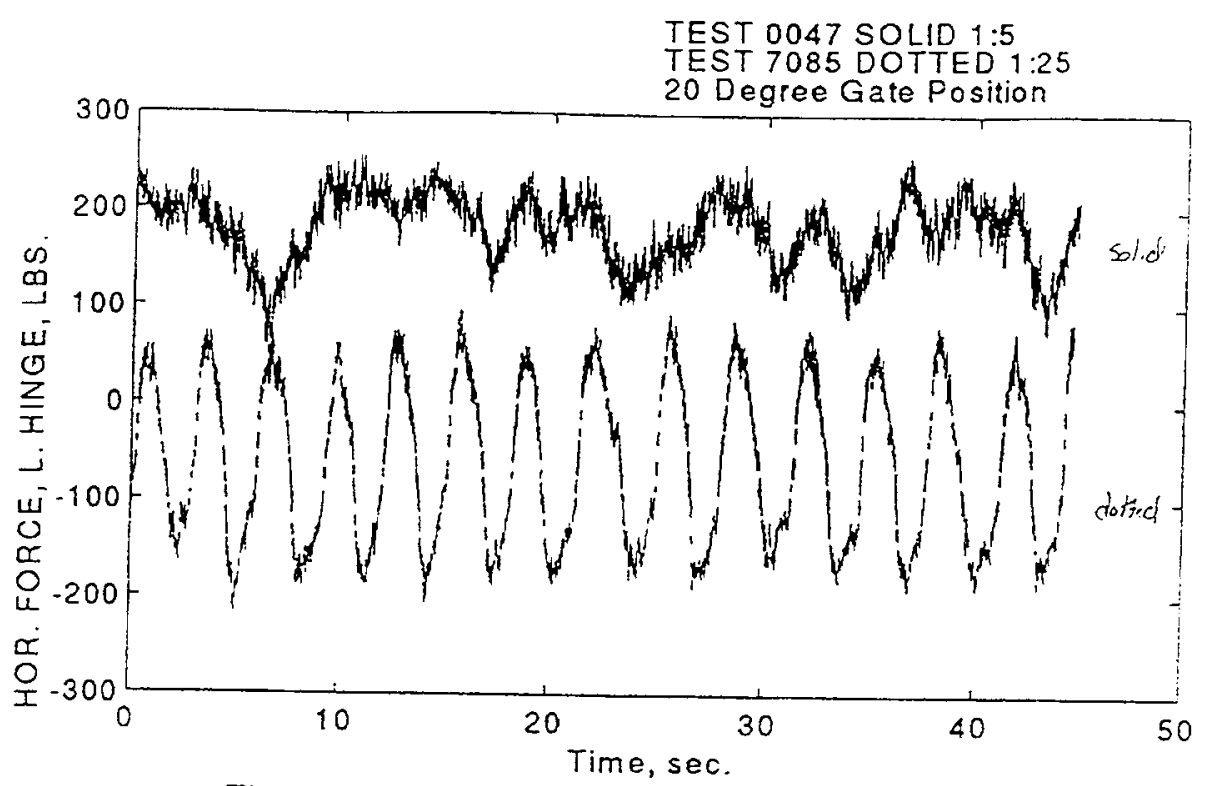

Time Domain Data Comparison for $1: 5$ and $1: 25$ Olmsted Flatgate Models.

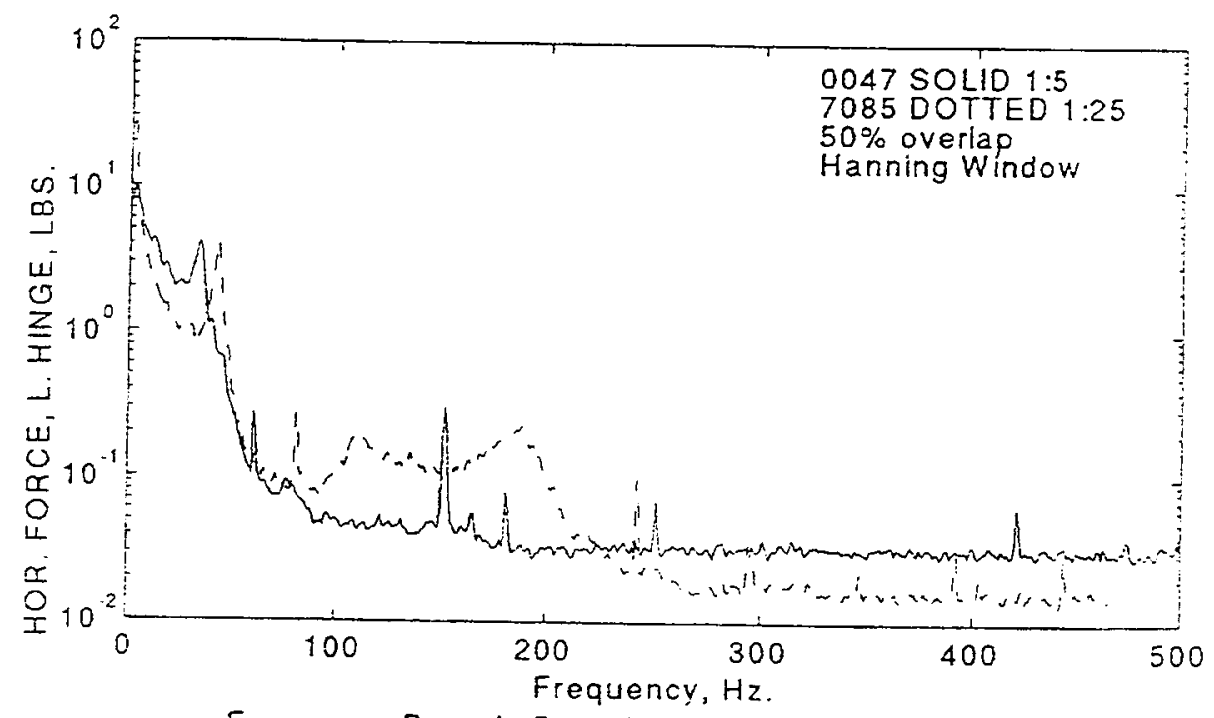

Frequency Domain Data Comparison for $1: 5$ and $1: 25$ Olmsted Flatgate Models.

e. Left horizontal hinge load

Figure 77. (Sheet 5 of 8) 


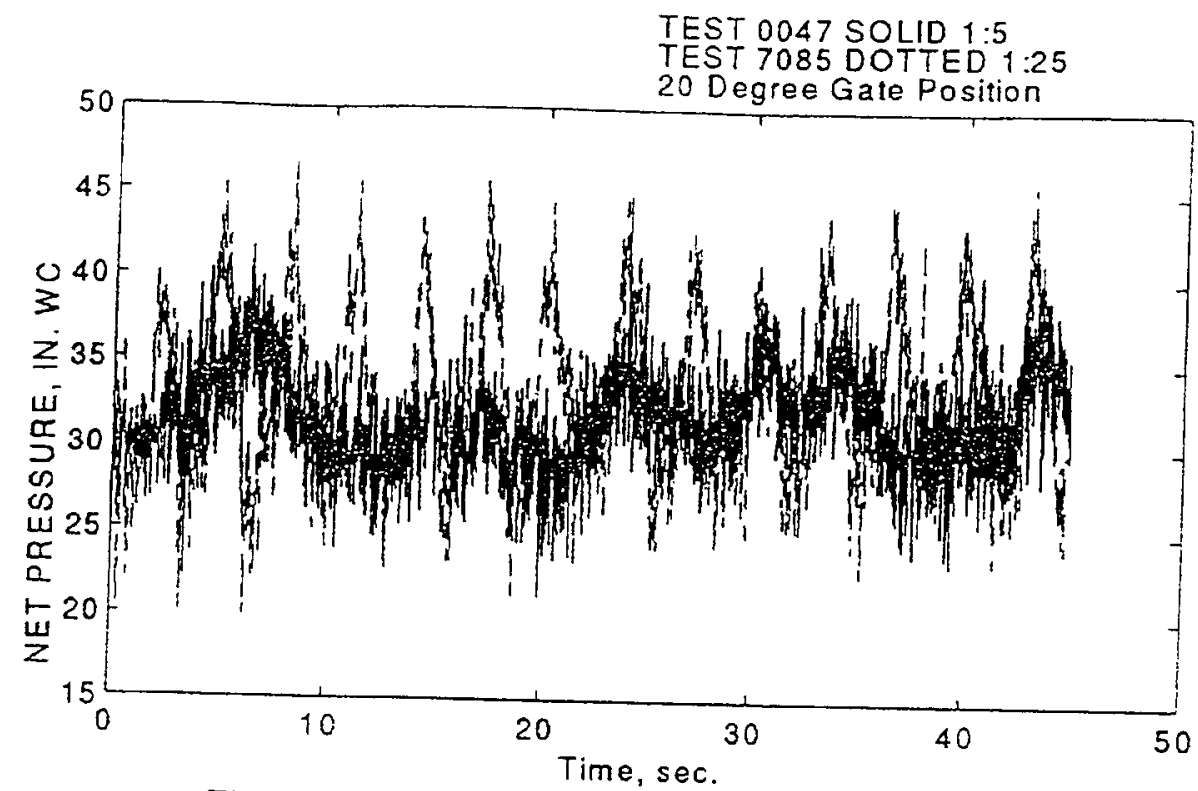

Time Domain Data Comparison for $1: 5$ and $1: 25$ OImsted Flatgate Models.

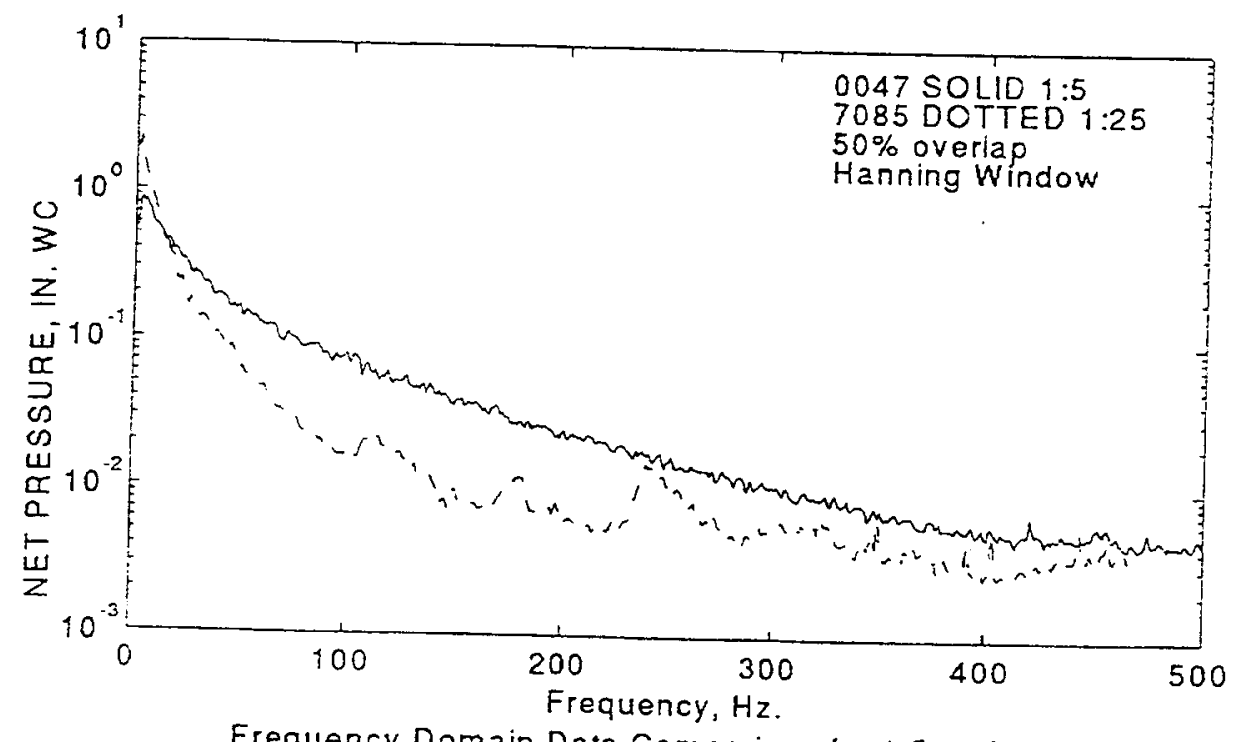

Frequency Domain Data Comparison for $1: 5$ and $1: 25$

f. Net pressure

Figure 77. (Sheet 6 of 8) 


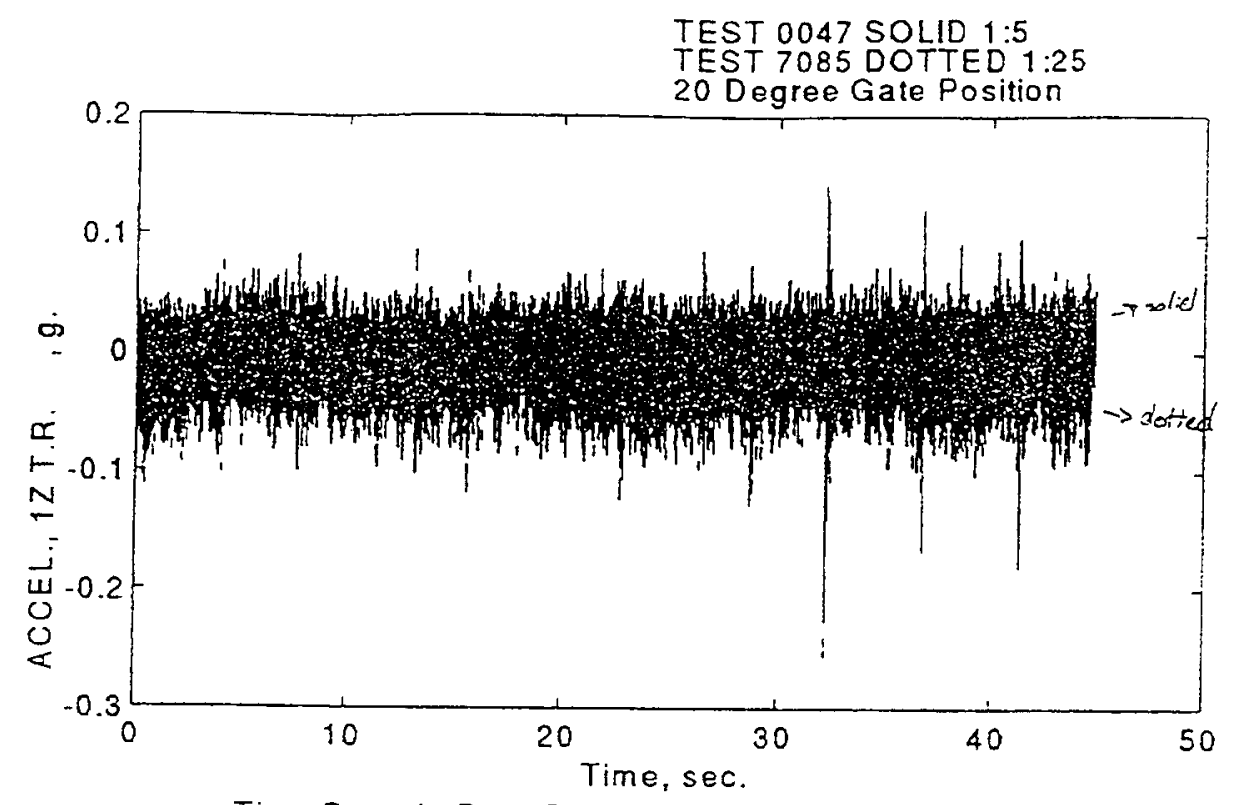

Time Domain Data Comparison for $1: 5$ and $1: 25$

Oimsted Flatgate Models.

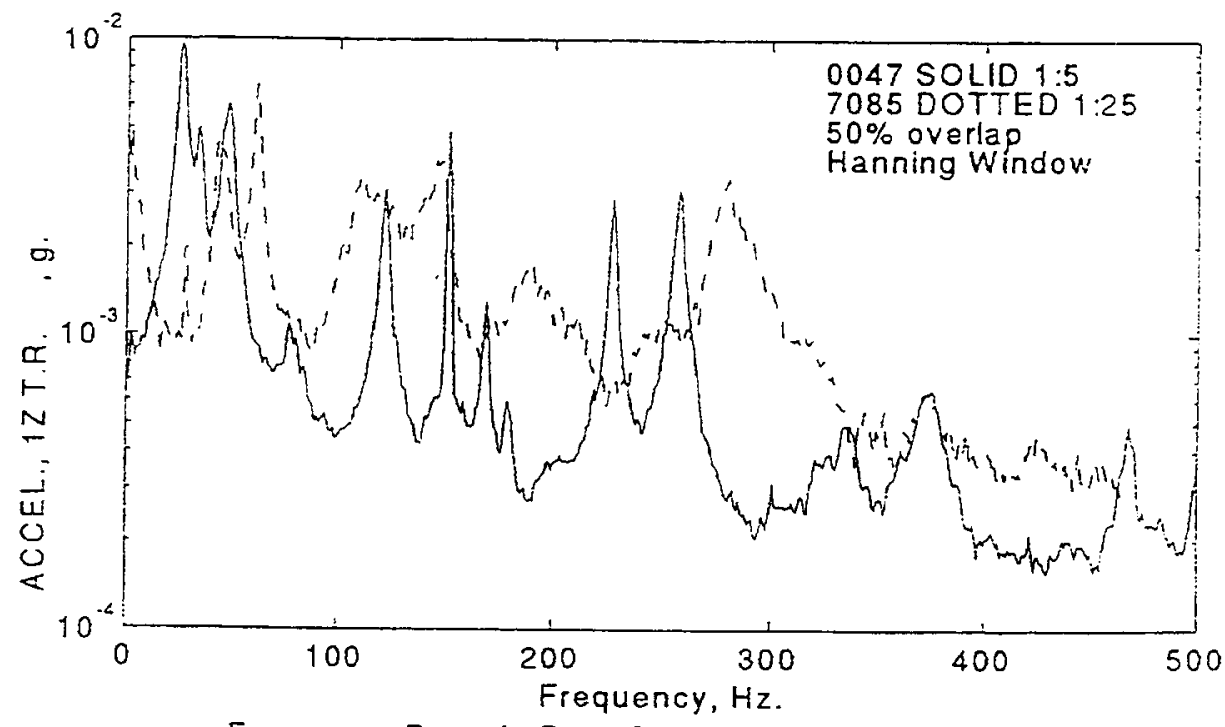

Frequency Domain Data Comparison for $1: 5$ and $1: 25$

Olmsted Flatgate Models.

g. Acceleration 12 (top right position on the gate)

Figure 77. (Sheet 7 of 8 ) 


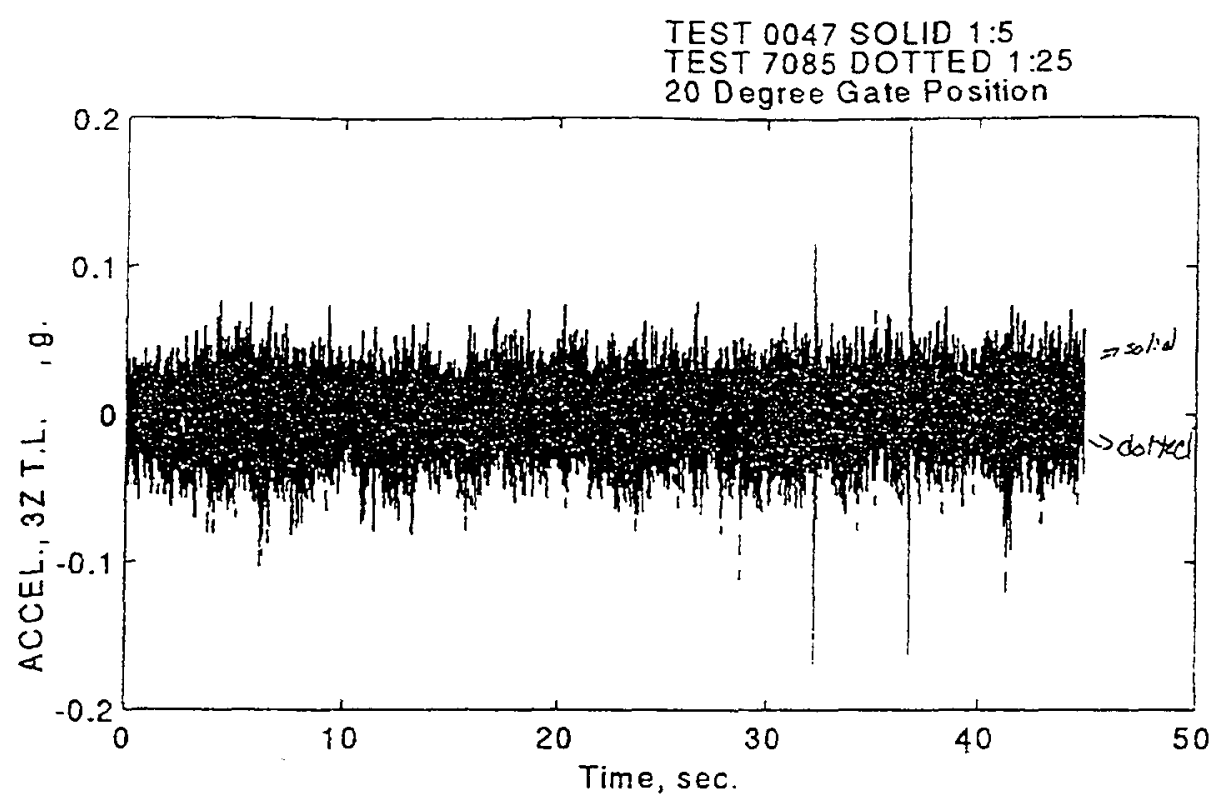

Time Domain Data Comparison for $1: 5$ and $1: 25$

Olmsted Flatgate Models.

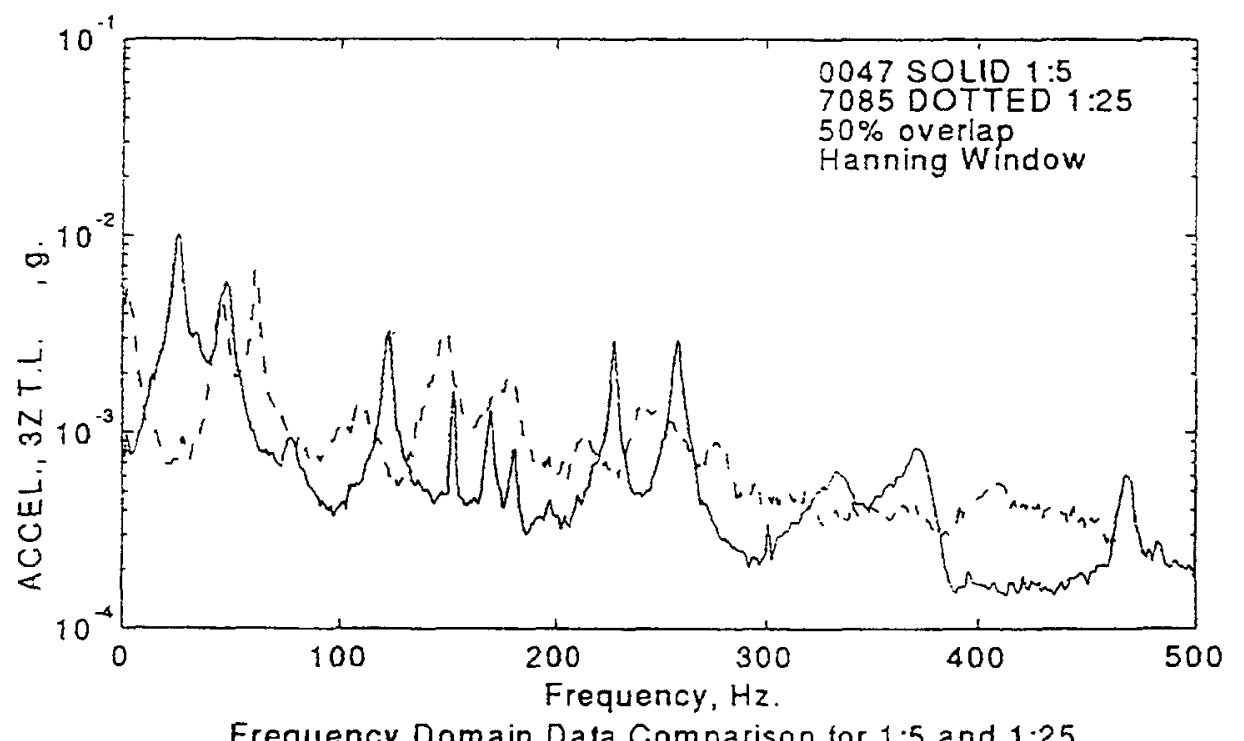

Frequency Domain Data Comparison for $1: 5$ and $1: 25$ Olmsted Flat'gate Models.

h. Acceleration $3 z$ (top left position on the gate)

Figure 77. (Sheet 8 of 8 )

upstream pressure. A comparison of responses for respective channels is presented in Table 14. The results that showed variations are printed in bold.

In general, the differences in the hinge reaction can be attributed to the mechanical distortion of the 1:25 model and the sensitivity of the measured low responses. Reduced sensitivity in reading the low magnitude accelerometer 


\begin{tabular}{|c|c|c|c|c|c|c|}
\hline \multicolumn{7}{|c|}{$\begin{array}{l}\text { Table } 14 \\
\text { Results for 1:25- and 1:5-Scale Flat Gate Models at 60-deg Position } \\
\text { (11-ft Head Difference, Three-Gate Gap Configuration) }\end{array}$} \\
\hline \multirow[b]{2}{*}{ Gauge Type } & \multicolumn{2}{|c|}{$\begin{array}{c}1: 5-S c a l e \\
\text { (Experiment No. 50) }\end{array}$} & \multicolumn{2}{|c|}{$\begin{array}{c}1: 25-S c a l e \\
\text { (Experiment No. 7088) }\end{array}$} & \multicolumn{2}{|c|}{ Ratio } \\
\hline & $\begin{array}{c}\text { Avg. } \\
\text { Reading } \\
\end{array}$ & $\begin{array}{c}\text { Std. } \\
\text { Deviation } \\
\end{array}$ & $\begin{array}{c}\text { Avg. } \\
\text { Reading } \\
\end{array}$ & $\begin{array}{c}\text { Std. } \\
\text { Deviation } \\
\end{array}$ & $\frac{(\bar{\mu}+\sigma)_{t \neq 50}}{(\bar{\mu}+\sigma)_{t+2088}}$ & $\frac{(\bar{\mu}+2 \sigma)_{\tau+50}}{(\bar{\mu}+2 \sigma)_{t+208}}$ \\
\hline Shaft Load, Ibf & 831.37 & 6.50 & 765.50 & 8.61 & 1.08 & 1.08 \\
\hline $\begin{array}{l}\text { Net Vertical } \\
\text { Force at Hinges, } \\
\text { Ibf }\end{array}$ & 253.76 & 6.41 & 534.37 & 2.99 & 0.48 & 0.49 \\
\hline $\begin{array}{l}\text { Net Horizontal } \\
\text { Force at Hinges, } \\
\text { lbf }\end{array}$ & 289.22 & 4.71 & 189.25 & 2.26 & 1.53 & 1.54 \\
\hline $\begin{array}{l}\text { Upstream } \\
\text { Pressure, in. of } \\
\text { water }\end{array}$ & -49.23 & 0.374 & -49.94 & 0.47 & 0.98 & 0.98 \\
\hline $\begin{array}{l}\text { Downstream } \\
\text { Pressure, in. of } \\
\text { water (ambient) }\end{array}$ & -63.70 & 1.43 & -54.27 & 1.26 & 1.17 & 1.17 \\
\hline Accel. T.R., g's & 0.017 & 0.035 & -0.012 & 0.0298 & 1.24 & 1.21 \\
\hline Accel. T.L., g's & 0.016 & 0.041 & 0 & 0.0318 & 1.79 & 1.54 \\
\hline Accel. M.L., g's & -0.009 & 0.018 & 0 & 0.129 & 0.209 & 0.174 \\
\hline
\end{tabular}

responses made it difficult to measure the correlation of the responses obtained from the two models. A reflection of the noisy ambient background during the tests could easily supersede the low accelerations caused by the flowing field. Therefore, the variation in the acceleration responses is not an ideal representation of the actual conditions. The recorded response (acceleration), however, showed that the 1:25-scale model could not predict the 1:5-scale model response quite accurately. A difference in elastic behavior could contribute to such error in gate responses.

Additionally, the limitations imposed by the 1:25-scale model, particularly for the hinge reaction forces and the fluctuation of navigation pool levels during data collection, also contributed to such a deviation in the results. These comparisons, however, indicate that despite crude geometric modeling using hydraulic similitude relations, the 1:25-scale model was capable of predicting the pressure envelope and the higher-order shaft load for the 1:5-scale pseudo-elastic model. The result also suggests that the higher the magnitude the better the correlation. 


\section{Parametric Study}

A systematic investigation of the gate responses for varied system parameters was performed to determine the importance of such parameters and the necessity of including them in a valid similitude model. As discussed earlier, an apparent distortion in reproducing the elastic properties of the prototype existed in the model due to not to meeting the Froude law criteria of $\lambda_{E}=\lambda_{\rho} \lambda_{L}$. A hybrid model, using a magnesium prop rod, was employed to understand the overall effects of such modeling distortion and to quantify the deviation encountered by not fulfilling this criteria. Adjustments of controlling parameters, such as oil column and stroke length for the hydraulic cylinder, enabled the model to simulate prototype frequency for different gate positions. The differences in the gate responses due to variation of modeling parameters are discussed below.

\section{Effects of prop-rod stiffness (Experiments No. 105 and No. 116)}

A comparison of measured responses for the steel and magnesium prop-rod supported gate are shown in Figure 78. For both cases, the pool difference was maintained at $11 \mathrm{ft}$ with a three-gate gap configuration and the gate was the middle gate. The data were recorded for $45 \mathrm{sec}$ with a low-pass filter at $500 \mathrm{~Hz}$. The time and PSD for selected sensors are compared in Figure 78.

There is a clear difference in the time-domain responses for the gate with steel and magnesium prop rods, but the difference is negligible for all practical purposes. The time and PSD comparison plots show that the z-reactions for bottom binges, prop-rod force, and upstream and downstream pressures for both cases agreed reasonably well. Although the PSDs for the $y$-reactions for bottom hinges were identical for both cases, the time-domain responses possessed different magnitudes. This is attributed to a biased error induced due to the very low magnitudes of the measured responses. PSDs for the gate accelerometer response show that the peaks for both cases slightly deviated from each other while maintaining an identical pattern in the overall distribution of the dominant operating modes (peaks). As identified in the previously discussed ODS analysis, the modal participation for the first four to five peaks are dominant in the gate vibration. The accelerometer PSDs also show that, except for mode two (torsion at about $68 \mathrm{~Hz}$ ), all steel rod modes appeared at higher frequencies than those of the magnesium rod. This variation is a measure of the sensitivity of the prop-rod elastic modulus in the global dynamic behavior of the gate. The comparison of the results suggests that though there are differences in magnitudes, a change in prop material for the flow-field under consideration did not substantially change the dynamics of the gate. An examination of the proprod PSD, however, showed that the operating modes for the magnesium rod had substantially changed in the z-direction. 

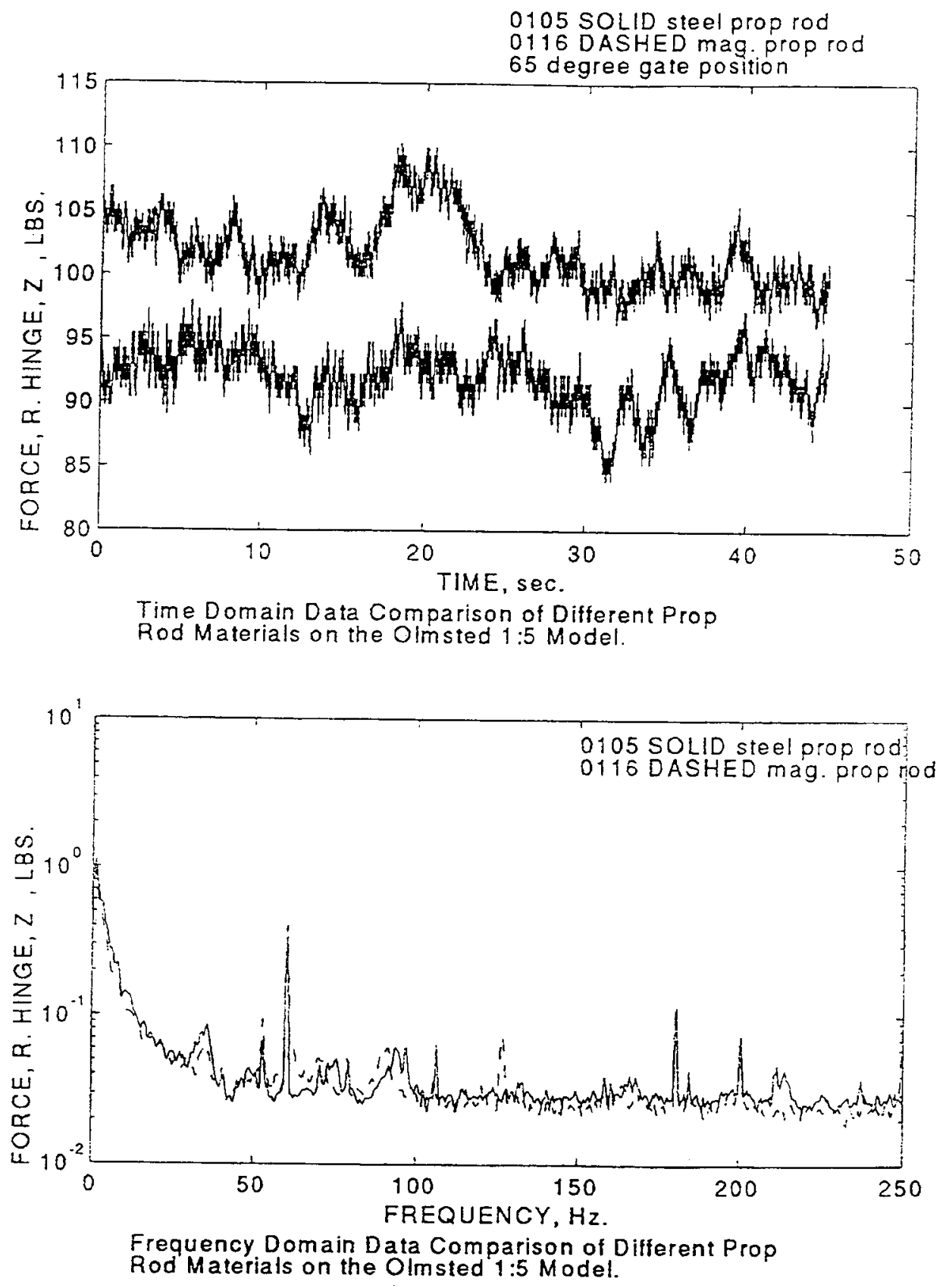

a. Right vertical hinge load

Figure 78. Fixed gate response comparison for the steel and magnesium propsupported gate, 11-ft head difference, 3GG configuration (Sheet 1 of 14) 


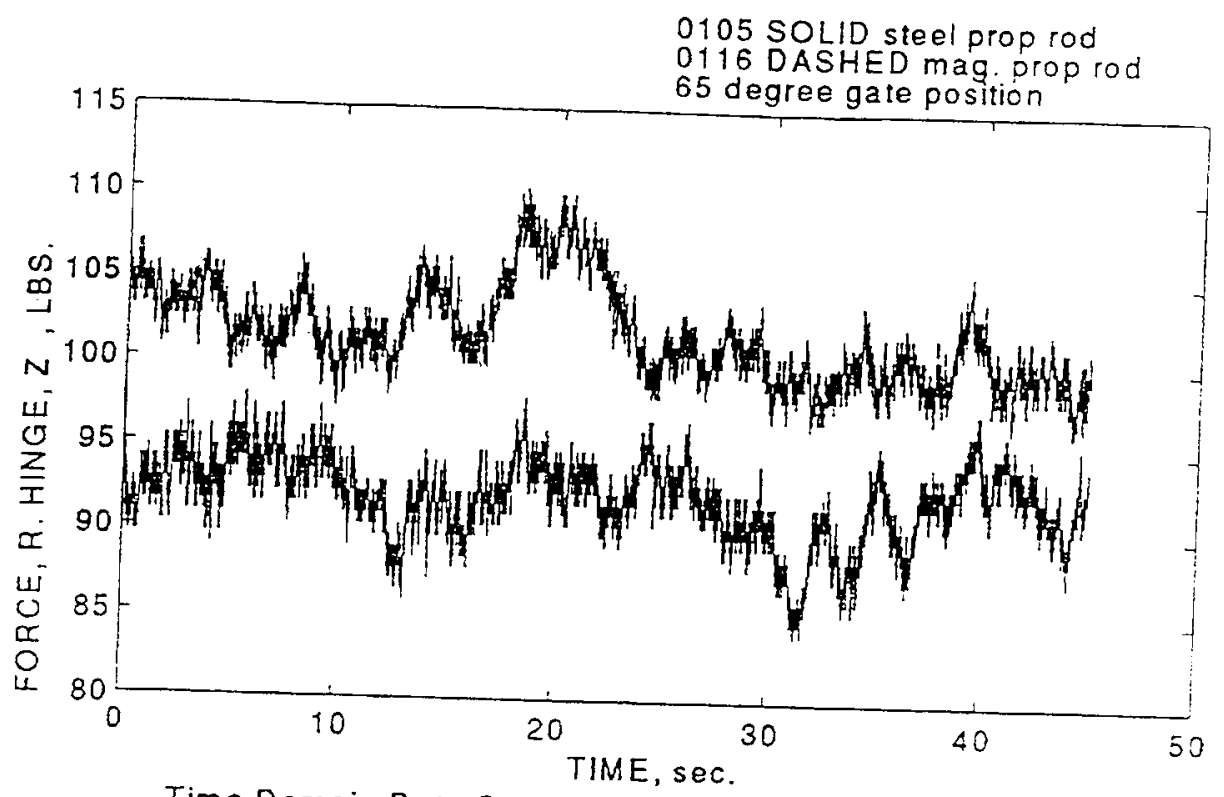

Time Domain Data Comparison of Different Prop

Rod Materials on the Olmsted 1:5 Model.

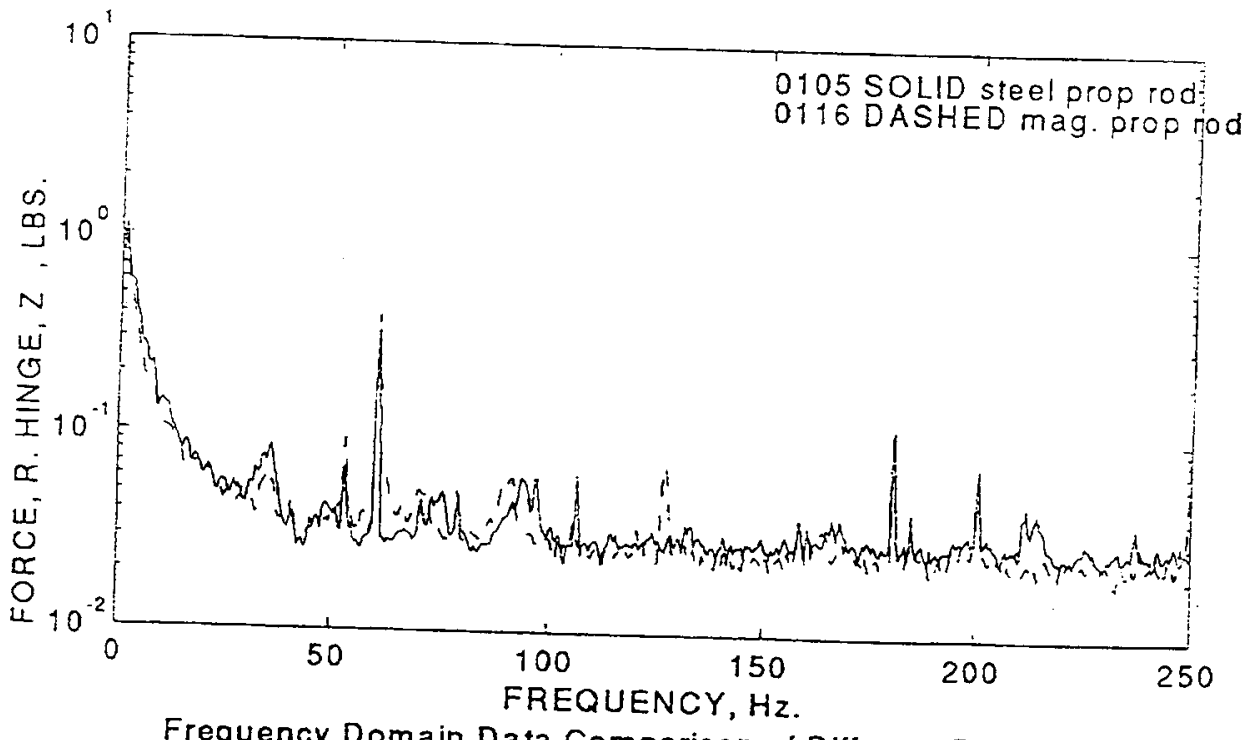

Frequency Domain Data Comparison of Different Prop
Rod Materials on the Oimsted $1: 5 \mathrm{M}$ odel.

b. Right horizontal hinge load

Figure 78. (Sheet 2 of 14) 


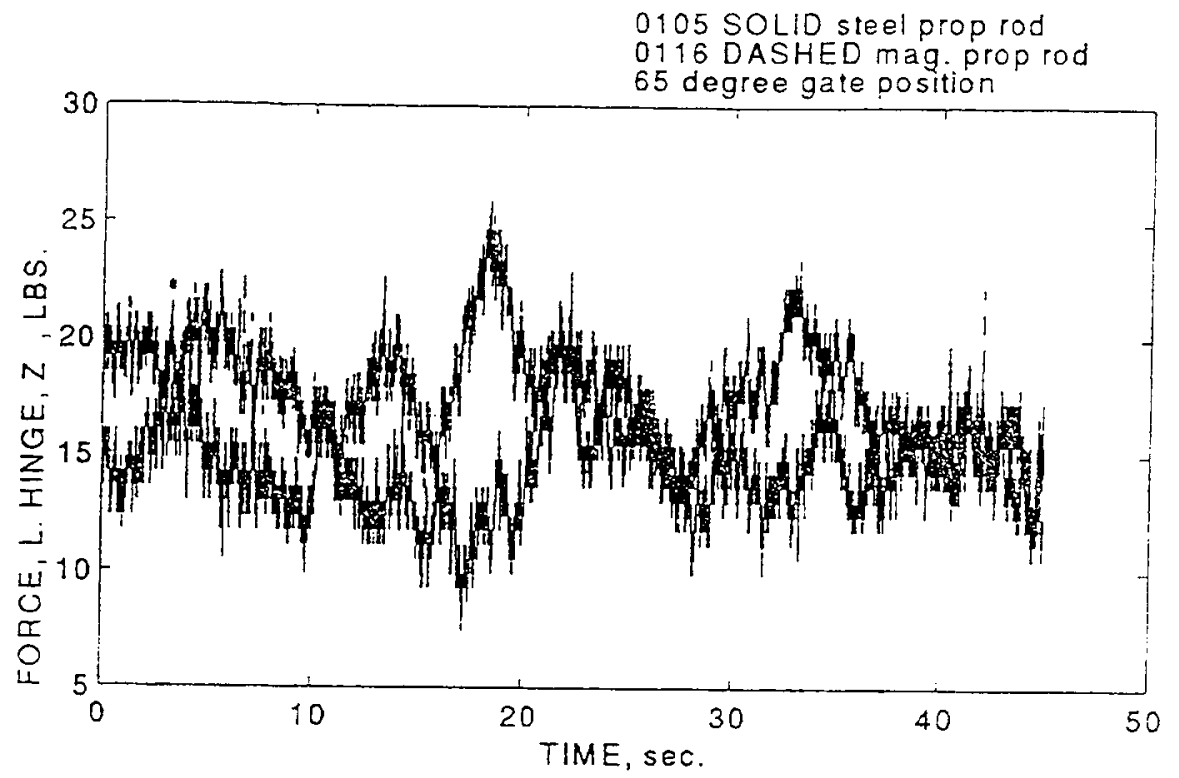

Time Domain Data Comparison of Different Prop

Rod Materials on the Olmsted 1:5 Model.

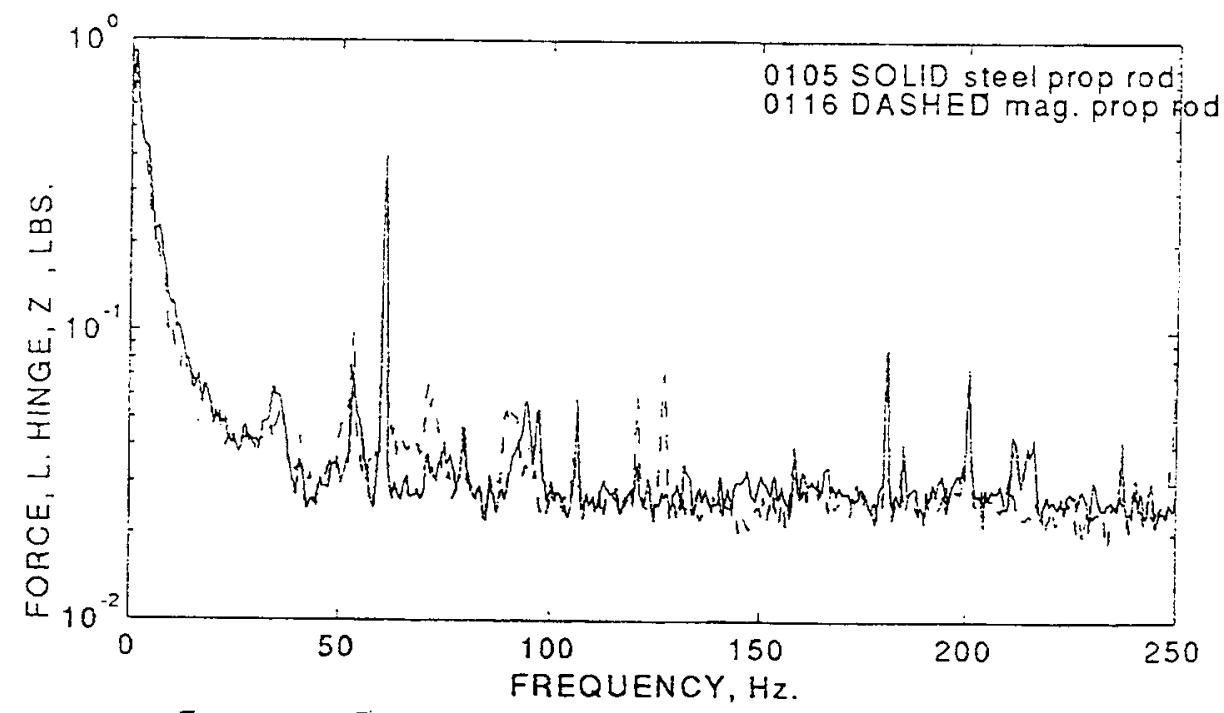

Frequency Domain Data Comparison of Different Prop Rod Materials on the Olmsted $1: 5 \mathrm{Model}$.

\section{c. Left vertical hinge load}

Figure 78. (Sheet 3 of 14) 


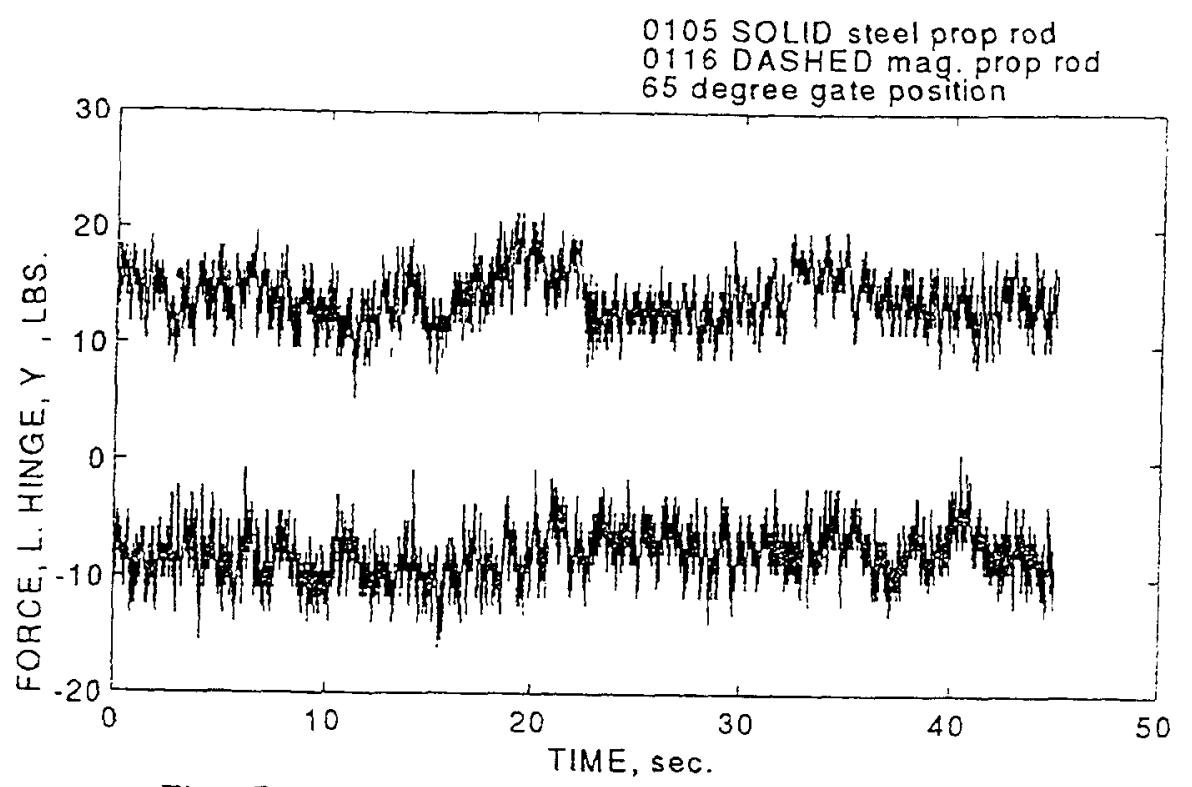

Time Domain Data Comparison of Different Prop

Rod Materials on the Oimsted 1:5 Model.

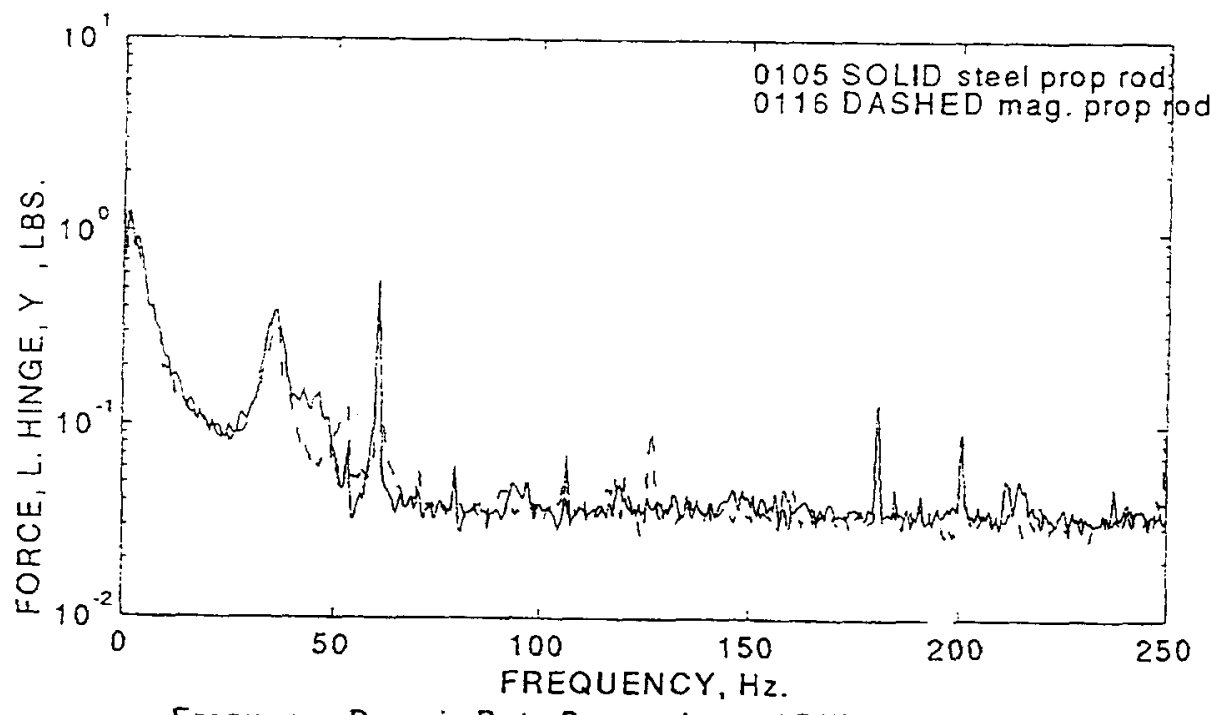

Frequency Domain Data Comparison of Different Prop

Rod Materials on the Olmsted 1:5 Model.

d. Left horizontal hinge load

Figure 78. (Sheet 4 of 14) 


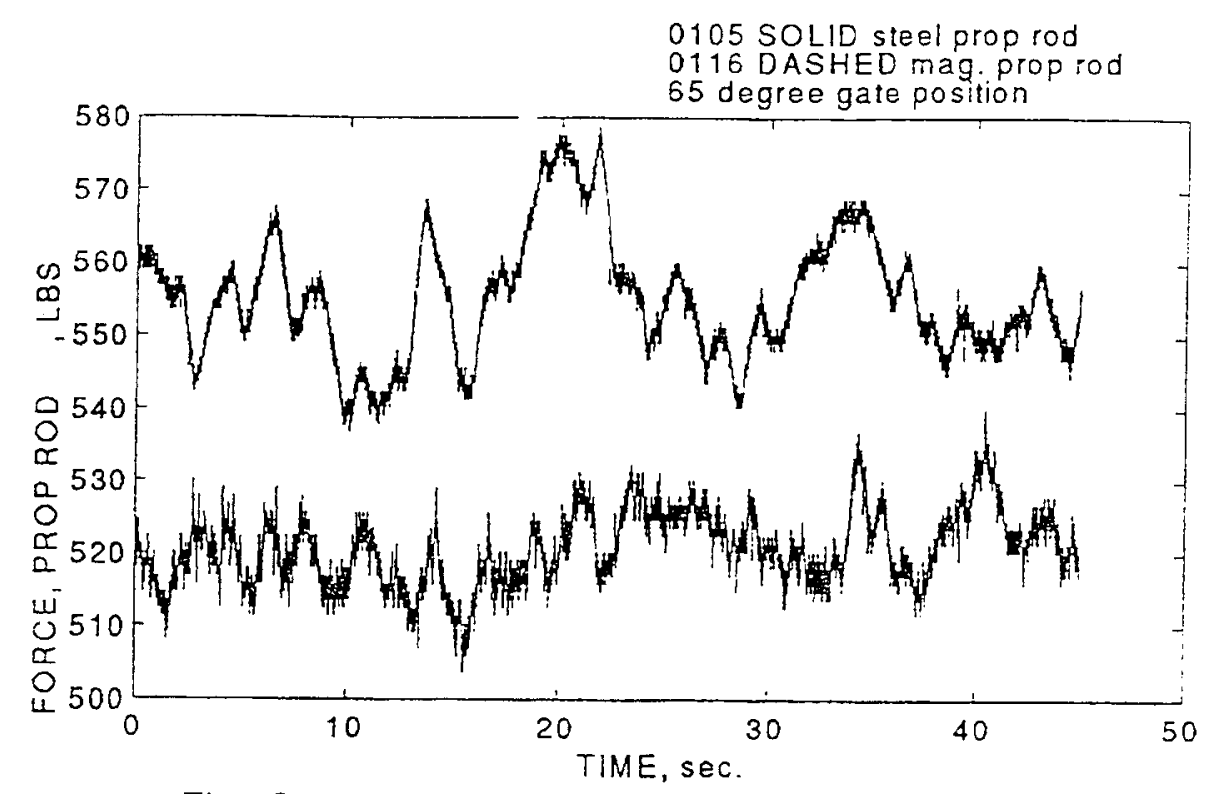

Time Domain Data Comparison of Different Prop

Rod Materials on the Oimsted 1:5 Model.

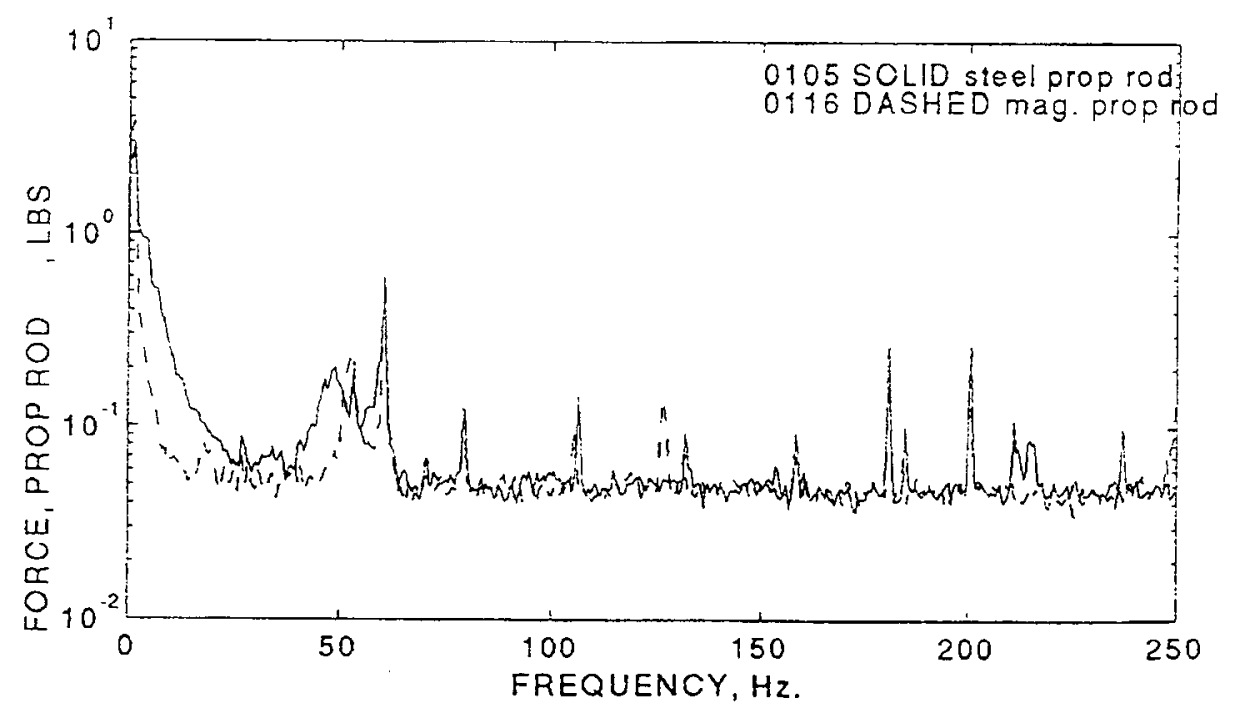

Frequency Domain Data Comparison of Different Prop Rod Materials on the Olmsted 1:5 Model.

\section{e. Prop load}

Figure 78. (Sheet 5 of 14) 


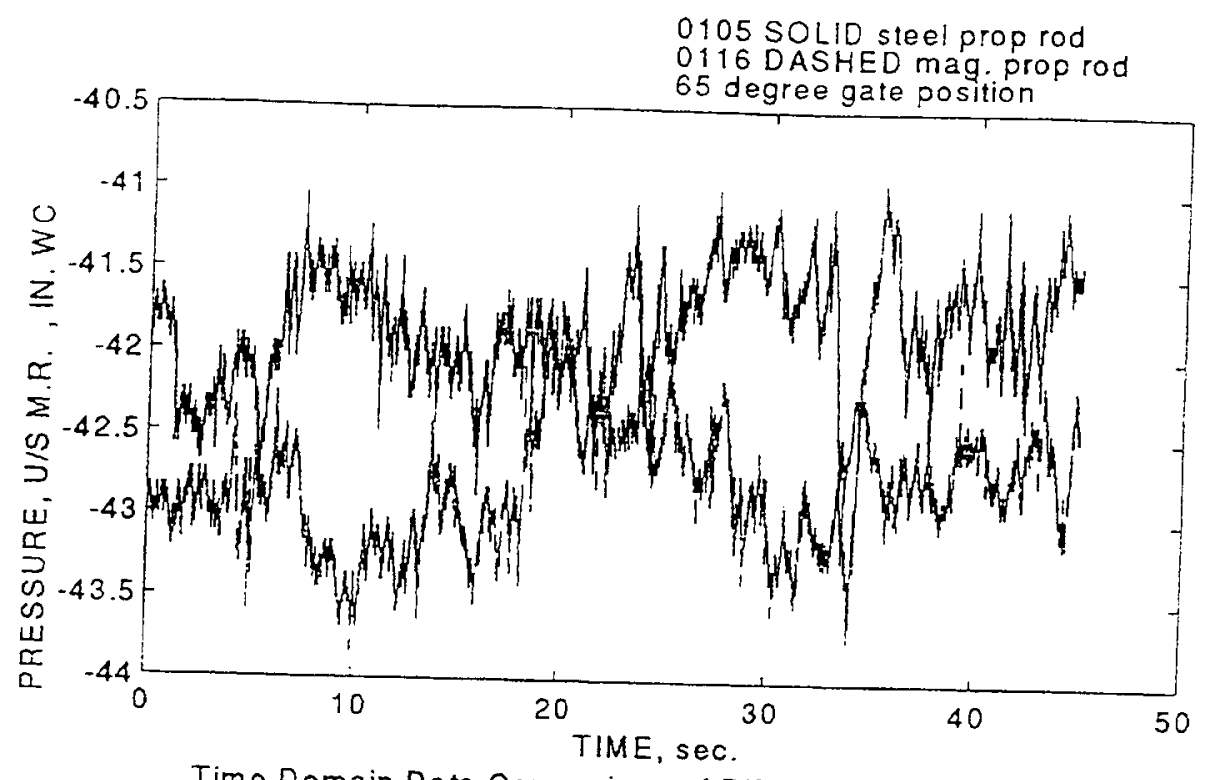

Time Domain Data Comparison of Different Prop

Rod Materials on the Olmsted 1:5 Model.

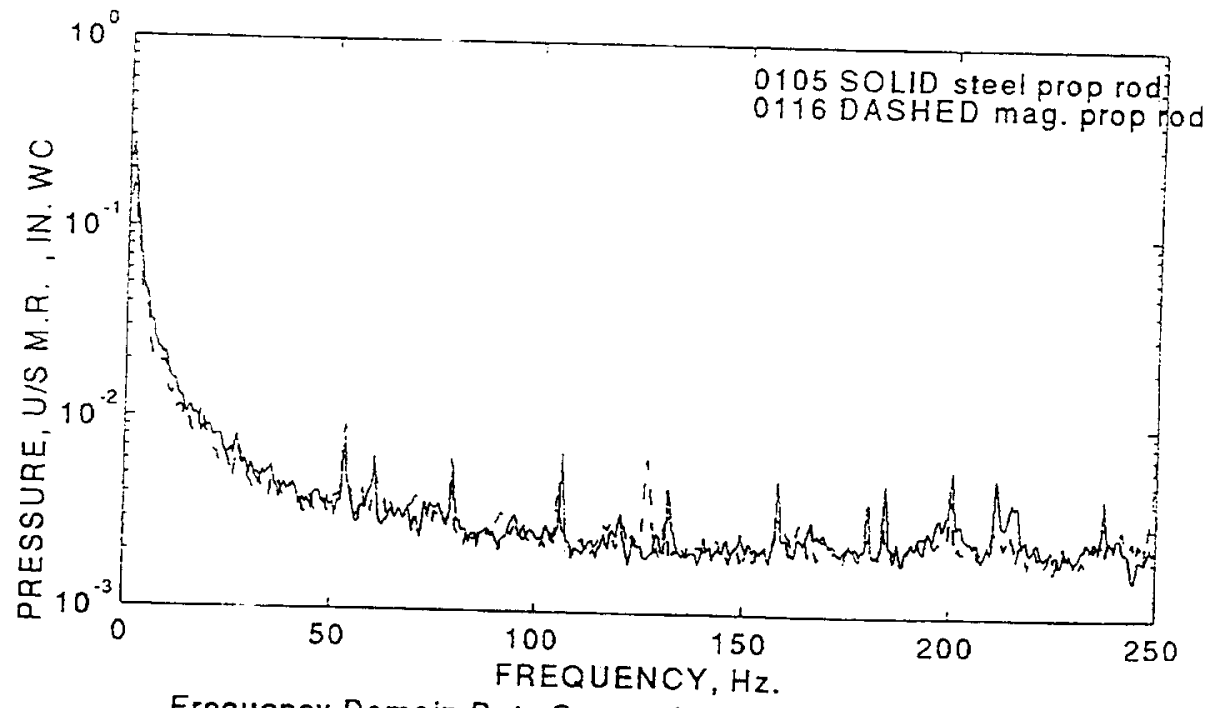

Frequency Domain Data Comparison of Different Prop

Rod Materials on the OImsted 1:5 Model.

\section{f. Upstream pressure, middle right position on gate}

Figure 78. (Sheet 6 of 14) 


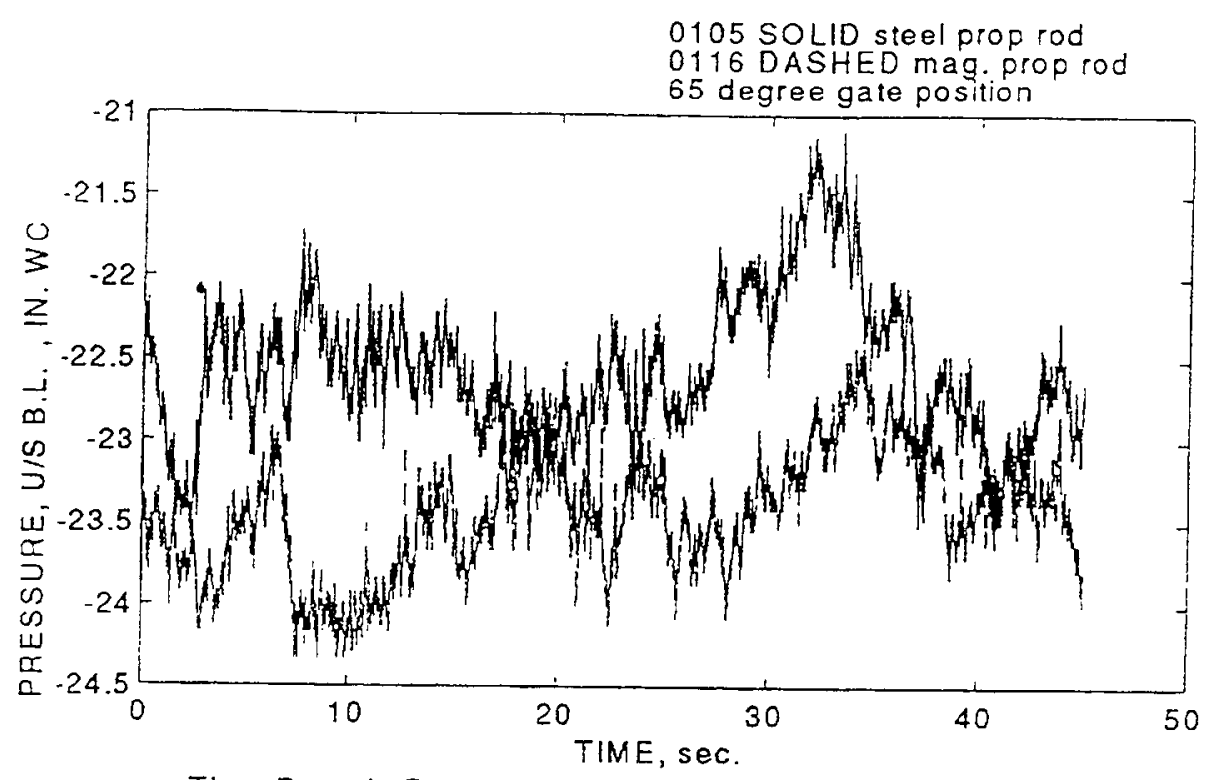

Tir : Domain Data Comparison of Different Prop

Rod Materials on the Olmsted 1:5 Model.

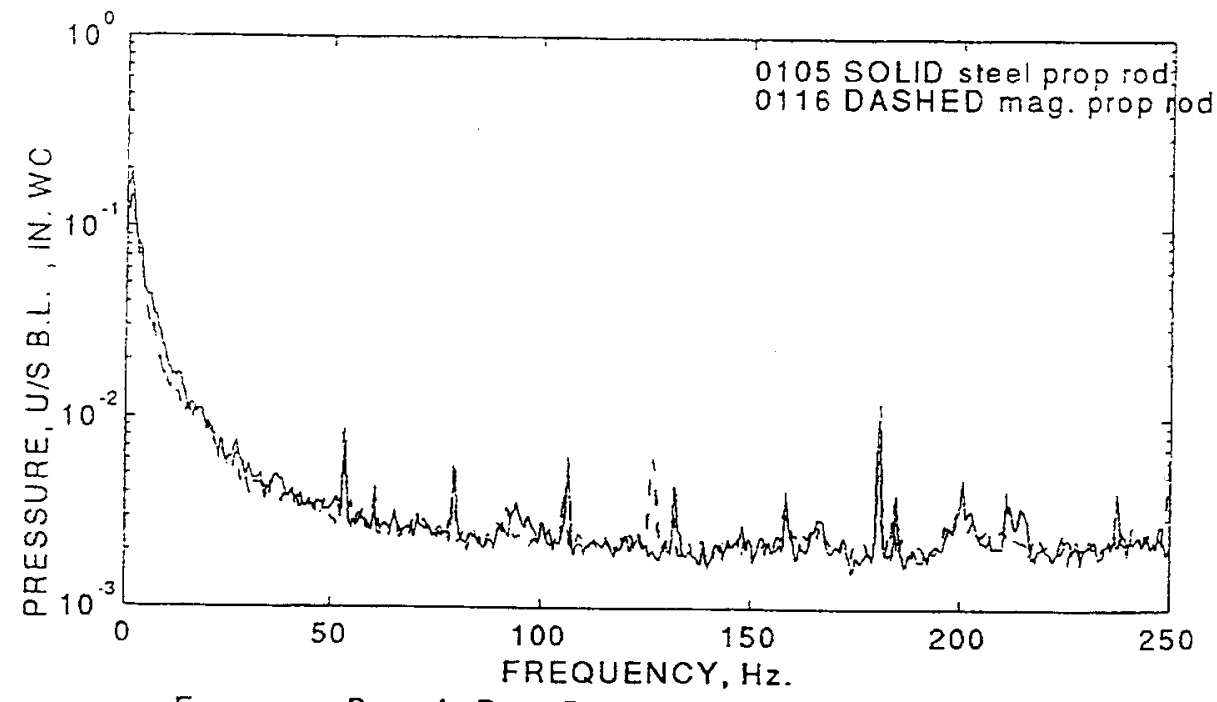

Frequency Domain Data Comparison of Different Prop Rod Materials on the Olmsted $f: 5$ Model.

\section{g. Upstream pressure, bottom left position on gate}

Figure 78. (Sheet 7 of 14) 


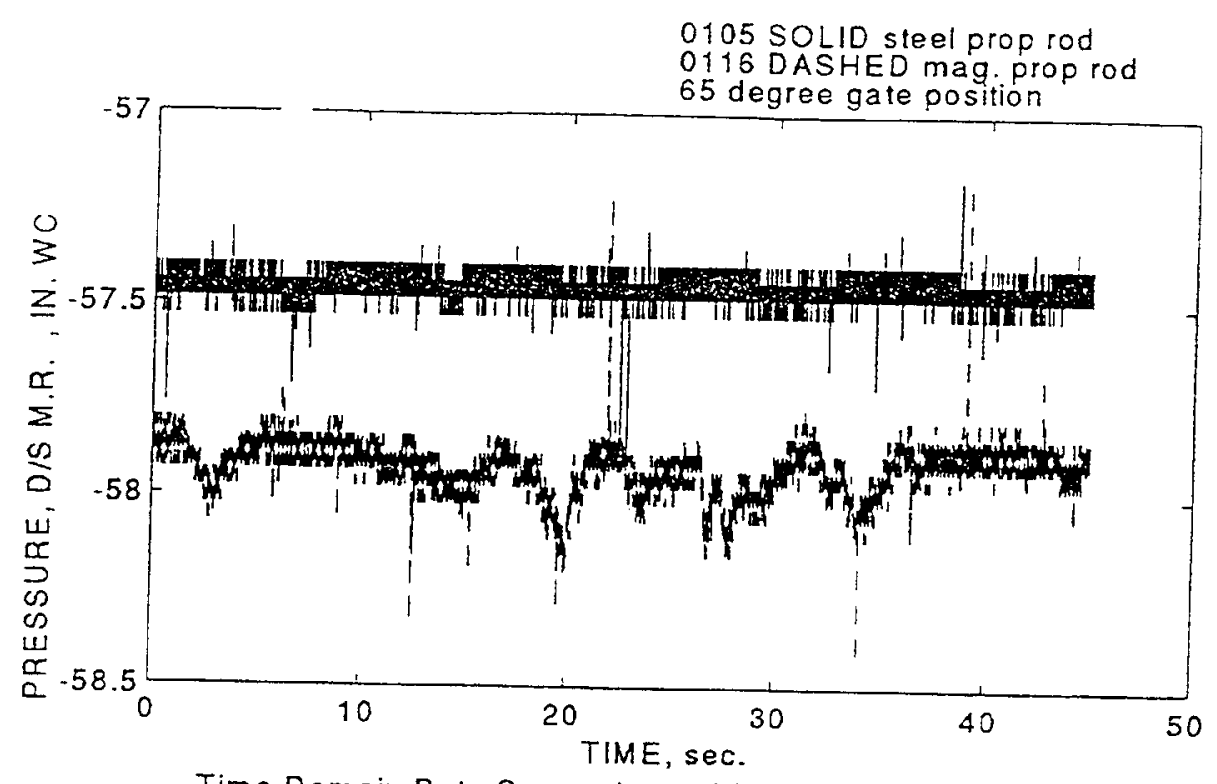

Time Domain Data Comparison of Different Prop Rod Materiais on the Olmsted 1:5 Model.

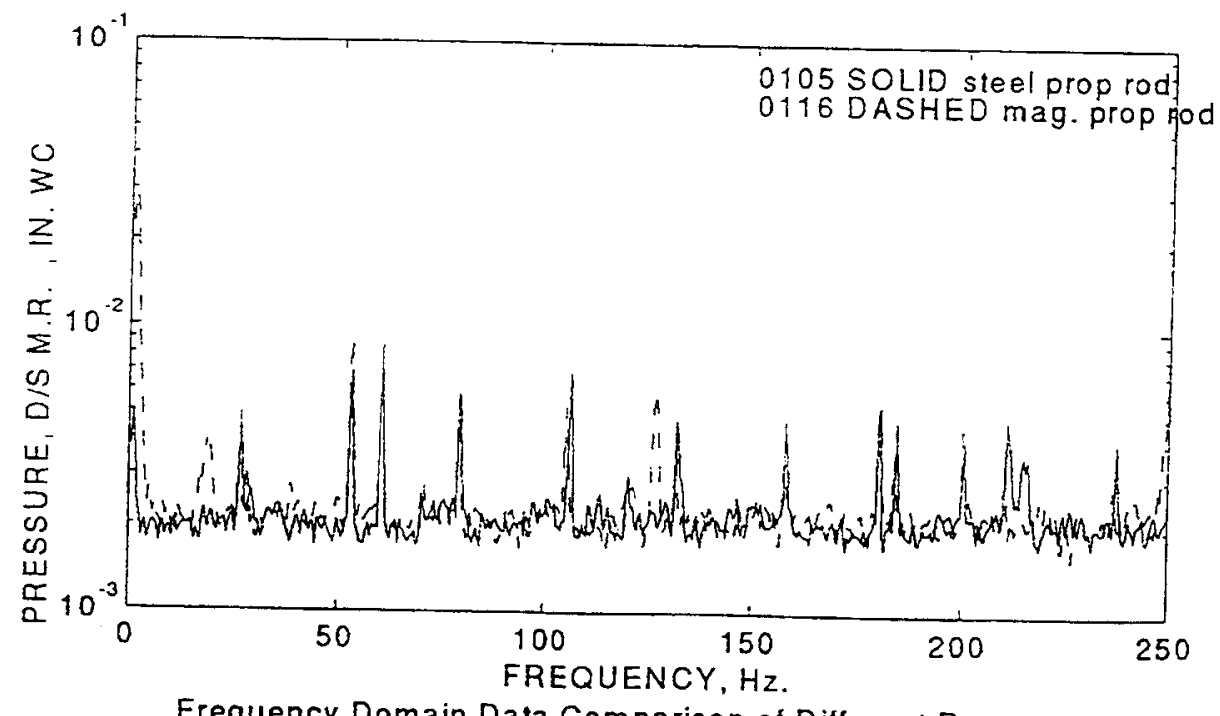

Frequency Domain Data Comparison of Different Prop Rod Materials on the Olmsted 1:5 Model.

h. Downstream pressure, middle right position on gate

Figure 78. (Sheet 8 of 14) 


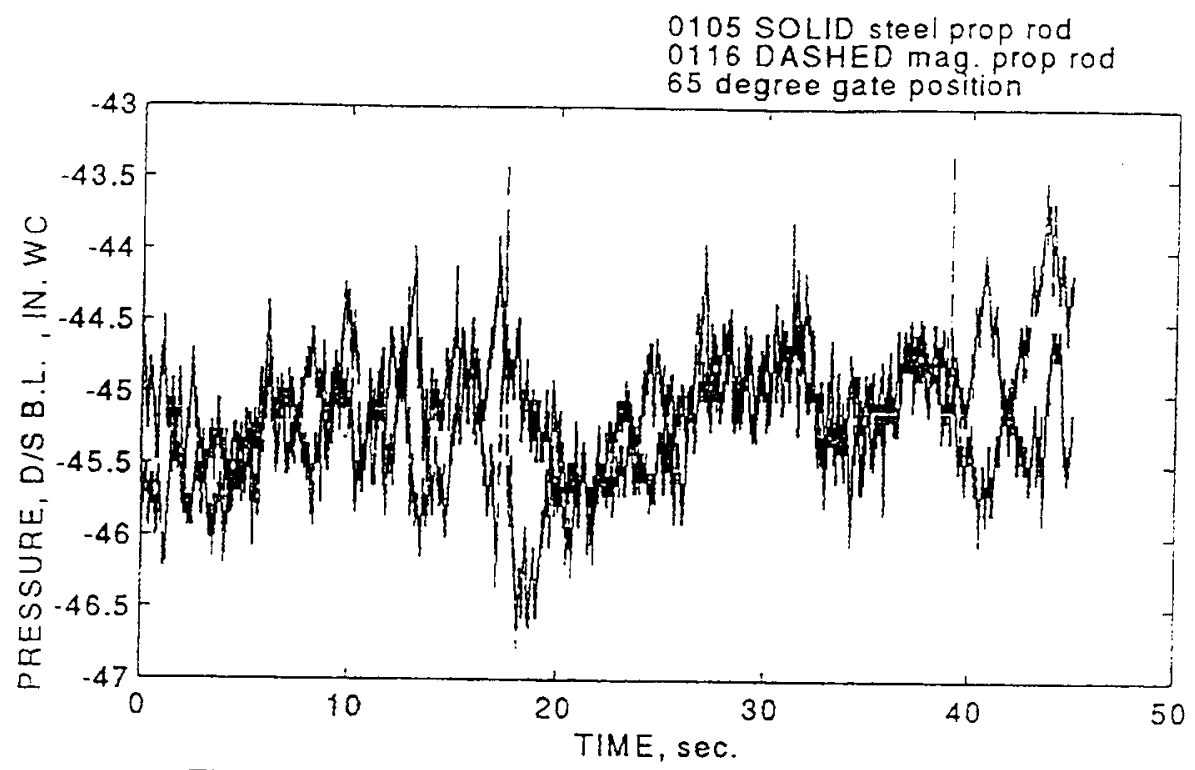

Time Domain Data Comparison of Different Prop

Rod Materials on the Olmsted 1:5 Model.

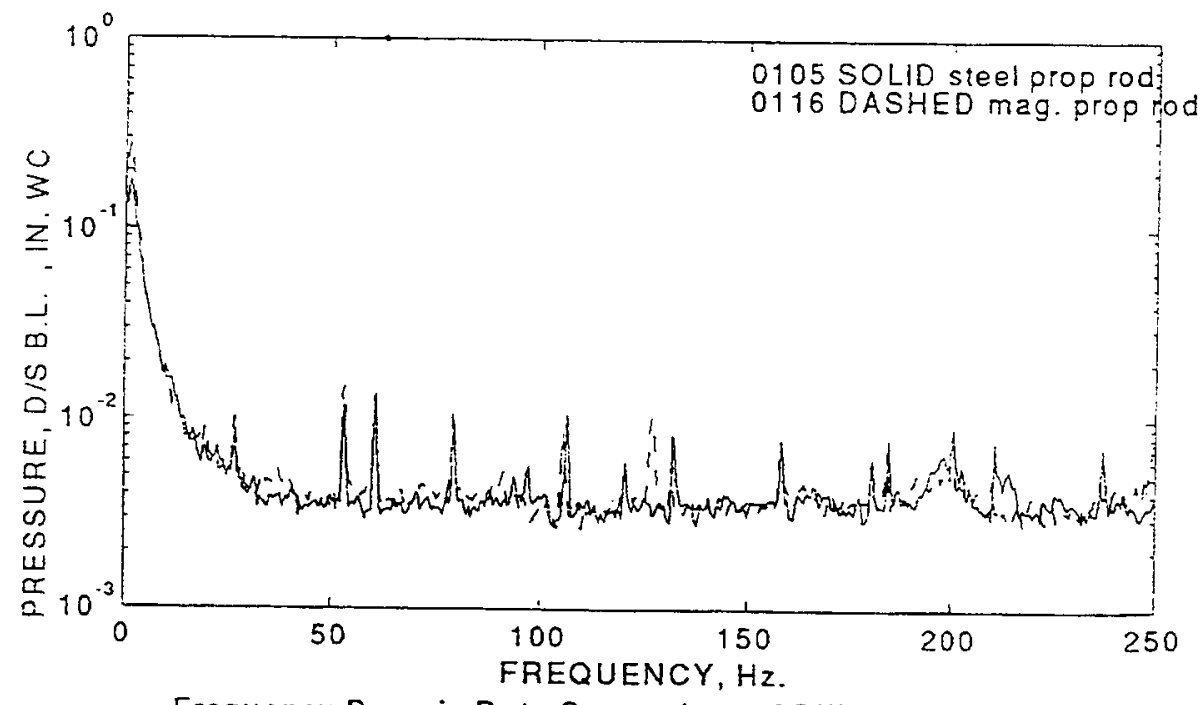

Frequency Domain Data Comparison of Different Prop

Rod Materials on the Olmsted 1:5 Model.

i. Downstream pressure, bottom left position on gate

Figure 78. (Sheet 9 of 14) 


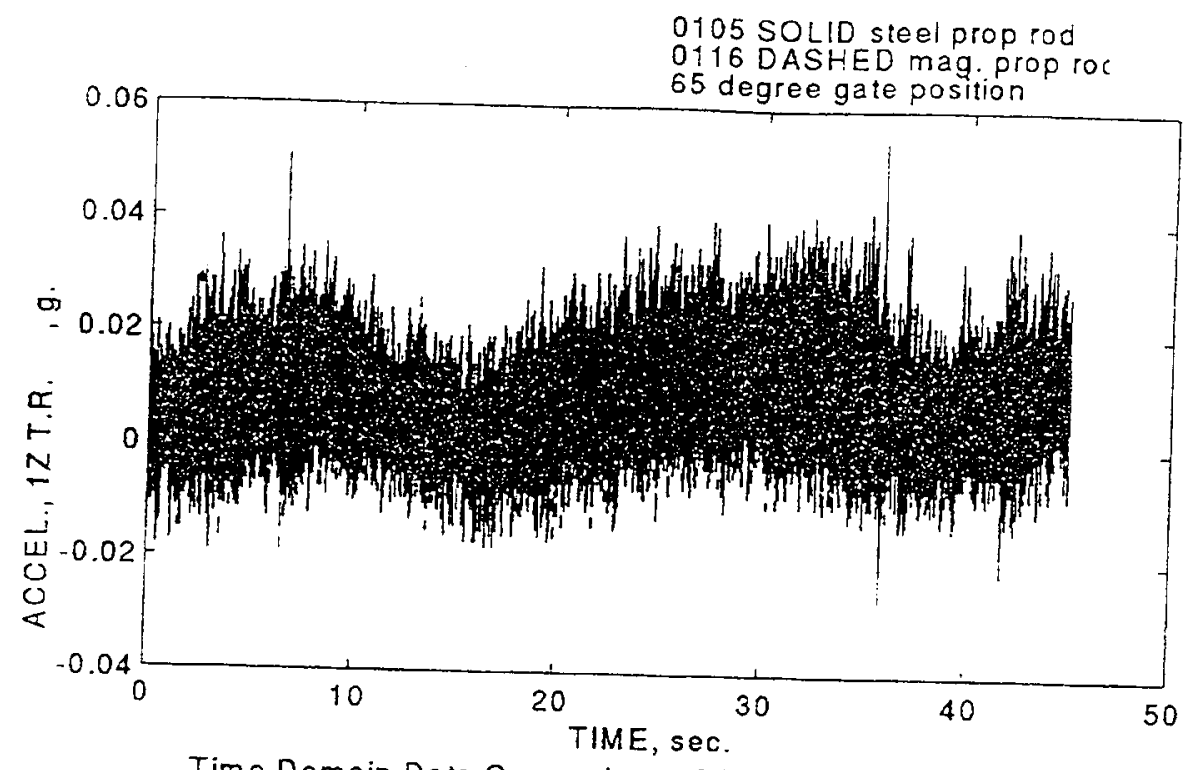

Time Domain Data Comparison of Different Prop

Rod Materials on the Oimsted 1:5 Model.

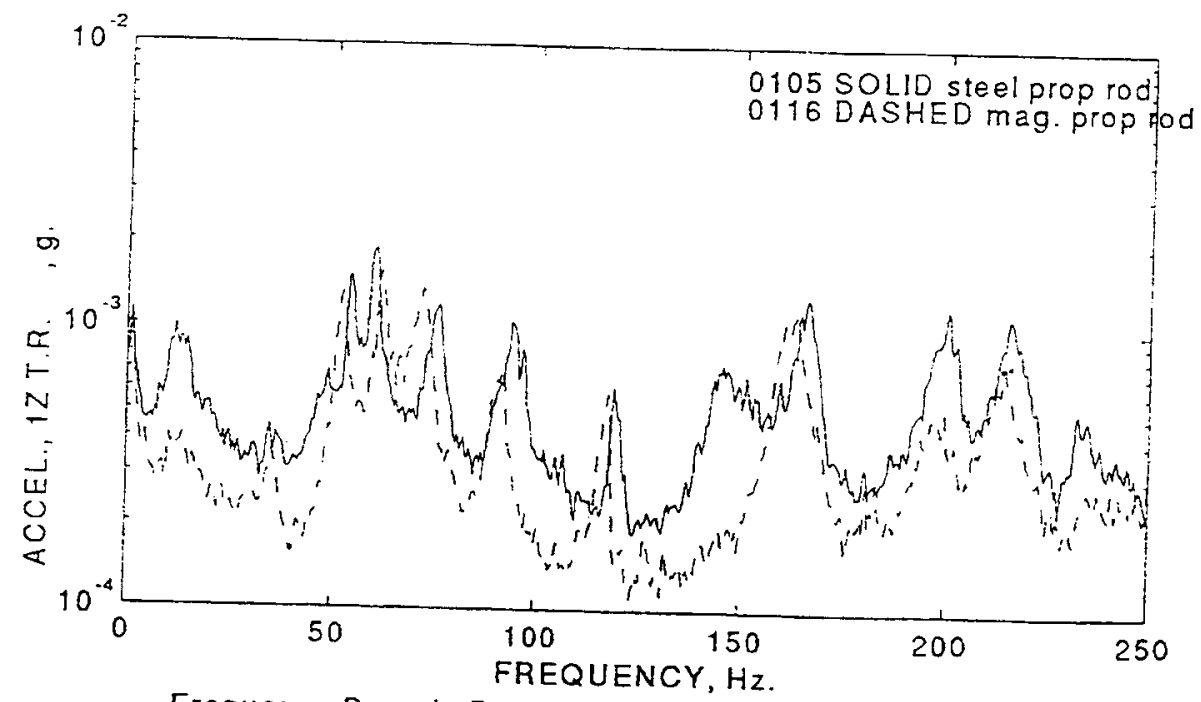

Frequency Domain Data Comparison of Different Prop

Rod Materials on the Olmsted 1:5 Model.

\section{j. Acceleration $1 z$ (top right position on gate)}

Figure 78. (Sheet 10 of 14) 


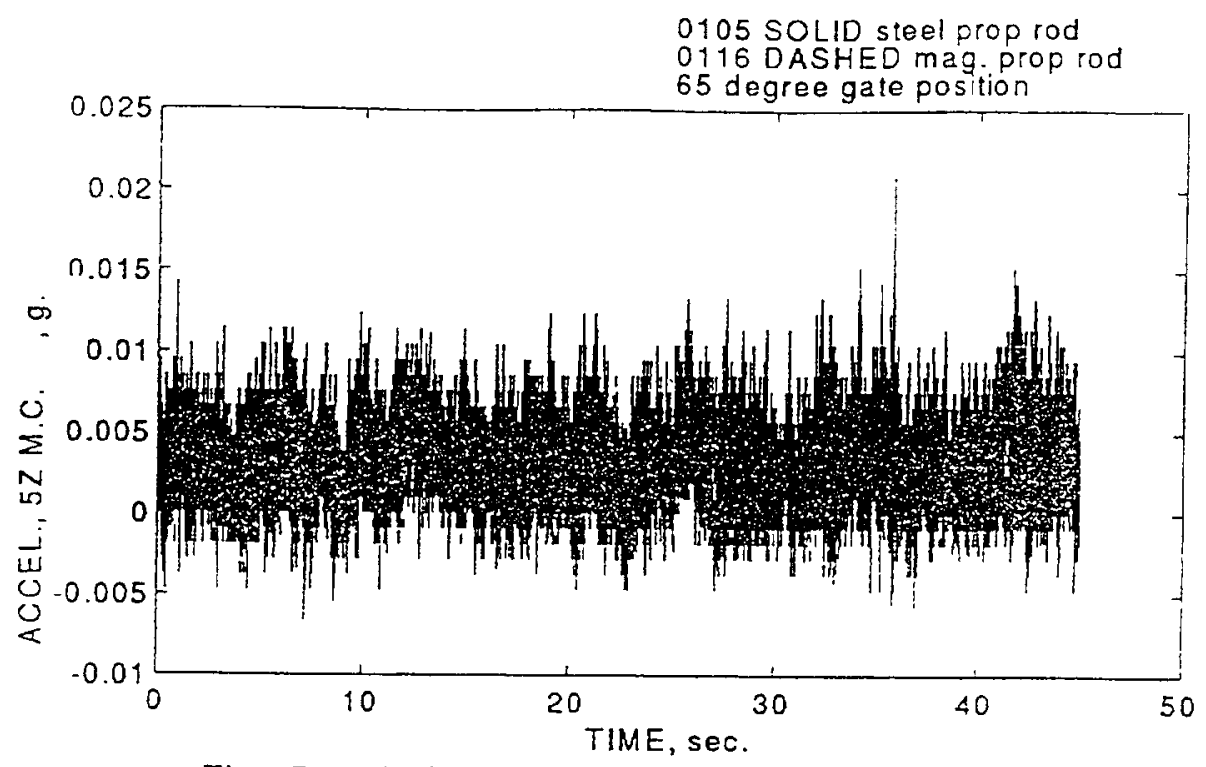

Time Domain Data Comparison of Different Prop Rod Materials on the Olmsted 1:5 Model.

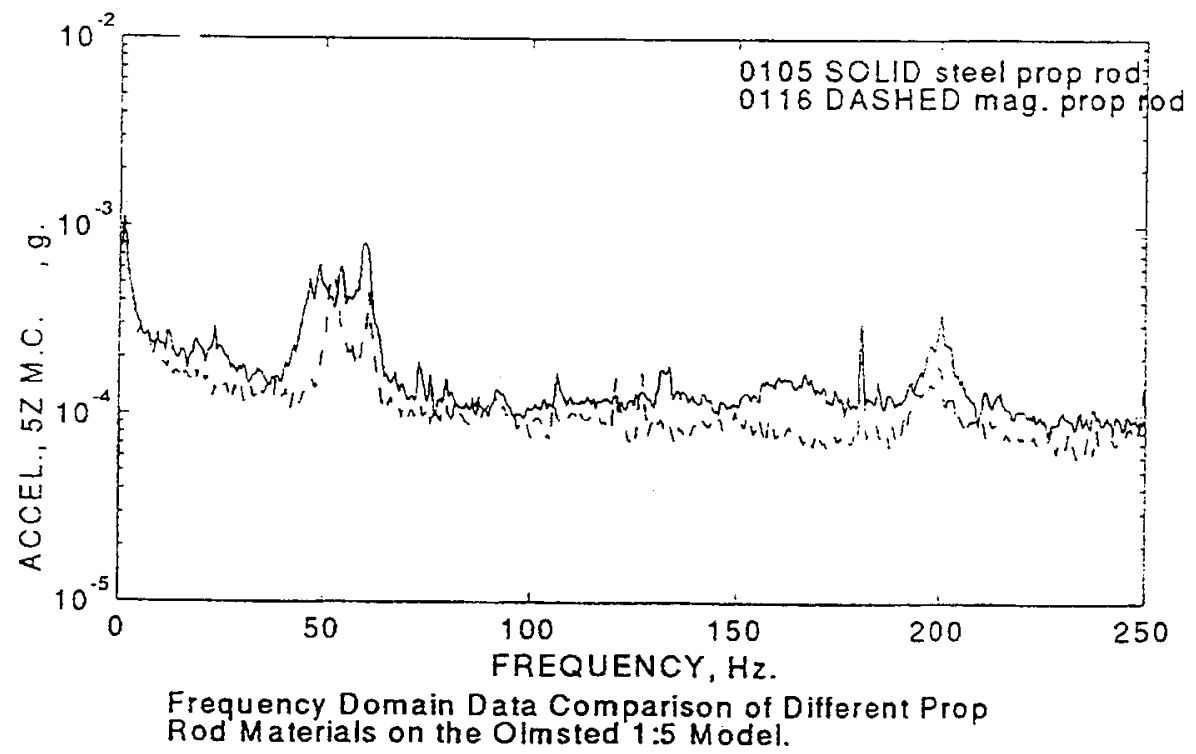

\section{k. Acceleration $5 z$ (midcenter position on gate)}

Figure 78. (Figure 11 of 14) 


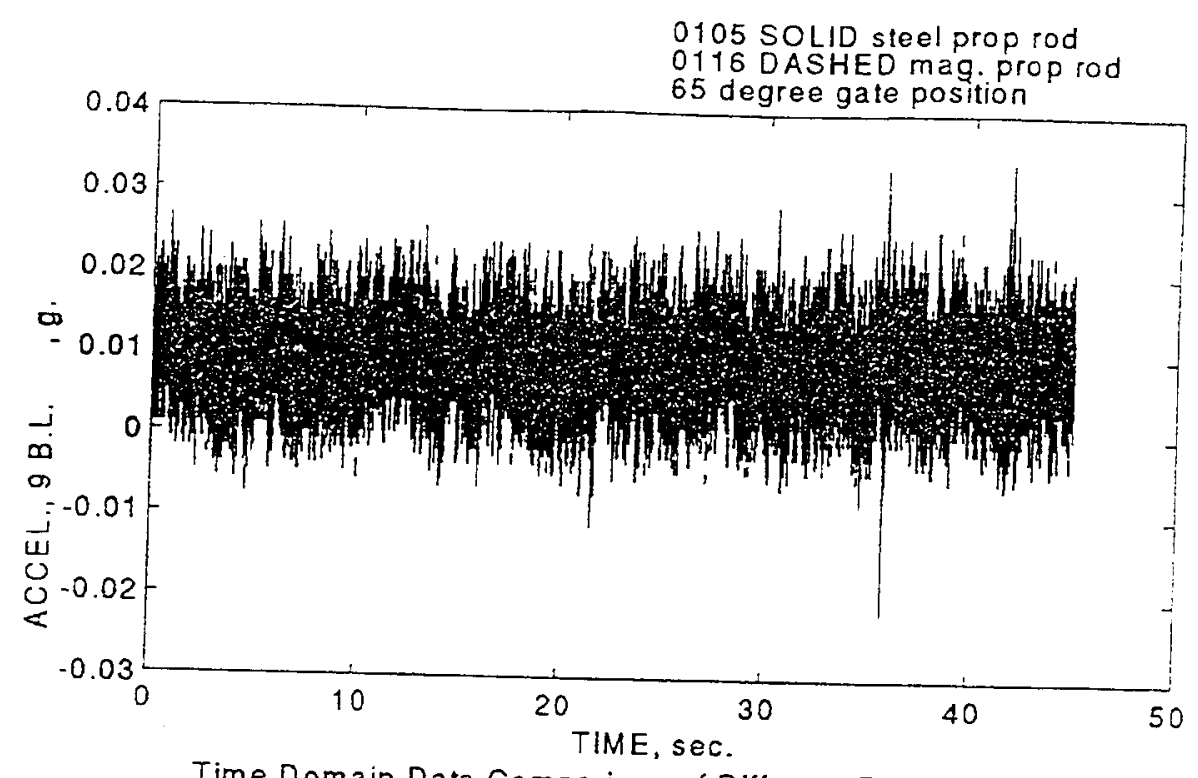

Time Domain Data Comparison of Different Prop

Rod Materials on the Oimsted 1:5 Model.

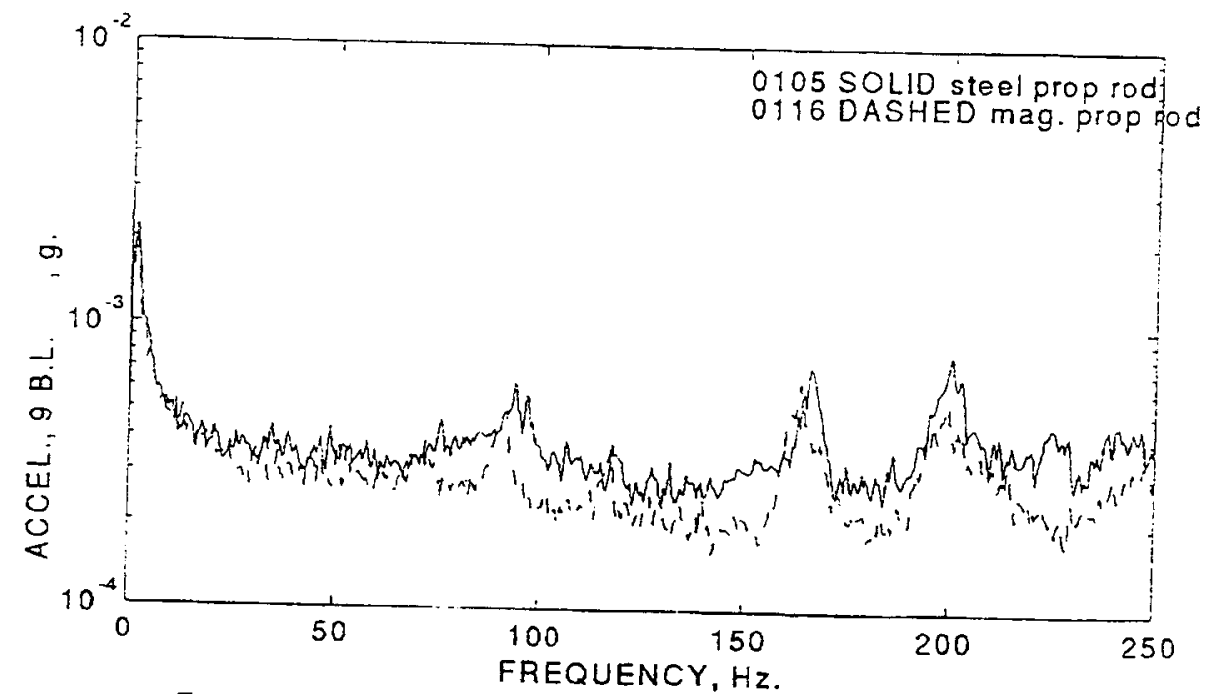

Frequency Domain Data Comparison of Different Prop

Rod Materials on the Olmsted 1:5 Model.

I. Acceleration $9 z$ (bottom left position on gate)

Figure 78. (Sheet 12 of 14) 


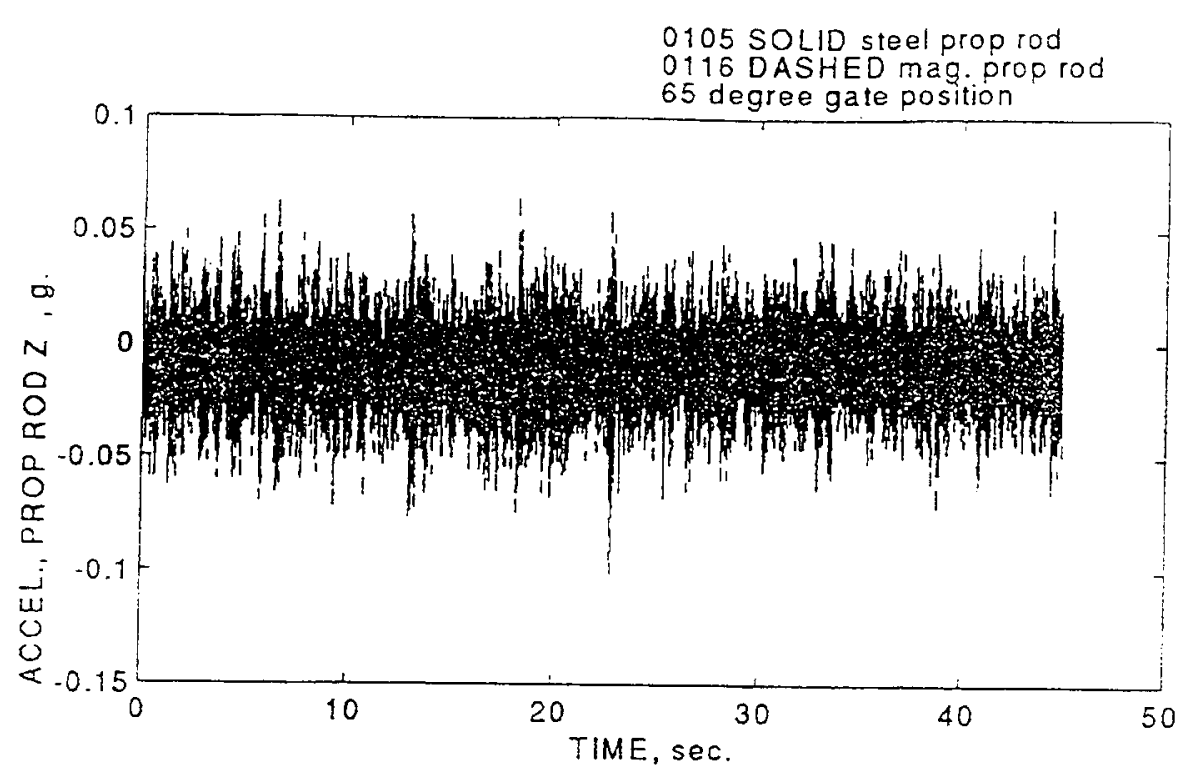

Time Domain Data Comparison of Different Prop

Rod Materials on the Olmsted 1:5 Model.

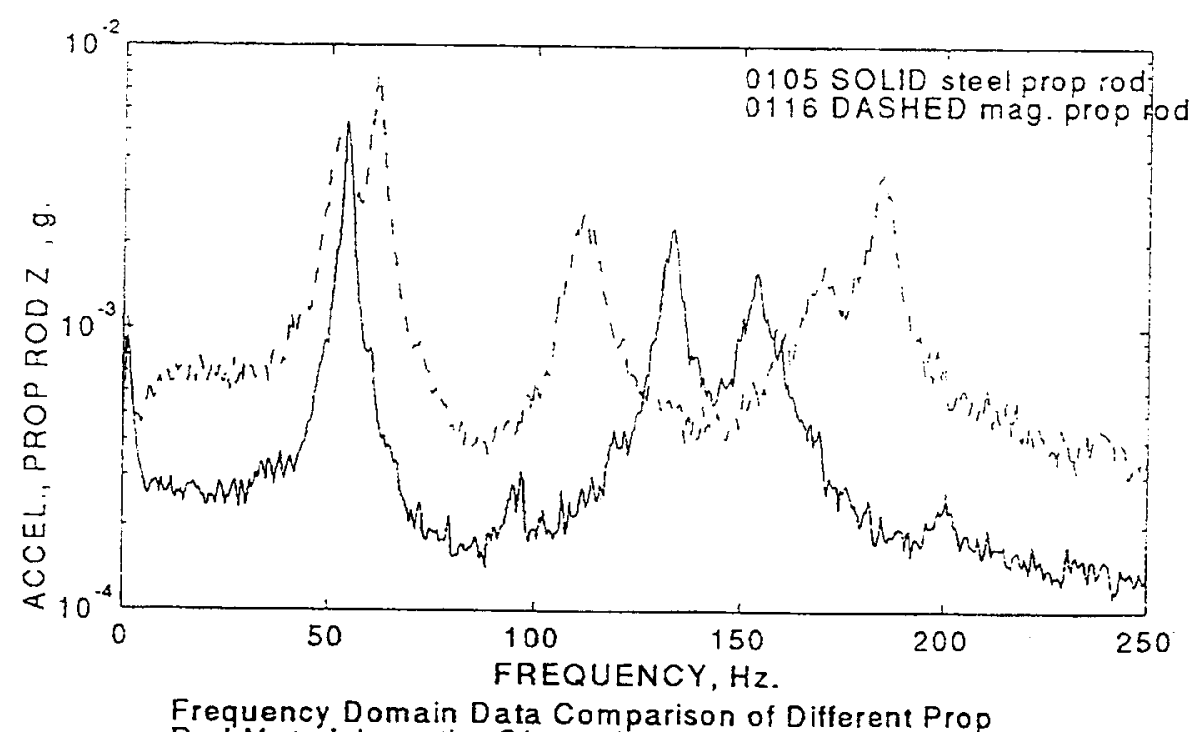

Rod Materials on the Olmsted $1: 5 \mathrm{Model}$.

m. Prop-rod acceleration along z-direction

Figure 78. (Sheet 13 of 14) 


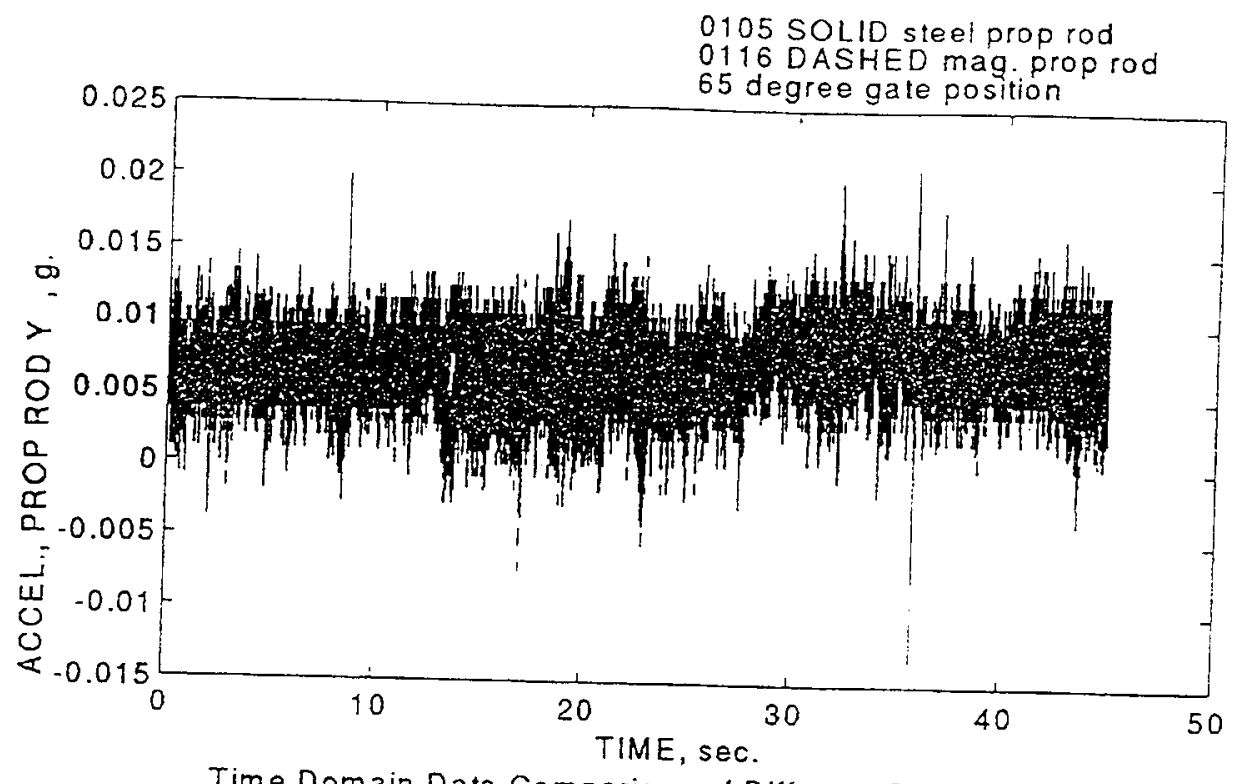

Time Domain Data Comparison of Different Prop

Rod Materials on the OImsted 1:5 Model.

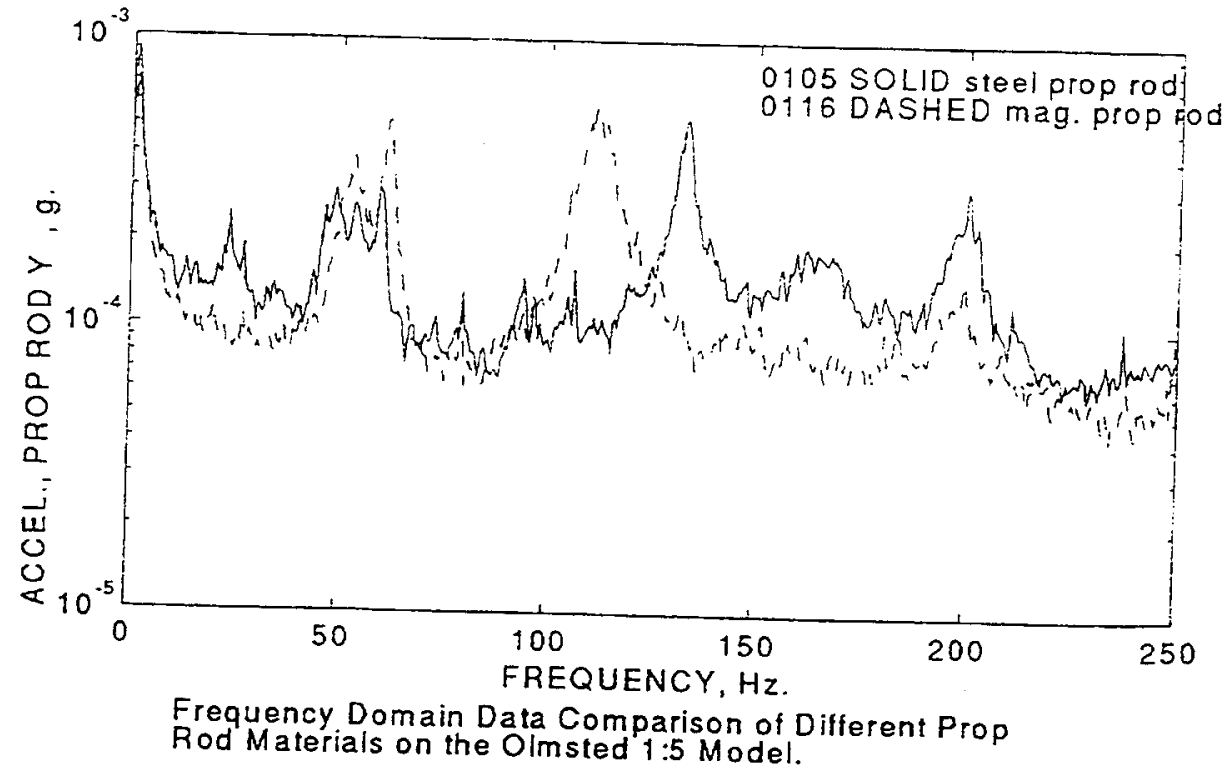

\section{n. Prop-rod acceleration along y-direction}

Figure 78. (Sheet 14 of 14) 
Although there are minor differences in the gate response due to a change in the modeling assumption, for all practical purposes these differences can be neglected with knowledge of Olmsted model operating field. A distortion of elastic properties in the Froude model thus creates no threat to the validity of the hydroelastic model in predicting the dynamics of the prototype wicket.

Therefore, all subsequent tests to determine the design envelops for measured responses were conducted using the steel prop rod.

\section{Effect of oil column volume}

A series of driving point (DP) responses was recorded for the dry gate for various oil column positions using the Scanning Laser Doppler Vibrometer (SLDV) system. A 50-lbf MB electro-dynamic shaker was used to excite the gate and the DP velocity was recorded using the laser system. FRF for different oil columns are compared in Figure 79. Six oil volumes were used be varying the auxiliary stroke length from 0 in. to the full extent of $21 \mathrm{in}$. As shown in the figure, the first mode and the peaks in the frequency range of 150 to $200 \mathrm{~Hz}$ were more sensitive to the hydraulic cylinder stiffness than the others. The first mode is the bouncing mode of the gate and depends primarily on the stiffness of the hydraulic lifting mechanism. The peaks between 150 and $200 \mathrm{~Hz}$ provided an interaction of the local lifting cylinder modes with those of the gate modes. The first peaks corresponding to the 0-, 7-, 14-, 20 -in., ( 0 in. + accumulator), and (20 in. + accumulator) auxiliary stroke positions were $46.75,46.02,48.34,49.74$, 48.17 , and $50.05 \mathrm{~Hz}$, respectively.

At the 24-deg gate position, the above experiment was repeated for the same oil volume levels. This experiment indicated that the oil columns are less sensitive to peak position at lower gate positions that at the raised position mentioned above. The peaks for different oil volumes did not deviate from each other as observed in the raised position.

Flow-induced dynamic responses for the 1:5-model with different oil columns were recorded to determine the sensitivity of hydraulic lifting system stiffness on the dynamic response of the gate. For an 11-ft head difference, three-gate gap configuration, three full-range tests were conducted for different volumes of oil column by setting the auxiliary stroke lengths to 0,10 , and 20 in. The gate for each case was raised from the down to up position at two different rates. The results showed that the dynamic response of the gate was independent of the gate raising rate. A selected number of responses for three different oil columns are presented in Figure 80. As seen in this figure, the dynamic responses for all cases closely match each other, thus showing that the gate responses are independent of the oil column volume changes.

Gate responses for a 20-deg fixed position were also recorded and compared for three different oil volumes. This comparison also indicated that the gate responses are relatively less sensitive to the oil column stiffness. The ratio of mean + two standard deviations of respective channel responses for three oil volumes were within 90 percent of each other. 


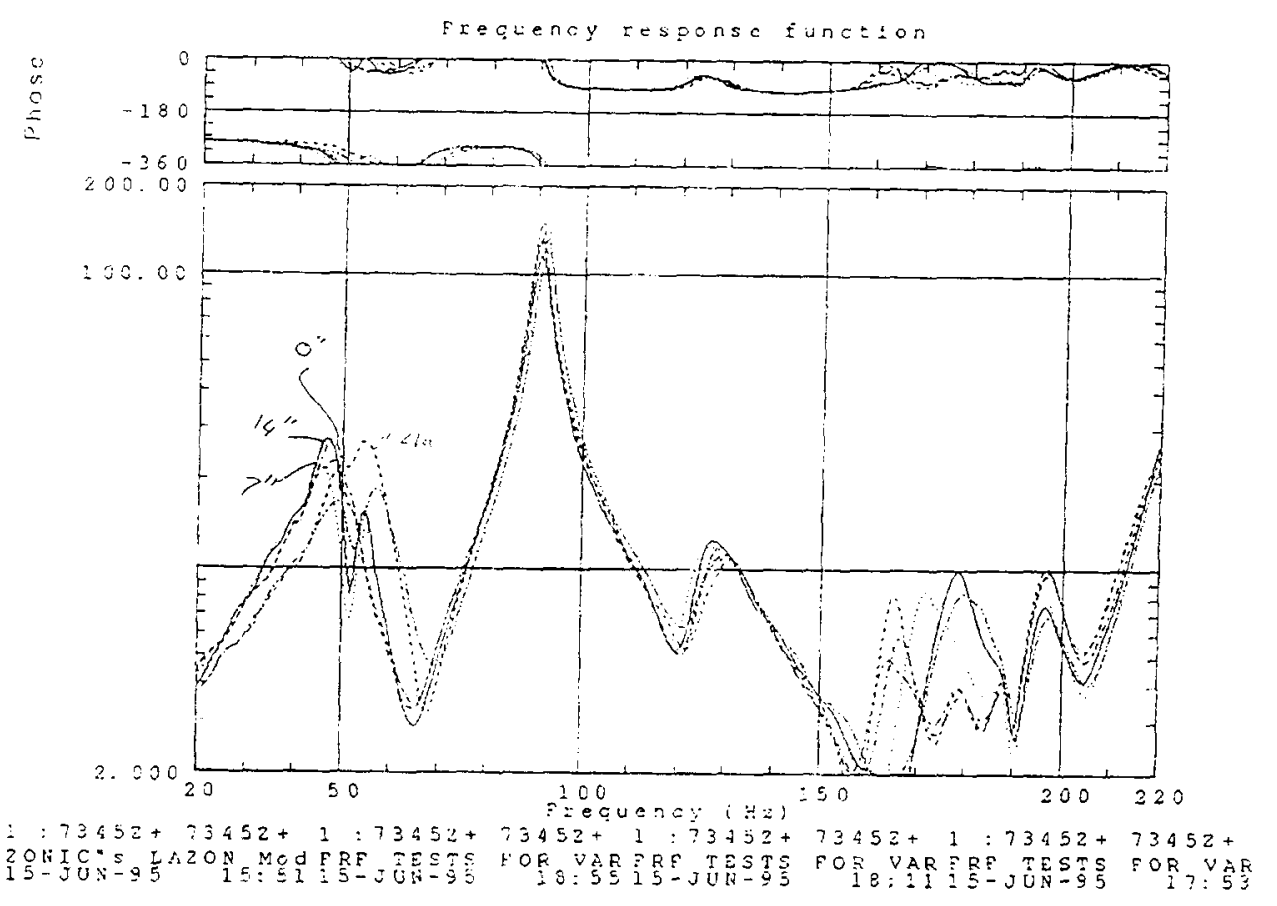

Figure 79. Driving point FRF comparison for different oil column levels

\section{Effect of gate-raising rate (144 sec vs $313 \mathrm{sec}$ )}

Two sets of experiments, for two different oil volumes, were conducted to measure the effects of gate raising rate corresponding to structural and hydraulic similitude laws. As mentioned earlier, the hydraulic law requires that time should be scaled by a factor of $\sqrt{\lambda_{L}}$, whereas the structural law requires a time scaling factor of $\lambda_{L}$. Corresponding to the prototype raising rate, these rates were 144 and $323 \mathrm{sec}$, respectively. Test data for these two different rates were recorded for full-range tests of the gate, with a $11-\mathrm{ft}$ head difference, and threegate gap configuration. A comparison of the respective responses in the timeand frequency-domain showed that the respective gate responses for both rates matched very well. A selected number of response comparisons for two different gate-raising rates is shown in Figure 81 . All experiments subsequently presented in this report were conducted using the hydraulic time scaling factor.

\section{Effects of prop-rod dragging (Experiments No. 42 and No. 87)}

A quantitative measurement of the flow-induced response with and without the prop rod was taken to measure the dragging effect of the prop blade during the raising of the gate. A comparison of the PSD showed no indication of a change in gate dynamics due to the dragging of the blade through the hurter track. A comparison of respective ratios of mean + standard deviation for both 


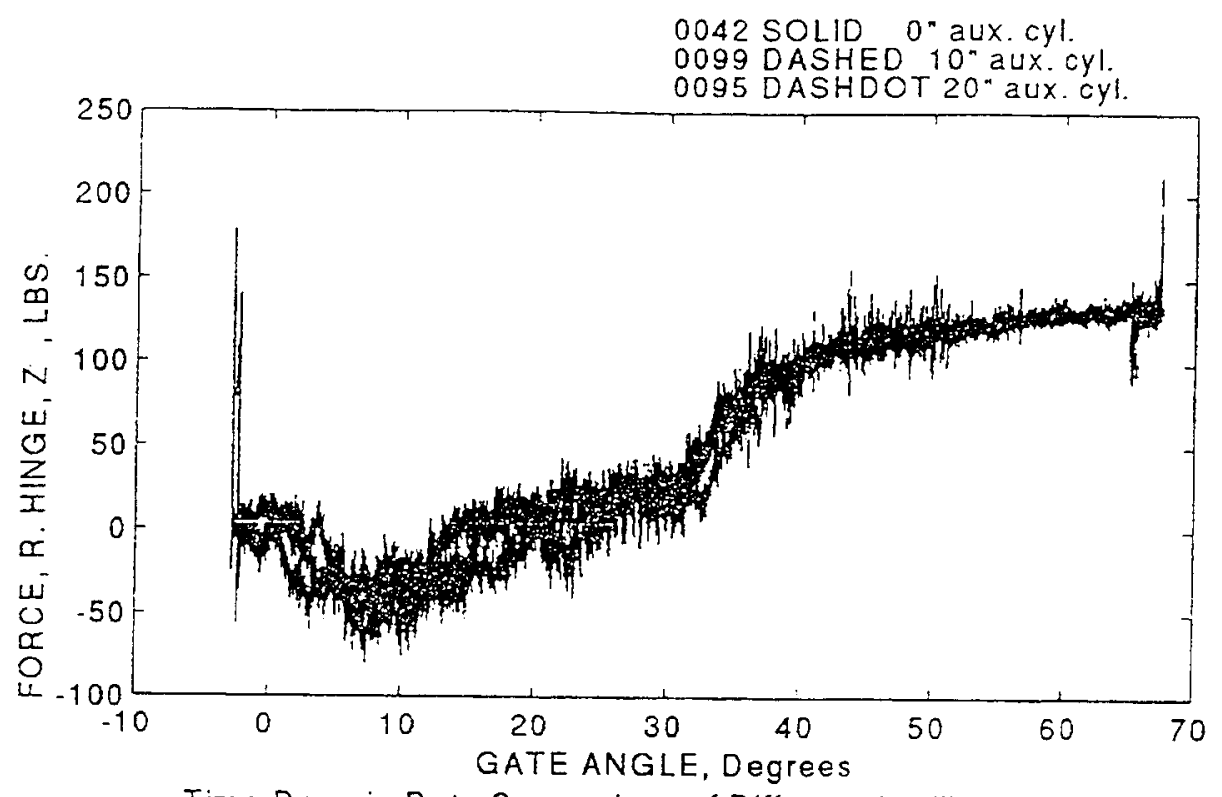

Time Domain Data Comparison of Different Auxillary Cylinder Lengths on the Olmsted 1:5 Model.

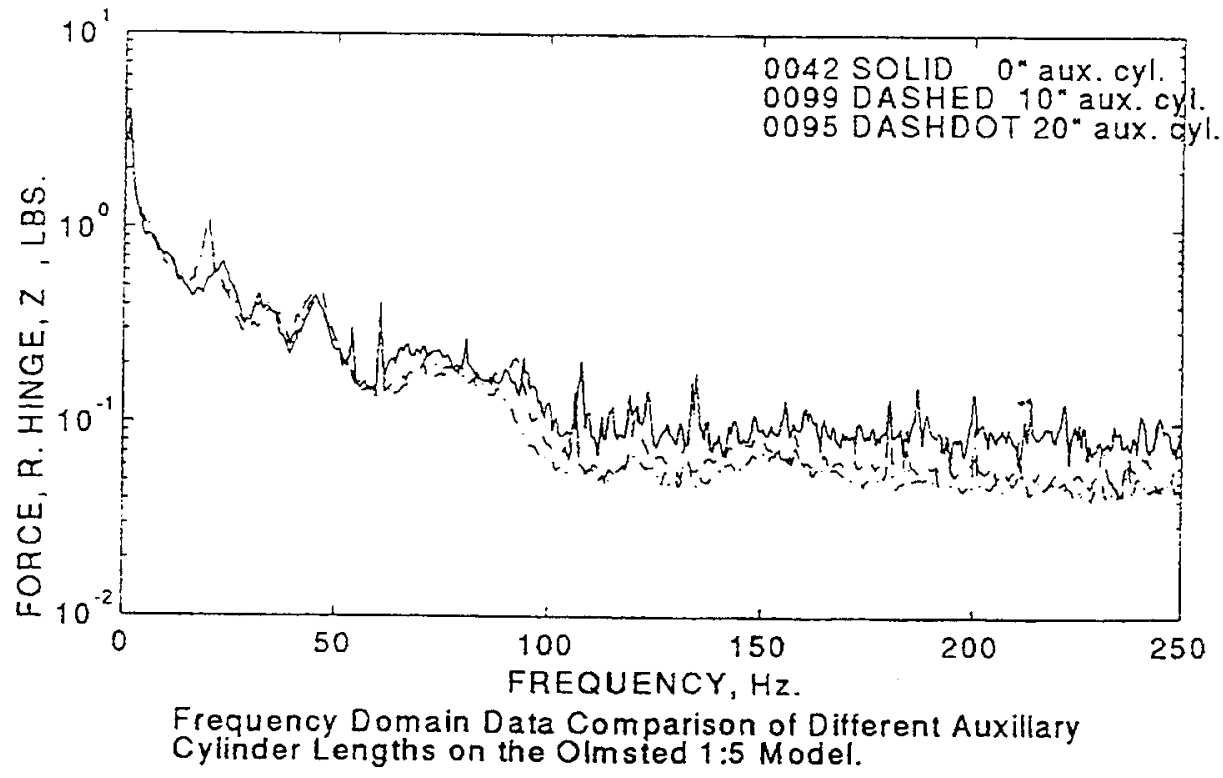

a. Right vertical hinge load

Figure 80. Effect of oil columns on flow-induced dynamic responses for instrumented gate, 11-ft head difference, 3GG configuration (Sheet 1 of 6 ) 


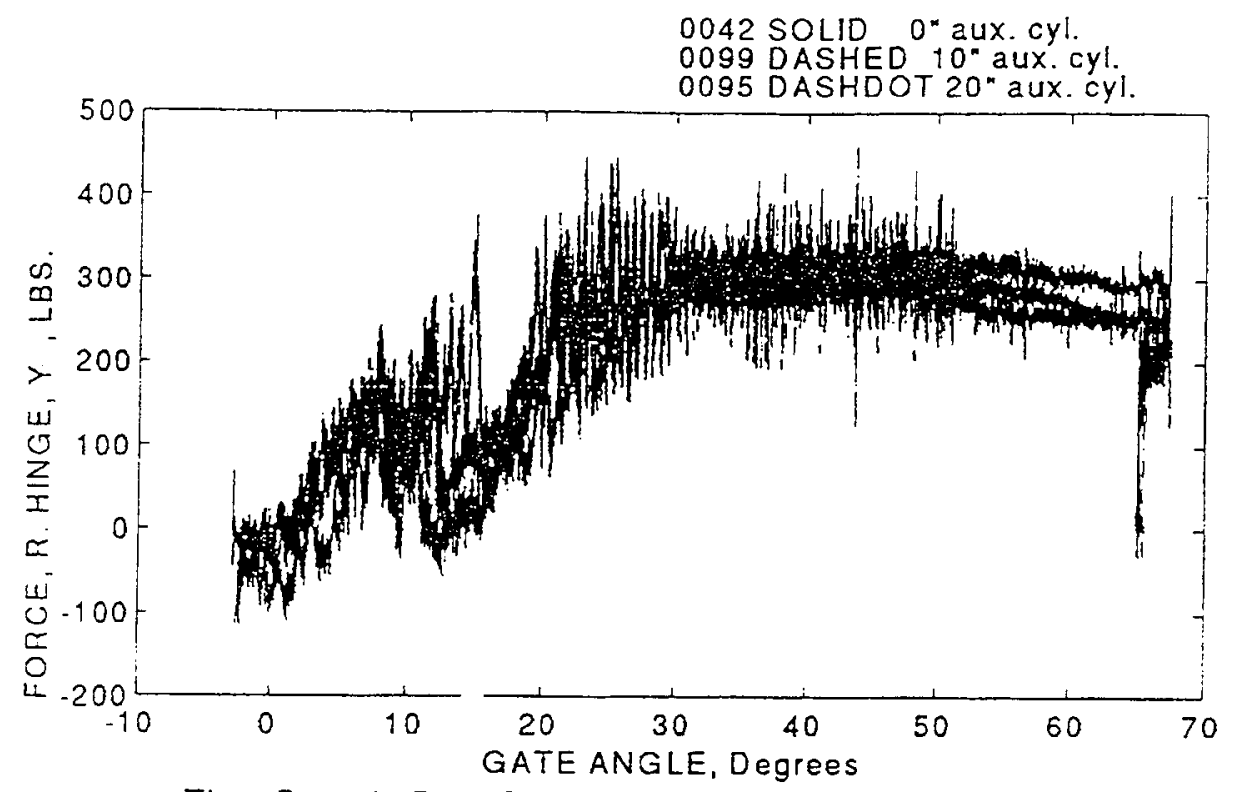

Time Domain Data Comparison of Different Auxillary

Cylinder Lengths on the OImsted 1:5 Model.

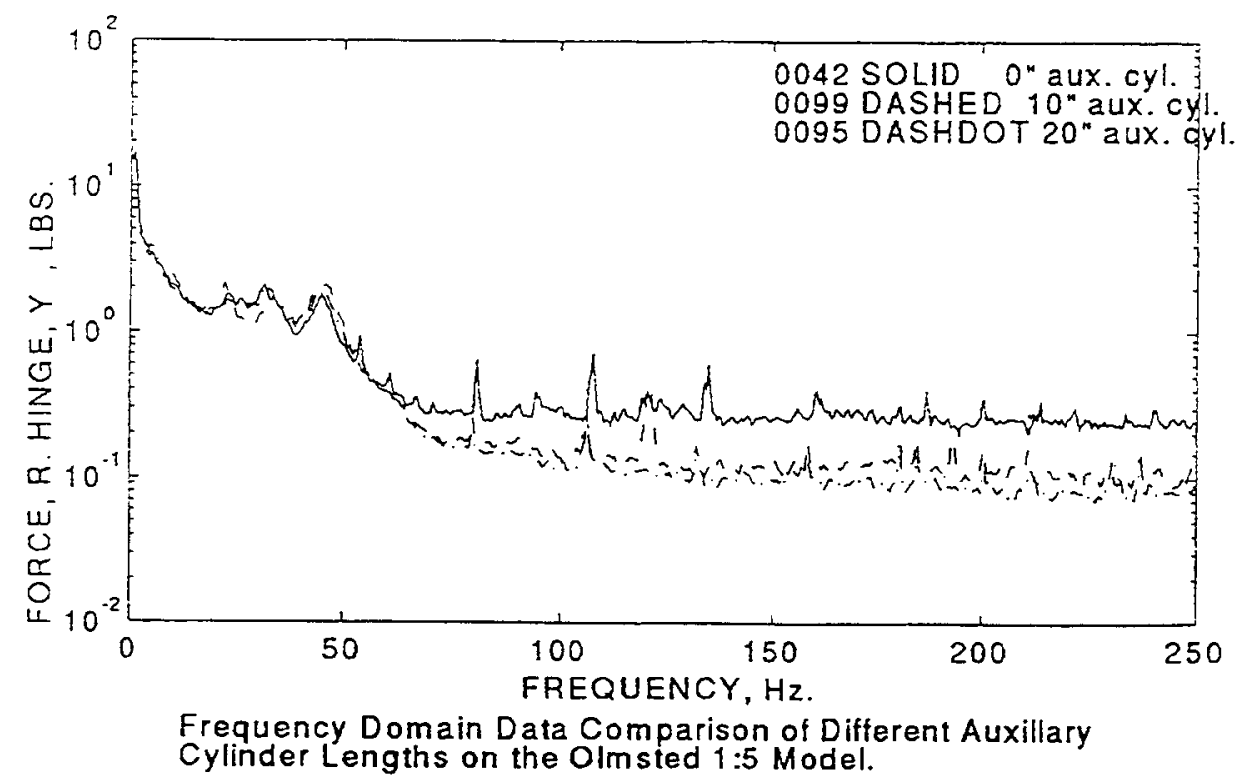

b. Right horizontal hinge load .

Figure 80. (Sheet 2 of 6 ) 

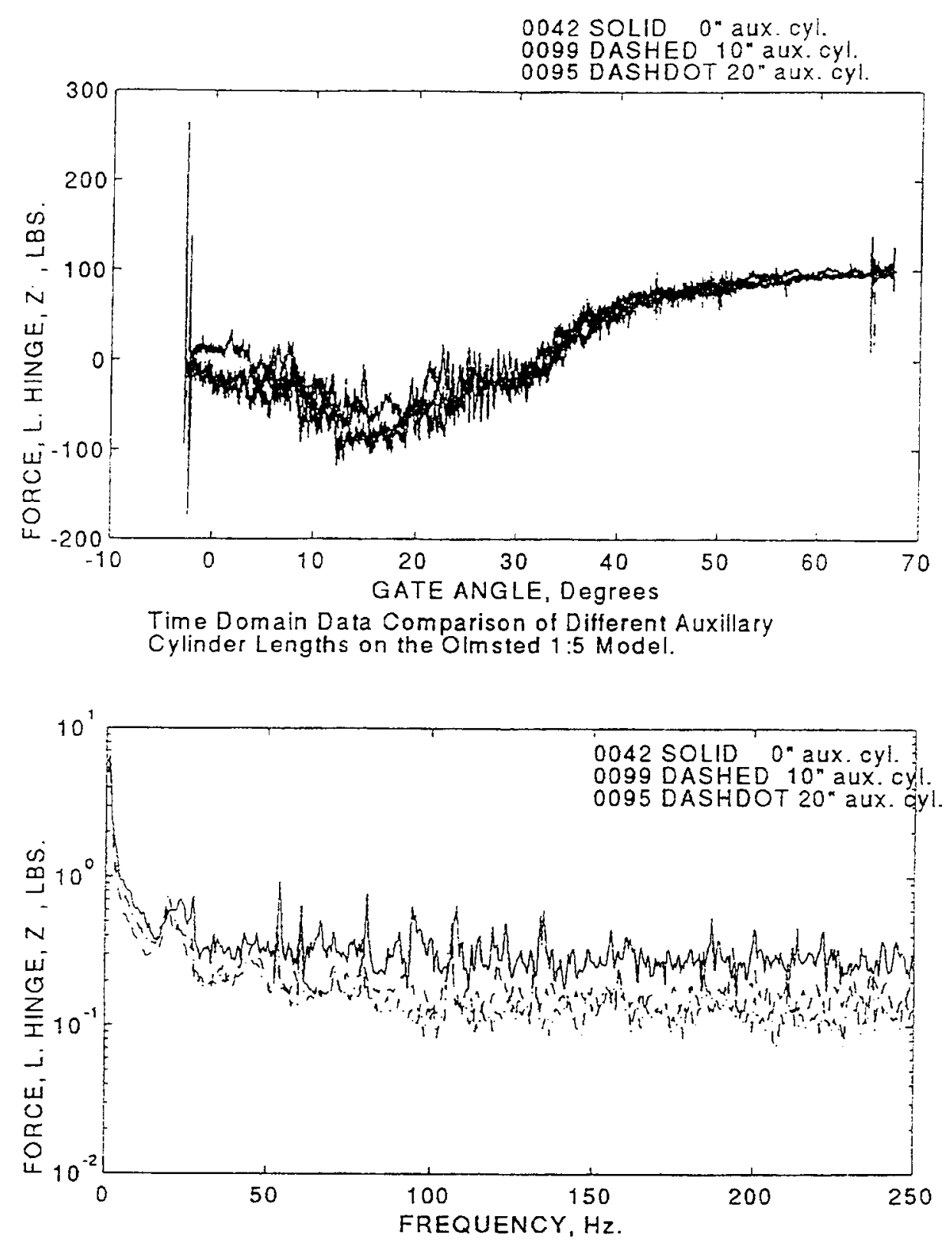

Frequency Domain Data Comparison of Different Auxillary Cylinder Lengths on the Oimsted 1:5 Model.

c. Left vertical hinge load

Figure 80. (Sheet 3 of 6 ) 


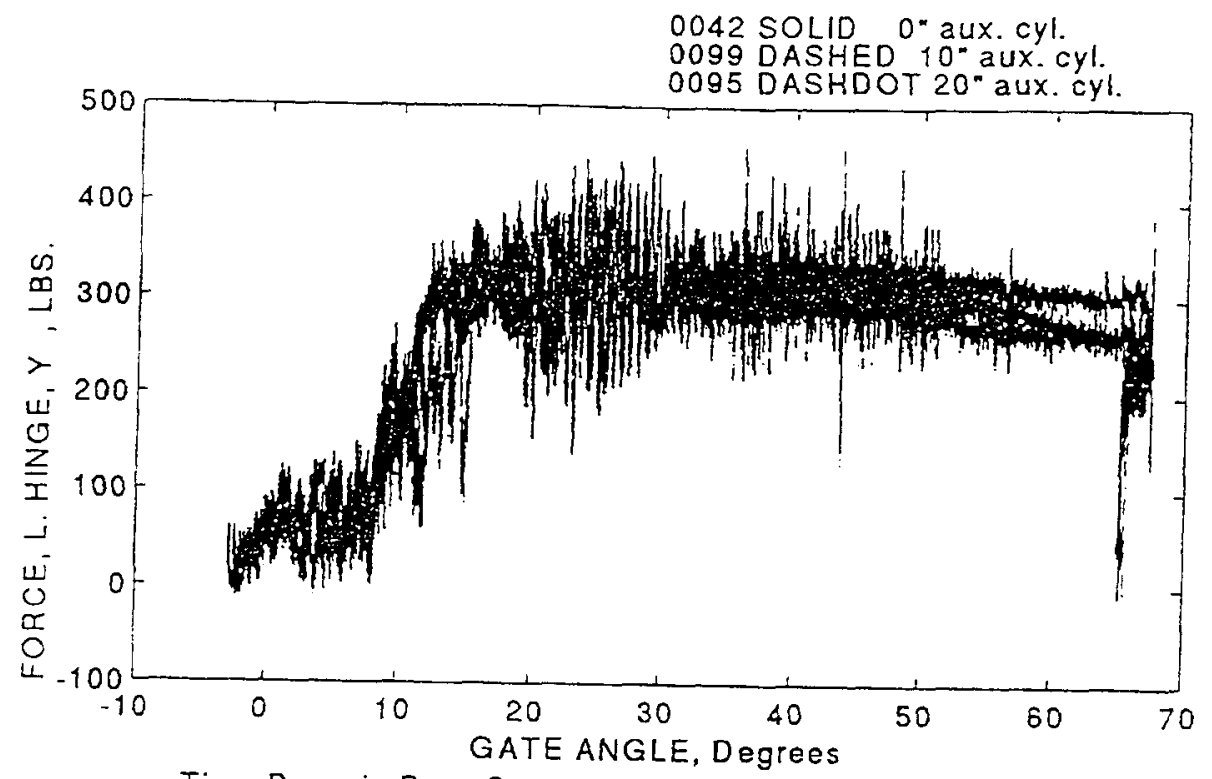

Time Domain Data Comparison of Different Auxillary Cylinder Lengths on the Oimsted 1:5 Model.

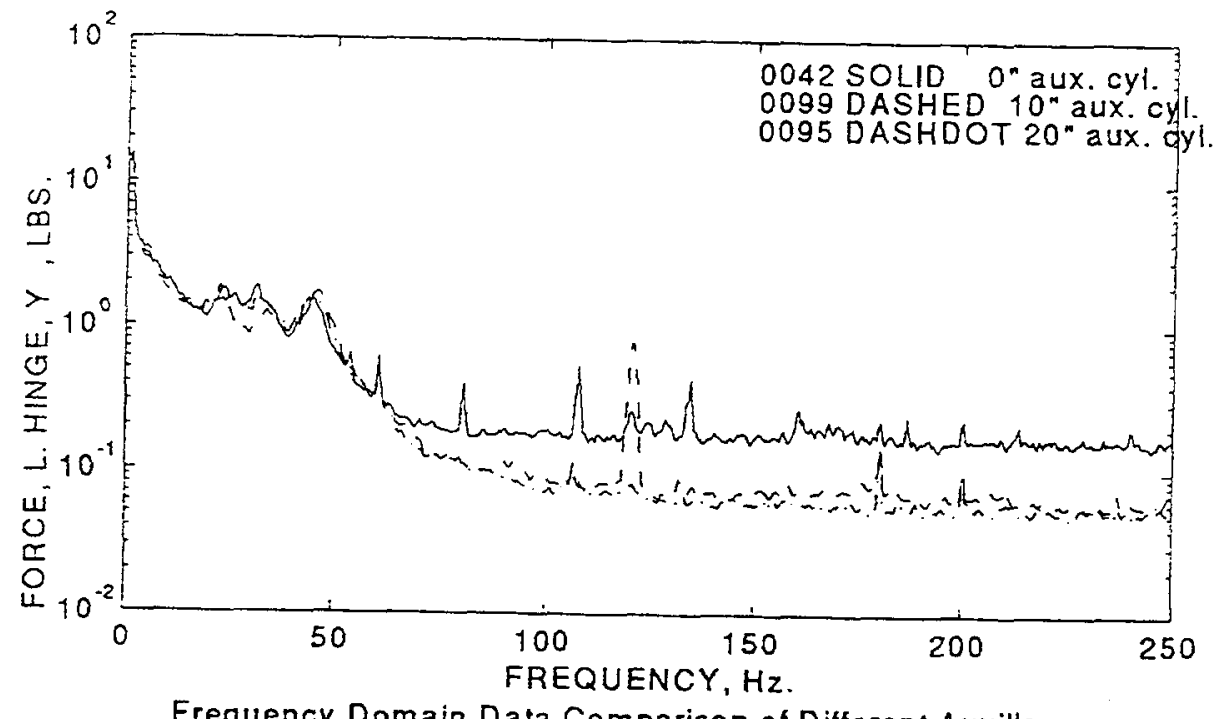

Frequency Domain Data Comparison of Different Auxillary Cylinder Lengths on the Olmsted 1:5 Model.

d. Left horizontal hinge load

Figure 80. (Sheet 4 of 6 ) 


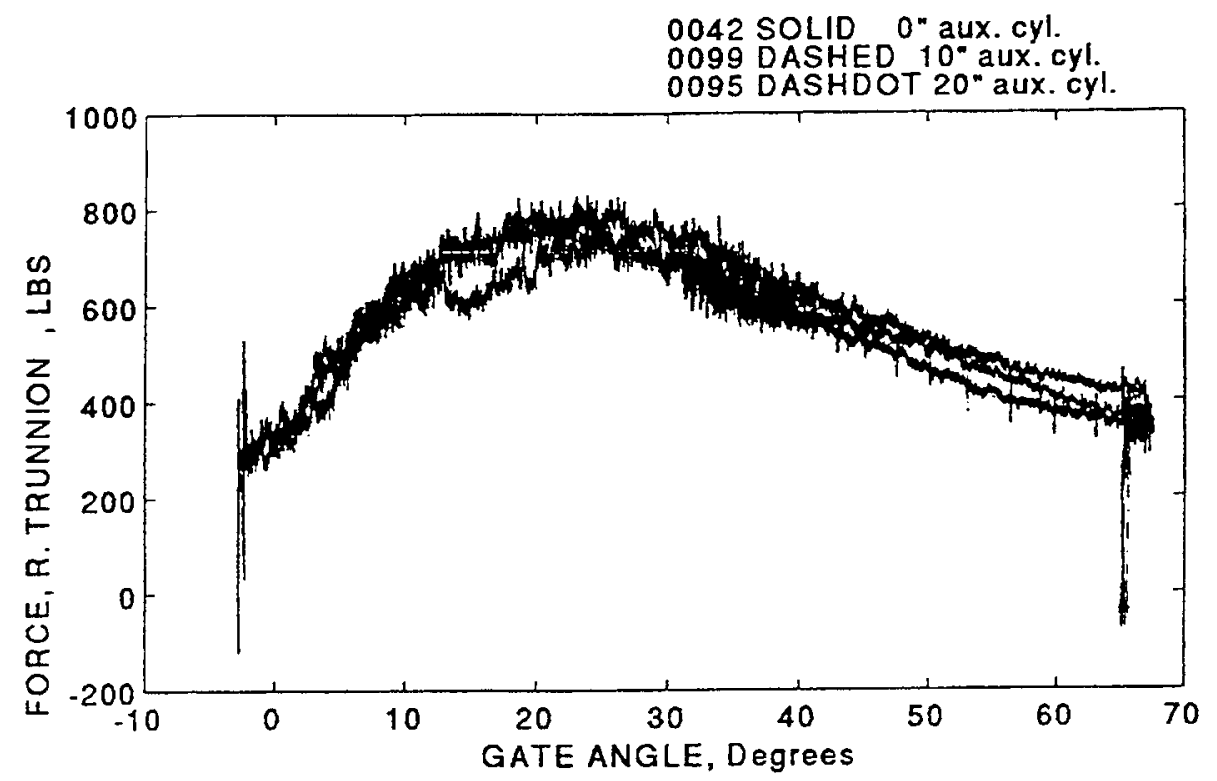

Time Domain Data Comparison of Different Auxillary Cylinder Lengths on the Olm sted 1:5 Model.

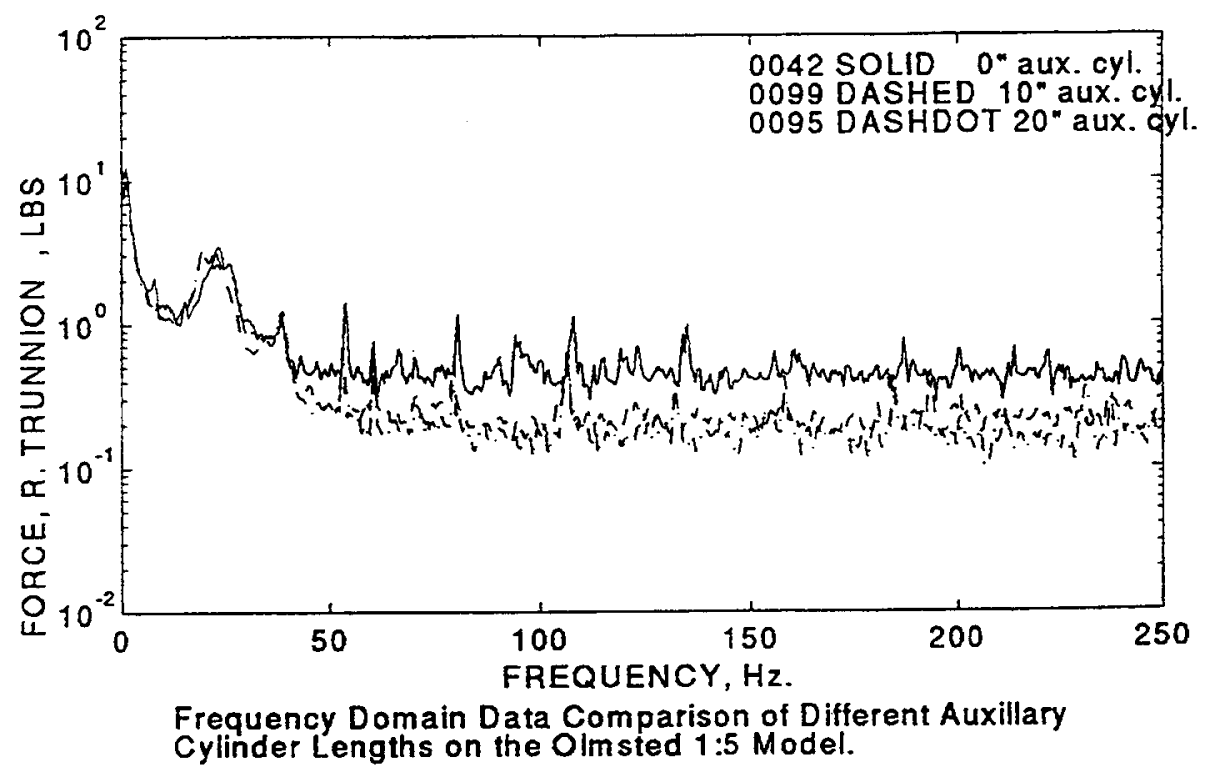

e. Trunnion load

Figure 80. (Sheet 5 of 6 ) 


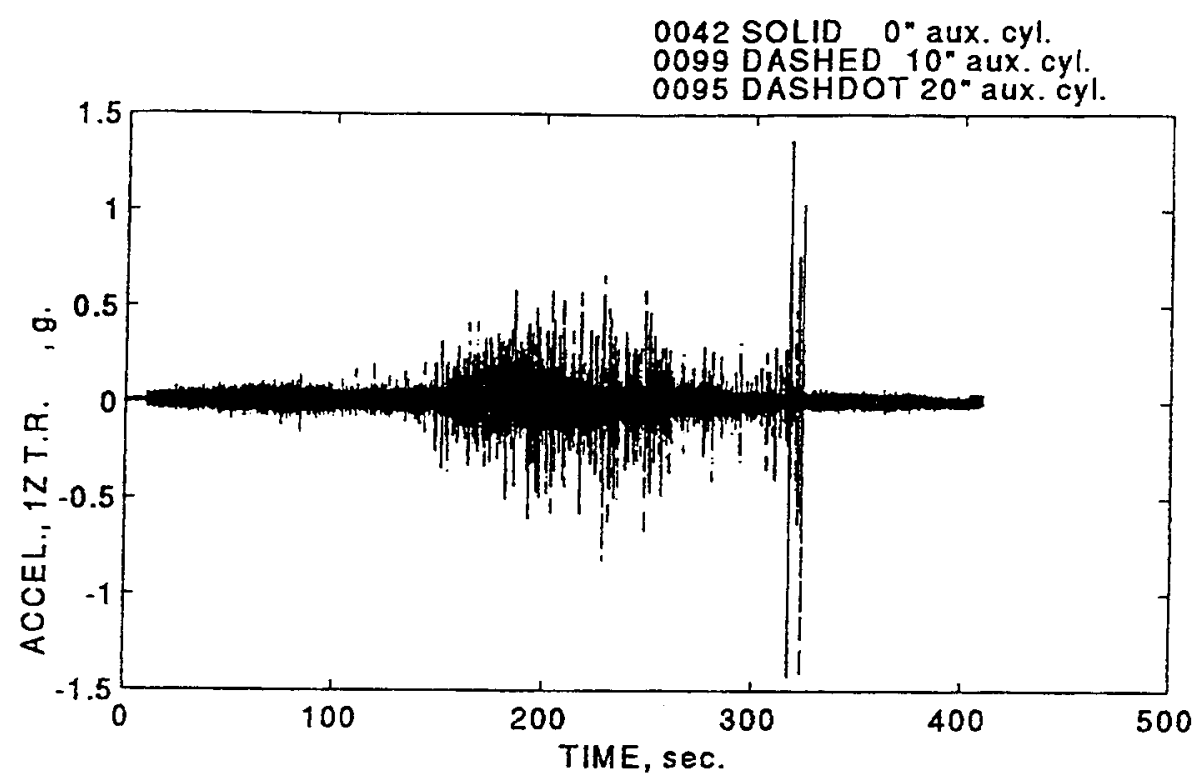

Time Domain Data Comparison of Different Auxillary Cylinder Lengths on the Oimsted 1:5 Model.

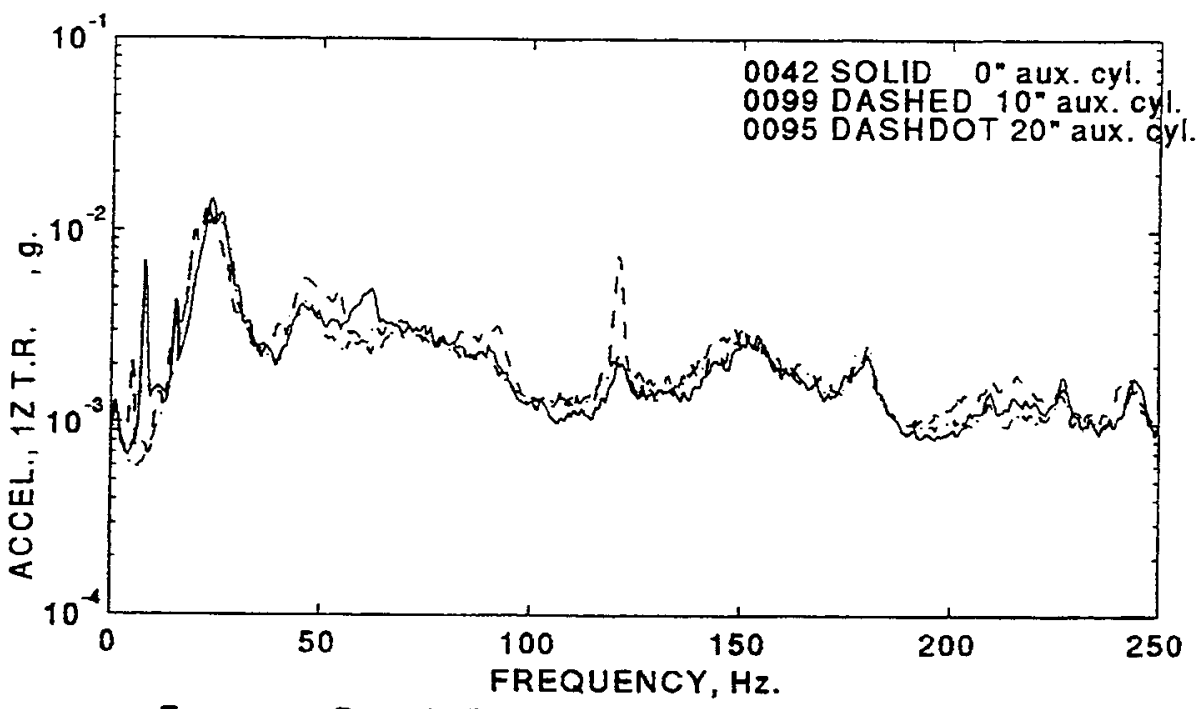

Frequency Domain Data Comparison of Different Auxillary Cylinder Lengths on the OImsted 1:5 Model.

f. Acceleration $1 z$ (top right position on gate)

Figure 80. (Sheet 6 of 6 ) 


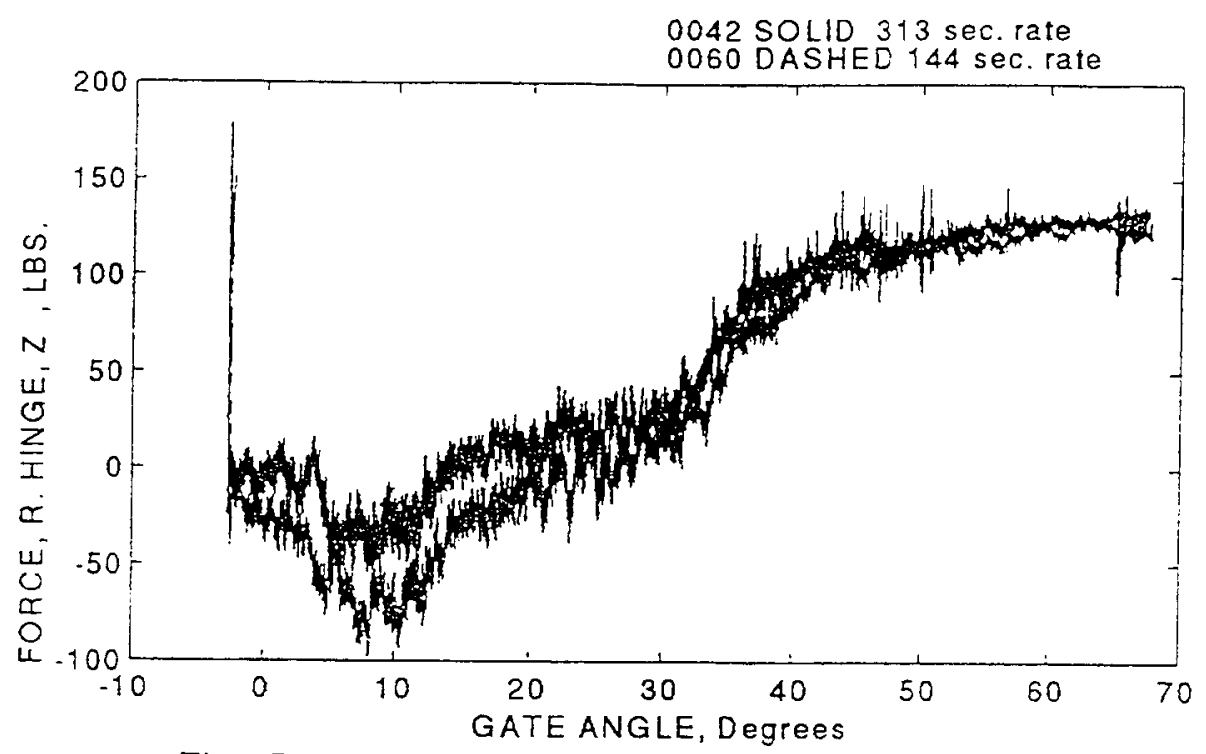

Time Domain Data Comparison of Different Gate Raising Rates on the Oimsted 1:5 Model.

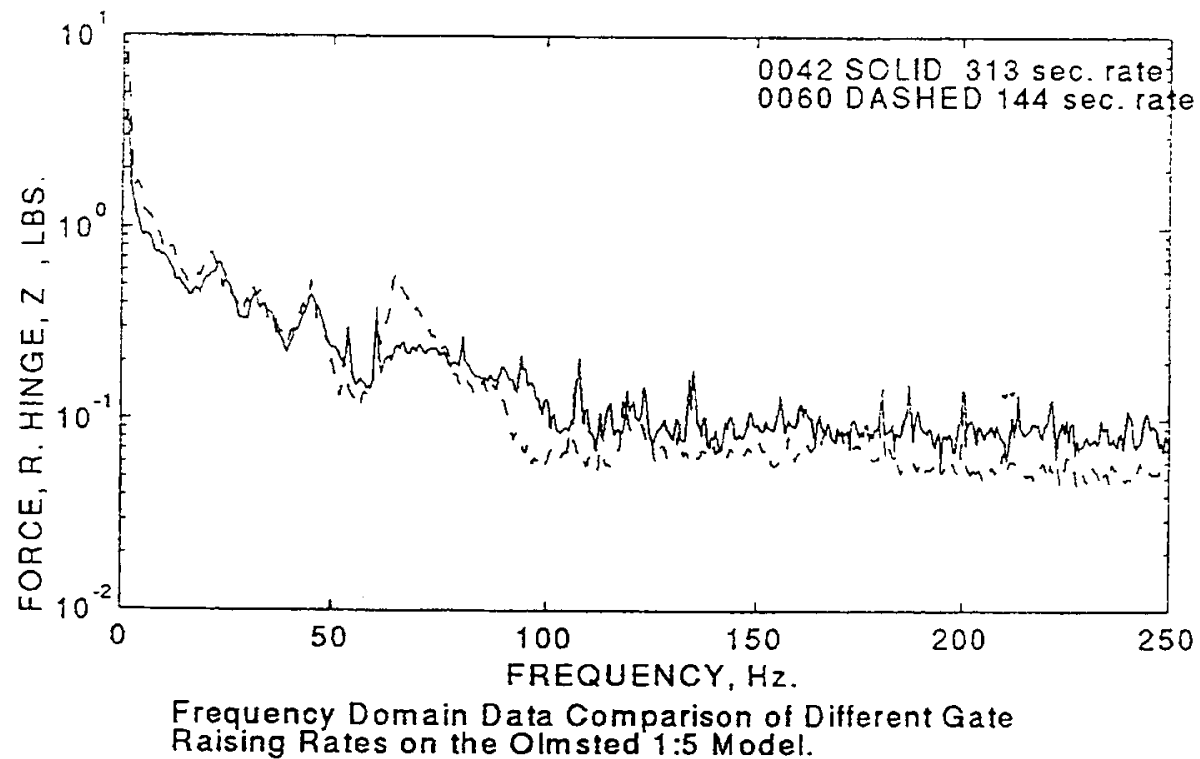

a. Right vertical hinge load

Figure 81. Effect of gate-raising rate on flow-induced dynamic responses for instrumented gate, 11-ft head difference, 3GG configuration

(Sheet 1 of 6 ) 


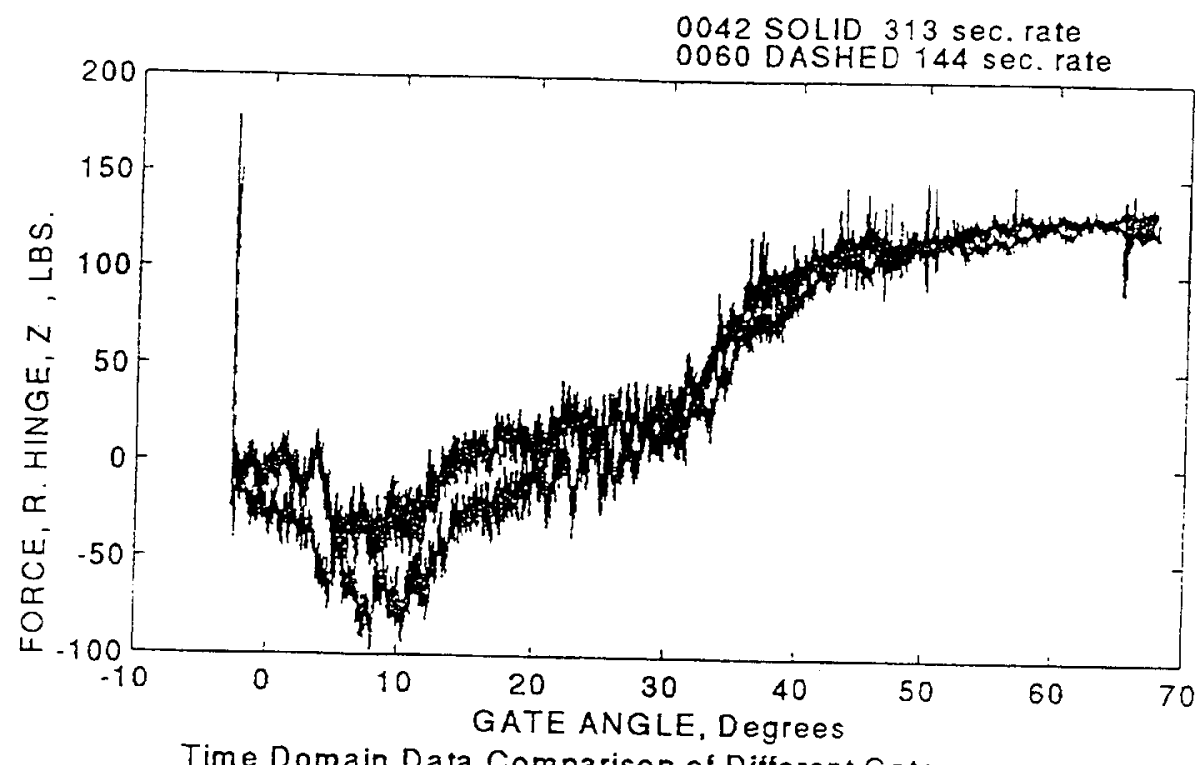

Time Domain Data Comparison of Different Gate Raising Rates on the Olmsted 1:5 Model.

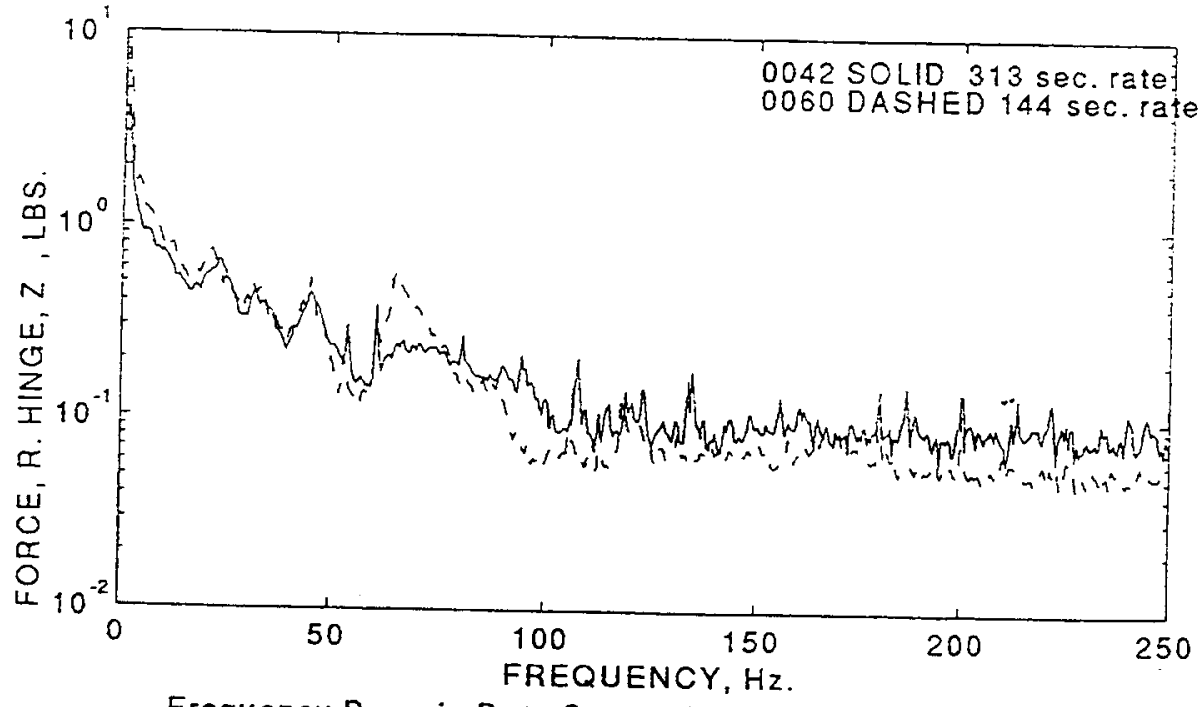

Frequency Domain Data Comparison of Different Gate

Raising Rates on the Olmsted 1:5 Model.

b. Right horizontal hinge load

Figure 81. (Sheet 2 of 6 ) 


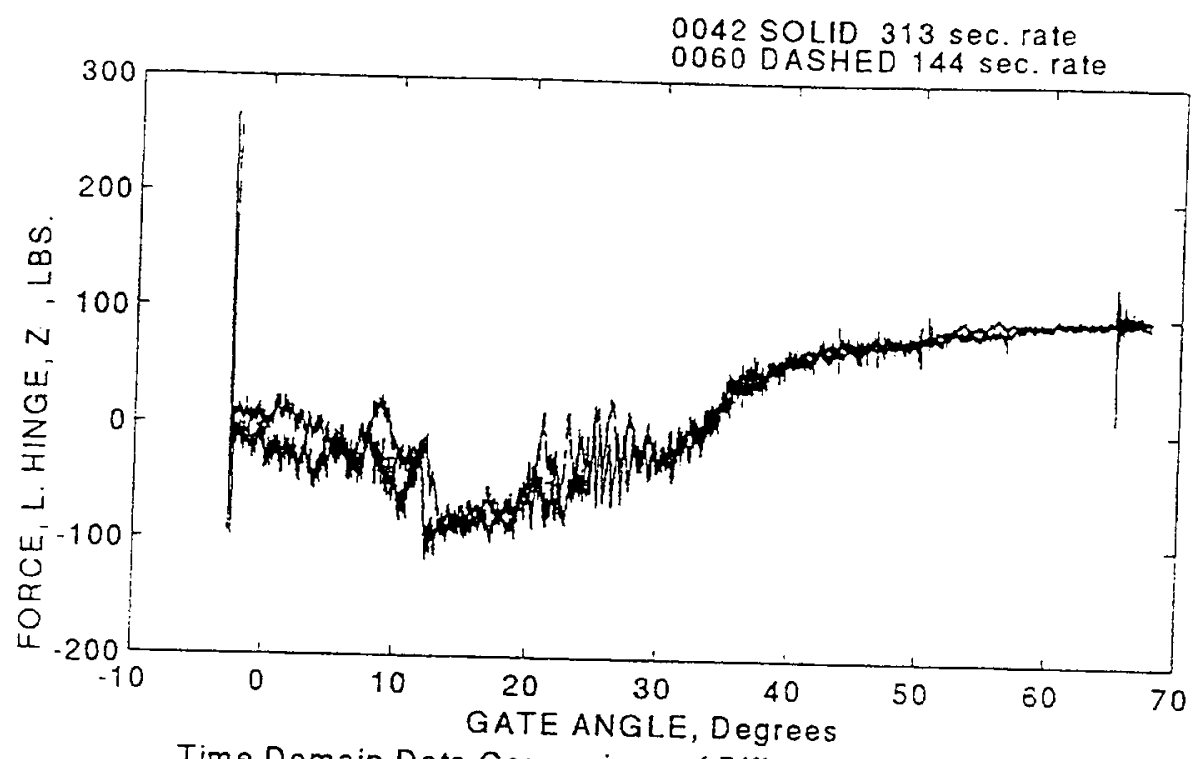

Time Domain Data Comparison of Different Gate Raising Rates on the OImsted 1:5 Model.

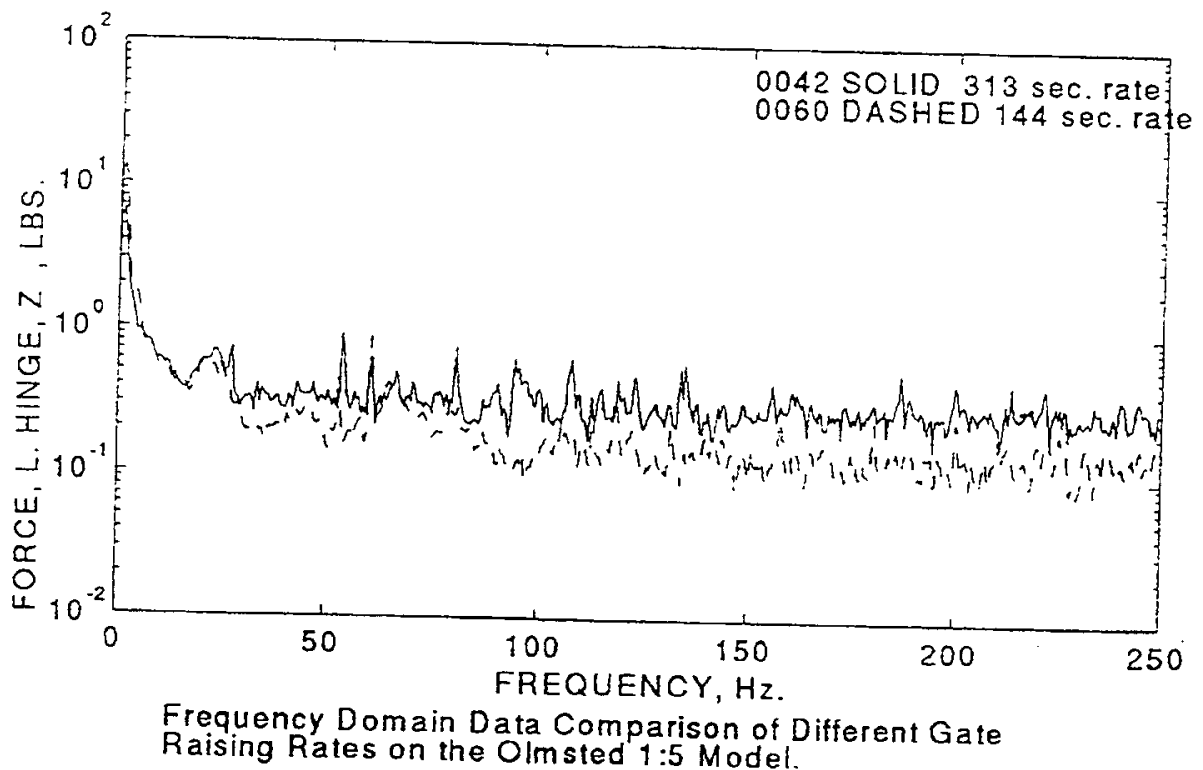

c. Left vertical hinge load

Figure 81. (Sheet 3 of 6 ) 


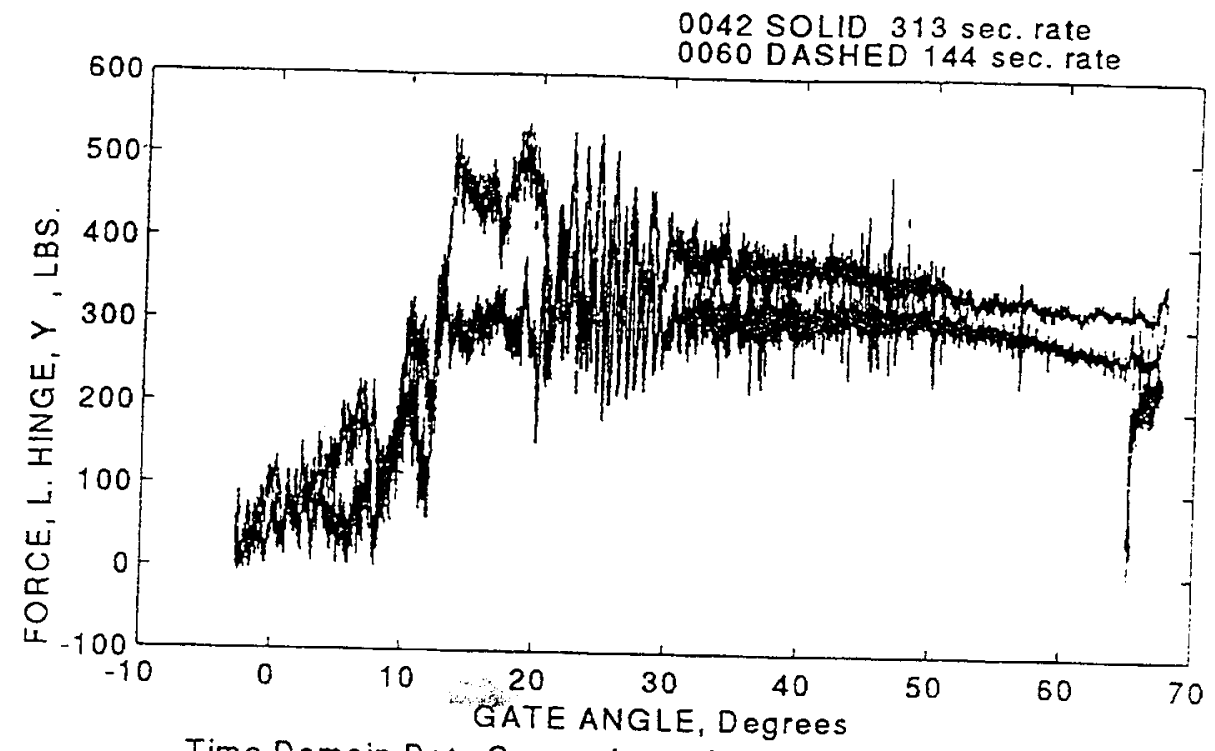

Time Domain Data Comparison of Different Gate

Raising Rates on the Olmsted 1:5 Model.

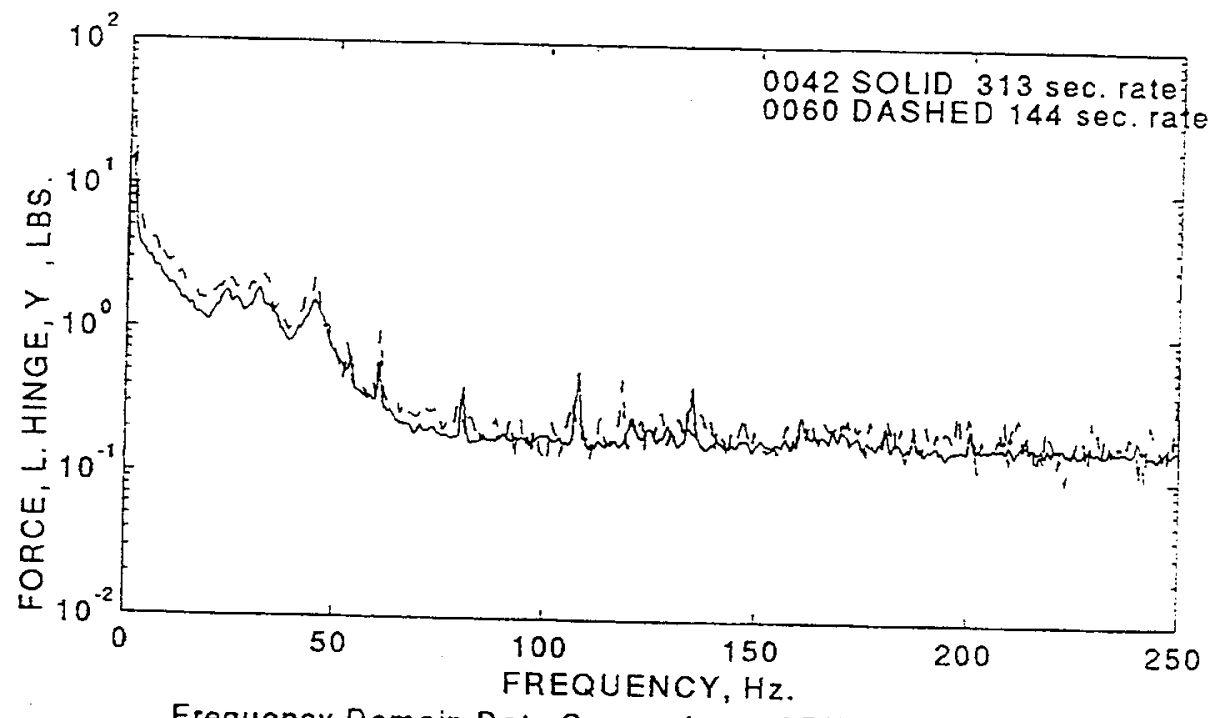

Frequency Domain Data Comparison of Different Gate Raising Rates on the Oimsted 1:5 Model.

d. Left horizontal hinge load

Figure 81. (Sheet 4 of 6 ) 


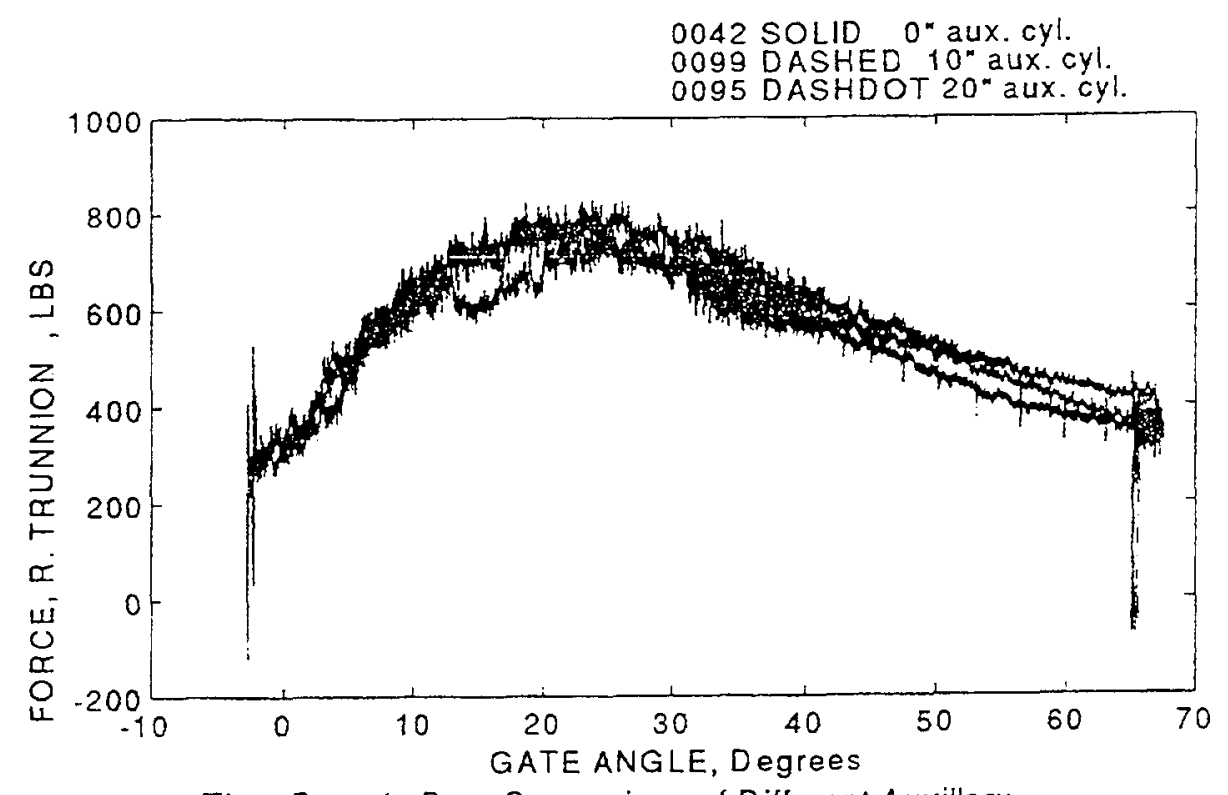

Time Domain Data Comparison of Different Auxillary

Cylinder Lengths on the OImsted 1:5 Model.

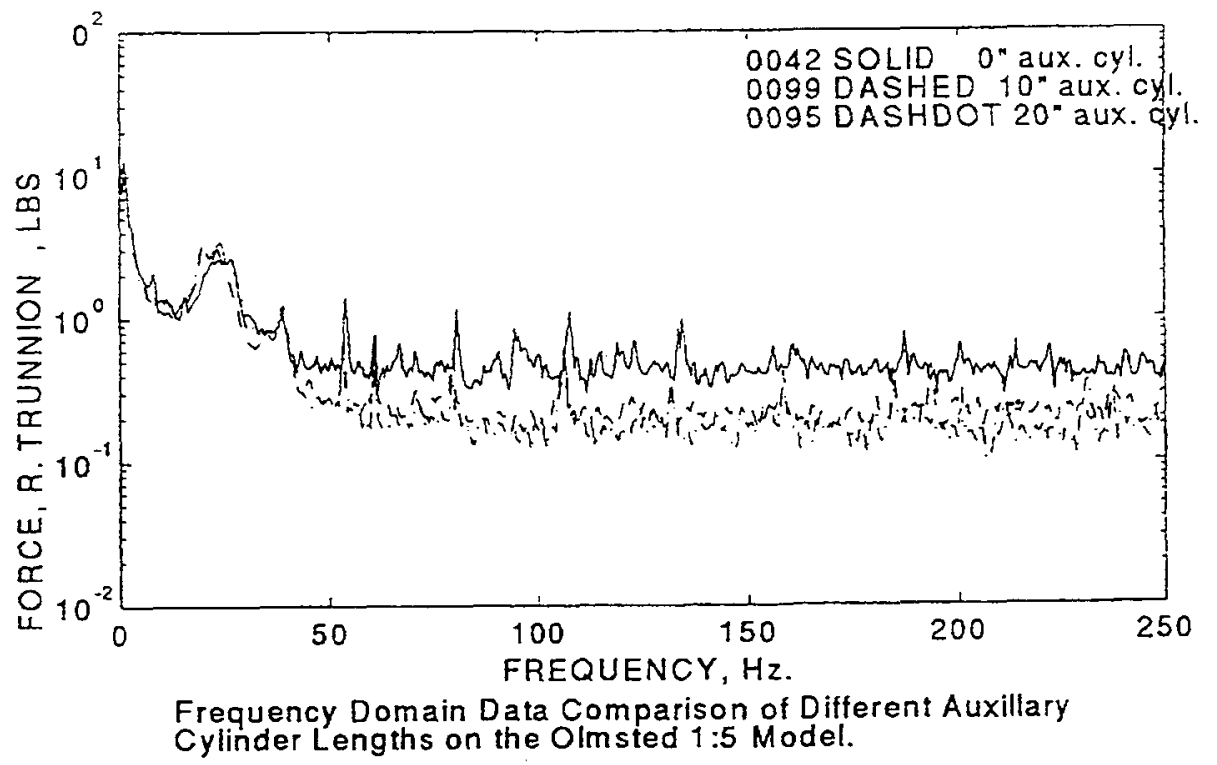

e. Trunnion load

Figure 81. (Sheet 5 of 6 ) 


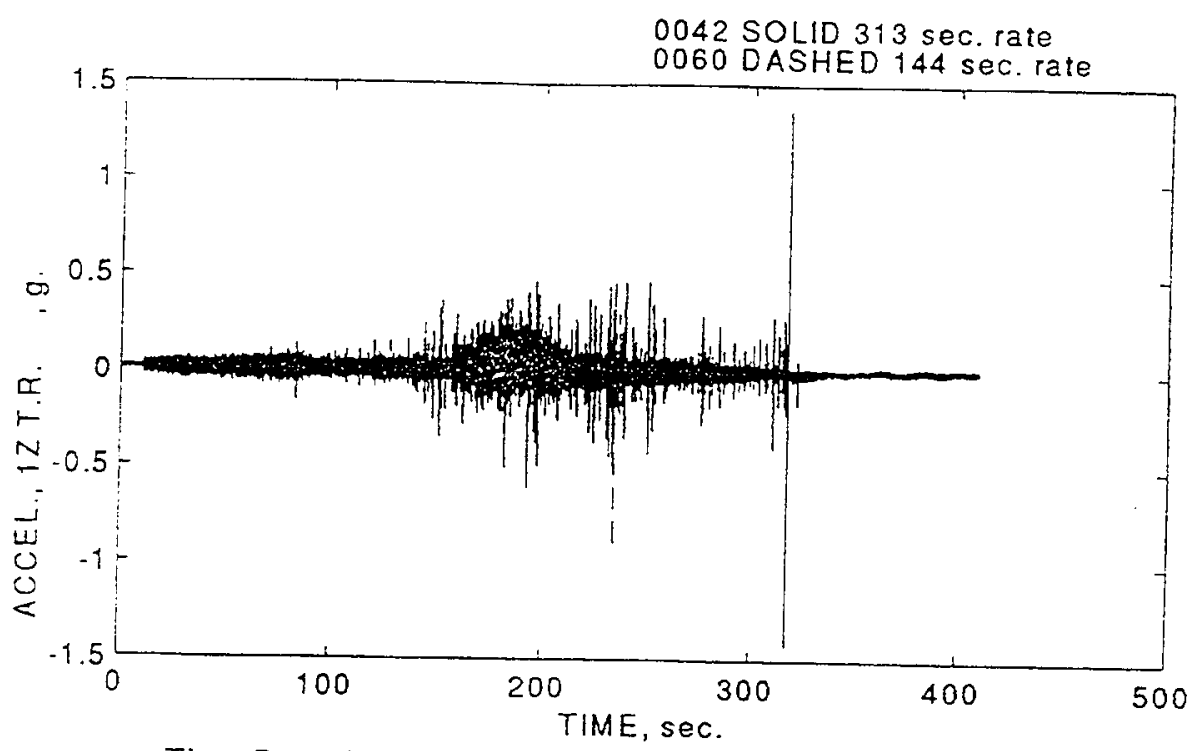

Time Domain Data Comparison of Different Gate

Raising Rates on the Olm sted 1:5 Model.

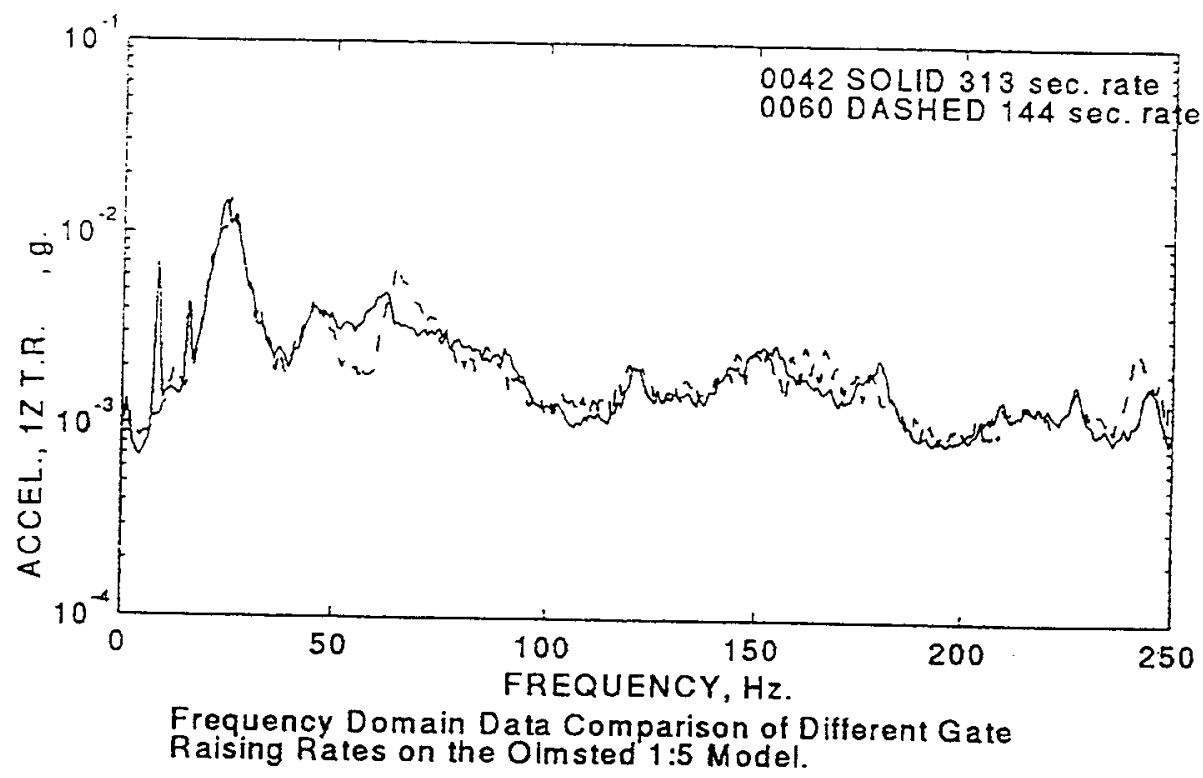

f. Acceleration 12 (top right position on gate)

Figure 81 . (Sheet 6 of 6 )

cases is presented in Table 15. The results that showed variations are printed in bold. As shown in the table, the dynamic changes in the reaction forces are not substantial due to the addition of prop blade on the system. 


\section{Observations}

For all practical purposes, the geometrically scaled model with hydraulic similitude relations is capable of predicting the prototype response pattern for the flow fields investigated in this report. As demonstrated previously in this chapter, the structural dynamic characteristics are preserved in the Froude model with the exception that the Froude law itself ignored the mass density-elastic modulus relationship. Although the effect of such modeling distortion is not completely understood, the error in responses due to such distortion is very minimal for the wicket investigated in this report. A variation in the measured gate response due to a change in prop-rod stiffness indicated the sensitivity of the prop rod on the global dynamic behavior of the gate. This observation essentially identifies the modeling error due not to meeting the Froude criteria in the hydraulic model. The hydraulic cylinder modeling was effectively used to simulate the prototype hydraulic lifting system. Effectiveness of the modeling technique for simulating the prototype hydraulic lifting system could not be verified at this stage until the prototype results are available. A comparison of the 1:5- and 1:25-scale models indicates that the overall response for the wicket is fairly independent of the stiffness of the lifting mechanism.

\begin{tabular}{|c|c|c|c|c|c|c|}
\hline \multirow{2}{*}{\multicolumn{3}{|c|}{ 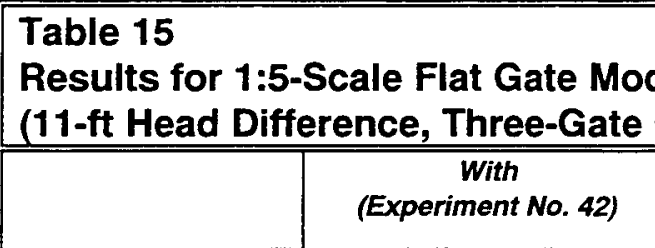 }} & \multirow{2}{*}{\multicolumn{2}{|c|}{$\begin{array}{c}\text { without } \\
\text { (Experiment No. 87) }\end{array}$}} & \multirow{2}{*}{\multicolumn{2}{|c|}{ Ratio }} \\
\hline & & & & & & \\
\hline Gauge Type & $\begin{array}{c}\text { Avg. } \\
\text { Reading } \\
(\bar{\mu})\end{array}$ & $\begin{array}{c}\text { Std. } \\
\text { Deviation } \\
(\sigma)\end{array}$ & $\begin{array}{c}\text { Avg. } \\
\text { Reading } \\
(\bar{\mu})\end{array}$ & $\begin{array}{c}\text { Std. } \\
\text { Deviation } \\
(\sigma)\end{array}$ & $\frac{(\bar{\mu}+\sigma)_{t * 42}}{(\bar{\mu}+\sigma)_{t * 87}}$ & $\frac{(\bar{\mu}+2 \sigma)_{t \# 42}}{(\bar{\mu}+2 \sigma)_{t \# 87}}$ \\
\hline Net hinge force, $\mathrm{z}$ lbf & 7.854 & 97.323 & 18.256 & 99.415 & 0.894 & 0.9328 \\
\hline Right hinge, $z$ lbf & 60.276 & 60.701 & 53.503 & 72.081 & 0.963 & 0.9191 \\
\hline Left hinge, $z \mathrm{lbf}$ & -52.422 & 76.073 & -35.247 & 68.467 & 1.239 & 1.1881 \\
\hline Net hinge force, y lbf & 321.127 & 187.389 & 379.715 & 146.267 & 0.967 & 1.0352 \\
\hline Right hinge, $y$ lbf & 140.755 & 134.503 & 180.296 & 122.584 & 0.909 & 0.9631 \\
\hline Left hinge, $y \mathrm{lbf}$ & 180.372 & 130.474 & 199.419 & 79.794 & 1.113 & 1.2293 \\
\hline Net shaft force, lbf & 920.691 & 426.523 & $1,122.493$ & 257.609 & 0.976 & 1.0831 \\
\hline Right trunnion, lbf & 476.84 & 299.093 & 604.025 & 200.382 & 0.965 & 1.0699 \\
\hline Left trunnion, lbf & 443.851 & 304.081 & 518.468 & 161.894 & 1.099 & 1.249 \\
\hline
\end{tabular}




\section{Wicket Gate Response}

\section{Background}

One of the most dangerous physical phenomena of hydraulic gates with overflow and underflow is self-excited vibrations (Goncharov and Semenkov 1974, Ishii and Knisely 1992). Self-excitation can be characterized by in-flow vibrations in which the vibrating gate and the excitation forces form a closedloop feedback vibration mechanism. With the development of such a flowstructure interaction, the gate generates an alternating pressure field that causes the gate to vibrate. The auto-oscillations in a self-exciting system cause radical changes in the nature and intensity of vibrations such that the random vibrations are replaced by almost periodical vibrations with constant frequency or amplitude, and their amplitude is further amplified. An amplification of the self-excited vibration may result from the coincidence of its frequency with the resonant frequency of the system. The potential destructive consequence of such a resonant mechanism could severely damage the system by developing dynamic instability or overburdening of responses.

Several investigators have studied the interaction of the hydrodynamic and structural responses to identify the causes and effects of flow-induced structural vibration problems in gates or gate-like structures (March and Elder 1992, Chowdhury, Hall, and Pesantes 1997, Ishii and Knisely 1992, Goncharov and Semenkov 1974, and Jongeling 1989). These studies have been conducted to investigate hydroelastic interaction between the flow and structure (Skladnev and Sheinin 1974, Jongeling 1989), to develop rational procedures for investigating highly complex and uncertain interaction of flow and structure (Goncharov and Semenkov 1974), to develop mathematical models of flowinduced vibration (Thang 1982, Treiber 1974, and Parkinson 1974), and to examine the vibration criteria or to determine the parameters of importance which effects the dynamic behavior of hydrodynamically loaded gate structures (Jongeling 1989 and Ishii and Knisely 1992). Formulation of hydrodynamic loads and other flow-induced physical phenomenon have also been studied by several investigators for describing the nature of flow fields and their behavior (Wille 1974, Treiber 1974, and Blevins 1990).

Blevins (1990) provides a generalized formulation for determining the flowinduced vibration of one-dimensional elastic structure. In his approach, the fluid 
forces consist of inertial force and drag force. The inertial force is caused by the buoyancy and the added mass effects of the flowing fluid. The drag component of the fluid force on the structure is the sum of the viscous drag and pressure drag produced by the relative velocity between the structure and the flow. Use of Blevins' formulation for computing the flow-induced vibration would be reasonably adequate if the fluid forces are accurately quantified for the specific flow and the structure. A valid representation of fluid force, particularly the added mass, is structure specific and depends on size and shape of the geometry, the boundary conditions, the flow-field, and the time-dependent vibration conditions. Therefore, experimental data are essential, even to use a simpler model for assessing its performance due to flow-induced vibration.

Potential flow models could be realistic and informative for obtaining a better representation of fluid forces (Blevins 1990). However, such estimation involves mathematically intensive and complex formulation with no guarantee of an increase in useful information without proper experimental validation (Vethamony et al. 1992, Parkinson 1974, and Thang 1982). A correlated simpler formulation, based on experimental verification, for an intricate flow-structure interaction problem could, however, be useful for broadening the understanding of the specific problem, identifying significant parameters, and determining the overall effects of variables and parameters. In the latter part of this report, a simpler model based on the Blevins (1990) formulation is developed for the Olmsted wicket.

Ishii and Knisely (1992) have studied the flow-induced dynamic characteristics of long-span model gates to determine the effects of operating conditions on the gate vibrations. The model gate was flat-bottomed with a simple rectangular shape. In their study, they related the effects of fluid behavior on the vibration of the gate by two nondimensional parameters: added mass of water and the fluid-excitation coefficient (negative damping coefficient). It was shown that a particular range in the gate opening could cause self-excited vibration on the gate. Also shown are the effects of different flow conditions, such as submergence of the downstream side or free discharge, on the dynamics of the gate. A flow-rate variation for a submerged downstream condition could induce a violent self-excited streamwise vibration known as vortex-induced vibration on the system. Parameters that influenced the vortex-induced vibrations were gate openings, gate vibration frequency, head difference between the upstream and downstream sides, and the gate width.

Several other experimental investigations also indicate a great variety of unpredictable dynamic characteristics with uncertain behaviors depending on numerous variables, ranging from flow conditions to the structural size and shape (Goncharov and Semenkov 1974, Skladnev and Sheinin 1974, and Parkinson 1974). The unique capabilities of experimental investigation make it the best candidate for understanding the complicated nature of interaction between the flow and structure. In the Olmsted wicket studies, therefore, the need for experimentation could not be overemphasized without sacrificing the understanding of the fundamental problem. 
A systematic investigation of flow-induced vibration of a single oscillator type model consisting of a vertical stiff gate plate with flow underneath was conducted by Jongeling (1989). The primary aim of this investigation was to establish the hydraulic and structural conditions at which in-flow vibrations occurred and to identify possible vibration domains. More intensive studies were performed at stationary hydraulic conditions (fixed gate position) to determine the inflow vibration mechanism that caused the gate to vibrate. A number of measures were taken to prevent damaging vibration in gate structures by: (a) changing the flow pattern by reshaping the structure, (b) changing the stiffness/mass combination such that the natural frequency is changed, and (c) increasing the structural damping. He showed that the inflow vibrations could be prevented by beveling the upstream face or the downstream face of the gate edge.

Similar effects on reducing in-flow vibration by changing the flow pattern due to reshaping the structure were observed during the Olmsted wicket model studies (Chowdhury, Hall, and Pesantes 1997). It was shown that the rounded tubular edge of the gate, instead of a sharp edge, at the flow-separation boundary reduced the vortex shedding excitation in the 1:25-scale flat gate (USAEWES 1992, Chowdhury, Hall, Pesantes 1997). Like the Jongeling's (1989) test methodology, WES examined the gate vibration in two stages. In the first stage, the vibration domains during a full-range experiment of the wicket were identified for various gate and operating configurations, and in the second stage, the gate vibration at fixed position was intensively studied for determining the vibration mechanisms of the wicket models.

In the Olmsted wicket model study, operational and modal dynamic parameters were extracted to understand the vibration mechanism and identify any potential resonance problems in the system. Operational and modal dynamic behavior analysis has been extensively used by different researchers to detect the causes of excessive vibration and improve design quality (Neyrinck et al. 1992, Mouch and Myers 1991, and Tonosaki and Nakada 1994). The ODS approach has the capability to analyze the system during operation such that the real operating forces, vibrations, and deformations are well preserved in the measured dynamics. A dominant peak at a particular frequency can occur due to a structural resonance and/or due to a corresponding peak in the active force spectra. A comparison of ODS with real mode shapes permitted identification of the reason for such a dominant peak occurring in a response spectra.

\section{General Outline}

A wide range of experiments were conducted in the model to measure the applied loading, physical conditions, and dynamic characteristics of the instrumented gate due to various operating conditions. These experiments were conducted to measure the forced response of the hydraulically lifted instrumented wicket during lifting as well as at fixed (parked) positions. The measured responses included both the input applied loading history and the 
output reaction and response history. Detailed information regarding the measured responses, including the transducer locations, calibrations, and functional descriptions are presented in Chapter 3 of this report.

\section{Overall plan}

A four-step plan was executed in this investigation to acquire data for analyzing the dynamic performance of the Olmsted wickets. These are:

a. Full-range vibration experiments.

b. Fixed gate vibration experiments.

c. Modal experiments - dry.

d. Modal experiments - wet.

Full-range experiments for all possible operational conditions, based on the current operating schedule for the Olmsted wickets, were used to identify the critical configuration for the hydraulically lifted wicket. A critical configuration provided the maximum flow-induced dynamic reactions for the test boundaries. Scanning the time-history responses for a full-range experiment identifies the gate orientation corresponding to the critical configuration. Further experimentation of the model at the critical arrangement provided data for determining the vibrational mechanism of the operating gate.

Second-round experimentation at the critical orientation and configuration was conducted for fixed gate positions. Operational dynamic behavior in these critical arrangements was examined to determine the resonance in the system. A resonance can exist in the system if one of the dominant peaks in the flowinduced response spectra of the gate coincides with one of the natural vibrating modes of the wet wicket. This required the measurement of dry and wet natural characteristics of the system.

Dynamic characteristics of the dry and wet gate were extracted using the SLDV system. All modal experiments were conducted for a fixed orientation of the gate. A wet gate refers to an operational state for which the flow conditions are preserved in the system during the measurement. A transient vibration of the gate in running water due to excitation from a known source was used to extract the wet modal parameters of the wicket. "Wet mode" terminology used in the literature refers to the natural characteristics of a structure in which the mechanical properties and the fluid actions are considered during measurement (Bishop and Price 1976). 


\section{Experimental boundaries and flow configurations}

The operational sequence for regulating the Olmsted navigational pass was preestablished by the ORL. Based on the current operational schedule, a wide range of conditions has been investigated to determine the most severe configuration for the 1:5-scale operating wicket. As presented in Table 16, a total of 10 test sets for two pool conditions and four gate configurations were recorded in the 1:5-scale model. The pool elevations shown in the table are in reference to the Olmsted levels and were scaled in the model based on the linear geometric scaling factor. Both of the five-gate gap configuration experiments were recorded for a reduced head difference of $4 \mathrm{ft}$ instead of $7 \mathrm{ft}$ as outlined in the original proposal. A 7 - $\mathrm{ft}$ head difference in a five-gate gap configuration could not be attained in the flume because of insufficient capacity of the hydraulic pumps. This change was in agreement with the revised wicket operational elevations for the boat-operated wicket. Table 16 also presents the gate positions, gap configuration, and the pool elevations corresponding to each experimental case.

A gap in the flume indicates an open space in the dam due to lowering of certain number of wickets. A five-gate gap creates a five-gate width bay with maximum flow through the flume, while a one-gate gap provides the least flow. Each gap type produced three operating configurations based on the position of the test gate on the channel. An "end gate" refers to a configuration in which the instrumented gate is located at the edge of an opening with n gates. A "gap gate" represents a configuration in which the wickets adjacent to the test gate are in the raised position. A lone gate indicates that the test gate is centered in an opening of $(2 n+1)$ gates, where $n$ is an integer number, and $z 1$.

At the bottom part of Table 16, also shown are the gate numbers and their respective positions in the flume. As shown by the arrow, gate No. 6 is the instrumented gate and the remaining are dummy gates.

Figures 82 through 84 show three different types of flow configurations in the 1:5-scale Olmsted model. Figure 82 displays a one-gate gap flow condition in which the instrumented gate (No. 6) rests down at a position of $-3 \mathrm{deg}$. This setup shows the condition of the gate immediately before initiation of full-range data recording. During the data acquisition in a full-range experiment, the gate would be raised to close the gap. Figure 83 presents a two-gate gap flow configuration with an end gate arrangement. In this figure the instrumented gate is at a 24-deg position, an intermediate orientation in between the down and the up positions. A three-gate gap flow case with a lone gate arrangement is shown in Figure 84. In this case, the instrumented gate is held at a $65-\mathrm{deg}$ position at the end of data acquisition. 


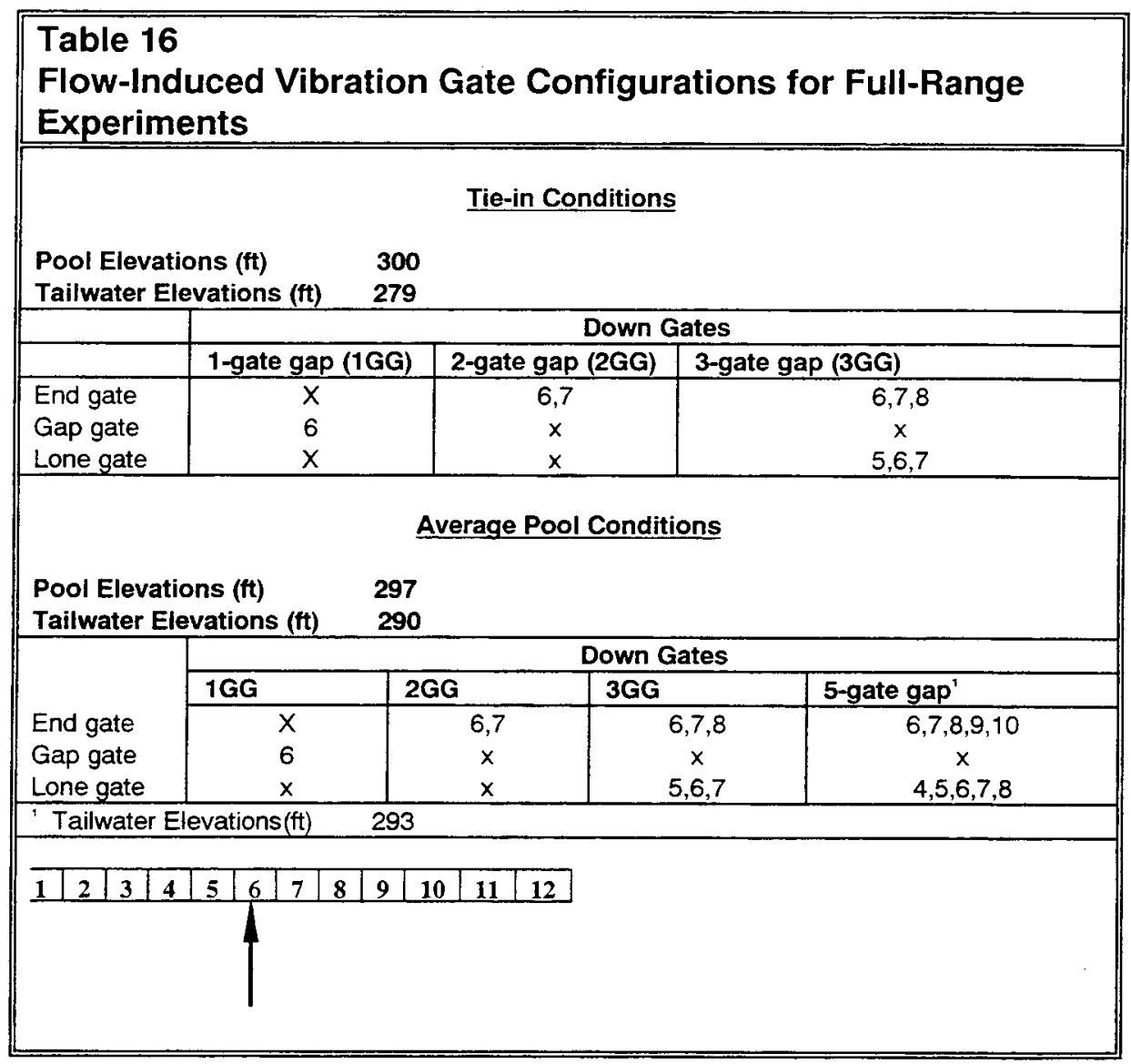

\section{Flow-Induced Response}

The data were recorded as the gate was raised from down to the up position using the automated hydraulic lifting system. In all cases mentioned here, the lifting cycle lasted for $410 \mathrm{sec}$. Out of the total hydraulic actuator operating period, actual time elapsed for raising the gate from a position of -3 to $65 \mathrm{deg}$ was $323 \mathrm{sec}$. This raising time was a scaled-down magnitude of the estimated prototype lifting time of $12 \mathrm{~min}$. The type 2 blade on the steel prop rod was connected to the gate during these experiments. As mentioned earlier in the data acquisition section of this report, a zero-reference was established at the beginning of a day to initialize the transducers installed on the gate. All experiment numbers were sequentially ordered by dates.

Table 17 presents the experiment numbers corresponding to the various flow configurations considered in this investigation. Two identical experiments were repeated for each test case for measuring the consistency and repeatability of the measured data. This was done by overlaying the respective force time-history of 


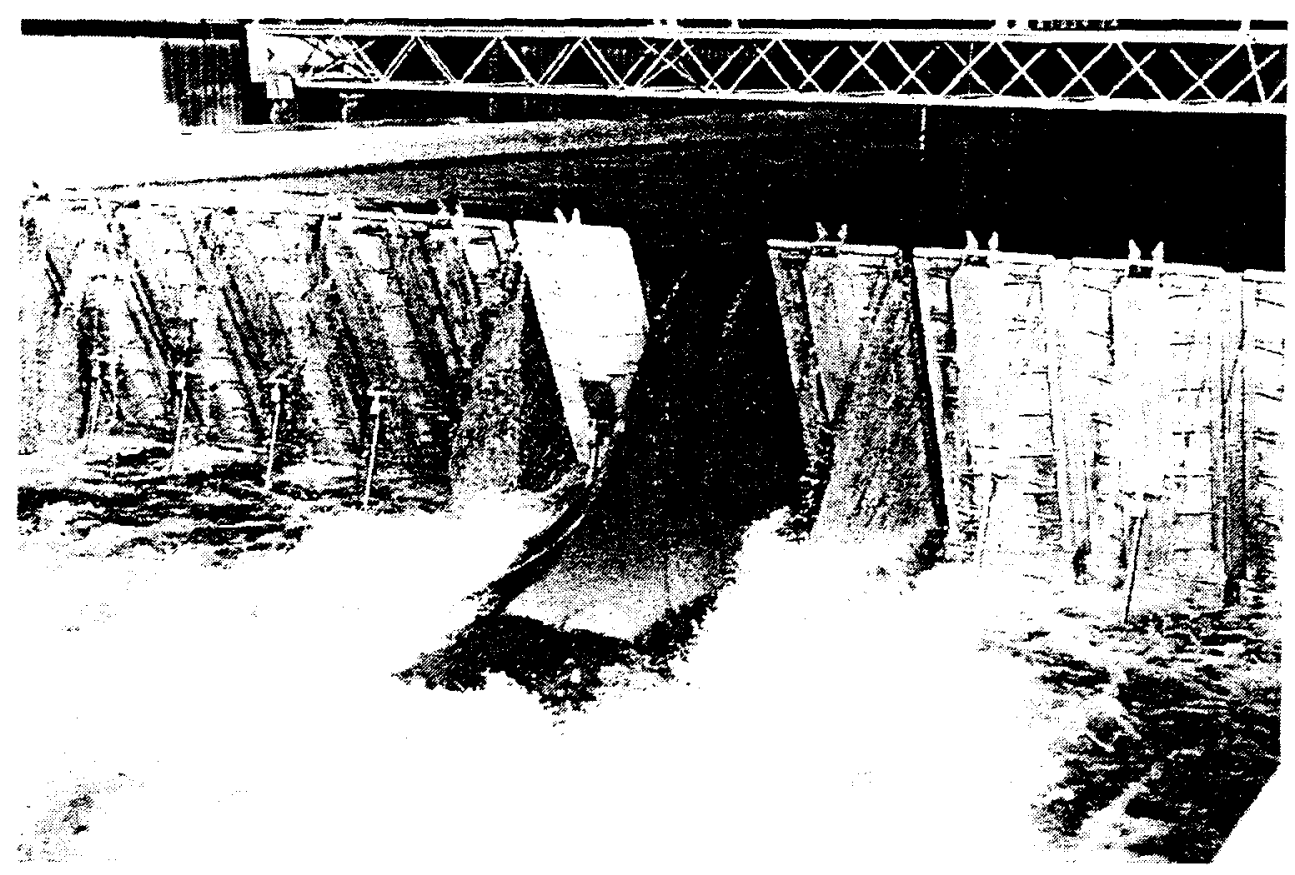

Figure 82. A 1GG flow configuration with instrumented gate at down position (Pool elevations: $300 \mathrm{ft}$ and $279 \mathrm{ft}$ )

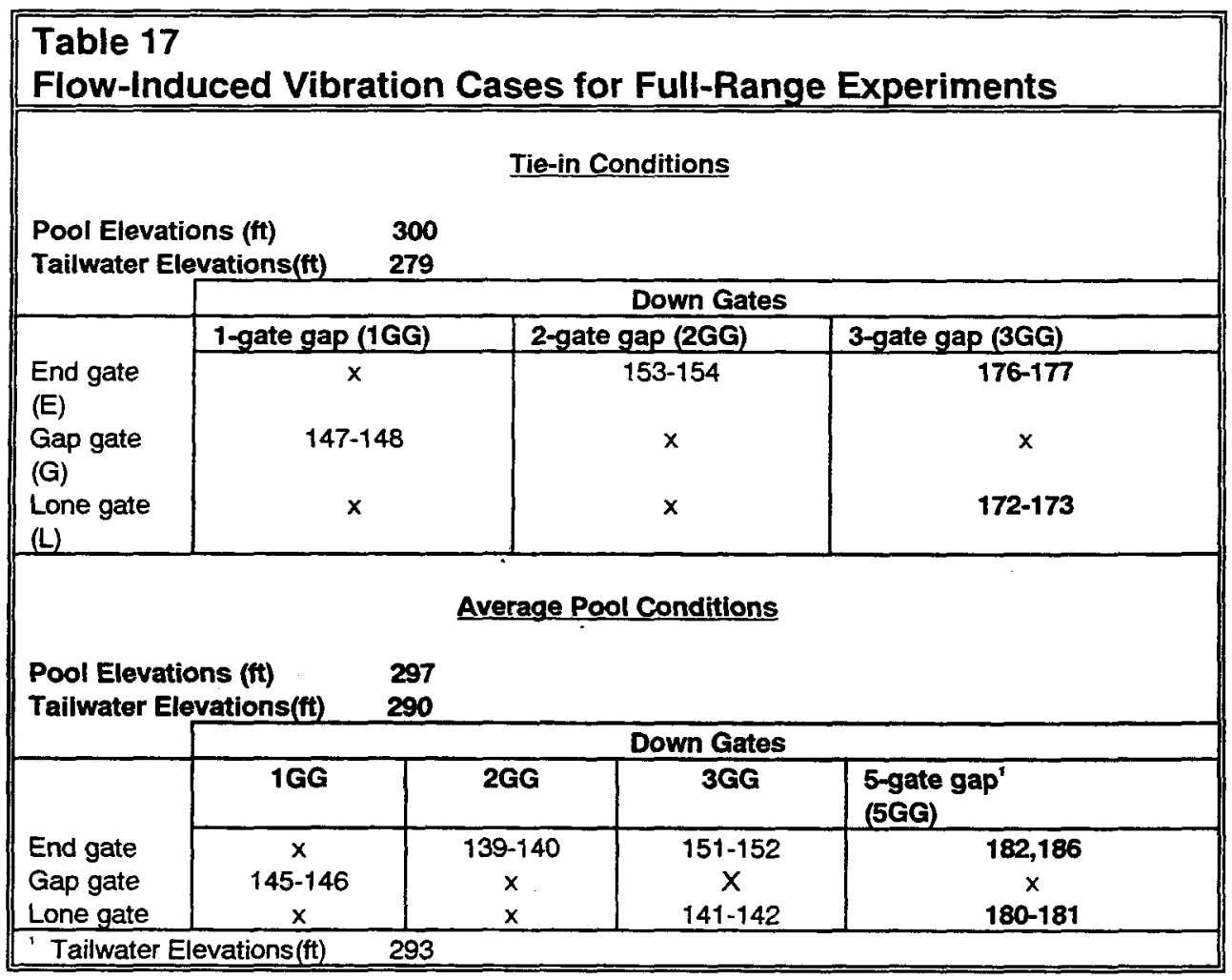




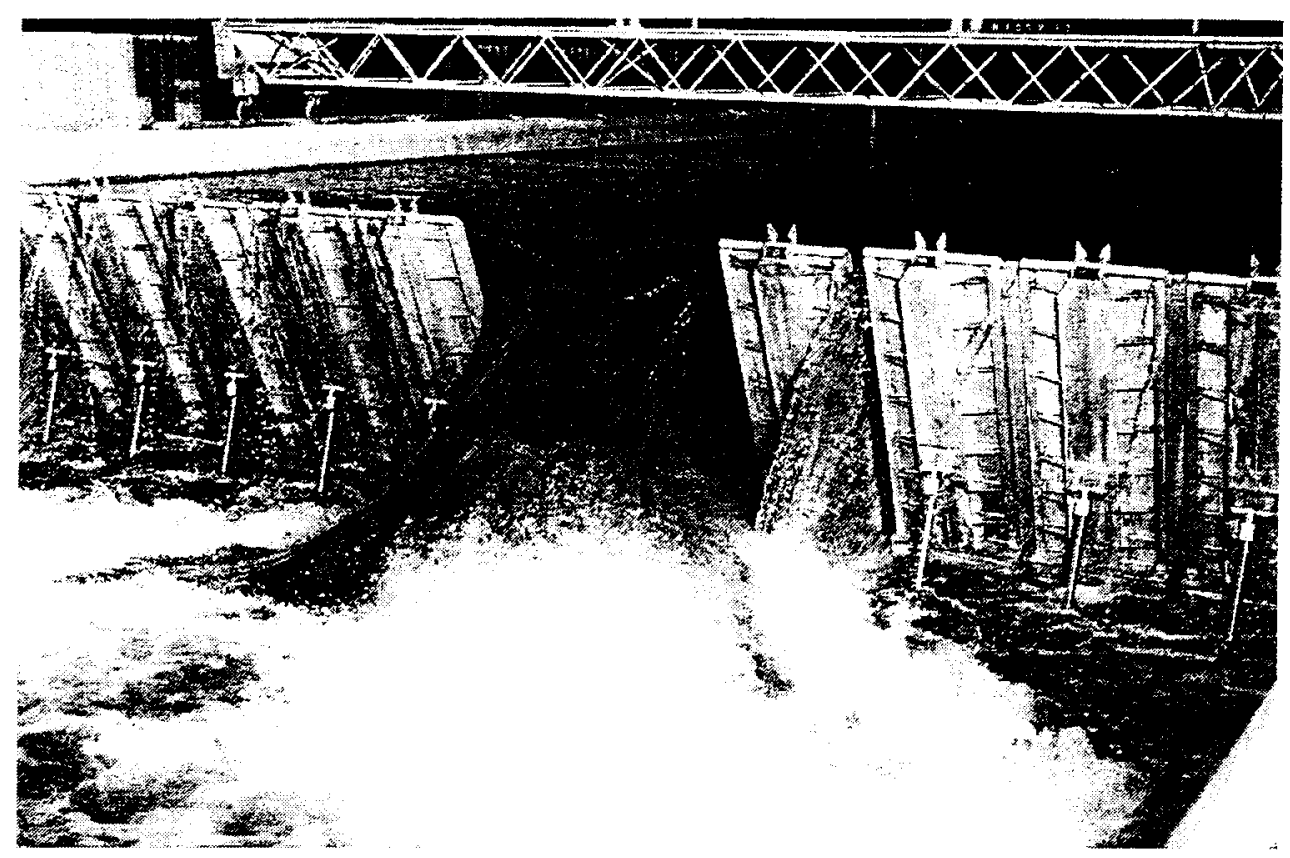

Figure 83. A 2GG flow configuration with end gate arrangement, instrumented at 24-deg position (Pool elevations: $300 \mathrm{ft}$ and $279 \mathrm{ft}$ )

identical experiments and observing their deviation from each other. It was noticed that the experiments were redundant and matched well with each other, as observed during a comparison of the time-history responses for the eight force channels (four bottom hinge reactions, two trunnion forces, and two ball forces) installed on the gate. Therefore, only one of the experimental data was used in the report for further analysis and investigation. Note that no prop-rod axial load data were recorded for the remaining experiments following number 142. A gap in recorded information for the prop rod was due to a malfunctioning of the transducer installed in it. The defective prop-rod transducer was not replaced for the rest of the study.

During the experiments, no auxiliary oil column was used to increase the oil volume in the cylinder chamber of the hydraulic lifting system. As mentioned in the previous chapter, a minimum volume of oil column in the hydraulic system simulated the dynamics of the most critical position of the wicket. All channels sampled data at a rate of 500 samples/sec/channel, except as noted otherwise. The sampled data were amplified and filtered at $250 \mathrm{~Hz}$ during the digitization process. Acceleration data for all tests succeeding test number 154 were sampled at 1000 samples/sec/channel and filtered at $500 \mathrm{~Hz}$. 


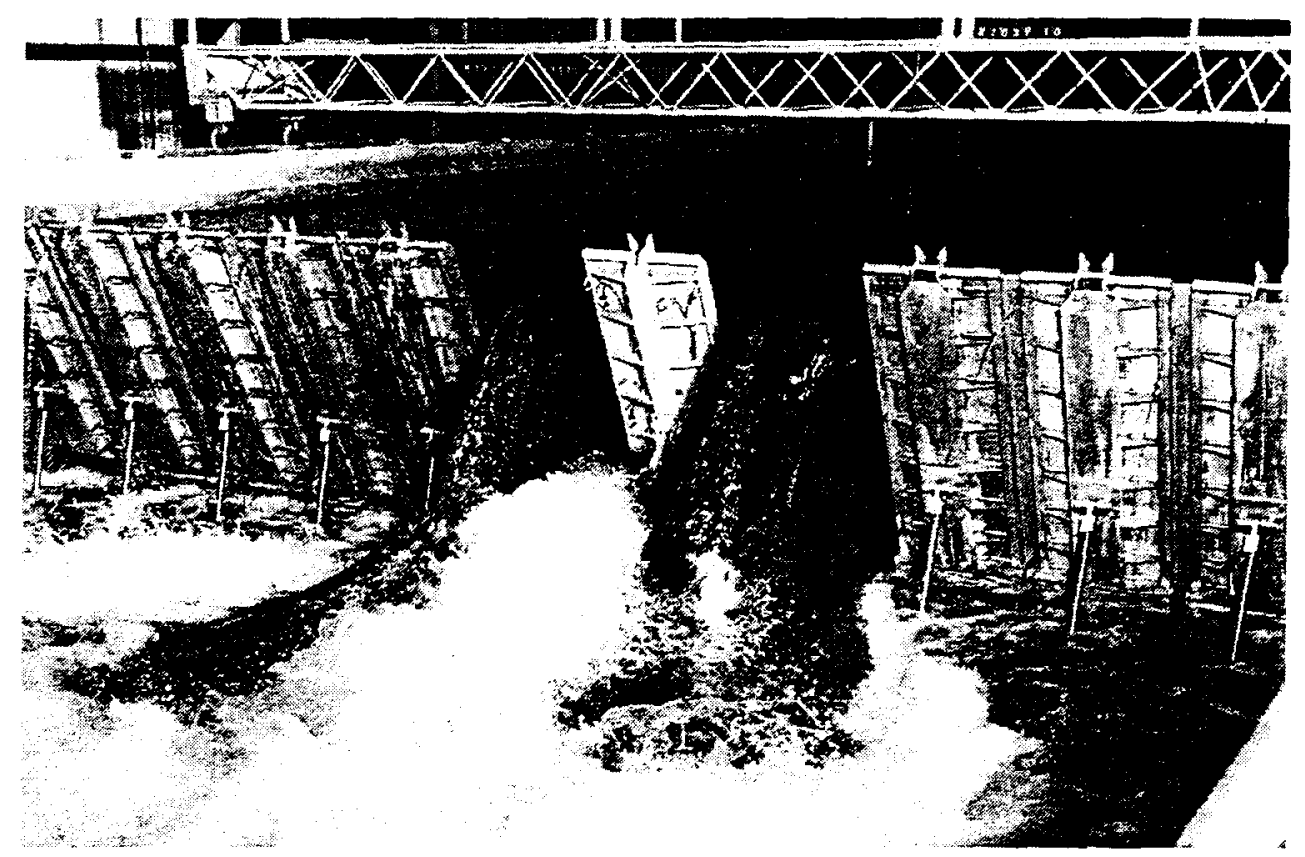

Figure 84. A 3GG flow case with lone gate arrangement, instrumented gate at up position (Pool elevations: $299 \mathrm{ft}$ and $279 \mathrm{ft}$ )

\section{Experimental results}

\section{1- ft head difference}

\section{Reaction forces}

Results for the 21-ft head-difference pool elevations are compared in Figures 85a through $\mathrm{x}$. In these figures, respective channel responses for four conditions are overlaid as a function of gate angle. Figures 85 a through $d$ show the time-domain orthogonal reaction forces for both of the bottom hinges. A schematic diagram of the model showing the directional sense of the reaction forces is presented in Figure 86. As shown in the figure, a positive horizontal force ( $y$-direction) results due to the pulling of the gate away from the sill. A positive vertical reaction (z-direction) resists the downward motion of the gate. Figures $85 \mathrm{a}$ through $\mathrm{d}$ indicate that the reaction forces for both hinges are asymmetrically distributed and their directions are reversed as the gate is raised from the down to the up position.

A sum of hinge reaction forces for both directions along with their corresponding PSD are shown in Figures $85 \mathrm{e}$ through $h$. During the onset of the raising cycle, negative $z$ - and positive $y$-reaction forces resist the thrust of rushing water which tend to pull the gate bottom away from the sill. This early overturning mechanism about the pivotal shaft support reverses its direction as the gate is raised. At about 16 to $40 \mathrm{deg}$ (Figure 85e) above the down position, the positive reaction forces act upon the gate which tend to push the gate bottom toward the sill (Figure 86.) 
1:5 Olmsted Model

Tests: $148,154,177,173$

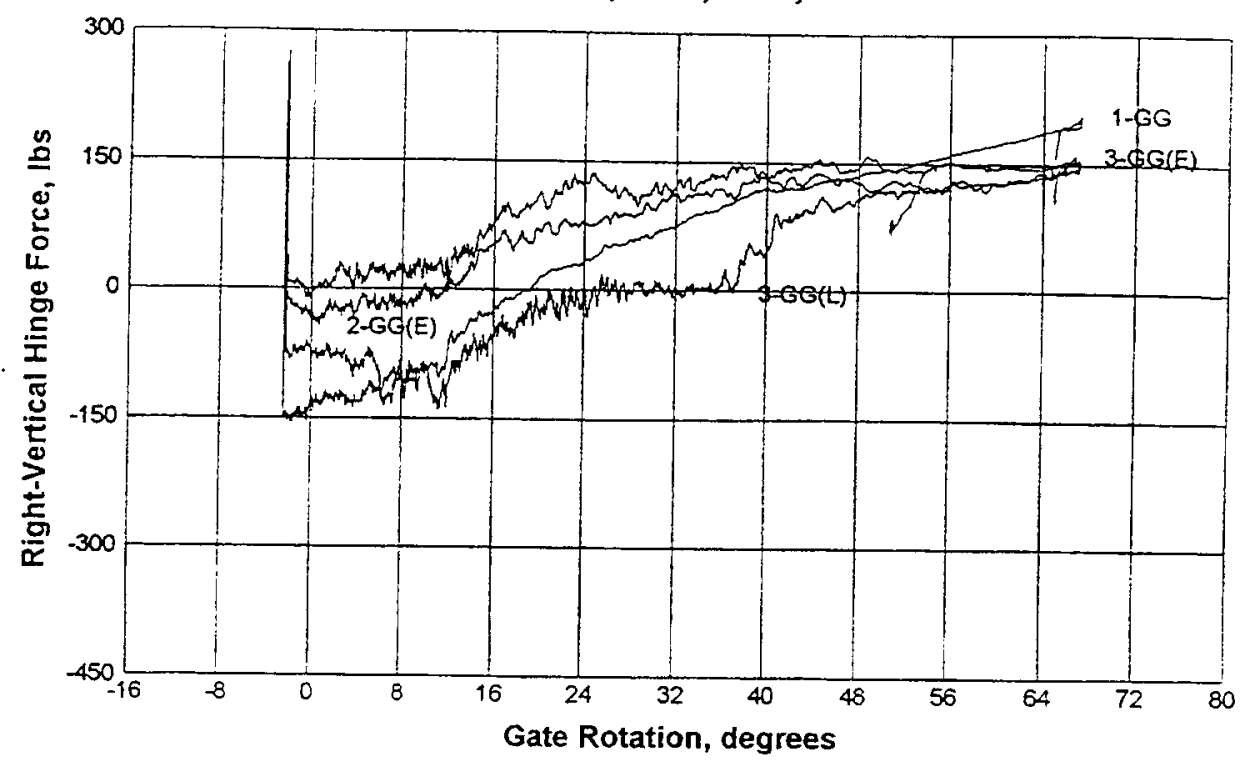

a. Right vertical hinge load

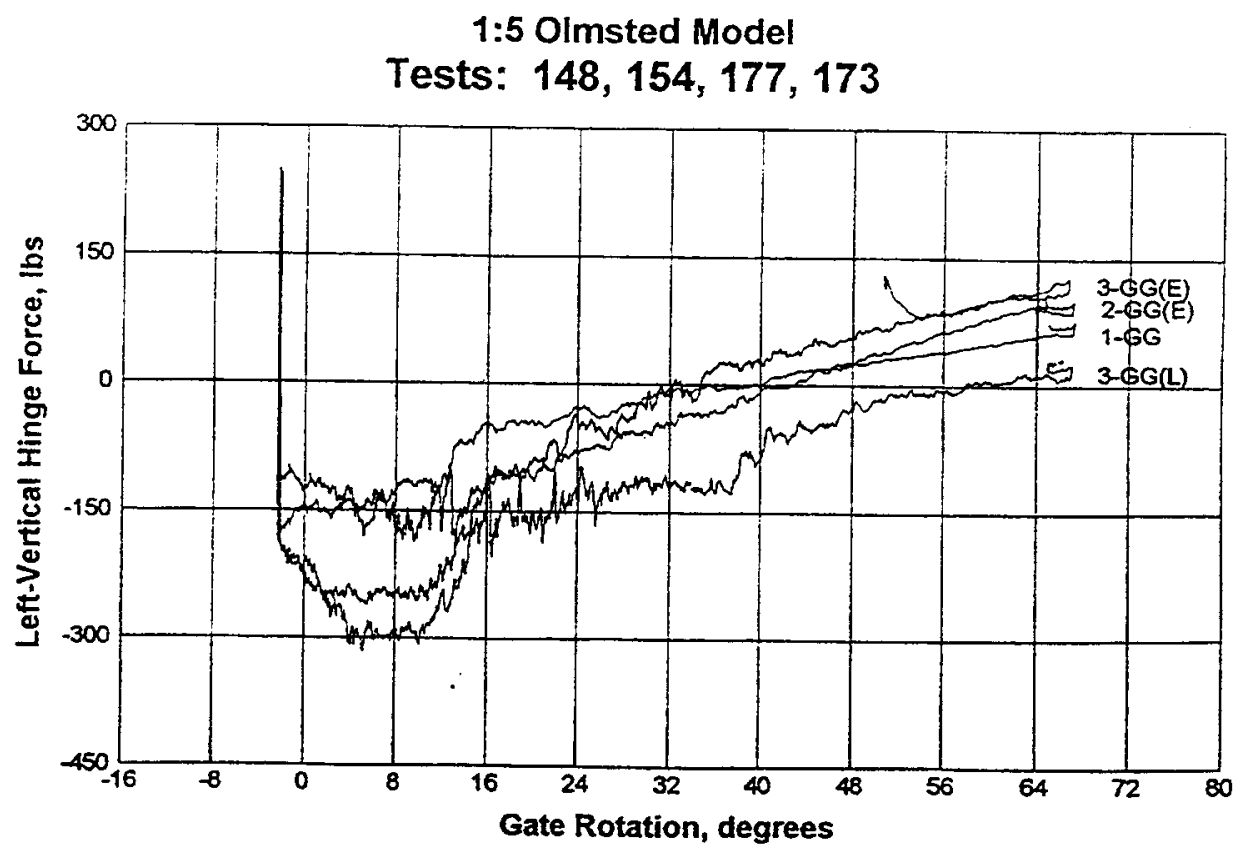

b. Left vertical hinge load

Figure 85. Comparison of responses over full range of operation for various gate configurations, 21-ft pool difference (Sheet 1 of 12) 


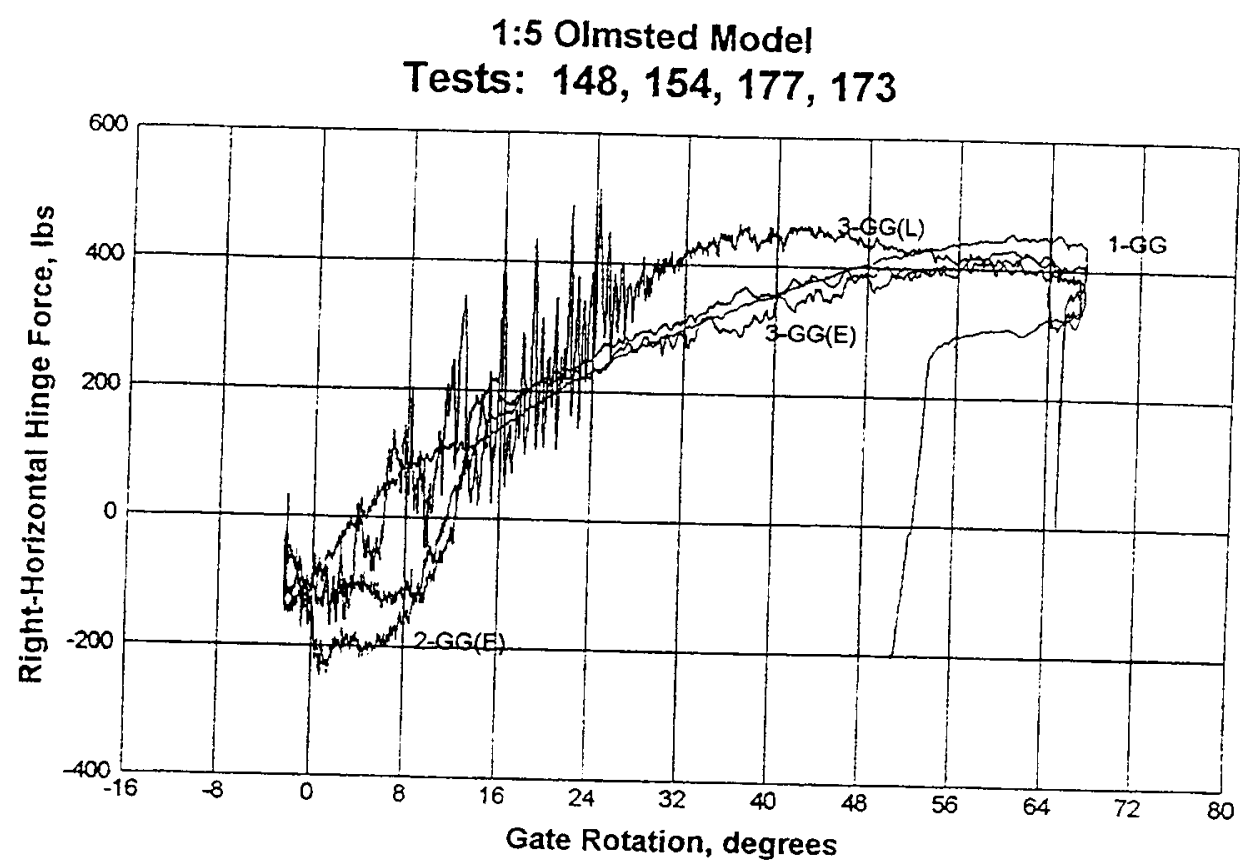

c. Right horizontal hinge load

1:5 Olmsted Model

Tests: $148,154,177,173$

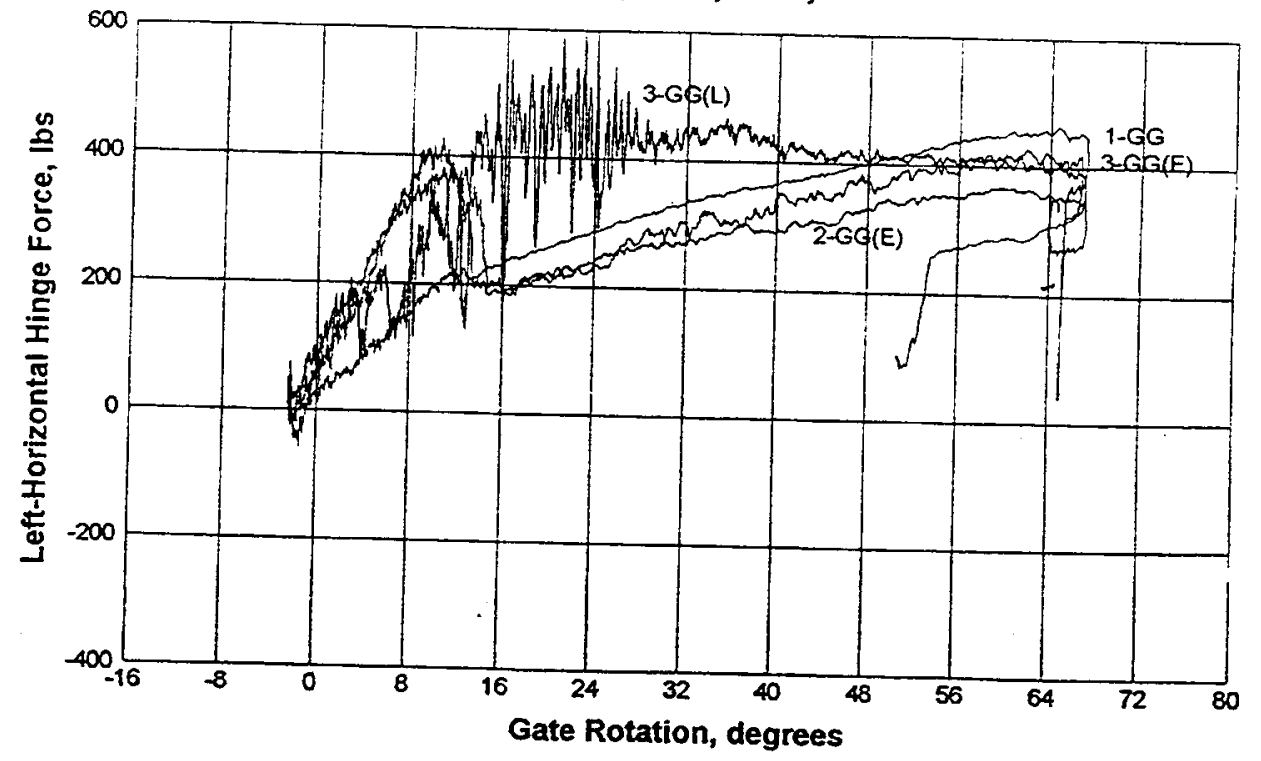

d. Left horizontal hinge load

Figure 85. (Sheet 2 of 12) 


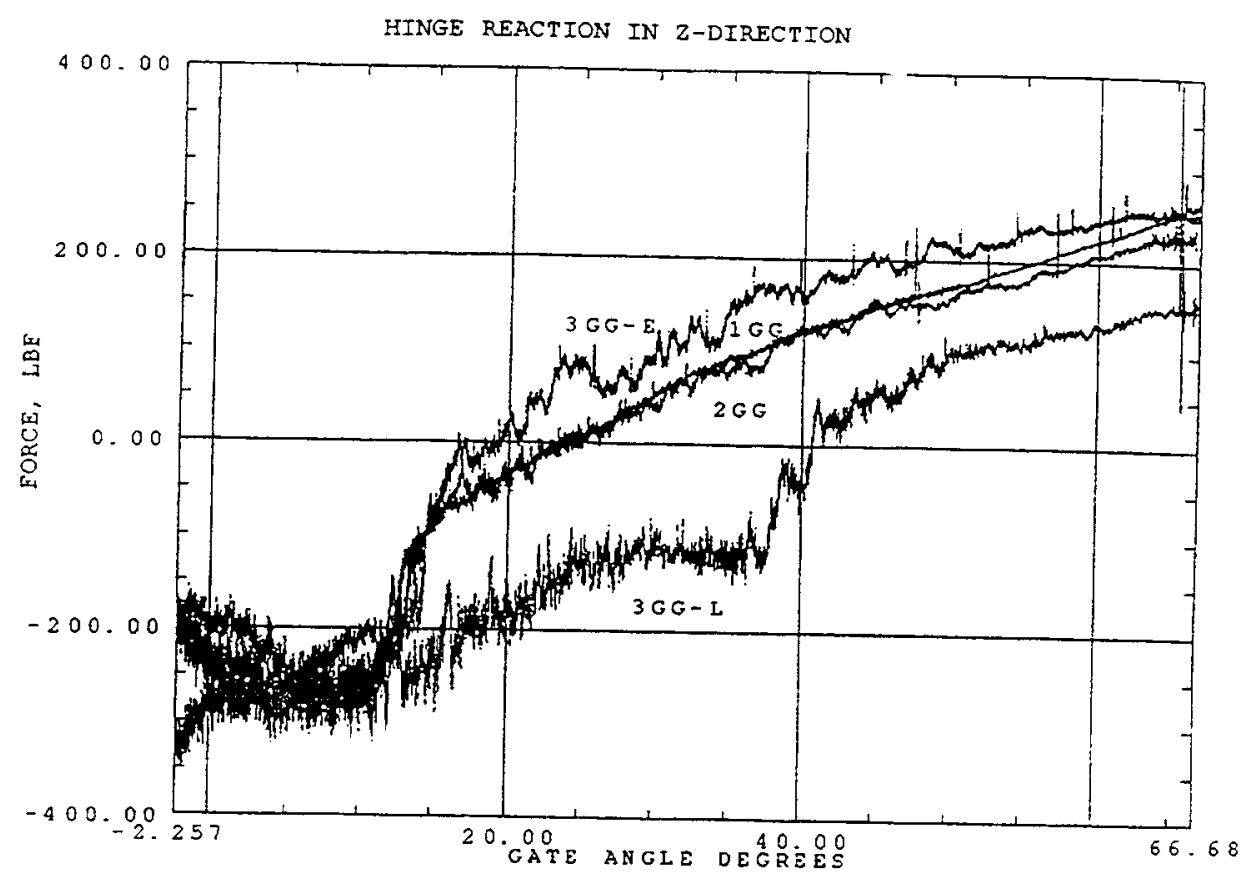

e. Time-domain total vertical hinge load

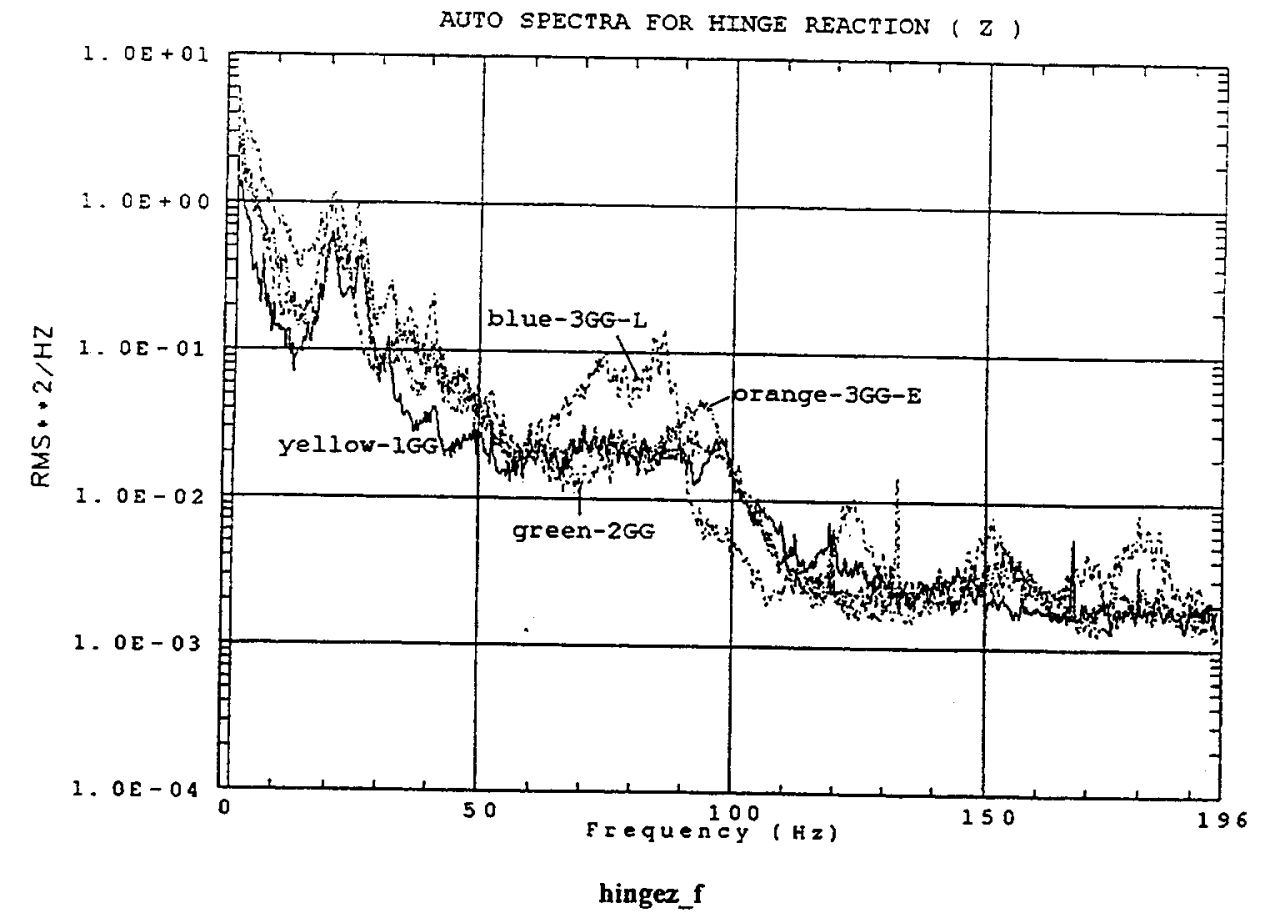

f. Frequency-domain vertical hinge load

Figure 85. (Sheet 3 of 12) 


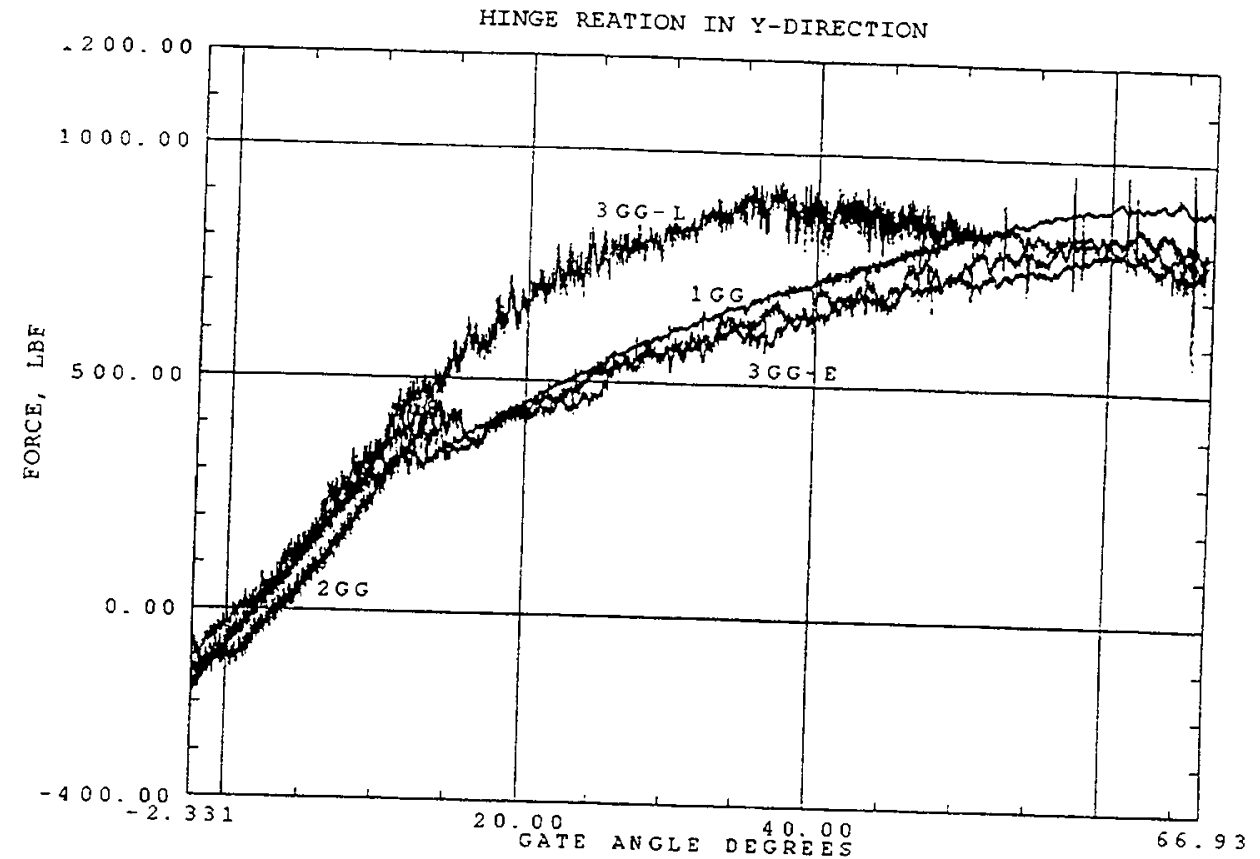

g. Time-domain total horizontal hinge load

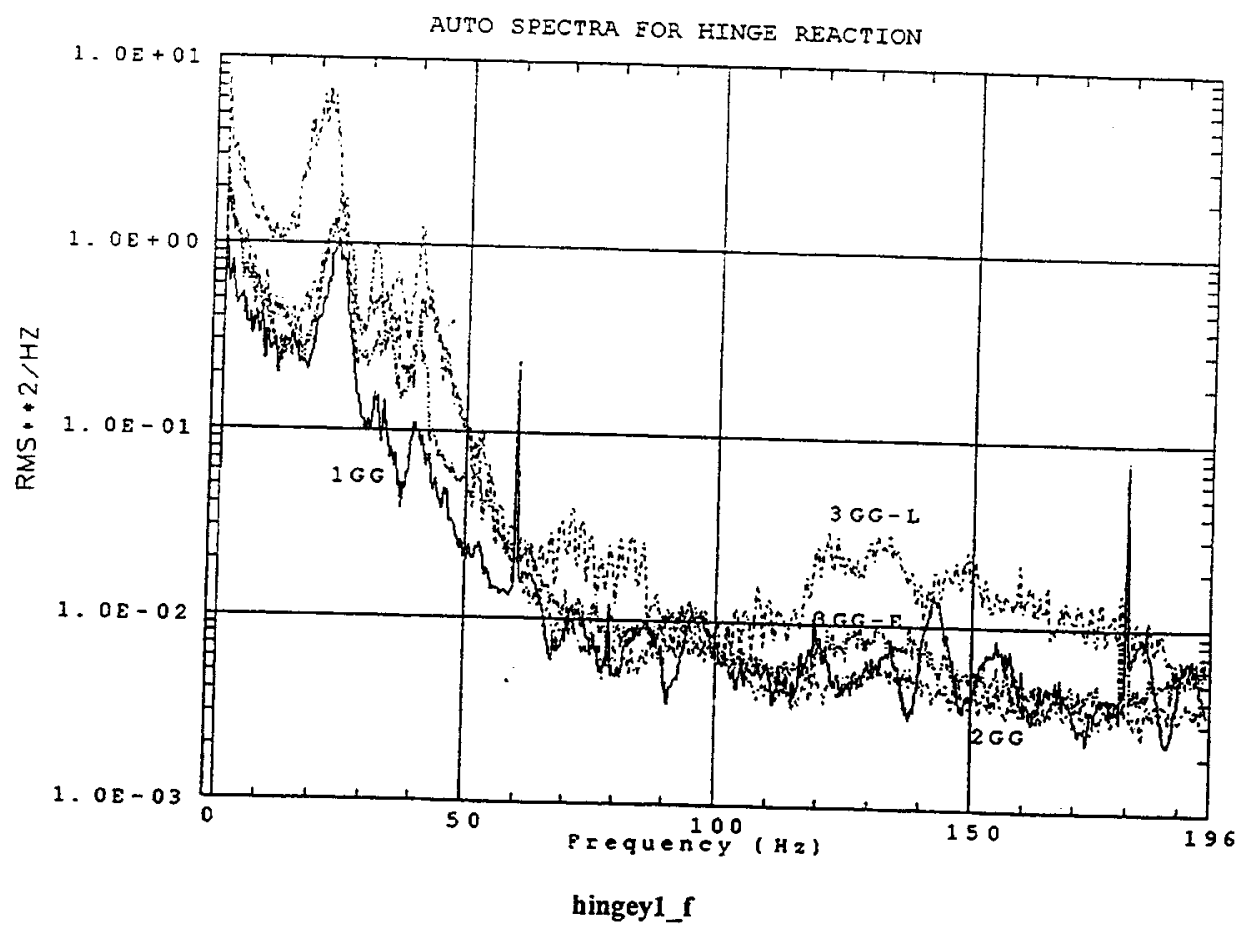

h. Frequency-domain horizontal hinge load

Figure 85. (Sheet 4 of 12) 


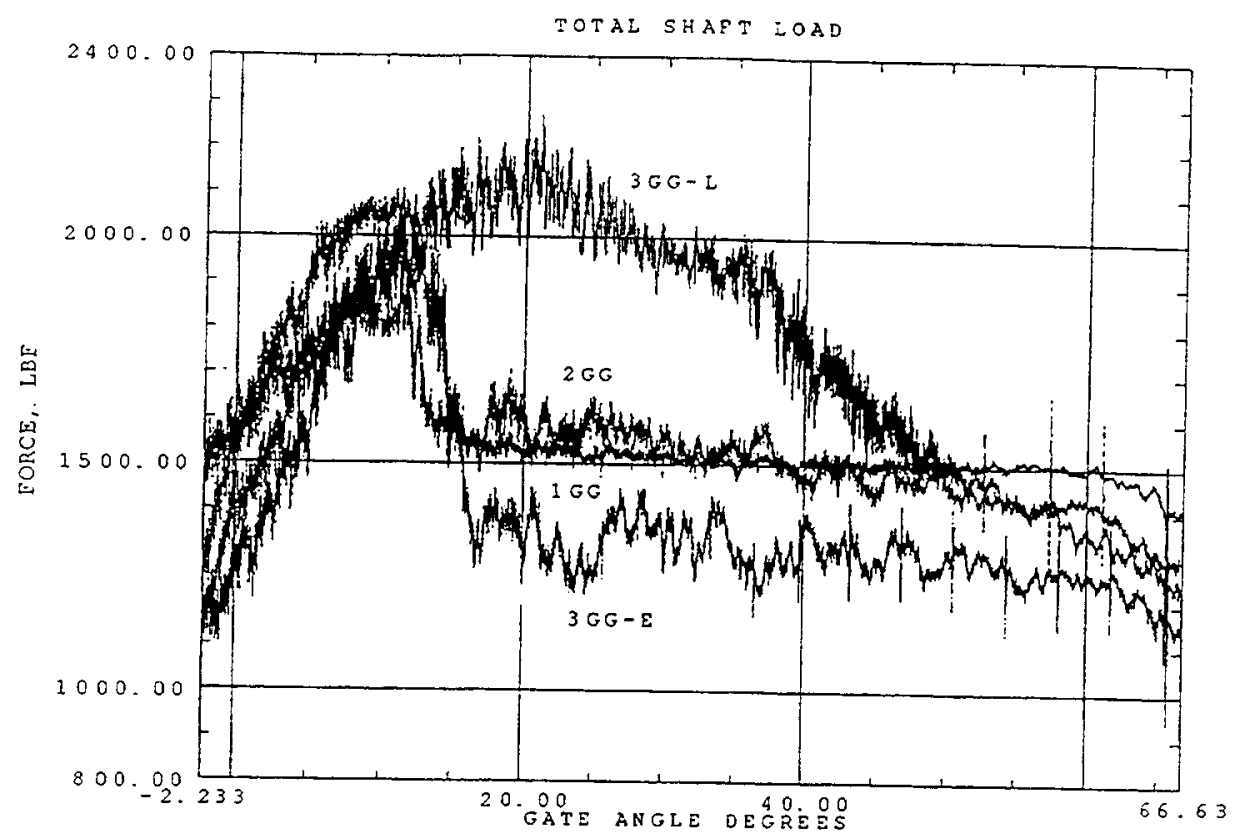

i. Time-domain total shaft load

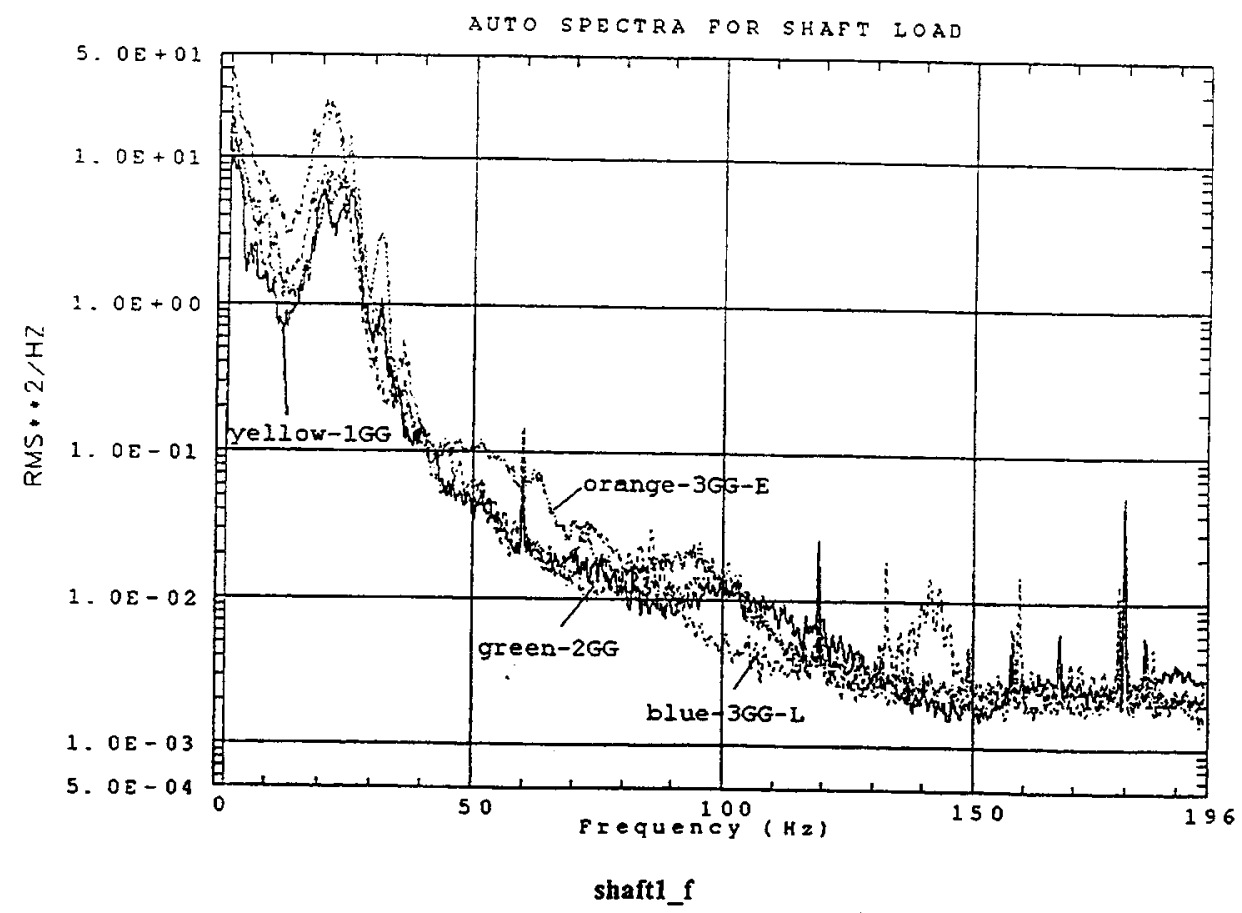

j. Frequency-domain total shaft load

Figure 85. (Sheet 5 of 12) 


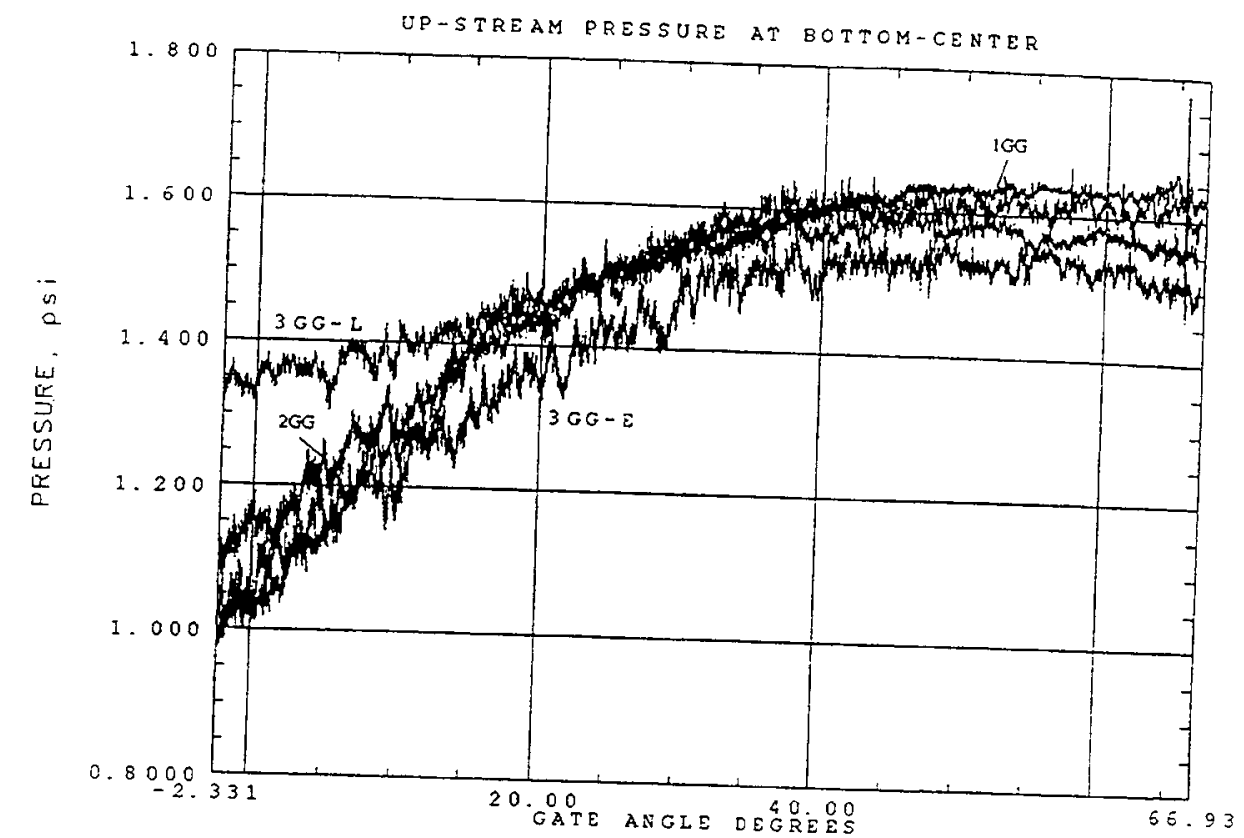

k. Upstream pressure history at bottom center of gate

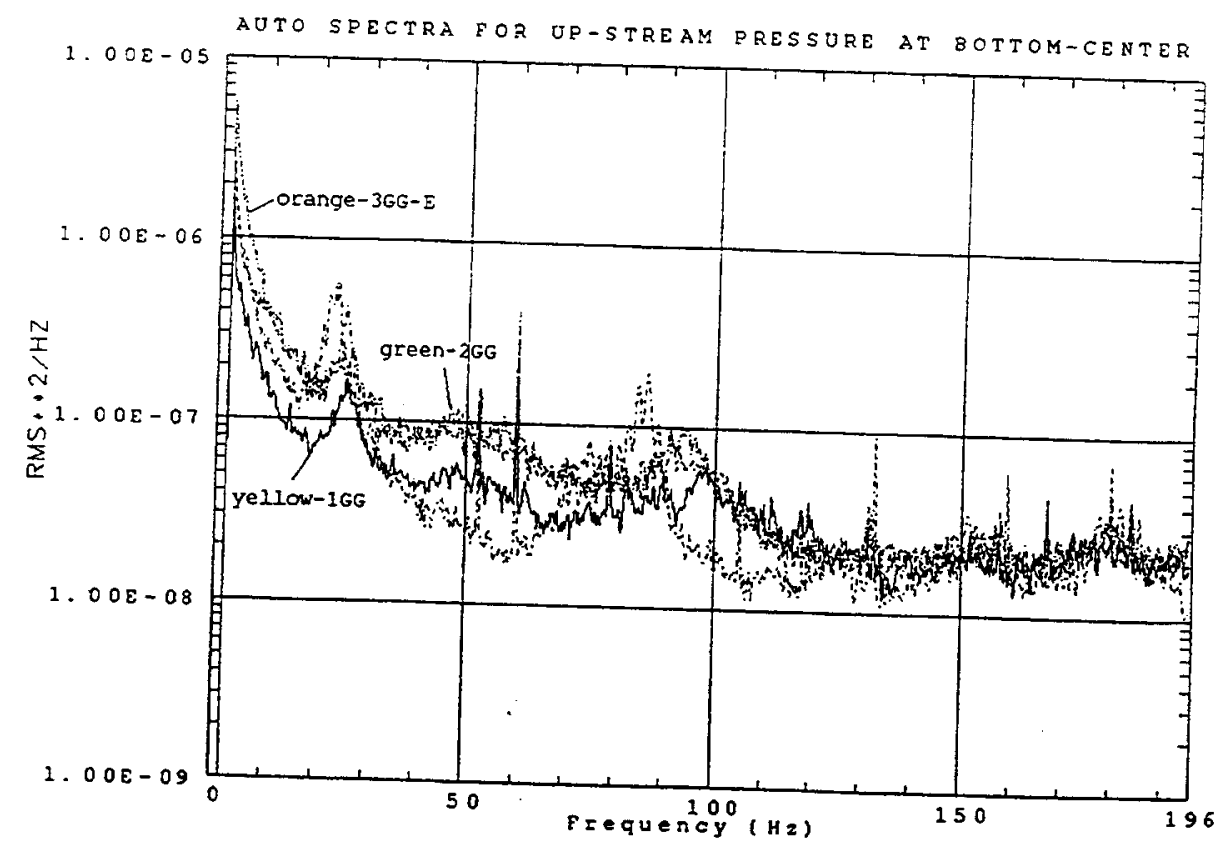

upbc1

\section{Frequency-domain upstream pressure at bottom center of gate}

Figure 85. (Sheet 6 of 12) 


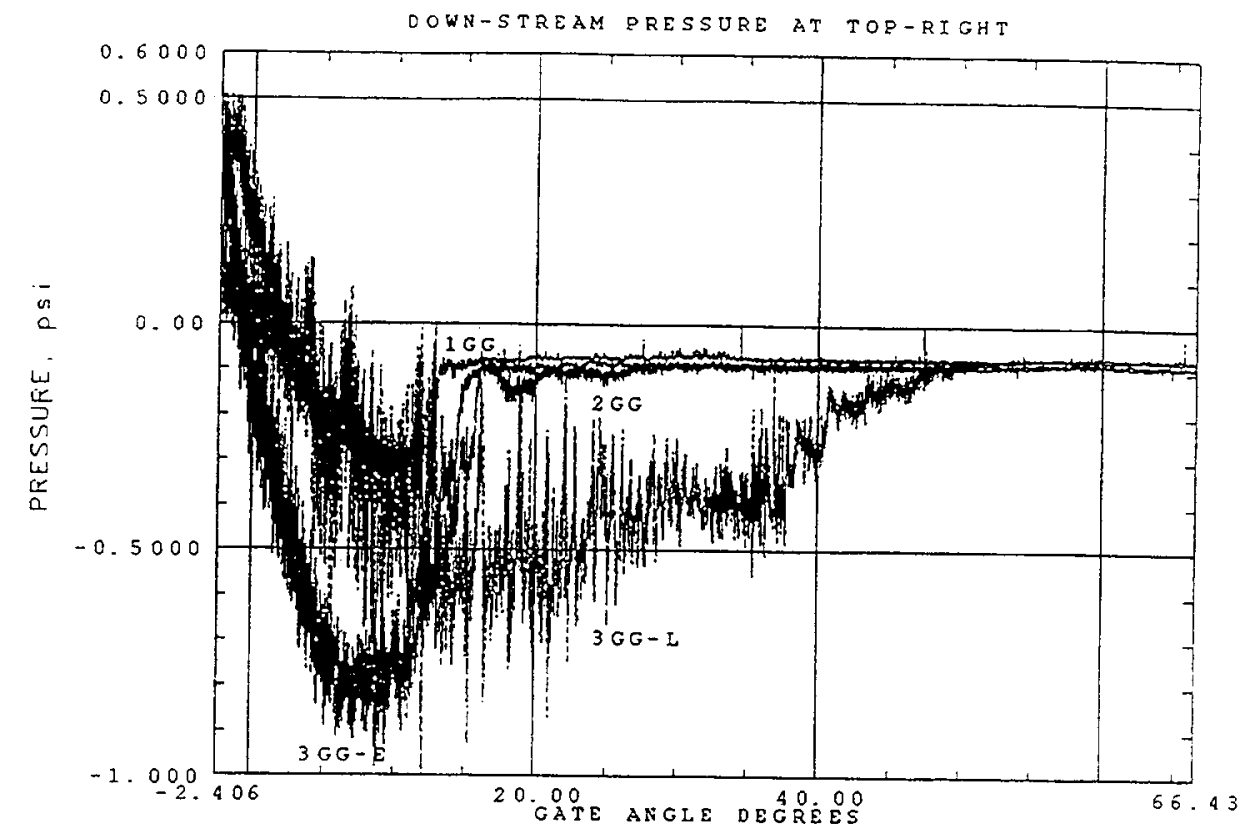

m. Downstream pressure history at top right of gate

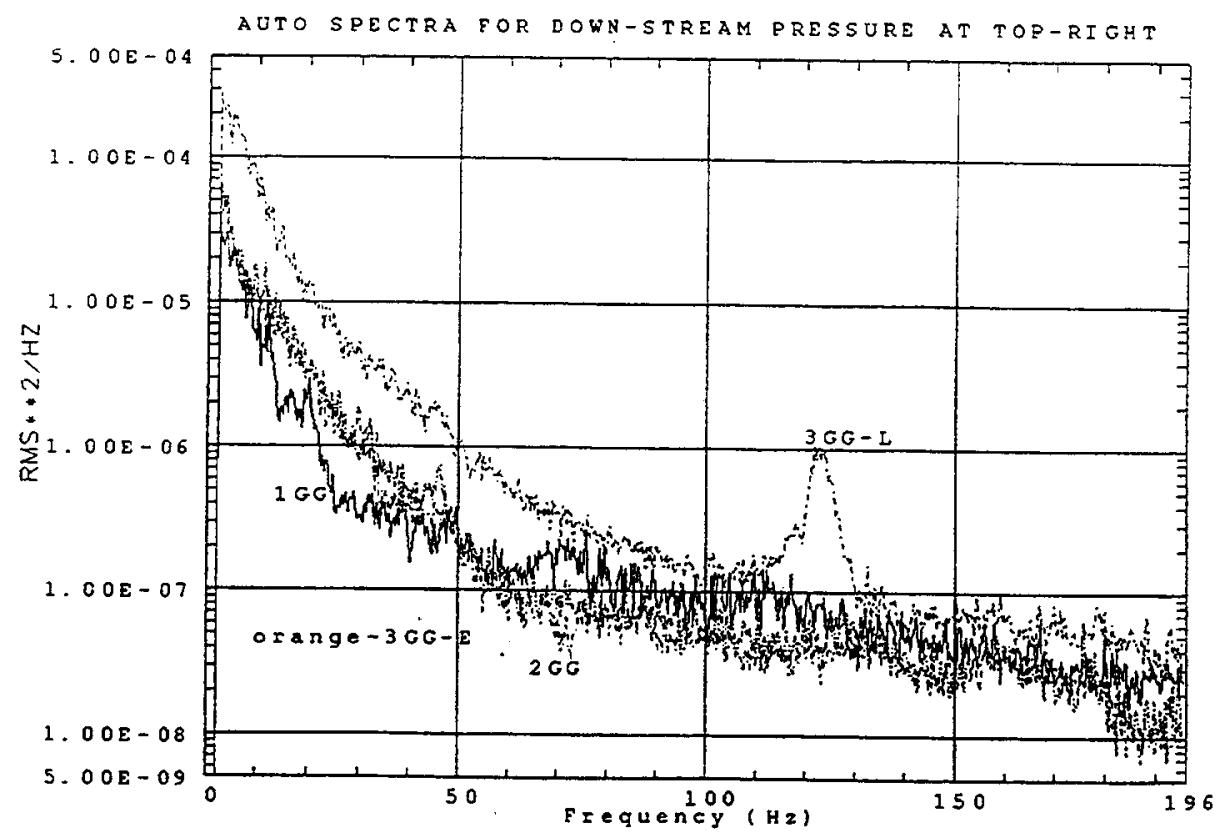

dptr1

n. Frequency-domain downstream pressure at top right of gate

Figure 85. (Sheet 7 of 12) 


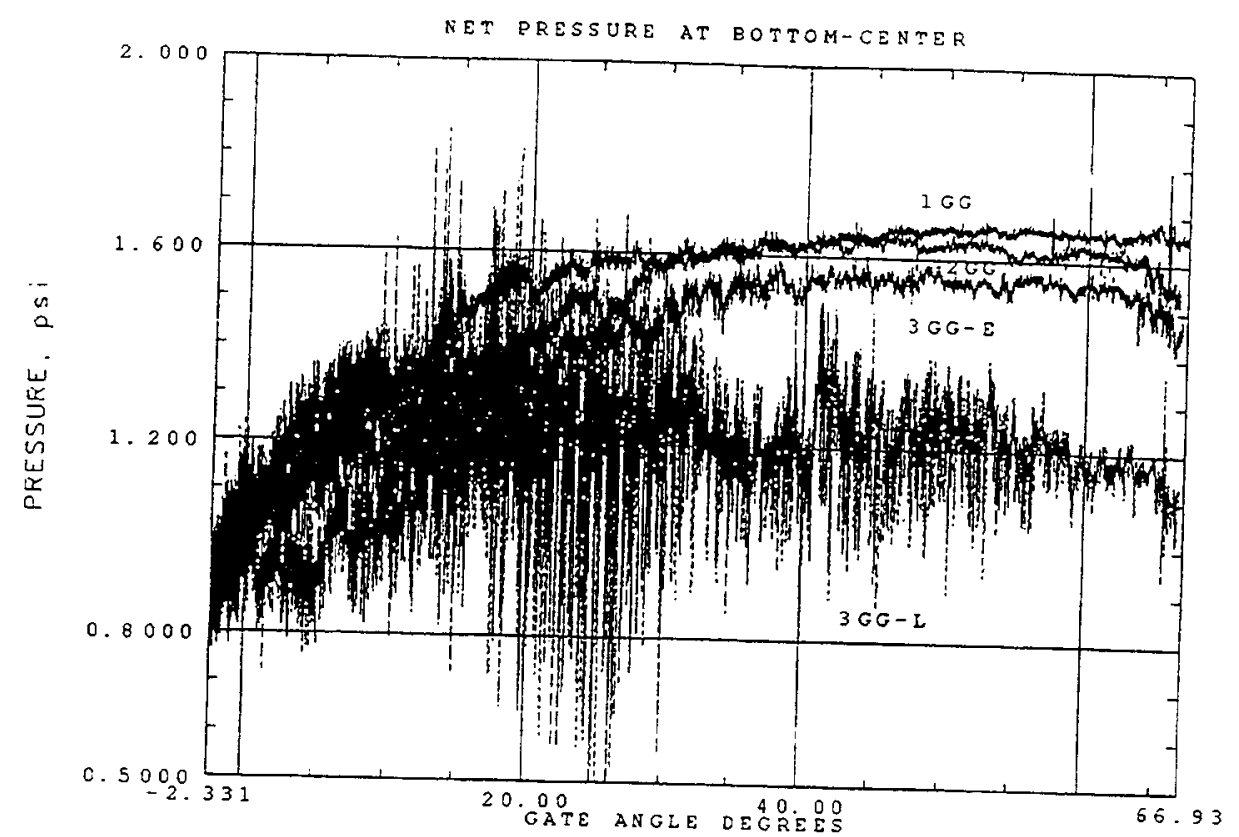

o. Net pressure at bottom center on gate

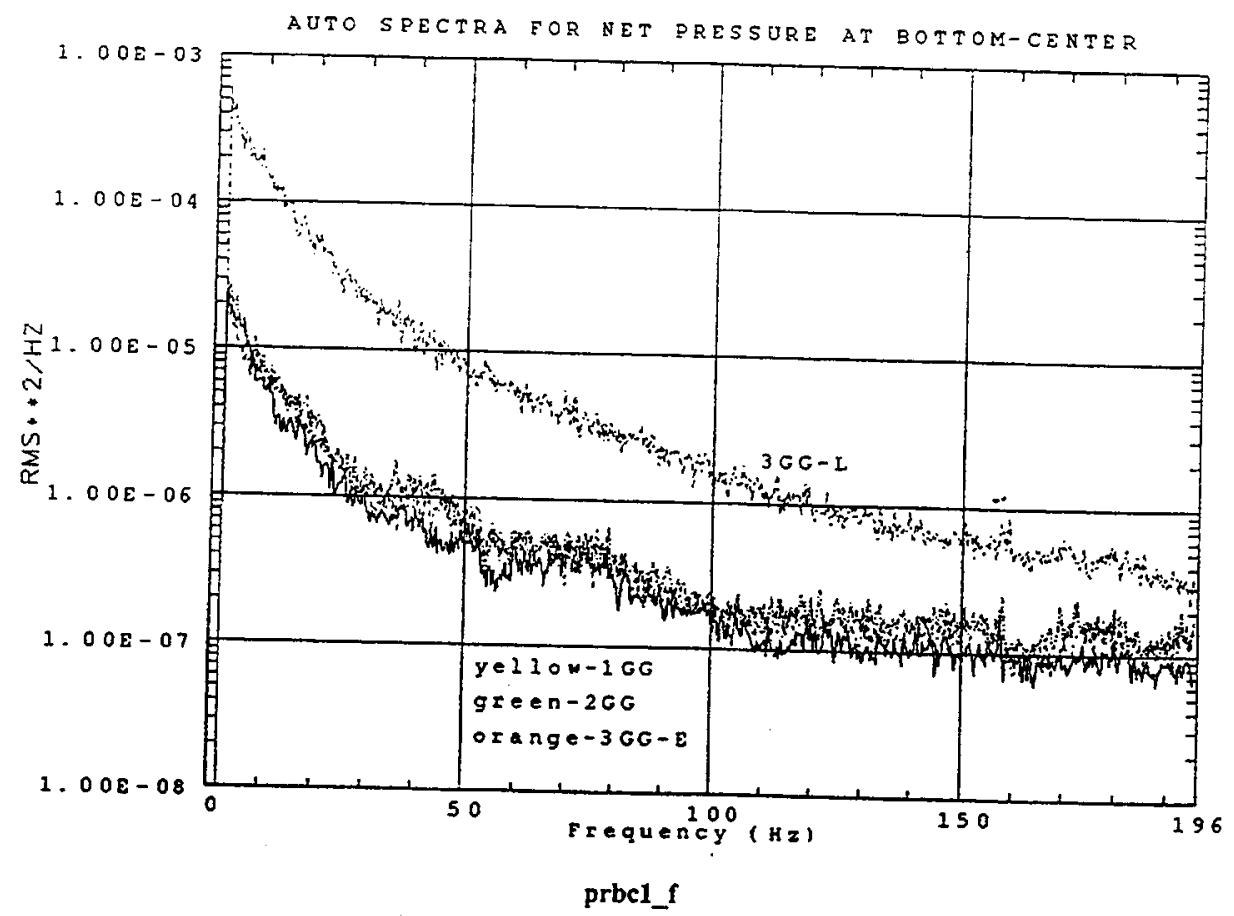

p. Frequency-domain net pressure at bottom center on gate

Figure 85. (Sheet 8 of 12) 


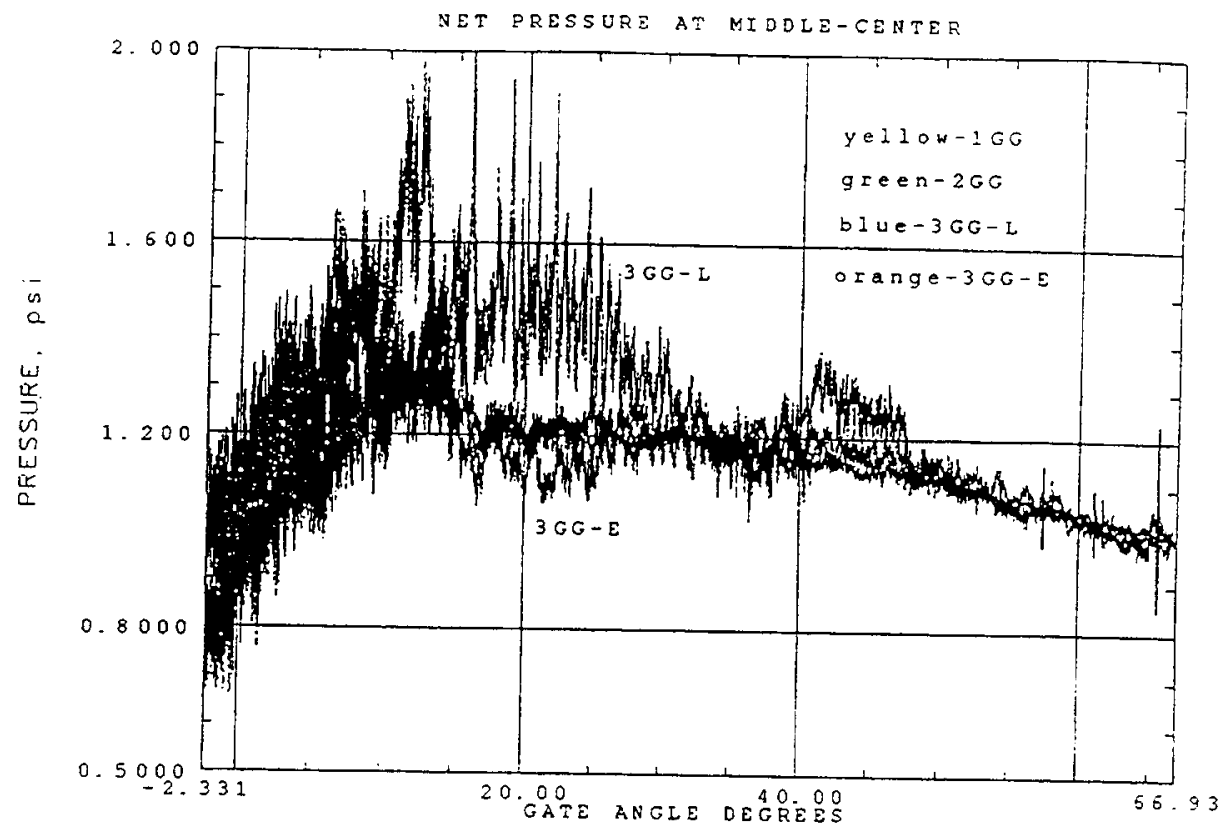

q. Net pressure at middle center on gate

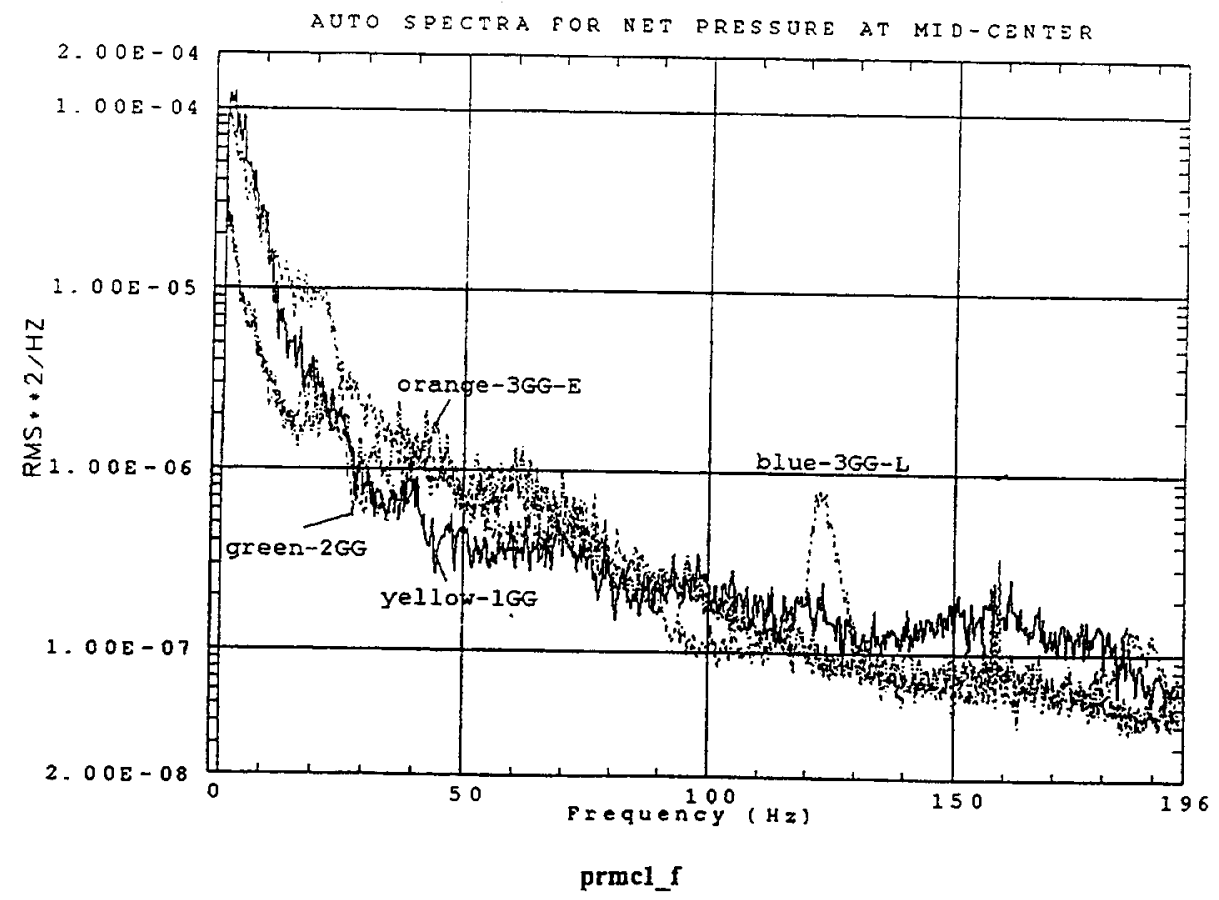

\section{r. Frequency-domain net pressure at middle center on gate}

Figure 85. (Sheet 9 of 12) 


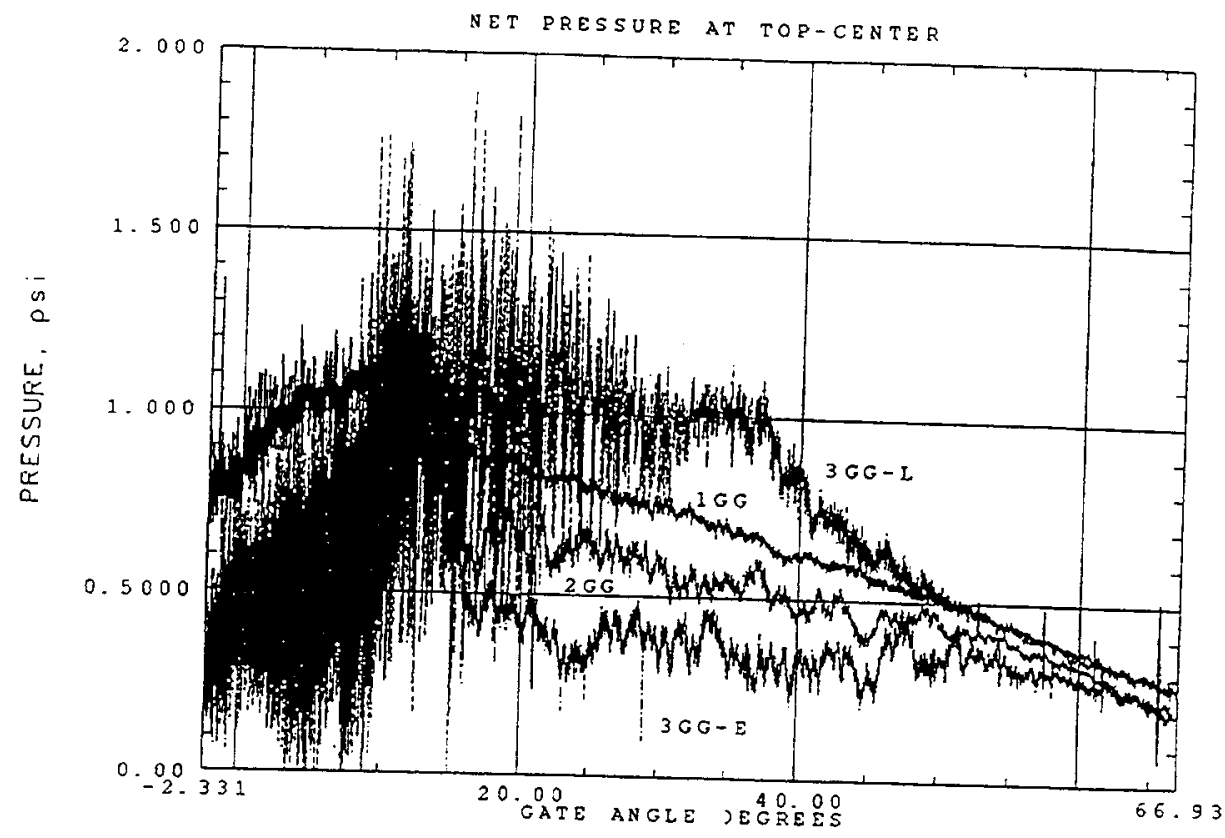

s. Net pressure at top center on gate

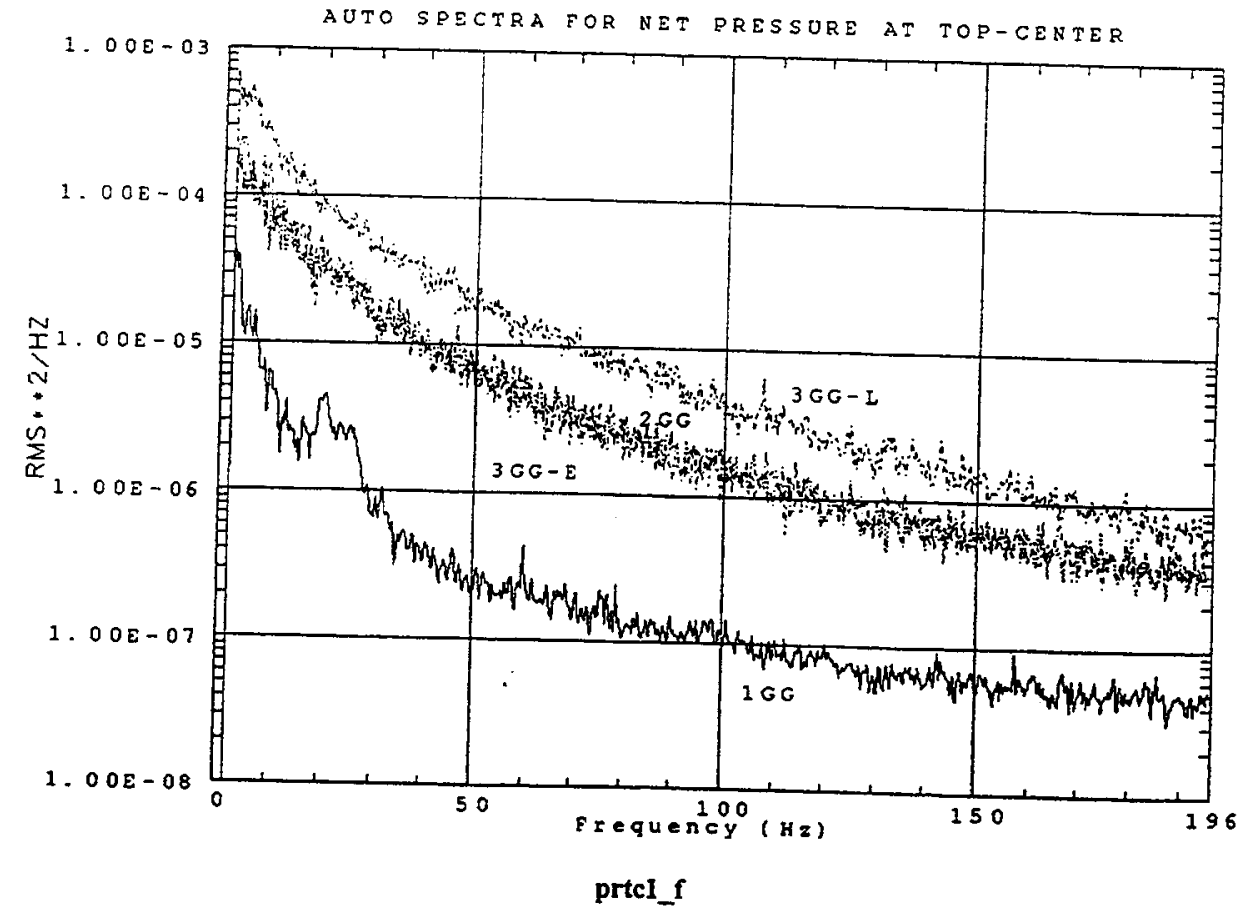

t. Frequency-domain net pressure at top center on gate

Figure 85. (Sheet 10 of 12) 


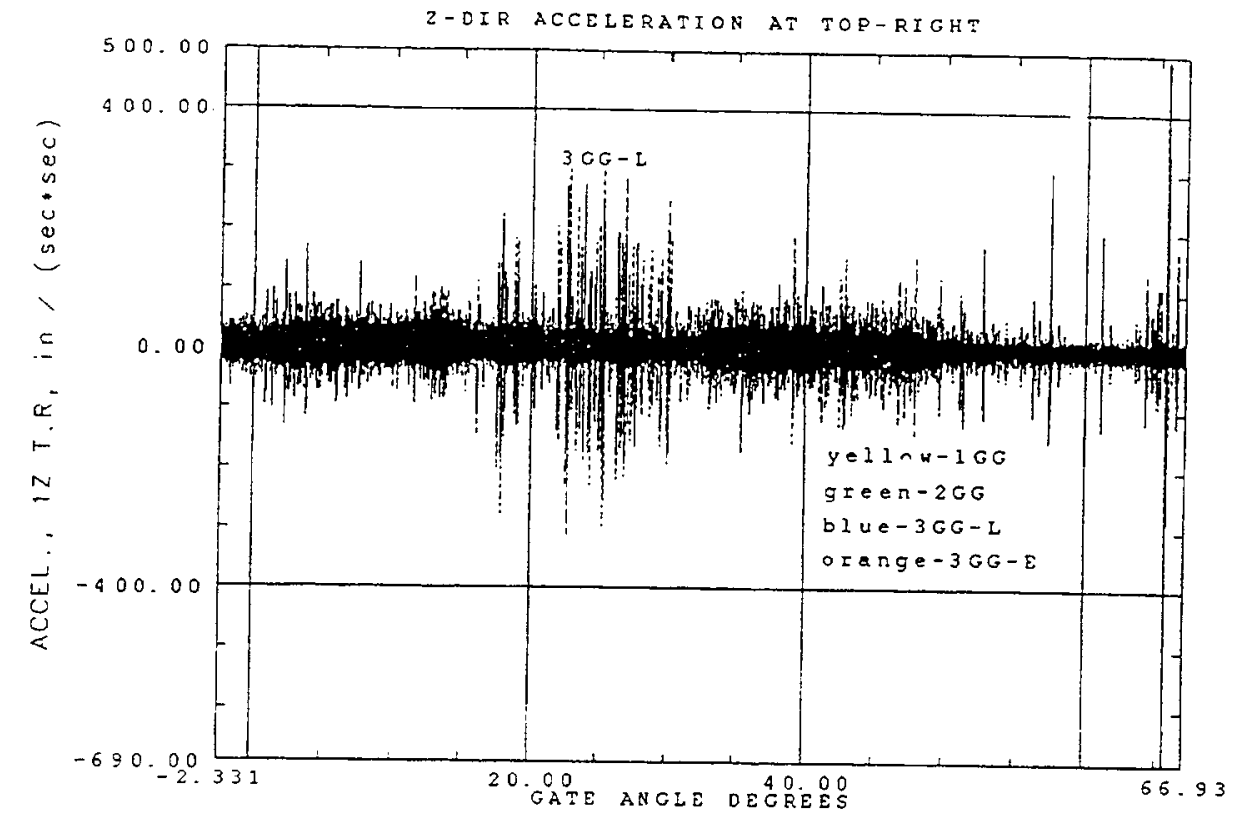

u. Z-directional acceleration at top right

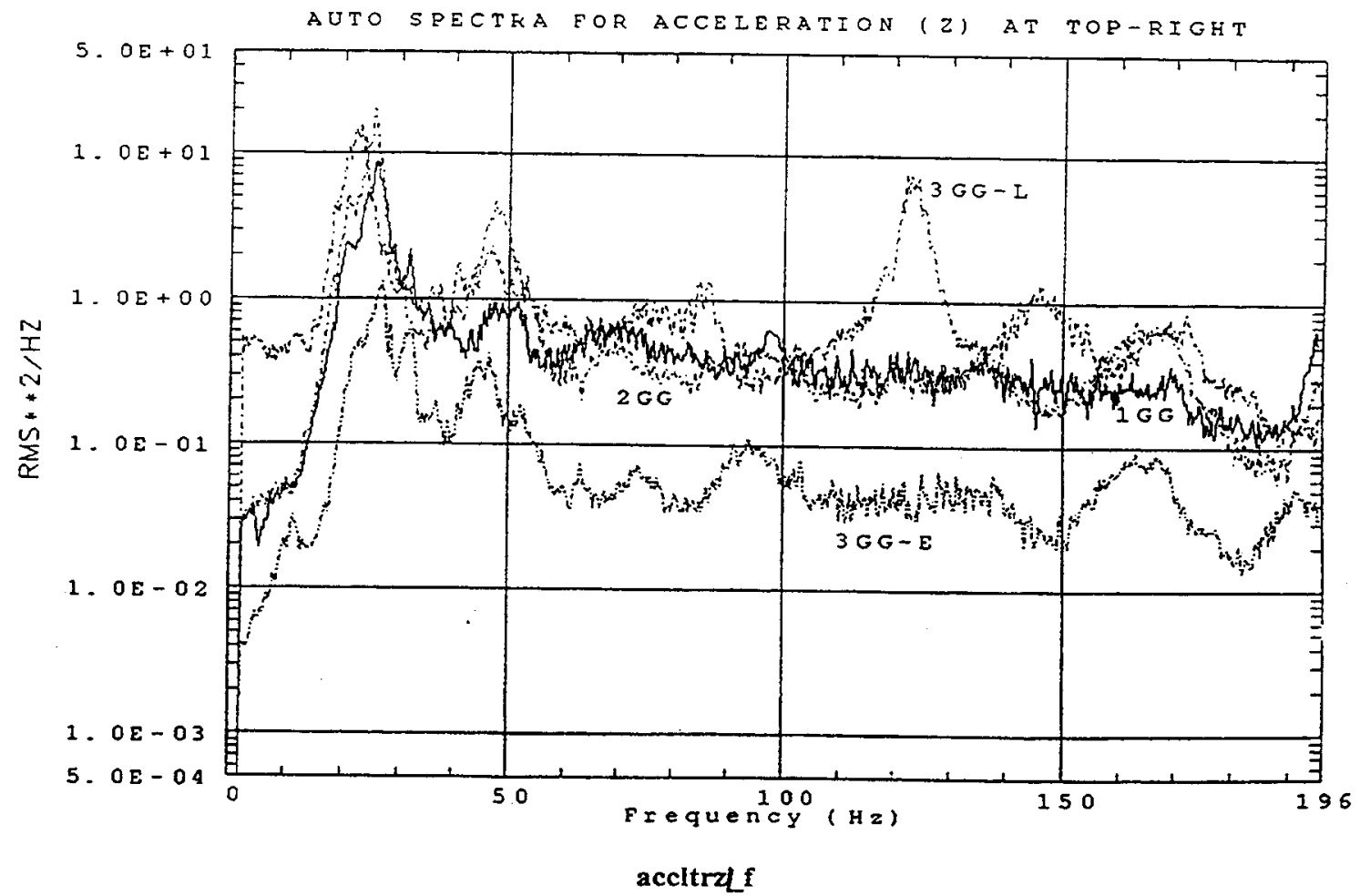

v. Frequency-domain z-acceleration at top right

Figure 85. (Sheet 11 of 12) 


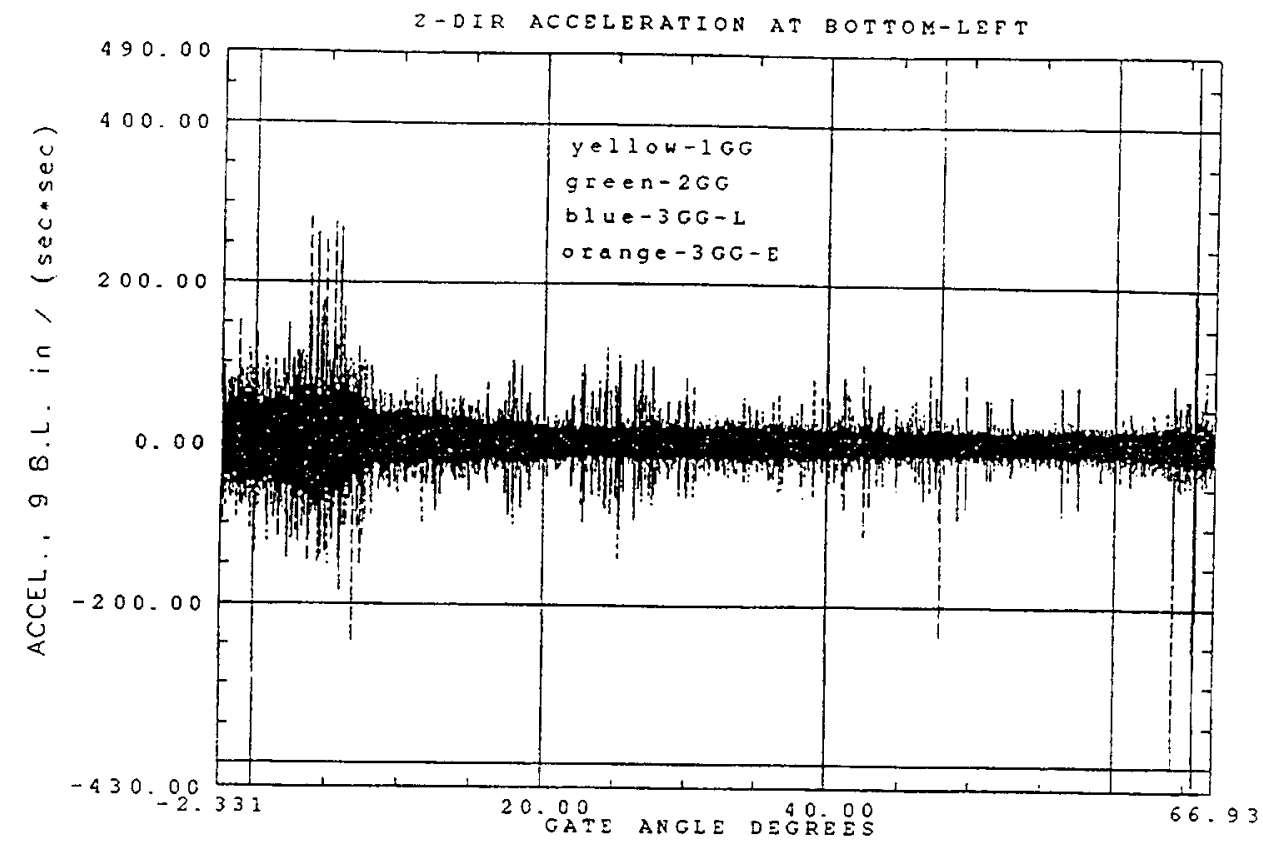

w. Z-directional acceleration at bottom left

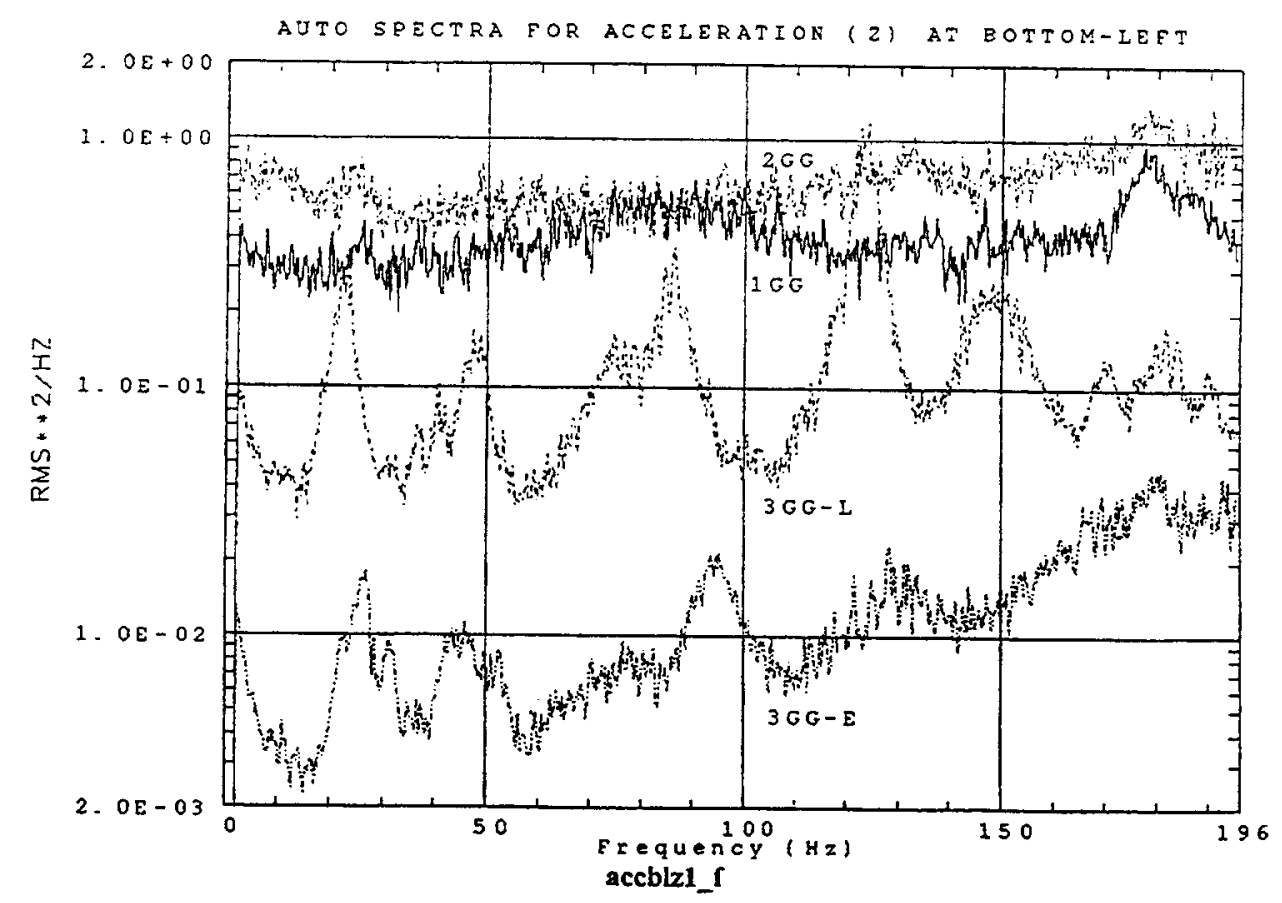

$x$. Frequency-domain z-acceleration at bottom left

Figure 85. (Sheet 12 of 12) 


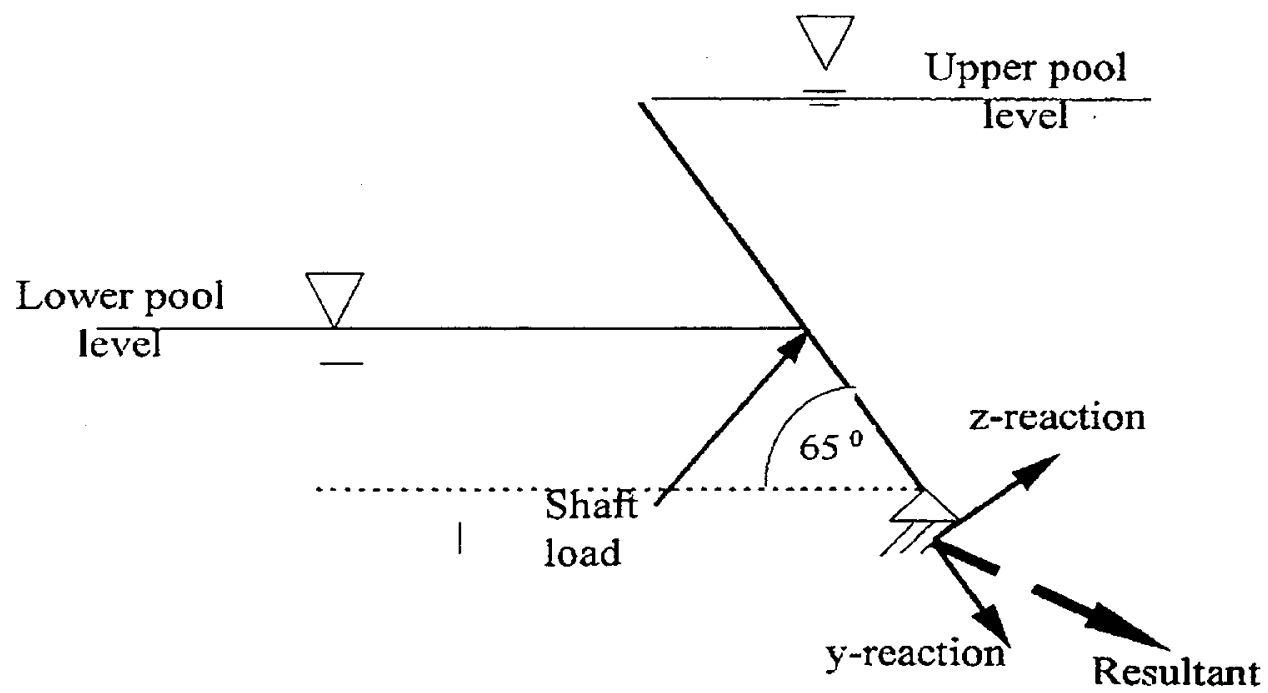

Figure 86. Sign convention for reaction forces

Time- and frequency-domain information for the shaft load obtained by summing the trunnion forces are presented in Figures $85 \mathrm{i}$ and $\mathrm{j}$. Similar to the hinge reactions, a maximum shaft load occurred during $3 \mathrm{GG}$, lone condition.

The PSD shown in Figures 85f, $\mathrm{h}$, and $\mathrm{j}$ were generated using the IDEASMaster series module with Hanning narrow window and 50 percent overlapping. A PSD provides the frequency description of the original time function and has units of $\left(f^{2} / \omega\right)$. These plots indicate that the largest mean square value of the response was developed due to three-gate gap configuration with lone condition (3GG-L). A high peak in the spectra affirms a higher order excitation in the system. Also, shown in the PSD plot are the distribution of peaks which represent the vibratory mode of the gate base for different flow configurations. A lightly loaded hinge reactions spectra seem to show more peaks than the heavily loaded shaft spectrum. This is due to relative magnitudes of the peaks in the spectra. Higher peaks were not distinctly visible in the spectra for responses with heavier responses. The frequency corresponding to each of the major vibratory modes (peaks) remained almost constant as the gate configuration was changed. A lower-order $20-\mathrm{Hz}$ mode was dominant in the reactions and shaft load spectra. This mode corresponded to a bouncing mode of the gate as evidenced during display of ODS using the Ideas-Master-series Test module. Therefore, the major vibration of the supporting devices was controlled by the first bouncing mode.

\section{Pressure field}

An upstream and a downstream pressure response and their corresponding PSD are shown in Figures $85 \mathrm{k}$ through $\mathrm{n}$. As shown in the PSD for the upstream pressure response, the flow field has a dominant mode at about $20 \mathrm{~Hz}$. The 
downstream pressure PSD has the random frequency distribution of the fluctuating air gap beneath the gate. The downstream pressure shows negative air pressure due to air-vacuum developed during the lifting of the gate above the downstream water surface. A sheet of water profile running down over the edges of the gate provided the shield for creating vacuum underneath the gate (Figure 83). A negative pressure sucks the gate down until an aeration allows to break the sheet of water profile around the gate. A fluctuation of the downstream pressure field is more visible due to turbulence of the flow and the random squeezing tendency of the atmospheric pressure to occupy the vacuum space underneath the gate. This pressure field may cause self-excited vibration such as the one noticed during experimentation of the 1:25-scale model Olmsted curved gate.

Net pressure responses and their corresponding PSD for bottom-center, middle-center, and top-center transducers are plotted in Figures 850 through $t$. A net pressure represents the actual pressure field exerted on the gate due to combined pressure fields on both upstream and downstream sides. A comparison of the net pressure spectra suggests that the $3 \mathrm{GG}-\mathrm{L}$ provided the highest total energy among the cases investigated. It also indicates that the random nature of fluctuation of the flow field with little or no interaction with the structural mode.

\section{Accelerometer response}

Acceleration (time- and frequency-domain plots) for two locations are presented in Figure 85u through $\mathrm{x}$. PSD for the accelerations show the dominant modes of flow-induced vibration for the gate corresponding to each of the flow configurations presented in the plots. A three-gate gap configuration caused the gate to vibrate at several modes. Multiple modes were excited by the variable frequency content of the flow field during the lifting of the gate from the down to up position. Corresponding modes of vibration at different frequency levels will be presented in the latter part of this chapter.

\section{7- ft pool difference}

Results for the 7-ft head-difference pool, with four different configurations, are compared in Figure 87. As in the previous case, the time- and frequencydomain orthogonal total reaction forces for both bottom hinges and the sum of trunnion forces representing the shaft load are presented in Figures 87a through f. An identical trend in the frequency distribution and the time-history plots for three responses are observed as in the $21-\mathrm{ft}$ experimental case presented above. In all cases, the maximum responses occurred for the 3GG-L case. The bottom hinge reactions indicate that the base of the gate tends to pull apart from the sill during the early stage of lifting, while after raising the gate about 22 deg above the flat position, the mechanism reverses its direction. A different configuration of the gate arrangement did not shift the peaks in the frequency distribution of the measured responses. A low-order peak at about $20 \mathrm{~Hz}$ was dominant for all 


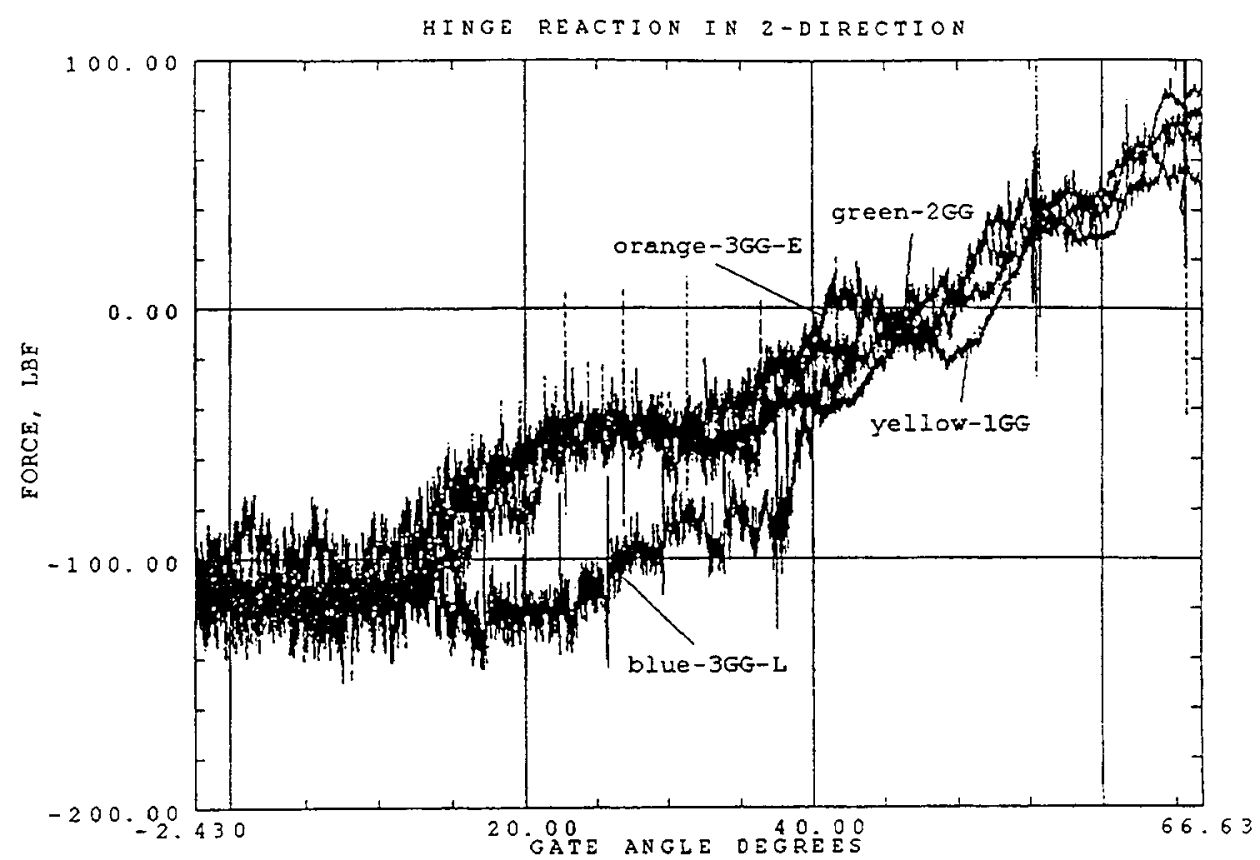

a. Total vertical hinge load

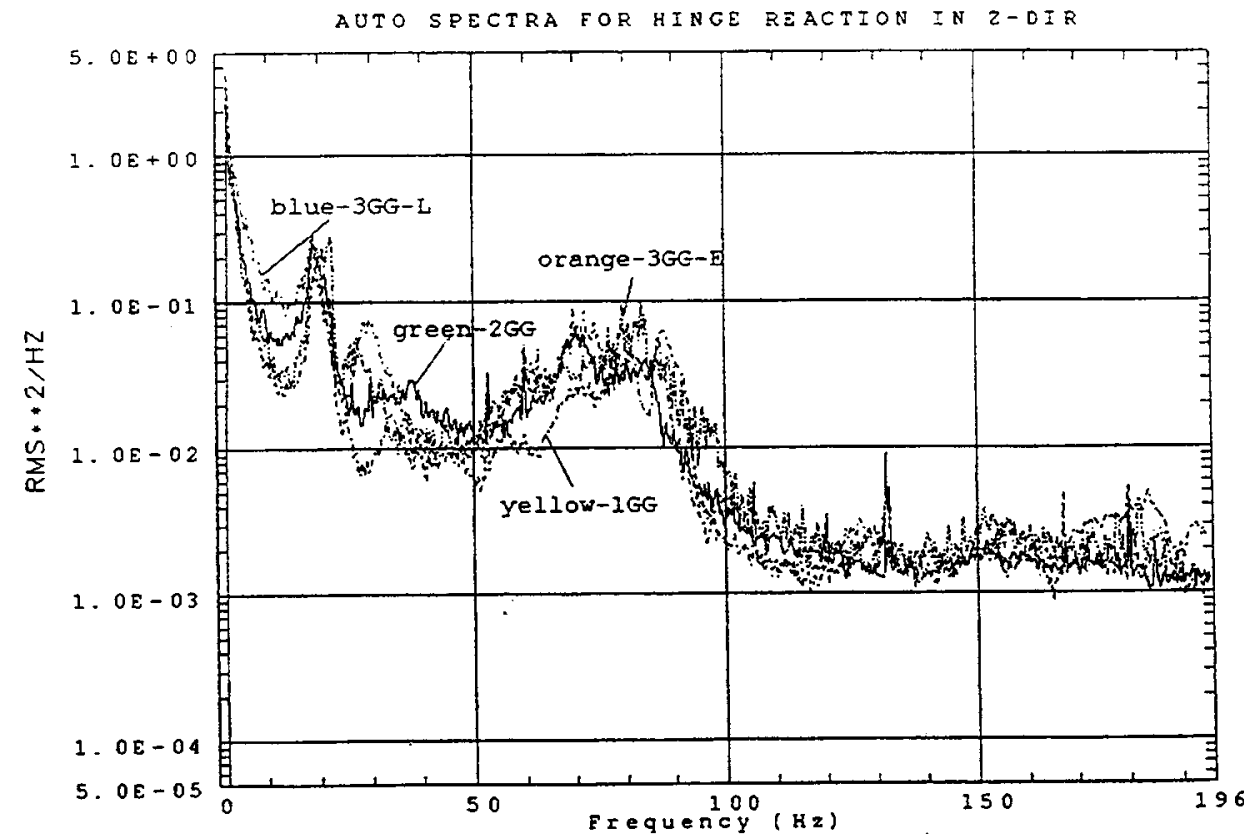

hingez2_f

b. Frequency-domain total vertical hinge load

Figure 87. Comparison of responses over full range of operation of various gate configurations, 7 - $\mathrm{ft}$ pool difference (Sheet 1 of 10) 


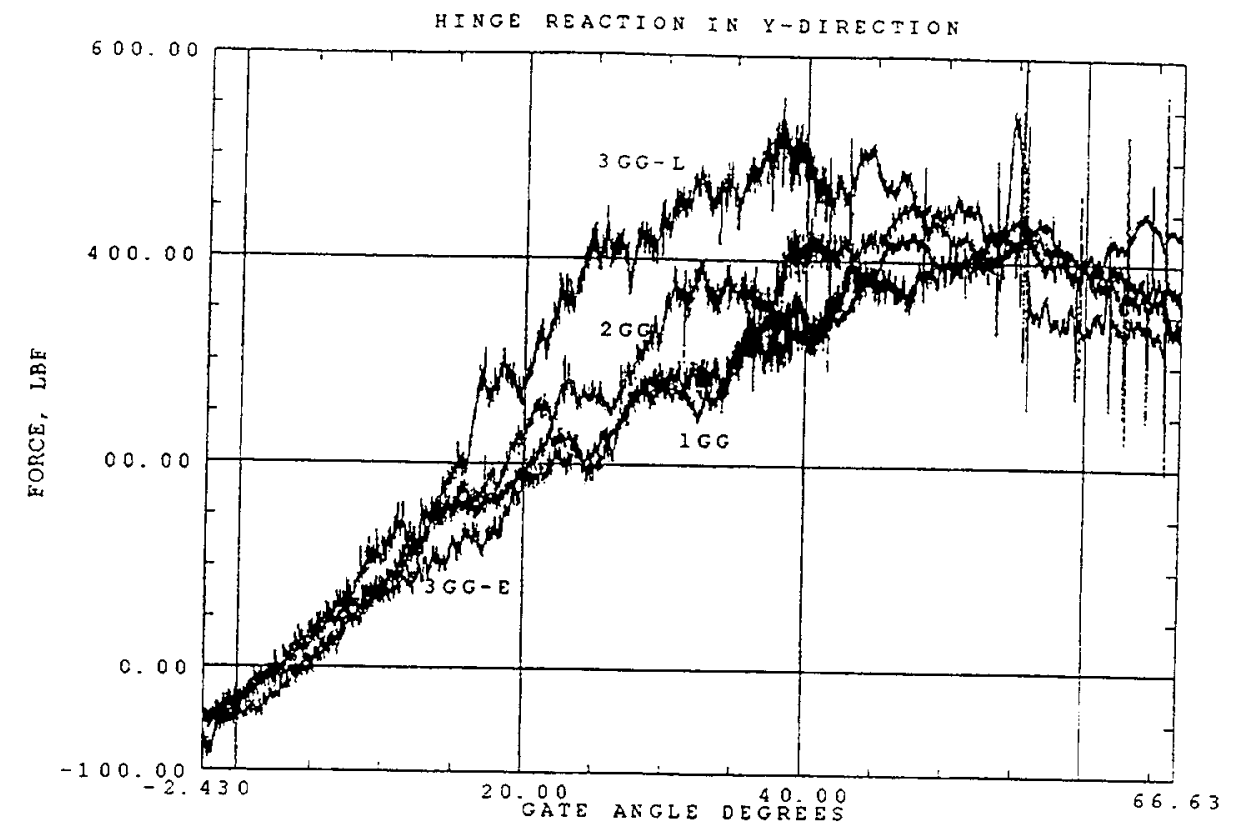

c. Total horizontal hinge load

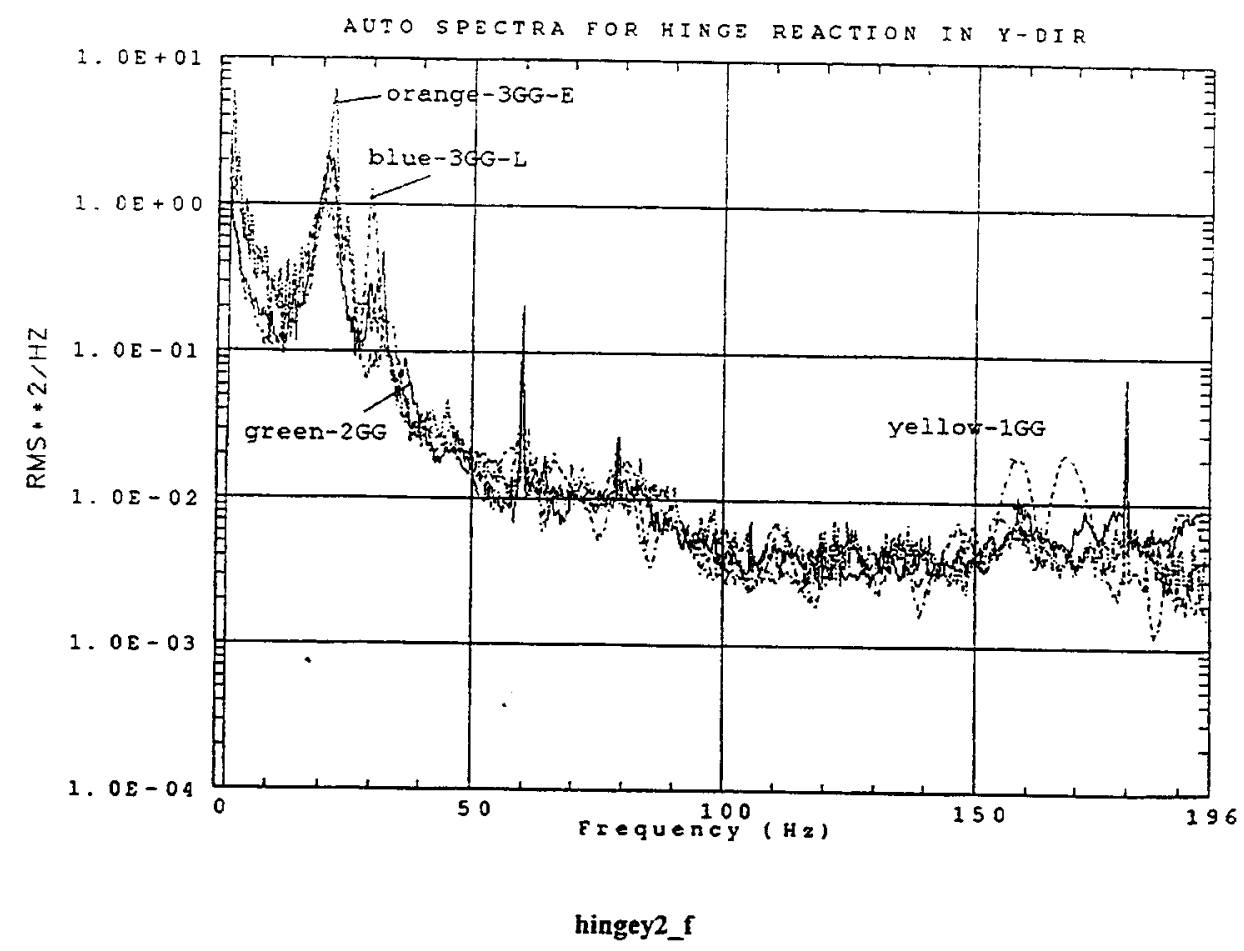

\section{d. Frequency-domain total horizontal hinge load}

Figure 87. (Sheet 2 of 10) 


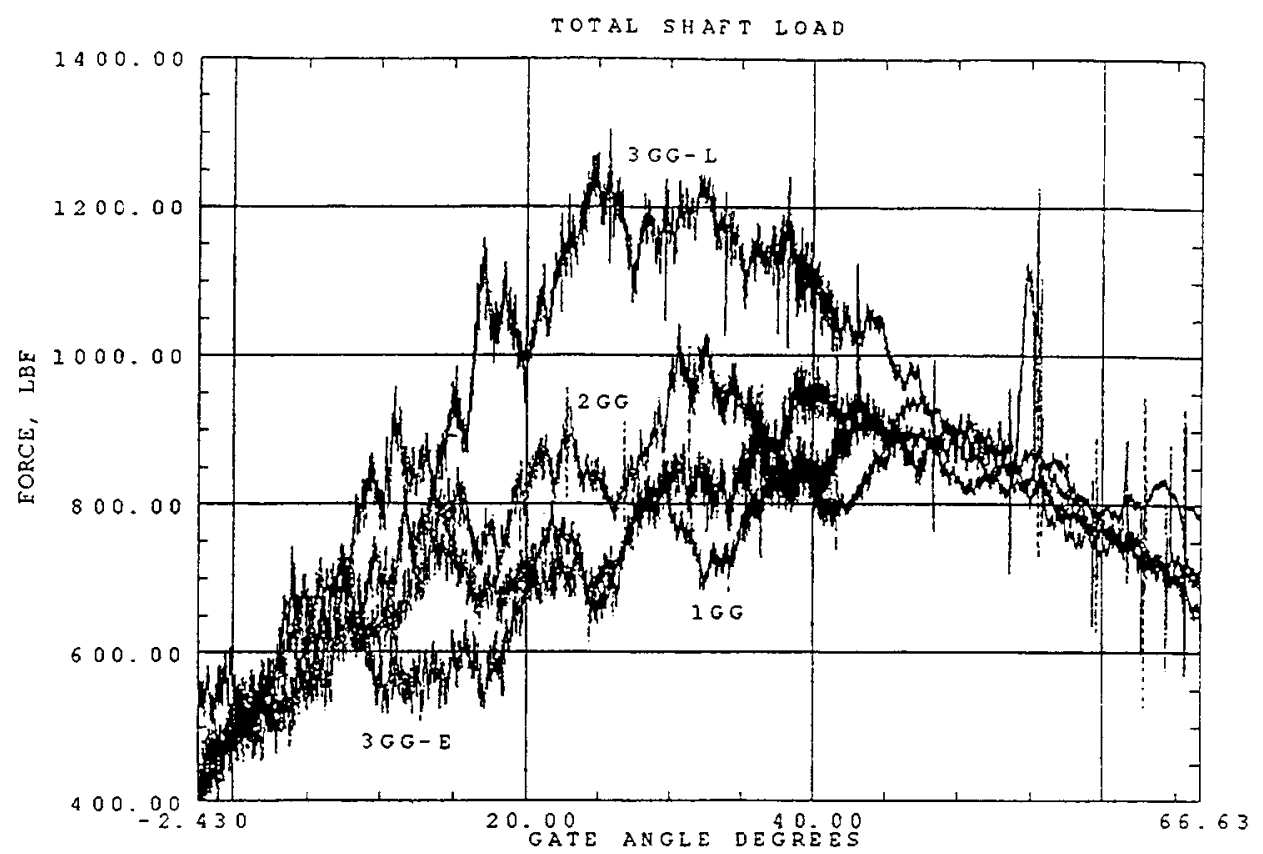

e. Total shaft load

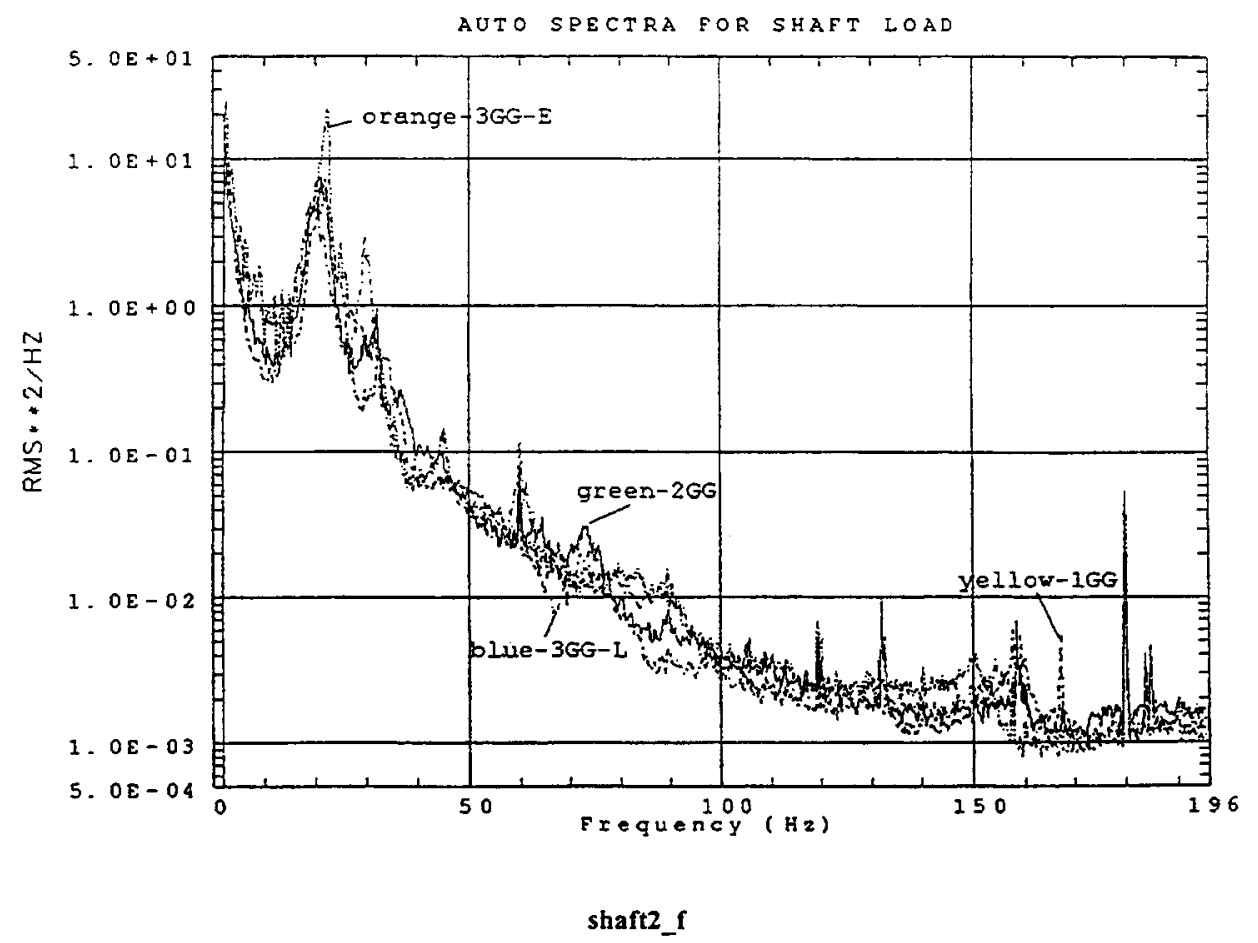

f. Frequency-domain total shaft load

Figure 87. (Sheet 3 of 10) 


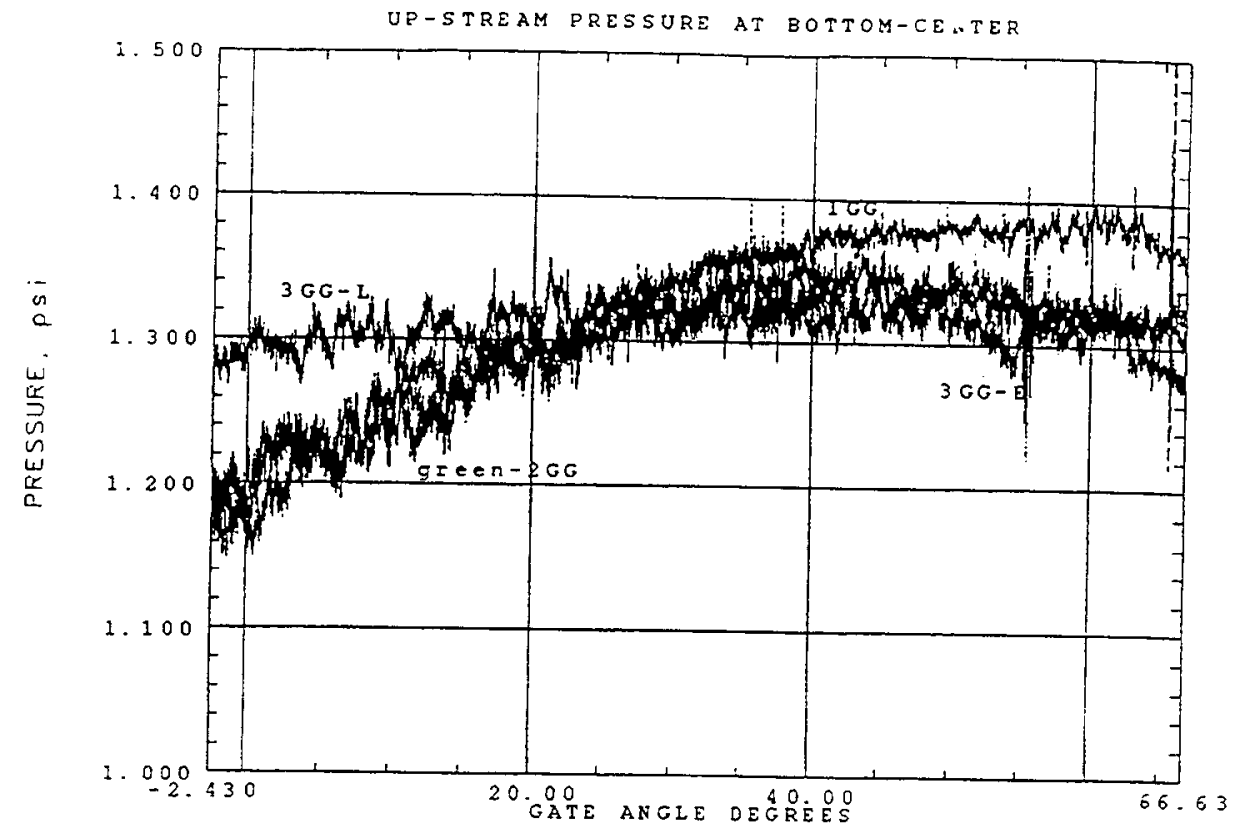

g. Upstream pressure history at bottom center of gate

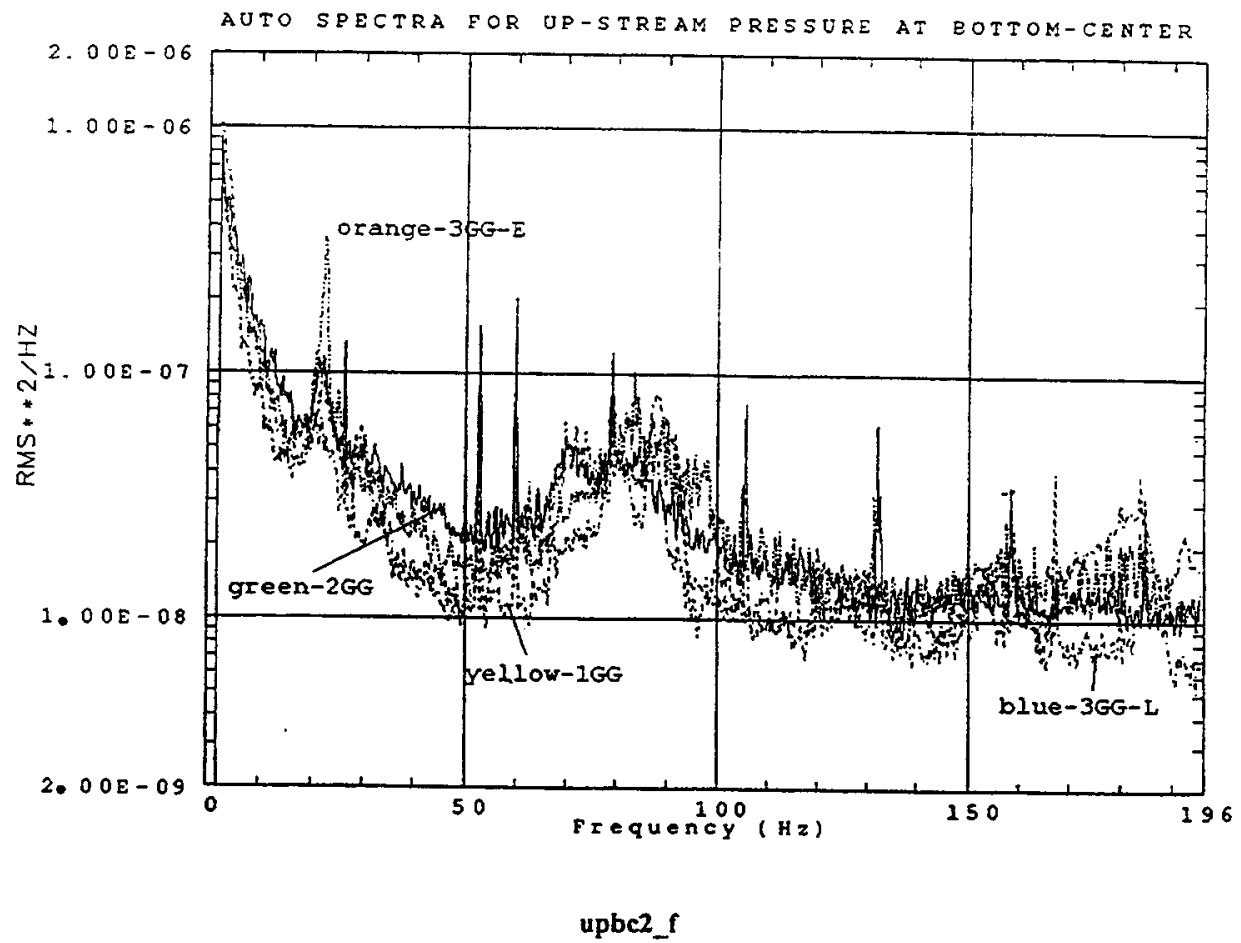

h. Frequency-domain upstream pressure at bottom center of gate

Figure 87 . (Sheet 4 of 10 ) 


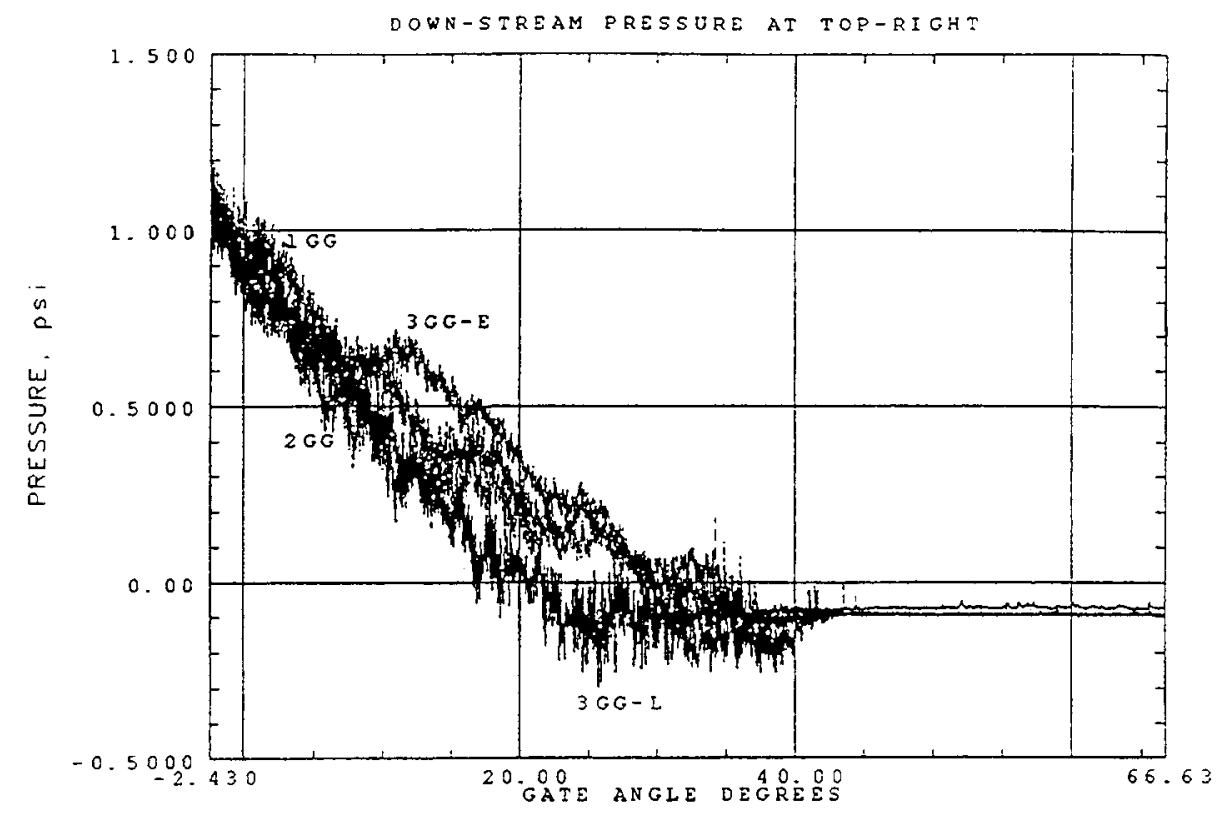

i. Downstream pressure history at top right of gate

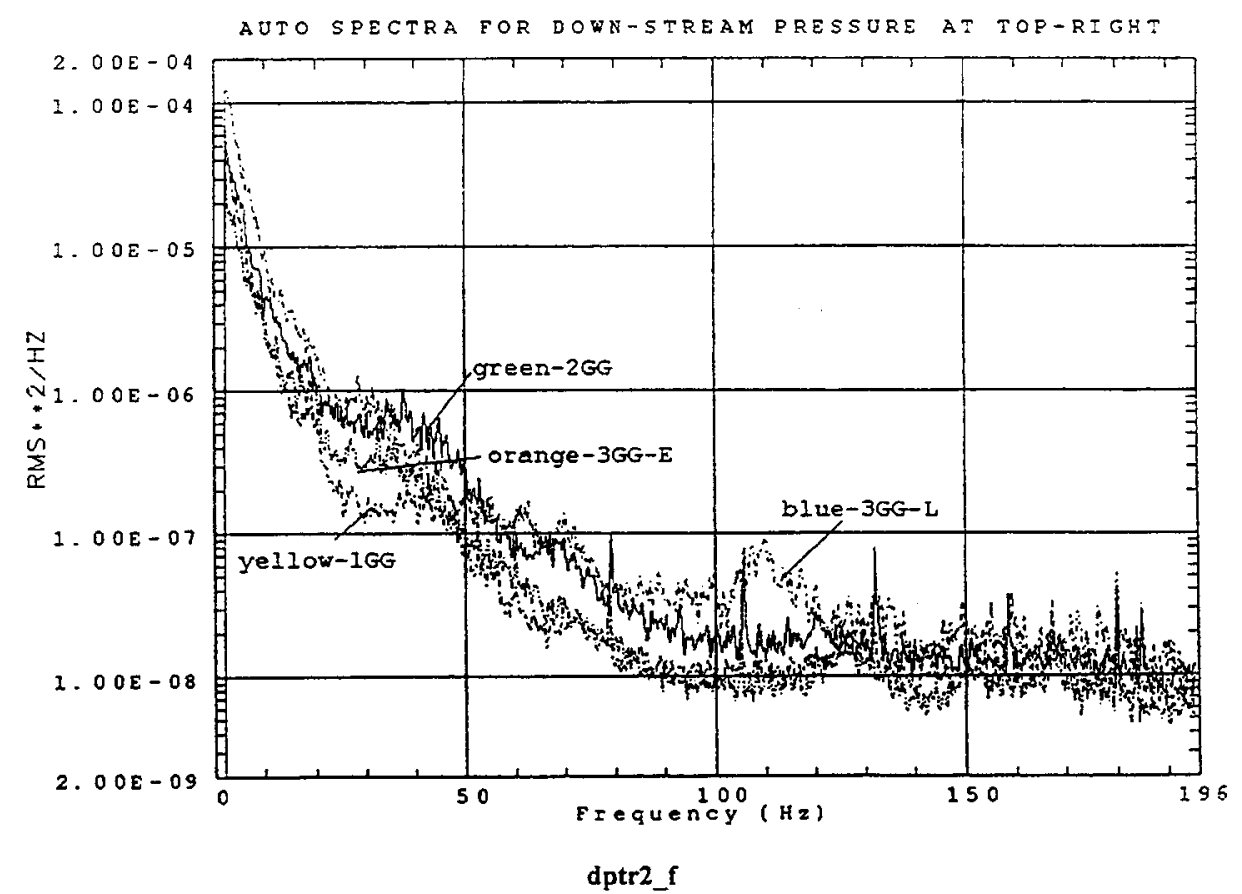

\section{j. Frequency-domain downstream pressure at top right of gate}

Figure 87. (Sheet 5 of 10 ) 


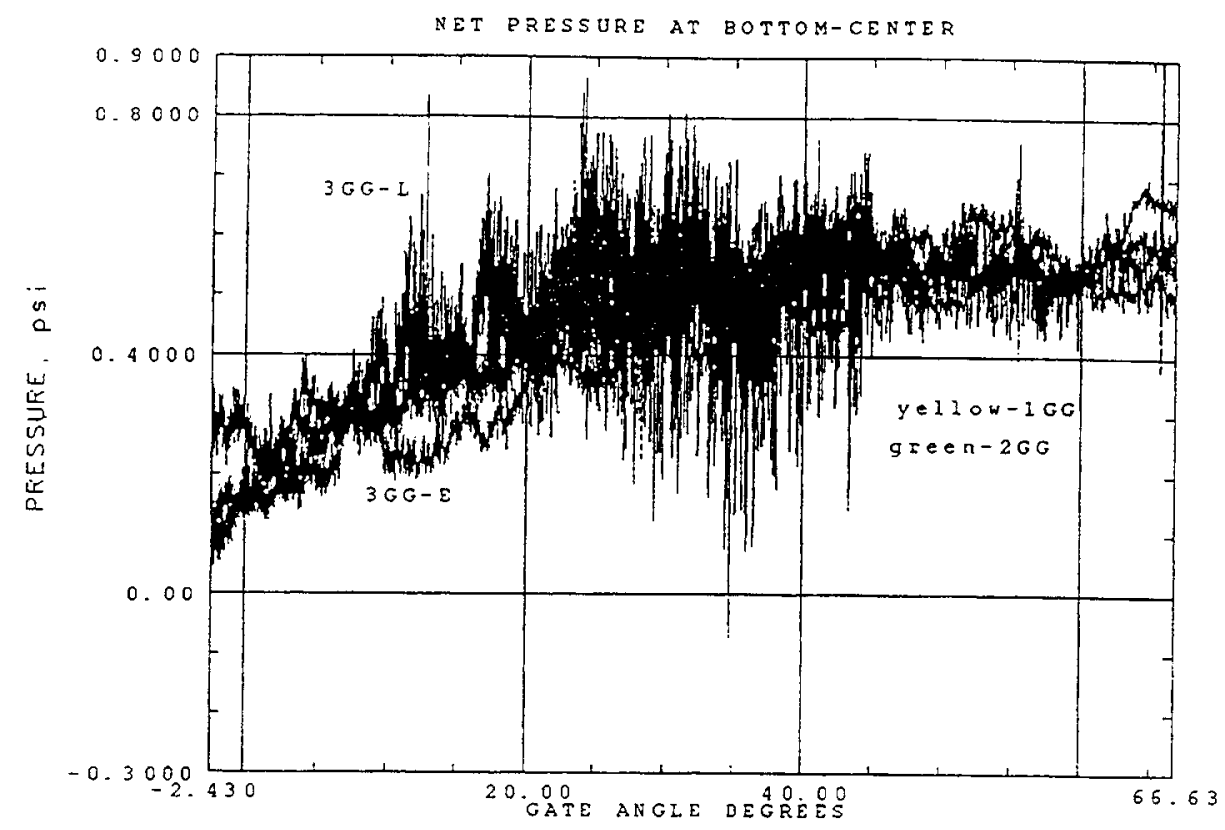

k. Net pressure at bottom center on gate

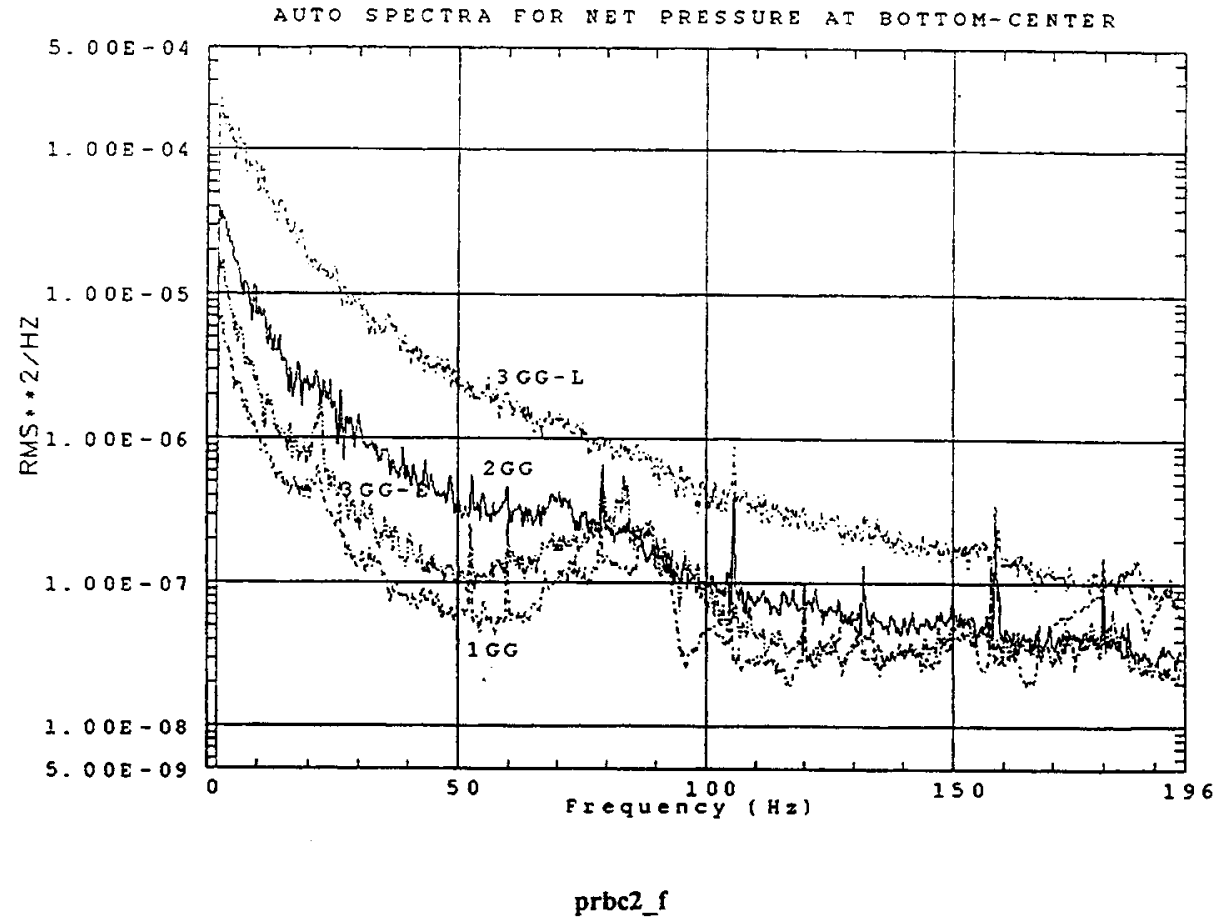

\section{Frequency-domain net pressure at bottom center on gate}

Figure 87. (Sheet 6 of 10) 


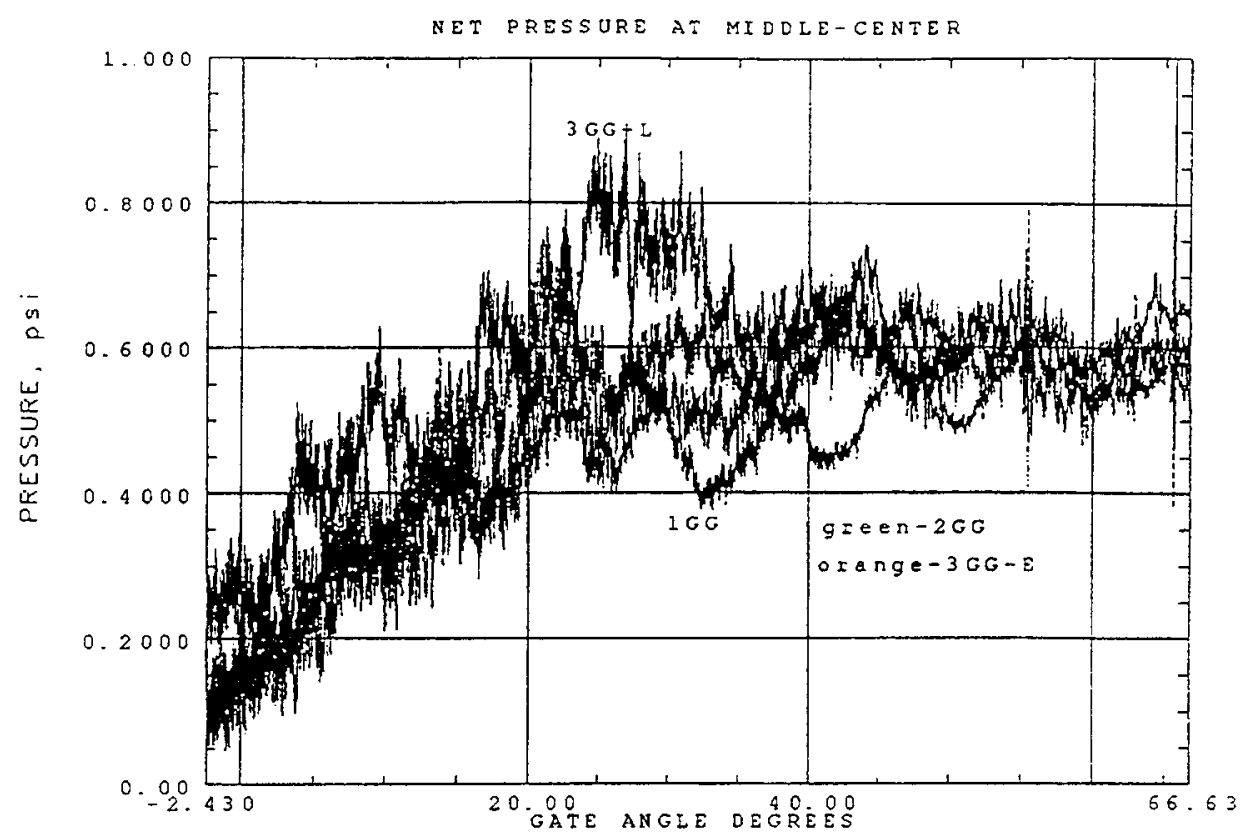

m. Net pressure at middle center on gate

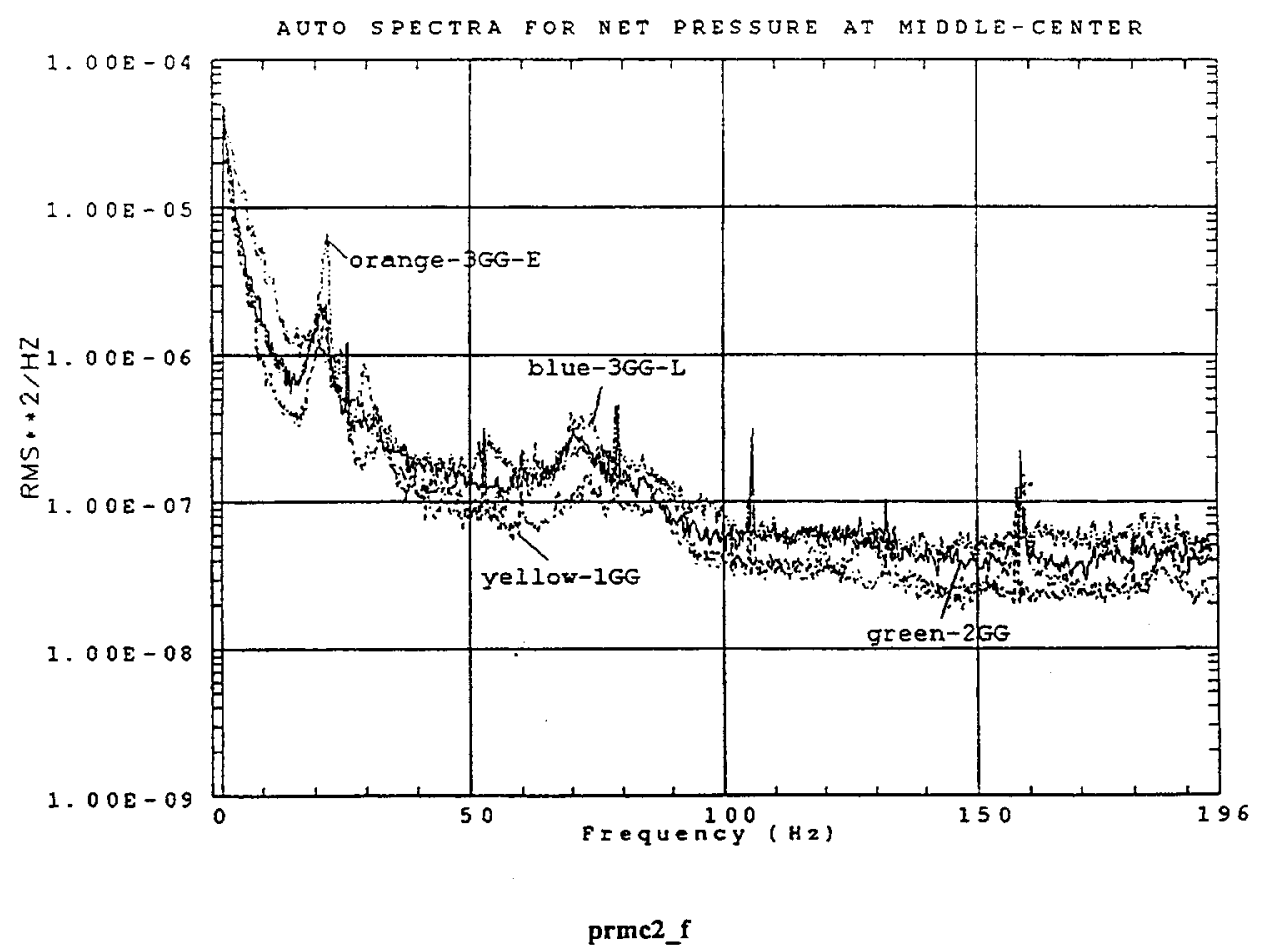

n. Frequency-domain net pressure at middle center on gate

Figure 87. (Sheet 7 of 10) 


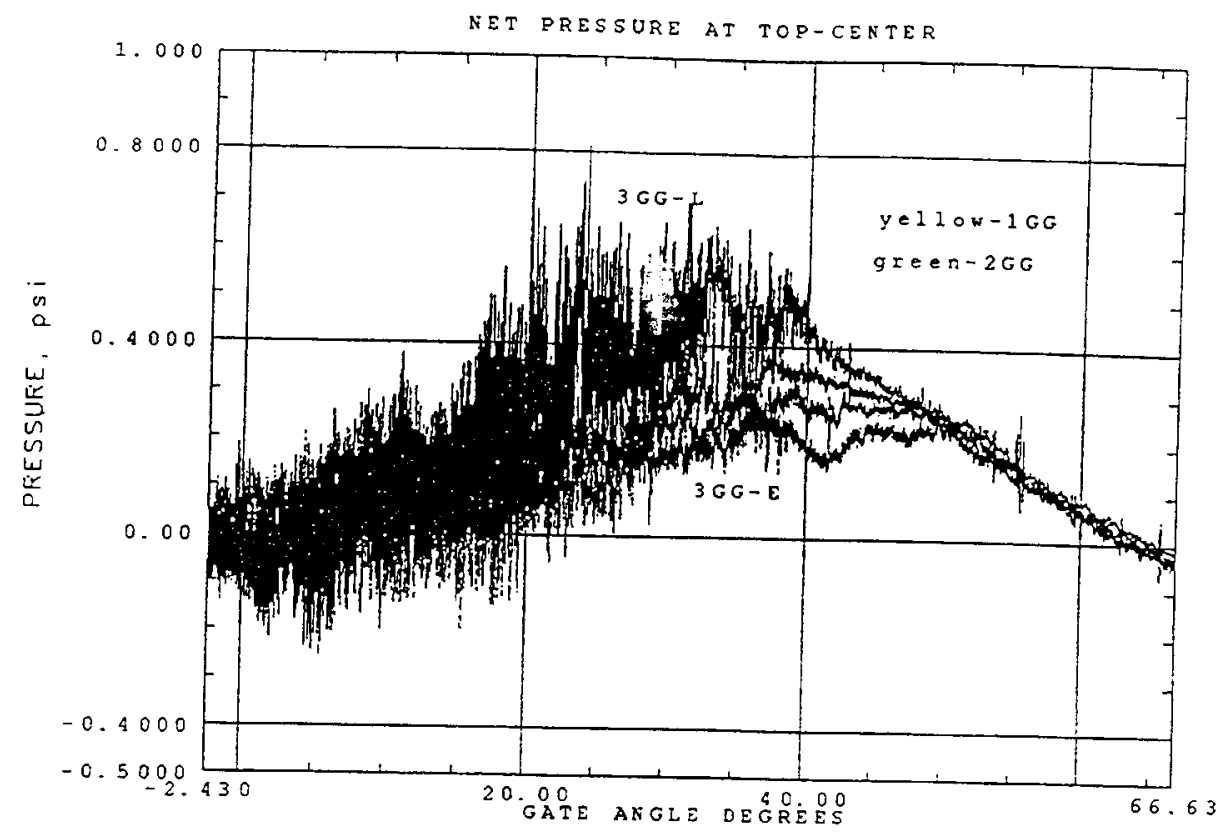

o. Net pressure at top center on gate

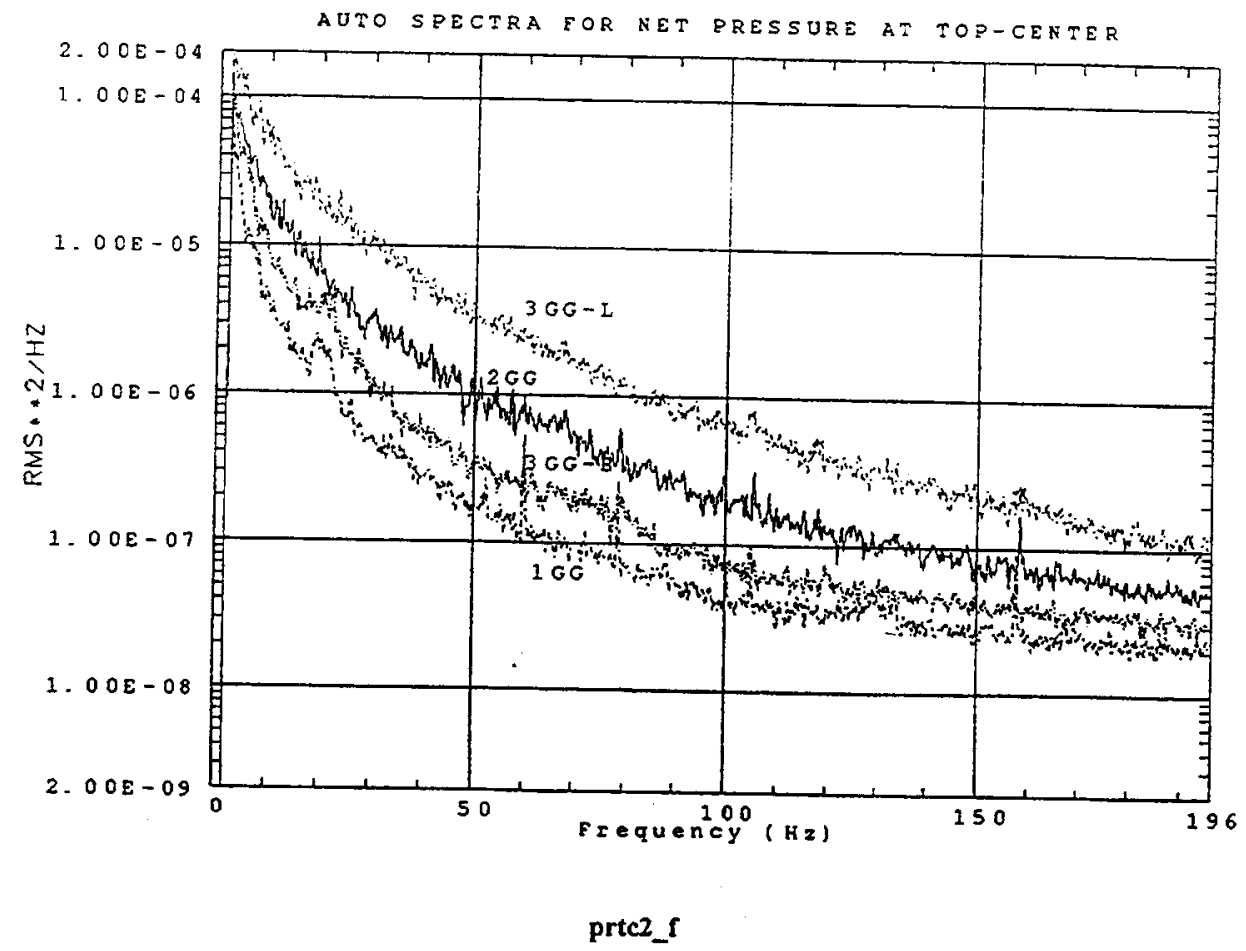

p. Frequency-domain net pressure at top center on gate

Figure 87. (Sheet 8 of 10 ) 


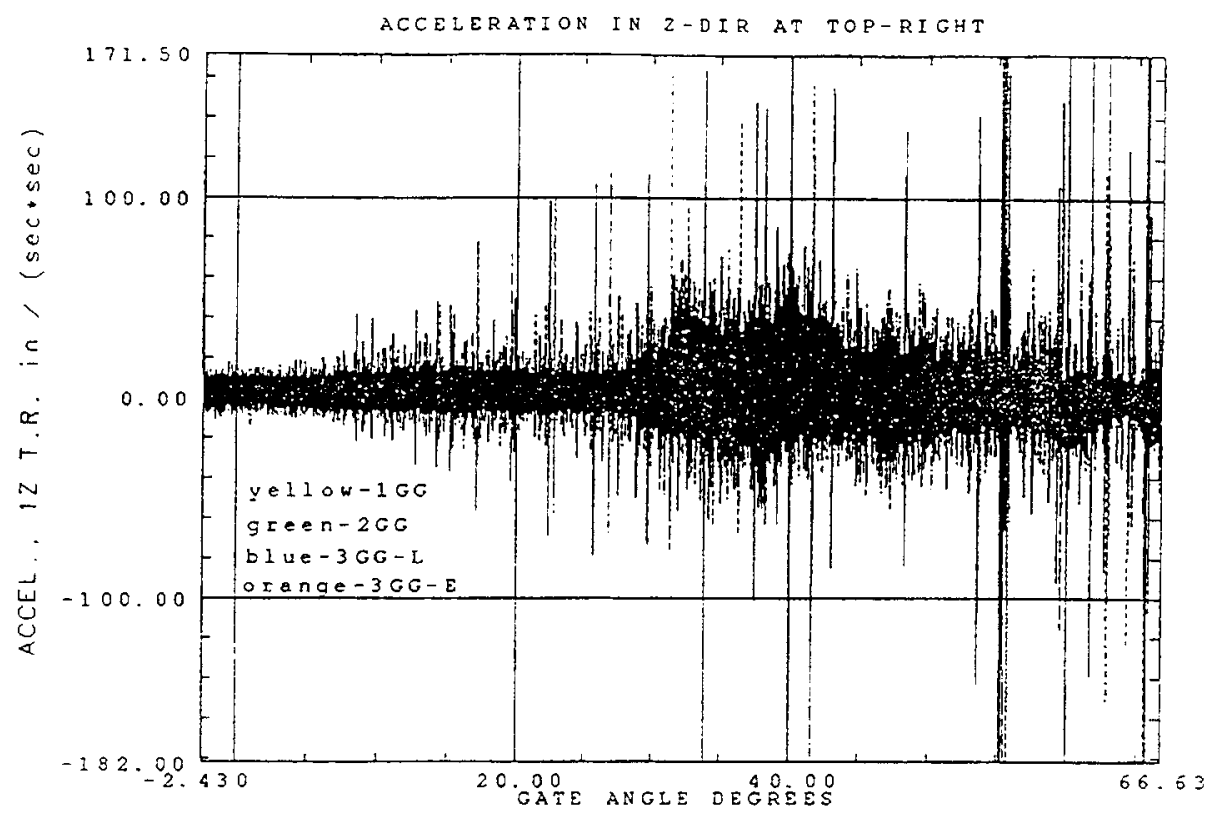

q. Z-directional acceleration at top right

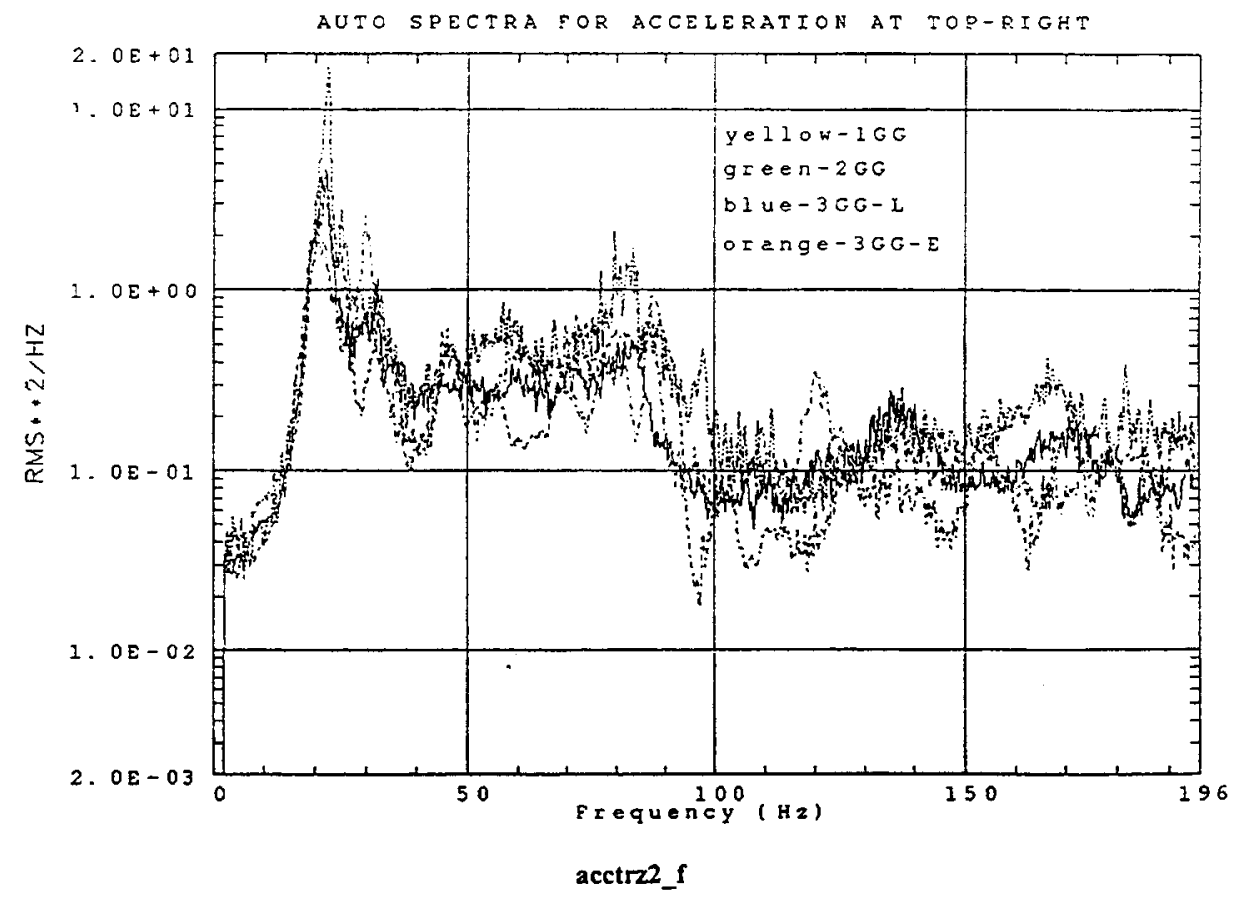

\section{r. Frequency-domain z-acceleration at top right}

Figure 87. (Sheet 9 of 10) 


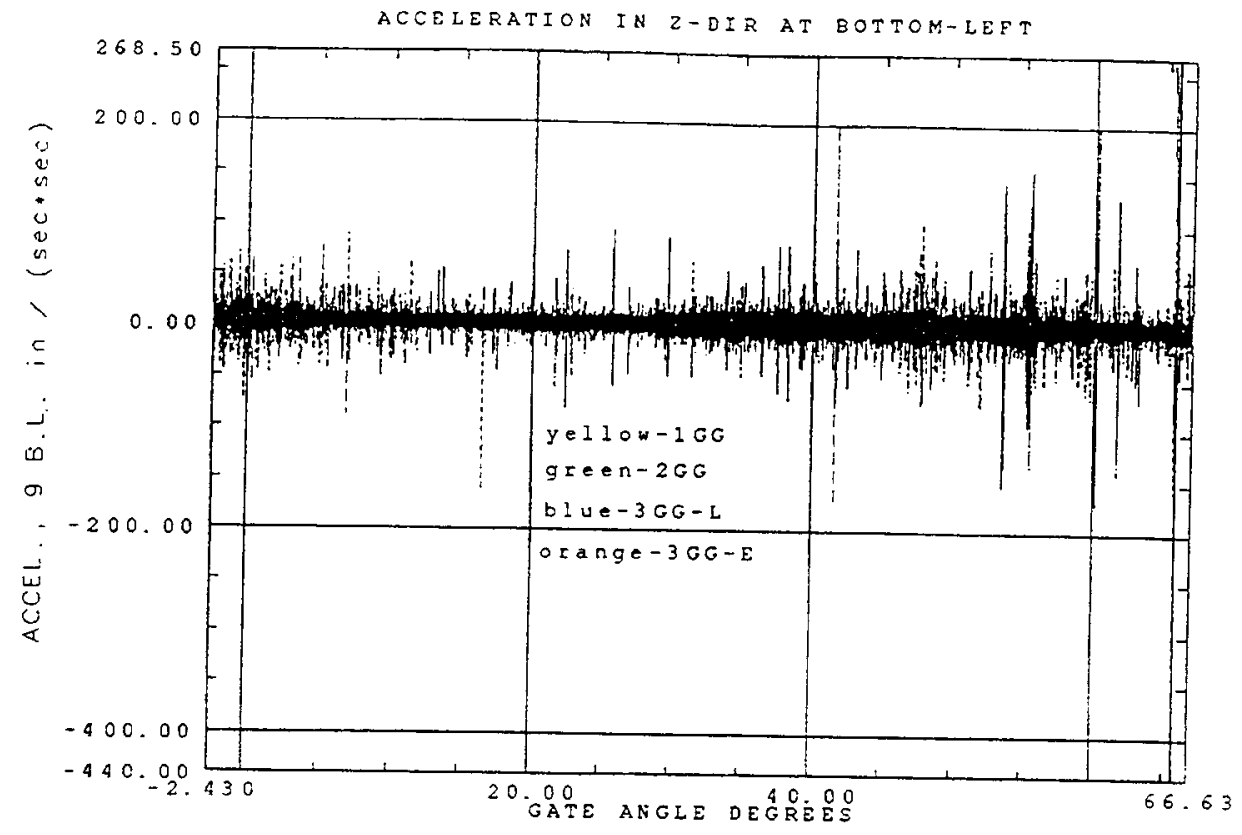

s. Z-directional acceleration at bottom left

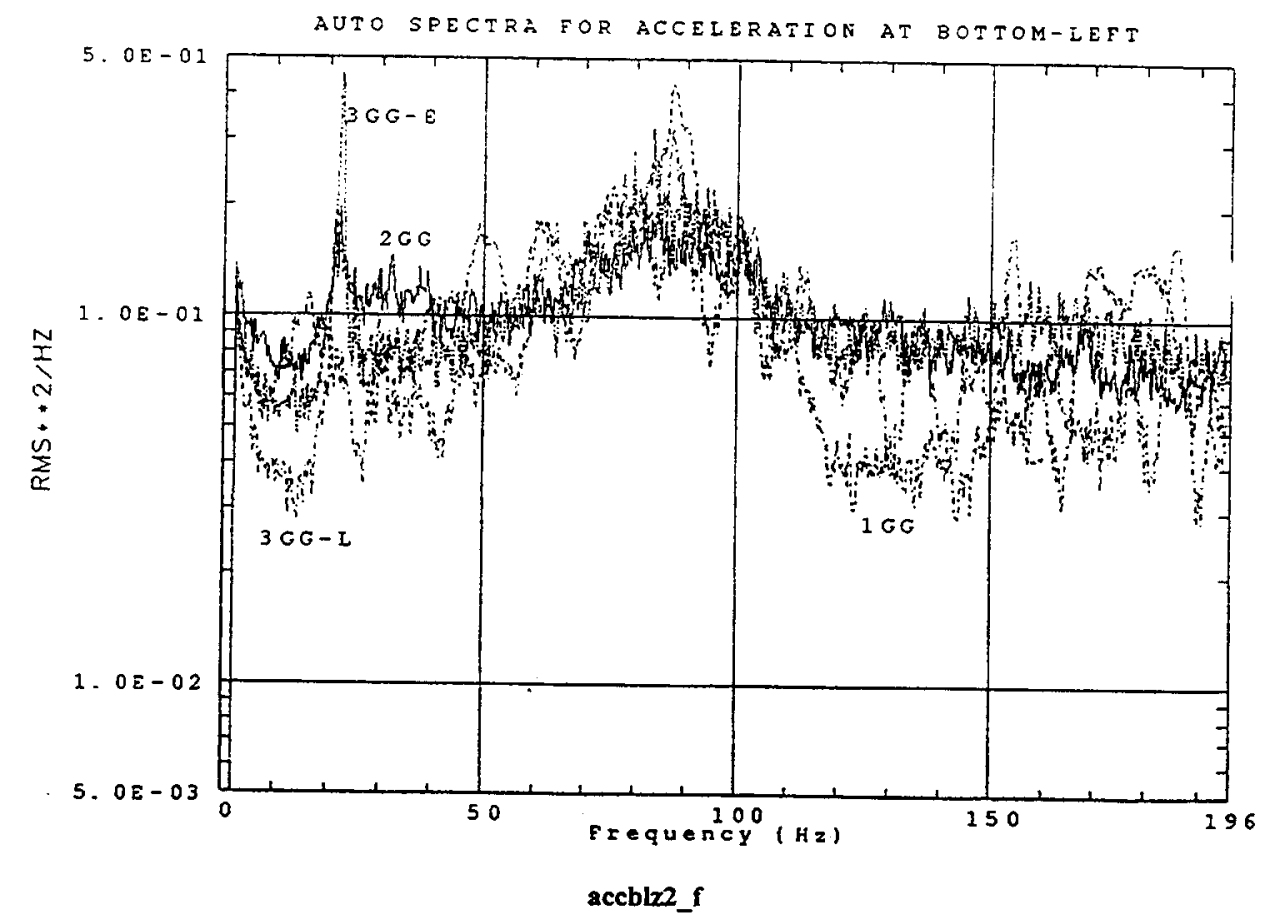

t. Frequency-domain z-acceleration at bottom left

Figure 87. (Sheet 10 of 10) 
three responses. Lightly loaded hinge transducers showed more peaks due to a relatively lower magnitude of the dominant peak.

Upstream and downstream pressure responses and their corresponding PSD are shown in Figure 87g through j. As shown in the PSD for the upstream pressure response, the flow field has two dominant modes at about 20 and $80 \mathrm{~Hz}$. Unlike the 21-ft head-difference downstream pressure distribution, the fluctuation of the downstream negative pressure for $7-\mathrm{ft}$ pool case is minimal and the vacuum duration beneath the gate was for relatively short. The downstream pressure PSD has the random frequency distribution of the fluctuating air gap beneath the gate.

Net pressure responses and their corresponding PSD for bottom-center, middle-center, and top-center transducers are plotted in Figures $87 \mathrm{k}$ through $\mathrm{p}$. A comparison of the net pressure spectra suggests that the $3 \mathrm{GG}-\mathrm{L}$ provided the highest total energy among the cases investigated. It also indicates that a random nature of fluctuation of the flow field dominated the response with little or no interaction with the structural mode.

Two of the largest acceleration responses, at top-right and bottom-left positions, are presented in Figures $87 \mathrm{q}$ through $\mathrm{t}$. PSD for the accelerations show the dominant modes of flow-induced vibration for the gate corresponding to each of the flow configurations presented in the plots. A random flow field of the gate raising cycle, with a broad band frequency content in the three-gate gap (lone), excited the gate at several distinct modes. A display of the operating shapes at dominant peaks would indicate the nature of vibration of the gate. Frequency matching for similar operating modes and "wet modes" would cause a resonant vibration in the system.

\section{4-ft pool difference}

Figure 88a through $v$ compares the results for the five-gate gap configurations with a 4-ft head difference. Time-domain responses for both hinges and shaft load are compared in Figures 88a through g. Figure 88a, b, d, and e present the orthogonal reactions for both hinges. Total vertical and horizontal reactions are shown in Figures $88 \mathrm{c}$ and $\mathrm{f}$. A sum of both trunnion forces representing the shaft load are presented in Figure 88g. For each case, 5GG lone condition provided the maximum responses.

Eighteen pressure gauge responses for both gate conditions are presented in Figures $88 \mathrm{~h}$ through $\mathrm{m}$. In each of these figures, directly recorded pressures in terms of height of water column are shown for three pressure transducers lying in a vertical plane transverse to the upright gate surface (see Figure 57 for gauge locations). Eighteen pressure-gauge records on a 3-by-3 grid adequately scan the pressure distribution on the surface of the wicket. These pressure records can be used to reconstruct the surface distribution of pressure on the wicket as a function of time. These readings represent the actual depth traversed by each gauge from the initial zero datum. As mentioned in the data acquisition chapter, 


\section{1:5 Olmsted Model}

Tests: 180,186

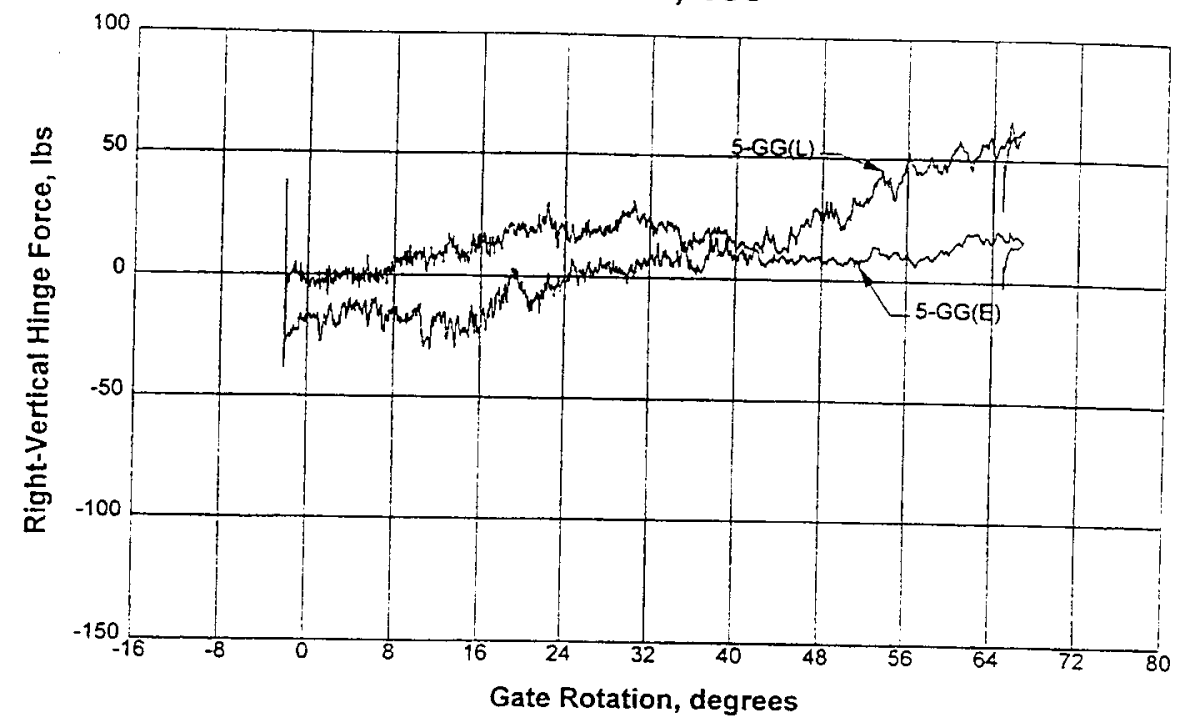

a. Right vertical hinge load

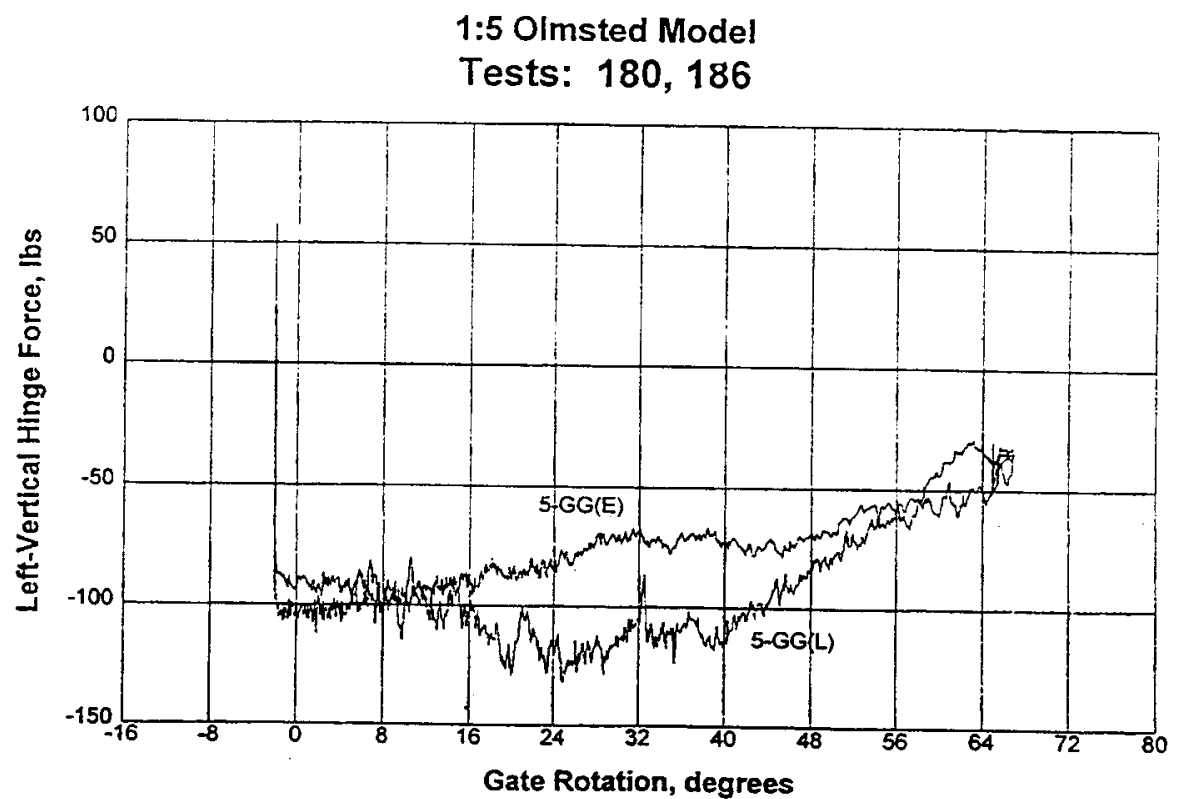

b. Left vertical hinge load

Figure 88. Comparison of reasponses over full range of operation for various gate configurations, 4-ft pool difference (Sheet 1 of 10) 


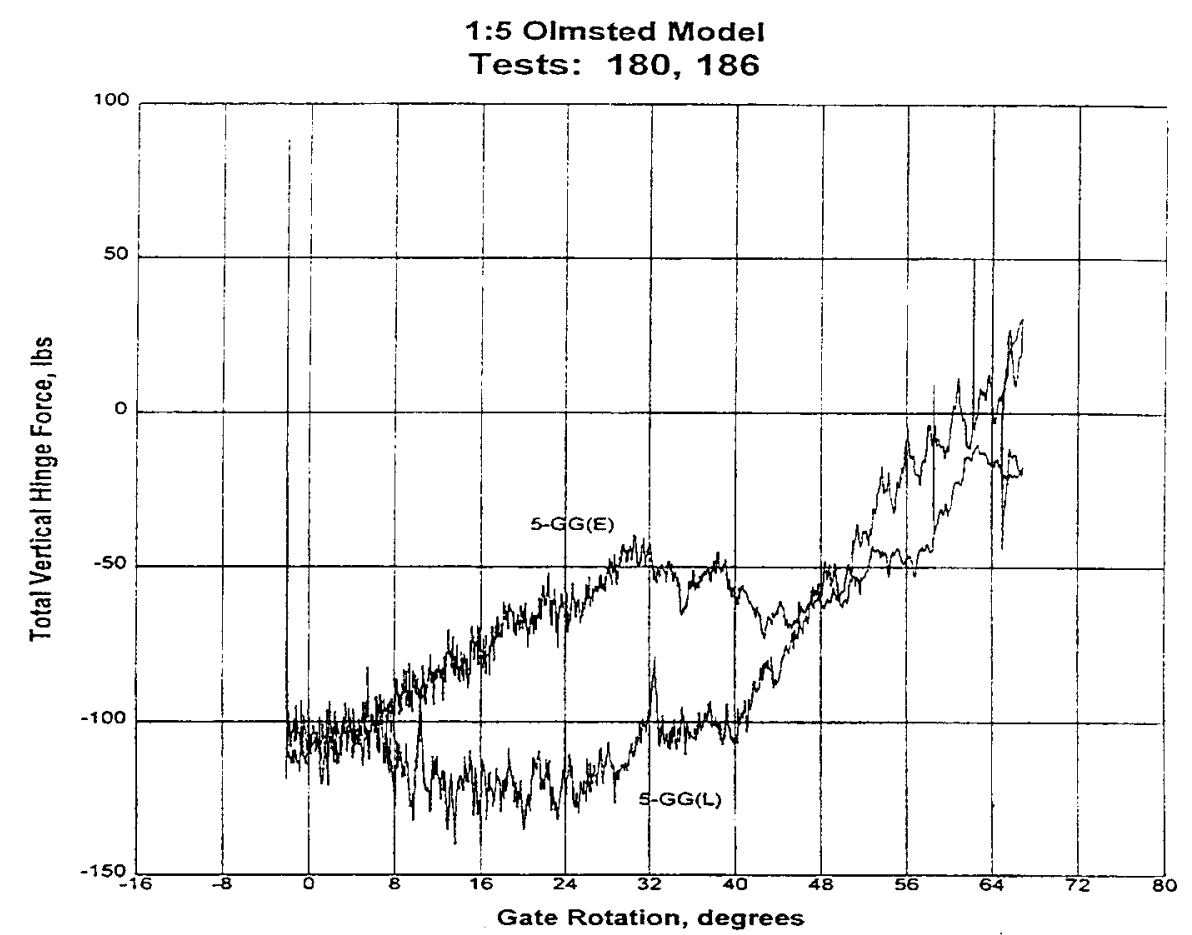

c. Total vertical hinge load

1:5 Olmsted Model

Tests: 180,186

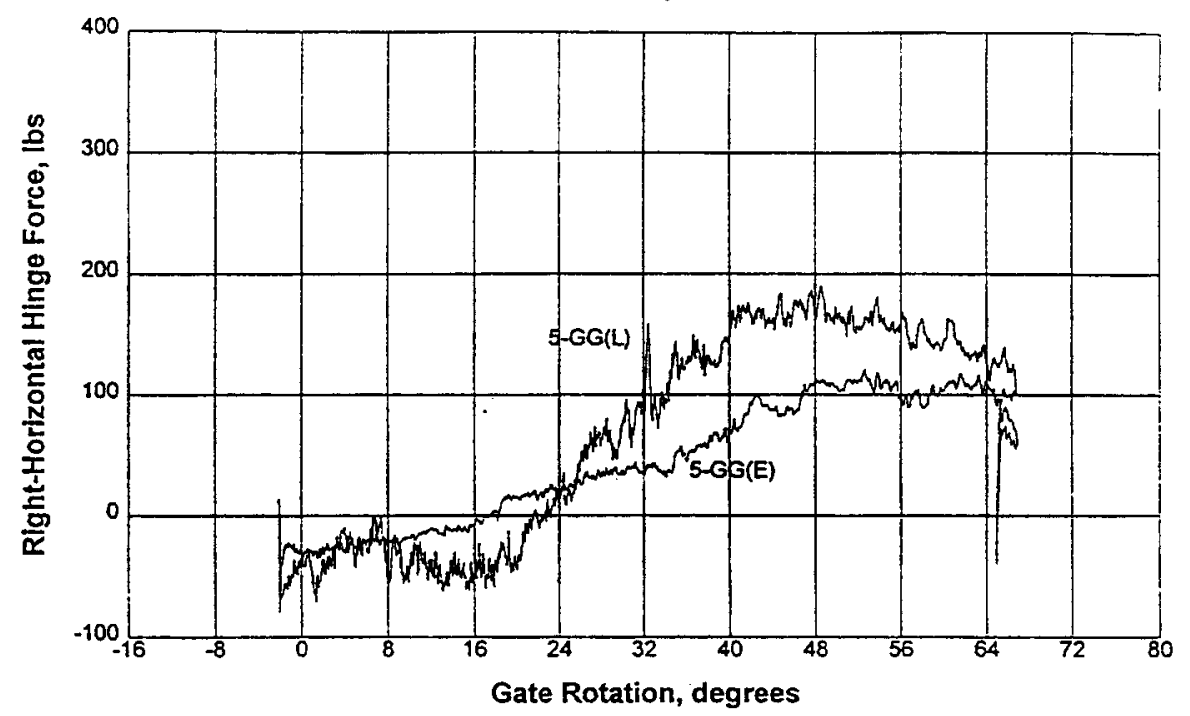

d. Right horizontal hinge load

Figure 88 . (Sheet 2 of 10 ) 


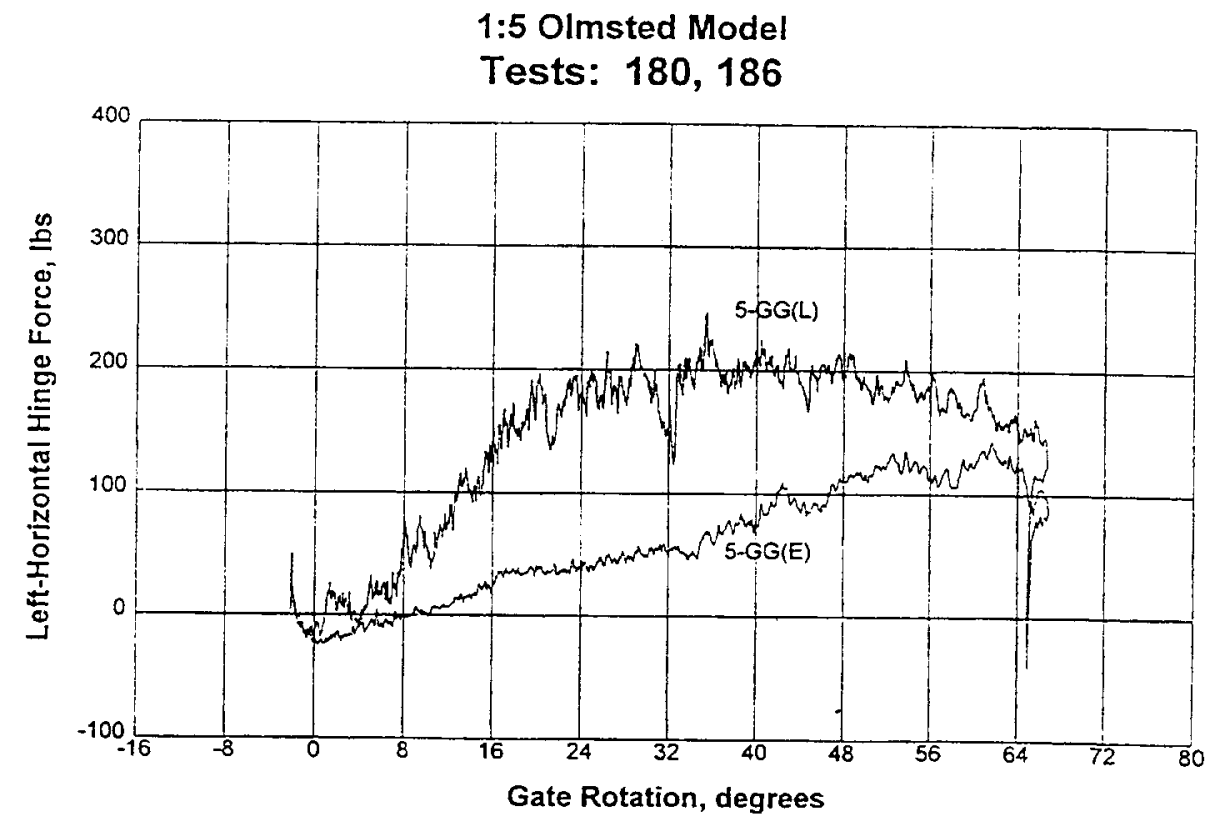

e. Left horizontal hinge load

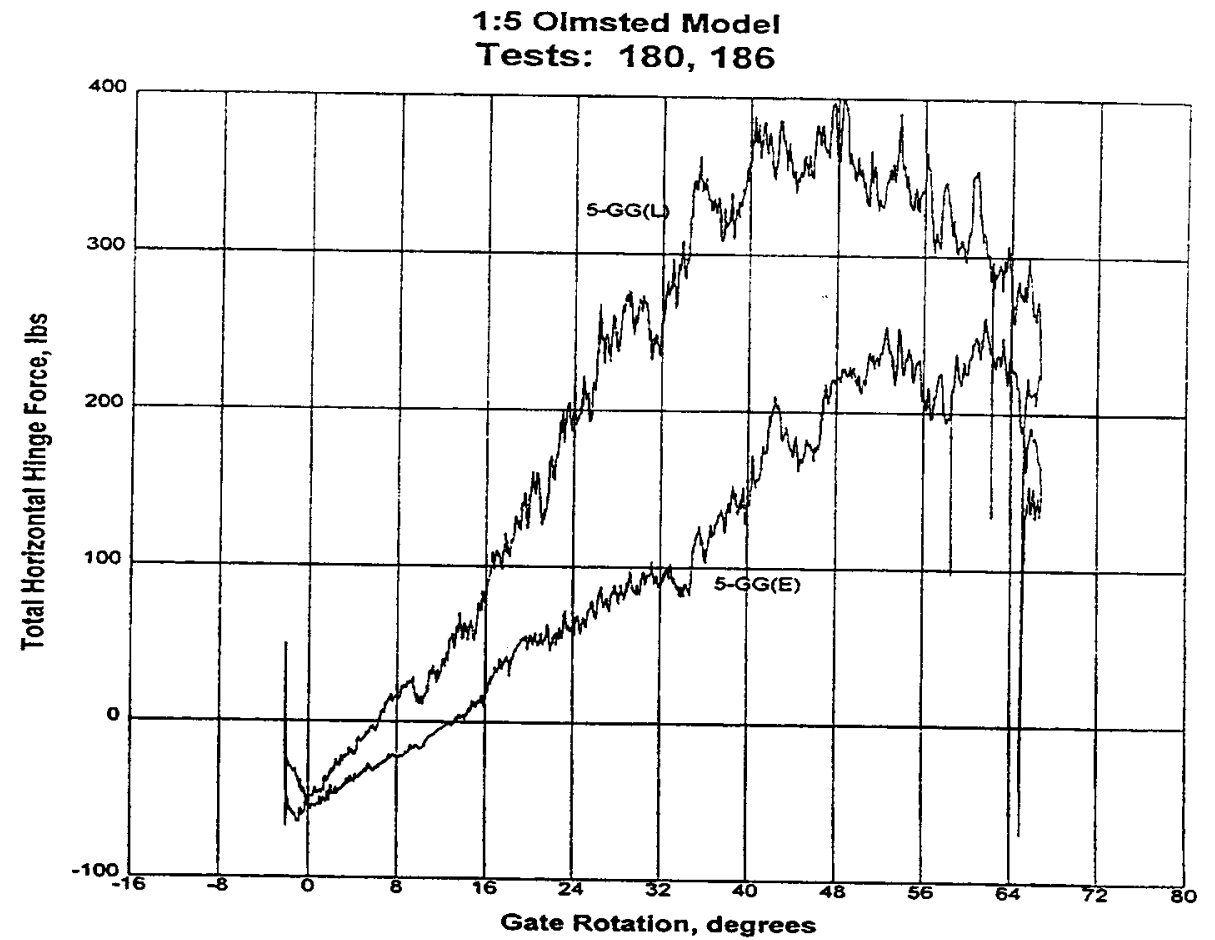

f. Total horizontal hinge load

Figure 88. (Sheet 3 of 10 ) 


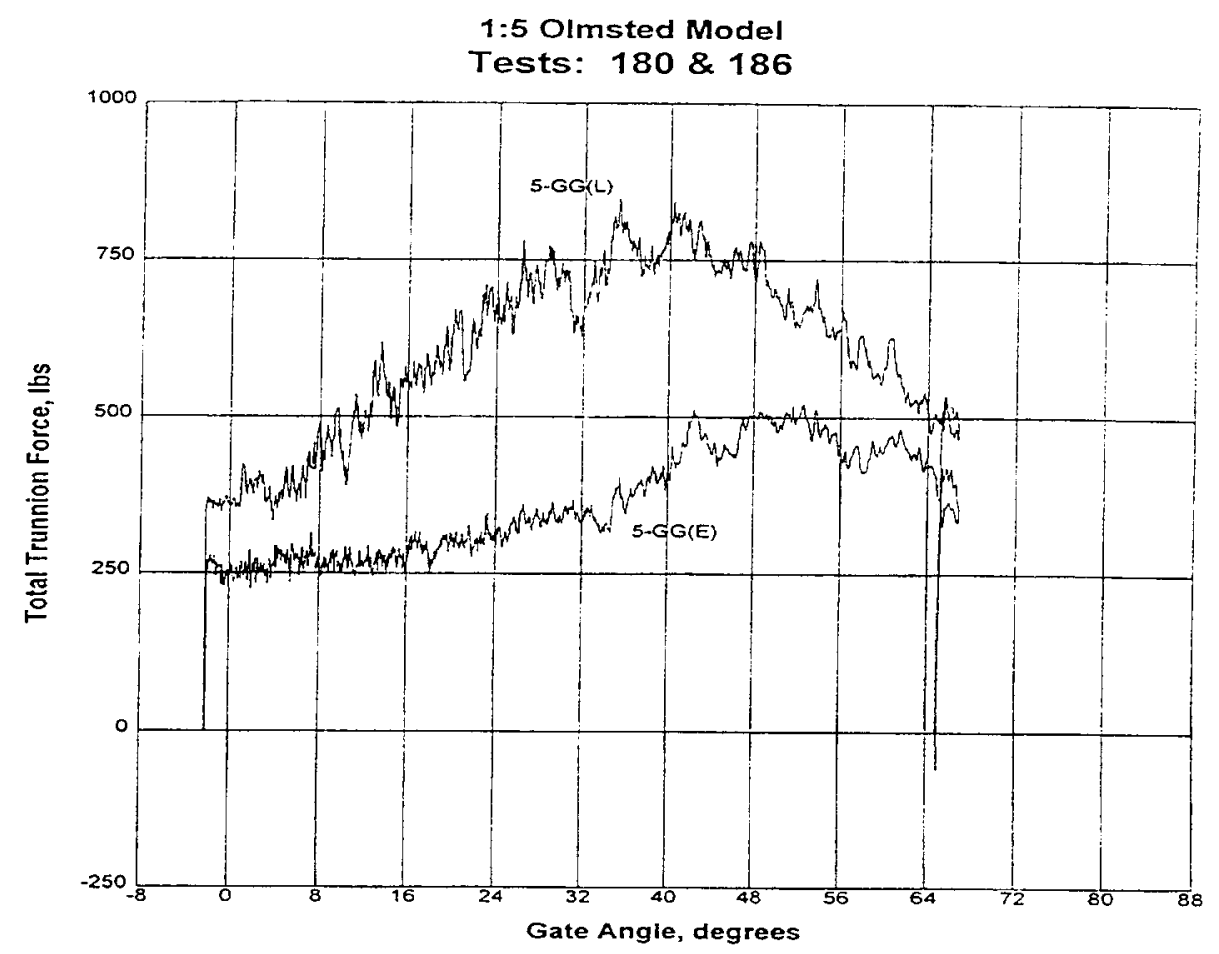

g. Total trunnion load

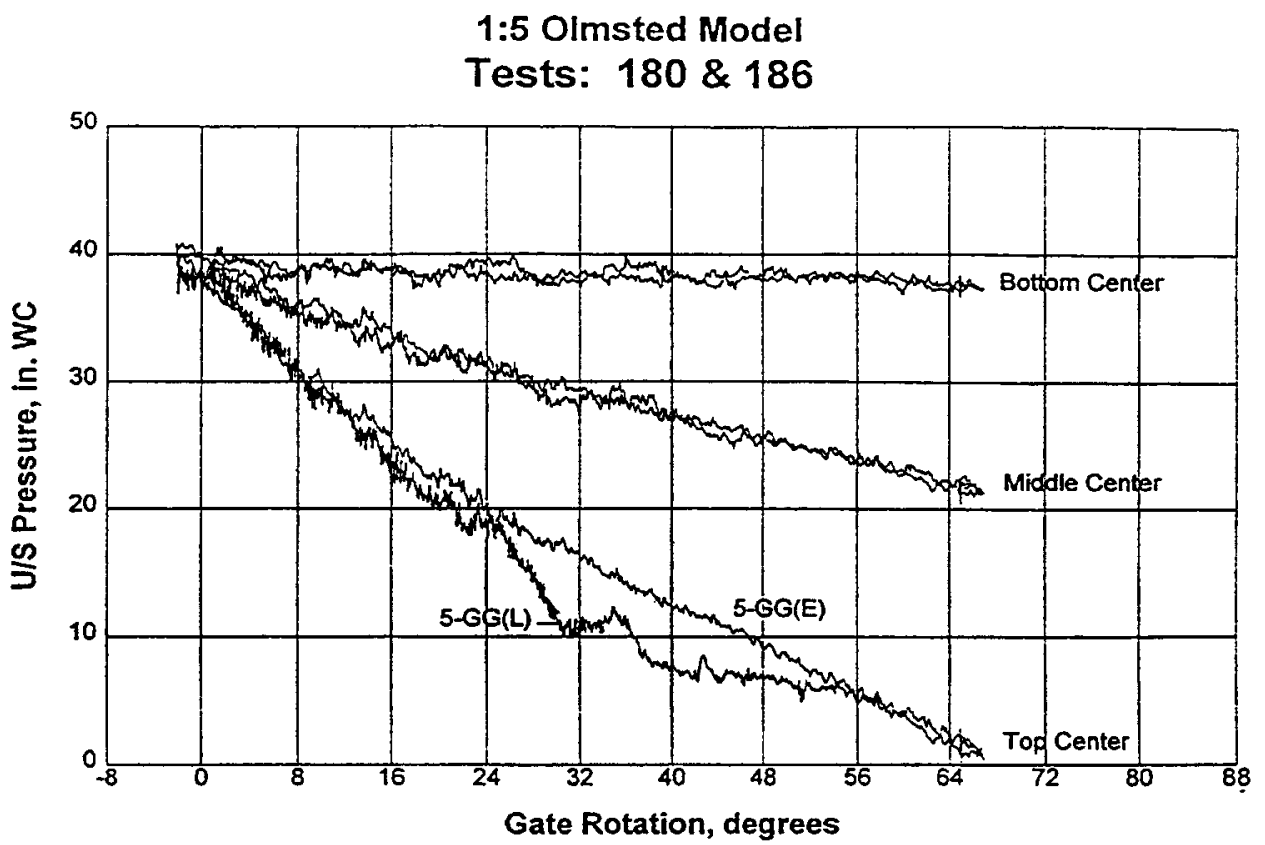

h. Upstream pressure records of transducers placed along center line of gate

Figure 88. (Sheet 4 of 10) 
1:5 Olmsted Model

Tests: $180 \& 186$

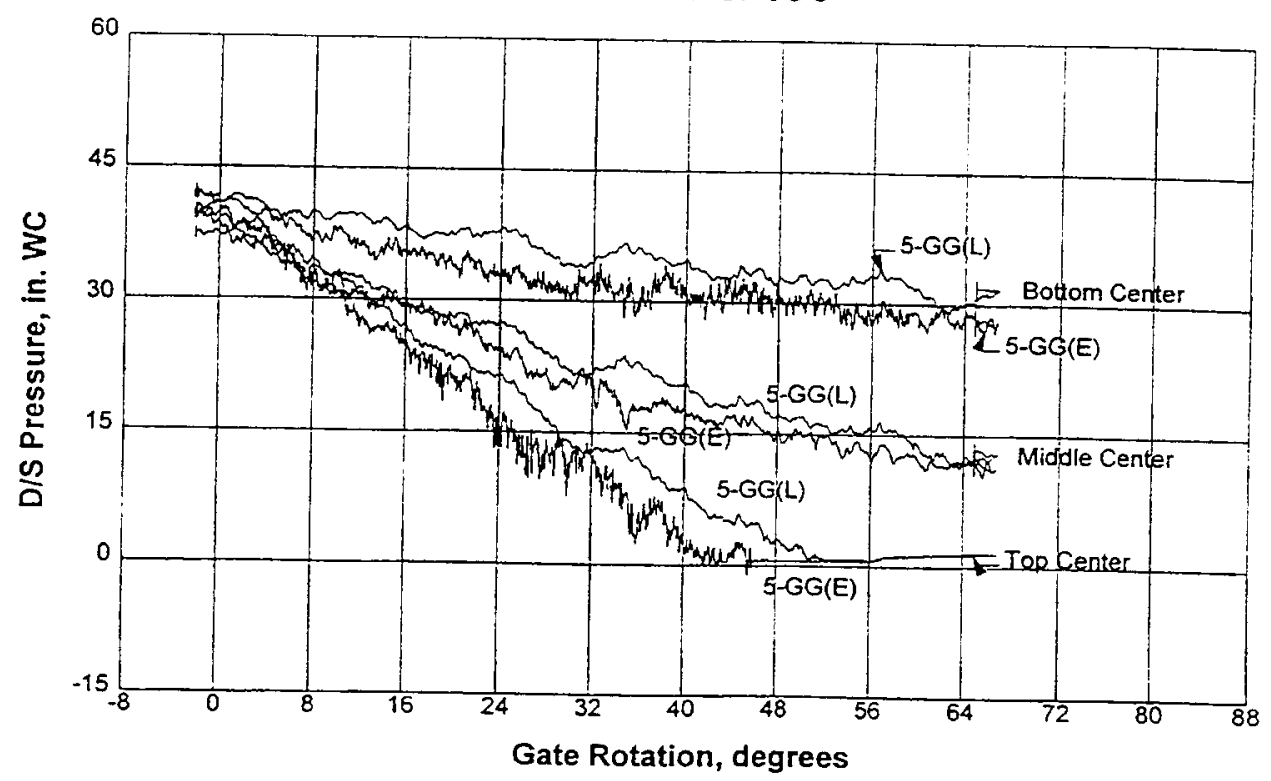

i. Downstream pressure records of transducers placed along center line of gate

\section{1:5 Olmsted Model}

Tests: $180 \& 186$

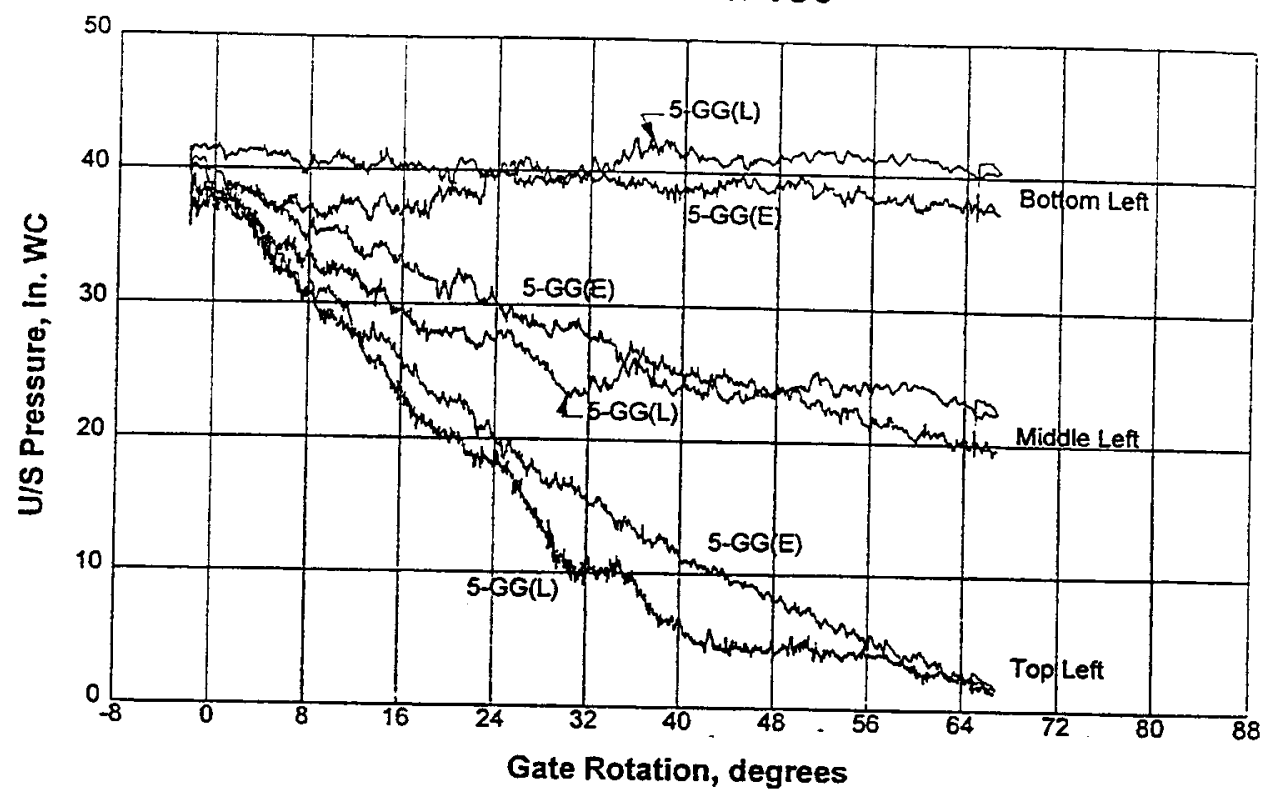

j. Upstream pressure records of transducers placed on left side of gate

Figure 88. (Sheet 5 of 10) 
Tests: $180 \& 186$

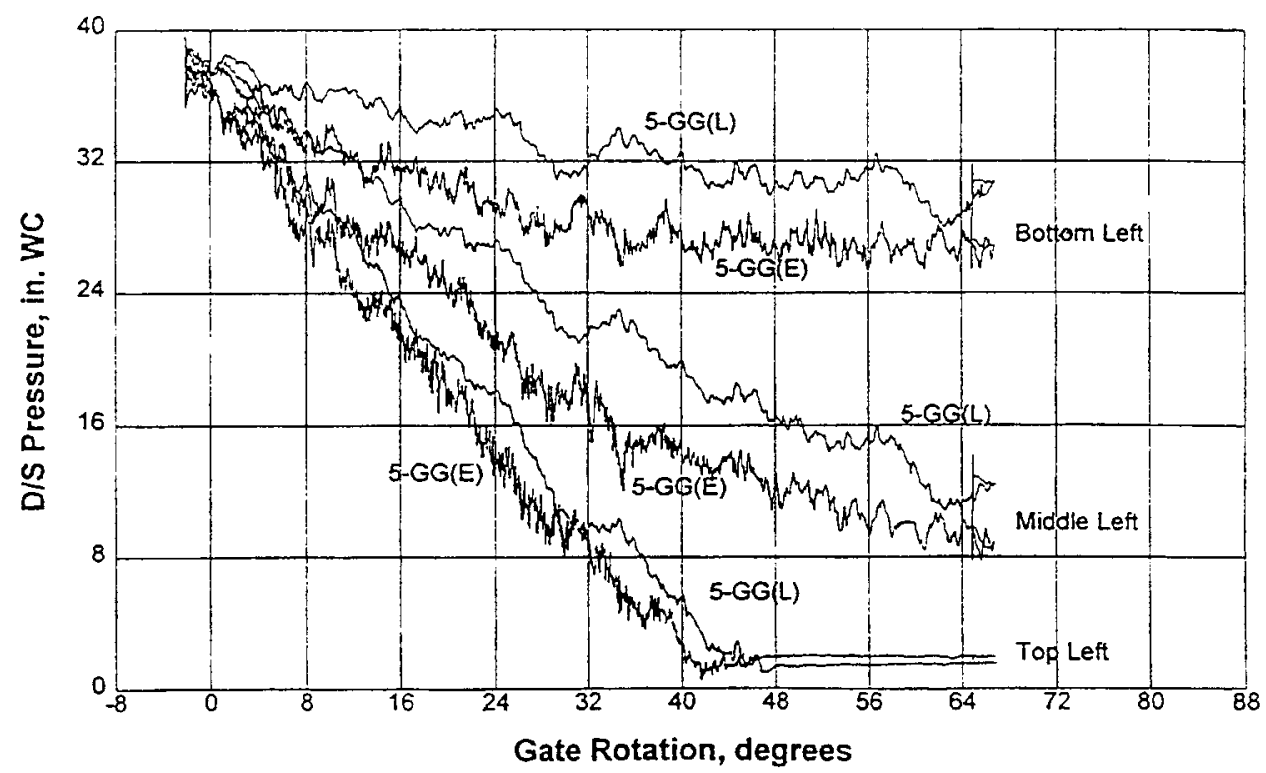

k. Downstream pressure records for transducers placed on left side of gate

1:5 Olmsted Model

Tests: $180 \& 186$

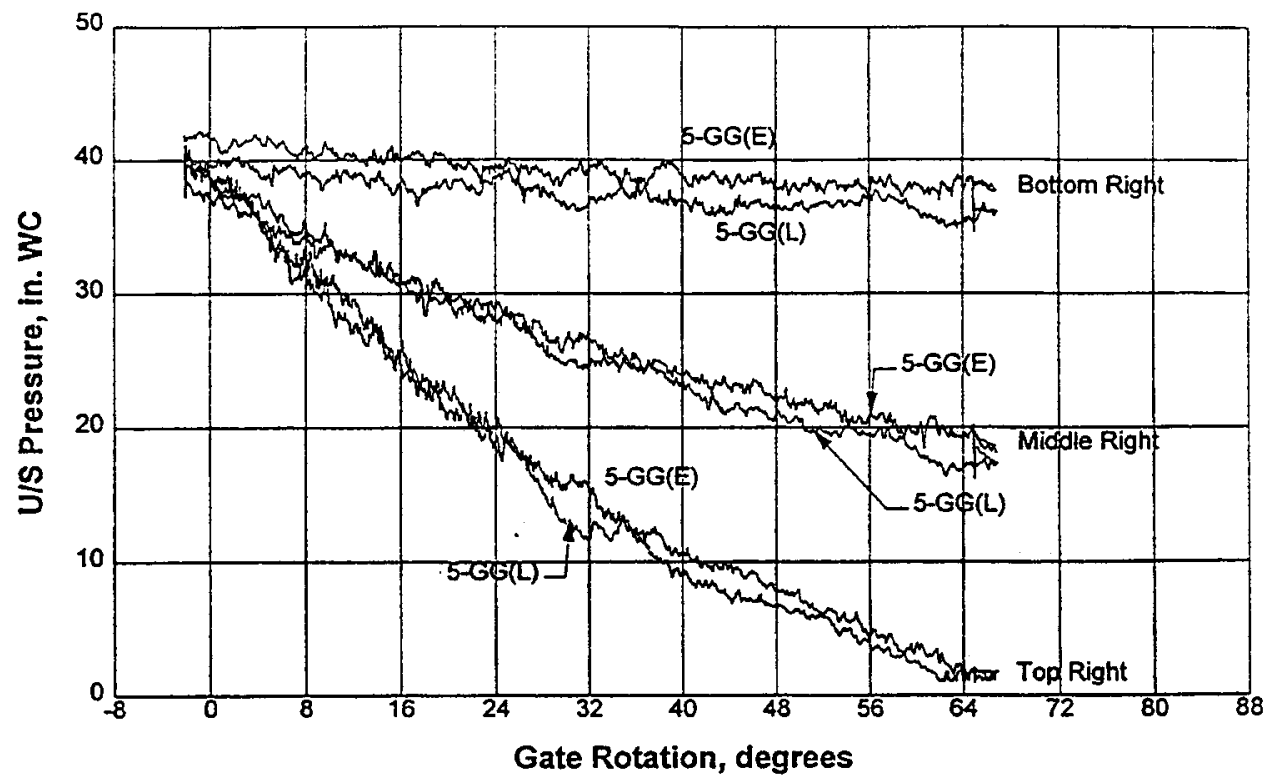

I. Upstream pressure records of transducers placed on right side of gate

Figure 88. (Sheet 6 of 10 ) 


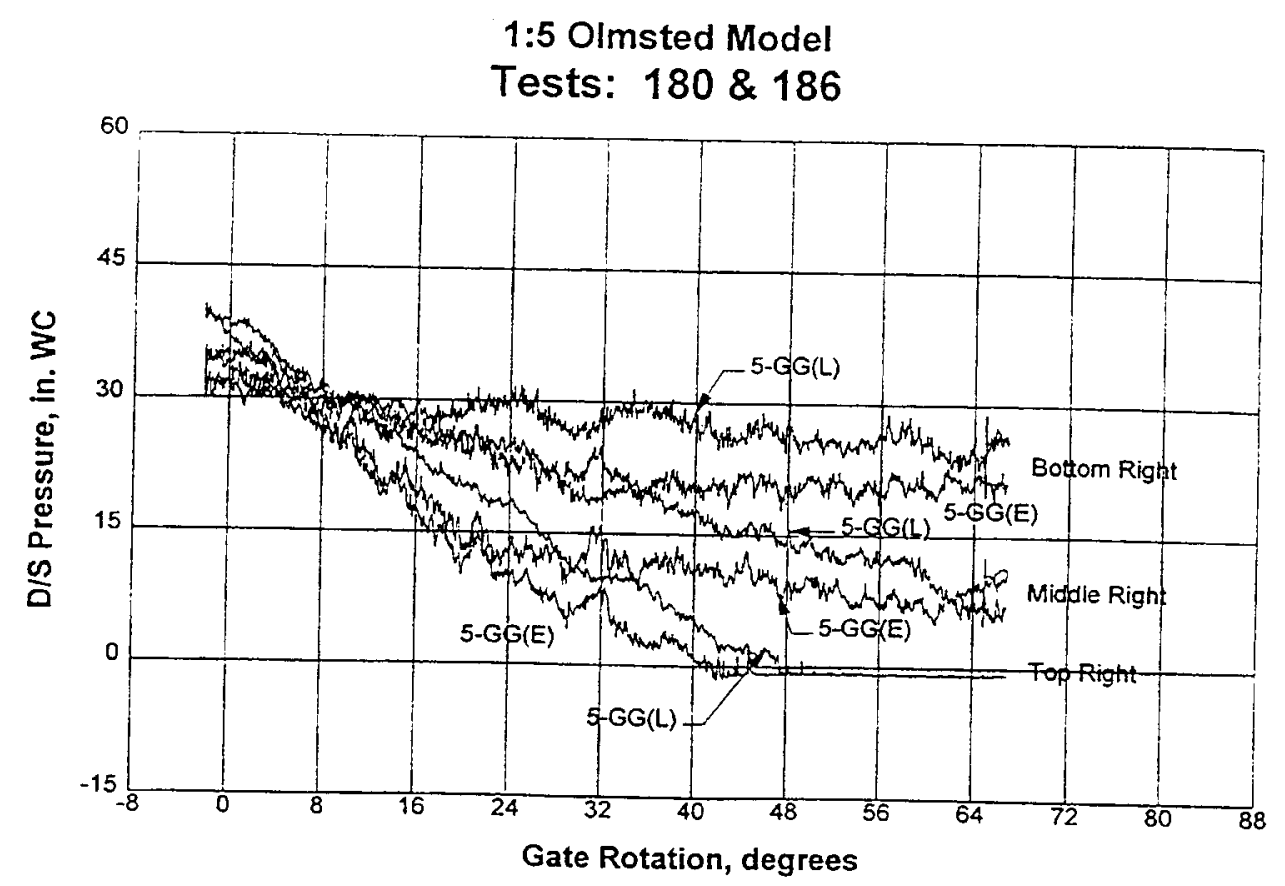

m. Downstream pressure records for transducers placed on right side of gate

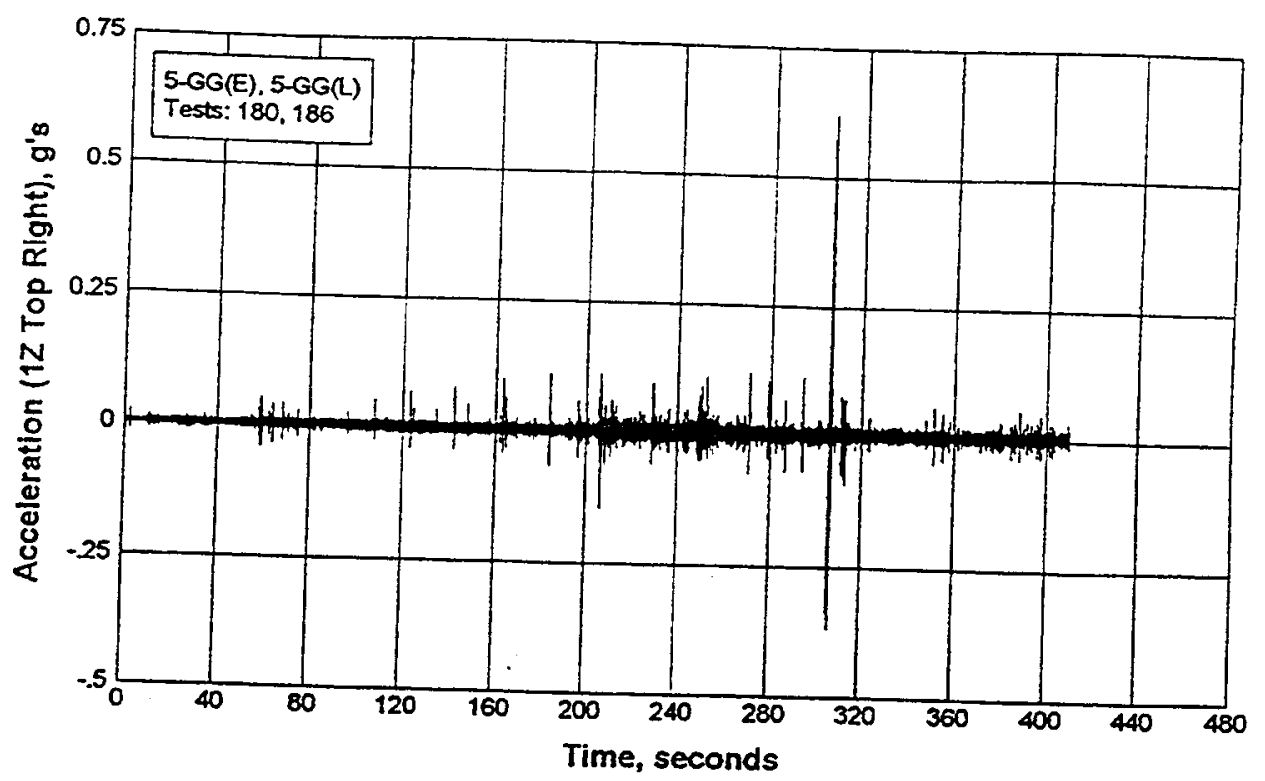

n. Z-directional acceleration at top right

Figure 88. (Sheet 7 of 10) 


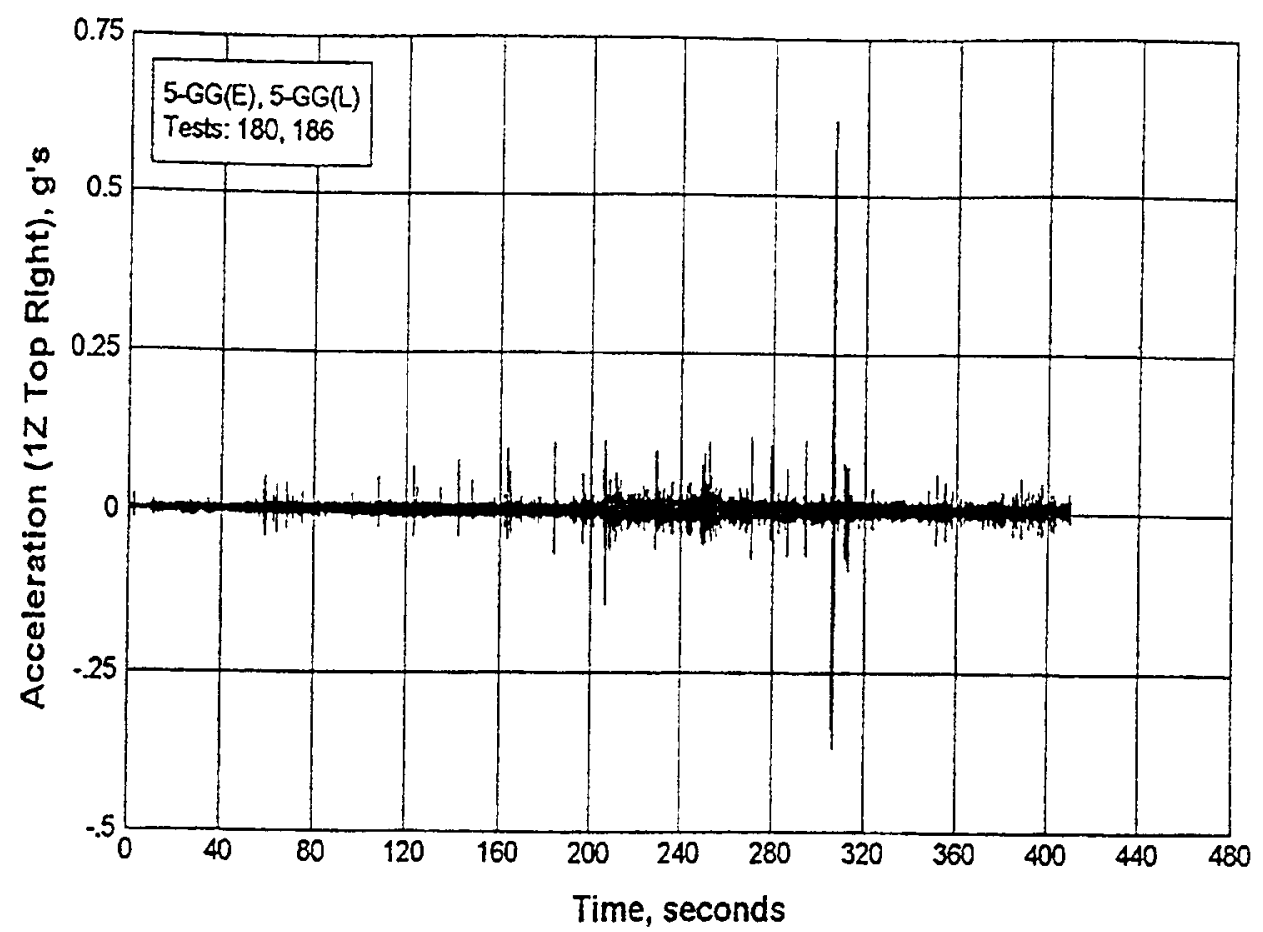

o. Z-directional acceleration at left corner of wicket

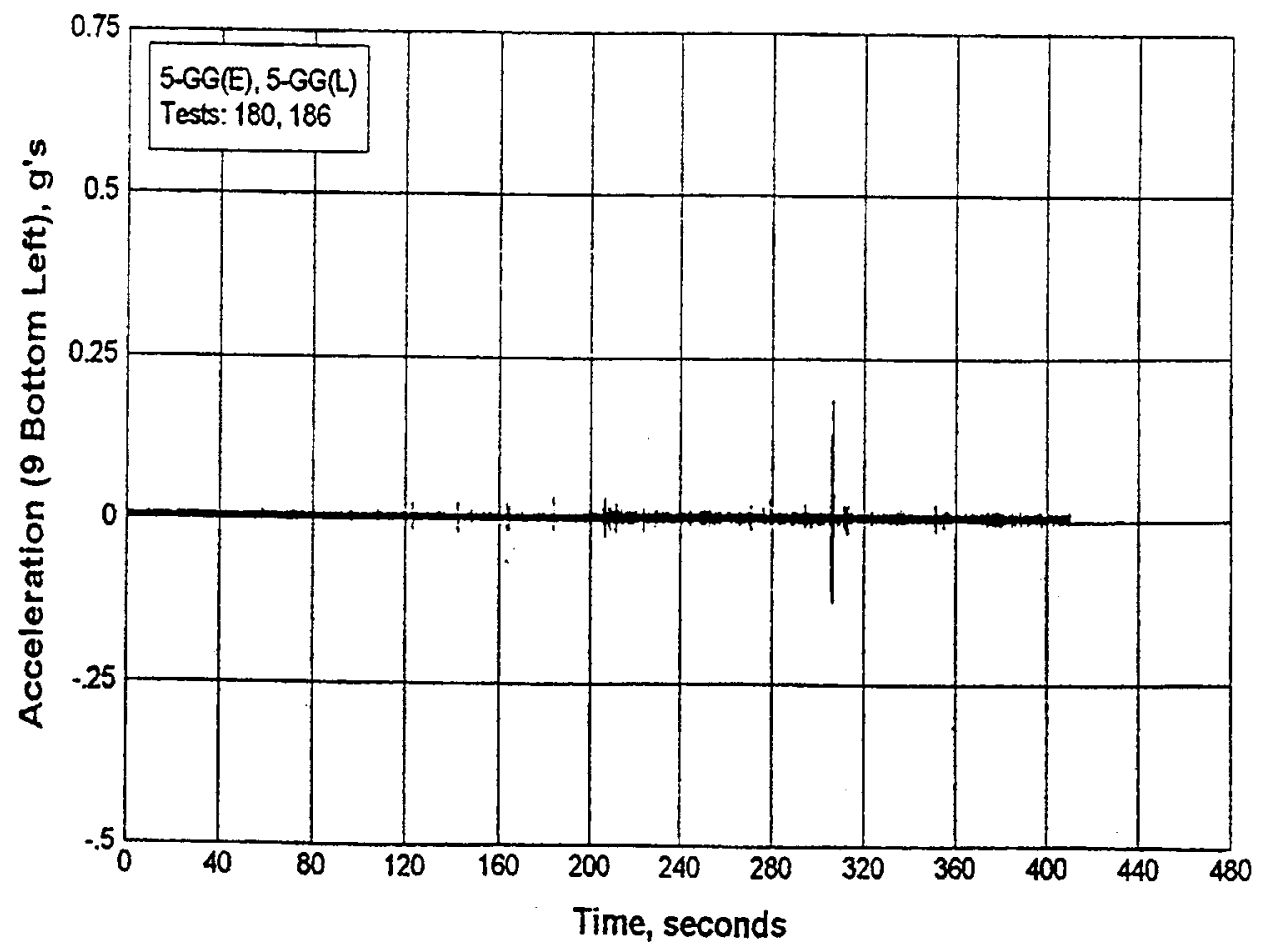

p. Z-directional acceleration at bottom left corner of gate

Figure 88. (Sheet 8 of 10) 


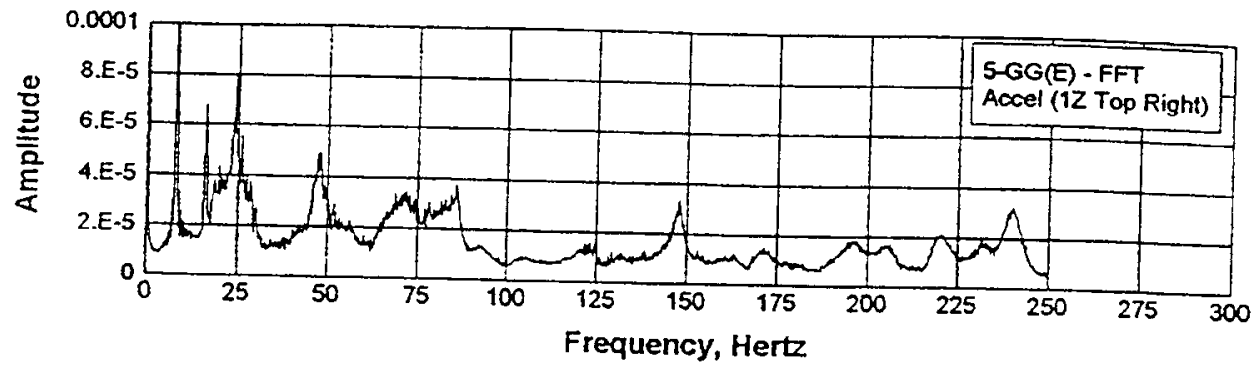

q. Frequency-domain z-acceleration at top right of wicket for $5 G G(E)$ condition

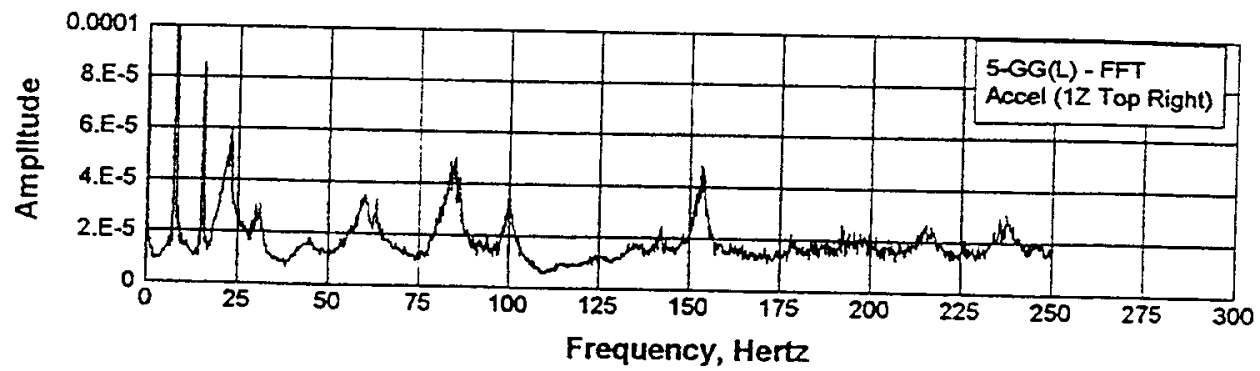

r. Frequency-domain z-acceleration at top right top of wicket for $5 G G(L)$ condition

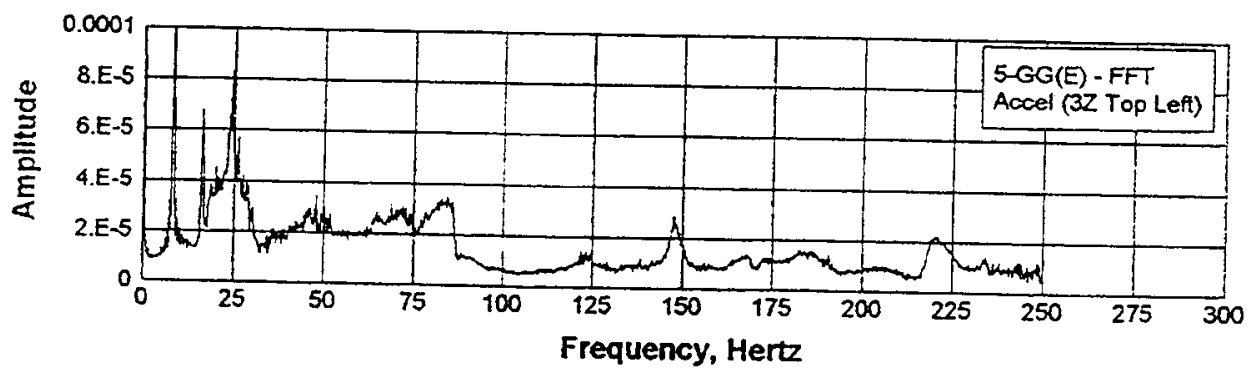

s. Frequency-domain z-acceleration at top left of wicket for $5 G G(E)$ condition

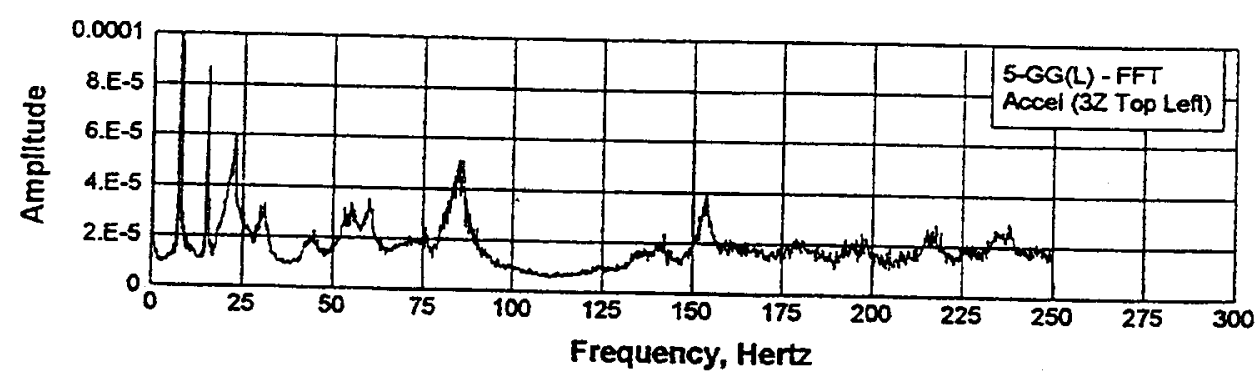

t. Frequency-domain z-acceleration at top left of wicket for $5 G G(L)$ condition

Figure 88. (Sheet 9 of 10) 


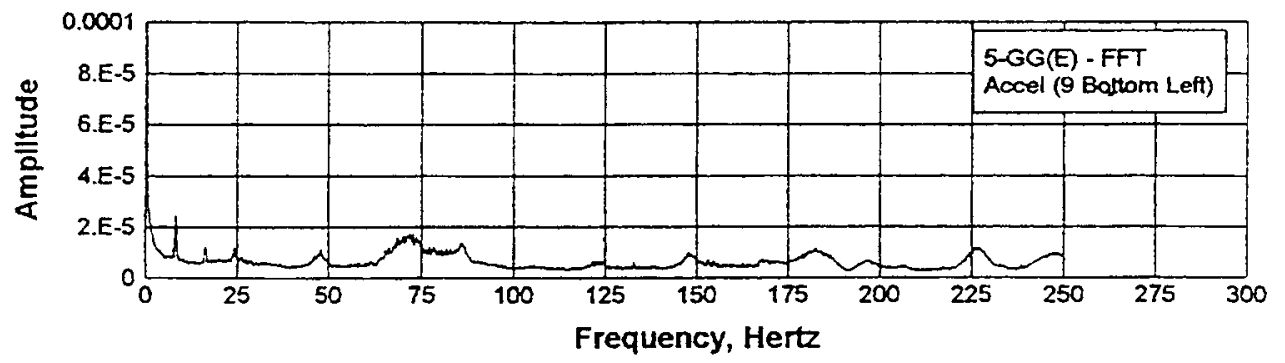

u. Frequency-domain z-acceleration at bottom left of wicket for $5 \mathrm{GG}(\mathrm{E})$ condition

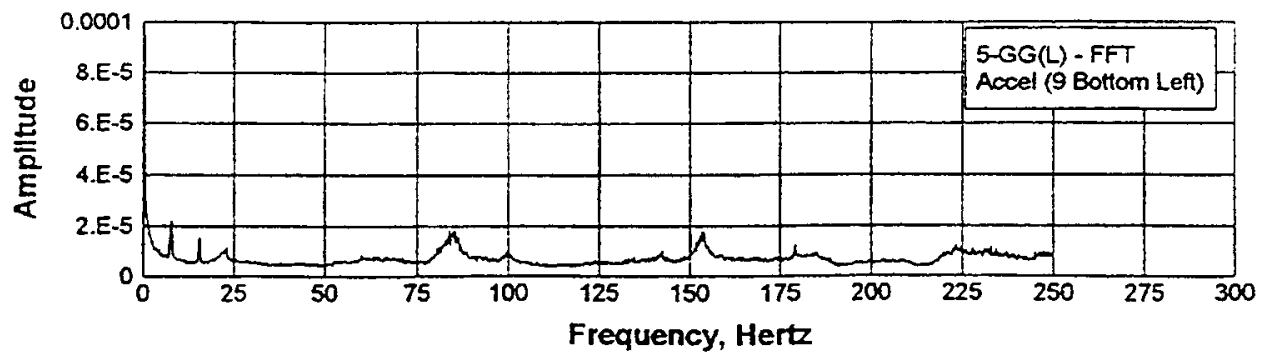

v. Frequency-domain z-acceleration at bottom left of wicket for $5 \mathrm{GG}(\mathrm{L})$ condition

Figure 88. (Sheet 10 of 10 )

by subtracting the respective initial zero depth from gauge readings, the absolute pressure exerted on the gate surface could be obtained. As seen in the plots, the upstream pressure responses did not differ due to a change in gate position. The downstream pressures on both edges of the gate, however, show a slight variation.

Three acceleration responses in the time- and frequency-domain are presented in Figures $88 \mathrm{n}$ through $\mathrm{v}$. Frequency-domain plots (Figures $88 \mathrm{q}$ through $\mathrm{v}$ ) for both gate conditions are separately plotted for three accelerometer plots presented in Figures $88 \mathrm{n}$ through p. As seen in the PSD plots, the gate positions in a five-gate gap configuration did not change the frequency distribution for respective channels.

\section{Critical operation}

A critical position corresponds to a gate location which provided the maximum amplitude for the responses measured for the gate configurations compared above. As observed in the full operation, a three-gate gap is the critical configuration regardless of head difference in the pool condition. In a 3GG configuration, the lone position of the instrumented gate provided the highest overall response. A scanning of the responses also shows that at about the 16 to 24 -deg position of the gate, maximum fluctuation occurred in the 
responses. For the 7 -ft pool condition, a critical position occurred at about the 20- to 30-deg position.

From the dynamics point of view, a critical operating shape could be the one in resonance with the natural mode shapes of the wet gate. Each of the peaks in the acceleration response represent an operating shape. A display of the operating shapes at dominant peaks would indicate the nature of vibration of the gate. Peaks in the acceleration response signify their participation in the overall flow-induced dynamics of the gate. The higher peaks indicate greater dynamic participation of the associated operating mode. Therefore, a frequency response function with distinctly featured peaks is the most critical feature for identifying dynamic problems in a system. As seen in the acceleration PSD, the three-gate gap lone-condition shows the distinct peaks for both pool conditions.

\section{Fixed Gate Response at Critical Operation}

As mentioned above, the critical dynamics of the gate occurred when the instrumented gate was positioned as the center gate of a three-gate gap. A statistical summary of the experimental results for two pool conditions at critical configurations are summarized in Table 18. The values presented in this table are the root mean-square $\left(\mathrm{RMS}_{\mathrm{x})}\right.$ and the standard deviation $(\sigma)$ of random response, $f(t)$. The $R^{2} S_{x}$ of a random function $f(t)$ can be defined as:

$R M S_{x}=\sqrt{\bar{x}^{2}}$, where $\bar{x}^{2}=\frac{1}{T} \int_{0}^{T} f^{2}(t) d t$. The standard deviation may be obtained by using, $\sigma_{x}=\sqrt{\left(\bar{x}^{2}-(\bar{x})^{2}\right)}$, where $\bar{x}$ is the mean value of the response function and $\mathrm{T}$ is the duration of time record.

By keeping the gate fixed at the critical location, the test gate responses were recorded for both pool conditions. For the $21-\mathrm{ft}$ pool condition, the data were recorded at 65- (prop-supported) and 24-deg positions. Note that the 24-deg position is a transient phase of the wicket operation which will be encountered only during the raising and lowering of the wicket. A prop-supported, 65-deg position, on the other hand, is the design condition of the wicket. At $65 \mathrm{deg}$, the wicket will be used to regulate the water depth on the upstream side of the river. For the 7-ft pool condition, two positions at 21.5 and $31.5 \mathrm{deg}$ were used for fixed gate data acquisition. The pressure gauge readings are presented in terms of water-column (wc) head above respective transducer. A negative pressure reading indicates that a suction due to vacuum underneath the gate pulls the gate downward.

As seen in the table, the upstream pressure remains steady about its mean with a minimum fluctuation not exceeding 10 percent of the RMS value. The downstream negative pressure, however, substantially varied about the respective RMS value. The accelerations of the gate due to flow-induced vibration remain substantially low for the test range presented in the table. A graphical display of measured responses for the 21 -ft head difference, three-gate gap flow configuration for two gate positions is presented in Figures 89 and 90 . 

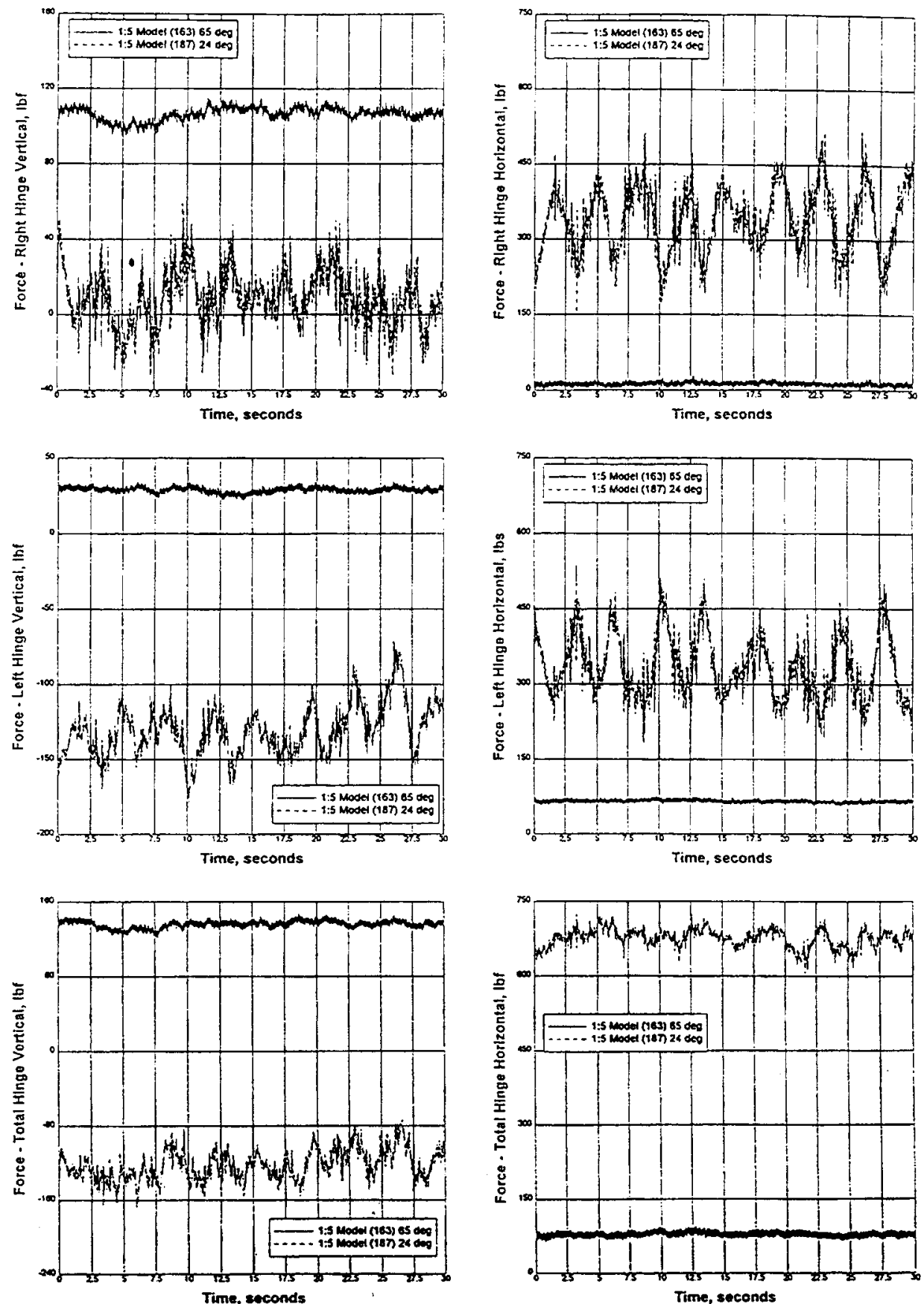

\section{a. Bottom hinge reactons}

Figure 89. Comparisons of fixed gate reaction forces as a function of time for two gate positions (Continued)

Figure 89 shows the variations of reaction forces due to a change in wicket positions. Figure 90 compares the pressures and accelerations of the wicket for both gate positions. 


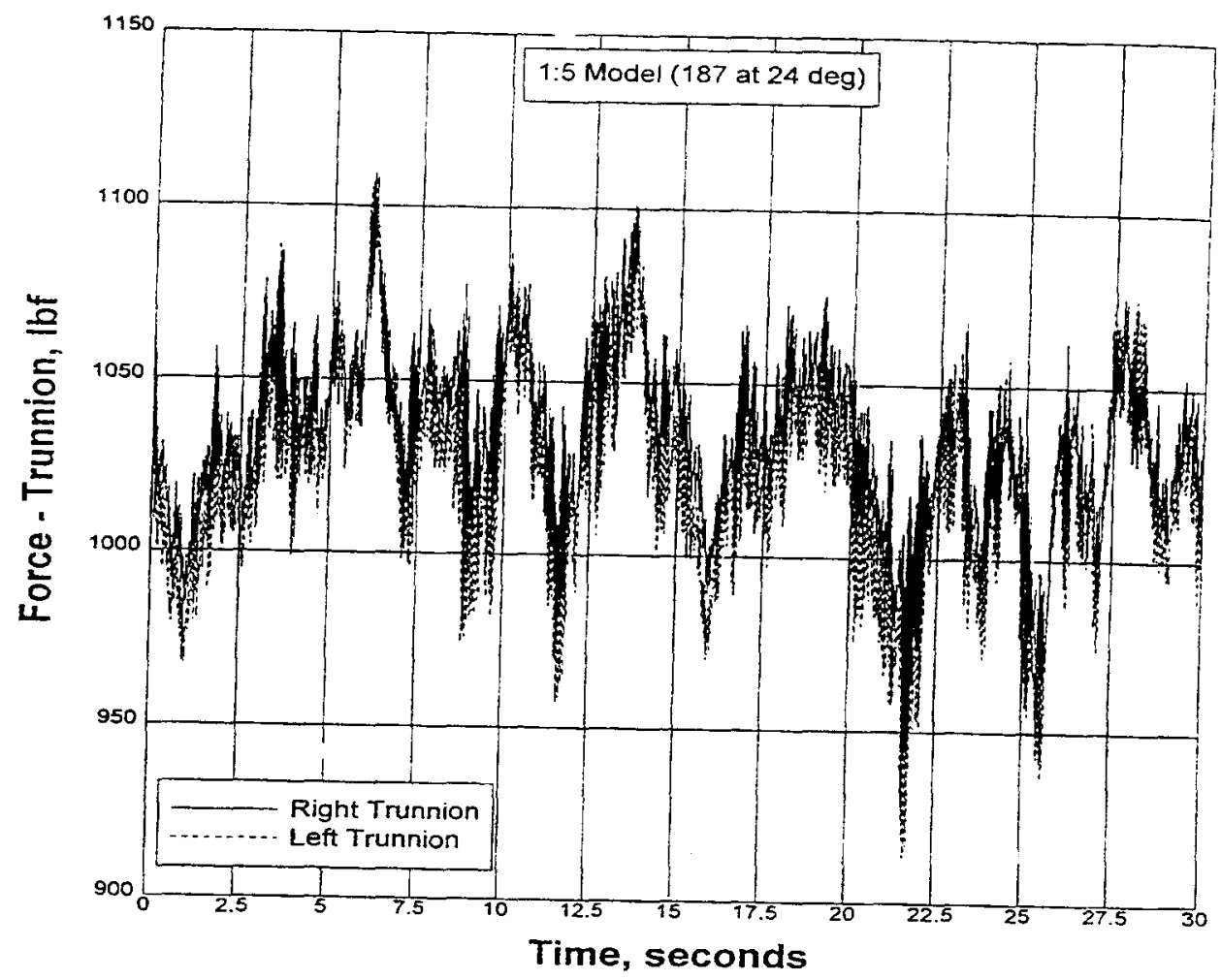

b. Trunnion force

Figure 89. (Concluded)

\section{Modal Experiments}

\section{Overview of modal analysis}

Modal analysis is the process of characterizing the dynamic behavior of an elastic structure in terms of its modes of vibration. The purpose of a modal analysis is to construct a mathematical model of the vibrational properties and behavior of the physical structure. Experimental modal analysis has been extensively used to verify analytical models, to predict the effects of structural modifications, to improve model performance, and to troubleshoot noise and vibration problems (Mouch and Myers 1991; Rogers 1989; Leuridian 1992; Lin, $\mathrm{Du}$, and Ong 1993; Dascotte 1991; and Petrick 1993). A successful implementation of modal modeling requires a basic understanding of the modal analysis technique which greatly depends on the quality of the measured responses and the effectiveness of the analysis technique used to extract modal parameters.

The theory behind modal analysis using the frequency-response functions is well documented in the literature (Ewins 1984, Hewlett-Packard 1986, SMS 1988, 


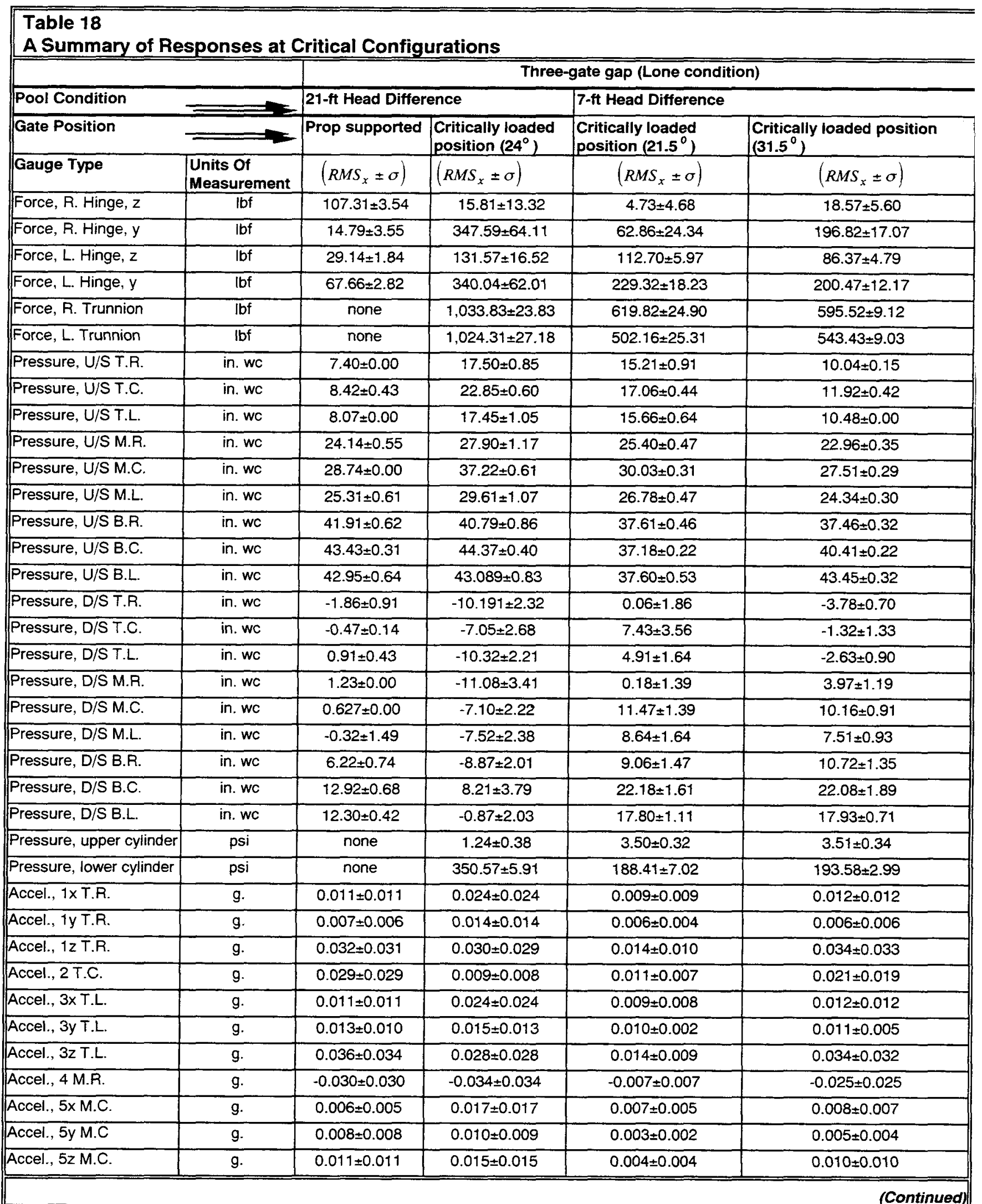




\begin{tabular}{||l|c|c|c|c|c}
\hline Table 18 (Concluded) & $\begin{array}{l}\left(R M S_{x} \pm \sigma\right) \\
\text { Measurement }\end{array}$ & $\left(R M S_{x} \pm \sigma\right)$ & $\left(R M S_{x} \pm \sigma\right)$ & $\left(R M S_{x} \pm \sigma\right)$ \\
\hline \hline Gauge Type & $g$. & $-0.029 \pm 0.027$ & $\begin{array}{c}-0.559 \pm 0.438 \\
\text { (clipped) }\end{array}$ & $-0.021 \pm 0.019$ & $-0.023 \pm 0.021$ \\
\hline Accel., 6 M.L. & g. & $0.027 \pm 0.026$ & $0.022 \pm 0.021$ & $0.007 \pm 0.004$ & $0.012 \pm 0.011$ \\
\hline Accel., 7 B.R. & g. & $0.027 \pm 0.024$ & $0.011 \pm 0.006$ & $0.013 \pm 0.004$ & $0.015 \pm 0.007$ \\
\hline Accel., 8 B.C. & g. & $0.033 \pm 0.032$ & $0.022 \pm 0.021$ & $0.009 \pm 0.004$ & $0.013 \pm 0.010$ \\
\hline Accel., 9 B.L. & g. & $-0.020 \pm 0.017$ & none & $0.013 \pm 0.009$ & $0.019 \pm 0.016$ \\
\hline Accel., Prop rod $z$ & g. & $0.011 \pm 0.010$ & none & $0.007 \pm 0.003$ & $0.009 \pm 0.006$ \\
\hline Accel., Prop rod y & ft, wc & $299.98 \pm 0.00$ & $299.80 \pm 0.00$ & $296.91 \pm 0.00$ & $296.93 \pm 0.00$ \\
\hline Elevation, U/S pool & ft, wc & $279.07 \pm 0.00$ & $279.09 \pm 1.81$ & $289.87 \pm 2.26$ & $289.93 \pm 3.68$ \\
\hline Elevation, D/S pool & & & & & \\
\hline
\end{tabular}

and SEM 1995) and, therefore, will not be repeated here. In general, it is important to recognize the basic assumptions that are required for singlereference point modal investigation. These assumptions include: (a) the structure's motion is linear and symmetric, (b) all of the modes are adequately excited at the reference point, and (c) only one mode exists at each pole location (i.e., the frequency and damping of each mode are "sufficiently" different from the other modes).

Assumption (a) requires that the structural response be adequately defined by a set of second-order differential equations ( Richardson 1975), with symmetric mass, damping, and stiffness matrices. This also implies that the FRF matrix is a symmetric. By using the random excitation technique, frequency spectrum averaging, and the required inversion of the input power spectrum matrix, a best linear approximation of the nonlinear system can be obtained (SMS 1988, Ewins 1984). Assumption (b) requires that the reference points are not near their nodal points (or zero points). The validity of assumption (c) depends on the ability of the curve fitting method to correctly estimate the modal parameters of very closely spaced modes.

The measurement and analysis techniques, however, are project dependent, case specific, and hardware and software sensitive. A valid modal experiment, therefore, fundamentally depends upon the ability to prevent sources of errors during the measurement and analysis processes. Three distinct stages of modal experiment, namely the setup, data acquisition, and the parameter extraction, are the primary levels that require careful evaluation for a successful implementation of the modal investigation. A variety of measurement and analysis flaws during these stages could easily provide misleading information about the dynamic behavior of the tested structure.

An illustrative system block diagram, for example, showing the experimental process for the modal investigation, could be used to identify the sources of errors that could exist during the measurement process. In an experimental modal analysis, the input and output responses are transformed to the frequencydomain and the ratio of the response to force signal in the frequency domain is used to extract the system information (Figure 91). 

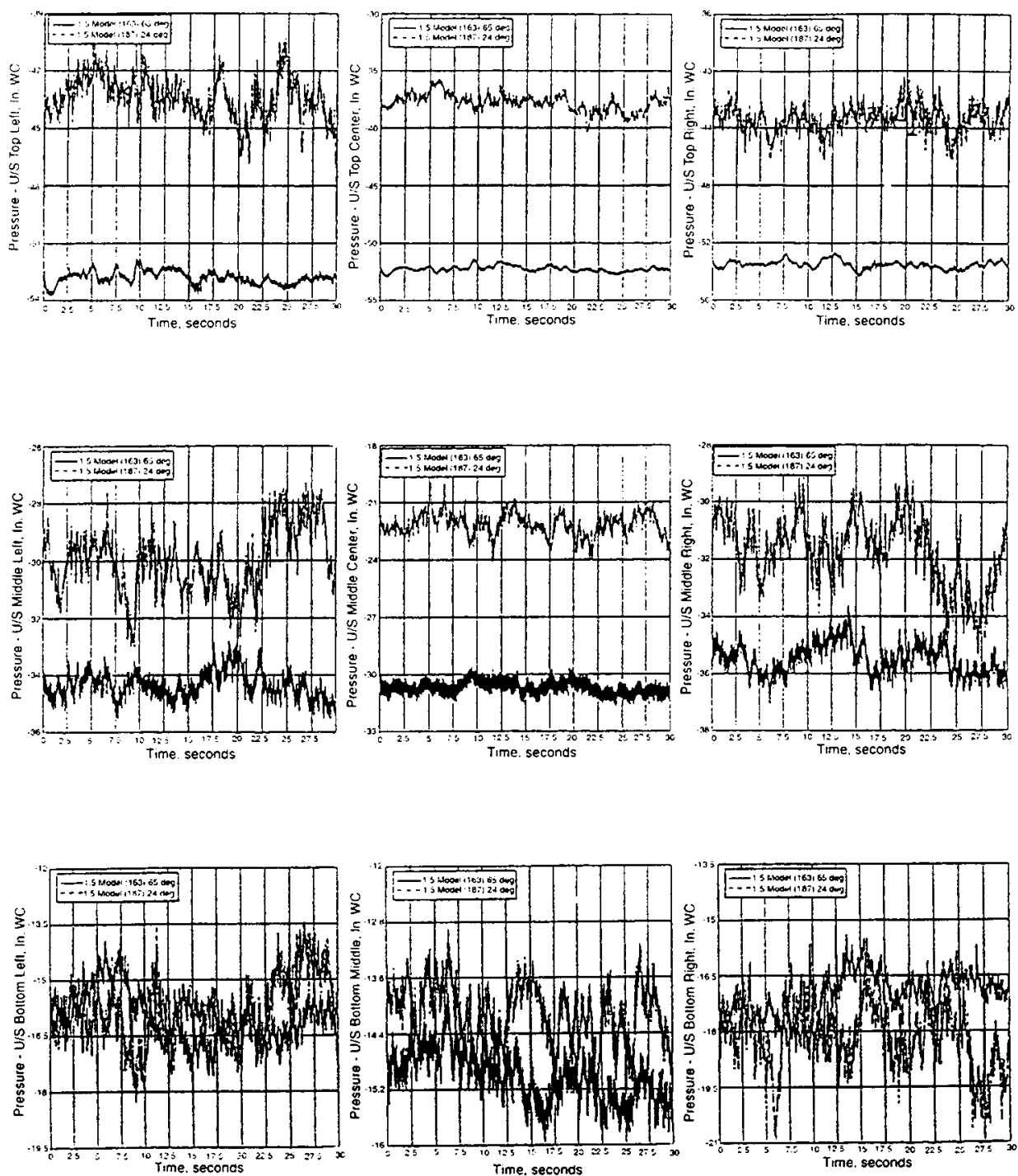

a. Upstream pressures

Figure 90. Comparisons of pressures and accelerations for two gate positions (Sheet 1 of 3)

This system information includes a set of modes defined by frequency, damping, mode shape, and residue. The two most obvious features of a mode are its resonance frequency and peak magnitude. Damping is the rate at which the structure returns to equilibrium. And, the residue is a complex number expressing the response amplitude and its phase relationship to the applied force. Mathematical values for the modal properties can be estimated from the frequency response functions (FRF) taken from the original modal test data. The unit forced response functions, commonly referred as the Frequency Response Function (FRF), are generated using the formulation, $G(\omega)=\frac{Y(\omega)}{X(\omega)}$. 

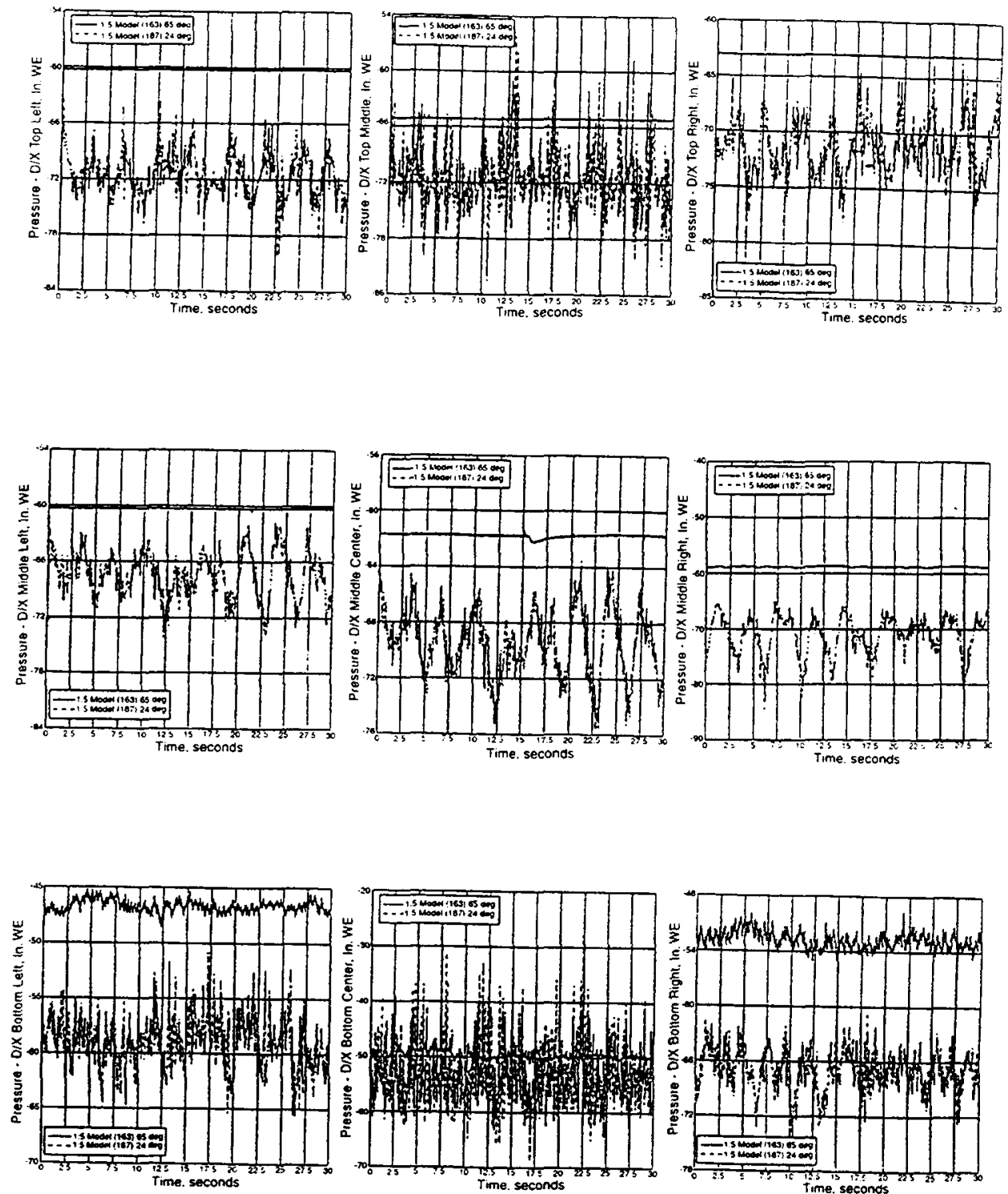

\section{b. Downstream pressures}

Figure 90. (Sheet 2 of 3 )

Measurement errors may result because of poor acquisition of input and output responses, poor spatial resolution of the proposed structure, and poor processing of the acquired data.

A preliminary analysis of the structure is needed to overcome many of the data acquisition problems and select the exciter and measurement locations on the proposed structure. A detailed description on using a pretest analysis to set up a modal experiment is available in the literature (LMS 1991, Jarvis 1991, and Kientzy and Richardson 1989). The objectives of such a preliminary FE model are to determine the possible nodal points for modes of interests and avoid 

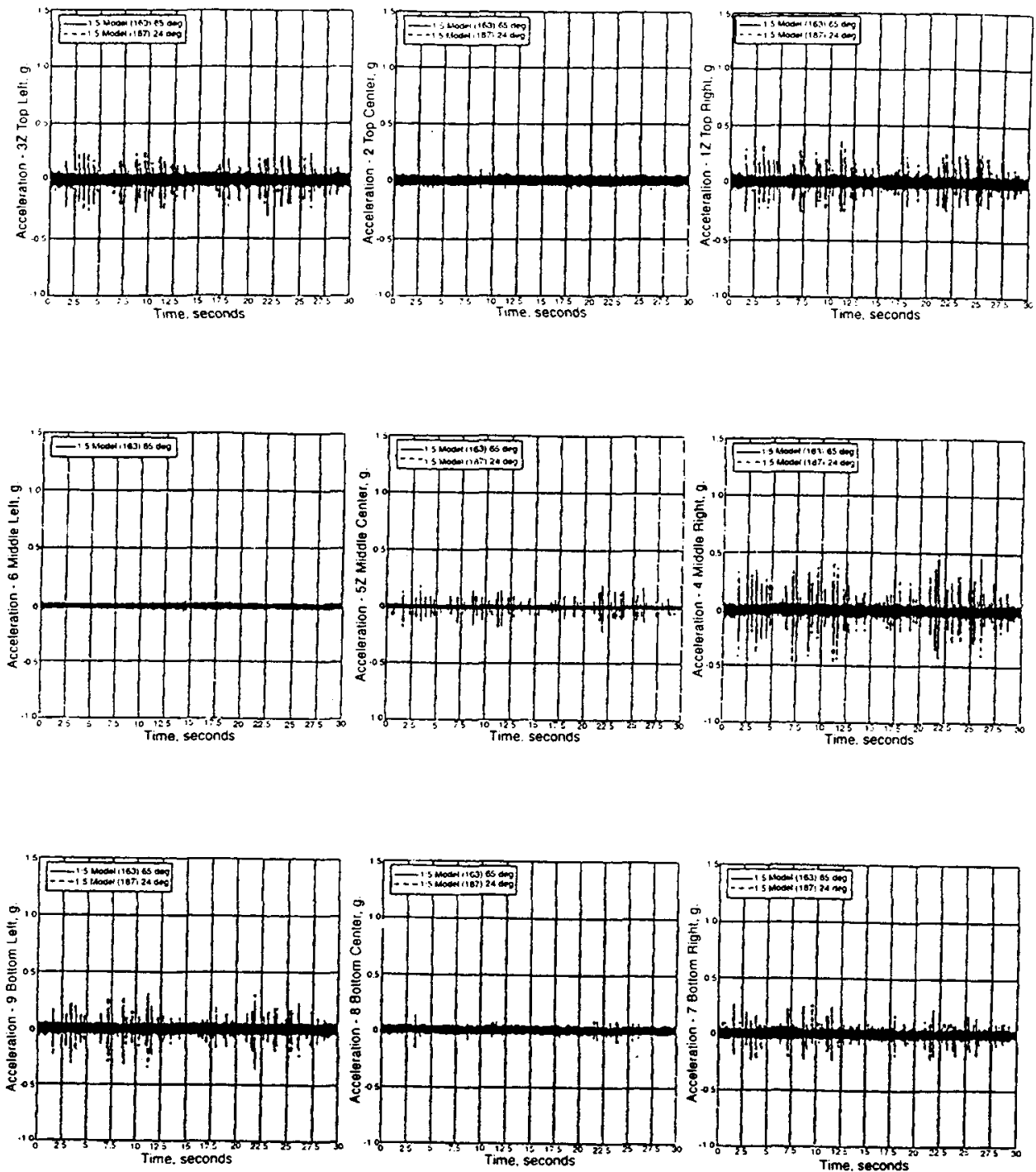

\section{c. Accelerations}

Figure 90 . (Sheet 3 of 3 )

locating the transducers at such nodal lines. Knowing the pattern of the highest modes, the number of measurement locations could be selected to sufficiently define the spatial resolution of the modes of interest. Prior knowledge of the frequency range is needed to select appropriate transducers for recording the input and output responses as well. Selection of excitation techniques ranging from impact hammer to shakers is dependent upon the characteristics of the system under consideration.

Data analysis is another important step in the modal experiment which requires substantial knowledge of digital signal analysis using the FFT analyzer. An understanding of signal aliasing and leakage errors is important to ensure correct frequency-domain conversion of the time-histories. Ramsey (1975) 


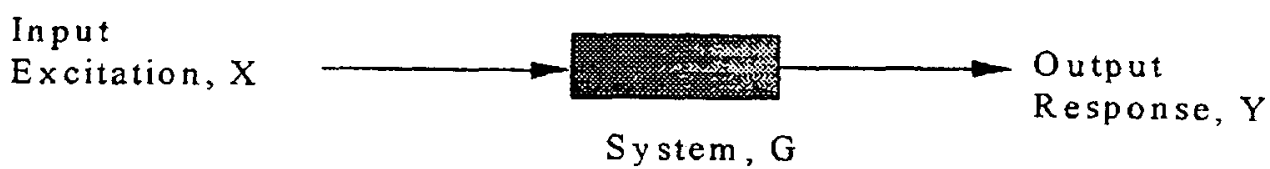

Figure 91. System block diagram for modal experiment

explicitly explains such dynamic analysis problems and suggests ways for effective measurements. More information regarding proper signal analysis can be found elsewhere (SMS 1988, Hewlett-Packard 1986, Ewins 1984, Ramsey 1975, and Richardson 1975).

Several parameter estimation techniques are available to extract modal parameters from the FRF measurements. Generally, there are two categories of curve-fitting techniques, single and multiple degrees of freedom, (SDOF, MDOF). General assumptions and background knowledge in postprocessing of the data are necessary to calculate parameters using these techniques. An SDOF technique, for example, is suitable for extracting information for relatively uncoupled modal peaks, while a direct parameter estimation technique using the MDOF algorithm can be quite useful for accurately defining closely spaced modes within a narrow frequency range (increased frequency resolution $(\Delta f)$ ), (Shye, VanKarsen, and Richardson 1988). Since this method works directly on the spectra in the frequency domain, it can account for modes outside the frequency range of interest.

\section{Experimental procedure and data analysis}

A preliminary FE analysis of the wicket was used to select the exciter and measurement locations for the modal experiment. Figure 92 shows the modal and FE grid layout for the upstream side of the wicket. Initially, 112 points with 16 rows and 7 columns were used for exploratory measurements. The grid rows were located in each of the bays spanning the transverse stiffeners and on the center line of each transverse stiffener supporting the skin plate. These measurements were conducted to determine the general system characteristics such as the frequency range of interest, modal density, and nonlinearity of the structure, and to measure the effectiveness of the newly acquired laser doppler scanning vibrometer (SLDV) - data acquisition system.

One such experiment showed that the inherent thermally induced scanner drift of the laser beam considerably degrades the quality of the data with an extended data acquisition time. In an uncontrolled environment, the thermal differential is expected to be proportional to the time interval. The longer the data acquisition time the greater the temperature differentials - resulting in an obvious drift of the laser beam from the intended locations. This is because a thermal drift erroneously points the laser beam away from the intended nodes. If the laser positions are incorrectly placed, then the corresponding modal information will be in error. Agee, Zeng, and Mitchell (1992) reported such a scanner drift of the laser beam due to the expansion and contraction of internal electronics and galvanometers. 


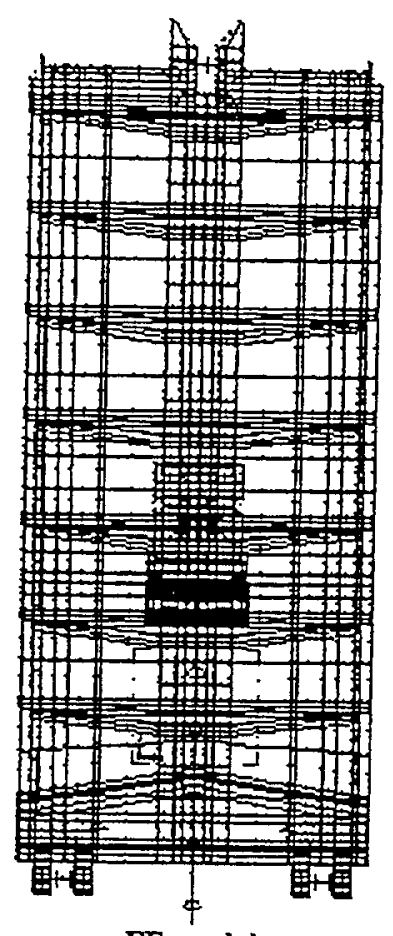

FE model

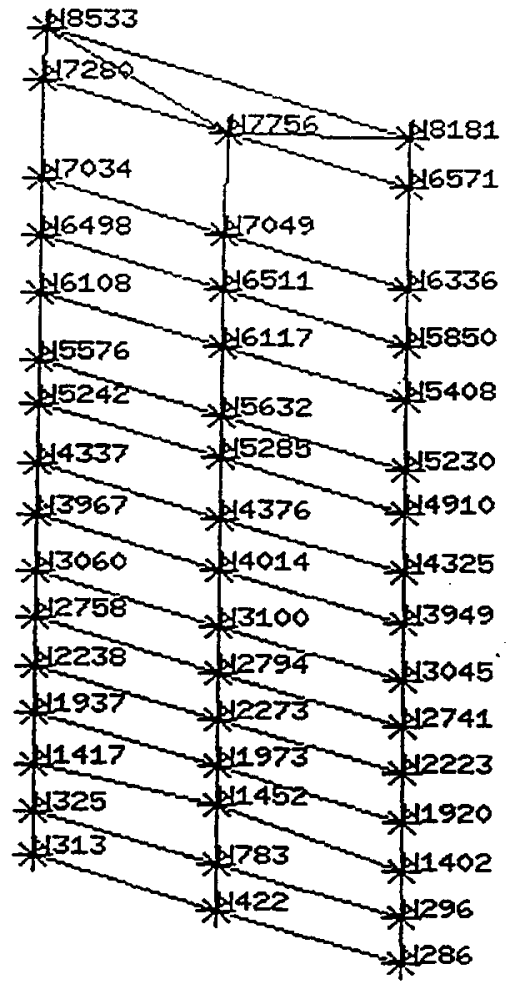

down_48_view

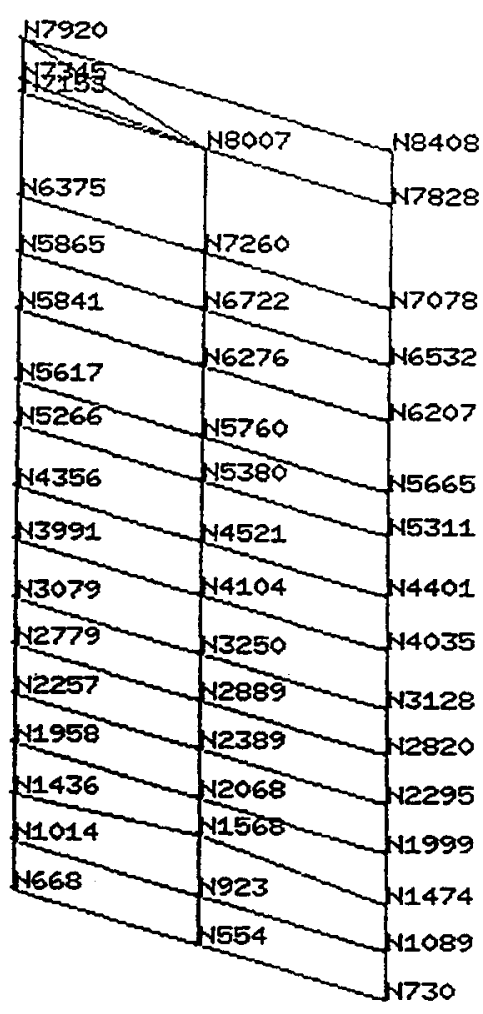

dry_48_nodes

Figure 92. Modal and FE grid layout 
An experiment was conducted to measure the thermal drift error for the SLDV system used in this project. Optical drift as a function of temperature for the SLDV system was determined by using a least square curve-fitting of the experimental data inclusive of temperature ranges of 78 to $90^{\circ} \mathrm{F}$. The angular drift $(\delta \theta)$ of the laser beam from principal focus line in degrees is related to the temperature $(F)$ by an expression, $\delta \theta=(0.0029 F-0.2278)^{0}$. This linear equation suggests that the offset $(\partial x=L(\delta \theta)$ ) of the laser beam from the intended position will be greatly increased as the laser head is placed further apart from the test structure as shown in Figure 93.

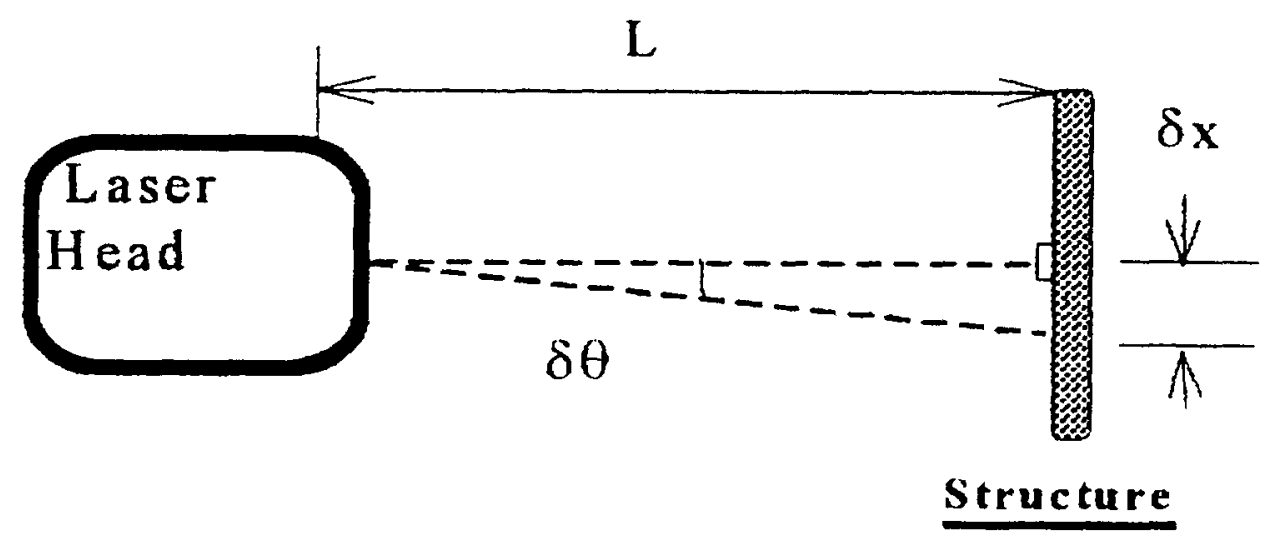

Figure 93. Schematic diagram of angular drift error in the SLDV system

A thermal drift error was obvious during the modal experiments performed on a long summer day. A scanning of 112 points with 30 ensembles could easily take 4 to 8 hours depending on the number of block rejections defined in the autorange process to set the input channel voltages. One way to minimize thermal drift error was to reduce the total scanning time for the wicket. In this regard, a modal grid with fewer nodes was used for scanning the remaining experiments. A 47-node grid instead of 112 nodes, with 16 rows and 3 columns, was used for scanning the wicket from the downstream position. Such a reduction in number of scanning nodes did not show any spatial resolution problems for the interested modes. As will be shown later, a three-column grid adequately identified the first eight modes of the wicket. Downstream scanning of the wet wicket was needed when water passed through the flume. In operation, the upstream nodes could not be accessed by the laser beam due to water flow. A majority of nodes in a 112-node grid, however, could not be accessed from the downstream side by the laser beam focusing from an oblique position on the bank of the flume. A reduced modal model with fewer nodes was therefore considered adequate for measuring global wicket information. 


\section{Experimental setup}

A typical experimental setup for the modal experiments using the SLDV system is shown in Figure 94. In this figure - referring back to the modal experiment block diagram (Figure 91) - the input excitation was provided by an electro-magnetic shaker and the output response was measured by a noncontact velocity transducer. Unique capabilities of both measurement systems provided an opportunity to record the modal data in the best possible manner.

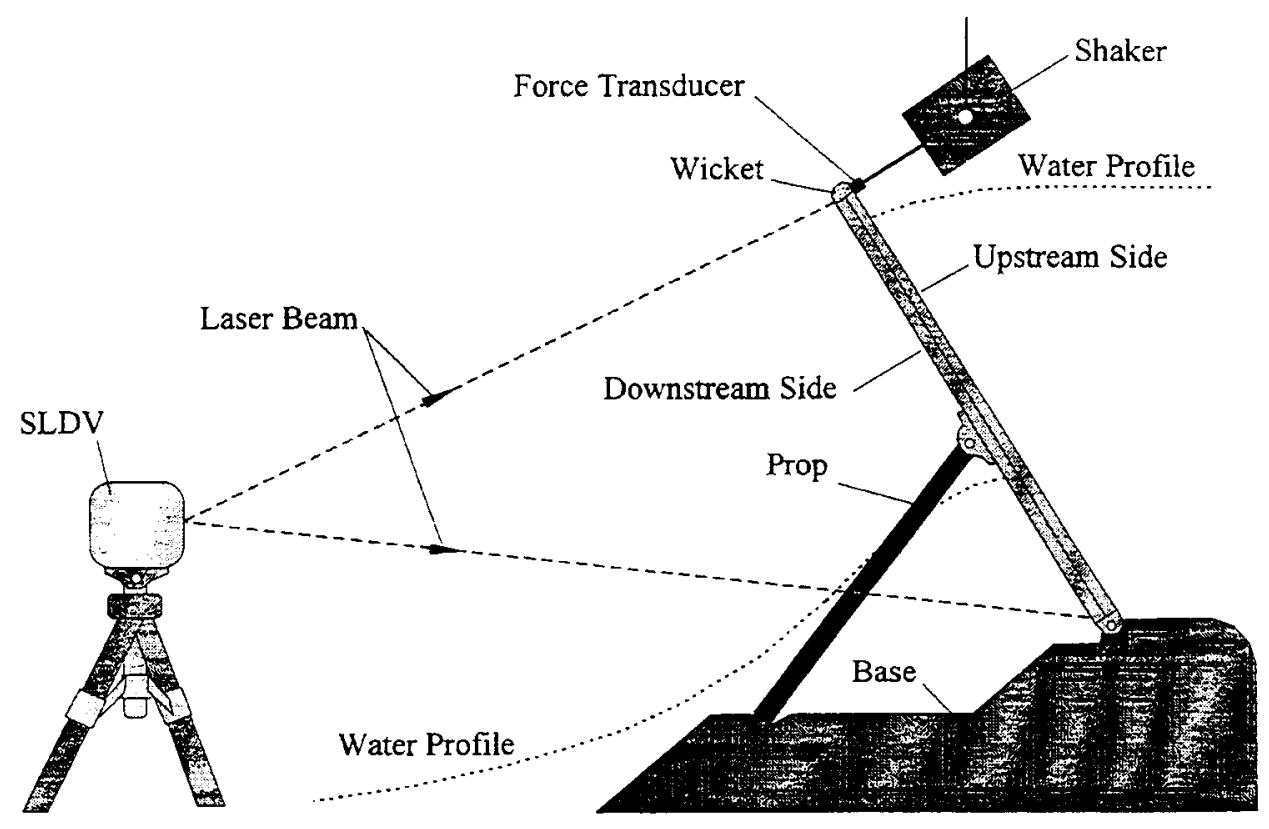

Figure 94. Experimental modal test setup

A shaker, for example, could eliminate many uncertainties in the acquisition process by providing a consistent and controlled force input. The flexibility of using different excitation functions in a shaker provides additional advantages for linearizing the system response of a nonlinear system. A burst random signal could minimize leakage, improve signal-to-noise ratio, and yields the best linearized model of a nonlinear system by removing distortion from the measured responses (SMS 1988, Ewins 1984). Nonlinearity of the wicket performance existed in the test structure, particularly due to the nature of the supporting devices. A slippage and the uncertain frictional behavior in the prop connections and the lifting cylinder's cup-ball mechanisms could easily distort the linear assumption involved in the modal experiment. Thus, a burst random excitation was used in this experiment to drive the shaker for a fixed frequency band that would provide a best linear model of the gate. In all modal experiments, an MB Modal 50A exciter (shaker) with an added 30-lb inertia block was used to excite the wicket with a burst random signal.

A PCB 208A02 force transducer, SN No. 8368, effective for a range 0 to $100 \mathrm{lbf}$, with a calibration factor of $19.608 \mathrm{lbf} / \mathrm{V}$, was used to measure the 
imparted force on the structure. The shaker was suspended with four turnbuckels from the overhung crane. The force transducer was epoxy glued onto the reference point and was connected to the shaker by a 1/8-in.-diam stinger using the procedure described in MB Dynamics Manual (1990). Alignment of shaker's thrust axis with that of the load cell was critical to the success of a shaker test; in particular, bending of the stinger could provide erroneous information during the stinger compression loading. Care was taken so that the thrust axis of the shaker was aligned and the stinger would not bent during exciter operation.

The state-of-the-art laser vibrometer provided a noncontact, high accuracy, high spatial resolution, and efficient data acquisition system by eliminating the need for multiple transducers. In addition to being the most effective and efficient data acquisition system, a laser does not mass load the structure, thus making the proposed structure time invariant. In a traditional modal experiment of roving accelerometers on the structure, such a condition of time invariance is difficult to attain. A functional description of laser operations is well documented in the literature (Agee, Zeng, and Mitchell 1992 and Zonic 1995). In a laser operation, however, to obtain the best sensor performance, it is important to understand the operating principles of the SLDV. A Zonic Lazon $^{\mathrm{TM}}$ system was used in this experiment.

An overview of the hardware setup for the Lazon system is presented in Figure $95 a$ and $b$. To achieve the best quality of velocity measurements, optimum working distance, correct focusing of laser beam, and a stable test surface must be maintained. Working distance should be such that the maximum angular deviation to cover the longer dimension of the structure remains less than $\pm 25 \mathrm{deg}$, as shown in the figure. This could be obtained by choosing a working distance at least 2.5 times the greater dimension of the structure over which measurement is required. Keeping in mind that the longer the working distance the less the reflection, thus for a long distance, a better surface preparation is necessary for enhancing the laser Doppler signal. A retroreflective coating on the surface of the instrumented gate could enhance laser Doppler signals of sufficient amplitude to ensure stable operation of the sensor. In this experiment, a locally available retroreflective tape was pasted on the grid nodes as shown in Figure 95b. Maintenance of optimum focus was accomplished by minimizing the spot size of the laser beam on the surface of the gates. An optimum focus could also be checked through the LED bargraph on the side panel of the laser head.

In Figure 95a, also shown is the Zonic system 7000 analyzer that controls the laser functions. Zonic system 7000 has two input and output modules. The analog signal conditioning input module (ASC-I) digitizes the excitation force and velocity signals in channels 1 and 2 , while the analog signal conditioning output (ASC-O) module generates the digitized signals to control the laser functions and drive the shaker. An internally generated random analog signal from channel 3 in the ASC-O module transmits through the power amplifier to drive the shaker at reasonable strength. 


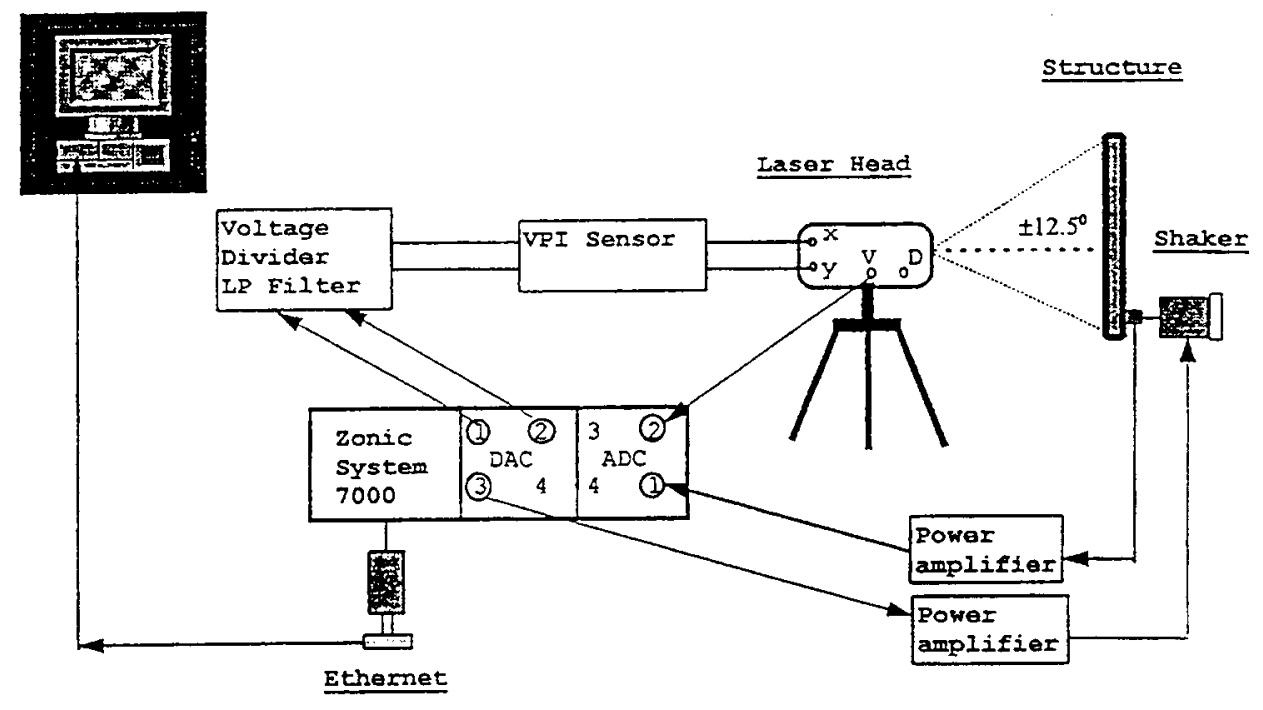

a. A typical zonic Lazon system hardware setup

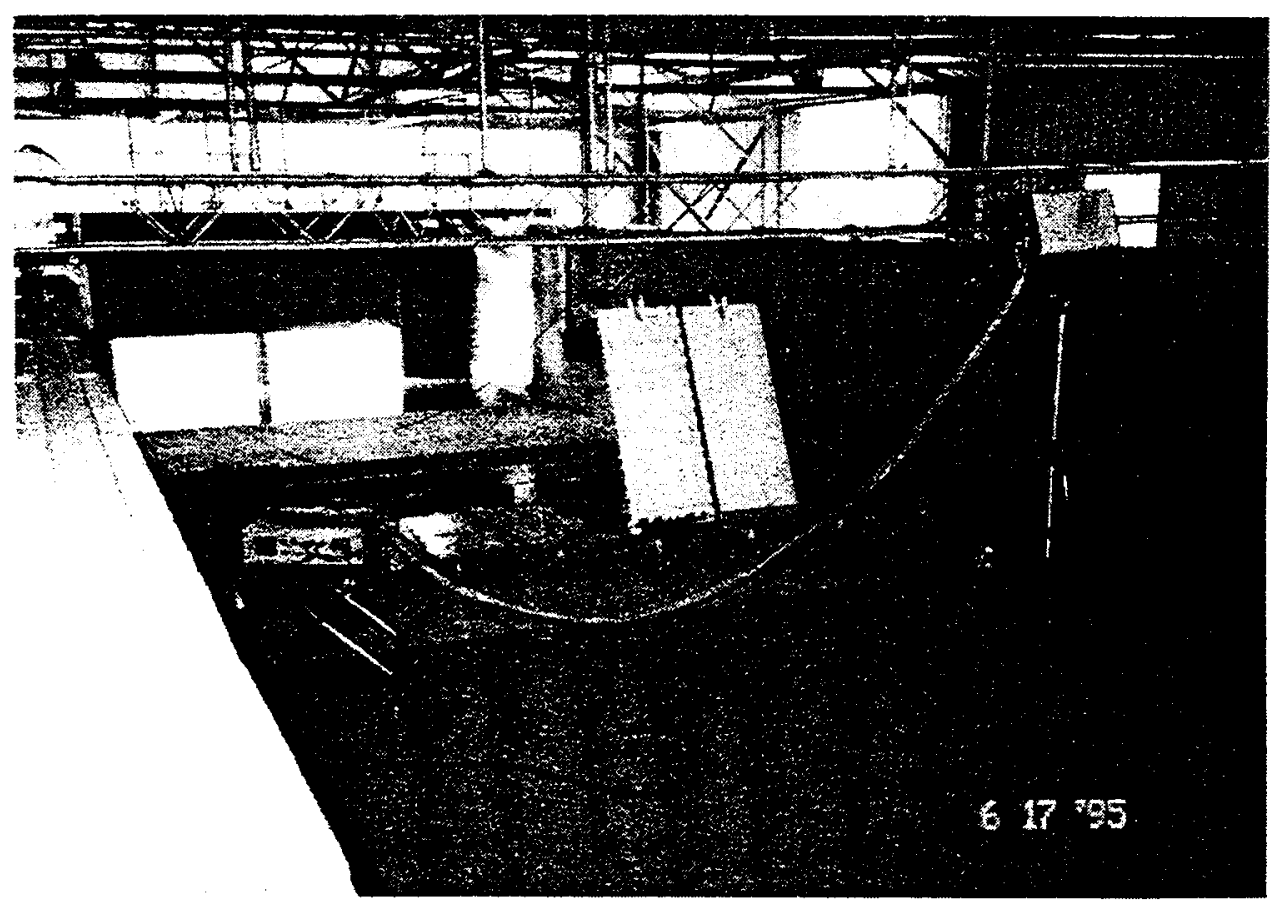

\section{b. A typical Lazon test setup}

Figure 95. Overview of hardware setup for Lazon system

An HP 712/80 workstation was networked with system 7000 for remotely operating the data acquisition of the SLDV system. The Zeta 4.0 computer program was used to operate the SLDV system and acquire the mobility frequency-response functions for each scanned point. The acquired mobility 
functions for the entire geometry would be used to extract the modal parameters for the test structure.

\section{Data acquisition and modal analysis}

The analyzer setup for the Lazon system is presented in Table 19. An ordered list of tasks would be executed to identify and set the laser on nodes to be measured and acquire the data. A detailed significance of the specifications used to set up the experiment can be found in the Zonic Lazon Manual (1995).

A sequential three-phase operation for the modal analysis using the SLDV data acquisition system is explained through the flowchart shown in Figure 96. These three phases include: (a) pretest modeling of the geometry using IDEASMaster Series/Simulation and Test modules, (b) data acquisition for the prescribed nodes using the Lazon system, and (c) estimation of modal parameters using IDEAS/Test module. In this figure, long horizontal dashed lines mark the phase boundaries. As seen in the flowchart, the geometry universal file created by the IDEAS/Test module provides the basic layout of the geometry to be scanned automatically by the Lazon system.

The laser head is positioned such that the scanners in the $\mathrm{X}$ and $\mathrm{Y}$ directions are driven close to their $25-\mathrm{deg}$ scan range. This process minimizes scan errors in subsequent steps. A manual laser beam positioning routine yields the global placement of the laser head with respect to the gate. Four registration points are used by the program for positioning the laser head in space with respect to a plane passing through the nodal points being scanned. This permitted determination of all point locations in the scan group. The laser scan setup allows correct positioning of the laser beam by adjusting the location of any point in the single verification task.

Subsequent to the correct identification of nodes by the scanner, the data acquisition system can then be used to acquire the average mobility FRF for each scanned node. Note that the analyzer setup is critical to the success of the digitization of the signals processed during this operation. The windowing and the signal types (burst or continuous) must be matched so that the leakage errors are minimized. Table 19 presents the analyzer setup for the modal experiment presented in this report. Total acquisition time is somewhat directly proportional to the quality of the acquired data which demands more measurement ensembles and adequately ranged voltage scales for the I/O channels. The selection of the numbers of blocks set for the autoranging and measurement ensembles needed careful adjustment depending upon the expected temperature changes over the interval of modal data acquisition time. As mentioned earlier, a thermal-drift error of the laser beam could provide misleading information with poor measured data. The mobility FRF and coherence $(\mathrm{COH})$ functions are stored as a function file (associated data file, ADF) for later retrieval using the IDEAS/Test module for modal analysis. 


\begin{tabular}{|c|c|c|}
\hline \multicolumn{3}{|c|}{$\begin{array}{l}\text { Table } 19 \\
\text { Lazon System Data Acquisition Setup }\end{array}$} \\
\hline Unit & Specification & Remarks \\
\hline $\begin{array}{l}\text { Laser Head Setup } \\
\text { a. Lens }\end{array}$ & $\begin{array}{l}\text { Short range lens, } \\
\text { working distance } 16.4 \mathrm{ft}\end{array}$ & $\begin{array}{l}\text { The short-range lens provides } \\
\text { adequate sensitivity for } \\
\text { measurements at working distances } \\
\text { up to } 16.4 \mathrm{ft} \text {. }\end{array}$ \\
\hline $\begin{array}{l}\text { b. Velocity and } \\
\text { frequency range }\end{array}$ & $\begin{array}{l}\text { Low-velocity range ( } 1,000 \mathrm{mV} \text { per } \\
\text { mms } 1 \text { ), } \# 3 \mathrm{FRF} \text { filter (A low-pass } \\
\text { filter of } 500 \mathrm{~Hz} \text { for the analog } \\
\text { velocity output) }\end{array}$ & $\begin{array}{l}\text { This velocity range provided the } \\
\text { greatest detection sensitivity of the } \\
\text { sensor for the working distance } \\
\text { used in this experiment. }\end{array}$ \\
\hline$\frac{A S C-O \text { and } A S C-1 \text { setup }}{\text { a. Output Channel-3 }}$ & $\begin{array}{l}\text { Waveform type: } \\
\text { Frequency range: } \\
\text { Noise type: } \\
\text { Waveform amplitude: }\end{array}$ & $\begin{array}{l}\text { Random } \\
0 \text { to } 650 \mathrm{~Hz} \\
\text { Pure burst, } 0.2 \mathrm{sec} \text {-on } / 3.0 \mathrm{sec} \text {-off. } \\
\pm 2.0 \mathrm{~V}\end{array}$ \\
\hline $\begin{array}{l}\text { b. Input channels } \\
\text { i. Channels }\end{array}$ & $\begin{array}{l}\text { Channel } 1 \\
\text { Calibration factor: } \\
\text { Full-scale volts: } \\
\text { Channel } 2 \\
\text { Calibration factor: } \\
\text { Full-scale volts: }\end{array}$ & $\begin{array}{l}\text { Force } \\
18.083 \mathrm{lbf} \\
6.0 \mathrm{~V} \\
\text { Velocity } \\
0.04 \mathrm{in} . / \mathrm{sec} \\
5.0 \mathrm{~V}\end{array}$ \\
\hline ii. Auto ranging & $\begin{array}{ll}\text { Auto type: } & \text { Transient } \\
\text { No. of blocks: } & 4 \\
\text { Upscale: } & 1.5\end{array}$ & $\begin{array}{l}\text { Auto ranging sets the input channel } \\
\text { voltages automatically during the } \\
\text { data acquisition process. Four } \\
\text { consecutive blocks of data must be } \\
\text { good before data acquisition could } \\
\text { start. If overload is sensed, the full- } \\
\text { scale voltage will be adjusted by the } \\
\text { upscale factor. }\end{array}$ \\
\hline iii. Window type & Exponential & - for transient-type input signal \\
\hline & $\begin{array}{l}\text { Hanning window with a } 50 \\
\text { percent overlap on responses, } \\
\text { and cosine tapped window on } \\
\text { force }\end{array}$ & - for continuous input signal \\
\hline $\begin{array}{l}\text { iv. Sampling } \\
\text { condition }\end{array}$ & $\begin{array}{l}\text { Analysis frequency: } \\
\text { Block size: } \\
\text { Measurement ensembles: }\end{array}$ & $\begin{array}{l}0 \text { to } 600 \mathrm{~Hz} \\
2,048 \\
30 \text { to } 50\end{array}$ \\
\hline v. Acquisition mode & $\begin{array}{l}\text { Pretriggered on channel } 1 \text {. Data } \\
\text { acquired after } 10 \text { samples on } \\
\text { positive slope at } 15 \text { percent of } \\
\text { the input level. }\end{array}$ & \\
\hline $\begin{array}{l}\text { TDAS setup \& function } \\
\frac{\text { storage }}{\text { a. Active Channels }} \\
\text { b. Function storage }\end{array}$ & $\begin{array}{l}\text { Channel } 1 \\
\text { Channel } 2 \\
\text { FRF and } \mathrm{COH}\end{array}$ & $\begin{array}{l}\text { Reference node } \\
\text { Laser channel - velocity response }\end{array}$ \\
\hline
\end{tabular}




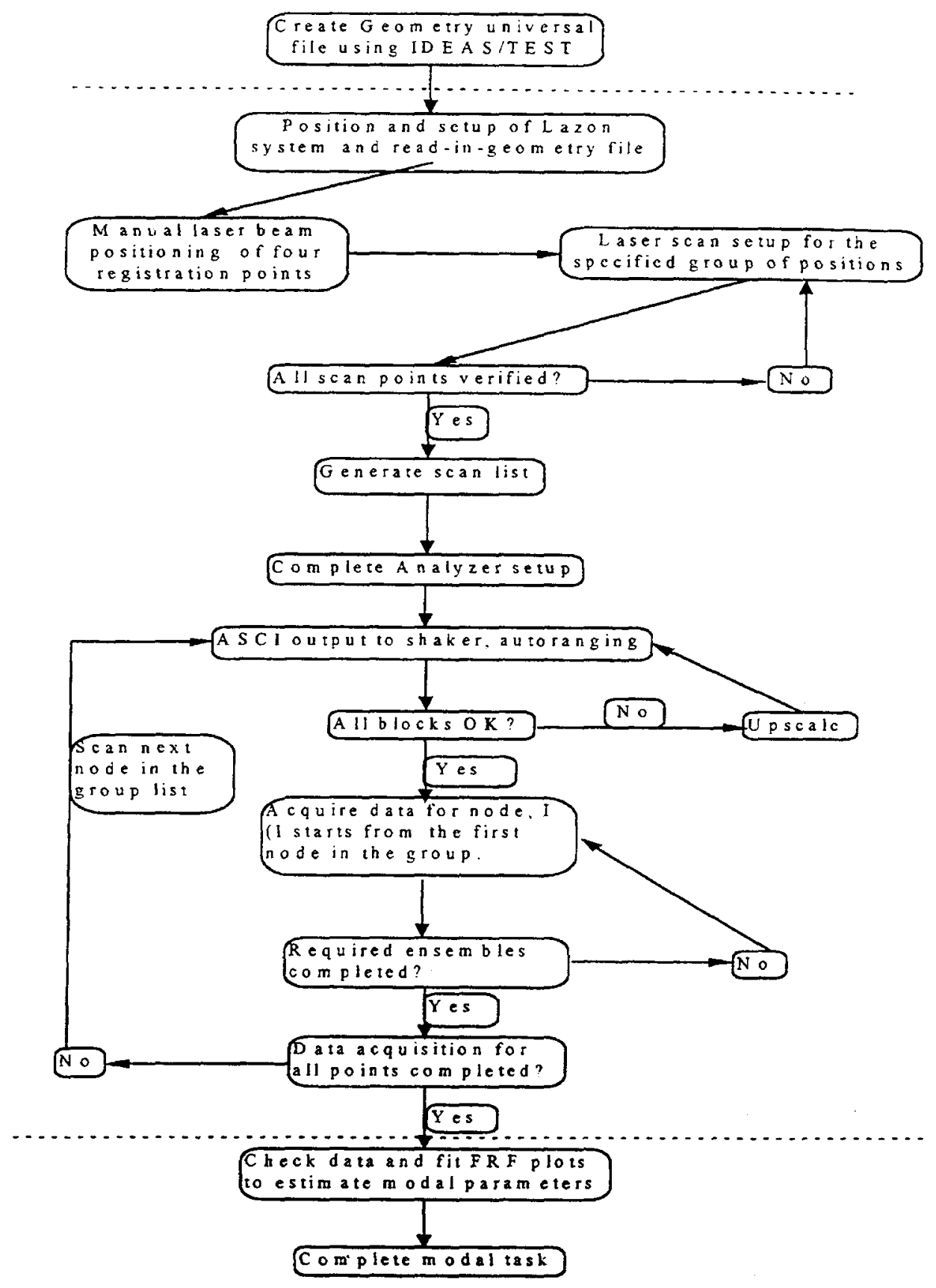

Figure 96. A flowchart for modal analysis using SLDV system

IDEAS-Master Series curve-fitting algorithms in the modal module were used to extract the modal parameters of the acquired data. In this regard, a number of algorithms ranging from SDOF to polyreference MDOF could be used to process the modal data. The mode shapes extracted using different algorithms were compared and the best-fit mode shape is reported here. Differences in the estimated modal parameters is very dependent on the choice of modal extraction algorithm, user interaction and the quality of the measurement data (SMS 1988). 


\begin{tabular}{|c|c|c|}
\hline \multicolumn{3}{|c|}{$\begin{array}{l}\text { Table } 20 \\
\text { Dry Modal Experiments }\end{array}$} \\
\hline Case Designation & Grid Pattern & Remarks \\
\hline A. Laser_down & $\begin{array}{l}\text { 48-node grid with the shaker } \\
\text { connected at node } 8181 \text { (Figures } \\
92 \text { and } 94 \text { ) }\end{array}$ & $\begin{array}{l}\text { Downstream face was scanned for } \\
\text { the prop-supported gate }\end{array}$ \\
\hline B. Laser_up_48 & $\begin{array}{l}\text { 48-node grid with the shaker } \\
\text { connected at node } 7345 \text { (Figures } \\
92 \text { and } 95 \mathrm{~b} \text { ) }\end{array}$ & $\begin{array}{l}\text { Upstream face was scanned for the } \\
\text { prop-supported gate }\end{array}$ \\
\hline C. Laser_shaft_112 & $\begin{array}{l}\text { 112-node grid with the shaker } \\
\text { connected at node } 7345 \text { (Figure } \\
95 \mathrm{~b} \text { ) }\end{array}$ & $\begin{array}{l}\text { Upstream face was scanned for the } \\
\text { lifting cylinder (shaft) supported } \\
\text { gate at } 65-\text { deg position. }\end{array}$ \\
\hline D. Position_24 deg_48 & $\begin{array}{l}\text { 48-node grid with the shaker } \\
\text { connected at node } 7345 \text { (Figure } \\
95 \text { b) }\end{array}$ & $\begin{array}{l}\text { Upstream face was scanned for the } \\
\text { lifting cylinder (shaft) supported } \\
\text { gate at } 24-\text { deg position. }\end{array}$ \\
\hline E. Laser_Multiref_112 & $\begin{array}{l}\text { 112-node grid with two shakers } \\
\text { connected at nodes } 7345 \text { and } \\
5311 \text { (Figure } 95 \mathrm{~b} \text { ) }\end{array}$ & $\begin{array}{l}\text { Upstream face was scanned for the } \\
\text { lifting cylinder (shaft) supported } \\
\text { gate at } 65 \text {-deg position. }\end{array}$ \\
\hline
\end{tabular}

\begin{tabular}{|c|c|c|c|c|c|c|c|c|c|c|}
\hline \multicolumn{11}{|c|}{$\begin{array}{l}\text { Table } 21 \\
\text { Correlation Matrix for First } 10 \text { Modes for Upstream and Downstream Laser Scanning }\end{array}$} \\
\hline $\mid \begin{array}{l}\text { Down- } \\
\text { stream }\end{array}$ & \multicolumn{10}{|c|}{ Upstream Modal Order (Column) } \\
\hline $\begin{array}{l}\text { Modal } \\
\text { Order } \\
\text { (Row) }\end{array}$ & $\begin{array}{c}1 \\
(49 \mathrm{~Hz})\end{array}$ & $\begin{array}{c}2 \\
(62 \mathrm{~Hz})\end{array}$ & $\begin{array}{c}3 \\
(96 \mathrm{~Hz})\end{array}$ & $\begin{array}{c}4 \\
(152 \mathrm{~Hz})\end{array}$ & $\begin{array}{c}5 \\
(171 \mathrm{~Hz})\end{array}$ & $\begin{array}{c}6 \\
(202 \mathrm{~Hz})\end{array}$ & $\begin{array}{c}7 \\
(232 \mathrm{~Hz})\end{array}$ & $\begin{array}{c}8 \\
(313 \mathrm{~Hz})\end{array}$ & $\begin{array}{c}9 \\
(416 \mathrm{~Hz})\end{array}$ & $\begin{array}{c}10 \\
(502 \mathrm{~Hz})\end{array}$ \\
\hline $\begin{array}{c}1 \\
\left(43^{\mathrm{Hz}}\right)\end{array}$ & & & 0.745 & & & & & & & \\
\hline $\begin{array}{c}2 \\
(58 \mathrm{~Hz})\end{array}$ & 0.847 & 0.977 & & & & & & & & \\
\hline $\begin{array}{c}3 \\
(91 \mathrm{~Hz})\end{array}$ & & & 0.971 & & & & & & & \\
\hline $\begin{array}{c}4 \\
(136 \mathrm{~Hz})\end{array}$ & & & & 0.612 & 0.480 & & & & & \\
\hline $\begin{array}{c}5 \\
(141 \mathrm{~Hz})\end{array}$ & & & & 0.601 & 0.845 & & & & & \\
\hline $\begin{array}{c}6 \\
(231 \mathrm{~Hz})\end{array}$ & & & & & & 0.52 & 0.807 & 0.637 & & \\
\hline $\begin{array}{c}7 \\
(291 \mathrm{~Hz})\end{array}$ & & & & & & & 0.908 & 0.916 & & \\
\hline $\begin{array}{c}8 \\
\left(400^{\circ} \mathrm{Hz}\right)\end{array}$ & & & & & & & & & 0.881 & \\
\hline $\begin{array}{c}9 \\
(512 \mathrm{~Hz})\end{array}$ & & & & & & & & & & 0.934 \\
\hline $\begin{array}{c}10 \\
(603 \mathrm{~Hz})\end{array}$ & & & & & & & & & & \\
\hline
\end{tabular}

Row Source: Laser_down, Column Source: Laser_up_48a

For relatively uncoupled modes, either an SDOF polynomial or circle-fit algorithm was used to best fit the mode shapes of the wicket. In most cases, the circle fit provided the best quality of the mode shape. A complex exponential and direct parameter algorithms were used to check the estimated mode shapes for closely coupled modes. The quality of a curve fitting method was measured by comparing the analytical function which approximates the original FRF with that of the original FRF. 


\begin{tabular}{|c|c|c|c|c|c|c|c|c|c|c|}
\hline \multirow{2}{*}{\begin{tabular}{|l|} 
Table 22 \\
Correlatio \\
$\begin{array}{l}\text { Shaft- } \\
\text { Supported } \\
\text { (Row) }\end{array}$ \\
\end{tabular}} & n Matri & for $\mathbf{P}$ & - anc & Shaft-S & upporte & d Mode & & & & \\
\hline & \multicolumn{10}{|c|}{ Prop-Supported (Column) } \\
\hline $\begin{array}{l}\text { Modal } \\
\text { Order }\end{array}$ & $\begin{array}{c}1 \\
\left(49^{\mathrm{Hz}}\right)\end{array}$ & $\begin{array}{c}2 \\
2 \\
(62 \mathrm{~Hz})\end{array}$ & $\begin{array}{c}3 \\
(96 \mathrm{~Hz})\end{array}$ & $\begin{array}{c}4 \\
(152 \mathrm{~Hz})\end{array}$ & \begin{tabular}{|c|}
5 \\
$(171 \mathrm{~Hz})$ \\
\end{tabular} & $\begin{array}{c}6 \\
(202 \mathrm{~Hz})\end{array}$ & $\begin{array}{c}7 \\
(232 \mathrm{~Hz})\end{array}$ & \begin{tabular}{|c|}
8 \\
$(313$ \\
$\mathrm{Hz})$ \\
\end{tabular} & \begin{tabular}{|c|}
9 \\
$(416$ \\
$\mathrm{Hz})$ \\
\end{tabular} & \begin{tabular}{|c|}
10 \\
$(502$ \\
$\mathrm{Hz})$ \\
\end{tabular} \\
\hline $\begin{array}{c}1 \\
(57 \mathrm{~Hz}) \\
\end{array}$ & 0.953 & 0.819 & & & & & & & & \\
\hline $\begin{array}{c}2 \\
\left(711^{-} \mathrm{Hz}\right)\end{array}$ & & 0.501 & & & & & & & & \\
\hline $\begin{array}{c}3 \\
(76 \mathrm{~Hz})\end{array}$ & & & 0.816 & & & & & & & \\
\hline $\begin{array}{c}4 \\
(95 \mathrm{~Hz}) \\
\end{array}$ & & & 0.891 & & & & & & & \\
\hline $\begin{array}{c}5 \\
(142 \mathrm{~Hz}) \\
\end{array}$ & & & 0.731 & 0.232 & & & & & & \\
\hline $\begin{array}{c}6 \\
(196 \mathrm{~Hz}) \\
\end{array}$ & & & & & & & 0.630 & 0.830 & & \\
\hline $\begin{array}{c}7 \\
(222 \mathrm{~Hz})\end{array}$ & & & & & & 0.583 & 0.944 & 0.804 & & \\
\hline $\begin{array}{c}8 \\
(296 \mathrm{~Hz}) \\
\end{array}$ & & & & & & 0.375 & 0.819 & 0.920 & & \\
\hline $\begin{array}{c}9 \\
\left(409^{\mathrm{Hz}}\right) \\
\end{array}$ & & & & & & & & & 0.855 & \\
\hline $\begin{array}{c}10 \\
(507 \mathrm{~Hz}) \\
\end{array}$ & & & & & & & & & & 0.902 \\
\hline
\end{tabular}

Row Source: Laser_shaft_112a, Column Source: Laser_up_48a

An illustration of such curve fitting effects is presented in Figure 97. In this figure, the analytical FRF estimated using the four curve-fitting algorithms are compared with the original FRF. The estimated parameters, frequency and damping in percent, and the corresponding mode shapes extracted using the methods are also presented in this plots. The SDOF polynomial and circle fit methods are based on SDOF curve fitting techniques, and the direct parameter and complex exponential methods are based on MDOF estimation technique. Despite the fact that the direct parameter algorithm is quite useful for accurately defining closely spaced modes within a narrow frequency range, this method extracted noninvariant parameters. As seen in the plots, the parameters estimated using the MDOF (direct parameter) system depended upon the consideration of different number of poles, matrix size and frequency band width. This direct parameter method, however, accounts for modes outside the frequency range of interest, since it works directly on the spectra in the frequency domain. In Figure 97c, although the estimated FRF fitted well with the measured FRF, the complex exponential method extracted poor mode shapes for both cases. In general, the mode shapes of the structure are relatively stable; that is, they are not sensitive to the extraction method. However, the natural frequencies and damping ratios are very sensitive to the curve-fitting techniques, which depend not only on the modal parameter extraction methods but also demand a great deal of patience, skill, and experience to obtain accurate results, especially when the modes are highly damped and closely coupled. Thus, the same mode shapes are obtained with each extraction method, but the indicated frequency where they occur may be shifted up or down from one method to another. 

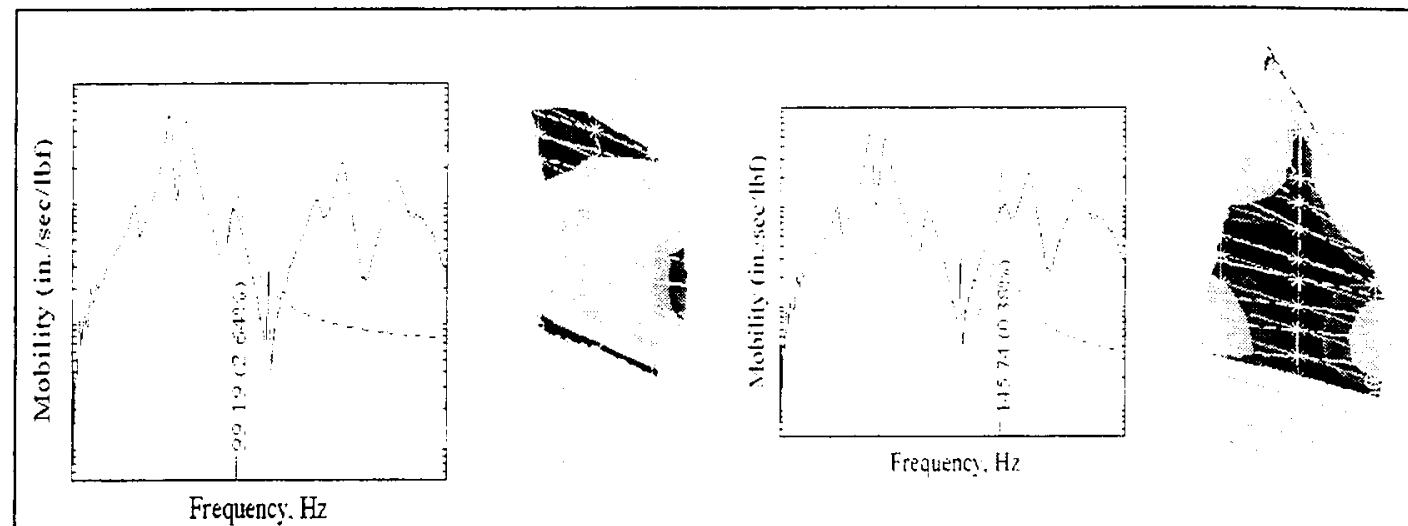

a. SDOF curve fitting

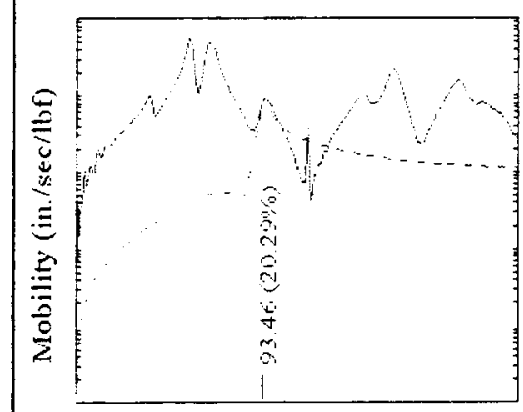

Frequency. $\mathrm{Hz}$

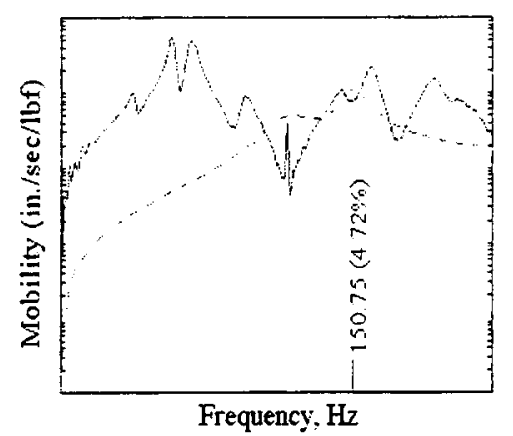

b. Direct parameter curve fitting

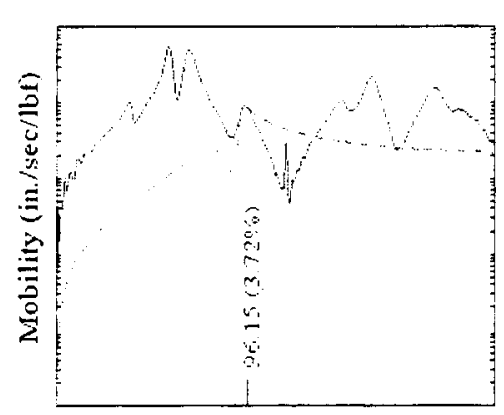

Frequency, $\mathrm{Hz}$

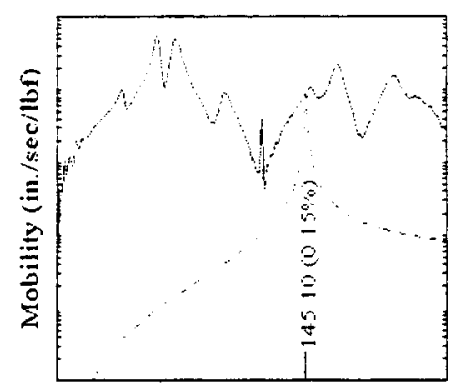

Frequency, $\mathrm{Hz}$
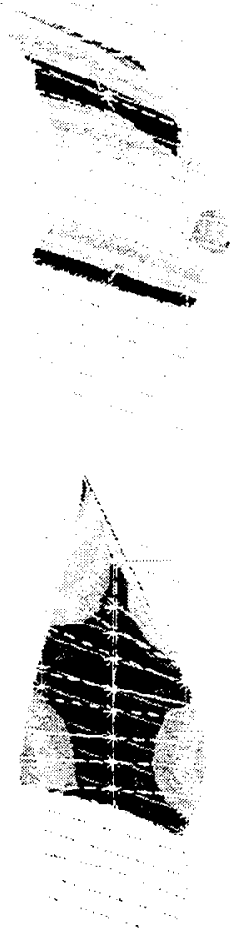

Figure 97. A comparison of SDOF and MDOF curve fitting methods (Continued)

\section{Dry condition}

A series of modal experiments were conducted for the dry wicket model to determine its system performance as a function of boundary conditions, supporting devices, and gate positions. Five such experimental cases are outlined in Table 20. Two grid patterns of 48 and 112 nodes were used to scan 


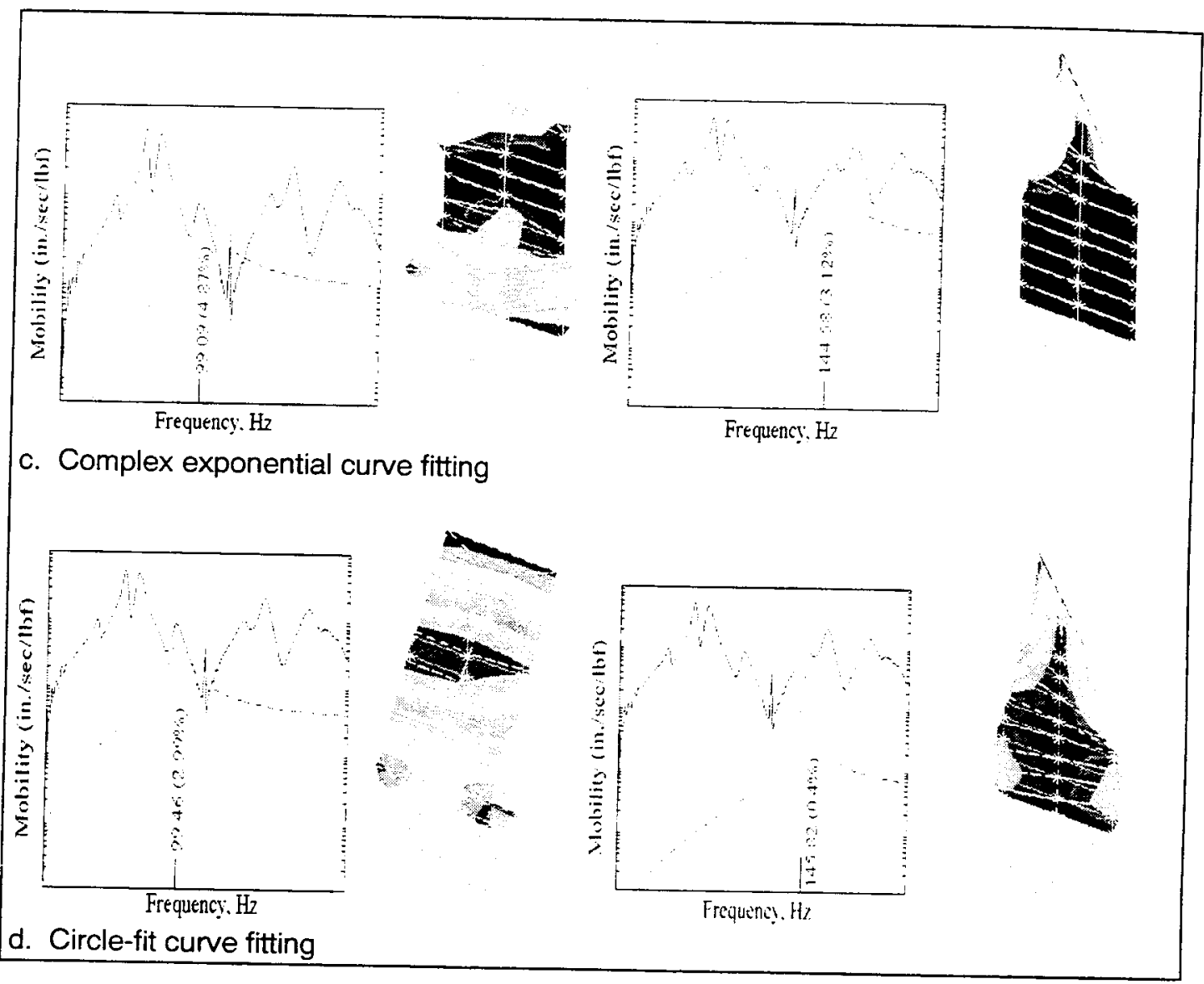

Figure 97. (Concluded)

the gate from downstream and upstream sides of the flume. Figure 92 shows the downstream scanning configuration for the modal experiment using the SLDV system. During the upstream scanning, the shaker and laser orientations were reversed from front to back.

\section{Prop supported}

A comparison of FRF for the wicket model when scanned from upstream and downstream sides of the flume is shown in Figure 98. Mode shapes corresponding corresponding to the peaks for both cases are presented in Figure 99. Figure 99a shows the dry mode shapes of the wicket when downstream face of the wicket was scanned. Figure $99 \mathrm{~b}$ presents the dry mode shapes when the wicket was scanned from the upstream platform.

These plots show that the corresponding modal frequencies for the upstream scanning are greater than those obtained from the downstream case. Despite the fact that the frequencies have shifted due to a change of scanning position, the global modes remain almost identical for both cases. The first rotational rigid body mode for the downstream scanning tends to become a translational mode during the upstream scanning. Modal densities are almost identical for both 


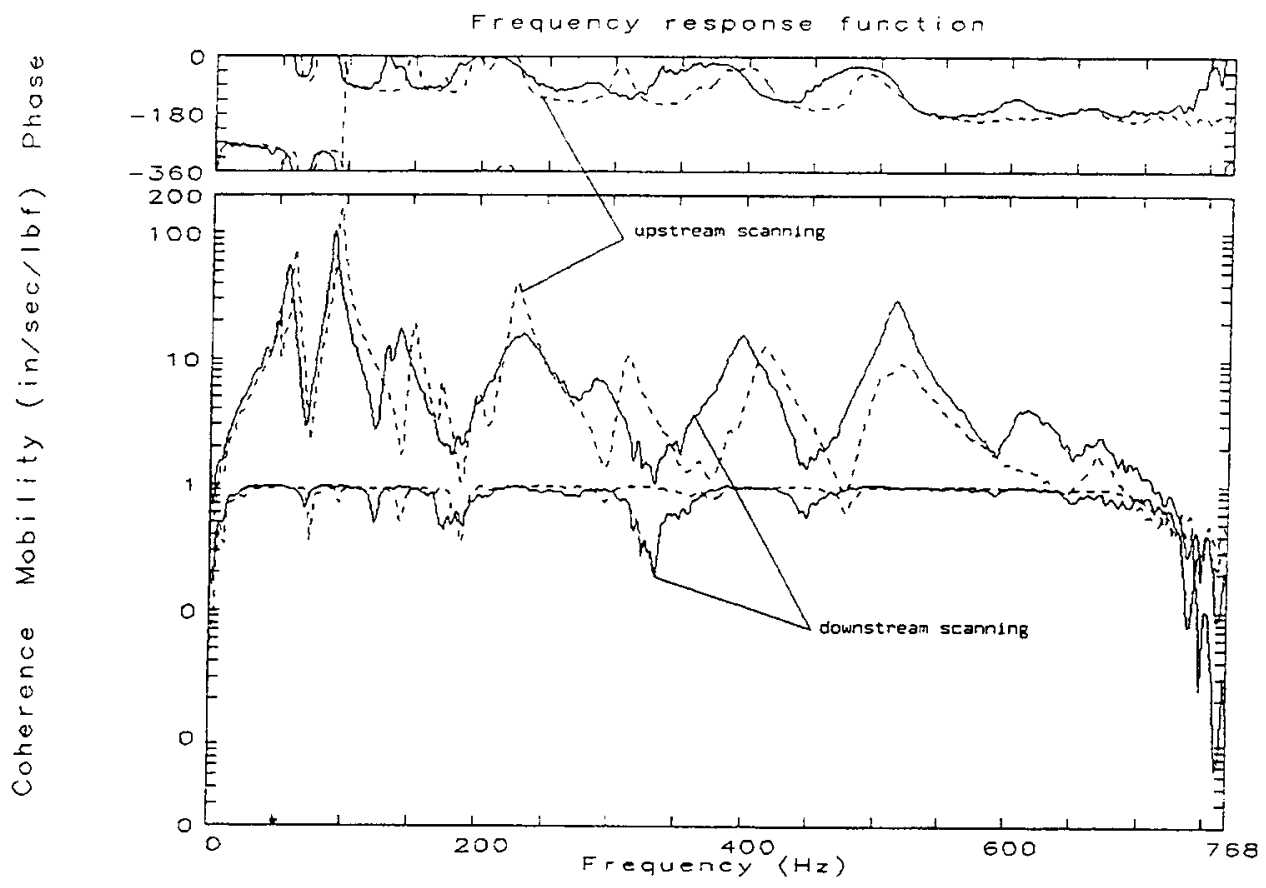

Figure 98. Mobility response functions for downstream and upstream results

cases except in the range of 100 to $200 \mathrm{~Hz}$. In this range, the upstream scanning provided three relatively separated peaks, while the downstream had only two closely coupled peaks.

A Modal Assurance Criteria (MAC) matrix was computed to measure the degree of statistical correlation between mode shapes obtained from two different sources. The MAC matrix provides a measure of the least square deviation of the points of Mode shape from the straight-line correlation. The MAC matrix between corresponding mode shapes obtained from source $\mathrm{A},\left\{\phi_{A i}\right\}$, and source $\mathrm{B},\left\{\phi_{B j}\right\}$, could be defined by:

$\operatorname{MAC}\left(\phi_{A i}, \phi_{B j}\right)=\frac{\left|\left\{\phi_{A}\right\}_{i}^{T}\left\{\phi_{B}\right\}_{j}\right|^{2}}{\left\{\phi_{A}\right\}_{i}^{T}\left\{\phi_{A}\right\}_{i}\left\{\phi_{B}\right\}_{j}^{T}\left\{\phi_{B}\right\}_{j}}$

The MAC matrix for the mode shapes obtained from upstream and downstream scanning is shown in Table 21. This table shows the degree of correlation between the corresponding modes for both cases. Similarity of multiple modes is found in the frequency range 96 to $313 \mathrm{~Hz}$. As explained in the latter part of this chapter, similar modes in the frequency range 100 to 200 $\mathrm{Hz}$ are influenced by the prop dynamics. Interaction of prop modes is also evident in the modes found in the frequency range 202 to $313 \mathrm{~Hz}$. The uncertain orientation of the clevis connection at the top of the prop rod caused the distortion of modes for the prop-supported gate. This observation indicates that there are seven distinct global mode shapes for the dry gate below $600 \mathrm{~Hz}$. 


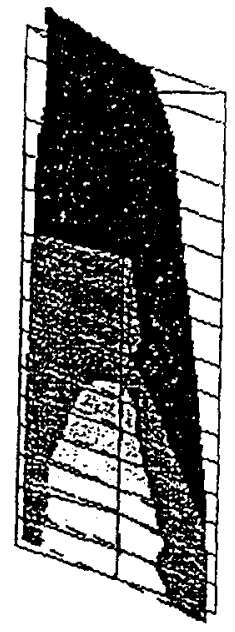

Freq: $43.11 \mathrm{~Hz}$ damping: $3.28 \%$ Peak 1

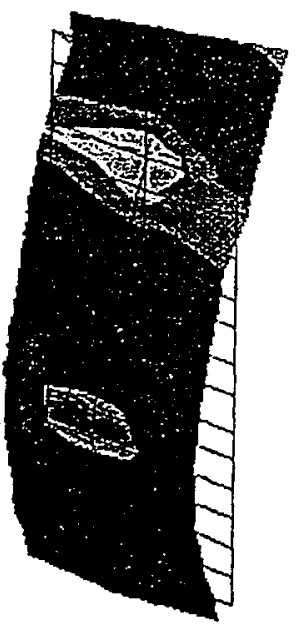

Freq: $141.20 \mathrm{~Hz}$ damping: $2.04 \%$ Peak 5

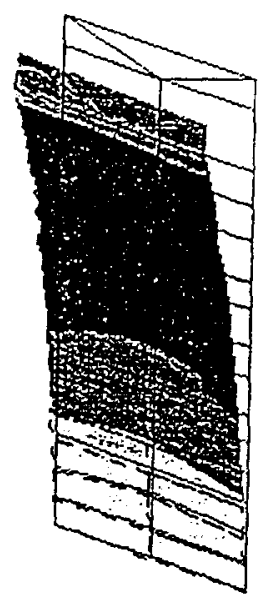

Freq: $58.00 \mathrm{~Hz}$ damping: $3.89 \%$ Peak 2

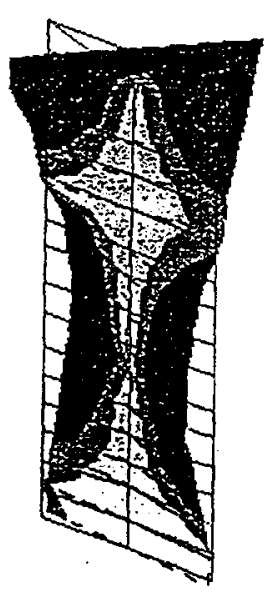

Freq: $231.6 \mathrm{~Hz}$ damping: $6.13 \%$ Peak 6

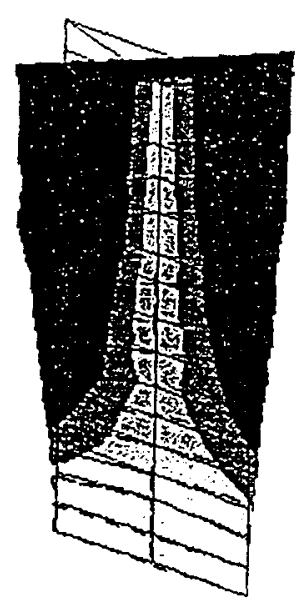

Freq: $91.40 \mathrm{~Hz}$ damping: $2.17 \%$ Peak 3

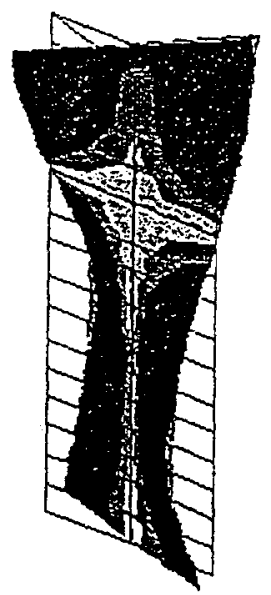

Freq: $291.90 \mathrm{~Hz}$ damping: $4.81 \%$ Peak 7

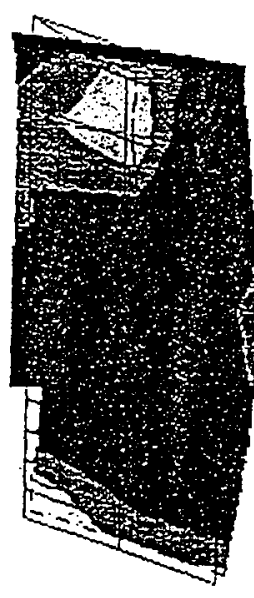

Freq: $136.24 \mathrm{~Hz}$ damping: $6.60 \%$ Peak 4

\section{a. Downstream face scanning}

Figure 99. Natural dry mode shapes of wicket using SLDV system (Continued)

A change in the FRF indicates that the system response for the gate is sensitive to the direction of the driving force. This change in frequency resulted primarily from nonlinearity of the no-tension supporting mechanisms (prop-rod blade support on the hurter recess) during the excitation of the gate. As seen in Figure 94, during downstream scanning the shaker exerted compressive force on the prop support. This condition is more stable and stiffer than the tensile resistance imparted on the prop rod during upstream scanning. The global 


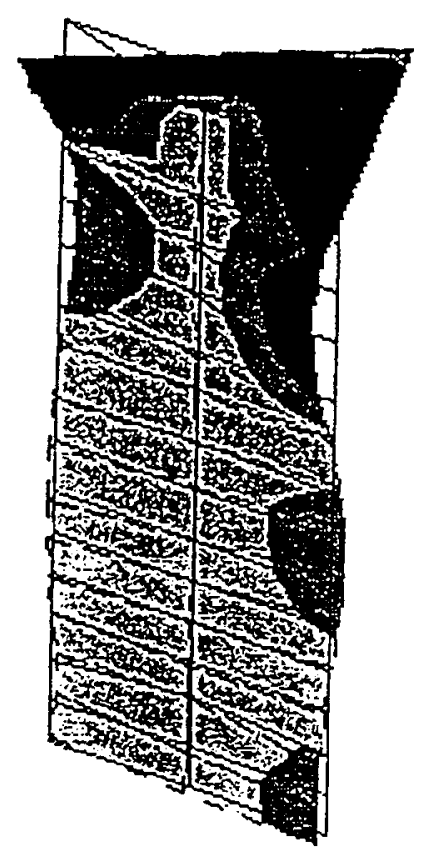

Freq: $512.53 \mathrm{~Hz}$ damping: $1.40 \%$ Peak 9

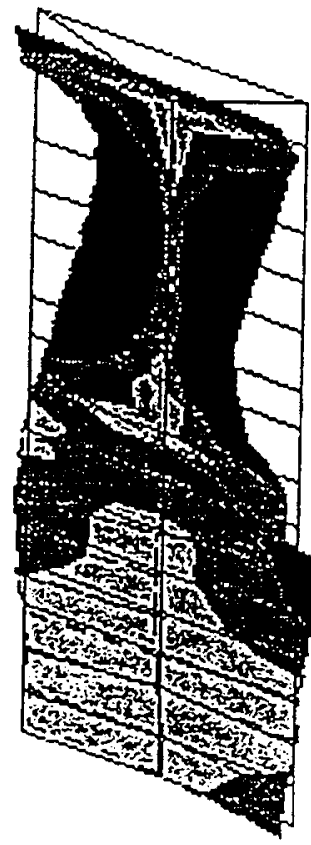

Freq: $603.08 \mathrm{~Hz}$ damping: $1.03 \%$ Peak 10

\section{b. Additional modes}

Figure 99. (Concluded)

responses for each case, however, are time invariant. This observation is made by looking at the variation of modal peaks for all responses. Figure 100a shows the superimposition of FRF plots for all response points for the downstream laser scanning case. A reciprocity test conducted on the gate also confirms the linearity of the gate response. Figure $100 \mathrm{~b}$ compares FRF for two measurement locations, such that the response node for the first case became the driving node for the second measurement and the driving point for the first case was the response node for the second measurement.

\section{Shaft supported}

FRF for the prop- and shaft-supported gate at $65 \mathrm{deg}$ are compared in Figure 101. This figure shows that the lower bending modes below $200 \mathrm{~Hz}$ are more sensitive to the alteration of the support from prop to shaft. The second and fourth global bending modes closely interacted with the shaft's local modes. A close look at the mode shapes reveals that the bending modes of the shaftsupported gate are closely coupled with the torsional modes. The ball-cup support mechanisms caused torsional modes to interact with the bending modes of the shaft-supported gate at the second and fourth positions in the modal order. Mode 2 for the shaft-supported gate (first bending at about $70 \mathrm{~Hz}$ ) was not adequately energized by the excitation function. Such an energy decline for the 


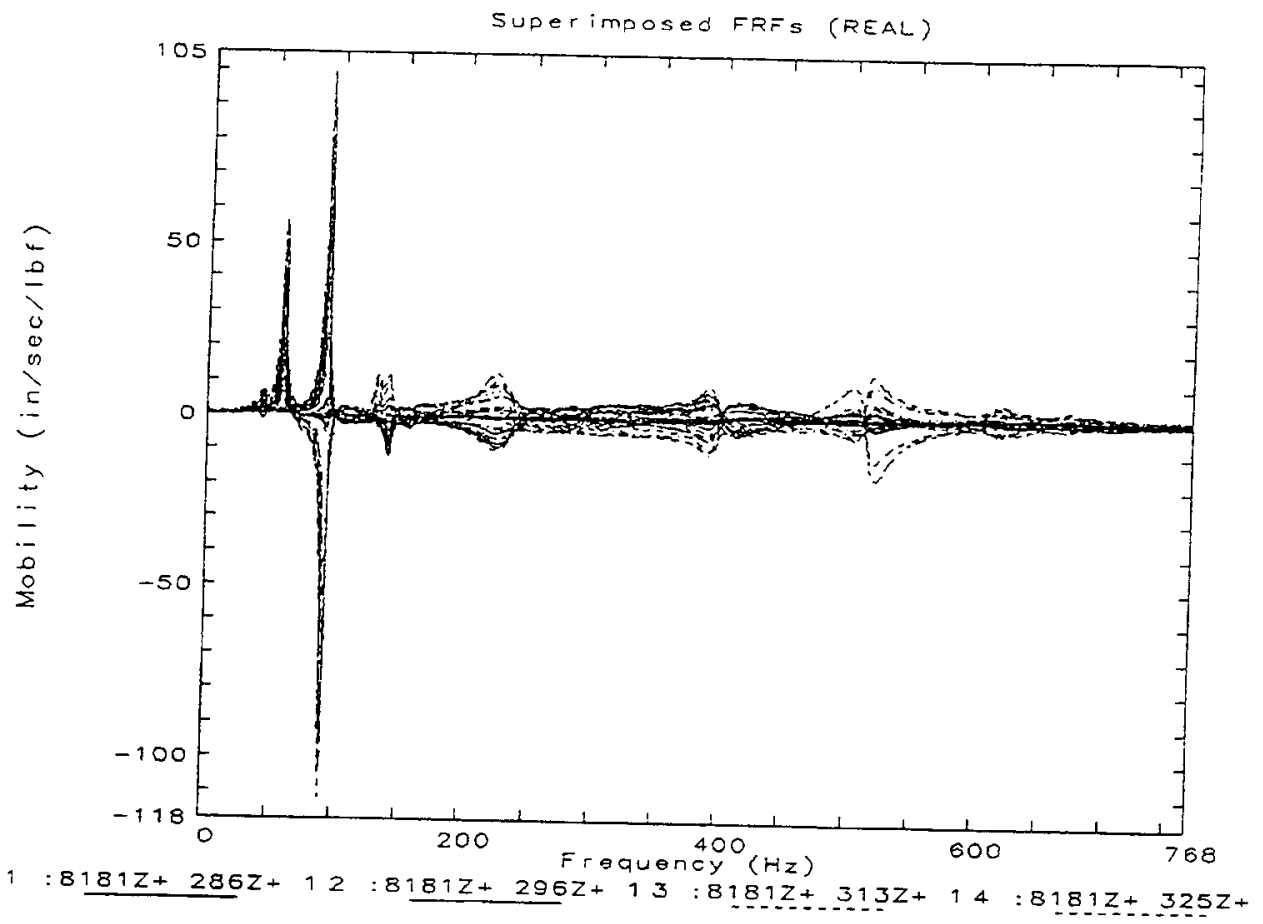

a. Superimposed FRF for all response locations

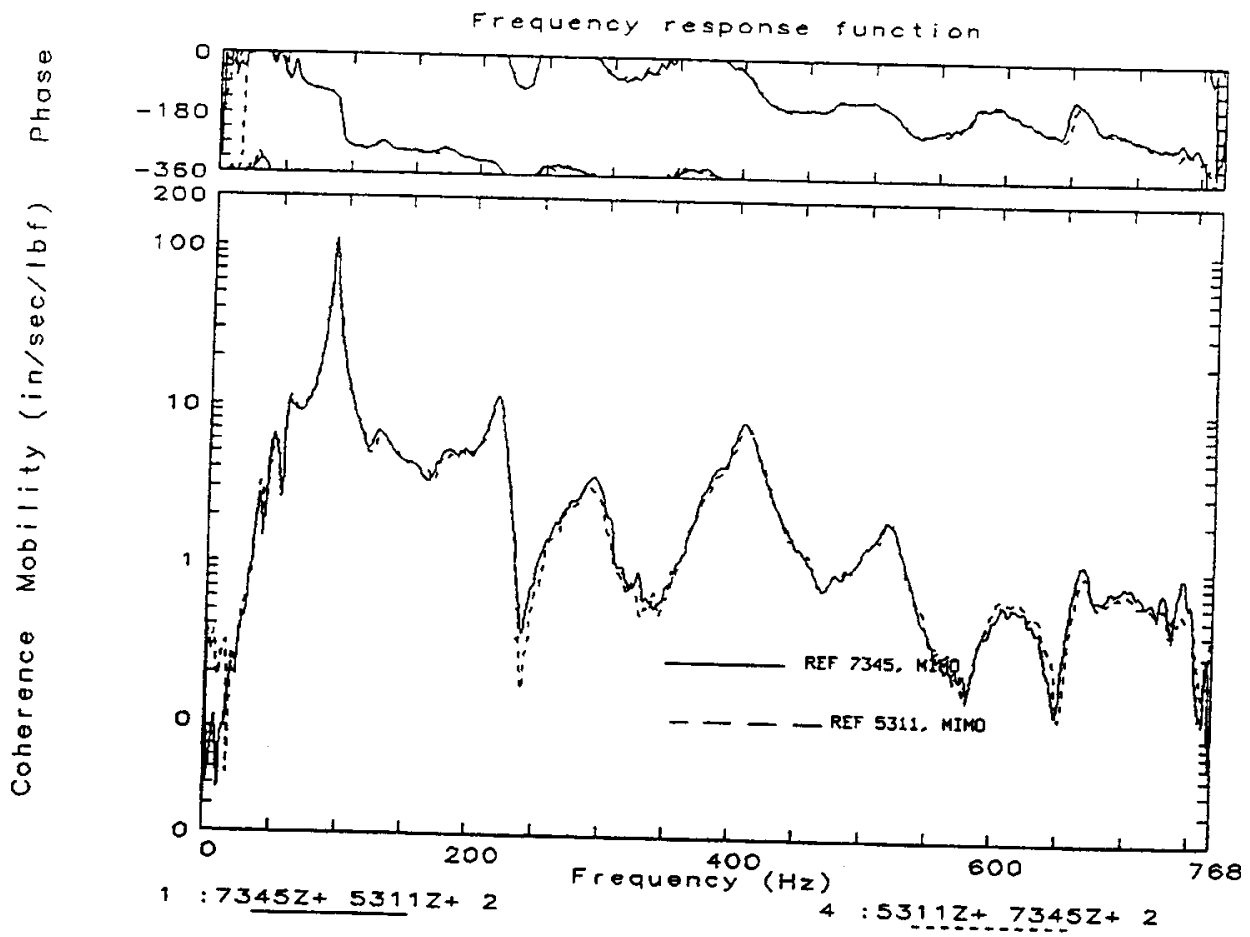

b. Superimposed driving point FRF for response at one node for excitation at another and vice-versa

Figure 100. Verifications of assumptions used in modal experiment 


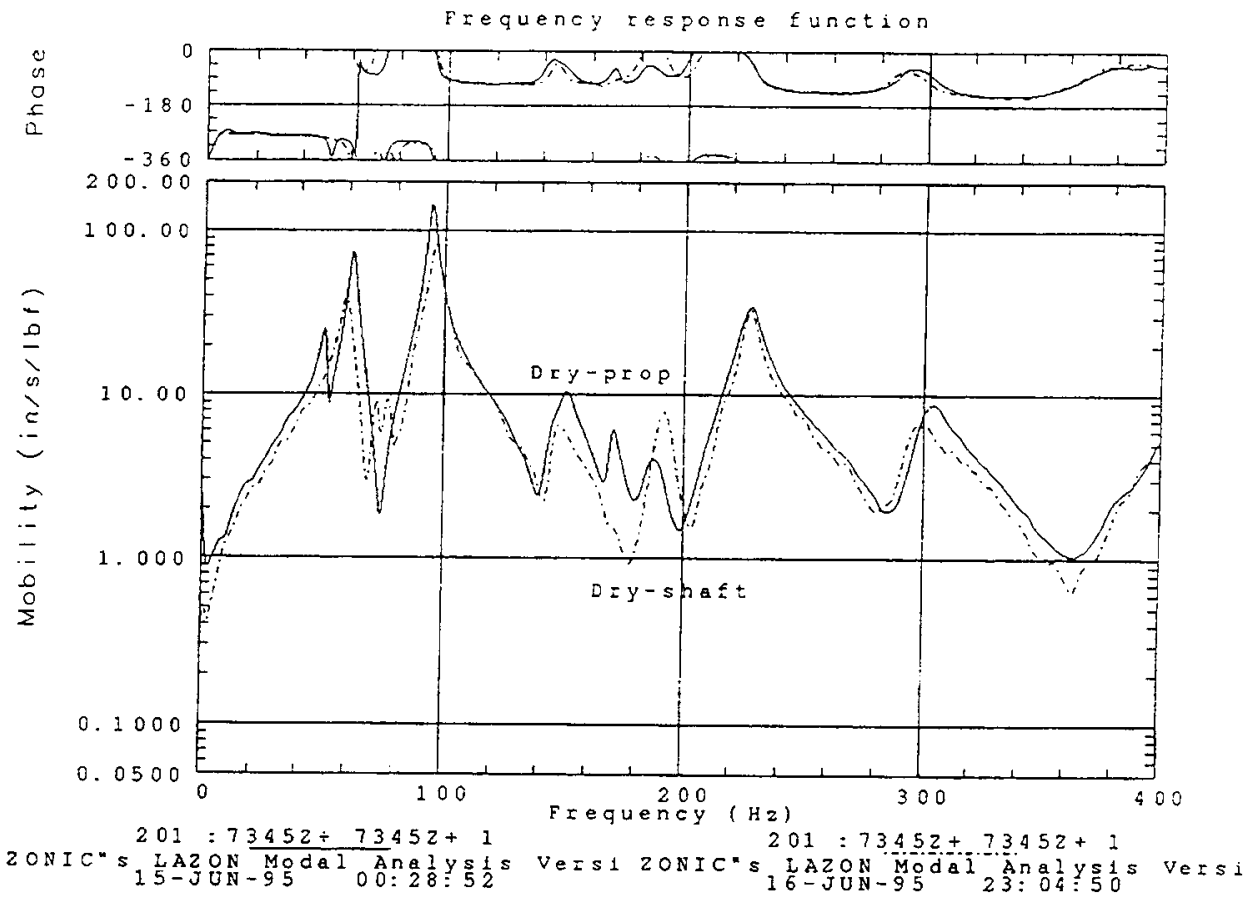

Figure 101. Mobility response function compariosn for prop- and shaft-supported dry wickets

second peak could be explained by the no-tension, ball-cup mechanism which did not restrain the gate from upward motion.

MAC numbers for modes corresponding to prop- and shaft-supported wickets are presented in Table 22 . The first rotational rigid body mode for the shaftsupported gate shows features of translational and rotational modes of the propsupported wicket. The bending modes at fourth and fifth columns in the MAC matrix did not correlate well with any shaft-supported wicket modes. The sixth, seventh, and eighth modes for both cases related well with the others.

Mode shapes for the shaft-supported dry gate on a 112-node grid are presented in Figure 102. This experiment also supports the notion that the spatial resolution for a less refined 48-node grid could adequately represent the modes of interest. This is due to the fact that a third-order transverse curve is not formed for the interested modes presented in Figure 102. A third-order curve could not be represented by three nodes along a transverse line on a 48-node grid.

A difference in the FRF for the 65-deg and 24-deg shaft-supported gate is shown in Figure 103. Modes for the lowered gate are relatively damper than those of the upright gate. Mode shapes for the 24-deg gate position are presented in Figure 104. Although the resonant frequencies shifted downward for the lowered gate, modes for the 24-deg position agreed well with the propsupported mode shapes presented in Figure 99 rather than the shaft-supported 


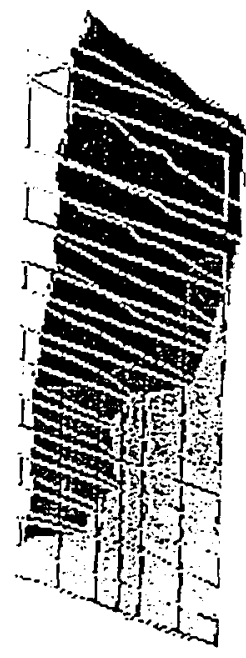

Freq: $57.66 \mathrm{~Hz}$ damping: $0.22 \%$ Peak 1

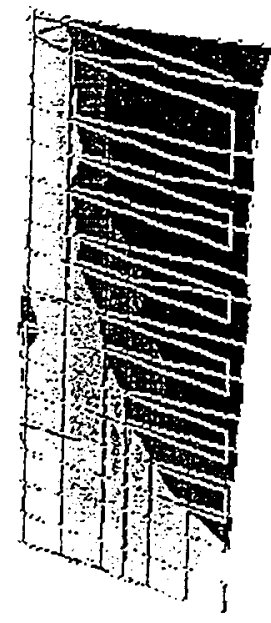

Freq: 70.78 damping: $15.43 \%$ Peak 2

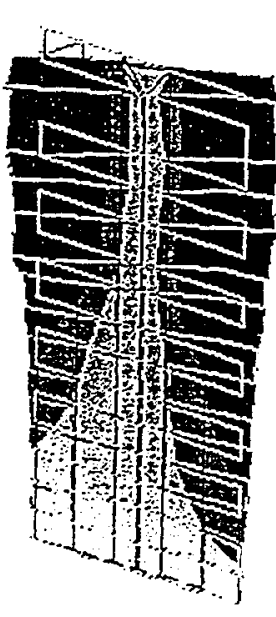

Freq: $76.12 \mathrm{~Hz}$ damping: $0.33 \%$ Peak 3

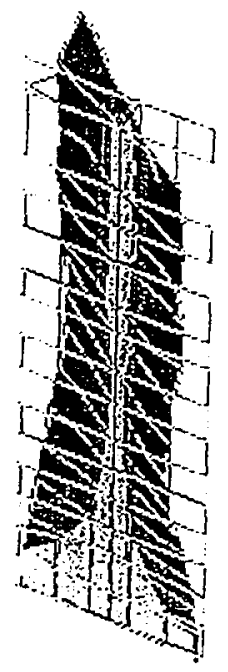

Freq: $94.95 \mathrm{~Hz}$ damping: $0.80 \%$ Peak 4

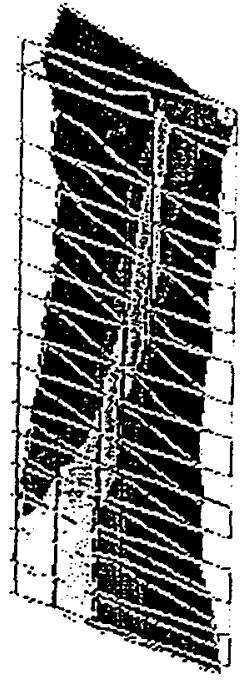

Freq: $142.23 \mathrm{~Hz}$ damping: $31.90 \%$ Peak 5

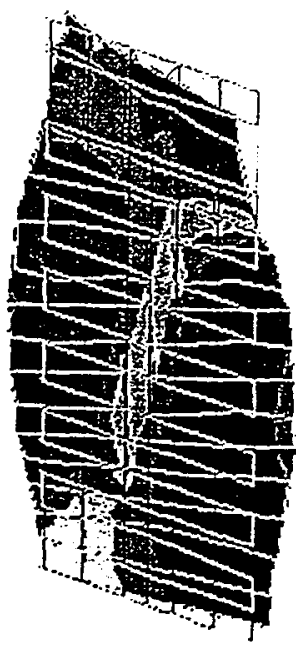

Freq: $195.84 \mathrm{~Hz}$ damping: $2.62 \%$ Peak 6 .

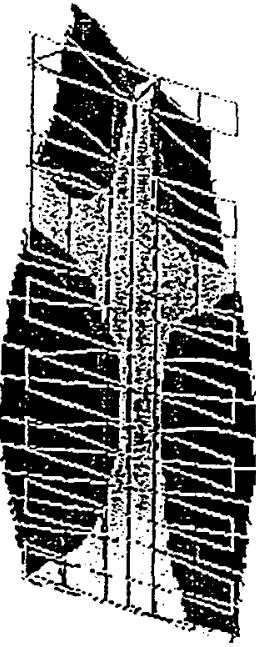

Freq: $222.18 \mathrm{~Hz}$ damping: $2.72 \%$ Peak 7

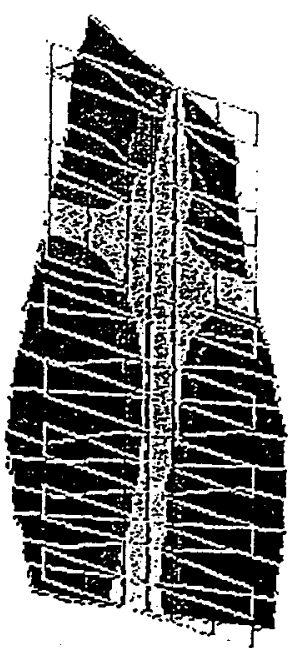

Freq: 296.31

damping: $3.79 \%$ Peak 8

Figure 102. Natural mode shapes from shaft-supported dry wicket (Continued)

fully raised wicket modes in Figure 102. A new torsional mode (at $126 \mathrm{~Hz}$ ) appeared for the 24-deg position wicket. The MAC numbers for modes corresponding to the two gate positions are shown in Table 23.

Multiple reference modal testing with two shakers was also conducted on the wicket model to extract the closely coupled modes which would otherwise be 


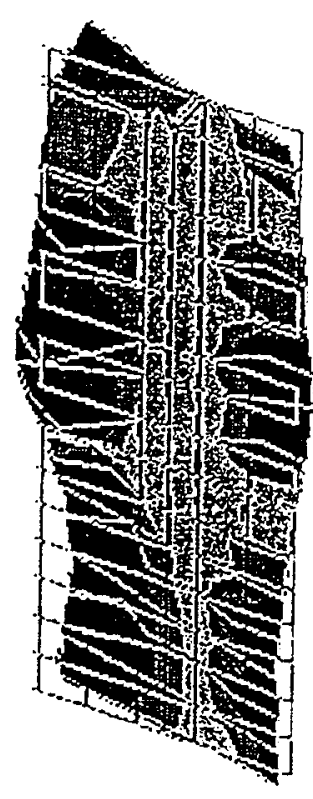

Freq: $409.93 \mathrm{~Hz}$ damping: $0.81 \%$ Peak 9

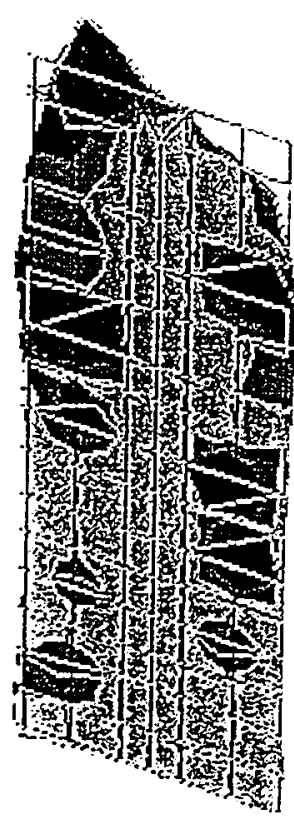

Freq: $507.57 \mathrm{~Hz}$ damping: $1.71 \%$ Peak 10

Figure 102. (Concluded)

difficult to extract using a single reference test. A multiple input multiple output (MIMO) test provides a more uniform distribution of energy to nonlinear structures, and use of random signals and frequency domain signal processing yield more consistent FRF measurements (Shye, VanKarsen, and Richardson 1988). The driving point (DP) responses of an MIMO test presented in Figure 105 shows the effectiveness of such testing in identifying closely coupled peaks. In this figure, for example, the first two peaks are well defined for DP5311, while the higher peaks are well excited by the shaker mounted at node 7345. Except for the first peak at $37.45 \mathrm{~Hz}$, modes obtained from the MIMO experiment agreed closely with those of the SIMO experiment. This new mode at $37.45 \mathrm{~Hz}$, with a 3.72 percent critical damping, was a rotational rigid body mode with a linear translational trend.

\section{Prop-rod vibrational behavior}

Modal experiments were conducted to determine the interaction of the dynamic characteristics of the prop rod with the global mode shapes of the gate presented above. Two modal experiments were performed to extract the natural characteristics of the prop rod along two major planes of orientation. A change in the mode shapes in two principal directions was expected since the support conditions at each end of the prop rod, one end with blade sitting on the hurter 


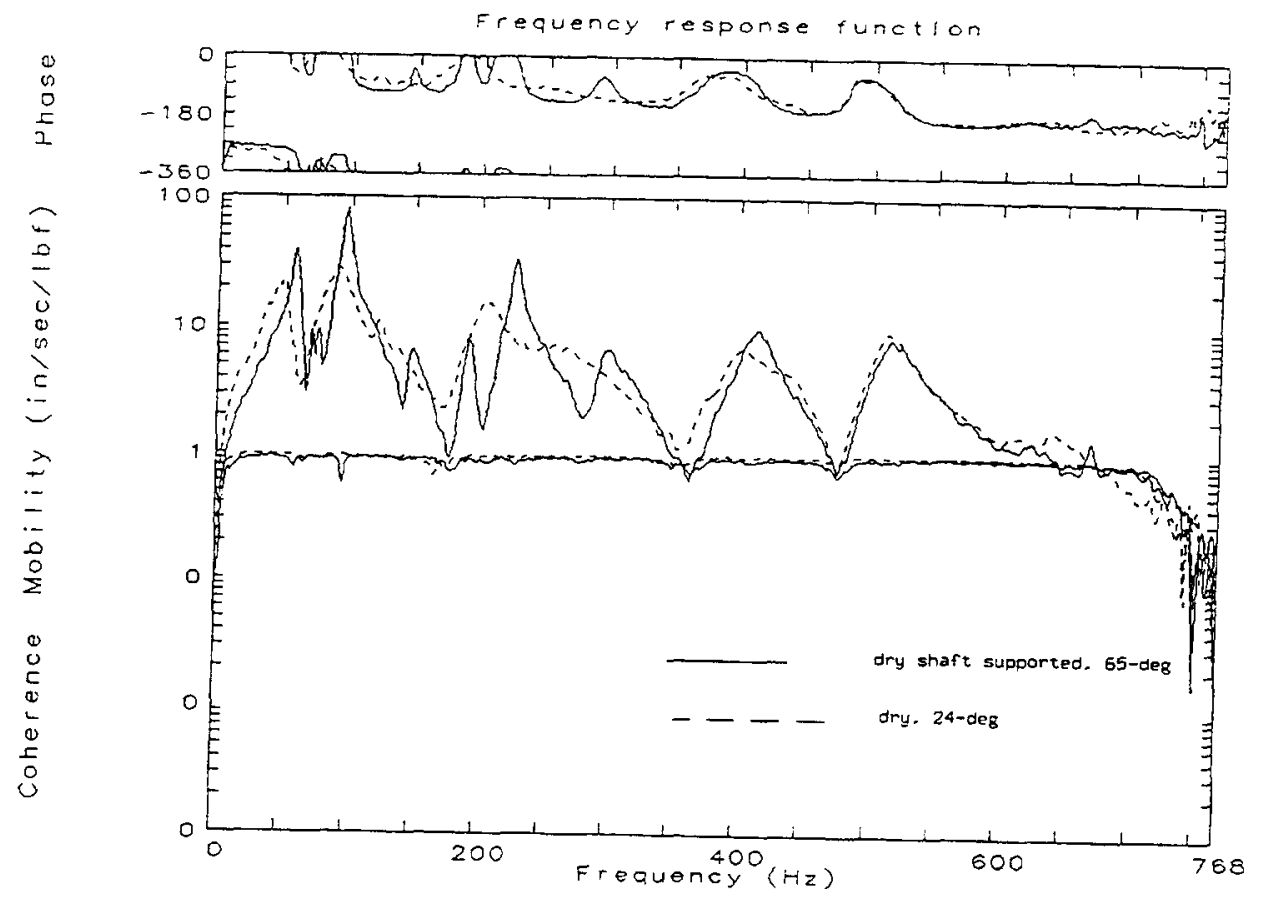

Figure 103. Mobility response function comparison for shaft-supported dry gate at 65- and 24-deg positions

recess and another with the clevis connection to the ball mounting plate, provided different resistance mechanisms along vertical and horizontal planes. Two setups as shown in Figure 106 were used to measure the FRF for the response nodes due to shaker excitation. The shaker was mounted on the gate and provided the force in a direction normal to the gate surface. The side-view setup measured the lateral responses of the upper portion of the gate and the prop rod. Upward and downward translational motions were captured during the topview scanning.

Mobility responses for the prop rod in two directions and the gate driving point response are compared in Figure 107. This figure clearly identifies the interaction of prop-rod major peaks with those of the gate peaks. Prop-rod mode shapes along the two orthogonal directions are presented Figure 108. In this figure, the prop rod and a transverse line on the top of the gate are used to display the multidirectional mode shapes of the wicket system. Figure 108a shows the natural mode shape of the top of the gate and the prop rod along the transverse direction normal to the side-view shown in Figure 106. Figure 108b presents the upward and downward motion, normal to the prop length in a vertical plane passing through the longitudinal axis of the gate. These mode shapes indicate the unrestrained boundary conditions in both ends of the prop rod. Occurrences of unstable modes (translation from the base), particularly at or near the first and second bending (at frequencies 60,150 and $200 \mathrm{hz}$ ), caused distortion in the global mode shapes of the wicket. 


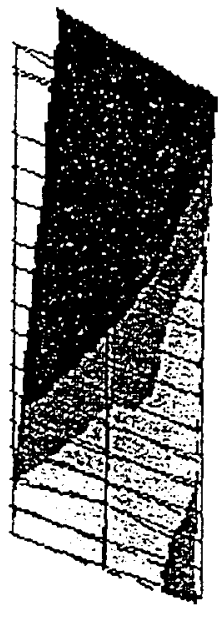

Freq: $21.06 \mathrm{~Hz}$ damping: $6.12 \%$ Peak 1

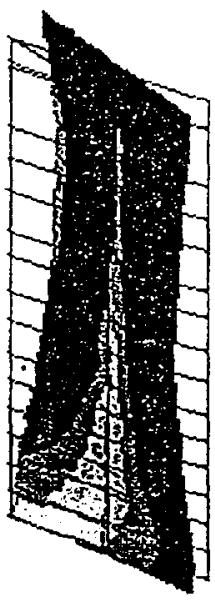

Freq: $126.29 \mathrm{~Hz}$ damping: $16.02 \%$

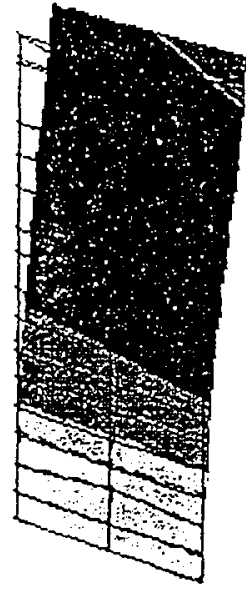

Freq: $53.22 \mathrm{~Hz}$ damping: $3.35 \%$ Peak 2

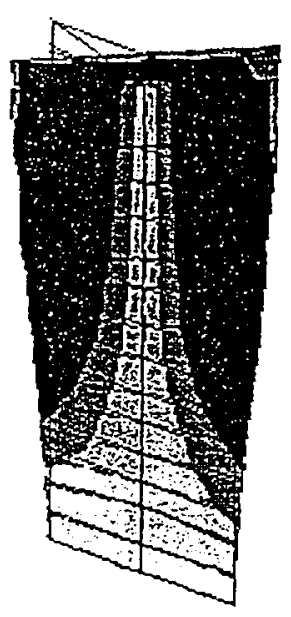

Freq: $93.87 \mathrm{~Hz}$ damping: $1.55 \%$ Peak 3

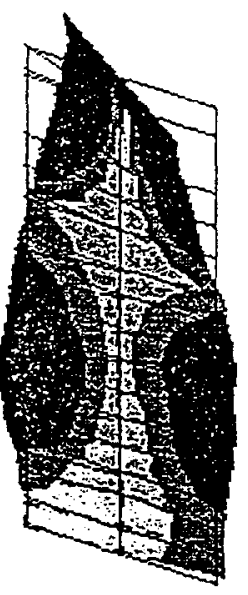

Freq: $209.41 \mathrm{~Hz}$ damping: $2.19 \%$ Peak 6

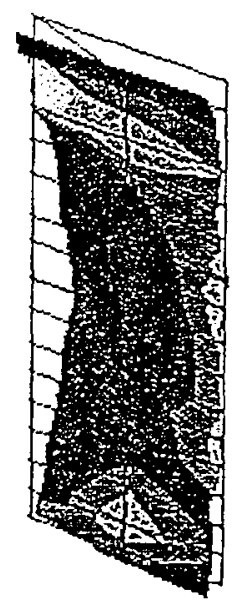

Freq: $124.28 \mathrm{~Hz}$ damping: $6.14 \%$ Peak 4

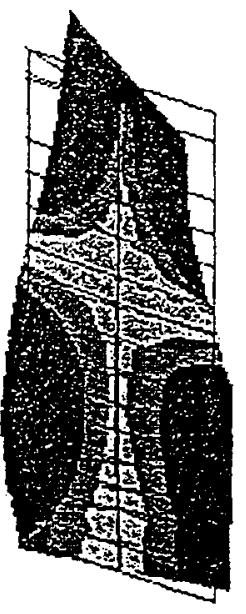

Freq: $251.33 \mathrm{~Hz}$ damping: $19.43 \%$ Peak 7

Figure 104. Natural mode shapes from downstream side of a 24-deg dry gate (Continued) 


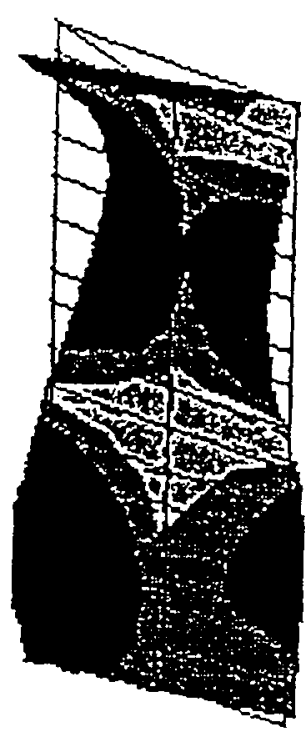

Freq: $387.80 \mathrm{~Hz}$ damping: $4.50 \%$

Peak 8

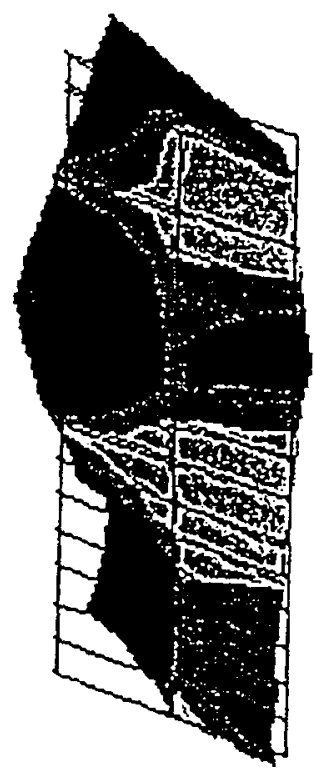

Freq: $398.06 \mathrm{~Hz}$ damping: $2.64 \%$ Peak 9

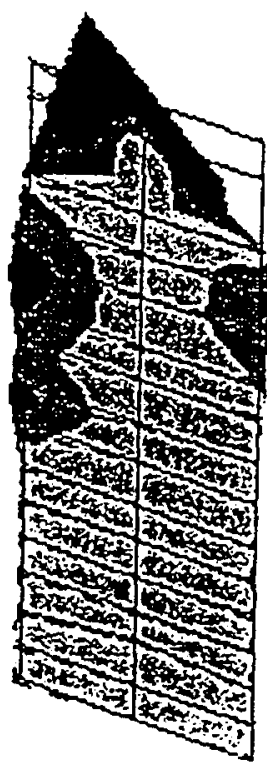

Freq: $502.58 \mathrm{~Hz}$ damping: $1.35 \%$ Peak 10

Figure 104. (Concluded)

\begin{tabular}{|c|c|c|c|c|c|c|c|c|c|c|}
\hline \multirow{3}{*}{$\mid$\begin{tabular}{|l} 
Table 2 \\
Correla \\
24 -deg \\
Position \\
(Row) \\
$\begin{array}{l}\text { Modal } \\
\text { Order }\end{array}$ \\
\end{tabular}} & $\begin{array}{l}3 \\
\text { tion } \mathrm{M}\end{array}$ & atrix fo & $r$ Prop & Suppol & ted and & 24-deg & Positio & Wicke & Modes & \\
\hline & \multicolumn{10}{|c|}{ Prop-Supported (Column) } \\
\hline & $\begin{array}{c}1 \\
\left(49^{\mathrm{Hz}}\right) \\
\end{array}$ & $\begin{array}{c}2 \\
(62 \mathrm{~Hz}) \\
\end{array}$ & $\begin{array}{c}3 \\
(96 \mathrm{~Hz}) \\
\end{array}$ & $\begin{array}{c}4 \\
(152 \mathrm{~Hz}) \\
\end{array}$ & $\begin{array}{c}5 \\
(171 \mathrm{~Hz}) \\
\end{array}$ & $\begin{array}{c}6 \\
(202 \mathrm{~Hz}) \\
\end{array}$ & $\begin{array}{c}7 \\
(232 \mathrm{~Hz})\end{array}$ & $\begin{array}{c}8 \\
(313 \mathrm{~Hz}) \\
\end{array}$ & $\begin{array}{c}9 \\
(416 \mathrm{~Hz}) \\
\end{array}$ & $\begin{array}{c}10 \\
(502 \mathrm{~Hz}) \\
\end{array}$ \\
\hline $\begin{array}{c}1 \\
\left(21^{1} \mathrm{~Hz}\right)\end{array}$ & 0.938 & 0.759 & & & & & & & & \\
\hline $\begin{array}{c}2 \\
(53 \mathrm{~Hz})\end{array}$ & 0.799 & 0.964 & & & & & & & & \\
\hline $\begin{array}{c}3 \\
(94 \mathrm{~Hz})\end{array}$ & & & 0.989 & & & & & & & \\
\hline $\begin{array}{c}4 \\
(124 \mathrm{~Hz})\end{array}$ & & & & 0.250 & & & & & & \\
\hline $\begin{array}{c}5 \\
(135 \mathrm{~Hz})\end{array}$ & & & & & 0.519 & & & & & \\
\hline $\begin{array}{c}6 \\
\left(209^{-} \mathrm{Hz}\right)\end{array}$ & & & & & & 0.730 & 0.924 & 0.640 & & \\
\hline $\begin{array}{c}7 \\
\left(251^{\mathrm{Hz}}\right)\end{array}$ & & & & & & 0.642 & 0.806 & 0.607 & & \\
\hline $\begin{array}{c}8 \\
(388 \mathrm{~Hz})\end{array}$ & & & & & & & & & & \\
\hline $\begin{array}{c}9 \\
(398 \mathrm{~Hz})\end{array}$ & & & & & & & & & 0.751 & \\
\hline $\begin{array}{c}10 \\
(503 \mathrm{~Hz})\end{array}$ & & & & & & & & & & 0.913 \\
\hline
\end{tabular}

Row Source: Position_24deg_48, Column Source: Laser_up_48a 


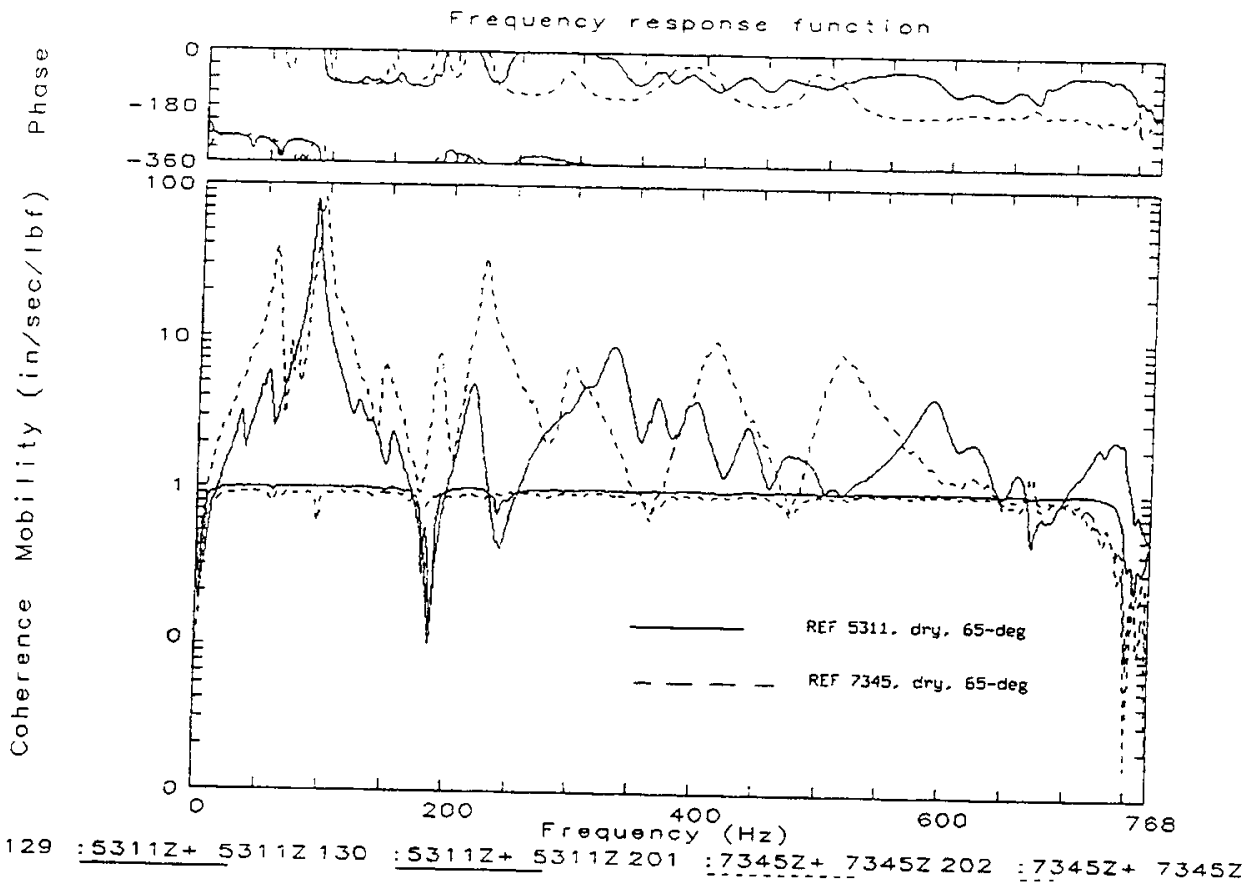

Figure 105. Mobility response frunctions of prop-supported wicket for two different reference points

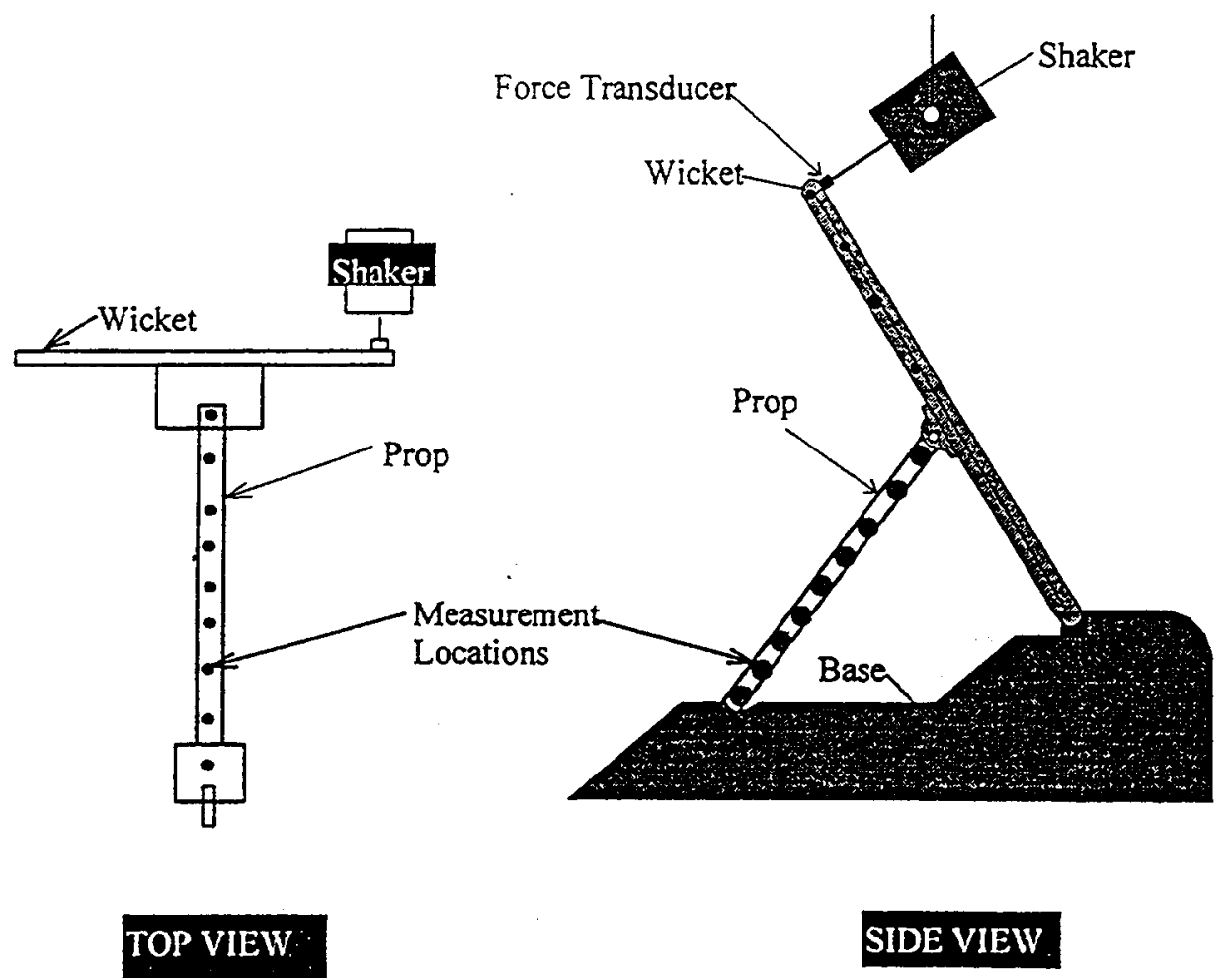

Figure 106. Prop-rod modal experiment setup 


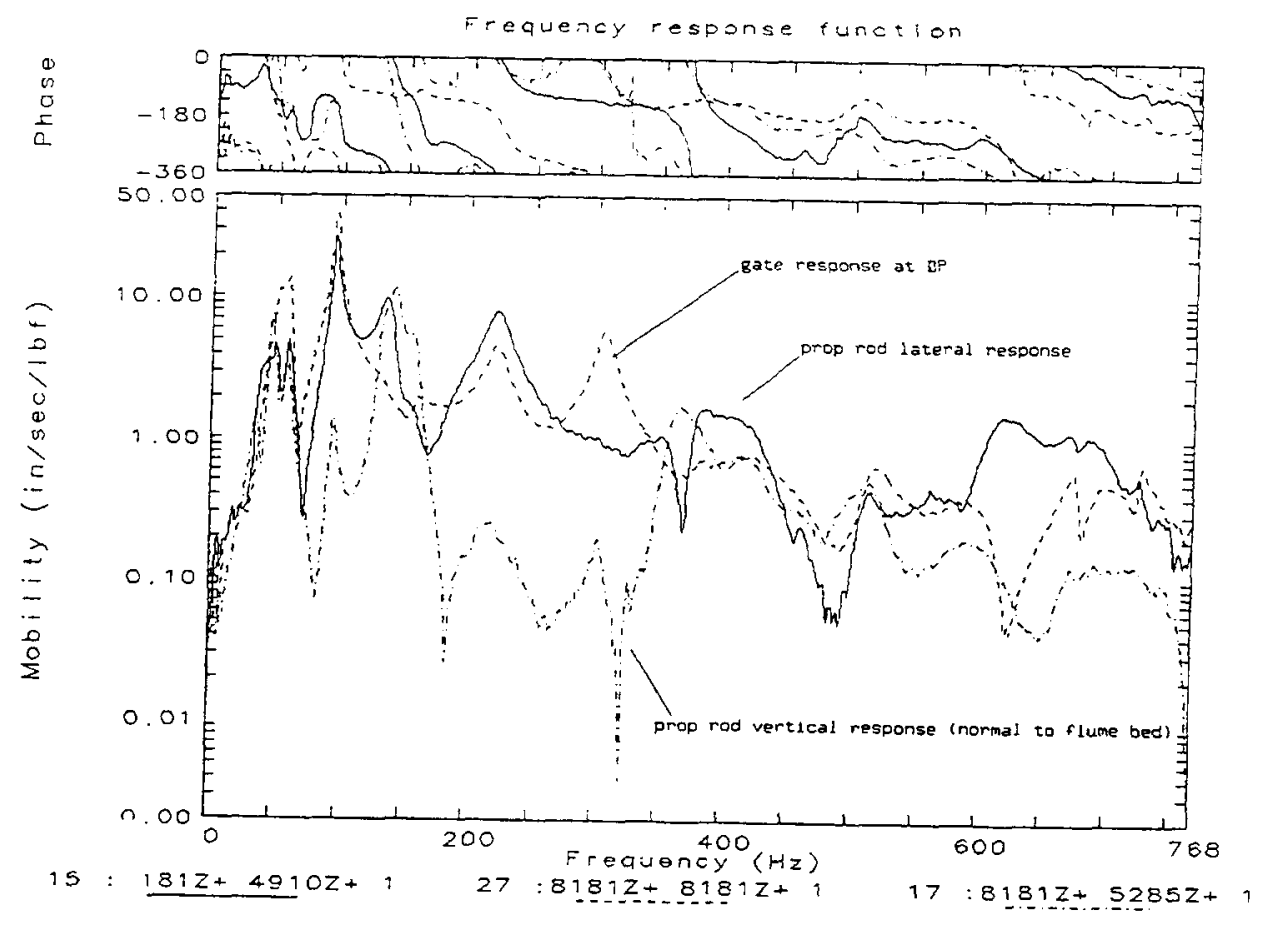

Figure 107. Directional sensitivity of mobility response functions for the prop rod

Superposition of FRF responses of nodes along the vertical plane (top-view scanning) shows major disturbances in the frequency ranges containing the first two bending modes of the gate (Figure 109). As a result of such a nonlinear interaction between the prop rod and gate, the modal frequencies shifted randomly as evinced in the FRF plots presented earlier. This interaction of prop dynamics also explains the reason for the existence of similar-looking global mode shapes at different frequencies. As a result, the global mode shapes, (for example modes 6 and 8 in Figure 99) look identical in two different frequencies, but a detailed study of the three-dimensional system reveals the difference between the shapes. This difference can be attributed to the multidirectional mode shapes of the prop rod.

\section{Wet condition}

A set of modal experiments for the wet wicket model was conducted to determine its system performance as a function of operating conditions, gate configuration and pool differences. Five such experimental cases are outlined in Table 24. For all cases, the 48-nodes grid was used to scan the fully raised gate from the downstream side of the flume. Figure 92 shows the downstream scanning nodal configuration for the modal setup. This configuration caused water to flow through the gap on both sides of the wicket. Water flowed around the edges of the wicket model, thus preventing the laser signal from acquiring responses from some nodes on the back side. Therefore, only about 30 out of 48 nodes were scanned for the wet condition. 


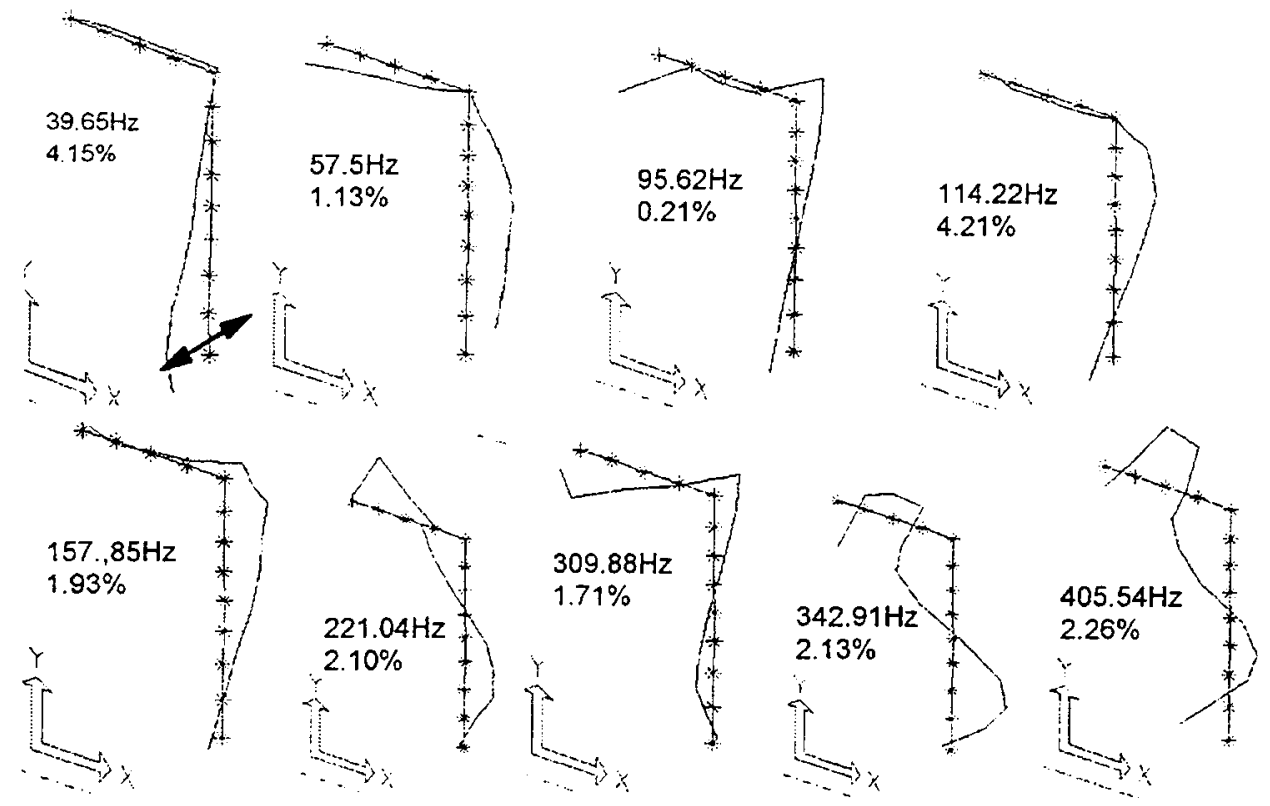

a. Mode shapes in transverse plane (side-sway)

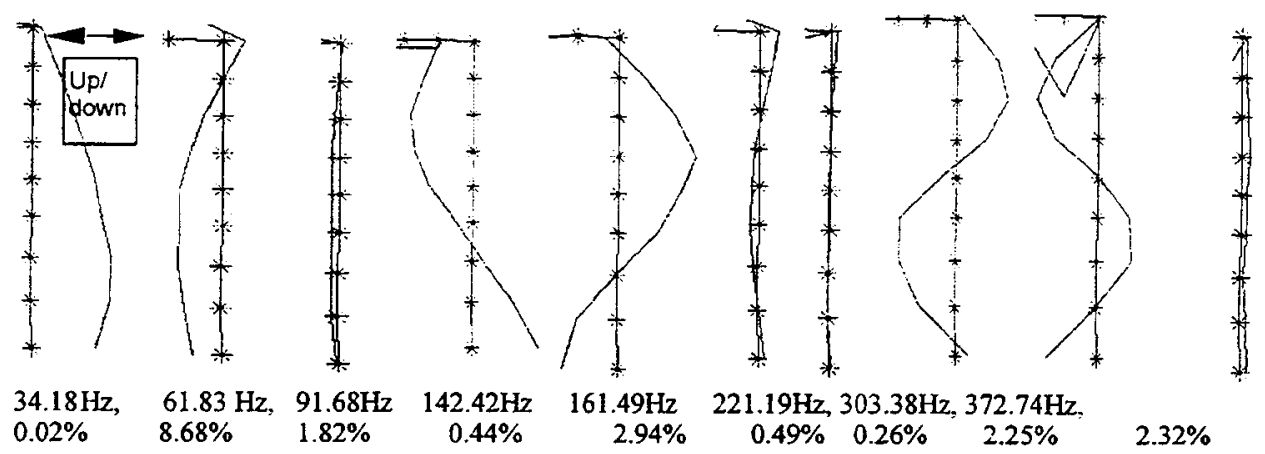

b. Mode shapes in vertical plane (up and down motion)

Figure 108. Prop-rod mode shapes

\begin{tabular}{|c|c|}
\hline \multicolumn{2}{|c|}{$\begin{array}{l}\text { Table } 24 \\
\text { Wet Modal Experiments }\end{array}$} \\
\hline Case Designation & Remarks \\
\hline A. Laser_21ft_no & $\begin{array}{l}\text { Prop-supported gate in a no-gate gap configuration with a 21-ft pool } \\
\text { difference }\end{array}$ \\
\hline B. Laser_21ft_2gdn & $\begin{array}{l}\text { Prop-supported gate in a 3-gate gap configuration with a 21-tt pool } \\
\text { difference }\end{array}$ \\
\hline C. Laser_21ft_sft & $\begin{array}{l}\text { Shaft-supported gate in a 3-gate gap configuration with a 21-ft pool } \\
\text { difference }\end{array}$ \\
\hline D. Laser_11ft_2gdn & $\begin{array}{l}\text { Shaft-supported gate in a 3-gate gap configuration with a 11-ft pool } \\
\text { difference }\end{array}$ \\
\hline E. Laser_7ft_2gdn & $\begin{array}{l}\text { Shaft-supported gate in a 3-gate gap configuration with a 7-ft pool } \\
\text { difference }\end{array}$ \\
\hline
\end{tabular}




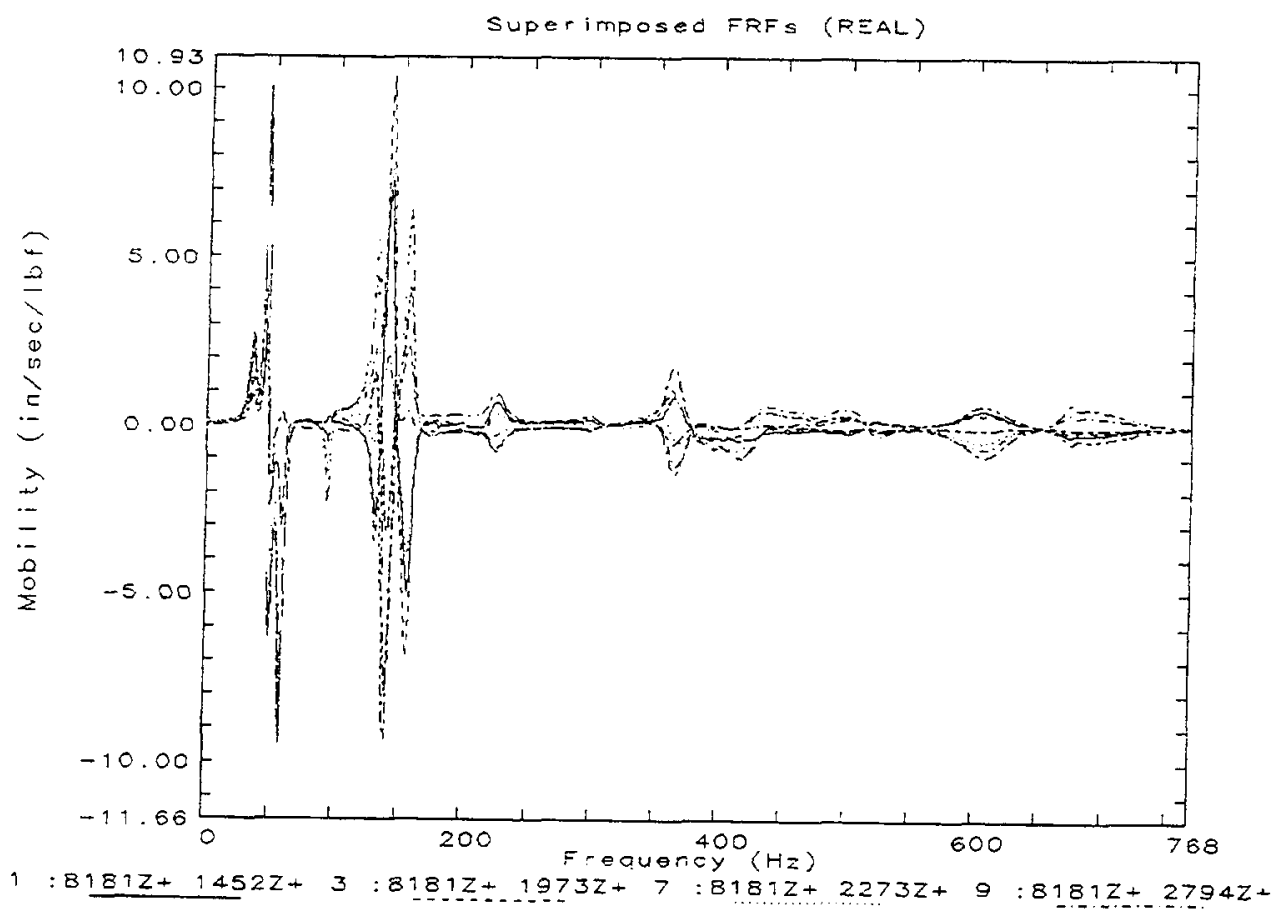

Figure 109. Superimposed prop-rod FRF responses

During the wet modal experiment, the operating boundary conditions and the flow characteristics were well preserved. Thus, the gate had random vibration during the modal experiment due to the background noise. In the midst of the flow-generated background noise, the shaker excited random input force adequately energized the natural modes of the wet gates. The accuracy of the measured FRF was measured by examining the coherence functions between the input and output responses for each nodal test point. For all FRF records used to extract the modal information, the output response sensed by the laser beam was well correlated to the excitation functions at the resonant points.

Figure 110 compares the FRF and coherence functions for the prop-supported dry and wet wickets. Wet gate response was measured for a $21-\mathrm{ft}$ pool difference with a no-gap gate configuration. This figure shows that the resonant frequencies decreased during the wet condition, as expected, because of the added mass effects of the surrounding water. The mobility function for the wet condition shows more peaks than the dry condition. Note that the dry FRF shows the resonant peaks of dry wicket due to shaker excitation. The wet FRF, however, indicates the forced vibrational modes due to water flow and the resonant modes of the wet wicket. Thus, the additional peaks in the wet FRF are indicative of flow-induced motion of the wicket due to water flow. Similar trends are also noticeable in the comparison plots of the wet and dry gates for shaft-supported fully raised and 24-deg positions (Figures 111 and 112). 


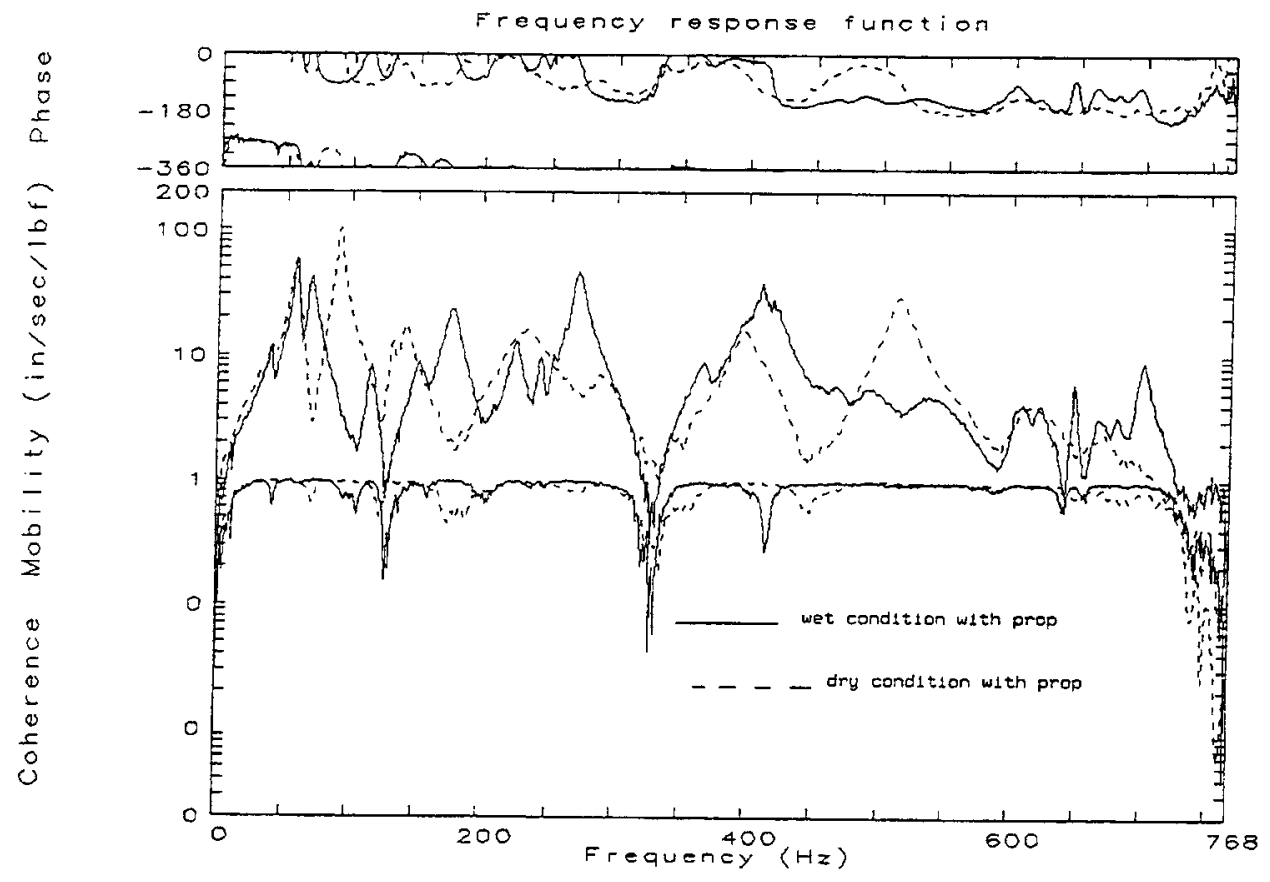

Figure 110. Comparison of FRF and coherence functions for prop-supported wet and dry gates

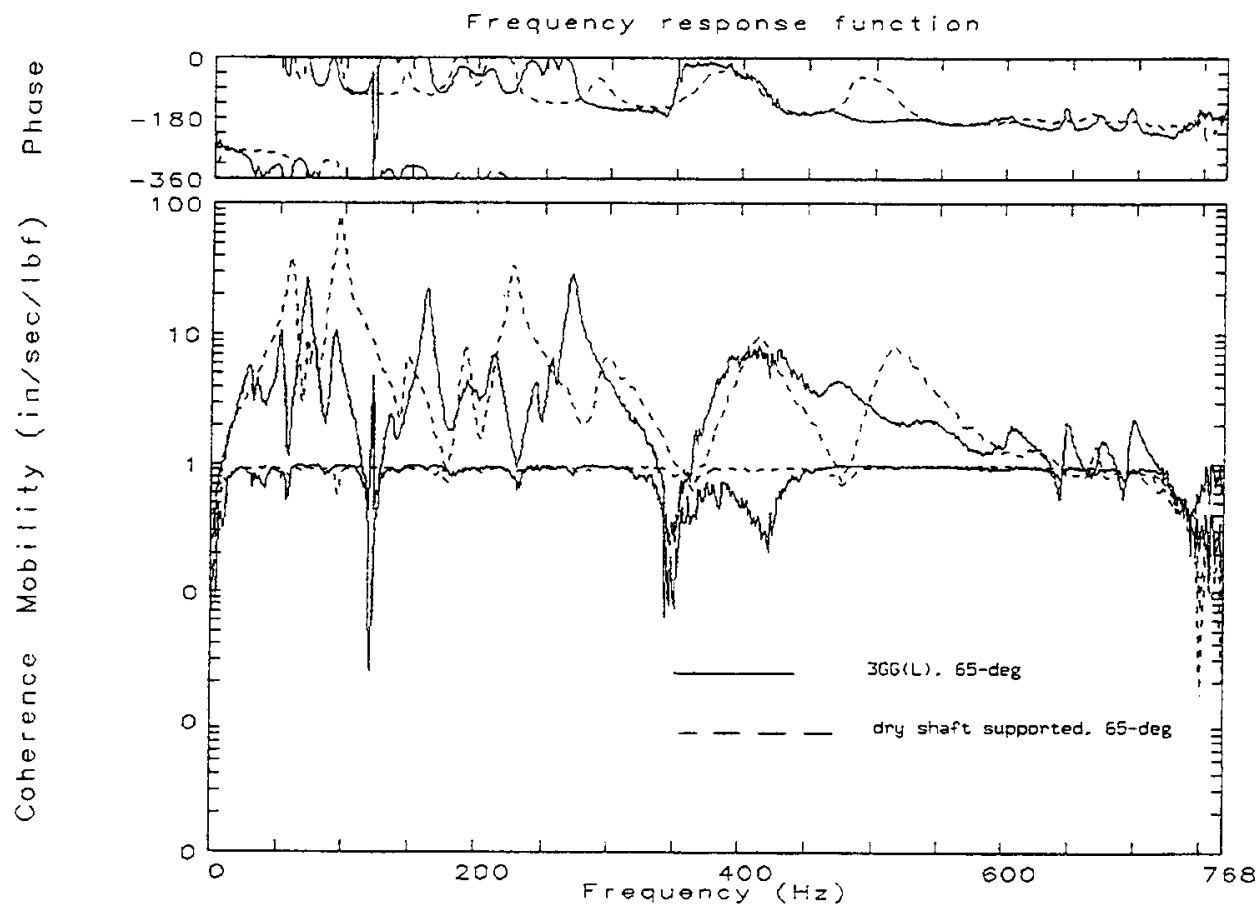

Figure 111. Comparison of FRF and coeherence functions for shaft-supported dry and wet wickets (3GG), 65-deg position 


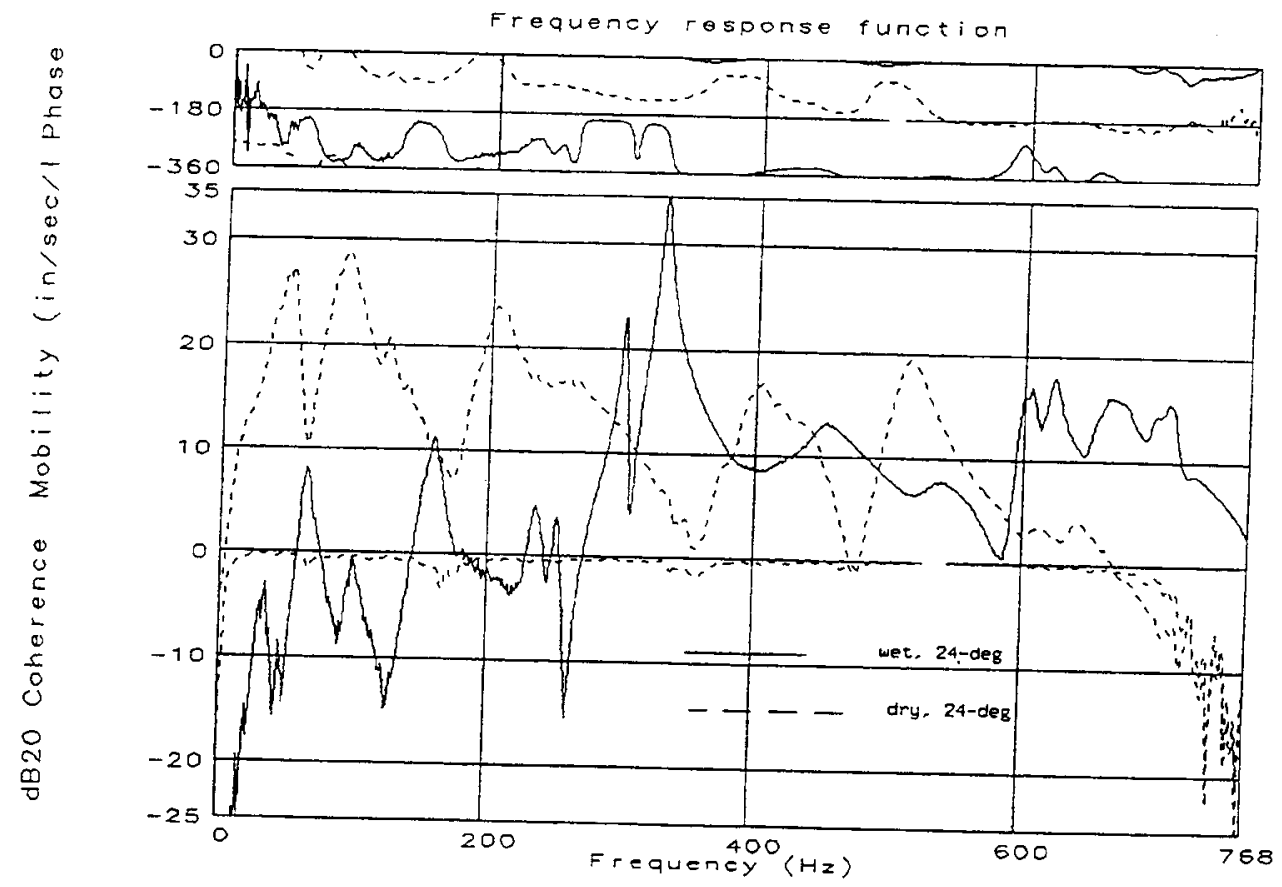

Figure 112. Comparison of FRF and coherence functions for shaft-supported dry and wet wickets (3GG), 24-deg position

Mode shapes for the prop- and shaft-supported fully raised wicket model during a three-gate gap, 21 -ft head difference flow configuration are presented in Figures 113 and 114. These figures indicate that similar mode shapes existed for both wet and dry cases. New modes representative of the boundary conditions also appeared in the wet case. These higher modes indicate the exposed gate area above the water surface by forming a distinct vibratory shape of the gate as shown in Figures 113 and 114 (see modes corresponding to frequencies 255.85 and $274 \mathrm{~Hz}$ in Figure 113 and $272.12 \mathrm{~Hz}$ mode in Figure 114). In general, all but the second mode showed a reduction in the resonant frequencies and a slight increase in the damping ratios for the wet case over the other mode.

The correlation matrix for the prop-supported dry and wet wickets for the critical configuration at $65 \mathrm{deg}$ is presented in Table 25 . In this table, modes that are considered fairly related to each other are in bold print. As shown in the table, the first four modes correlated well with each other. The sixth and seventh modes are likely to be the same global torsional mode which have interacted with the local prop modes. For identifying similar modes between the dry and wet gates, therefore, only one mode in this range would be used in added mass computation. The eighth mode for the wet gate, a bending mode, was not found in the dry gate. Note that for the wet condition a few of the localized modes showing the motion of the gate surface above water level - in the frequency ranges 215 to $400 \mathrm{~Hz}$ - are not compared in the MAC table.

As mentioned earlier in the analysis of prop-rod dynamics, the second global mode was dominated by the prop resistance. A minimum interference of 


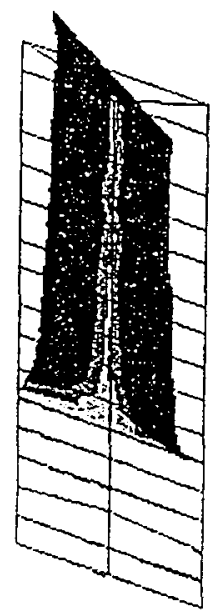

Freq: $38.90 \mathrm{~Hz}$ damping: $2.60 \%$ Peak 1

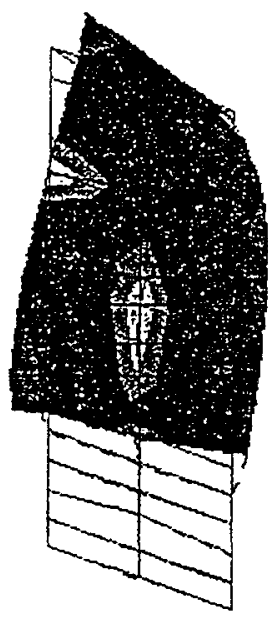

Freq: $115.45 \mathrm{~Hz}$ damping: $5.02 \%$ Peak 5

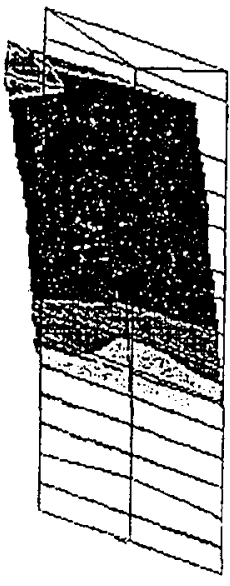

Freq: $58.57 \mathrm{~Hz}$ damping: $1.90 \%$ Peak 2

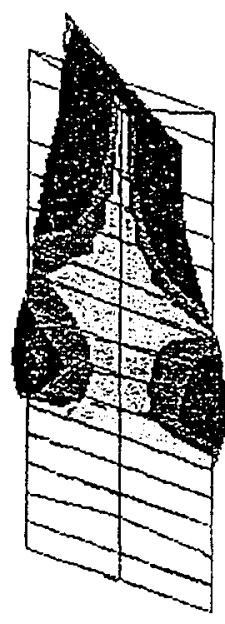

Freq: $148.47 \mathrm{~Hz}$ damping: $2.90 \%$ Peak 6

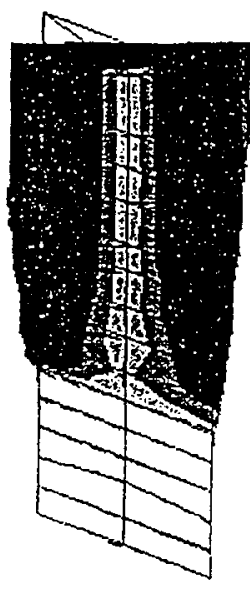

Freq: $68.69 \mathrm{~Hz}$ damping: $3.00 \%$ Peak 3

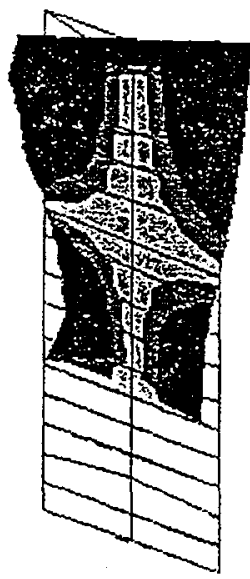

Freq: $164.68 \mathrm{~Hz}$ damping: $1.97 \%$ Peak 7

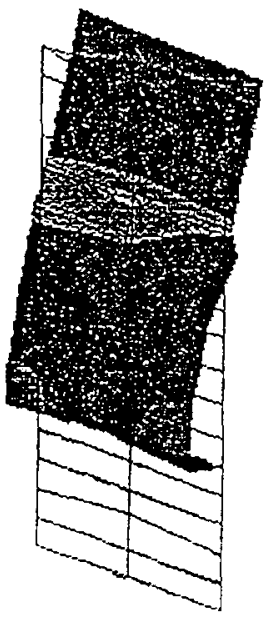

Freq: $96.86 \mathrm{~Hz}$ damping: $3.10 \%$ Peak 4

Figure 113. Natural wet mode shapes of prop-supported wicket in 3GG condition with 21-ft head difference (Continued)

the second mode due to water flow was expected, since the prop-rod dynamics had not been affected by the flow. Note in Figure 111, a sharp spike appears at about $120 \mathrm{~Hz}$ for the wet experiment. This was caused by the power harmonics from the pumps that had been running from an unfiltered power source independent of the one used to power the data acquisition system. A constant voltage power transmission filtering device was used for the data acquisition system. 


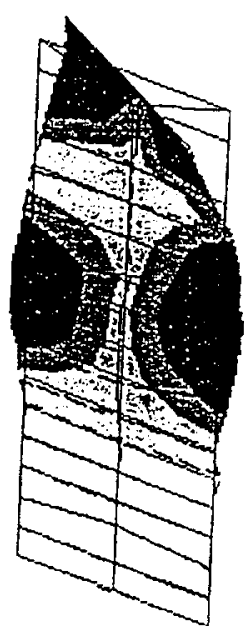

Freq: $215.28 \mathrm{~Hz}$ damping: $3.58 \%$ Peak 9

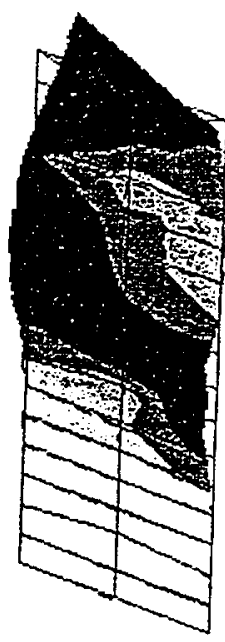

Freq: $385.74 \mathrm{~Hz}$ damping: $0.59 \%$ Peak 13

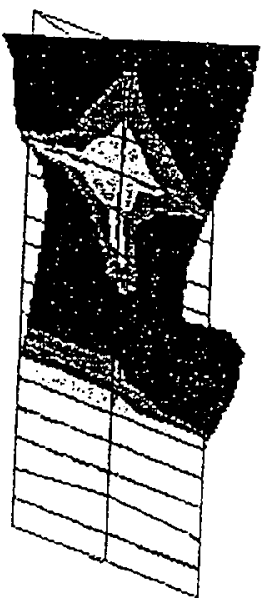

Freq: $246.29 \mathrm{~Hz}$ damping: $1.33 \%$ Peak 10

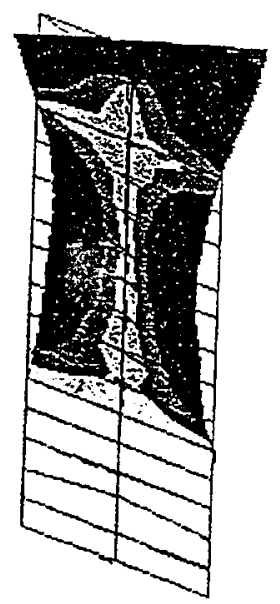

Freq: $408.04 \mathrm{~Hz}$ damping: $1.39 \%$ Peak 14

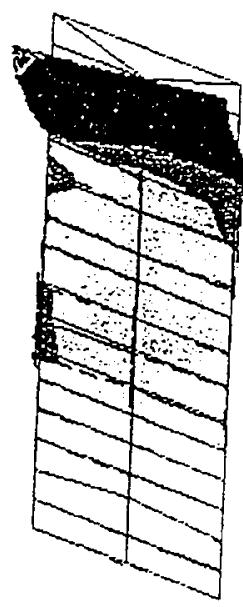

Freq: $255.85 \mathrm{~Hz}$ damping: $0.23 \%$ Peak 11

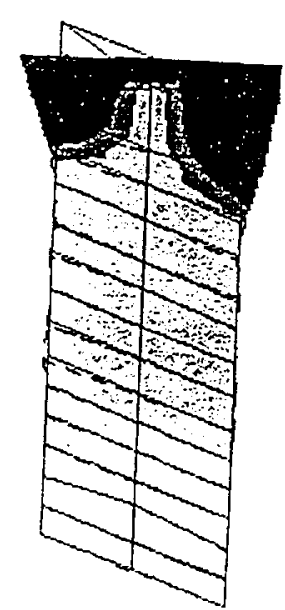

Freq: $274.00 \mathrm{~Hz}$ damping: $1.23 \%$ Peak 12

Figure 113. (Concluded)

The MAC relation between the shaft-supported dry and wet cases also shows a similar relationship as in the prop-supported condition. In this case, the global modes - less sensitive to the prop support - remain unchanged due to the added mass effect.

MAC relations for the dry and wet gate modes for the critical position at $24 \mathrm{deg}$ are presented in Table 26 . Note that the mode shapes for the completely submerged gate at $24 \mathrm{deg}$ were generated using the built-in accelerometer 


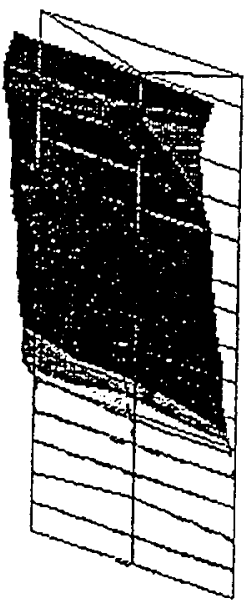

Freq: $26.84 \mathrm{~Hz}$ damping: $1.91 \%$ Peak 1

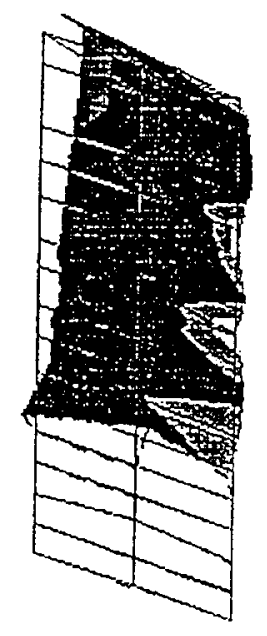

Freq: $43.91 \mathrm{~Hz}$ damping: $17.12 \%$ Peak 2

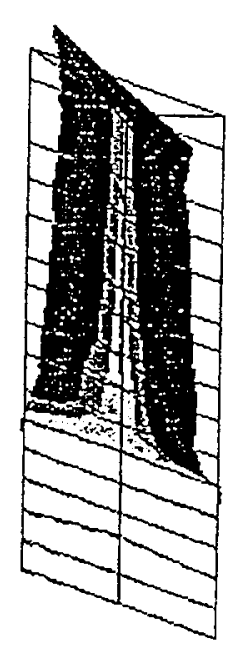

Freq: $50.95 \mathrm{~Hz}$ damping: $0.01 \%$ Peak 3

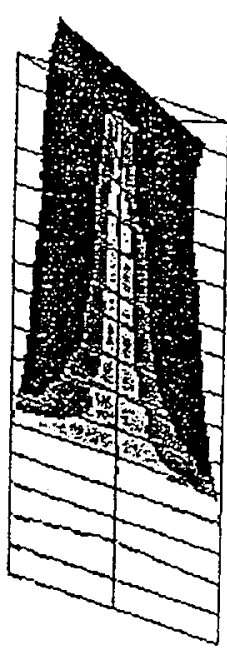

Freq: $70.79 \mathrm{~Hz}$ damping: $1.71 \%$ Peak 4

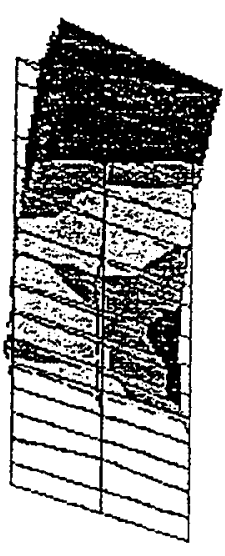

Freq: $87.21 \mathrm{~Hz}$ damping: $0.52 \%$

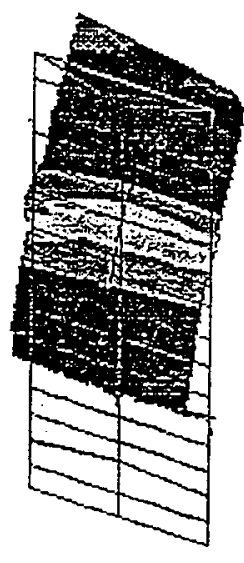

Freq: $94.43 \mathrm{~Hz}$ damping: $1.84 \%$ Peak 5

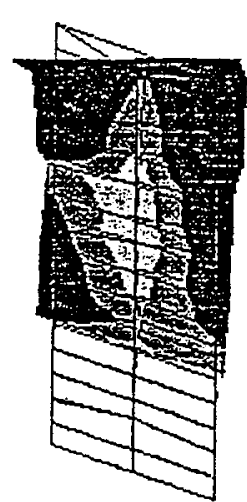

Freq: $121.79 \mathrm{~Hz}$ damping: $2.74 \%$ Peak 6

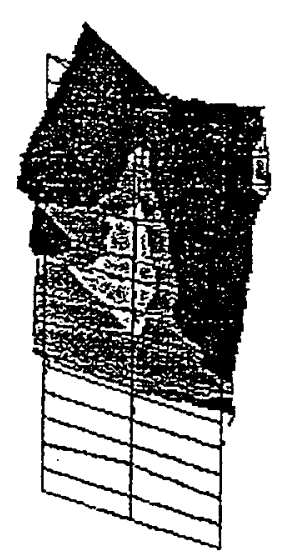

Freq: $136.23 \mathrm{~Hz}$ damping: $11.22 \%$ Peak 7

Figure 114. Natural wet mode shapes of shaft-supported gate in $3 G G$ condition with 21-ft head difference (Continued)

records instead of scanned laser signals. The shaker-excited nine-accelerometer responses were used to extract the modal parameters of the operational wicket. Mode shapes for the 24-deg wet gate for a 2 GG configuration are shown in Figure 115. As shown in the figure, a nine-node grid reasonably identified the first-order bending and torsional modes of the wet wicket. It also indicated the higher modes representative of the boundary conditions of the dry surface above the water level. A dispersion of MAC numbers throughout the table indicates a 


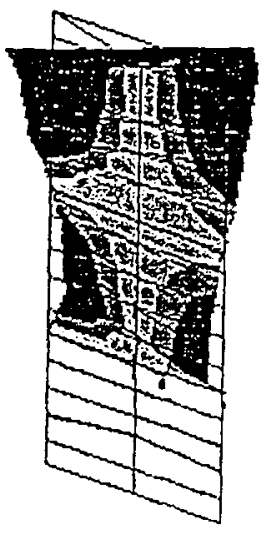

Freq: $162.45 \mathrm{~Hz}$ damping: $0.74 \%$ Peak 8

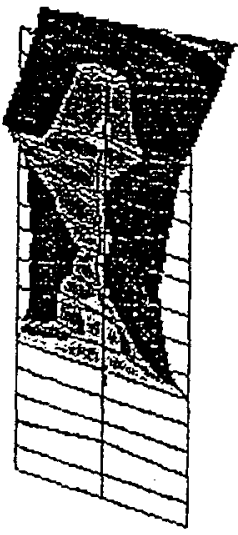

Freq: $395.63 \mathrm{~Hz}$ damping: $2.65 \%$

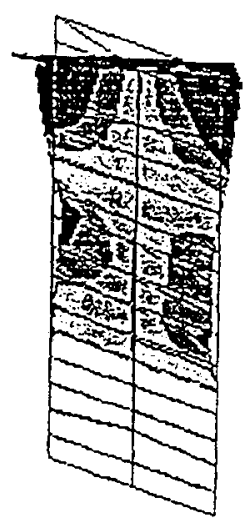

Freq: $214.50 \mathrm{~Hz}$ damping: $0.52 \%$ Peak 9

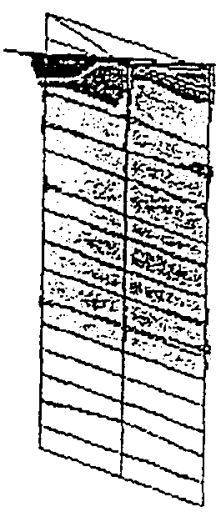

Freq: $258.93 \mathrm{~Hz}$ damping: $0.41 \%$ Peak 10

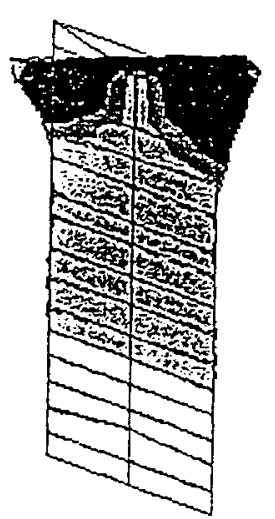

Freq: $272.12 \mathrm{~Hz}$ damping: $0.76 \%$ Peat 11

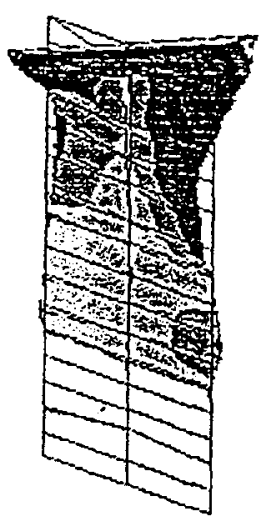

Freq: $419.86 \mathrm{~Hz}$ damping: $0.05 \%$

Figure 114. (Concluded)

similarity of one mode with multiple modes from the comparing case. This is due to the lack of spatial resolution of the nine-node grid which could not adequately interpolate the higher-order modes. For instance, the first bending mode for the dry gate matched well with the first, fourth, eighth, and ninth wet modes (see MAC numbers in Table 26). In this table, nodes that are considered fairly related to each other are in bold print. Referring to the dry mode shapes for the wicket at $24 \mathrm{deg}$ (Figure 104), one can easily identify the reason for having such multiple correlation of the first dry bending mode with the four wet modes. In this case, all related modes with higher MAC numbers have similarity with the first-order bending modes for the dry wicket. The third and sixth wet modes correspond to the first and second torsional global modes of the dry gate. 


\begin{tabular}{|c|c|c|c|c|c|c|c|c|c|c|}
\hline \multicolumn{11}{|c|}{$\begin{array}{l}\text { Table } 25 \\
\text { Correlation Matrix for Prop-Supported Dry and Wet Gates (21-ft Head Difference, } \\
\text { 3GG Flow Configuration) }\end{array}$} \\
\hline Dry Gate & \multicolumn{10}{|c|}{ Wet Wicket (Column) } \\
\hline $\begin{array}{l}\text { Modal } \\
\text { Order } \\
\text { (Row) }\end{array}$ & $\begin{array}{c}1 \\
\left(39^{\mathrm{Hz}}\right)\end{array}$ & $\begin{array}{c}2 \\
(58 \mathrm{~Hz})\end{array}$ & $\begin{array}{c}3 \\
(68 \mathrm{~Hz})\end{array}$ & $\begin{array}{c}4 \\
(97 \mathrm{~Hz})\end{array}$ & $\begin{array}{c}5 \\
(115 \mathrm{~Hz})\end{array}$ & $\begin{array}{c}6 \\
(148 \mathrm{~Hz})\end{array}$ & $\begin{array}{c}7 \\
(165 \mathrm{~Hz})\end{array}$ & $\begin{array}{c}8 \\
(198 \mathrm{~Hz})\end{array}$ & $\begin{array}{c}9 \\
(215 \mathrm{~Hz})\end{array}$ & $\begin{array}{c}10 \\
(408 \mathrm{~Hz})\end{array}$ \\
\hline $\begin{array}{c}1 \\
(43 \mathrm{~Hz})\end{array}$ & 0.783 & & 0.719 & & & & & & & \\
\hline $\begin{array}{c}2 \\
(58 \mathrm{~Hz})\end{array}$ & & 0.935 & & & & & & & & \\
\hline $\begin{array}{c}3 \\
(91 \mathrm{~Hz})\end{array}$ & 0.933 & & 0.970 & & & & & & & \\
\hline $\begin{array}{c}4 \\
(136 \mathrm{~Hz})\end{array}$ & 0.395 & & & & & & & & & \\
\hline $\begin{array}{c}5 \\
(141 \mathrm{~Hz})\end{array}$ & & & & 0.700 & & & & 0.535 & & \\
\hline $\begin{array}{c}6 \\
(231 \mathrm{~Hz})\end{array}$ & & & & & & 0.568 & 0.678 & & & \\
\hline $\begin{array}{c}7 \\
(291 \mathrm{~Hz})\end{array}$ & & & & & & 0.501 & 0.731 & & & \\
\hline $\begin{array}{c}8 \\
(400 \mathrm{~Hz})\end{array}$ & & & & & & & & & 0.590 & 0.789 \\
\hline $\begin{array}{c}9 \\
(512 \mathrm{~Hz})\end{array}$ & & & & & & & & & & 0.566 \\
\hline $\begin{array}{c}10 \\
(603 \mathrm{~Hz})\end{array}$ & & & & & & & & & & \\
\hline
\end{tabular}

Row Source: Laser_down, Column Source: Laser_21ft_2g_dn

\begin{tabular}{|c|c|c|c|c|c|c|c|c|c|c|}
\hline $\begin{array}{l}\text { Table } 2 \\
\text { Correla } \\
\text { Differer }\end{array}$ & $\begin{array}{l}\text { tion } M \\
\text { ce, } 2 G\end{array}$ & $\begin{array}{l}\text { trix fo } \\
\text { Flov }\end{array}$ & $\begin{array}{l}\text { Dry } \\
\text { Conf }\end{array}$ & $\begin{array}{l}\text { nd Wet } \\
\text { guratio }\end{array}$ & ate Mo & des at & 24-deg & osition & (21-ft F & lead \\
\hline Dry Gate & & & & Wet W & cket (C) & Slumn) & & & & \\
\hline \begin{tabular}{|c|} 
Modal \\
Order \\
(Row)
\end{tabular} & $\begin{array}{c}1 \\
(39 \mathrm{~Hz})\end{array}$ & $\begin{array}{c}2 \\
(65 \mathrm{~Hz})\end{array}$ & $\begin{array}{c}3 \\
(74 \mathrm{~Hz})\end{array}$ & $\begin{array}{c}4 \\
(110 \mathrm{~Hz})\end{array}$ & $\begin{array}{c}5 \\
(162 \mathrm{~Hz})\end{array}$ & $\begin{array}{c}6 \\
(219 \mathrm{~Hz})\end{array}$ & $\begin{array}{c}7 \\
(237 \mathrm{~Hz})\end{array}$ & $\begin{array}{c}8 \\
(250 \mathrm{~Hz})\end{array}$ & $\begin{array}{c}9 \\
(303 \mathrm{~Hz})\end{array}$ & $\begin{array}{c}10 \\
(410 \mathrm{~Hz})\end{array}$ \\
\hline $\begin{array}{c}1 \\
(21 \mathrm{~Hz})\end{array}$ & 0.801 & & & 0.621 & & & & 0.708 & 0.734 & \\
\hline $\begin{array}{c}2 \\
(53 \mathrm{~Hz})\end{array}$ & 0.548 & & & & & & & & 0.535 & \\
\hline $\begin{array}{c}3 \\
\left(93^{\mathrm{Hz}}\right)\end{array}$ & & 0.763 & 0.857 & • & & & & & & \\
\hline $\begin{array}{c}4 \\
(124 \mathrm{~Hz})\end{array}$ & & & & & & & & & & 0.465 \\
\hline $\begin{array}{c}5 \\
(135 \mathrm{~Hz})\end{array}$ & & & & & & & & & & \\
\hline $\begin{array}{c}6 \\
(209 \mathrm{~Hz})\end{array}$ & & & & & 0.774 & & 0.728 & & & \\
\hline $\begin{array}{c}7 \\
(251 \mathrm{~Hz})\end{array}$ & & 0.628 & 0.629 & & 0.666 & & 0.591 & & & \\
\hline $\begin{array}{c}8 \\
(387 \mathrm{~Hz})\end{array}$ & & & & & & & & & & 0.512 \\
\hline $\begin{array}{c}9 \\
(398 \mathrm{~Hz})\end{array}$ & & & & & & & & & & 0.356 \\
\hline $\begin{array}{c}10 \\
(502 \mathrm{~Hz})\end{array}$ & & & 0.608 & & 0.700 & & 0.583 & & & \\
\hline
\end{tabular}

Row Source: Position_24deg_48, Column Source: Acc_24deg_21ft2 


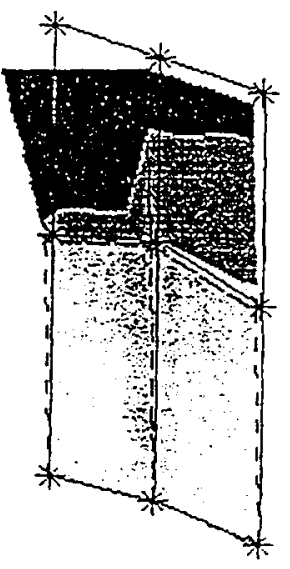

Freq: $38.19 \mathrm{~Hz}$ damping: $7.00 \%$ Peak 1

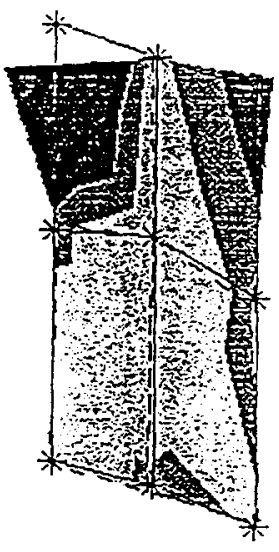

Freq: $162.57 \mathrm{~Hz}$ damping: $1.70 \%$ Peak 4

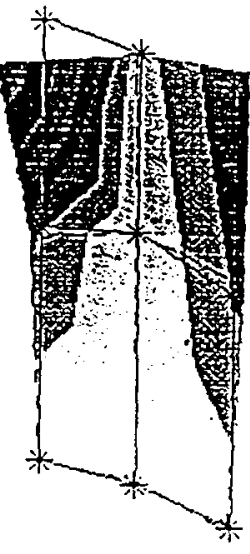

Freq: $65.11 \mathrm{~Hz}$ damping: $3.92 \%$ Peak 2

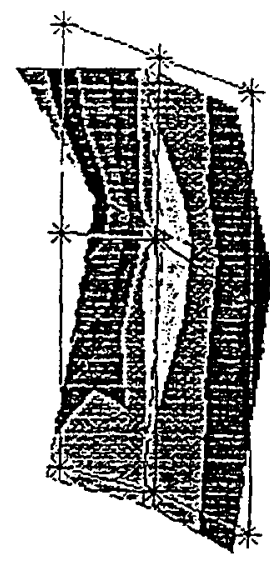

Freq: $219.81 \mathrm{~Hz}$ damping: $1.10 \%$ Peak 5

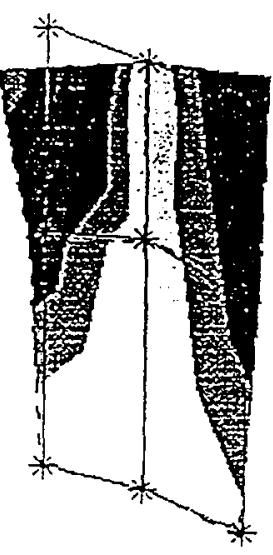

Freq: $74.17 \mathrm{~Hz}$ damping: $1.54 \%$

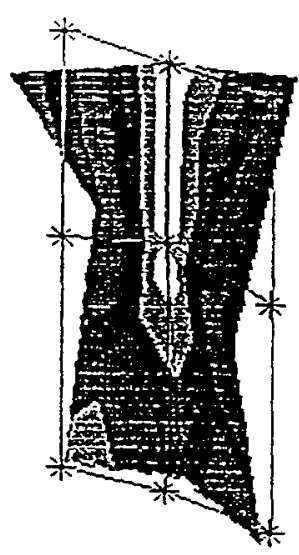

Freq: $237.82 \mathrm{~Hz}$ damping: $0.58 \%$ Peak 6

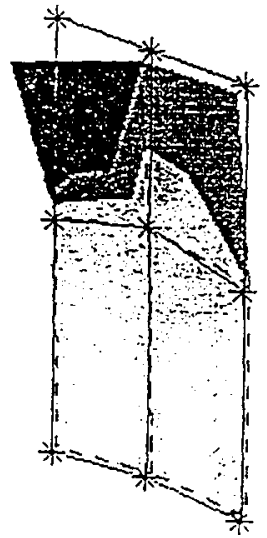

Freq: $110.75 \mathrm{~Hz}$ damping: $3.73 \%$ Peak 3

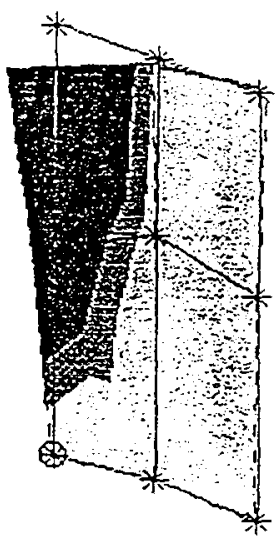

Freq: $250.59 \mathrm{~Hz}$ damping: $0.84 \%$ Peak 7

Figure 115. Natural mode shapes for 24-deg wet gate with 21-ft head difference (Continued)

Mode 9 shows the localized bending of the wicket above the water surface. In general, the sequential order of global dry modes - those not affected by the prop dynamics - remained identical for the wet wicket. Unlike the shaft-supported dry case, the bending modes, however, for the wet gate at 24 deg were well energized by the driving force.

A comparison of FRF for the prop-supported no-gap and three-gate gap flows indicates that the frequency response for the fully raised wet gate is almost independent of the gate configuration. Figure 116 compares the FRF and coherence plots for no-gap and three-gate gap flow configurations for the wet 


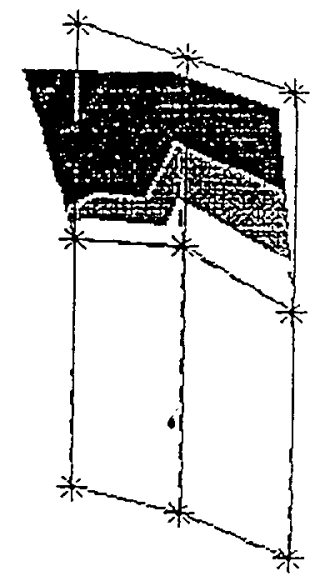

Freq: $303.96 \mathrm{~Hz}$ damping: $0.43 \%$ Peak 8

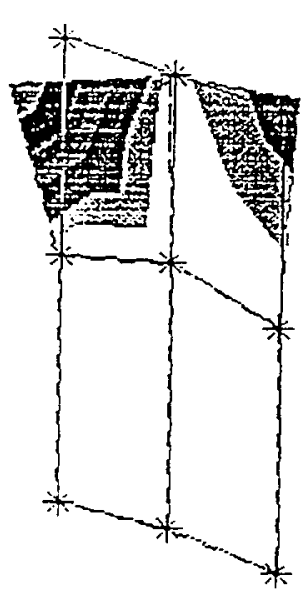

Freq: $329.80 \mathrm{~Hz}$ damping: $0.56 \%$ Peak 9

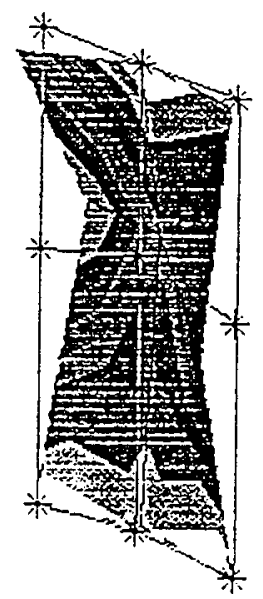

Freq: $410.17 \mathrm{~Hz}$ damping: $0.64 \%$ Peak 10

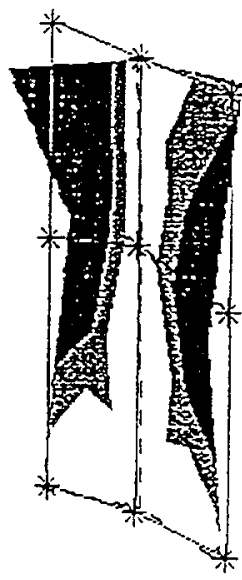

Freq: $449.05 \mathrm{~Hz}$ damping: $0.12 \%$ Peak 11

Figure 115. (Concluded)

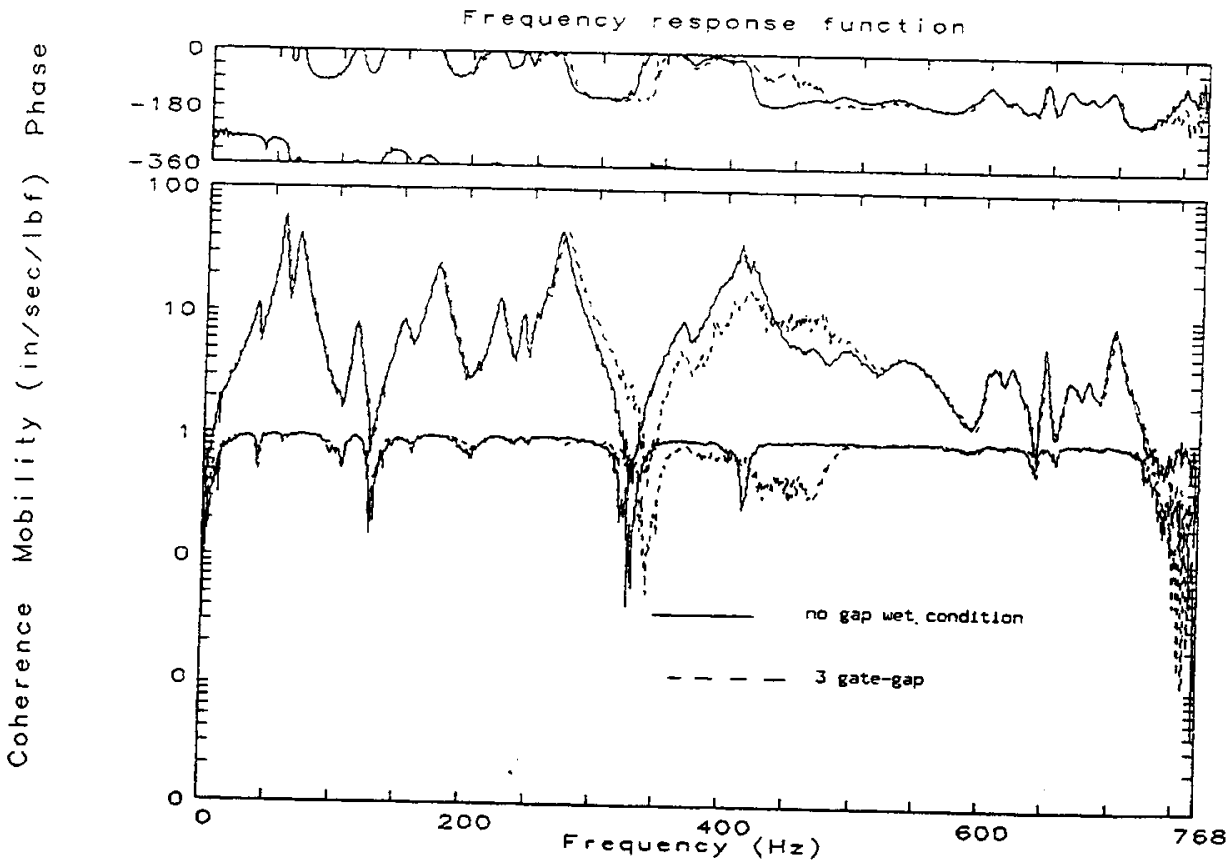

Figure 116. Comparison of FRF and coherence plots for no-gap and 3GG flow configurations for wet gate at $65 \mathrm{deg}$

gate at $65 \mathrm{deg}$. Good correlation between the MAC numbers of corresponding modes for both cases indicated their similarity and good linear correlation. This observation also suggests that the external disturbance of the flow during $3 \mathrm{GG}$ flow condition did not change the physical characteristics (modal behavior) of the wet gate. The primary factor that determines the modal behavior of a wet 
gate is the water level that comes in contact with the gate surface. Like the 65deg case, wet FRF responses at the 24-deg critical position for the $1 \mathrm{GG}$ and $3 \mathrm{GG}$ did not differ significantly from each other (Figure 117).

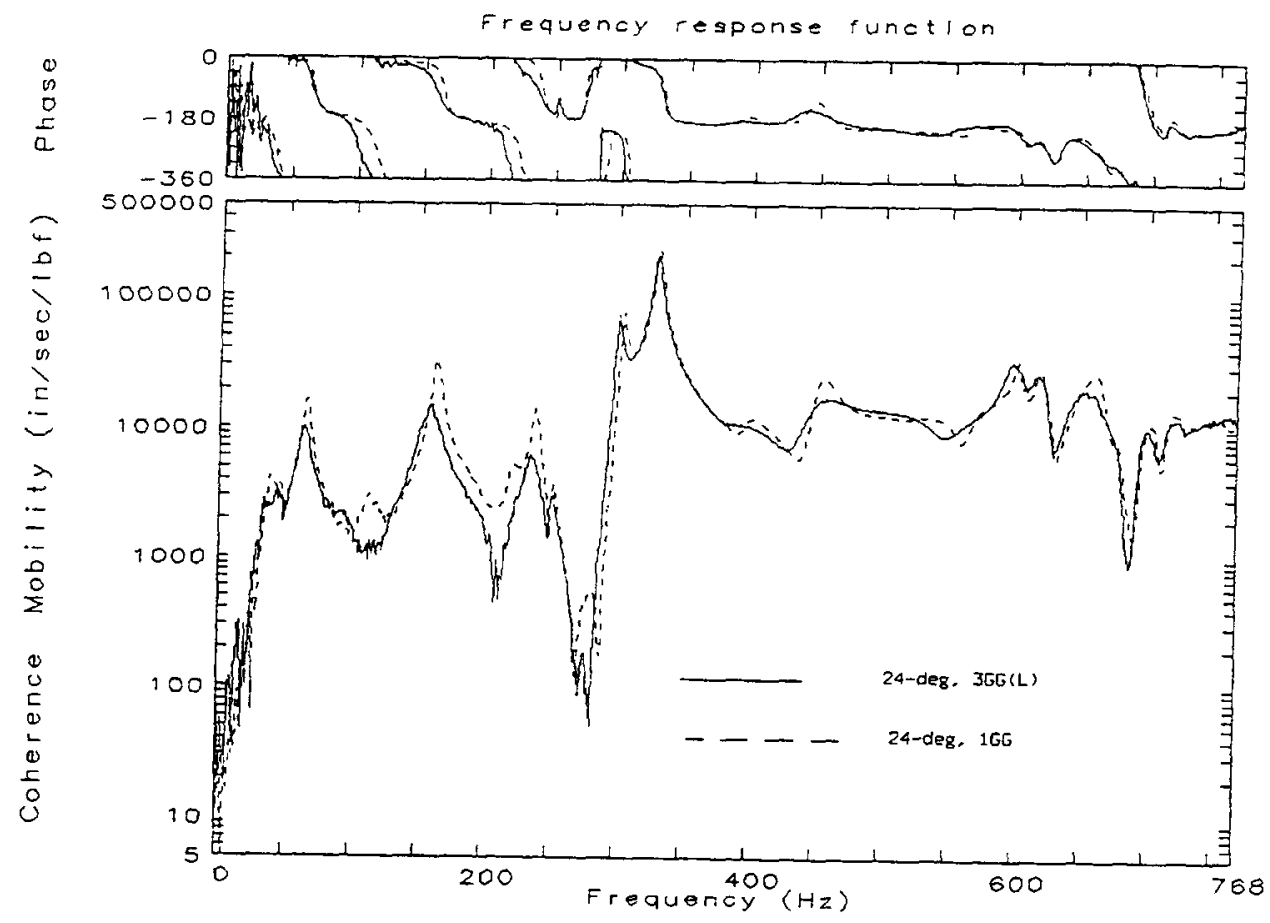

Figure 117. Mobility response function comparison of wet wicket at 24-deg critical position for 1GG and $3 G G$ configurations

A comparison of FRF for three pool levels for an identical three-gate gap flow condition is presented in Figure 118. As seen in the figure, the head differences caused the resonant frequencies to shift without drastically changing the overall pattern of the frequency responses. All three head differences at 7 , 11 , and $21 \mathrm{ft}$ generated similar modal patterns.

\section{Operating Deflected Shape}

The ODS for the model were analyzed to understand the actual response pattern of the gate during operation. ODS can be regarded as the modern "digital camera" equivalent of the traditional analog stroboscope. These shapes were animated using the IDEAS-TEST module by generating a frame of deflected shapes for each instance of time response. Nine accelerometer readings along the z-direction were used to generate the frame of accelerometer responses. The frames were animated and displayed sequentially as if the 


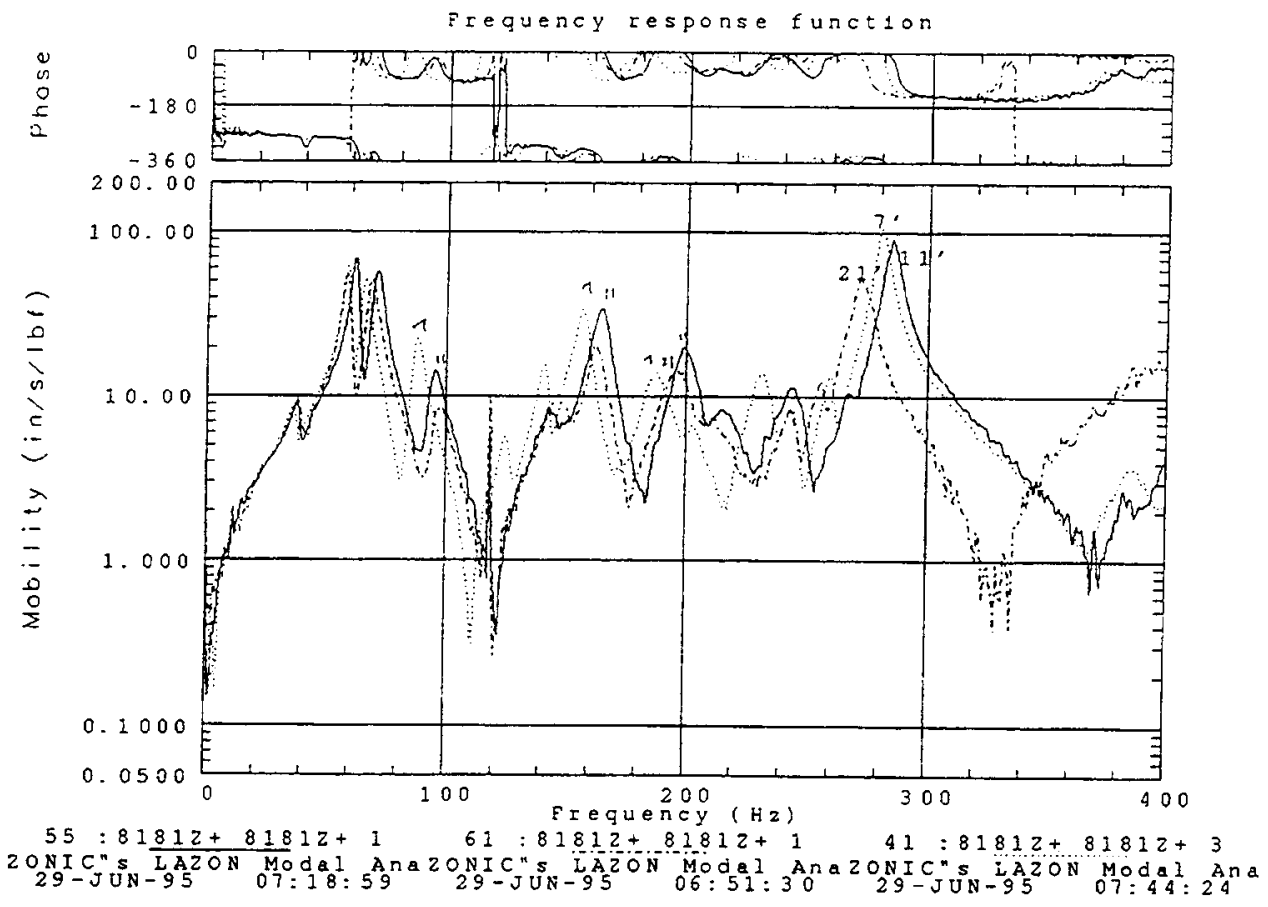

Figure 118. Mobility response functions for the wet wicket at 7-, 11-, and 21-ft head differences

structure were vibrating in real time. As shown in the previous section, a ninenode grid could adequately display the first-order bending and torsional modes of the wicket. Prior knowledge of modal patterns for higher modes could also be used to identify a few of the second-order modes as well.

The ODS for the gate in the frequency domain were examined to identify any resonant vibrations (potential trouble areas) due to flow-induced vibrations of the gate. Cross-spectra of the measured accelerometer responses for a fixed reference were considered to generate the transmissibility functions for the gate. A transmissibility function for each response accelerometer was obtained using the quasi FRF equation. Quasi FRF were calculated from the fixed reference point (j) and each response point (i) using the equation:

$$
\text { quasi } F R F_{i j}=\frac{G_{i j}(\omega)}{\sqrt{G_{i j}}(\omega)}
$$

where

$$
\begin{aligned}
& G_{i j}(w)=\text { cross-power spectrum between reference and response point } \\
& G_{i j}(w)=\text { auto-power spectrum of reference point }
\end{aligned}
$$

The transmissibility function has an amplitude of a response point itself and a phase angle that is normalized by the phase of the reference point. The operating 
shapes were identified by extracting the corresponding amplitude and phase data from each quasi frequency response function.

Two distinct effects of the flow, one due to added mass of the partially submerged gate and another due to the flow-induced forcing function, are required to detect and identify any resonant problem. This is due to the fact that the natural mode of vibration for the operating gate with added mass effect will shift from the corresponding dry modal frequencies. A resonance problem will exist if one of the operating modes of the gate coincides with one of the natural modes of vibration of the wet gate.

\section{Frequency-domain ODS at critical operation}

The ODS of the operating gate at three critical configurations are studied to determine the resonant problems in the gate operation. The flow-induced response measurements for a three-gate gap flow configuration with a $21-\mathrm{ft}$ head difference were used to construct the ODS for three critical configurations. These configurations include: (a) operating gate when lifted from down to up position (Experiment No. 173), (b) gate fixed at 65-deg position (Experiment No. 163), and (c) gate at 24-deg position (Experiment No. 187), which showed the most fluctuation in the measured responses.

The ODS for the operating gate and the transmissibility functions for each case are presented in Figures 119 through 121 in the frequency-domain. Peaks in this plot are the dominant frequency-dependent flow-induced operating deflections. Transmissibility functions for all response accelerations were consequently used to display the ODS in the frequency-domain. A scanning of the ODS for the operating gate, when raised fully from the down position, indicated that the gate vibrated with distinct operating shapes similar to the wet natural mode shapes identified previously in the experimental modal analysis. The first rigid-body translation ODS was found at a frequency of approximately $23 \mathrm{~Hz}$. As shown in the figure, a bending shape was noticeable at a frequency of $86 \mathrm{~Hz}$, torsion at 40 and $46 \mathrm{~Hz}$, and a second torsion at $96 \mathrm{~Hz}$. ODS at $243 \mathrm{~Hz}$ shows the water level effect on the gate's behavior. Though the amplification at the resonance point is marginal, a similarity in the wet mode shapes and the ODS suggests that the energy content of the random flow excites the natural wet modes of the gate. As seen in previous time-history plots (Figures 85 and 86) the fluctuation of the responses about the mean, however, is very low.

A correlation of selected ODS for the prop-supported fixed wicket and the operational gate is presented in Table 27. In this table, modal orders are based on the sequential numbering of peaks found in the transmissibility functions. In general, respective resonant frequencies for the raising and fixed gates differed from each other, indicating a difference in flow characteristics between them. As shown in Table 27, multiple correlations existed among the shapes compared. Operating shapes corresponding to the raising gate peaks at $46,86,150$, and 239 $\mathrm{Hz}$ agreed well with the shapes associated with the fixed gate peaks shown in the table. Similar peaks are printed in bold in the table. 


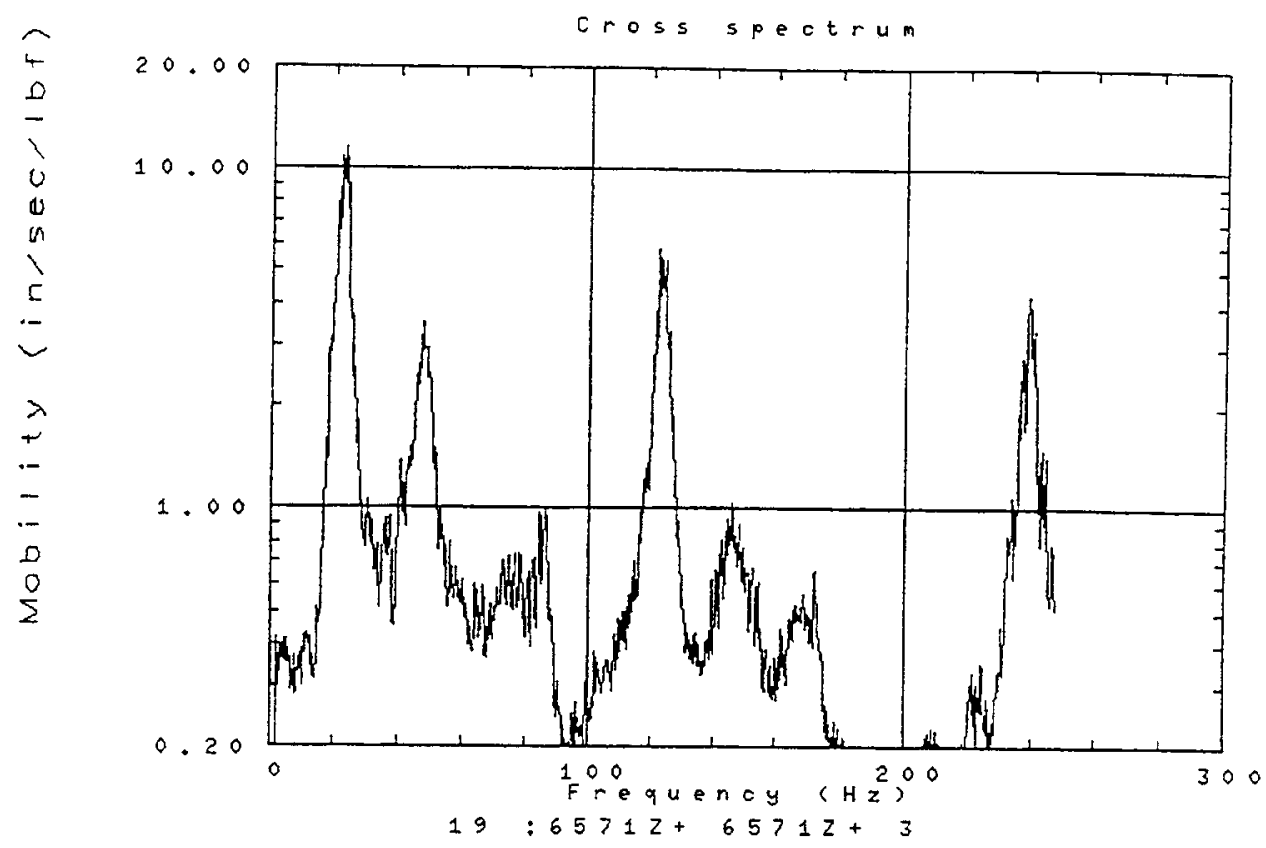

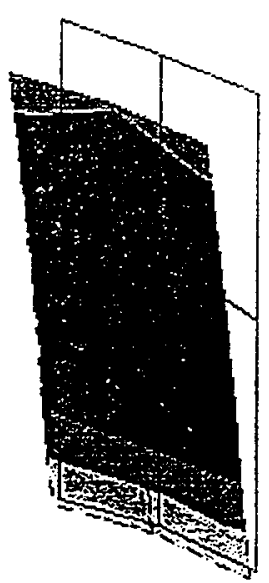

Freq: $22.72 \mathrm{~Hz}$ Peak 1

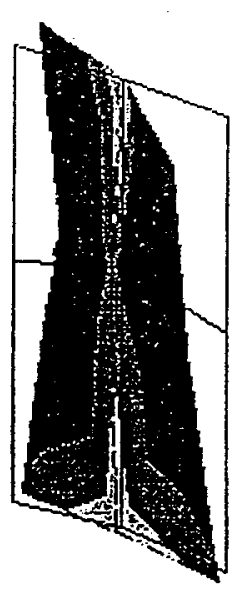

Freq: $40.56 \mathrm{~Hz}$ Peak 2

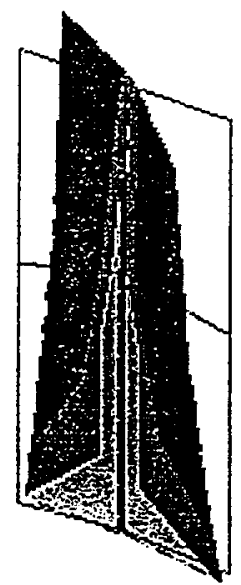

Freq: $46.91 \mathrm{~Hz}$ Peak 3

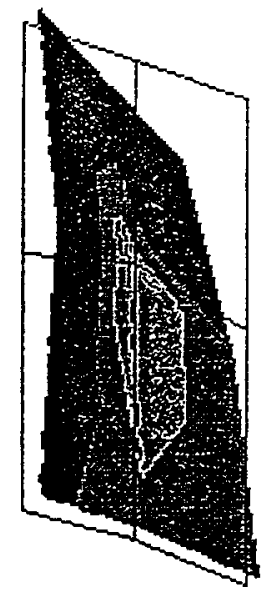

Freq: $70.12 \mathrm{~Hz}$ Peak 4

Figure 119. Transmissibility plot and operating shapes for raising gate during 21-ft head difference, 3GG flow configuration (Continued)

MAC numbers showing the ODS, corresponding to modal peaks, correlation for the fixed wicket at critical configuration ( $24 \mathrm{deg}$ ) and the operational gate are presented in Table 28 . In this table, modes that are considered fairly related to each other are in bold print. A good correlation between these values indicates that the ODS for the fixed gate agreed well with those of the raising gate. 


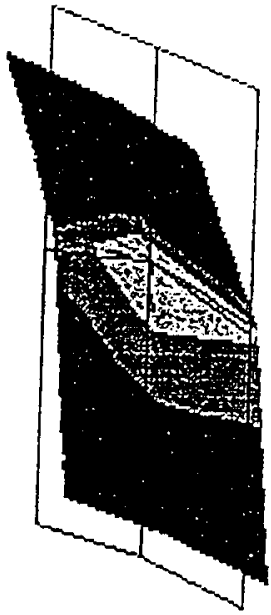

Freq: $86.25 \mathrm{~Hz}$ Peak 5

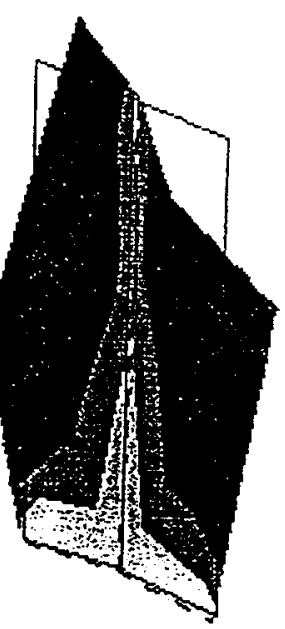

Freq: $172.25 \mathrm{~Hz}$ Peak 9

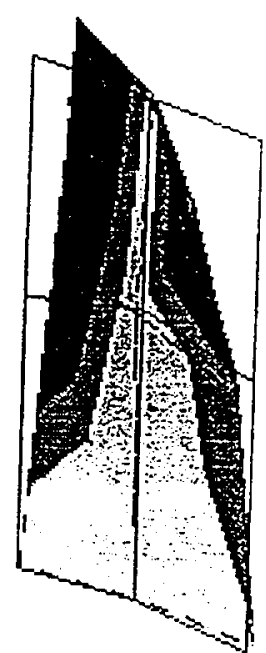

Freq: $96.51 \mathrm{~Hz}$ Peak 6

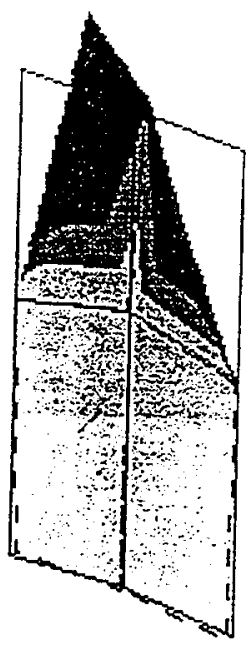

Freq: $239.44 \mathrm{~Hz}$ Peak 10

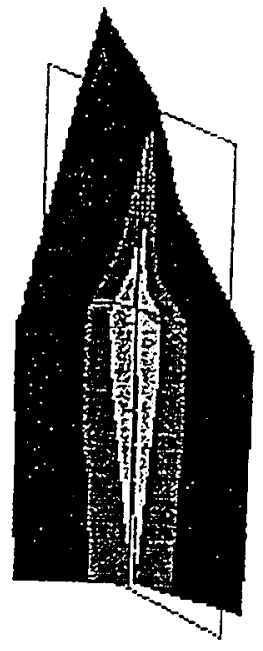

Freq: $122.65 \mathrm{~Hz}$ Peak 7

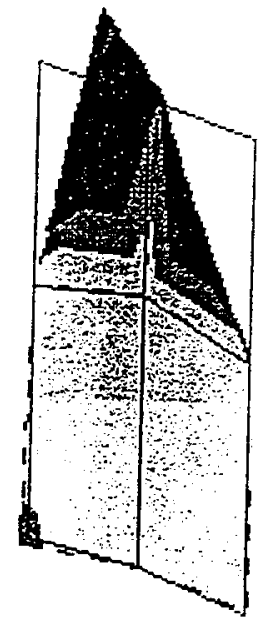

Freq: $243.59 \mathrm{~Hz}$

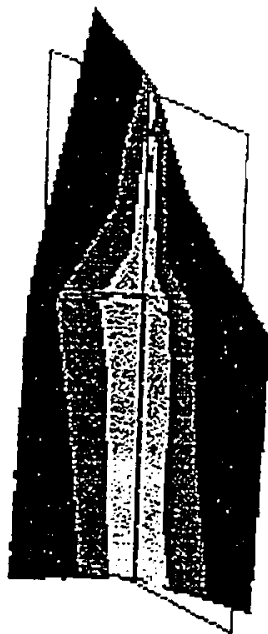

Freq: $150.01 \mathrm{~Hz}$ Peak 8

\section{Figure 119. (Concluded)}

Existence of identical frequencies with similar ODS for both cases also suggests that the major excitation of the wicket at the 24-deg position dominated the response over the entire range of operation.

MAC relation of operating shapes between the fixed gate at $24 \mathrm{deg}$ and 65 deg showed that shapes associated with corresponding peaks are not related to each other. Resonant peaks shifted from one to another, but the patterns appeared similar for both cases. 


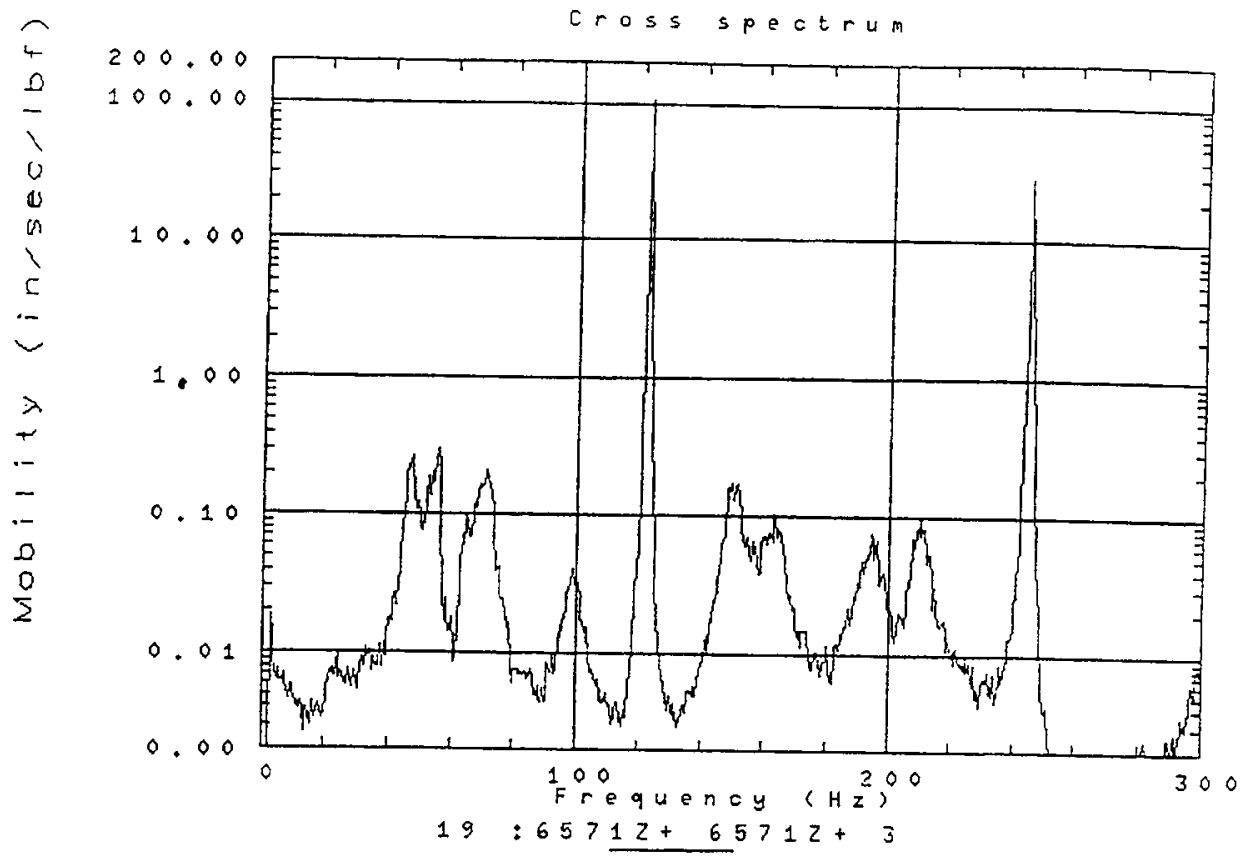

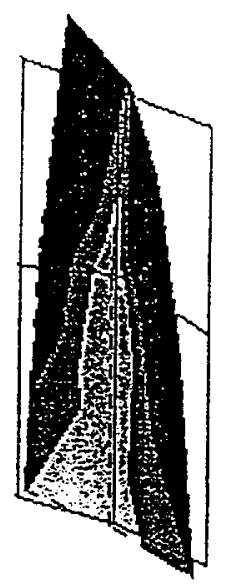

Freq: $46.91 \mathrm{~Hz}$ Peak 1

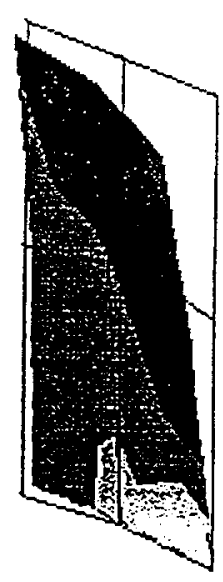

Freq: $55.71 \mathrm{~Hz}$ Peak 2

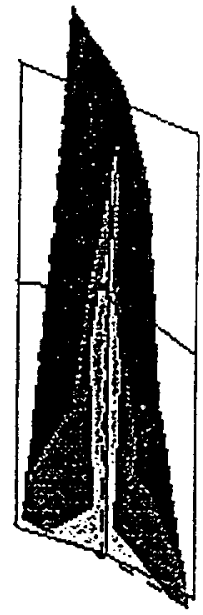

Freq: $71.34 \mathrm{~Hz}$ Peak 3

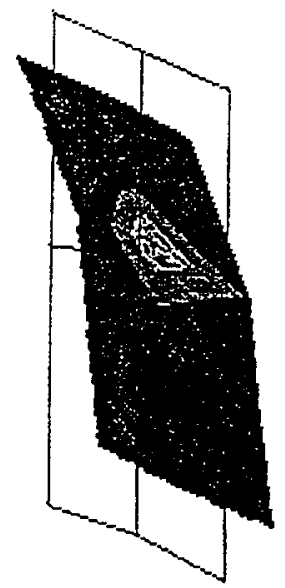

Freq: $99.20 \mathrm{~Hz}$ Peak 4

Figure 120. Transmissibility plot and operating shapes for prop-supported gate during 21-ft head difference, 3 GG flow configuration (Continued)

A comparison of the mode shapes and ODS for the prop-supported gate during a 21 -ft head pool condition with a three-gate gap flow configuration indicates that the transmissibility functions could adequately be used to predict the resonant frequencies and associated shapes of the wet wicket. Figure 113 shows the mode shapes of the wet wicket obtained using a shaker-excited laser 


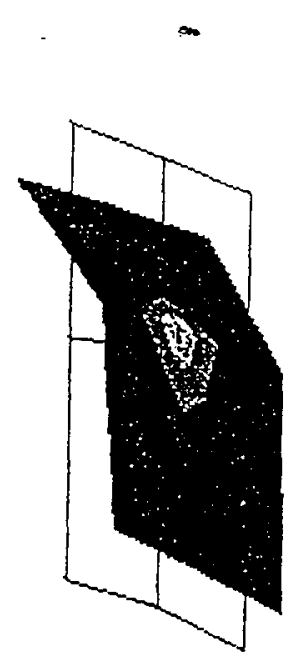

Freq: $122.16 \mathrm{~Hz}$ Peak 5

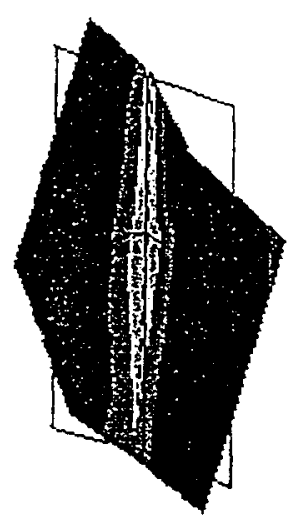

Freq: $211.58 \mathrm{~Hz}$ Peak 9

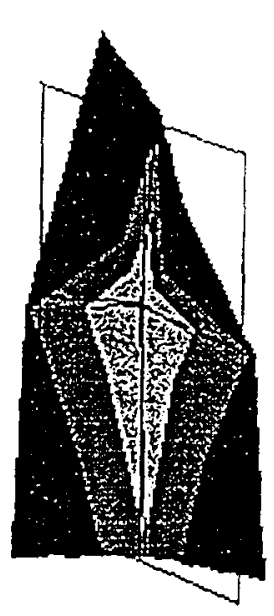

Freq: $151.00 \mathrm{~Hz}$ Peak 6

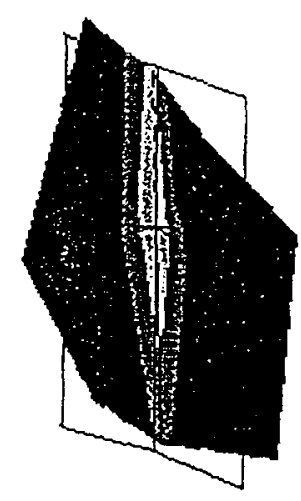

Freq: $244.32 \mathrm{~Hz}$ Peak 10

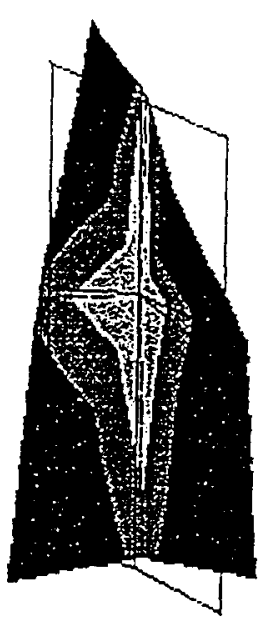

Freq: $163.21 \mathrm{~Hz}$ Peak 7

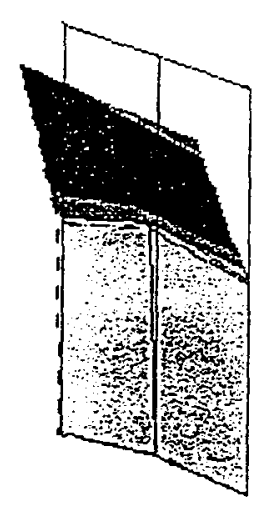

Freq: $304.43 \mathrm{~Hz}$ Peak 11

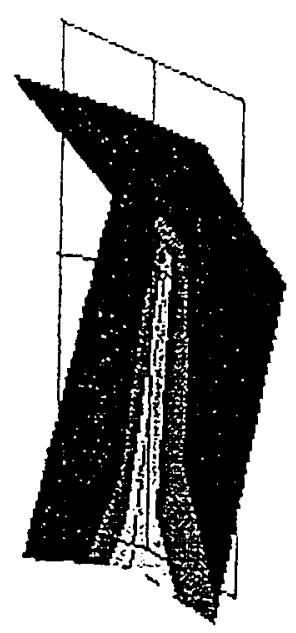

Freq: $195.95 \mathrm{~Hz}$ Peak 8

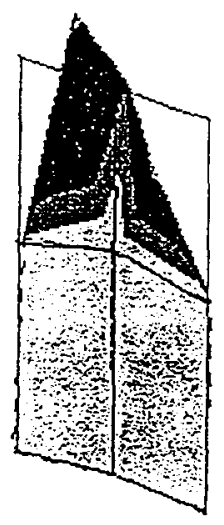

Freq: $334.24 \mathrm{~Hz}$ Peak 12

Figure 120. (Concluded)

scanning of response signals. Figure 120 shows the ODS for the gate due to flow-induced random vibration. Both experiments were conducted for an identical flow configuration with fixed-gate orientation. As discussed earlier, ODS was extracted using the built-in accelerometer responses of the gate due to ambient vibration of the flowing stream. A MAC relation, shown in Table 29, indicates a very good correlation among the respective shapes. In this table, modes that are considered fairly related to each other are in bold print. A similarity of the mode shapes with the ODS may exist due to resonance between natural frequencies and those produced by the flow with severe resulting effects, or this similarity may be due to excitation of the modes by the broad-band frequency signal of a weak flow. For the prop-supported case, the latter is true since the time-domain response signal does not show any indication of severe resulting response due to the resonant vibrations. The effectiveness of the ODS method suggests that for all practical purposes a good estimation of the modal parameters for an operating wicket could be developed from built-in response 


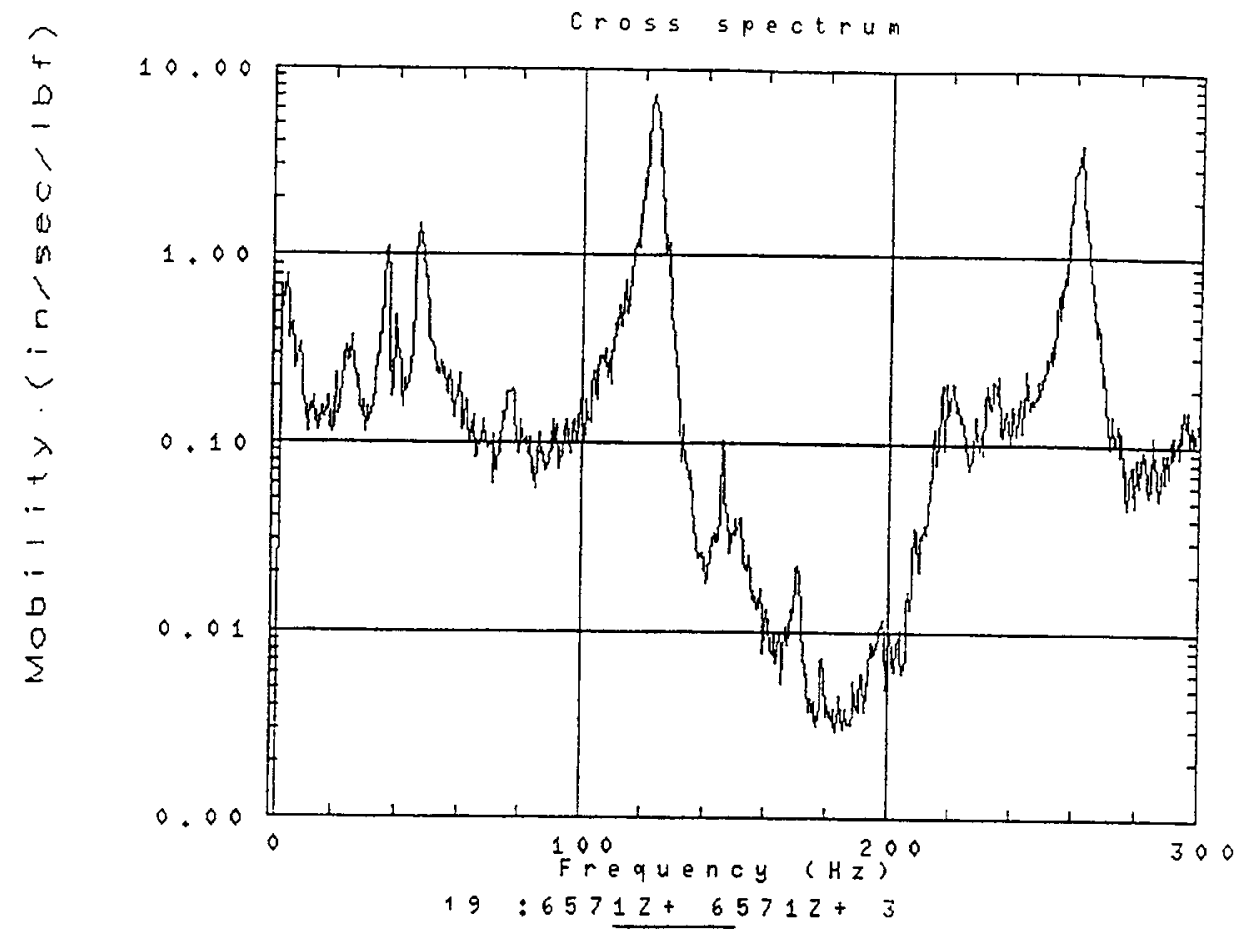

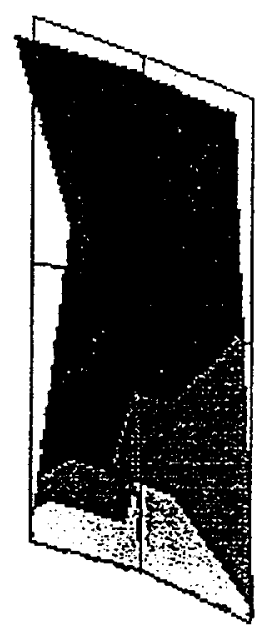

Freq: $25.41 \mathrm{~Hz}$ Peak 1

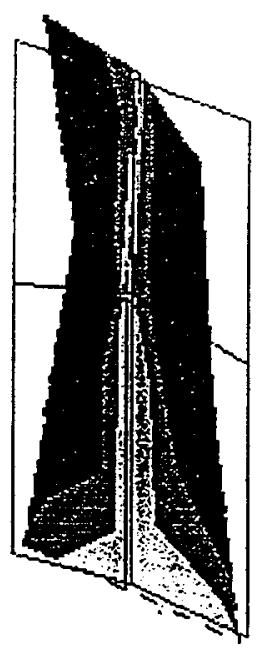

Freq: $36.16 \mathrm{~Hz}$ Peak 2

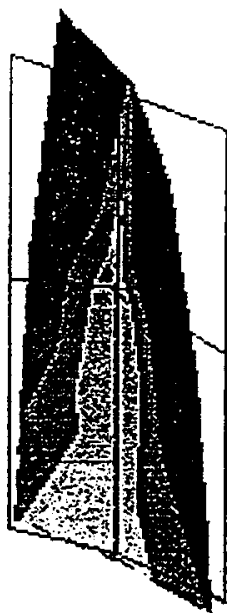

Freq: $46.91 \mathrm{~Hz}$ Peak 3

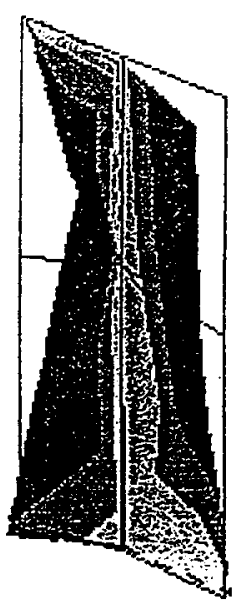

Freq: $76.72 \mathrm{~Hz}$ Peak 4

Figure 121. Transmissibility plot and operating shapes for fixed gate at 24-deg position during 21 -ft head difference, $3 G$ G flow configuration (Continued) 


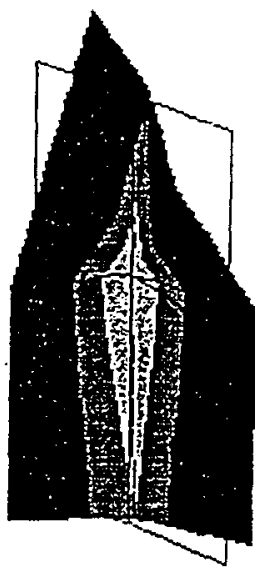

Freq: $123.14 \mathrm{~Hz}$ Peak 5

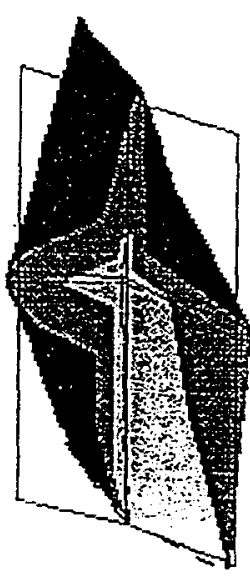

Freq: $218.43 \mathrm{~Hz}$ Peak 9

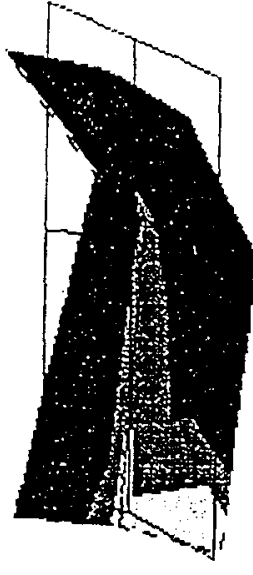

Freq: $145.62 \mathrm{~Hz}$ Peak 6

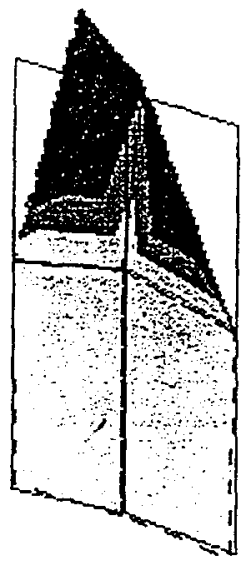

Freq: $261.43 \mathrm{~Hz}$ Peak 111

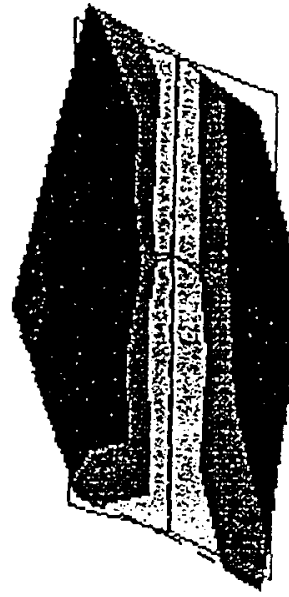

Freq: $170.05 \mathrm{~Hz}$ Peak 7

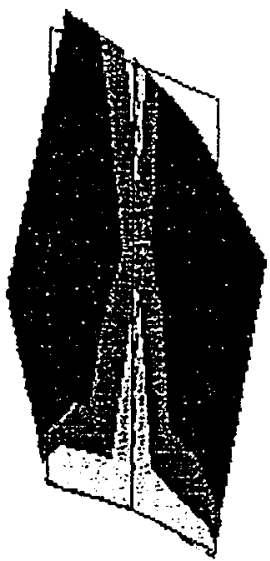

Freq: $333.26 \mathrm{~Hz}$ Peak 11

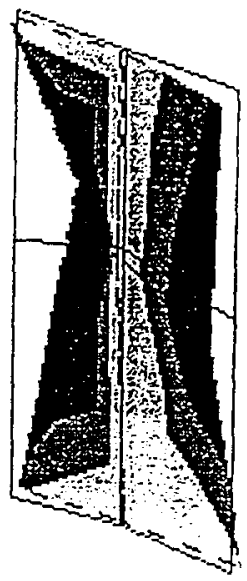

Freq: $178.85 \mathrm{~Hz}$ Peak 8

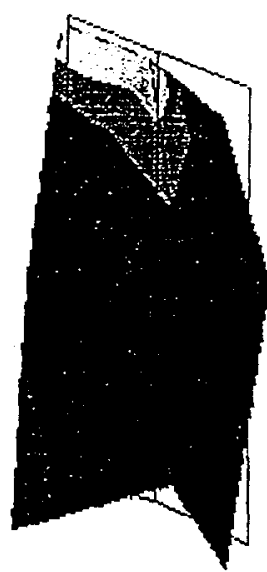

Freq: $413.40 \mathrm{~Hz}$ Peak 12

Figure 121. (Concluded)

measurements of the wet gate. This could be accomplished without conducting extensive modal experiment for known forced excitation, provided the random flow provides a reasonable energy distribution in the frequency range of interest.

For an identical gate configuration, resonant frequencies for the 24-deg wet gate differed from the peak frequencies obtained from the accelerometer signals due to flow-induced vibration. A MAC correlation of the natural wet mode shapes and the dominant forced operating shapes corresponding to the peaks in the transmissibility function is presented in Table 30. A poor correlation shown in the table suggests that operating shapes due to flow-induced motion differed from the natural characteristics of the wet gate. This is an indication of strong 


\begin{tabular}{|c|c|c|c|c|c|c|c|c|c|c|}
\hline $\begin{array}{l}\text { Table } 2 \\
\text { Correla } \\
\text { Gates }\end{array}$ & $\begin{array}{l}7 \\
\text { tion } M \\
\text { ?1-ft } H\end{array}$ & $\begin{array}{l}\text { atrix of } \\
\text { ead Dif }\end{array}$ & $\begin{array}{l}\text { Opera } \\
\text { fferenc }\end{array}$ & $\begin{array}{l}\text { ting } S \\
e, 3 G G \\
\end{array}$ & $\begin{array}{l}\text { napes } f \\
\text { Flow } \mathrm{C}\end{array}$ & $\begin{array}{l}\text { Prop- } \\
\text { onfigure }\end{array}$ & $\begin{array}{l}\text { Supporte } \\
\text { tion) }\end{array}$ & ed Fixed & and $\mathrm{Ra}$ & aising \\
\hline $\begin{array}{l}\text { ODS for } \\
\text { Fixed } \\
\text { Gate }\end{array}$ & & & & ODS & or Raisi & ng Wick & (Colun & nn) & & \\
\hline $\begin{array}{l}\text { Modal } \\
\text { Order } \\
\text { (Row) }\end{array}$ & $\begin{array}{c}(22 \mathrm{~Hz}) \\
1 \\
\end{array}$ & $\begin{array}{c}(40 \mathrm{~Hz}) \\
2\end{array}$ & $\begin{array}{c}(47 \mathrm{~Hz}) \\
3 \\
\end{array}$ & $\begin{array}{c}(70 \mathrm{~Hz}) \\
4\end{array}$ & $\begin{array}{c}(86 \mathrm{~Hz}) \\
5 \\
\end{array}$ & $\begin{array}{c}(96 \mathrm{~Hz}) \\
6 \\
\end{array}$ & $\begin{array}{c}(122 \mathrm{~Hz}) \\
7\end{array}$ & $\begin{array}{c}(150 \mathrm{~Hz}) \\
8 \\
\end{array}$ & $\begin{array}{c}(172 \mathrm{~Hz}) \\
9 \\
\end{array}$ & $\begin{array}{c}(239 \mathrm{~Hz}) \\
10\end{array}$ \\
\hline $\begin{array}{c}46 \mathrm{~Hz} \\
1\end{array}$ & & 0.960 & 0.980 & & & 0.801 & & & & \\
\hline $\begin{array}{c}55 \mathrm{~Hz} \\
2 \\
\end{array}$ & & & & 0.794 & & & & & & \\
\hline $\begin{array}{c}71 \mathrm{~Hz} \\
3\end{array}$ & & 0.858 & 0.977 & & & 0.805 & & & & \\
\hline $\begin{array}{c}99 \mathrm{~Hz} \\
4\end{array}$ & & & & & 0.779 & & & & & \\
\hline $\begin{array}{c}122 \mathrm{~Hz} \\
5 \\
\end{array}$ & & & & & 0.813 & & & & & \\
\hline $\begin{array}{c}151 \mathrm{~Hz} \\
6 \\
\end{array}$ & & & & & & & 0.953 & 0.925 & & \\
\hline $\begin{array}{c}163 \mathrm{~Hz} \\
7\end{array}$ & & & & & & & 0.873 & 0.924 & & \\
\hline $\begin{array}{c}195 \mathrm{~Hz} \\
8\end{array}$ & & & & & 0.253 & & & & & \\
\hline $\begin{array}{c}211 \mathrm{~Hz} \\
9 \\
\end{array}$ & & & & & & & & & 0.886 & \\
\hline $\begin{array}{c}244 \mathrm{~Hz} \\
10 \\
\end{array}$ & & & & & & & & & 0.594 & \\
\hline $\begin{array}{c}259 \mathrm{~Hz} \\
11\end{array}$ & & & & & 0.780 & & & & & 0.984 \\
\hline $\begin{array}{c}334 \mathrm{~Hz} \\
12 \\
\end{array}$ & & & & & & 0.813 & & & & 0.984 \\
\hline $\begin{array}{c}366 \mathrm{~Hz} \\
13 \\
\end{array}$ & & & & & & & & & 0.866 & \\
\hline $\begin{array}{c}389 \mathrm{~Hz} \\
14 \\
\end{array}$ & & 0.299 & & & & & & & & \\
\hline
\end{tabular}

Row Source: raw163, Column Source: raw 173

flow-induced forced motion at 24 deg which caused the gate to deflect in a shape different from the natural mode shapes. Energy content of the pressure field at wet resonant frequencies was not adequate to excite the gate to form natural shapes. As shown in the ODS for the wet gate in Figure 121, torsional shapes are more dominant during the flow at critical configuration.

Peak frequencies for the raising gate shown in Figure 119 differed from those of the resonant frequencies for the prop-supported and 24-deg wet gate. The peak frequencies for the raising gate represent the major frequencies of the gate motion which were excited during operation. Such a frequency distribution provides an overall frequency-domain behavior which was excited during gate operation. Frequency-domain operating shapes for the moving gate correlated better with the mode shapes of the prop-supported gate than those of the 24-deg wet gate. Table 31 shows the MAC numbers for the ODS of the lifting wicket and mode shapes for the prop-supported gate. 


\begin{tabular}{|c|c|c|c|c|c|c|c|c|c|c|c|c|}
\hline $\begin{array}{l}\text { Table } 28 \\
\text { Correlat } \\
\text { Gate (21 }\end{array}$ & $\begin{array}{l}n \mathrm{Ma} \\
\mathrm{Hea}\end{array}$ & $\begin{array}{l}\times 0 \\
\text { Diff } \\
\end{array}$ & $\begin{array}{l}\text { Ope } \\
\text { enc }\end{array}$ & $\begin{array}{l}\text { ing } S \\
3 G G\end{array}$ & $\begin{array}{l}\text { lapes } \\
\text { low } \\
\end{array}$ & nfi & $\begin{array}{l}\text { ed } \\
\text { rati }\end{array}$ & tte a & 24 & g anc & Rais & \\
\hline $\begin{array}{l}\text { ODS } \\
\text { for } \\
\text { Raising }\end{array}$ & & & & $S$ for & Fixed & Nicke & t at 2 & $4 \mathrm{deg}$ & (Col & umn) & & \\
\hline $\begin{array}{l}\text { Modal } \\
\text { Order } \\
\text { (row) }\end{array}$ & $\begin{array}{l}25 \\
\mathrm{~Hz} \\
1\end{array}$ & $\begin{array}{c}36 \\
\mathrm{~Hz} \\
2 \\
\end{array}$ & $\begin{array}{c}46 \mathrm{~Hz} \\
3\end{array}$ & $\begin{array}{c}76 \\
\mathrm{~Hz} \\
4\end{array}$ & $\begin{array}{c}123 \\
\mathrm{~Hz} \\
5\end{array}$ & $\begin{array}{c}145 \\
\mathrm{~Hz} \\
6 \\
\end{array}$ & $\begin{array}{c}170 \\
\mathrm{~Hz} \\
7 \\
\end{array}$ & $\begin{array}{c}178 \\
\mathrm{~Hz} \\
\mathbf{8} \\
\end{array}$ & $\begin{array}{c}218 \\
\mathrm{~Hz} \\
9 \\
\end{array}$ & $\begin{array}{l}261 \\
\mathrm{~Hz} \\
\mathbf{1 0}\end{array}$ & $\begin{array}{l}333 \\
\mathrm{~Hz} \\
11\end{array}$ & $\begin{array}{l}413 \\
\mathrm{~Hz} \\
12\end{array}$ \\
\hline $\begin{array}{c}22 \mathrm{~Hz} \\
1\end{array}$ & 0.166 & & & & & & & & & & & \\
\hline $\begin{array}{c}40 \mathrm{~Hz} \\
2\end{array}$ & & 0.94 & & & & & & & & & & \\
\hline $\begin{array}{c}47 \mathrm{~Hz} \\
3\end{array}$ & & 0.89 & 0.932 & & & & & & & & & \\
\hline $\begin{array}{c}70 \mathrm{~Hz} \\
4\end{array}$ & & & & 0.537 & & & & & & & & \\
\hline $\begin{array}{c}86 \mathrm{~Hz} \\
5\end{array}$ & 0.330 & & & & & & & & & & & \\
\hline $\begin{array}{c}96 \mathrm{~Hz} \\
6 \\
\end{array}$ & & & 0.860 & & & & & & & 0.801 & & \\
\hline $\begin{array}{c}122 \mathrm{~Hz} \\
7\end{array}$ & & & & & 0.99 & & & & & & & \\
\hline $\begin{array}{c}150 \mathrm{~Hz} \\
8 \\
\end{array}$ & & & & & 0.97 & & & & & & & \\
\hline $\begin{array}{c}172 \mathrm{~Hz} \\
9\end{array}$ & & & & & & & & & & & 0.864 & \\
\hline $\begin{array}{c}239 \mathrm{~Hz} \\
10\end{array}$ & & & & & & & & & & 0.993 & & \\
\hline
\end{tabular}

Row Source: raw173, Column Source: raw187 


\begin{tabular}{|c|c|c|c|c|c|c|c|c|c|c|c|c|}
\hline \multicolumn{13}{|c|}{$\begin{array}{l}\text { Table } 29 \\
\text { Correlation Matrix of Mode Shapes and ODS for Prop-Supported Gate (21-ft Head } \\
\text { Difference, 3GG Flow Configuration) }\end{array}$} \\
\hline ODS & \multicolumn{12}{|c|}{ Mode Shapes Using SLDV (Column) } \\
\hline $\begin{array}{l}\text { Modal } \\
\text { Order } \\
\text { (row) }\end{array}$ & $\begin{array}{c}38 \\
\mathrm{~Hz} \\
1\end{array}$ & $\begin{array}{c}58 \\
\mathrm{~Hz} \\
2 \\
\end{array}$ & $\begin{array}{c}68 \\
\mathrm{~Hz} \\
3\end{array}$ & $\begin{array}{c}96 \\
\mathrm{~Hz} \\
4\end{array}$ & $\begin{array}{c}115 \\
\mathrm{~Hz} \\
5\end{array}$ & $\begin{array}{c}148 \\
\mathrm{~Hz} \\
6\end{array}$ & $\begin{array}{c}164 \\
\mathrm{~Hz} \\
7\end{array}$ & $\begin{array}{c}198 \\
\mathrm{~Hz} \\
8\end{array}$ & $\begin{array}{c}215 \\
\mathrm{~Hz} \\
9\end{array}$ & $\begin{array}{c}246 \\
\mathrm{~Hz} \\
10\end{array}$ & $\begin{array}{c}255 \\
\mathrm{~Hz} \\
11\end{array}$ & $\begin{array}{c}274 \\
\mathrm{~Hz} \\
12\end{array}$ \\
\hline $\begin{array}{c}46 \mathrm{~Hz} \\
1\end{array}$ & 0.90 & & & & & & & & & & & \\
\hline $\begin{array}{c}55 \mathrm{~Hz} \\
2\end{array}$ & & 0.575 & & & & & & & & & & \\
\hline $\begin{array}{c}71 \mathrm{~Hz} \\
3\end{array}$ & 0.94 & & 0.956 & & & & & & & & & \\
\hline $\begin{array}{c}99 \mathrm{~Hz} \\
4\end{array}$ & & & & 0.965 & & & & 0.946 & & & & \\
\hline $\begin{array}{c}122 \mathrm{~Hz} \\
5\end{array}$ & & & & 0.975 & & & & 0.904 & & & & \\
\hline $\begin{array}{c}151 \mathrm{~Hz} \\
6\end{array}$ & & & & & & 0.957 & 0.964 & & & & & 0.917 \\
\hline $\begin{array}{c}163 \mathrm{~Hz} \\
7\end{array}$ & & & & & & 0.877 & 0.910 & & & & & 0.842 \\
\hline $\begin{array}{c}195 \mathrm{~Hz} \\
8\end{array}$ & & & & 0.921 & & & & 0.825 & & & & \\
\hline $\begin{array}{c}211 \mathrm{~Hz} \\
9 \\
\end{array}$ & & & & & & & & & 0.839 & 0.847 & & \\
\hline $\begin{array}{c}244 \mathrm{~Hz} \\
10\end{array}$ & & & & & & & & & & 0.702 & & \\
\hline $\begin{array}{c}304 \mathrm{~Hz} \\
11\end{array}$ & & 0.848 & & & & & & & & & 0.677 & \\
\hline $\begin{array}{c}334 \mathrm{~Hz} \\
12\end{array}$ & & & & & & 0.952 & 0.913 & & & & & 0.939 \\
\hline
\end{tabular}

Row Source: raw163, Column Source: laser_21ft_2g_dn 


\begin{tabular}{|c|c|c|c|c|c|c|c|c|c|c|}
\hline \multicolumn{11}{|c|}{$\begin{array}{l}\text { Table } 30 \\
\text { Correlation Matrix of Mode Shapes and ODS During Critical Flow Configuration at } \\
\text { 24-deg Gate Position (21-ft Head Difference, 3GG Flow Configuration) }\end{array}$} \\
\hline ODS & \multicolumn{10}{|c|}{ Mode Shapes Using SLDV (Column) } \\
\hline $\begin{array}{l}\text { Modal } \\
\text { Order } \\
\text { (row) }\end{array}$ & $\begin{array}{l}38 \\
\mathrm{~Hz} \\
1\end{array}$ & $\begin{array}{l}65 \\
\mathrm{~Hz} \\
2\end{array}$ & $\begin{array}{l}110 \\
\mathrm{~Hz} \\
3\end{array}$ & $\begin{array}{l}162 \\
\mathrm{~Hz} \\
4\end{array}$ & $\begin{array}{l}219 \\
\mathrm{~Hz} \\
5\end{array}$ & $\begin{array}{l}237 \\
\mathrm{~Hz} \\
6 \\
\end{array}$ & $\begin{array}{l}250 \\
\mathrm{~Hz} \\
7\end{array}$ & \begin{tabular}{|l}
303 \\
$\mathrm{~Hz}$ \\
8 \\
\end{tabular} & $\begin{array}{l}329 \\
\mathrm{~Hz} \\
9\end{array}$ & $\begin{array}{l}410 \\
\mathrm{~Hz} \\
10\end{array}$ \\
\hline $\begin{array}{l}25 \mathrm{~Hz} \\
1\end{array}$ & & & & & 0.419 & & & & & 0.486 \\
\hline $\begin{array}{l}36 \mathrm{~Hz} \\
2\end{array}$ & & 0.405 & & & & & & & & \\
\hline $\begin{array}{l}46 \mathrm{~Hz} \\
3\end{array}$ & & 0.676 & & & & & & & & \\
\hline $\begin{array}{l}76 \mathrm{~Hz} \\
4\end{array}$ & & & & & & & & & & 0.526 \\
\hline $\begin{array}{l}123 \mathrm{~Hz} \\
5\end{array}$ & & & & & & 0.714 & & & & \\
\hline $\begin{array}{l}145 \mathrm{~Hz} \\
6\end{array}$ & & & & & 0.750 & & & & & \\
\hline $\begin{array}{l}170 \mathrm{~Hz} \\
7\end{array}$ & & & & & & 0.541 & & & & 0.687 \\
\hline $\begin{array}{l}178 \mathrm{~Hz} \\
8\end{array}$ & & & & & & & & & & 0.366 \\
\hline $\begin{array}{l}218 \mathrm{~Hz} \\
9\end{array}$ & & & & 0.561 & & & & & & \\
\hline $\begin{array}{l}261 \mathrm{~Hz} \\
10\end{array}$ & & & & 0.742 & & & & & 0.768 & \\
\hline
\end{tabular}

Row Source: raw187_wc_ren, Column Source: acc_24deg_21ft2 


\begin{tabular}{|c|c|c|c|c|c|c|c|c|c|c|c|}
\hline \multicolumn{12}{|c|}{$\begin{array}{l}\text { Table } 31 \\
\text { Correlation Matrix of Mode Shapes for Prop-Supported Gate and ODS fol } \\
\text { Lifting Wicket (21-ft Head Difference, 3GG Flow Configuration) }\end{array}$} \\
\hline ODS & \multicolumn{11}{|c|}{ Mode Shapes Using SLDV (Column) } \\
\hline $\begin{array}{l}\text { Modal } \\
\text { Order } \\
\text { (row) }\end{array}$ & $\begin{array}{c}38 \\
\mathrm{~Hz} \\
1\end{array}$ & $\begin{array}{c}58 \\
\mathrm{~Hz} \\
2\end{array}$ & $\begin{array}{c}68 \\
\mathrm{~Hz} \\
3\end{array}$ & $\begin{array}{c}96 \\
\mathrm{~Hz} \\
4\end{array}$ & $\begin{array}{c}115 \\
\mathrm{~Hz} \\
5\end{array}$ & $\begin{array}{c}148 \\
\mathrm{~Hz} \\
6\end{array}$ & $\begin{array}{c}164 \\
\mathrm{~Hz} \\
7\end{array}$ & $\begin{array}{c}198 \\
\mathrm{~Hz} \\
8\end{array}$ & $\begin{array}{c}215 \\
\mathrm{~Hz} \\
9\end{array}$ & $\begin{array}{l}246 \\
\mathrm{~Hz} \\
10\end{array}$ & $\begin{array}{c}255 \\
\mathrm{~Hz} \\
11\end{array}$ \\
\hline $\begin{array}{c}22 \mathrm{~Hz} \\
1\end{array}$ & & 0.921 & & & & & & & & & \\
\hline $\begin{array}{c}40 \mathrm{~Hz} \\
2\end{array}$ & 0.902 & & 0.911 & & & & & & & & \\
\hline $\begin{array}{c}46 \mathrm{~Hz} \\
3\end{array}$ & 0.954 & & 0.962 & & & & & & & & \\
\hline $\begin{array}{c}70 \mathrm{~Hz} \\
4 \\
\end{array}$ & & & 0.530 & & & & & 0.520 & & & \\
\hline $\begin{array}{c}86 \mathrm{~Hz} \\
5\end{array}$ & & & & 0.785 & & & & 0.840 & & & \\
\hline $\begin{array}{c}96 \mathrm{~Hz} \\
6\end{array}$ & 0.790 & & 0.855 & & & 0.736 & & & & & \\
\hline $\begin{array}{c}122 \mathrm{~Hz} \\
7\end{array}$ & & & & & & 0.872 & 0.936 & & & & \\
\hline $\begin{array}{c}150 \mathrm{~Hz} \\
8\end{array}$ & & & & & & & 0.895 & & & 0.838 & \\
\hline $\begin{array}{c}172 \mathrm{~Hz} \\
9\end{array}$ & & & & & & & & & & 0.867 & \\
\hline $\begin{array}{c}239 \mathrm{~Hz} \\
10\end{array}$ & & & & & & 0.962 & 0.894 & & & & \\
\hline
\end{tabular}

Row Source: raw173, Column Source: laser_21ft_2g_dn 


\section{Structural Response Evaluation}

\section{Basics of Structural Evaluation}

Dynamic response is a sensitive indication of the physical integrity of any structure and has been extensively used to evaluate, assess, and improve design practices. An effective evaluation of structural responses could measure the adequacy, effectiveness, and integrity of a structural system. Analytical model and/or experimental responses could simultaneously be used in support of such an evaluation scheme. Understanding of technical failures or mechanisms is the key to the design evaluation scheme which could greatly enhance the confidence in the design standards (Petroski 1993). Design improvement could also be made by correcting such an anticipated mechanisms/failure during the development phase of a study. Mitigation of vibration problems in the Olmsted curved wicket, for example, could not have been possible without realization of the vortex-induced self-excited vibration during the physical model studies (Elder 1992).

For the Olmsted wicket, the measured responses and analytical models are used to evaluate the design standards by analyzing possible mechanisms. Many possible failure patterns could seriously affect the performance of the nonredundant prop or hydraulically lifted wicket system. Most obvious among them are the failure mechanisms for the prop support and the instability of the wicket gate. An inadvertently designed inadequate prop rod could buckle due to overstress, sheared along the pin connections, or could severely vibrate due to resonant problem. Fatigue stress of the highly sensitive parts (bottom hinges and the vicinity of the ball-mounting plate) could pose a threat to the reliability of the overall system. Dynamic overstressing or resonance of the structural components could well be regarded as dangerous possibilities that could exist during the flow-induced vibration.

In this part of the report, in addition to the determination of any existence of possible critical mechanisms mentioned above, the measured dynamic responses were evaluated for the wicket to measure the adequacy of the design parameters, to identify the effectiveness of the structural system, and determine the integrity and reliability of the Olmsted wicket system. Design improvements were made to mitigate anticipated problems during the operation of the wickets. A few of such mitigations include the change of gate geometry, change of prop-support 
mechanisms to strengthen its shearing capacity, and reconfiguration of the hurter assembly.

Self-excited vibration due to vortex shedding is one of the major problems that could exist in an operating wicket. During such a transitional phase, a rampant fluctuation about the mean value is expected. A major fluctuation of the measured responses could indicate the existence of such a serious dynamic problem. From the response measurement of the Olmsted wicket during critical configuration such type of excitation is insignificant. Table 18 in the previous chapter presents the maximum fluctuation of the resulting responses about the respective RMS magnitudes. As discussed earlier, the coefficients of variation ( $\operatorname{COV}=\frac{\sigma}{\mu}$, where $\sigma$ is the standard deviation and $\mu$ is the mean of the random variable. COV is a measure of dispersion and is less sensitive to the mean than $\sigma)$ for the hinges were relatively higher than those of the remaining responses because of the inadequate sensitivity of the hinge sensors in the measured ranges. Much smaller measured hinge responses provided less restraint against vibration, thus causing a relatively higher fluctuation of the hinge reactions. Also, the sensitivity of the hinge sensors was reduced because the measured force ranges were far below the calibrated magnitudes.

Resonance of a vibrating system could seriously threaten the survivability of a vibrating structure. Resonance problem for the flow-induced vibrating gate was identified by comparing the ODS of the operating gate with the wet gate natural characteristics of the wicket. ODS represented the actual dynamics of the system during the critical flow configuration, and the wet gate modal parameters characterized the natural behavior of the gate at that particular environment due to forced vibration. As shown in the previous chapter, wet gate natural mode shapes and the corresponding ODS well agree with each other for the prop-supported gate. For a moderate strength pressure field at the 65-deg position, the random energy content of the flow was sufficient to excite the natural modes of the wet gate. Interestingly, however, wet gate natural modes at critical configuration did not match with the ODS of the wicket at the 24-deg position. A difference in the natural shapes for the wet gate from the flowinduced operating shapes at critical configuration is a sign of relief from potential danger of dynamic resonant problem. Considering the existence of an resonant dynamic behavior at the 65-deg position, additional evaluation of the dynamic responses was needed to determine the consequences of such resonance in the overall performance of the wicket.

A random nature of the flow-field, with a broad-band frequency response, excites the wet natural modes during the flow. As shown in the pressure field PSD (see pressure field PSD in previous chapter), the energy level continued to be present in the frequency ranges containing the wet modes of vibration. An energy field at the resonant frequency was quite adequate in exciting the modes of the wet wicket.

Since the nature of the flow field could not be overturned to stop the resonant excitation of the prop-supported gate, there must be adequate margins of safety 
against the overstressing of the wicket from the resulting flow-induced pressure fields. Fatigue due to cyclic loading must be checked so that the fatigue stress due to the anticipated most likely applied stress could not exceed the allowable limits.

\section{Strength and Fatigue}

\section{Flow-induced gate strain}

Flow-induced strains for the operating wicket gate were measured using the strain transducers manufactured by the Bridge Diagnostics, Inc., Boulder, CO. Four transducers, each had a 3-in. gauge length, were mounted on the gate at about 16 in. up from the base of the gate using c-clamps at the highest stress point on the gate. Two transducers were mounted on the top skin plate on the upstream side, and the others were mounted on the bottom flanges on the downstream side of the gate (Figure 122). For a 21-ft head difference, three-gate gap configurations, the resulting strain on the gate during the raising of the gate from a 12.1- to 65-deg position is shown in Figure 123. A sampling frequency of $100 \mathrm{~Hz}$ was used to digitize the strains. All strains correspond to the axial strain along the longitudinal axis of the gate. In this plot, the angular position of the gate corresponding to the raise time are also plotted. As shown in the plot, strain data after $189.1 \mathrm{sec}$ represent the strain on the prop-supported gate. A positive magnitude of the data corresponds to the tensile strain on the gate and a negative for compression.

From the measured responses, an absolute maximum stress level of 11,250 psi in the prototype could be estimated for the most critical flow configuration. In a 100 -year life cycle, the gate operating sequences is very unlikely to exceed 2,000 cycles. Such a reasonably low cycle of gate operation and a low anticipated strain level does not pose a fatigue threat for the structural components unless environmental effects have harshly deteriorated the mechanical performance of the gate material. Moreover, the maximum strain level occurs only during the transitional phase of operation and lasts for a short duration as shown in the figure. For the intended purpose, a prop-supported fixed gate ( $65 \mathrm{deg}$ position) is the most active position during the course of operation. The strain level on the gate reduces as the wicket is raised and becomes stable for the prop-supported gate.

\section{Prop rod}

Mechanical strength of the prop rod were investigated to determine its buckling capacity, shearing strength of the connecting pins, and overall load carrying capacity. The allowable load capacity for the 10 -in.-diam steel rod was computed using the American Institute of Steel Construction (AISC) allowable stress formulation (AISC 1989). The analytically computed buckling load capacity of 1,123 kips much exceeded the predicted maximum prototype load of 150 kips. 


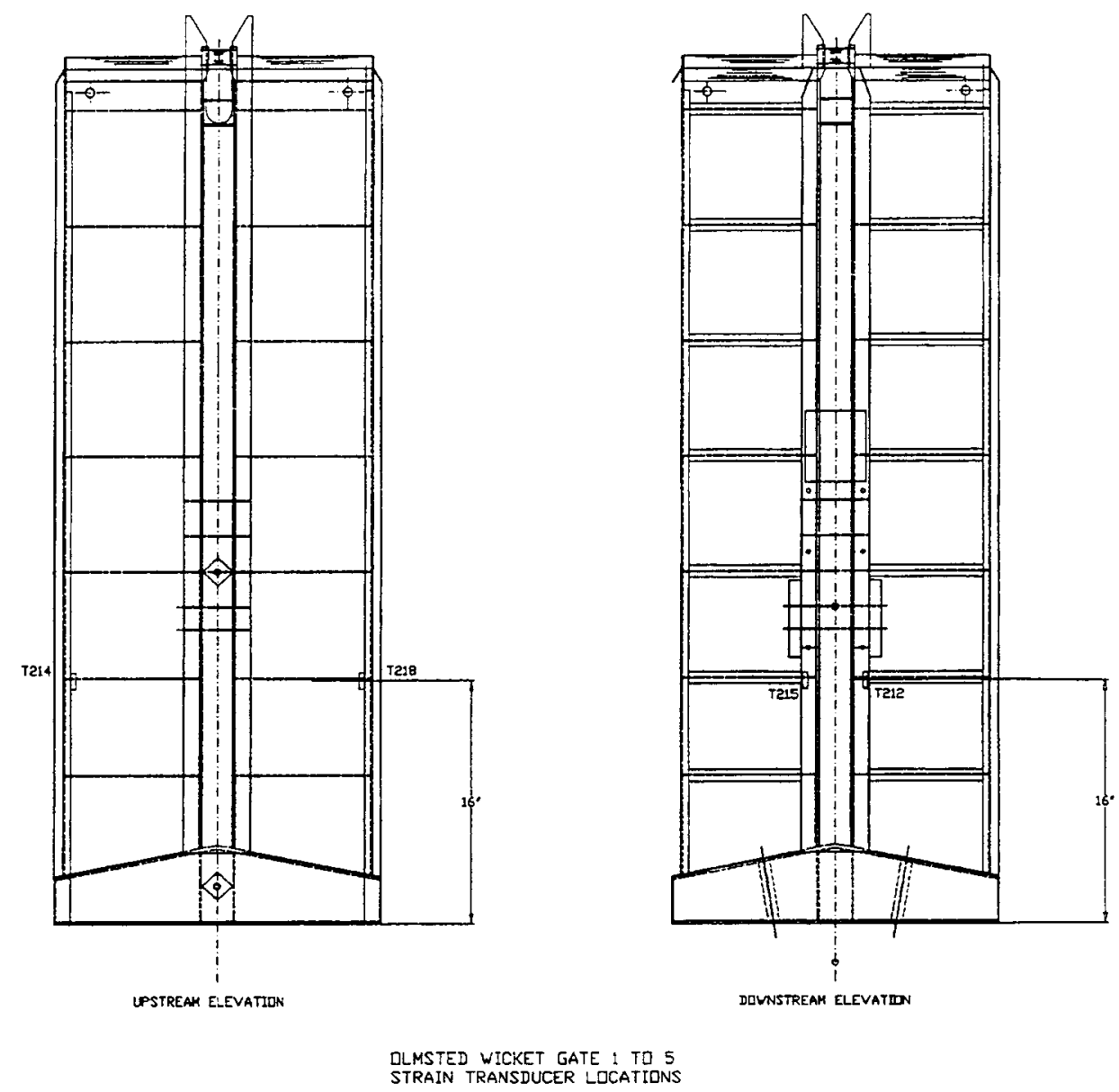

Figure 122. Strain transducer locations

The under-designed shear pin used to connect the prop-support blade with the prop rod sheared during the operation of the 2-GG flow configuration (Appendix A). It was found that the double shear, 1.25-in.-diam pin had a shear capacity of 78.5 kips which was below the actual prop load applied during the critical flow configuration. Revised double pins, each with a diameter of 2 in., provided a shear capacity of 201 kips. This new pin provided adequate strength to carry the implied loads during the course of the flow experiments.

\section{Base hinge assembly}

The shear pins and the connecting devices through which the loads are transferred from the gate to the bases are the most susceptible components in the wicket assembly. These parts may be subjected to fatigue failure due to reversed loading. A fatigue analysis of the pins and the base hinges (Figure 124) are performed to determine their usefulness against possible fatigue failure. The modified Goodman line method are considered for computing the acceptability of a component subject to a combination of static and reversed dynamic loading (Lindeburg 1986). In this method, criteria for failure are established by relating 


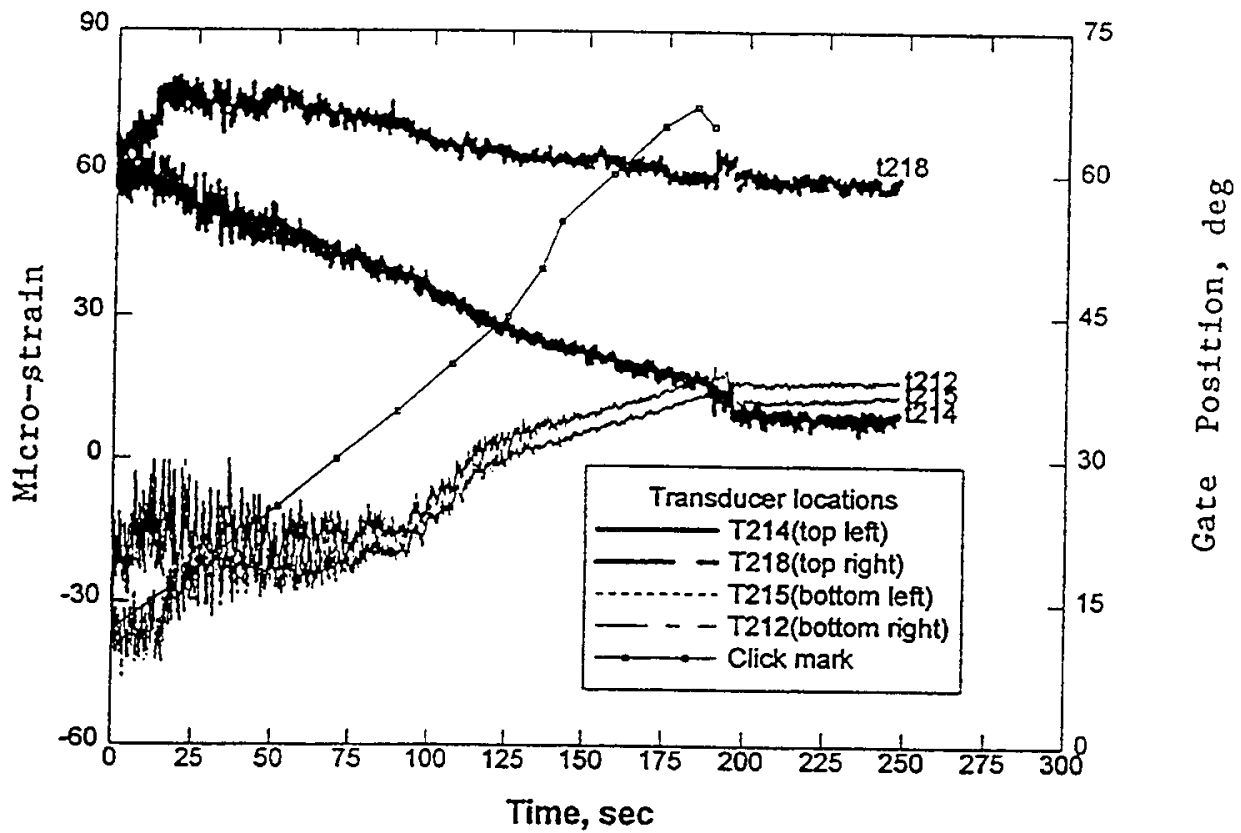

Figure 123. Flow-induced strain response for the wicket gate furing a $21-\mathrm{ft}$ head difference 3GG flow test

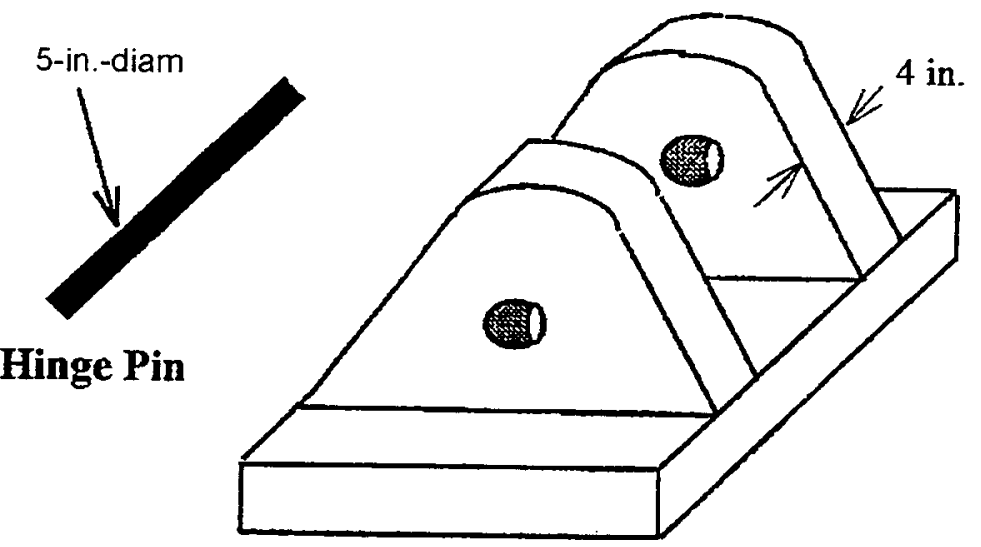

Figure 124. Gate frame hinge assembly

the tensile yield strength $\left(S_{y t}\right)$, the tensile ultimate strength $\left(S_{\mathrm{ut}}\right)$, and the endurance limit $\left(S_{e}\right)$. For steel, the endurance strength is approximately one-half of the ultimate tensile strength $\left(\mathrm{S}_{\mathrm{e}}=0.5 \mathrm{~S}_{\mathrm{u}}\right)$. Figure 125 illustrates the concepts of this method in identifying the safe operation area using the known material properties. In this figure, the equivalent normal stress $\left(\sigma_{e q}\right)$ is obtained by using the equation, $\sigma_{e q}=\sigma_{e}+\sigma_{m}\left(\frac{S_{e}}{S_{u t}}\right)$, where, $\sigma_{\mathrm{a}}$ and $\sigma_{\mathrm{m}}$ are the alternating and mean normal stress on the structure and are related to the maximum and minimum stress applied to the component. The mean and alternating stresses are obtained using, 


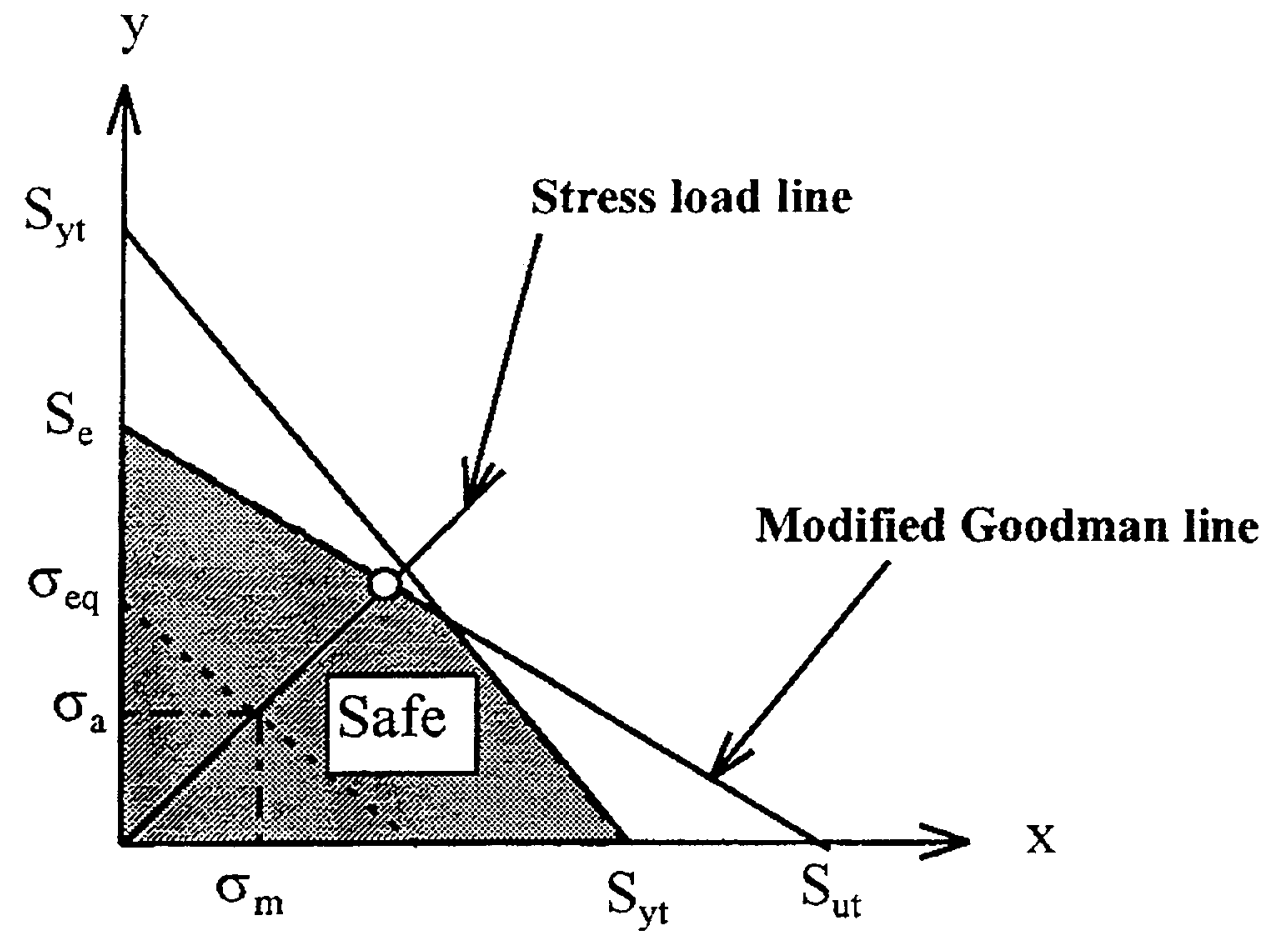

Figure 125. The envelope of fatigue failure using the Goodman line

$\sigma_{m}=\frac{\sigma_{\max }+\sigma_{\min }}{2}$, and $\sigma_{a}=\frac{\sigma_{\max }-\sigma_{\min }}{2}$

For a structure subjected to fluctuating shearing stress, the safe region could be indicated by the shaded area shown in Figure 125. The mean and alternating shearing stress could be obtained using similar equations as in the normal stress case presented above.

For the 5-in. nominal diameter hinge pin, the maximum shearing stresses were obtained from the experiments conducted for the $21-\mathrm{ft}$ head difference, three-gate gap configuration, with the gate being fixed at a 24-deg position (Experiment No. 187). The safe region for a part that has been stressed repeatedly in shear is shown in Figure 126. A minimum safety factor of 13.5 was obtained for the hinge pin for the most critical flow configuration. A safety factor is the ratio of the mean stress to the yield strength, or the alternating stress (shearing or normal) to the endurance strength of the material.

For the hinge base shown above, the critical normal loads were obtained from the same experiment as in the shear pin. The predicted loads were applied on a finite element (FE) model of the hinge base and the maximum and minimum Von-Mises stresses were used to determine the factor of safety against possible fatigue failure. Four-node linear tetrahedron solid elements were used to model the hinge bases as shown in Figure 127. The ABAQUS general-purpose FE code 


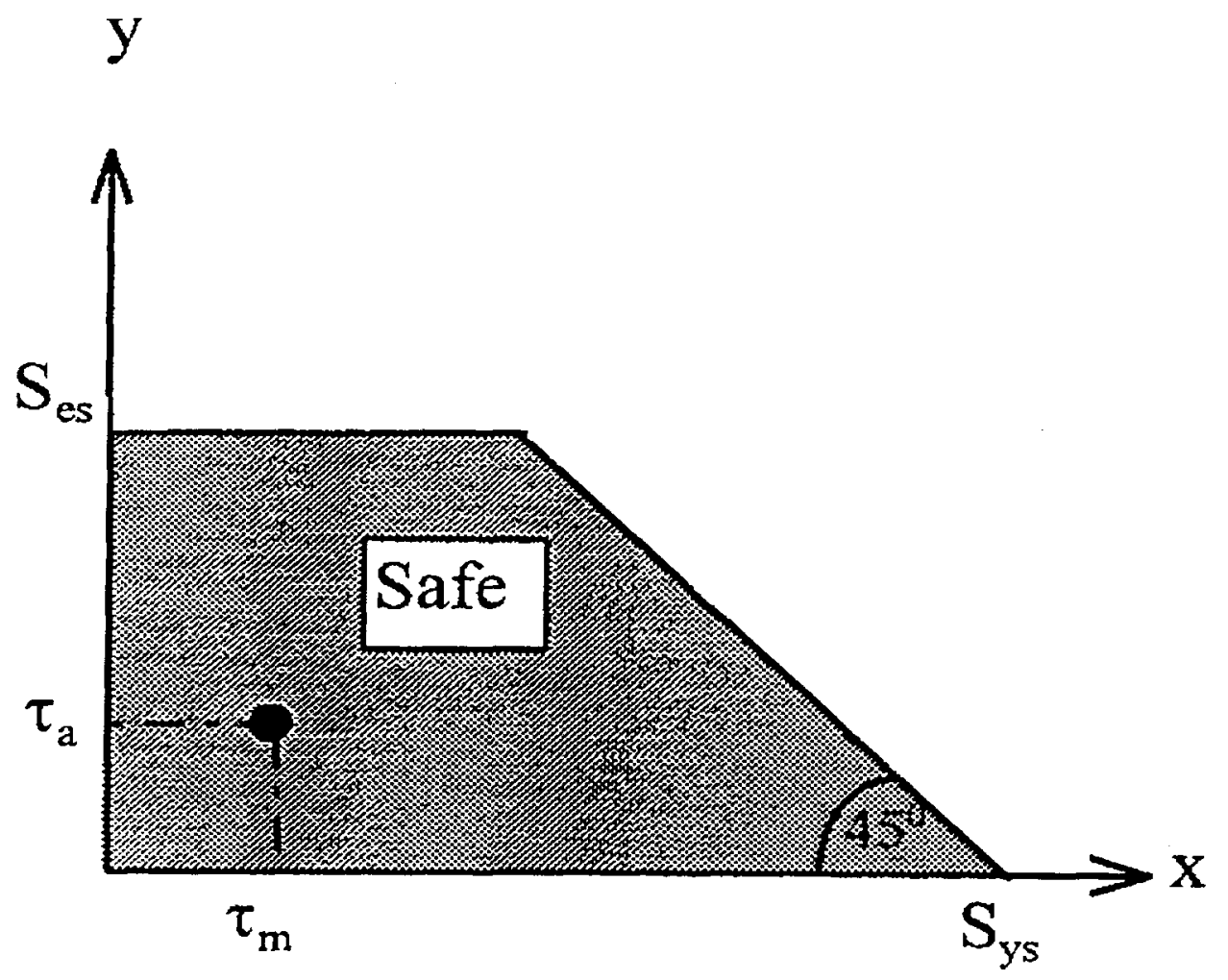

Figure 126. Safe region for fluctuating shear loads

was used to analyze the structure for the dynamic loads (reaction forces) applied along the two principal directions of the base. Hinge forces along the principal directions are obtained from the measured orthogonal hinge reactions shown in Figure 89. All base nodes were fully restrained to model the fixity of the hinges to the base plate. The maximum and minimum Von-Mises stress on the hinges were 8,000 psi and 5,000 psi, respectively. Applying the Goodman line method, a safety factor of 7.39 was obtained for the critical gate frame hinges.

Above computations also agree with the AISC requirements for the fatigue allowable stress for members subjected to reversed loading. Based on the AISC (1989) strength design consideration, the maximum allowable stress range for the wicket members subject to fatigue loading must not exceed $22 \mathrm{ksi}$. This allowable stress is higher than the applied mean stresses found for both cases presented above. The allowable fatigue stress range is estimated for the loading condition 1 (number of loading cycles in the ranges 20,000 to 100,000), and the type and location of member connections as in the hinge base. 

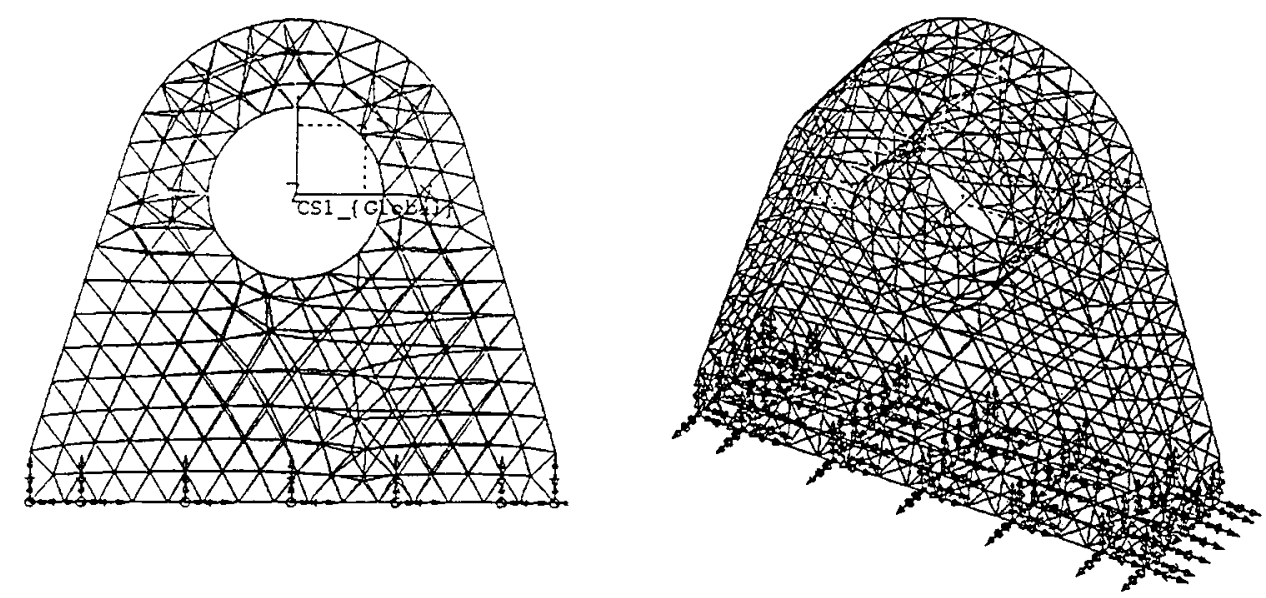

Figure 127. Finite element model for the base hinge 


\section{Physical Parameter Identification}

\section{Maximum Measured Responses}

The largest peak estimates using the Type - I Extreme Value Distribution of Maxima (EVD) are computed for the measured random responses. Test results for the 21-ft head difference pool condition with critical flow configuration were used to compute the "design" values at 99.99 percent level. At 99.99 percent confidence level, the design value estimates $(\hat{x})$ for a random variable $(\tilde{x})$ could be obtained by using the equation

$\hat{x}=\mu_{\tilde{x}}+6.728985\left(\sigma_{\tilde{x}}\right)$ where $\mu_{\tilde{x}}$ and $\sigma_{\tilde{x}}=$ mean and standard deviation

This equation was obtained by substituting the cumulative probability of 99.99 percent in the cumulative distribution function (cdf) for the Type - I, EVD method. The cdf for EVD is $F_{X}(\tilde{x})=e^{-e^{-\alpha(x-\beta)}}$ (Wirsching and Ortiz 1993)

where

$\alpha=\frac{1.283}{\sigma_{\tilde{x}}}$,and $\beta=\mu_{\tilde{x}}-\frac{0.577}{\alpha}$

The EVD is commonly used for modeling extreme environmental phenomena and is suitable for estimating design loads for the wicket components.

Table 32 presents the estimated design responses for the model and the prototype wicket. Model response estimates were based on the maximum of the corresponding experimental results presented in Table 18. Prototype estimates were predicted from the model responses using the Froude similitude relationships presented earlier. Note that in the table, the total trunnion force agrees well with the measured lifting cylinder force. Lifting cylinder force could be obtained by multiplying the cylinder bore area with the measured chamber pressure (oil pressure) shown in the table. A negative pressure on the downstream side of the gate provides a suction force on the gate which must be added to the respective upstream pressure gauge readings for computing the total flow-induced pressure on the gate surface. 


\begin{tabular}{|c|c|c|c|c|}
\hline \multicolumn{4}{|c|}{$\begin{array}{l}\text { Table } 32 \\
\text { Estimated Design Responses for Prototype Wicket }\end{array}$} & \\
\hline \multirow{2}{*}{$\begin{array}{l}\text { Gauge Type } \\
\text { (i) }\end{array}$} & \multirow[t]{2}{*}{$\begin{array}{l}\text { Uिrits of } \\
\text { Measurement }\end{array}$} & \multicolumn{2}{|c|}{$\begin{array}{l}\text { Design Estimates, TYPE - I, EVD } \\
\hat{x} \approx\left(\mu_{\bar{x}}+6.728985 \sigma_{\bar{x}}\right)\end{array}$} & \\
\hline & & $\begin{array}{c}\text { 1:5-scale } \\
\text { Model, } \tilde{x}_{\text {mad al }}\end{array}$ & $\begin{array}{l}\text { Prototype Wicket, } \\
\tilde{x}_{\text {protonpe }}=\lambda_{i} \tilde{x}_{\text {model }}\end{array}$ & \\
\hline Force, R. Hinge, $z$ & $\mathrm{Ibf}$ & 131.13 & $16,391.33$ & \\
\hline Force, R. Hinge, $y$ & $\mathrm{Ibf}$ & 778.92 & $97,364.74$ & \\
\hline Force, L. Hinge, $\mathbf{z}$ & $\mathrm{Ibf}$ & 242.73 & $30,341.60$ & \\
\hline Force, L. Hinge, $y$ & $\mathrm{lbf}$ & 757.30 & $94,663.04$ & \\
\hline Prop axial load at 65 deg & $\mathrm{bbf}$ & $1,192.00$ & $149,000.00$ & \\
\hline Force, R. Trunnion & Ibf & $1,194.18$ & $149,272.71$ & \\
\hline Force, L. Trunnion & $\mathrm{ibf}$ & $1,207.20$ & $150,900.48$ & \\
\hline Pressure, U/S T.R. & psi & 0.84 & 4.19 & \\
\hline Pressure, U/S T.C. & psi & 0.97 & 4.85 & \\
\hline Pressure, U/S T.L. & psi & 0.89 & 4.43 & \\
\hline Pressure, U/S M.R. & psi & 1.29 & 6.46 & \\
\hline Pressure, U/S M.C. & psi & 1.49 & 7.46 & \\
\hline Pressure, U/S M.L. & psi & 1.33 & 6.65 & \\
\hline Pressure, U/S B.R. & psi & 1.68 & 8.41 & \\
\hline Pressure, U/S B.C. & psi & 1.70 & 8.50 & \\
\hline Pressure, U/S B.L. & psi & 1.76 & 8.79 & \\
\hline Pressure, D/S T.R. & psi & -0.93 & -4.66 & \\
\hline Pressure, D/S T.C. & psi & -0.91 & -4.53 & \\
\hline Pressure, D/S T.L. & psi & -0.91 & -4.55 & \\
\hline Pressure, D/S M.R. & $\mathrm{psi}$ & -1.23 & -6.14 & \\
\hline Pressure, D/S M.C. & psi & -0.80 & -3.98 & \\
\hline Pressure, D/S M.L. & psi & -0.85 & -4.25 & \\
\hline Pressure, D/S B.R. & psi & -0.81 & -4.04 & \\
\hline Pressure, D/S B.C. & psi & 1.22 & 6.09 & \\
\hline Pressure, D/S B.L. & psi & -0.52 & -2.62 & \\
\hline Pressure, upper cylinder & psi & 3.80 & 19.00 & $\equiv 2,083.79 \mathrm{lbf}$ \\
\hline Pressure, lower cylinder & psi & 390.34 & $1,951.70$ & $\equiv 300,439.6 \mathrm{lbf}$ \\
\hline Accel., 1x T.R. & in. $/ \sec ^{\wedge} 2$ & 71.68 & 71.68 & \\
\hline Accel., 1y T.R. & in. $/ \sec ^{\wedge} 2$ & 41.81 & 41.81 & \\
\hline Accel., 12 T.R. & in./sec^2 & 92.97 & 92.97 & \\
\hline Accel., 2 T.C. & in. $/ \sec ^{\wedge} 2$ & 86.61 & 86.61 & \\
\hline Accel., 3x T.L & in./ $\sec ^{\wedge} 2$ & 71.68 & 71.68 & \\
\hline Accel., 3y T.L. & in./sec^2 & 39.60 & 39.60 & \\
\hline Accel., 3z T.L. & in./sec^2 & 102.31 & 102.31 & \\
\hline Accel., 4 M.R. & in./sec^2 & 101.54 & 101.54 & \\
\hline Accel., 5x M.C. & in./sec^2 & 50.77 & 50.77 & \\
\hline Accel., 5y M.C & in. $/ \sec ^{\wedge} 2$ & 27.26 & 27.26 & \\
\hline Accel., 5z M.C. & in. $/ \sec ^{\wedge} 2$ & 44.80 & 44.80 & \\
\hline
\end{tabular}




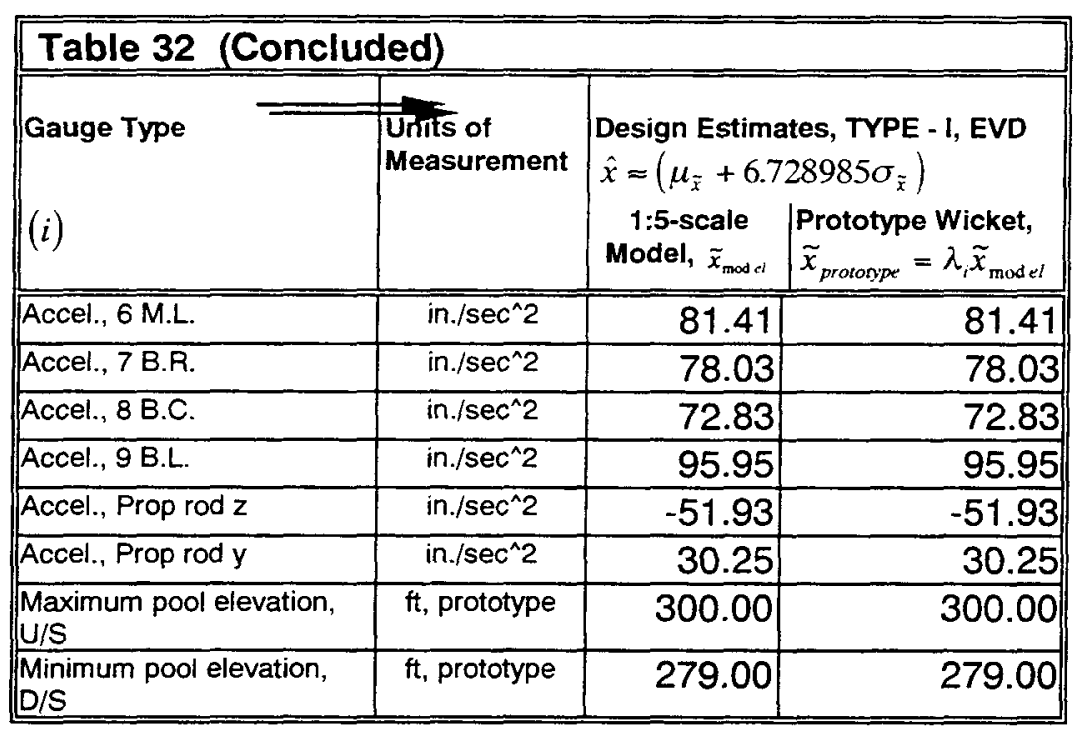

Estimated design responses presented in the table are limited to the flow boundaries mentioned in this report and could not be regarded as a substitute for dynamic response analysis of the wicket for final structural evaluation. These values merely provide an estimation of the upper boundaries which could be used for preliminary design checks or for a quick structural performance evaluation of wicket components.

\section{Pressure distributions on a prop-supported gate}

Pressure readings from two experiments were analyzed to understand the nature of spatial distribution of water pressure, dynamic amplification, and its fluctuation about the corresponding mean values. Test results from Experiment No. 111 and Experiment No. 163 were used to plot the pressure readings along three columns of the gate as a function of depths. Figure 128 shows the location of the pressure gauges and the three columns along which the data are plotted to determine the pressure variation as a function of water depth. Figure 129 shows the relative pool elevations which were used to determine the static water depths for each gauge location.

Experiment No. 111 represents the pressure readings of a prop-supported gate for a 11-ft head pool condition (upstream elevation, $296.5 \mathrm{ft}$, and downstream elevation, $285.5 \mathrm{ft}$ ) with no-gap flow configuration. Gaps among the gates were closed by needles to stop water flow through the channel. Experiment No. 163 represents data from a prop-supported gate on a 21 -ft head difference pool condition for a $3 \mathrm{GG}$ flow configuration. For the second set of experiments, the upstream pool elevation was at $300 \mathrm{ft}$ and the downstream elevation was set at $279 \mathrm{ft}$.

Figure 130 presents the upstream and downstream pressure distributions as a function of gauge depth for both cases. The pressure is presented in the unit of 


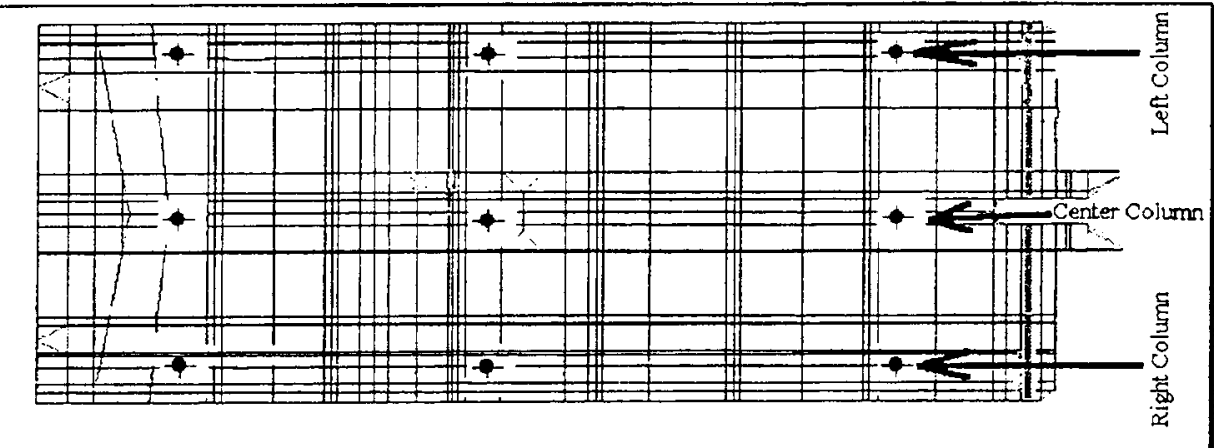

Figure 128. Pressure gauge locations and column lines for displaying pressure readings

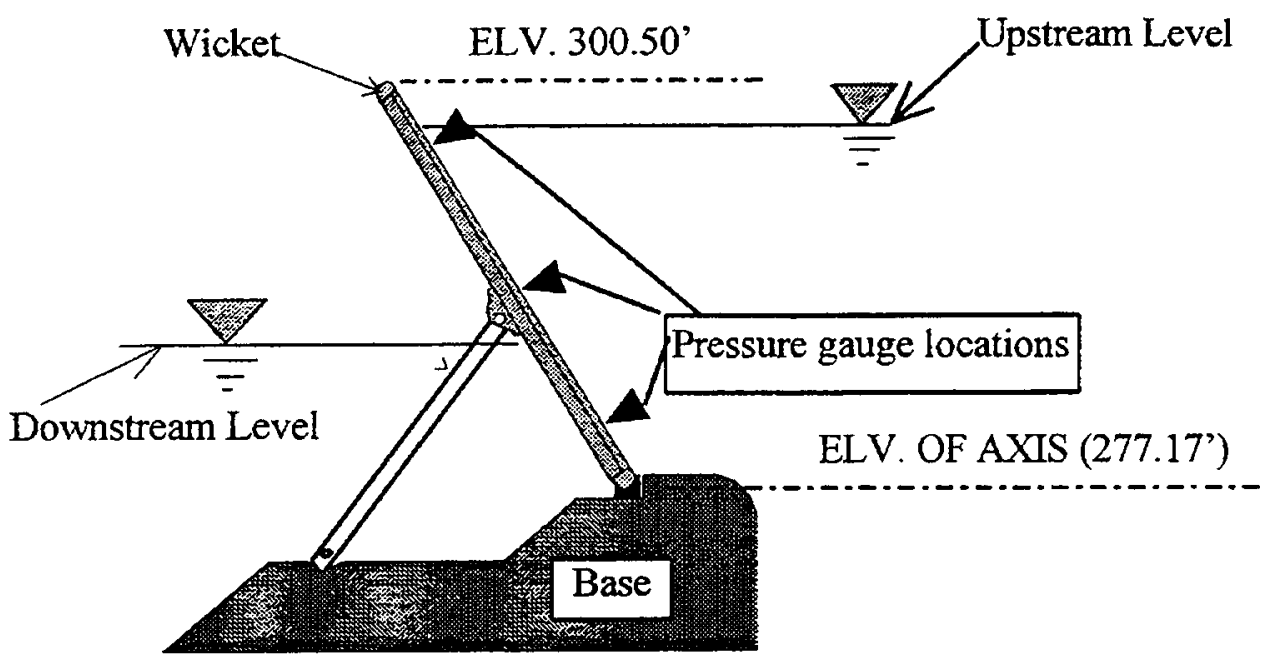

\section{SIDE VIEW}

Figure 129. Relative water elevation for pressure measurements

inches of water column and the gauge depth was measured from the hinge axis up. Each plot presents the static, average, and two-standard deviation away from the mean value. Static water depth is the submerged gauge depth from the water surface on respective sides of the wicket. Average and the deviation of random response (sigma) about the respective mean were obtained directly from the experimental record. Note that the mean and mean minus 2 -sigma $(\mu-2 \sigma)$ are plotted in each figure to show the fluctuation of the dynamic pressure about the mean. A mean plus 2 -sigma $(\mu+2 \sigma)$ limit, not shown in the plot, would provide the upper boundary of the random response for which about 97.5 percent of the data will be below or equal to that value. The upper boundary of the random 

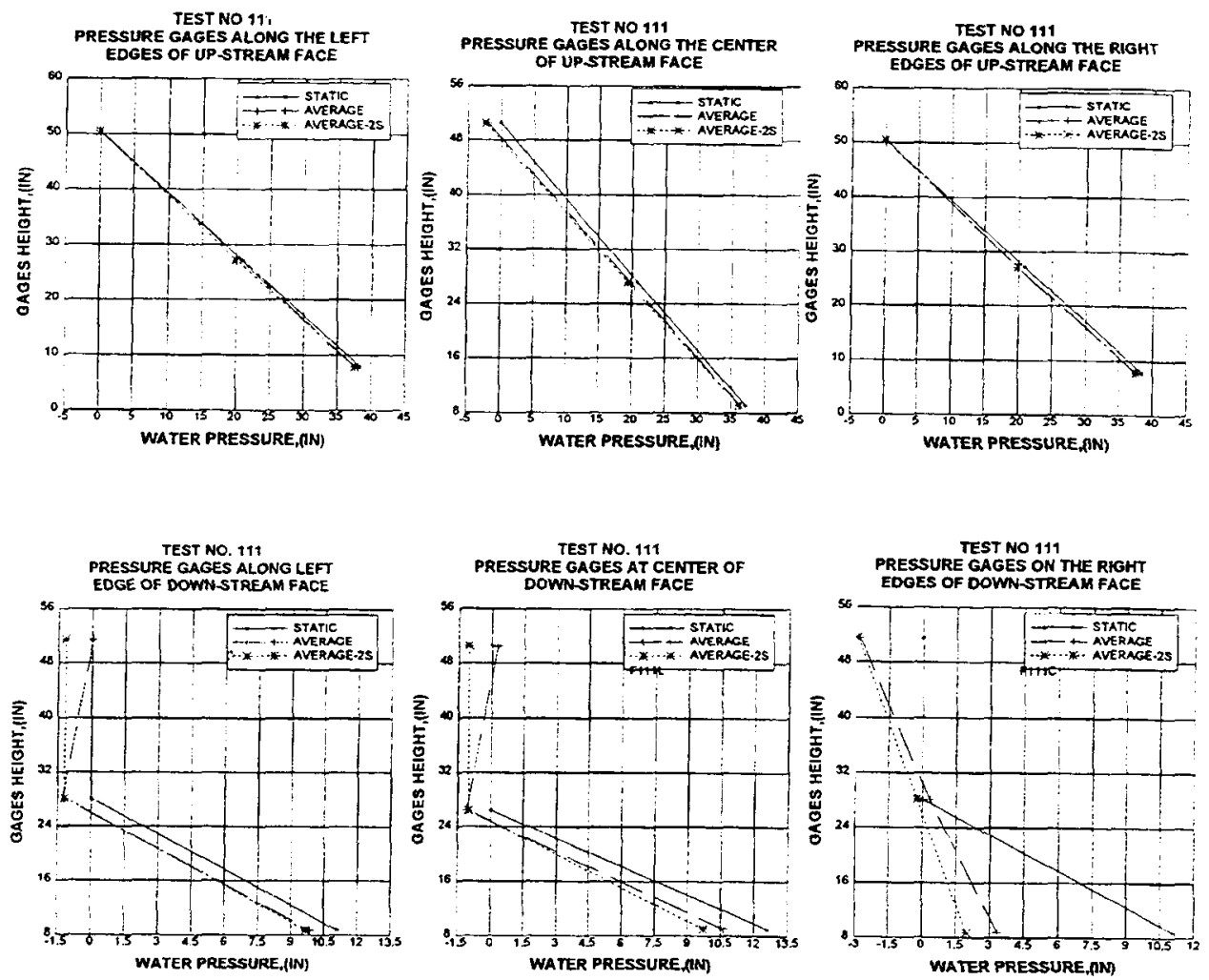

a. No-gap, 11-ft head difference

Figure 130. Pressure distributions on prop-supported gate as a function of depth (Continued)

response could easily be constructed by placing a symmetrically opposite line of the $(\mu-2 \sigma)$ value about the mean response.

Upstream pressure gauge readings for both cases show a linear trend in the pressure distribution as a function of water depth. This plot also indicates that the static pressure head is the conservative estimate of the maximum pressure readings on the upstream gate surface. Notice that for all cases the static head is greater than the flow-induced upper boundary at $9712^{\text {th }}$ percentile represented by $(\mu+2 \sigma)$ line. For the $21-\mathrm{ft}$ head difference pool condition, the deviation of the flow-induced maximum upstream pressure readings (at $97.5^{\text {th }}$ percentile) from the static head is distinctly noticeable. A drop in the measured pressure reading is due to the increase in velocity head through the adjacent down-gate openings. By using the Bernoulli's energy conservation equation, such a deviation in the pressure readings could be used to estimate the flow velocity through the openings.

A negative pressure indicating a suction on the downstream surface of the gate is noticeable for both cases. These plots also show that about a one-third of the gate, above the hinge axis, did not have any suction on the downstream gate surface. For the $21-\mathrm{ft}$ head pool condition, the downstream static pressure is zero. A maximum suction of 3.9 in. of water column on the downstream surface 

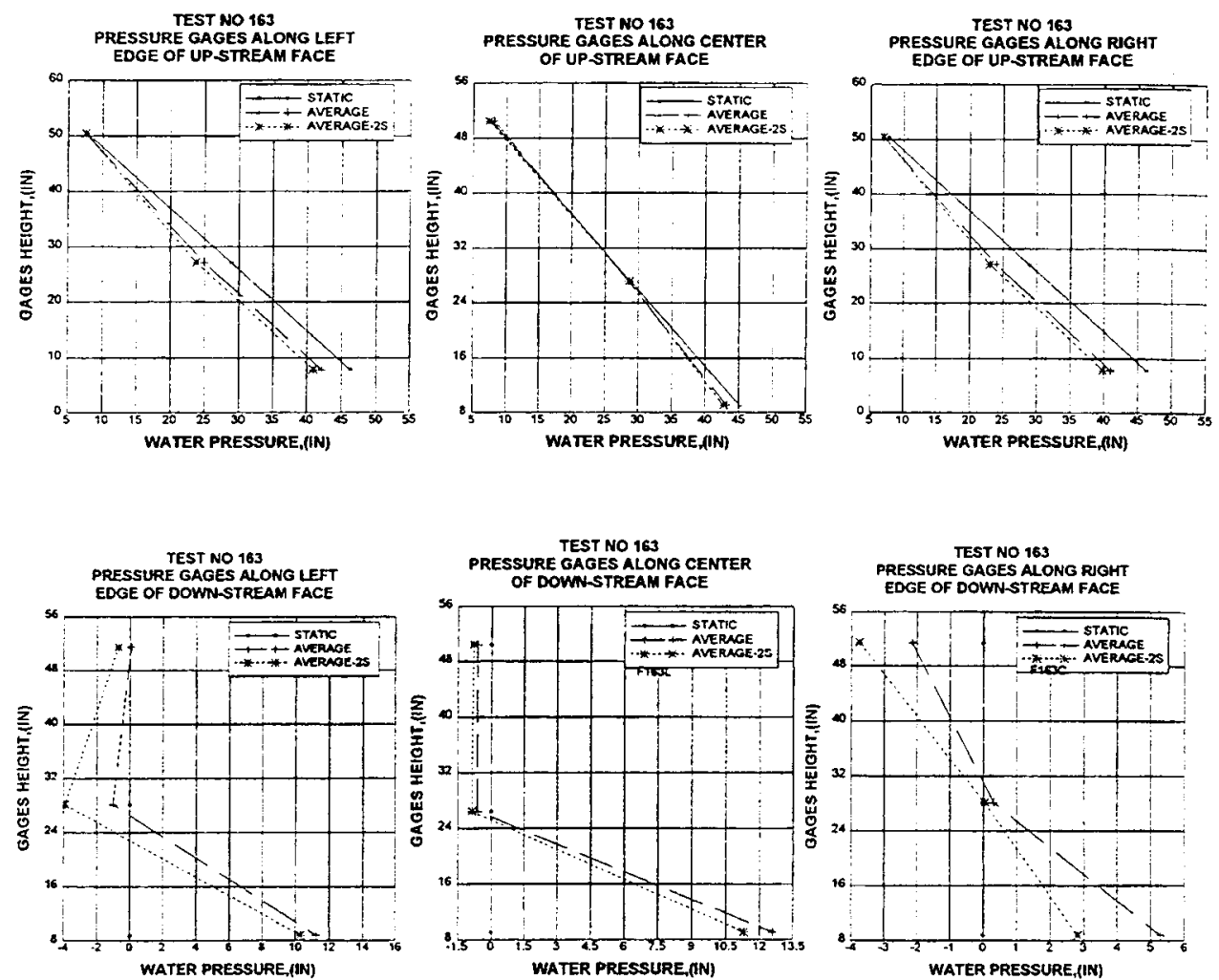

b. Three-gate gap, 21-ft head difference

Figure 130. (Conciuded)

resulted because of the interaction of the flowing water through the gate openings. A major fluctuation in the pressure readings is relatively more dominant on the gauges located on both edges of the wicket. This suggests that the turbulence of the flowing water through the adjacent gate openings caused fluctuation in the downstream pressure readings.

A study of the pressure distribution indicates that a static head on the upstream surface would conservatively estimate the maximum pressure peaks for the prop-supported gate. For design evaluation, a time-dependent pressure history could be reconstructed based on the measured normalized pressure timehistory, $\mathrm{P}_{\mathrm{n}}(\mathrm{t})$, with the maximum amplitude of pressure reading obtained during the operating cycle. The normalized pressure time-history indicative of the dynamic fluctuation of the anticipated pressure response could be obtained from the random pressure history, $\tilde{P}(t)$, using the equation, $\left(P_{n}(t)=\frac{\tilde{P}(t)}{|\tilde{P}(t)|}\right)$ 


\section{Representative pressure history}

The representative pressure history is one of the upstream pressure readings, since the PSD of upstream pressure history shows some existence of flowstructure interaction as shown in Figures 85 and 87 . As shown in the pressure PSD plots, the upstream pressure had peaks near or in the vicinity of structural modes, which suggest an interaction of the pressure and gate responses during the flow measurement. The downstream pressure PSD, however, are random and external in nature, thus, do not interact with the gate responses.

A critical design configuration, however, is different form the prop-supported condition presented above. Most critical pressure loading occurs when the gate is in a 20- to 24-deg position on its lifting cycle. A spatial distribution of the pressure records at fixed time instant during the raising of the wicket in a $21-\mathrm{ft}$ head pool condition, three-gate gap configuration, is presented in Figure 131. Eight pressure envelopes each for upstream and downstream gate surface were developed by plotting the pressure responses for all gauges corresponding to eight gate positions shown in the figure. For the upstream case, positive pressures indicate that the water exerts pressure along the gravitation direction normal to the gate surface. A negative water pressure indicates a suction during the lifting of the gate and is acting away from the gate surface towards the gravitational field.

This figure also confirms the earlier findings on the linear distribution of the pressures along the water depth. Therefore, the maximum estimate of pressure readings $\left(\hat{P}_{\max }\right)$ presented in Table 32 could be used in the normalized pressuretime history $\left(P_{n}(t)\right)$ to evaluate the design performance of the wicket. The equation for the design pressure filed would become $P(t)=\hat{P}_{\max } P_{n}(t)$.

\section{Added Mass Effects}

When a structure vibrates in a dense fluid, its dynamic behaviors could change substantially. This change, due to the coupled motions of the structure with the surrounding, generally can be simplified as the added mass effect. As most of the researchers in the hydroelasticity study have agreed, the added mass of a structure vibrating in a fluid is one of the most complicated issues, yet it is a very important factor to determine the dynamic characteristics of the structure in the fluid. Analytical solutions to the added mass with a number of assumptions, such as ideal incompressible and inviscid two-dimensional (2-D) flow, lowamplitude and linear vibration, and rigid body motion or fixed boundary conditions, are only able to yield accurate results for some very simple structures, such as plate or cylinder.

For a wicket gate model, the complicated boundary conditions and the complex geometry with unstable three-dimensional (3-D) flow conditions make the analytical method unrealistic. Thus, using experimental modal analysis to determine the added mass and it effects on the gate vibration was proposed. This 


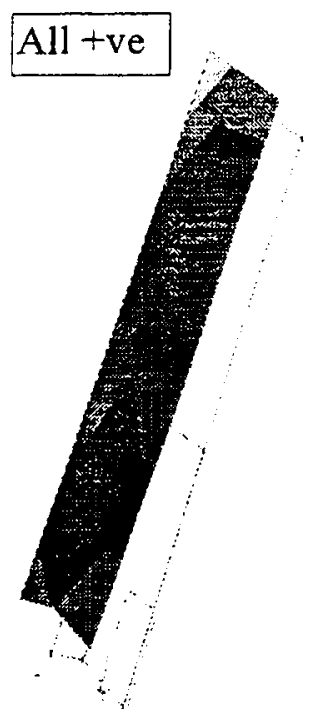

$\operatorname{up} 173\left(0^{0}\right)$

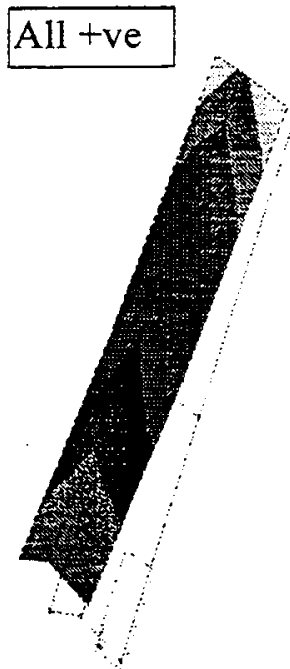

Up173 (400)

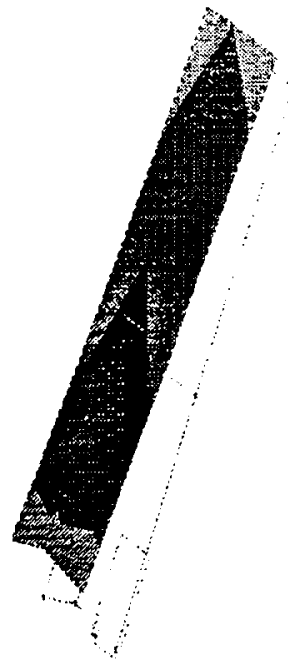

$\operatorname{up} 173\left(10^{0}\right)$

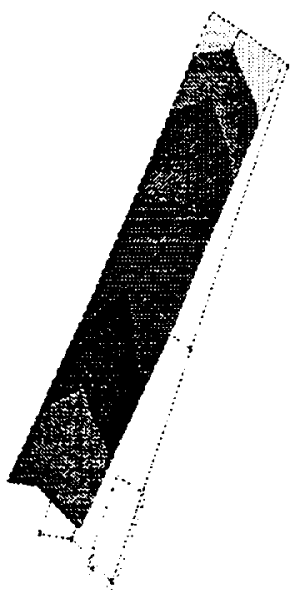

up173 (500)

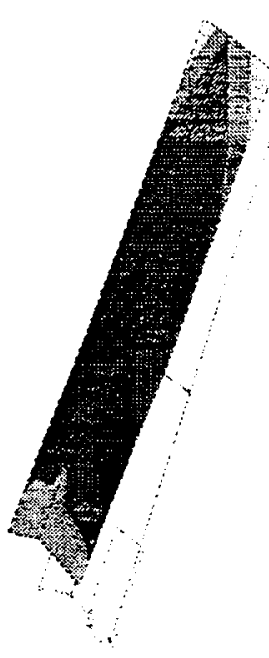

$\operatorname{up} 173\left(20^{0}\right)$

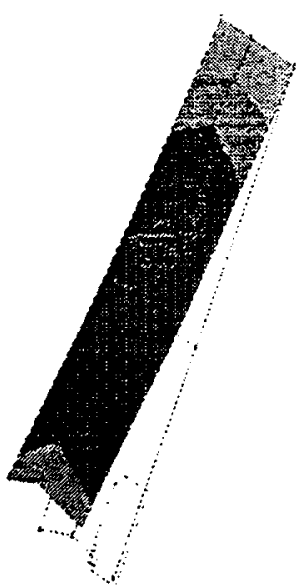

$\operatorname{up} 173\left(60^{0}\right)$

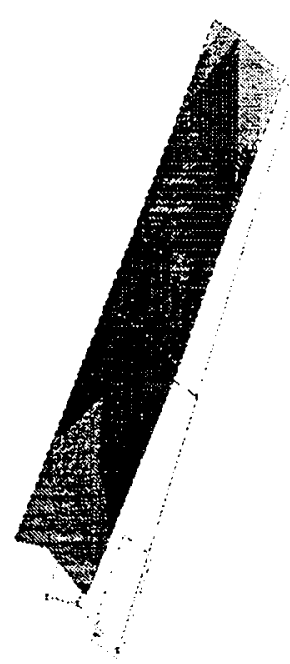

up173(300)

\section{a. Upstream face}

Figure 131. Pressure envelopes on raising wicket (Continued)

technique can count the effects of the complicated boundary conditions, geometry of the gate, and the flow conditions. The experiment results show that the added mass of the gate model could be several times greater than the structural mass. The effects of added mass on the gate vibrations are tremendous, which were indicated by the significant reductions in the natural frequencies. However, the experimentally determined added mass inherited a great deal of nonlinearity and irregularity due to a number of factors. 


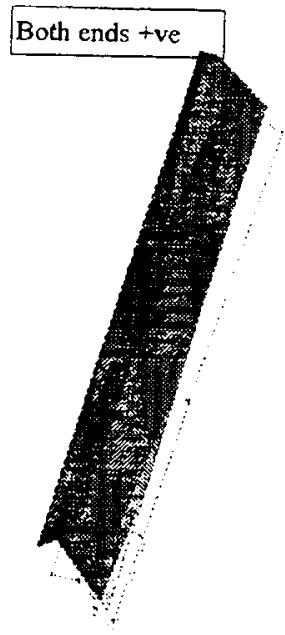

dp173 (-30)

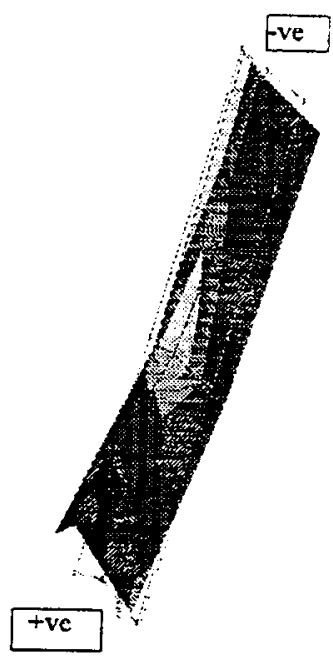

$\operatorname{dp} 173\left(40^{0}\right)$

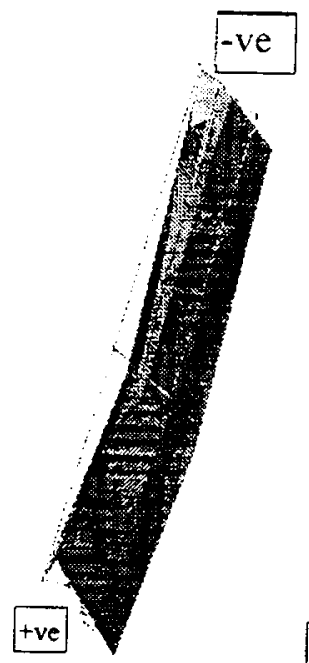

dp173 (100)

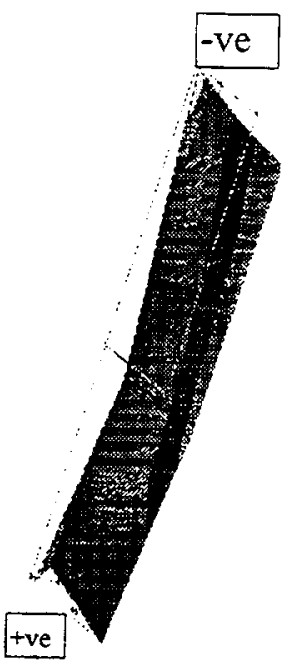

dp173(200)

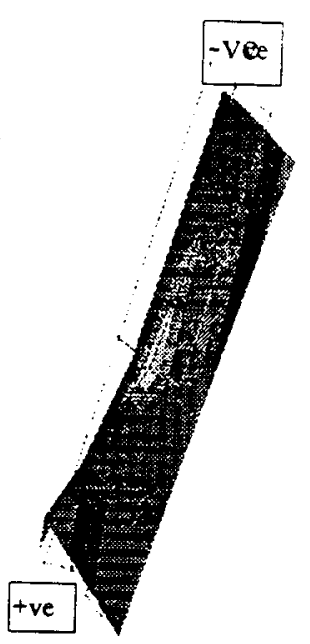

dp173 (300)

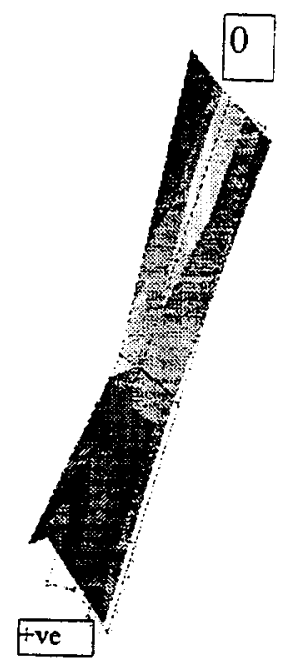

$\operatorname{dp} 173\left(50^{\circ}\right)$

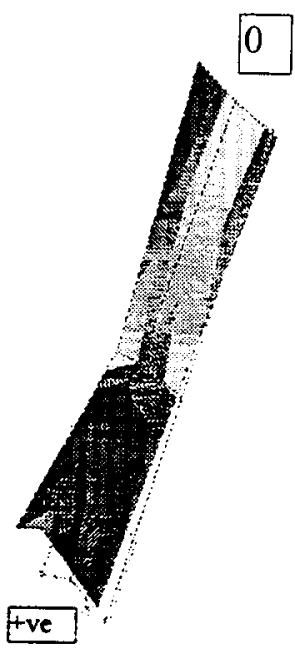

dp173 $\left(60^{\circ}\right)$

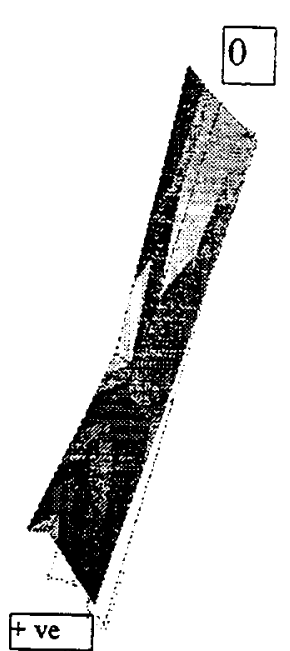

dp173 (650)

\section{b. Downstream face}

Figure 131. (Concluded)

\section{Background}

When a structure is fully or partially submerged in a fluid, the dynamic characteristics of the structure, mainly the natural frequencies and the damping ratios, will change substantially if the fluid density is comparable to the structure density, such as a steel wicket gate in water. This well-known phenomenon is due to coupled motions of the structure and the surrounding fluid. This coupled 
motion can be simplified, in most cases, as the structure vibrates with an added mass due to the fluid entrained by the vibrating structure.

In general, the added mass is believed to be a function of a number of variables, such as geometry of the structure, physical properties of the fluid, boundary conditions and mode shapes of the structure, and vibration amplitude and frequency. Theoretically determining this added mass and its effect has been a very challenging problem (Blevins 1993). The major difficulty comes from the fact that, in general, both a hydrodynamic model and a structural dynamic model are 3-D problems.

Chen, Wambsganss, and Jendrzejczyk (1976) used two-dimensional (2-D) potential flow theory and the linearized Navier-Stocks equation to obtain a closed-form solution for the added mass of a vibrating rod in confined viscous fluid. Yang and Moran (1979), with a similar approach as Chen, Wambsganss, and Jendrzejczyk (1976), determined the added mass of a hexagonal cylinder from a FE model. Vethamony et al. (1992) estimated the added mass of a tethered spherical float from wave-induced motion by using 2-D potential flow theory and linearized Bernoulli's equation. All these studies were limited to rigid body motion or the fundamental mode of the structures. For a 2-D potential flow model, the assumptions of the strip theory are applied. The strip theory neglects axial flow effects and assumes that flow at one cross section is independent of flow at other sections. Therefore, the 2-D potential flow theory can only be applied to the fundamental modes of the structures or simple and slender structures (Che, Riggs, and Ertekin 1994).

Despite complexity and computational intensity, some attempts have been made to solve structural-fluid coupled motion problems analytically by using a 3-D potential flow model. Ma (1993) and Ma and Xie (1993) used Rayleigh's method and Fourier Transformation to obtain a 3-D fluid model solution for a rectangular plate vibrating in water with one edge fixed, in which the wet mode shape was substituted by the fundamental dry mode shape. Yan and Yan (1990) applied the boundary-element method and FE method to the 3-D fluid model and 3-D structural model, respectively, to determine the added mass for a radius gate.

While the success of analytical methods is limited to simple structures and is constrained by the complexity and anomalous assumptions, experimentally determining the added mass and its effects sometimes is the only way to deal with flexible structures under complicated practical situations. Maheri and Severn (1992) showed the frequency and mode shape dependency of the added mass of flexible cylindrical structures by exciting the cylinders at each mode and measuring the accelerations and pressures at a number of points on the cylinders. They discovered that imperfections in the geometry of the cylinders could lead to large discrepancies between the finite element model prediction and the true frequencies of lower modes. Thang (1982) investigated a sluice gate model with adjustable fundamental frequency of the system to determine the added mass of the gate vibrating in vertical direction (normal to streamwise direction) under various conditions. Ishii and Knisely (1992) conducted an experiment on a longspan gate model of which lateral fundamental frequency is also adjustable. 
Unlike Thang (1982), Ishii and Knisely (1992) determined the added mass associated with the vibrating gate model in the streamwise direction by treating the gate as a rigid body.

\section{Equations for added mass}

For plate vibration, the natural frequencies of the plate $(f)$ can be found using the equation shown below

$$
f_{i}=\frac{\lambda_{i}^{2}}{2 \pi l^{2}} \sqrt{\frac{E A t^{3}}{12 m\left(1-v^{2}\right)}}, \quad i=1,2,3, \cdots
$$

where

$$
\begin{aligned}
& i=\text { index of mode } \\
& \lambda=\text { dimensionless parameter which is a function of mode shapes, Poisson's } \\
& \text { ratio, plate geometry, and boundary conditions } \\
& l=\text { plate length } \\
& E=\text { modulus of elasticity } \\
& A=\text { plate area } \\
& t=\text { plate thickness } \\
& m=\text { plate mass } \\
& v=\text { Poisson's ratio }
\end{aligned}
$$

When a structure vibrates in a fluid, the primary effect of the entrained fluid on the structural vibration can be approximately modeled using an additional effective mass and damping. With the added mass being $\Delta m$, the natural frequency of a plate vibrating in a fluid will be:

$$
\left(f_{i}\right)_{f l u i d}=\frac{\lambda_{l}^{2}}{2 \pi l^{2}} \sqrt{\frac{E A t^{3}}{12\left(m+\Delta m_{i}\right)\left(1-v^{2}\right)}}, i=1,2,3, \cdots
$$

Since the density of air is much less than the density of most structures, that is, $\Delta m<m$, the above equation for plate natural frequency can be used for a plate that vibrates in air. Assume $\lambda$ will not change by the presence of the fluid for each mode, then the ratio of the above equations becomes:

$$
\frac{\left(f_{i}\right)_{\text {air }}}{\left(f_{i}\right)_{\text {fluid }}}=\sqrt{\frac{\left(m+\Delta m_{i}\right)}{m}}, \quad i=1,2,3, \cdots
$$


The added mass of a plate vibrating in a fluid can be determined from the natural frequencies of the plate in air and in the fluid:

$$
\Delta m_{i}=m\left[\left(\frac{\left(f_{i}\right)_{\text {air }}}{\left(f_{i}\right)_{\text {fluid }}}\right)^{2}-1\right], i=1,2,3, \cdots
$$

\section{Experimental results for wicket gate model}

Under the normal operating condition, the upstream water flows around the side of the gate to the downstream. It is very clear that the nature of the gate construction, boundary conditions, and the flow condition would forbid any accurate analytical solution to the added mass of the gate. Thus, five sets of experimental modal results are used to investigate the added mass and its effects on the dynamic behaviors of the wicket model. These include the modal results for the dry gate and four sets of wet cases for the three-gate gap flow configuration with different pool elevations.

The nominal water pool elevation measurements, however, were not taken directly around the gate model but at some distance away from the gate where the flow surface was flat and stable. It can be seen from the water profile presented earlier that the actual level of gate submergence was different with the nominal water pool elevation. The water levels at the surfaces of the gate model were slightly lower on the upstream side and somewhat higher on the downstream side than the nominal water pool levels. The variations depended on the flow condition and the water head difference.

Modal frequencies for the prop-supported dry gate and four wet cases are presented in Figure 132. Four wet cases consisted of 21-ft, 11-ft, 7-ft, 0 -ft pool differences. As seen in the plot, the modal frequencies are not very sensitive to the head difference. Variations of added mass as a function of global mode order are shown in Figure 133. In this figure, only the global modes that correlated well with the respective dry modes were used to eliminate the uncertain modes that varied frequently during the modal experiments. These uncertain modes were highly sensitive to the prop/shaft dynamics and could not represent the global wicket behavior fairly. As shown in the plot, all but the first bending mode (mode order no. 2) were affected by the positive added mass. In general, added mass increased as a function of modal order.

Influenced by the complicated flow conditions, unusual boundary conditions, and complex 3-D geometry of the wicket gate model, experiment results indicate that the added mass of the wicket model were packed with nonlinearity and irregularity. To just make the matter worse and more complicated, the vibrations of the gate were coupled with the vibrations of the prop which obviously, from geometric point of view, were affected by the water in a quite different manner compared with the rectangular gate. It hardly showed any satisfactory relationship with the available classic theories if any such analytical prediction could be made. Blevins' (1993) strip theory for a slender submerged rectangular 


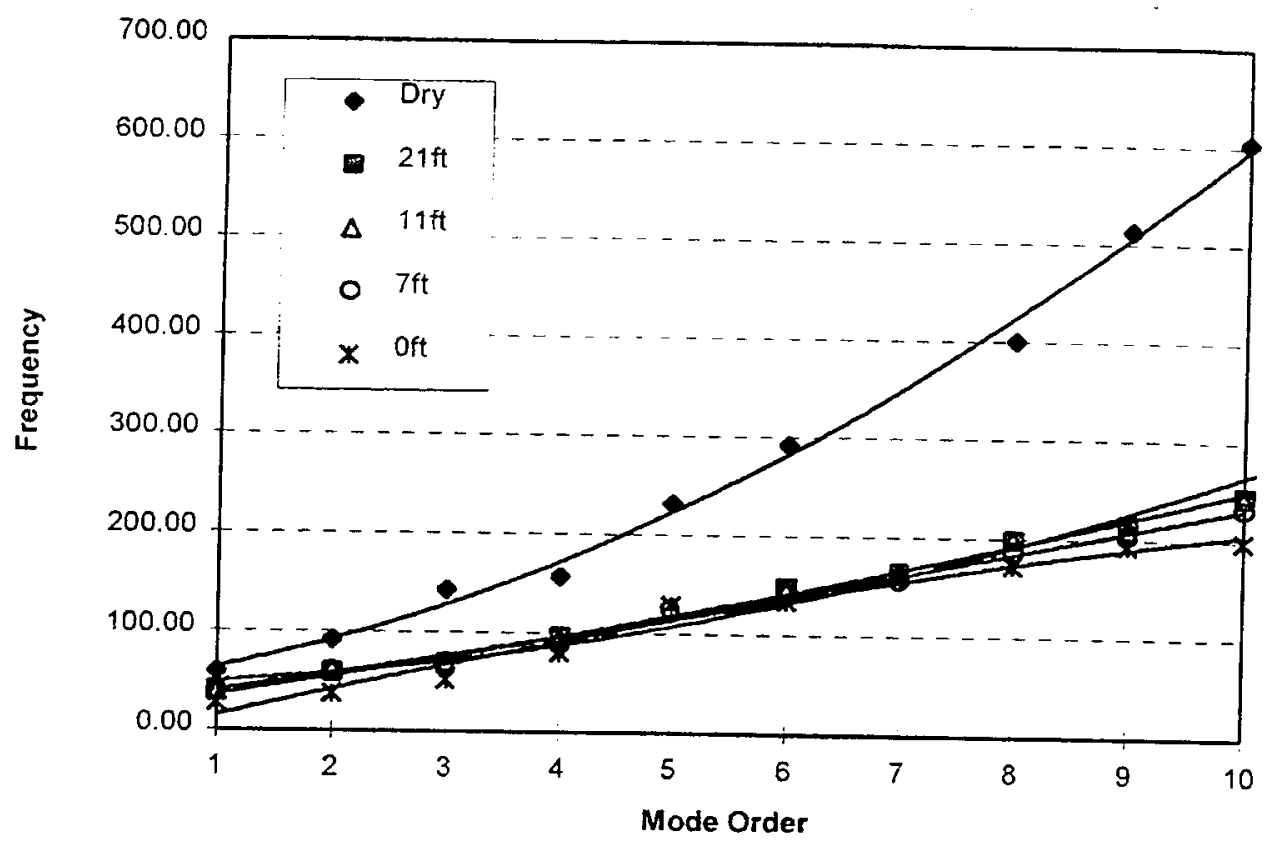

Figure 132. Wet frequency variation as a function of pool difference

\section{Added-mass for different water head}

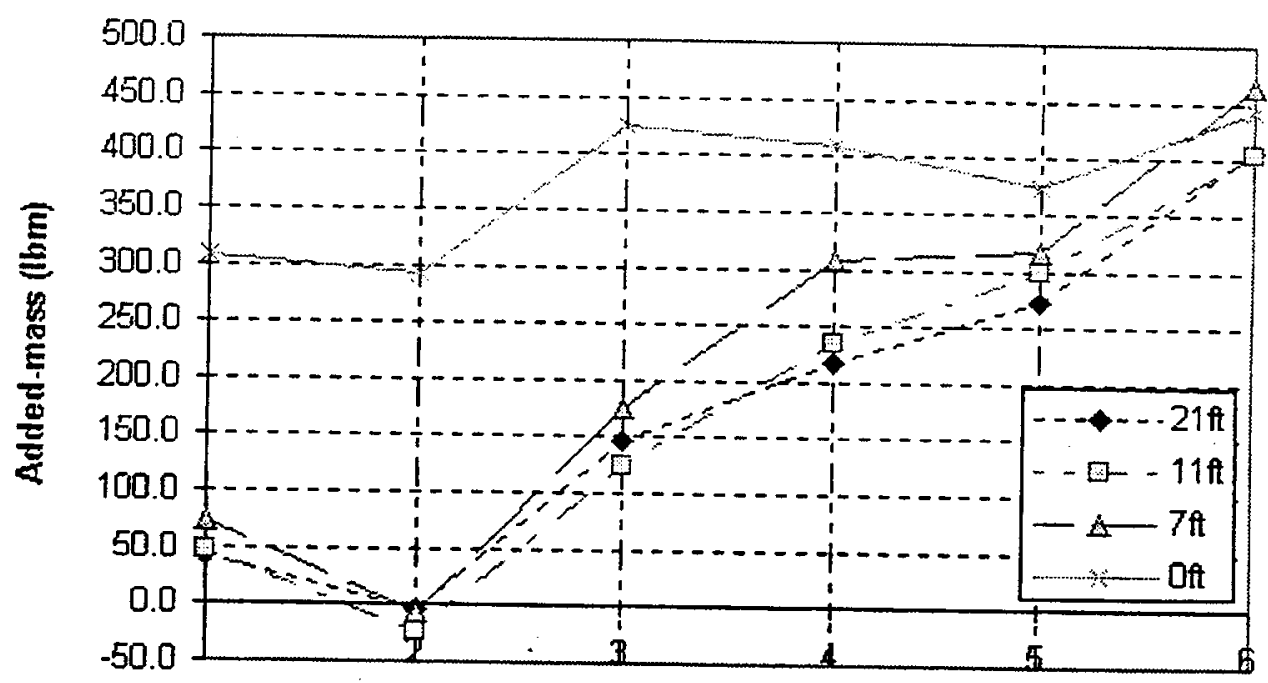

Made order

Figure 133. Added mass as a function of water heads

plate, for example, predicted a very high added mass compared to the experimental results. Subdividing both surfaces of the gate into rectangular components that were in contact with water and adding added masses for each component, $736 \mathrm{lb}$ of added mass was estimated using the formula proposed by Blevins. Figure 133 shows that the added mass for the interested domain could vary from -25 to $450 \mathrm{lb}$ depending upon the modal order. 
From an analytical point of view, a single value of added mass instead of a variable added mass would be convenient for computing global response for the wet wicket. In this regard, the modal peaks in the FRF function showing the contribution of each mode in the total response could be used to determine the weighted contribution. A weighted average of the added mass $\left(\Delta m_{x}\right)$ could be computed using the formula shown below.

$$
\Delta m_{w}=\frac{\left(\sum A_{i} \Delta m_{i}\right)}{\sum A_{i}}
$$

where

$$
\begin{aligned}
\mathrm{A}_{\mathrm{i}} & =\text { amplitude of the } \mathrm{i}^{\text {th }} \text { modal peak } \\
\Delta m_{i} & =\text { corresponding added mass. }
\end{aligned}
$$

Table 33 presents the modal mass $\left(\Delta \mathrm{m}_{\mathrm{i}}\right)$, peak amplitude $\left(\mathrm{A}_{\mathrm{i}}\right)$, and damping $\left(\zeta_{i}\right)$ for the four wet case used in the experimentation. The last row shows the

\begin{tabular}{|c|c|c|c|c|c|c|c|c|c|c|c|c|}
\hline \multicolumn{13}{|c|}{$\begin{array}{l}\text { Table } 33 \\
\text { Added Mass Parameters for Wet Gate }\end{array}$} \\
\hline \multirow{3}{*}{\begin{tabular}{|l|} 
Mode \\
Order
\end{tabular}} & \multicolumn{3}{|l|}{$21-\mathrm{ft}$} & \multicolumn{3}{|l|}{$\overline{11-\mathrm{ft}}$} & \multicolumn{3}{|l|}{$7-\mathrm{ft}$} & \multicolumn{3}{|c|}{ Completely Submerged } \\
\hline & $\cdot \mathrm{m}_{1}$ & $\bar{A}$ & $\cdot$ & $\cdot m$ & $\bar{A}$ & $\cdot$ & $\cdot \cdot_{\mathrm{i}}$ & $A_{1}$ & - & $\cdot m$ & $A$ & $\cdot$ \\
\hline & $\mathrm{Ib}$ & in./s/lbf & $\%$ & $\mathbf{I b}$ & in./s/lbf & $\%$ & Ib & $\ln . / \mathrm{s} / \mathrm{lbf}$ & $\%$ & $\mathrm{Ib}$ & in./s/lbf & $\%$ \\
\hline 1 & 43.4 & 56.3 & 2.60 & 47.4 & 35.9 & 2.26 & 73.1 & 35.6 & 3.41 & 308.0 & 115.2 & 11.64 \\
\hline 2 & -3.7 & 460.4 & 1.90 & -23.0 & 508.9 & 1.90 & -5.3 & 448.8 & 2.06 & 292.8 & 122.7 & 8.54 \\
\hline 3 & 146.4 & 477.8 & 3.00 & 125.2 & 640.0 & 2.35 & 175.3 & 523.5 & 2.34 & 426.0 & 319.7 & 2.95 \\
\hline 4 & 218.3 & 147.4 & 3.10 & 234.9 & 216.9 & 2.84 & 307.9 & 261.6 & 2.15 & 408.7 & 172.7 & 3.46 \\
\hline 5 & 272.3 & 203.7 & 2.90 & 298.8 & 140.9 & 2.90 & 316.4 & 131.4 & 1.13 & 373.6 & 136.9 & 2.48 \\
\hline 6 & 407.0 & 433.1 & 1.97 & 405.8 & 604.9 & 1.58 & 464.4 & 495.1 & 1.55 & 443.5 & 160.4 & 2.08 \\
\hline$\left(\Delta m_{2}\right)$ & \multicolumn{3}{|c|}{188.11} & \multicolumn{3}{|c|}{190.3} & \multicolumn{3}{|c|}{234.22} & \multicolumn{3}{|c|}{389.70} \\
\hline
\end{tabular}
weighted added mass representing the global changes in the virtual mass of the wet gate.

As most of the researchers in the hydroelasticity study have agreed, the added mass of a structure vibrating in a fluid is one of the most complicated issues, yet it is a very important factor to determine the dynamic behaviors of the structure in the fluid. Analytical solutions to the added mass with a number of assumptions, such as ideal incompressible and inviscid 2-D flow, low-amplitude and linear vibration, and rigid body motion or fixed boundary conditions, are only able to yield accurate results for some very simple structures, such as plate or cylinder.

For the wicket gate model under the real flow condition, using experimental modal techniques to determine the added mass is a suitable technique that can count the effects of the complicated boundary conditions, geometry of the gate, and the flow conditions. The experiment results show that the added mass of the gate model can be several times greater than the structural mass. The effects of 
added mass on the gate vibrations are tremendous, which are indicated by the significant reductions in the natural frequencies. The unavoidable reality was clearly reflected in the nonlinearity and irregularity of the experimentally determined added mass of the wicket gate model. 


\section{Conclusions and Significance of Study}

\section{Dynamic Design Development}

Numerous modifications during the progress of the model construction helped devlop a functional design drawing for the prototype wicket. Changes in the wicket design were made to facilitate fabrication, correct dislocation of fitting parts, adjust design faults, and ensure smooth operation by eliminating operational difficulties. Major changes were made in the supporting devices to adjust design faults and to improve on operational difficulties. The prop components had been reconstructed from a double-fork pin connection to a single-sided pin connection. The under-designed shear pin in the prop-supported blade was redesigned to provide adequate strength. The hurter had gone through substantial changes because of difficulties in keeping the prop blade on the track during raising and lowering of the gate.

Latest wicket design evolved as construction progressed and new ideas were incorporated to resolve detected as well as anticipated construction and operational difficulties. The 1:5-scale model construction provided a very effective testing ground for checking the accuracy of the prototype design drawings.

\section{Structural Similitude Model}

For all practical purposes, a geometrically scaled model with hydraulic similitude relations is capable of predicting the prototype response pattern for the flow fields investigated in this report. A comparison of 1:25- and 1:5-scale model responses suggests that the modeling distortion of Froude model by not meeting the mass-density-elastic modulus criteria has minimal effect on the predicted time-domain wicket response. A complete similarity of frequencydomain responses of these models is not attained by the Froude model because of modeling distortions in the 1:25-scale model. A lack of complete geometrical scaling of the 1:25-scale model was one of the factors that made it impossible to match the frequency responses.

Nonlinearities of the prop rod connections significantly changed the frequency-domain behavior of the wicket. Dissimilarities in frequency-domain 
responses, however, did not significantly reduce the effectiveness of the similitude models in predicting the time-domain responses. This is partially due to the lesser sensitivity of the low-amplitude flow field in the frequency range containing the structural modes of the wicket. PSD for the pressure field shows that the random pressure field asymptotically reduces as frequencies increase.

\section{Dynamic Performance Evaluation}

A $3 \mathrm{GG}$ flow configuration provided the most critical flow conditions among the proposed operating sequences. Highest fluctuation of the wicket response and the absolute maximum of the responses occurred at or near the breaking of the air pocket underneath the gate surface. For the highest possible head difference, this critical condition existed at about $24 \mathrm{deg}$ up from the lowered position of the wicket. A comparison of the upper bounds of the time-domain responses for the proposed operating scheme with that of the design limits proved the robustness of the wicket design.

Frequency-domain response analyses effectively identified the structural and flow fields interaction. Upstream pressure PSD indicate the signature of the flow field that slightly interacted with the global structural modes. Downstream pressure history formed a random field that was independent of the structural global modes. Accelerometer PSD identified the major peaks that were energized due to the flow-induced vibration. Existence of resonant problems due to the proximity of global structural wet modes and the resonant peaks due to flow-induced vibration were systematically analyzed for locating the source of potential vibration problem.

A shaker-excited modal experiment in the midst of random noise of the operating flow field successfully extracted the modal parameters of the wet wicket. The state-of-the art SLDV system effectively used to determine the dry and wet natural characteristic of the wicket. A comparison of the wet modes with the operating shapes corresponding to the peaks in the flow-induced accelerometer responses indicated the resonance of the prop-supported gate. Operating shapes are the actual dynamics of the system during the critical flow configuration, and the wet gate modal parameters represent the natural behavior of the gate at that particular environment due to forced vibration. For the propsupported gate, wet gate natural mode shapes and the corresponding ODS were related to each other. Resonant vibrations were measured due to the weaker pressure field at the 65-deg position, where the random energy content of the weak flow excited the natural modes of the wet gate. Interestingly, however, wet gate natural modes at critical configuration did not match with the ODS of the wicket at the 24-deg position. The wet gate ODS changed with varying wicket positions. A strong flow-induced operating shape at the critical conjunction caused the wicket to form shapes different from the natural shapes of the wet wicket. Additional strain measurements and fatigue analysis of the hinges and connecting pins showed no sign of overstressing of the wicket components due to the resulting flow-induced pressure fields. 
An absolute maximum stress level of 11,250 psi in the prototype was estimated for the most critical flow configuration. For the proposed reasonably low cycle of gate operation and a low anticipated strain level, such an applied stress does not pose a fatigue threat for the structural components unless environmental effects have harshly deteriorated the mechanical performance of the gate material. Moreover, the maximum strain level occurs only during the transitional phase of operation and lasts for a short duration. The strain level for the prop-supported gate, the most active position during the course of operation, is significantly low compared to the maximum stress at the critical configuration. The prop rod allowable load capacity of 1,123 kips far exceeded the predicted maximum prototype load of 150 kips.

\section{Design Parameter Identification}

Upper boundaries of the wicket responses are constructed using the Type - I EVD method for the proposed flow boundaries and the likely service conditions mentioned in this report. Experimental results for the most critical flow configuration were used to compute the "design" values at a 99.99-percent confidence level.

A linear spatial distribution of pressure envelopes similar to the static head was noticed for the upstream gate surface. Pressure distribution on the upstream surface indicates that a static head would conservatively estimate the maximum pressure peaks for the prop-supported gate. For design evaluation, a timedependent pressure history is proposed to reconstruct the design pressure history for the Olmsted wicket. A design pressure history consists of the pressure envelopes similar to the static head having the design amplitudes obtained using the Type-I EVD approach. The time dependency of the design amplitudes could take the shape of a typical normalized upstream-pressure time-history obtained from experimental results. A normalized accelerometer-response history could also be used for representing the time-history for the design pressure fields.

\section{Analytical Model Development}

Physical parameters obtained from the experimental results provided one of the most valuable information for updating the FE model of the proposed wicket. These included the static and dynamic parameters indicative of the actual performance of the operating wicket. Static parameters such as physical mass and static stiffness obtained from load-deflection measurements provided the basis for preliminary calibration of the FE model. Dynamic parameters included the dry and wet gate modal parameters and the physical input loading and output response history from the actual flow measurements.

Experimentally measured modal parameters for the dry gate supported the development of the FE model for simulating the dynamic characteristics of the dry gate. Global modes not sensitive to the prop dynamics were considered more reliable and stable for comparison and updating process. A number of higher 
unstable wicket modes varied greatly due to their interaction with the localized prop modes. Global modes that were not sensitive to the prop dynamics were identified and used for computing the dynamic parameter for the operating gate.

Wet gate natural characteristics were synthesized to determine the added mass contribution representing the effects of water upon the dynamics of the wicket. Experimental determination of added mass is one of the most effective and suitable methods, particularly because of its ability to account for the complicated flow conditions, unusual boundary conditions, and complex 3-D geometry of the wicket model. Theoretical determination of added mass using potential theory would have been unrealistically complex for the wicket gate considering the complexity and uncertainty of analytical formulation indicated by many of the researchers.

A simple, yet effective, experimental determination of added mass is possible from the built-in response measurements of the operating wicket in which the wet gate modal frequencies could be estimated from the transmissibility functions generated using the accelerometer responses. A similarity of the resonant frequencies for the prop-supported wet gate with those of the peaks in the transmissibility functions suggested the applicability of such a relatively easy method for estimating the added mass for an operating gate. Using the plate theory, the wet and dry natural frequencies were used to determine the added mass on the operating gate. For all practical purposes, such an easier method would eliminate the necessity of conducting an extensive externally activated modal experiment for the operating wicket. This method, however, could not be applicable for determining added mass for the gate during aggressive turbulent flow near the breaking of the air pocket.

The experimental results showed that the added mass of the gate model can be several times greater than the structural mass. The effects of added mass on the gate vibrations are tremendous, as indicated by the significant reductions in the natural frequencies. The unavoidable reality was clearly reflected in the nonlinearity and irregularity of the experimentally determined added mass of the wicket gate model. In general, the added mass varied as a function of modes and showed no consistent trend in its variation.

\section{Prototype Operational Adjustment and Cost Savings}

Safe operational configurations for the prototype gate were recommended based on the model operational behavior. This early detection of safe zones averted enormous operational difficulties of the prototype wicket. Several tests with various flow and gap configurations were conducted to demonstrate the anticipated problems that could have been expected during the operation of the prototype gate. It was noticed that a two-gate gap (instrumented gate being the end gate) operation could not engage the prop rod when the head difference was more than $11 \mathrm{ft}$. Two-gate gap flow experiments over $11 \mathrm{ft}$ in head difference resulted in flows causing the prop rod to float and not maintain proper location within hurter. For this flow condition, the prop blade would not sit on the hurter 
recess during the retraction of the cylinder from the up position. However, a three-gate gap with the instrumented gate being the center gate could be operated with a minor chance of disengagement of the prop rod when the head difference was more than $16 \mathrm{ft}$. All experiments were conducted without the guide bar on the prop blade.

Apart from providing a functional design drawing for the prototype wicket, a 1:5-scale model saved millions of dollars by not repeating experiments in the prototype covering the whole operational sequences. In addition to the cost savings on the model studies, which had a cost ratio of $1: 10$ relative to the prototype, the intermediate model provided greater flexibility to experiment with a variety of configurations which would otherwise be impossible to conduct on the prototype facility. 


\section{References}

Agee, B. L., Zeng, X., and Mitchell, L. D. (1992). "An automated system for acquiring modal analysis data," Sound and Vibration 26(6), 14-21.

American Society for Testing and Materials. (1982). "Specification for structural steel." 01.04, Annual book of ASTM standards. Philadelphia, PA.

American Institute of Steel Construction (AISC). (1989). Manual of steel construction. $9^{\text {th }}$ ed., Chicago, II.

Baker, W. E., Westine, P. S., and Dodge, F. T. (1973). Similarity methods in engineering dynamics. Hayden Book Company, Rochelle Park, NJ.

Batista, R. C., Carvalho, E. M. L., and Roitman, N. (1991). "Hydroelastic reduced scale model for wave-loaded compliant tower," Experimental Mechanics 31(3), 220-225.

Bazant, Z. P. (1993). "Scaling laws in mechanics of failure," ASCE Journal of Engineering Mechanics 119(9), 1828-1844.

Beckwith, T. G., and Marangoni, R. D. (1990). Mechanical measurements. $4^{\text {th }}$ ed., Addison-Wesley, New York, NY.

Bendat, J. S., and Piersol, A. G. (1980). Engineering_applications_of correlation and spectral analysis. Wiley-Interscience, New York, NY.

. (1986). Random data: Analysis and measurement procedures. $2^{\text {nd }}$ ed., Wiley-Interscience, New York, NY.

Biggs, J. M. (1964). Introduction to structural dynamics. McGraw-Hill, New York, NY.

Bishop, R. E. D., and Price, W. G. (1976). "On the relationship between 'dry modes' and 'wet modes' in the theory of ship response," Journal of Sound and Vibration 45(2), 157-164.

Blevins R. D. (1990). Flow-induced vibrations. $2^{\text {nd }}$ ed., Van Nostrand Reinhold, New York, NY. (1993). Formulas for natural frequency and mode shape. Krieger, Malabar, FL. 
Boyce L., and Kana, D. D. (1993). "Scale model study of pile foundations under earthquake excitation," Journal of Energy Resources Technology, Transactions of the American Society of Mechanical Engineers (ASME) 115(1), 47-55.

Caccese, V., and Harris, H. G. (1990). "Earthquake simulation testing of smallscale reinforced concrete structures," ACI Structural Journal 87(1), 72-80.

Chakrabarti, S. K. (1992). "Hydroelastic modeling of large structures in a wave tank" Offshore technology proceedings of the international offshore mechanics and Arctic engineering symposium. ASME, New York, NY, 1(B), 527-533.

Che, X., Riggs, H. R., and Ertekin, R. C. (1994). "Composite 2D/3D hydroelastic-analysis method for floating structures," ASCE Joumal of Engineering Mechanics 120(7), 1499-1520.

Chen, S. S., Wambsganss, M. W., and Jendrzejczyk, J. A. (1976). "Added mass and damping of a vibrating rod in confined viscous fluids," Journal of Applied Mechanics, Transactions of the ASME 14, 325-329.

Chowdhury, M. R., Garner S., Seda-Sanabria, Y., and Hall, R. L. (1997). “A Finite-Element Model for the Olmsted Wicket," Technical Report SL-97-06, U.S. Army Engineer Waterways Experiment Station, Vicksburg, MS.

Chowdhury, M. R., and Hall, R. L. (1995). "A flow-induced response study for a 1:25-scale wicket model." Proceedings of the 13th International Modal Analysis Conference (IMAC). Nashville, TN, 1, 675-682.

Chowdhury, M. R., Hall, R. L., Hoffman, P., McClellan, B., and Mundolch, J. (1997). "Composite wicket gate development at the Olmsted prototype dam." International composite expo. The Composite Institute of the Society of the Plastics Industry, Nashville, TN.

Chowdhury, M. R., Hall, R. L, and Pesantes, E. (1997). "Flow-induced vibrational test results for a 1:25 flat wicket gate," Technical Report SL-97-04, U.S. Army Engineer Waterways Experiment Station, Vicksburg, MS.

Chowdhury, M. R., Ross, D. G., and Hall, R. L. (1997). "Experimental comparison of prototype and 1:5 wicket gates," Technical Report SL-97-05, U.S. Army Engineer Waterways Experiment Station, Vicksburg, MS.

Culligan-Hensley, P. J., and Savvidou, C. (1995). "How can geotechnical engineers contribute to research in environmental engineering?" The Earth, Engineers, and Education: A Symposium in Honor of Robert $V$. Whitman. Massachusetts Institute of Technology, Cambridge, MA, 128-143.

Dascotte, E. (1991). “Applications of finite element model tuning using experimental modal data," Sound and Vibration 25(6), 22-26. 
DeGroot, P. H. (1992). "Observations and recommendations for the proposed hydraulically controlled Wicket gates and the model testing program, Olmsted Locks and Dam navigable gate pass," Informal Report, U.S. Army Engineer District, Louisville, KY.

Derucher, K. N., and Heins, C. P. (1981). Materials for civil and highway engineers. Prentice-Hall, Elglewood Cliffs, NJ.

Elder, R. A. (1992). "Review of the 1:25 scale hydraulic model Olmsted wicket dam," Consulting Hydraulics Engineering, Cincinnati, $\mathrm{OH}$.

Ewins, D. J. (1984). Modal testing: Theory and practice. Research Studies, Letchworth, England.

Farrar, C. R., Baker, W. E., and Dove, R. C. (1994). "Dynamic parameter similitude for concrete model," ACI Structural Journal 91(1), 90-99.

Goncharov, L. A., and Semenkov, V. M. (1974). "Field investigations of dam and gate vibrations." Flow-induced structural vibrations. E. Naudascher, ed., Springer-Verlag, Berlin, 297-310.

Hewlett-Packard. (1985). "The fundamentals of signal analysis," Application Note 243, Palo Alto, CA.

. (1986). "The fundamentals of modal testing," Application

Note 243-3, Palo Alto, CA.

HKS, Inc. (1992). “ABAQUS users manual,” version 5.2, Pawtucket, RI.

Ishii, N., and Knisely, C. (1992). "Flow-induced vibrations of long-span gates due to shed vortices," JSME International Journal, series 3 35(1), 1-8.

Jarvis, B. (1991). "Enhancements to modal testing using finite-elements," Sound and Vibration 25(3), 28-30.

Jongeling, T. H. G. (1989). "Flow-induced self-excited in-flow vibrations of gate plates," Journal of Fluids and Structures 2(10), 542-566.

Key, B. (1989). "Dynamic similitude theory: Key to understanding the ASME compressor-performance test," Journal of Petroleum Technology 41(8), 860-866.

Kientzy, D., and Richardson, M. (1989). "Using finite-element data to set up modal tests," Sound and Vibration 23(6), 16-23.

Kulite Semiconductor. (1994). "Kulite precision pressure transducers and transmitters,"Leonia, NJ. 
Lance, G. R. (1992). "Developing an operating plan for Olmsted Locks and Dam." Fourth water resource management workshop. ASCE, Mobile, AL.

Leuridan, J. (1992). "Modal analysis: A perspective on integration," Sound and Vibration 26(4).

Lin, R. M., Du, H., and Ong, J. H. (1993). "Sensitivity based method for structural dynamic model improvement," Computers \& Structures 47(3), 349-369.

Lindeburg, M. R. (1986). Civil engineering reference manual. $4^{\text {th }} \mathrm{ed}$., Professional Publications, San Carlos, CA.

. (1994). Mechanical engineering reference manual. $9^{\text {th }} e d$, Professional Publications, San Carlos, CA.

LMS Intl. (1991). "Large-scale modal testing of a space frame structure - from pretest analysis to FEA model validation," Sound and Vibration 25(3), 6-16.

Ma, J. (1993). "Hydroelastic coupled vibration of a plate facing an infinite strip of water," Journal of Hydraulic Engineering 3, 48-55.

Ma, J., and Xie, S. (1993). "Coupling vibration of an isolated plate with water," Journal of Hydraulic Engineering 9, 62-69.

Maheri, M. R., and Severn, R. T. (1992). "Experimental added-mass in modal vibration of cylindrical structures," Engineering Structures 14(3), 163-175.

March, T. A., and Elder, R. A. (1992). "Review of the 1:25-scale hydraulic model Olmsted Wicket Dam," U.S. Army Engineer Division, Ohio River, Cincinnati, $\mathrm{OH}$.

MathWorks, The. (1992). MATLAB for windows, user's guide. Natick, MA.

MB Dynamics. (1990). “Modal 50A vibration exciter manual," Manual No. Modal50A.Man, Cincinnati, $\mathrm{OH}$.

Merritt, H. E. (1967). Hydraulic control systems. Wiley, New York, NY.

Mouch, T. A., and Myers, W. D. (1991). "The use of dynamic analysis in the design of an automobile door," Sound and Vibration 25(4), 28-30.

Neyrinck, F., Bakkers, W., Parodi, M., Pallotti, P., Piombo, B., and Turino G. (1992). "Operational analysis and problem identification of a high performance racing engine." 10th International Modal Analysis Conference. Society For Experimental Mechanics, Bethel, CT, 1399-1405.

Novak, P., and Cabelka, J. (1981). Models in hydraulic engineering physical principles and design applications. Pitman, Boston, MA. 
Parkinson, G. V. (1974). "Mathematical models of flow-induced vibrations of bluff bodies." Flow-induced structural vibrations. E. Naudascher, ed., Springer-Verlag, Berlin, 81-127.

Paz, M. (1985). Structural dynamics theory and computation. Von Nostrand Reinhold, New York, NY.

PCB Piezotronics. (1993). "Vibration \& shock: Sensor selection guide," Depew, $N Y$.

Peterson, E. L., and Snyder, R. C. (1987). "Modal excitation made easy," Sound and Vibration 21(3).

Petrick, L. (1993). "Correlation of experimental and analytical models of a cross-country ski," Sound and Vibration 27(6), 18-23.

Petroski, H. (1993). "Failure as source of engineering judgment: Case of John Roebling," Journal of Performance of Constructed Facilities 7(1), 46-58.

Ramsey, K. A (1975). "Effective measurements for structural dynamics testing: Part I," Sound and Vibration 9(11), 24-35. . (1976). "Effective measurements for structural dynamics testing: Part II," Sound and Vibration 10(4).

Richardson, M. (1975). "Modal testing using digital test systems." Seminar on understanding digital control and analysis in vibration test systems (part 2). The Shock and Vibration Information Center, NRL, Washington, DC, 43-64.

Rogers, P. (1989). "Modal modeling for dynamic analysis confidence," Sound and Vibration 23(8).

Roitman, N., Andrade, R. F. M., and Batista, R. C. (1992). "Dynamic response analysis of a small-scale model tension leg platform," Marine Structures 5(6), 491-513.

Schofield, A. N. (1981). "Dynamic and earthquake geotechnical centrifuge modelling." Inermational conference on recent advances in geotechnical earthquake engineering and soil dynamics. Vol 3. University of MissouriRolla, Rolla, MO, 1081-1100.

Seda-Sanabria, Y. (1994). "Dynamic behavior study of a wicket gate of the Olmsted Dam in Ohio River," M.S. thesis, University of Puerto RicoMayaguez.

Shye, K., VanKarsen, C., and Richardson, M. (1988). "Modal testing using multiple references." Practical aspects of modal analysis. Structural Measurement Systems, San Jose, CA. 
Sibert, W. L. (1972). "The Improvement of the Ohio River." Transactions of ASCE 63(1104), 388-425.

Simitses, G. J., and Razaeepazhand, J. (1992). "Structural similitude and scaling laws for laminated beam-plates." Topics in composite materials and structures. ASME, Aerospace Division, New York, NY, 37-45.

Simpson, M., and Solomon, A. (1992). "Summary report: Olmsted Wicket Dam project review of $1 / 25$ scale model," Structural Dynamics Research Corporation, Milford, $\mathrm{OH}$.

Skladnev, M. F., and Sheinin, I. S. (1974). "On flow-induced structural oscillations." Flow-induced structural vibrations. E. Naudascher, ed., Springer-Verlag, Berlin, 250-264.

Snowberger, R. B. (1995). "Olmsted prototype at Smithland, Kentucky." Proceedings of the Corps of Engineers structural engineering conference. San Antonio, TX.

Soast, A. (1994). "Record size lock and dam project gets a soggy launch as rains swell the lower Ohio River for months," Engineering News Record 24-26.

Society of Experimental Mechanics (SEM). (1995). "Modal analysis: Theory and application course," Bethel, CT.

Solignac, J. L., Pagan, D., and Molton, P. (1989). "Experimental study of incompressible flow on the upper surface of a delta wing," Recherche Aerospatiale (English Edition) 6, 47-65.

Stearns, S. D. (1975). Digital signal analysis. Hayden Book Company, Rochelle Park, NJ.

Stockstill, R. L. (1992). "Literature review of flat gate aeration, Olmsted Locks and Dam; Design analysis: Olmsted Locks and Dam and prototype, Vol 3 of 3," U.S. Army Engineer District, Louisville, Louisville, KY.

Structural Dynamics Research Corporation. (1993a). "Final report of the finiteelement analysis of the 1:25-scale Olmsted wicket gate and 1:25-scale model design package," U.S. Army Engineer District, Louisville, Louisville, KY. (1993b). “I-DEAS Master Series 1.2," Milford, OH.

Structural Measurement System (SMS). (1988). "Practical aspects of modal analysis," Course Notes, San Jose, CA.

Tanaka, H. (1990). "Similitude and modeling in wind tunnel testing of bridges," Journal of Wind Engineering and Industrial Aerodynamics 33(1-2), 283-300. 
Tao, H. (1990). "Characteristics of flow pressure pulsation and similitude law of model." Hydraulic engineering - Proceedings of the 1990 national conference. ASCE, Boston Society of Civil Engineering Section, Boston, MA, 743-748.

Teledyne Engineering Services. (1993). "Shear pin transducer," Manufacturers Pamphlet, Brewer Engineering, Marion, MA.

Thang, N. D. (1982). “Added mass behavior and its characteristics at sluice gates." Flow induced vibrations in fluid engineering. BHRA Fluid Engineering, Cranfield, England, 13-28.

Thoft-Christensen, P., and Baker, M. J. (1982). Structural reliability theory and its applications. Springer-Verlag, New York, NY.

Tonosaki, H., and Nakada, T. (1994). "Experimental considerations of the operating vibration behavior on 4 cylinder power train." 12th International modal analysis conference. Society For Experimental Mechanics, Bethel, CT, 1633-1637.

Treiber, B. (1974). "Theoretical study of nappe oscillations." Flow-induced structural vibrations. E. Naudascher, ed., Springer-Verlag, Berlin, 34-46.

U.S. Army Engineer District, Louisville. (1993). "Design analysis: Olmsted Locks and Dam prototype, Ohio River, Smithland, KY," 1-3, SFB No. DACA27, Louisville, KY.

USAEWES. (1989). "Model study of scour protection for Olmsted Locks and Dam," U.S. Army Engineer Waterways Experiment Station, Vicksburg, MS. (1992). "Data report, model study of Olmsted navigation pass," U.S. Army Engineer Waterways Experiment Station, Vicksburg, MS.

(1995). "DPLOT version 1.2.0.3," Structures Laboratory, U.S. Army Engineer Waterways Experiment Station, Vicksburg, MS.

U.S. Department of Interior, Bureau of Reclamation. (1992). “An implementation plan for dam safety," Denver, $\mathrm{CO}$.

. (1994). "Hydraulic laboratory techniques," Technical Publication, Water and Power Resources Services, Denver, CO.

Vethamony, P., Chandramohon, P., Sastry, J. S., and Narasimhan S. (1992). "Estimation of added mass and damping coefficients of a tethered spherical float using potential flow theory," Ocean Engineering - An International Journal of Research and Development 19(5), 427-436.

Wille, R. (1974). "Generations of oscillatory flows." Flow-induced structural vibrations. E. Naudascher, ed., Springer-Verlag, Berlin, 1-16. 
Wirsching, P., and Ortiz, K. (1993). "Reliability methods in mechanical and structural design." $14^{\text {th }}$ Annual seminar and workshop. College of Engineering and Mines, Aerospace and Mechanical Engineering Department, University of Arizona, Tucson, AZ.

Yan, G., and Yan, S. (1990). "Study of hydro-elastic couple natural characteristics for radius gate," Journal of Hydraulic Engineering 7, 49-55.

Yang, C. I., and Moran, T. J. (1979). "Calculations of added mass and damping coefficients for hexagonal cylinders in a confined viscous fluid." Flow induced vibration symposium presented at the National Congress of pressure vessel and piping technology. ASME, New York, NY, 97-103.

Zonic Corporation. (1991). "Workstation 7000 users manual," Milford, OH.

(1995). "Lazon user manual," Milford, OH. 


\section{Appendix A Selected Prototype Design Sheets}




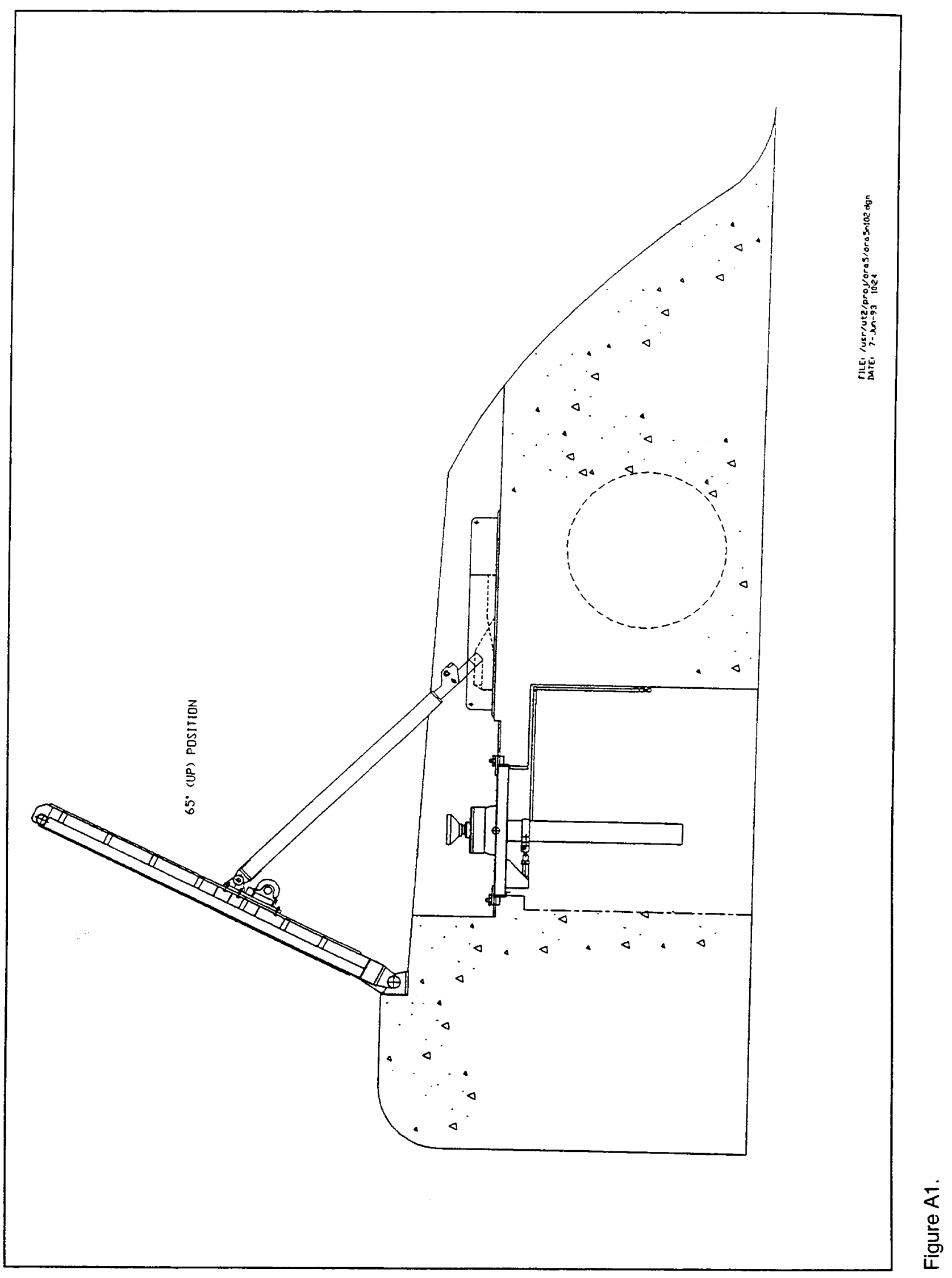




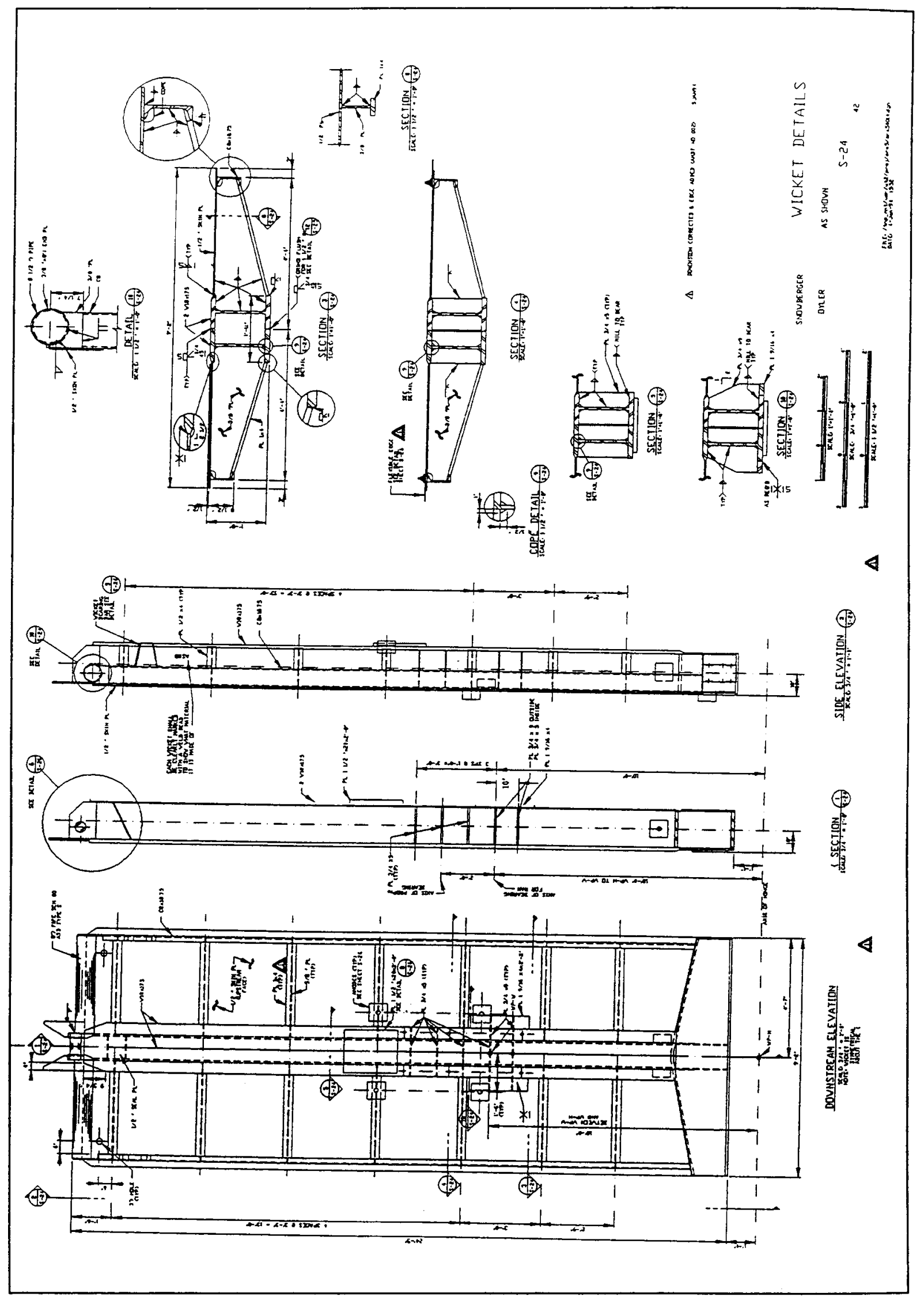

نั 


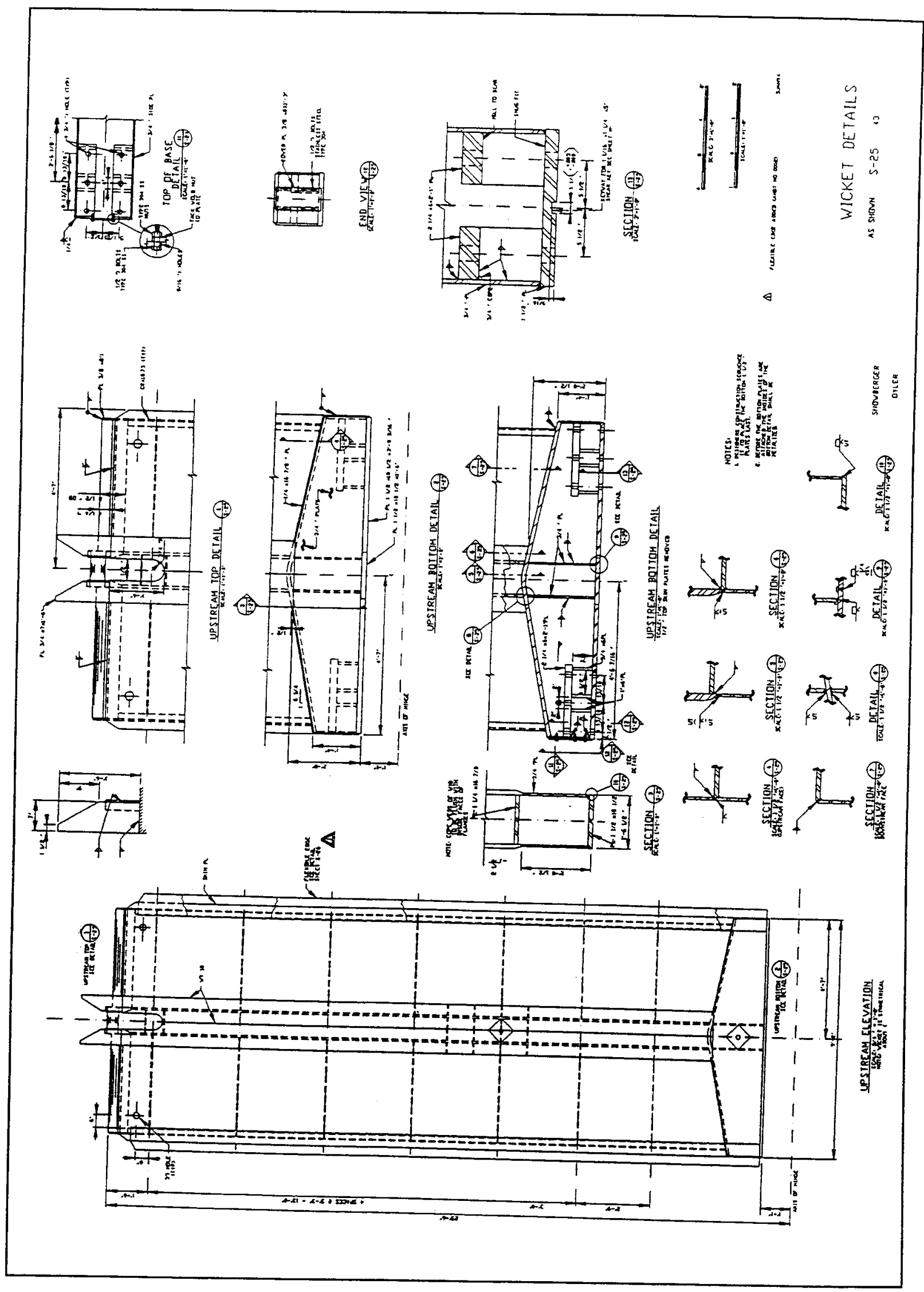

$\frac{\text { o }}{0}$
o
产 


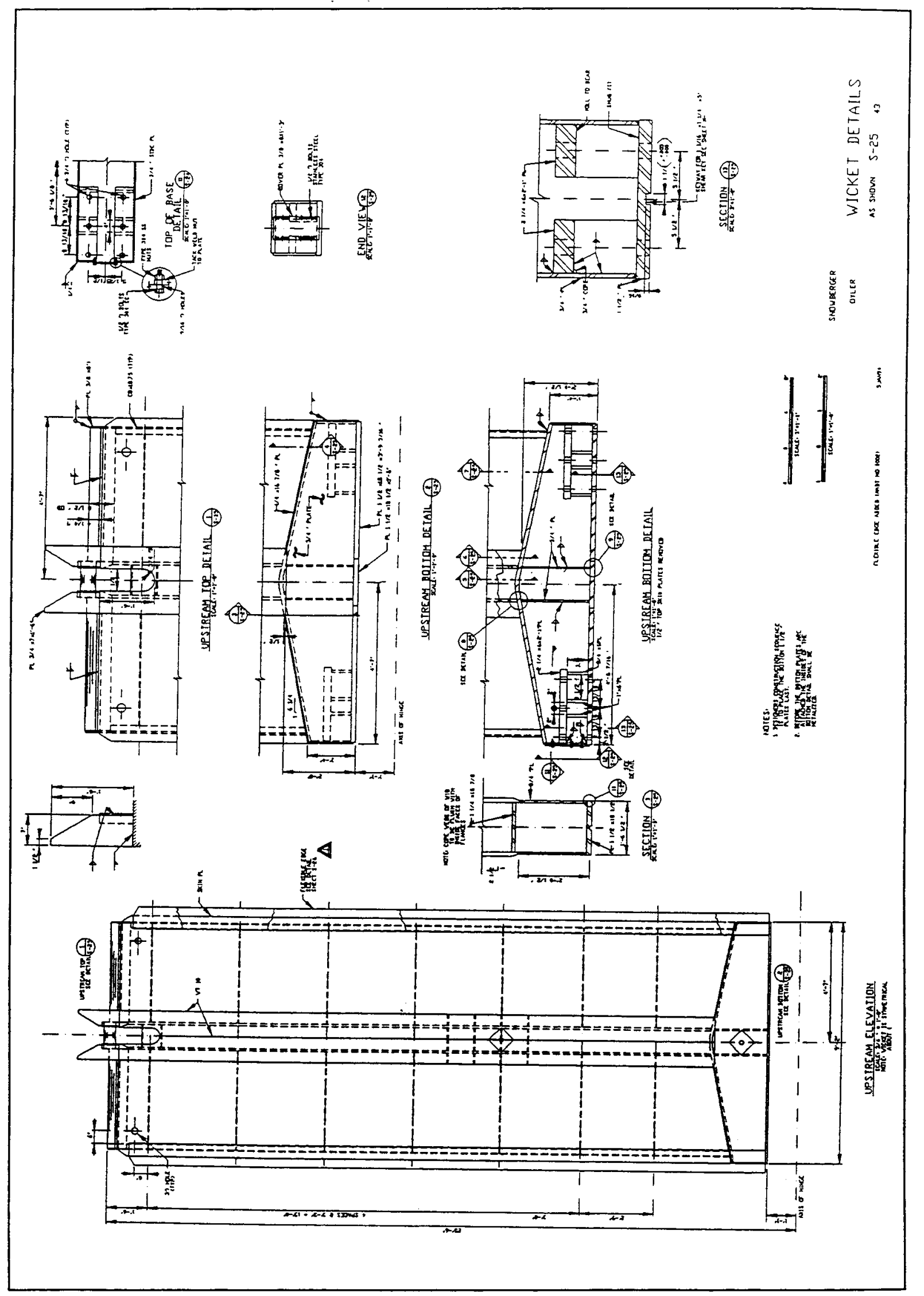

$\frac{\dot{\alpha}}{0}$
$\frac{0}{3}$
$\frac{0}{4}$ 


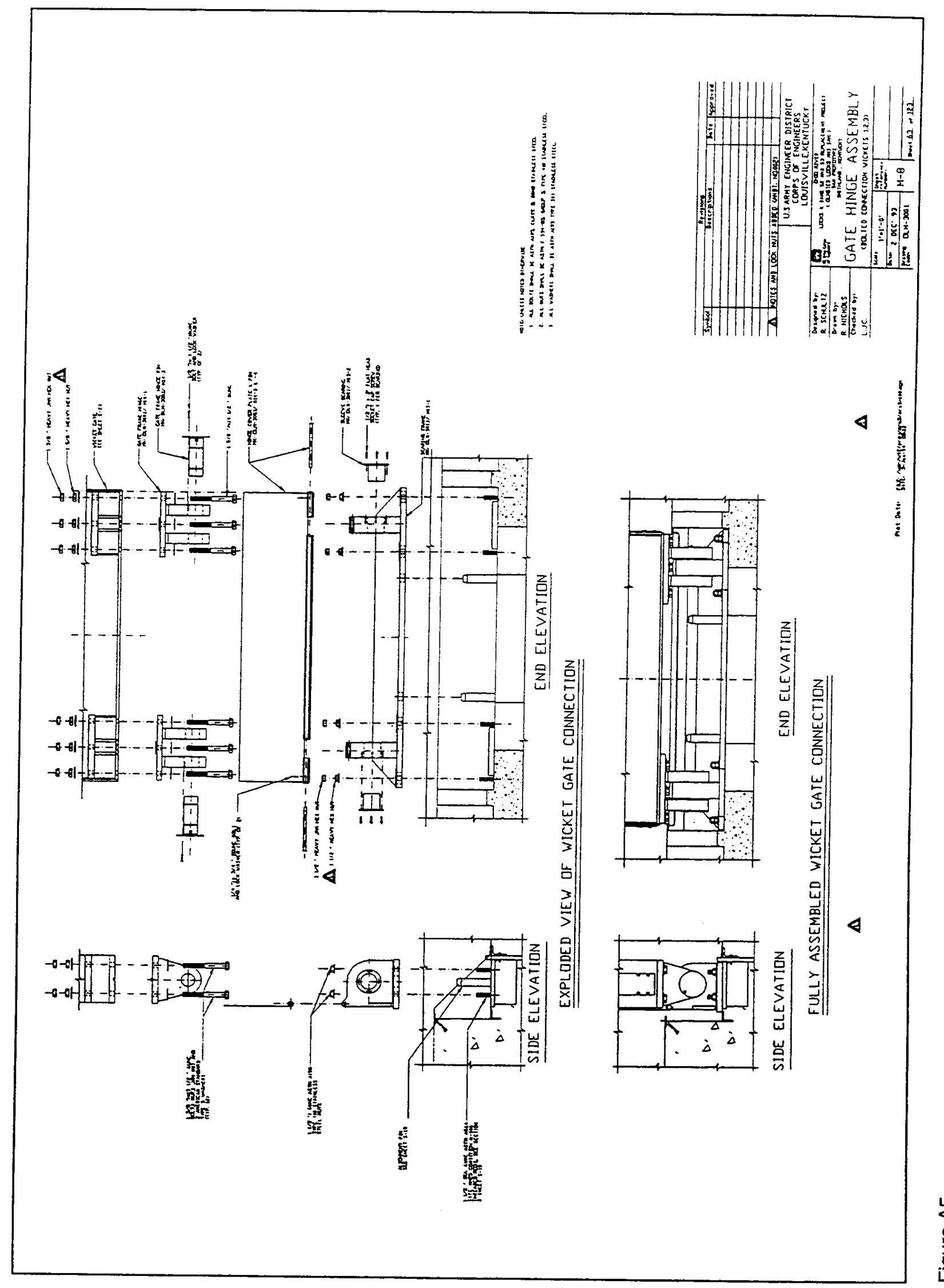

$\frac{\stackrel{20}{\frac{1}{0}}}{\frac{0}{5}}$ 


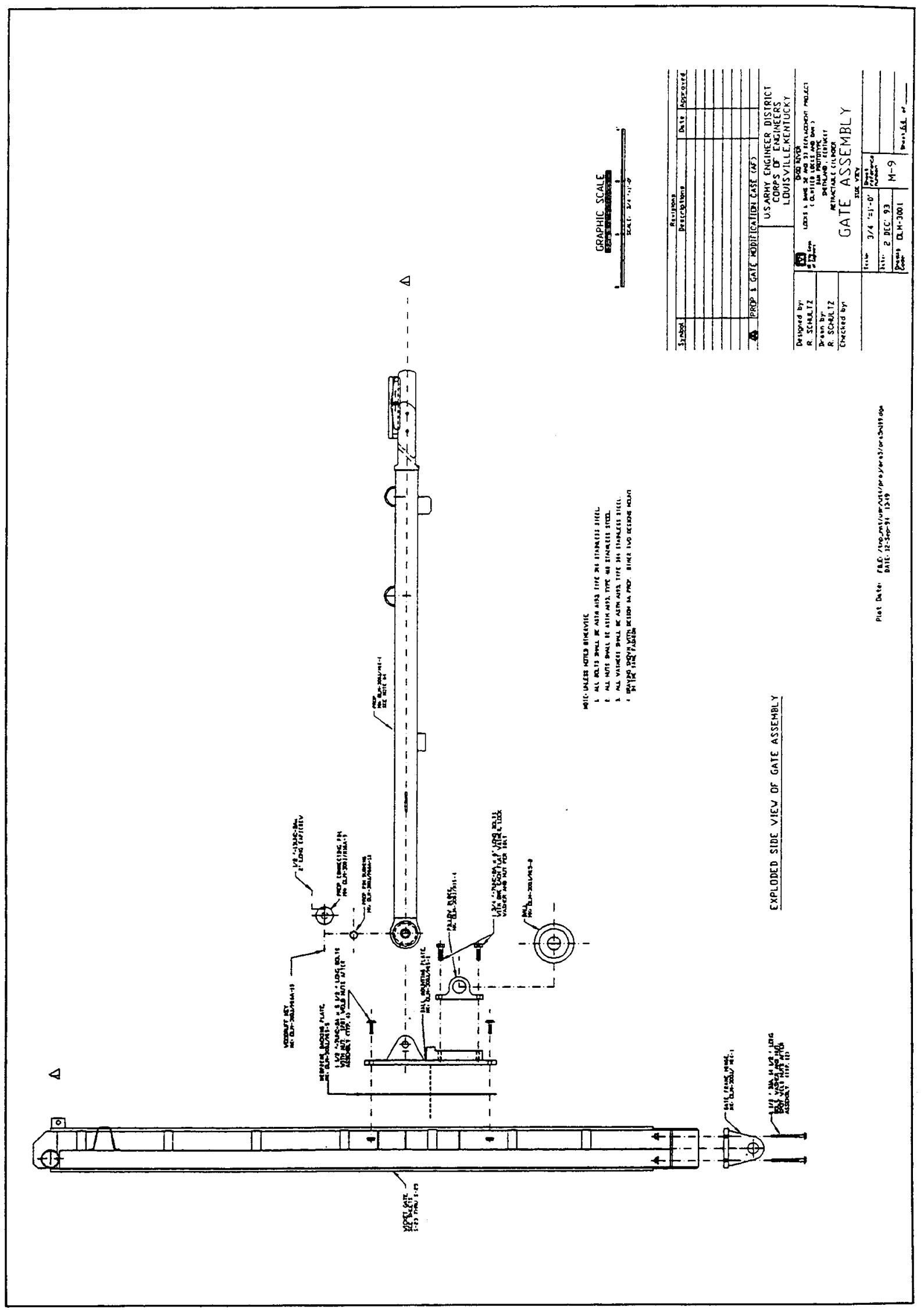

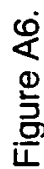




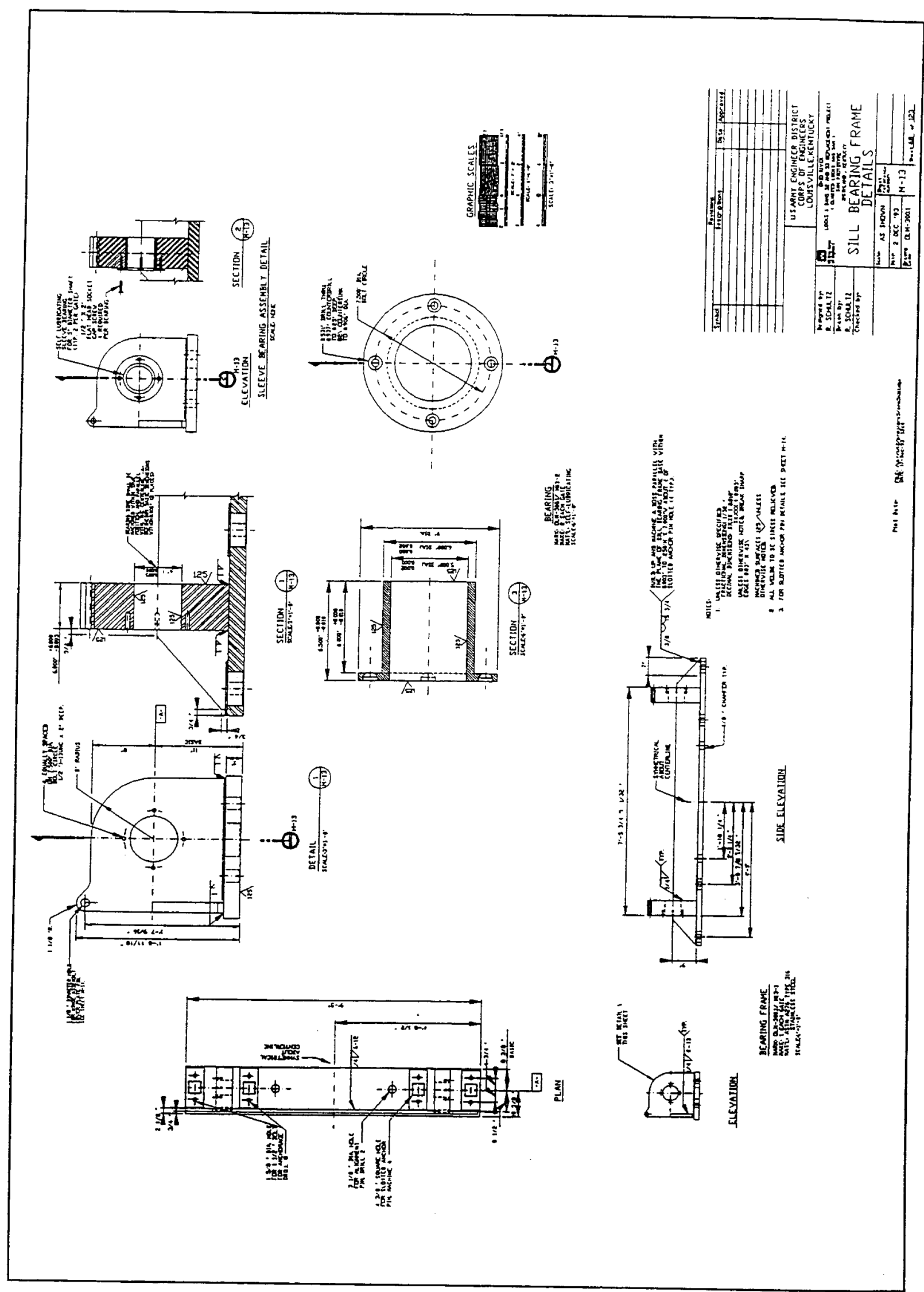

$\frac{1}{4}$
$\frac{1}{5}$
$\frac{5}{4}$ 


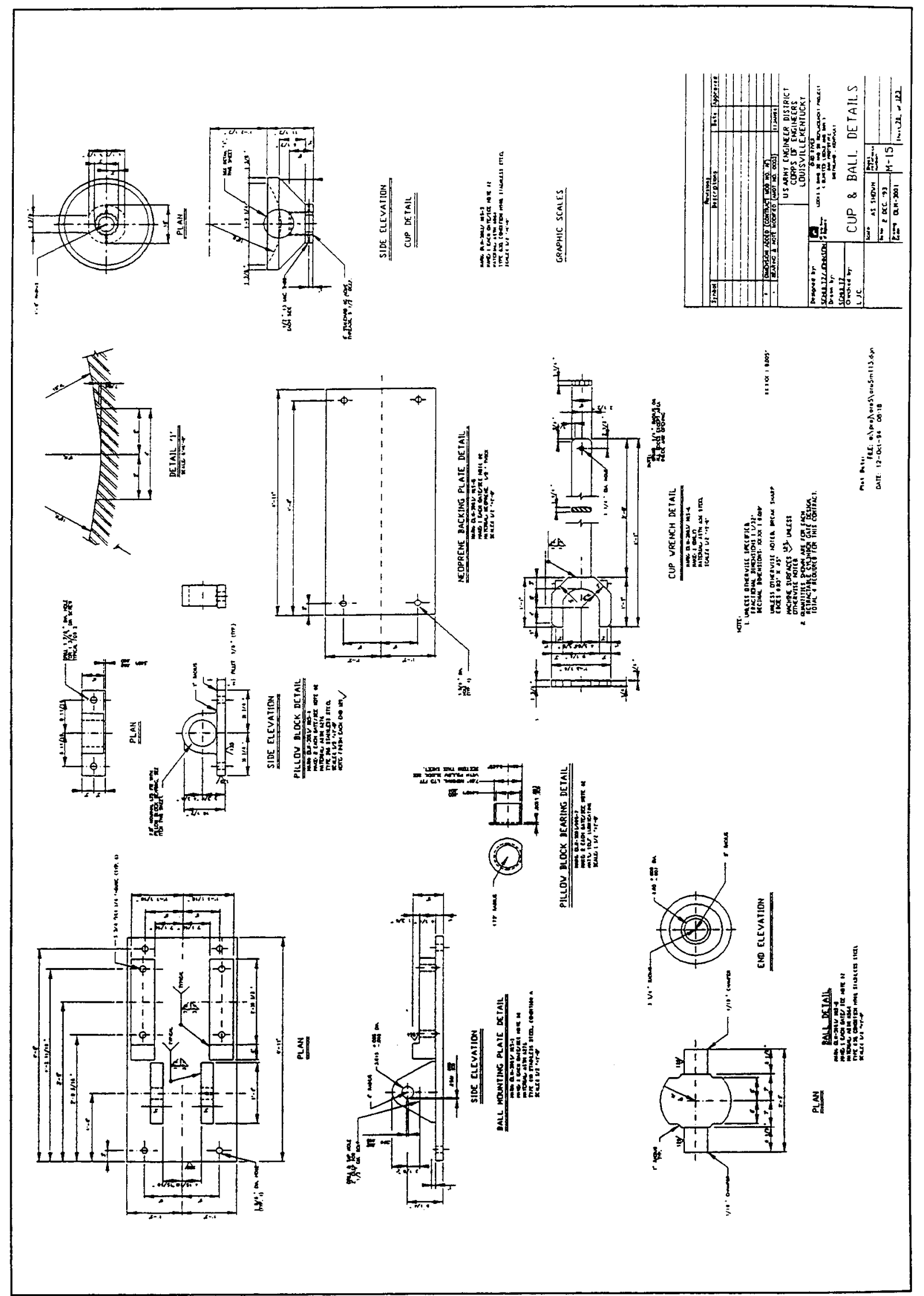

$\frac{\infty}{\frac{\infty}{2}}$ 


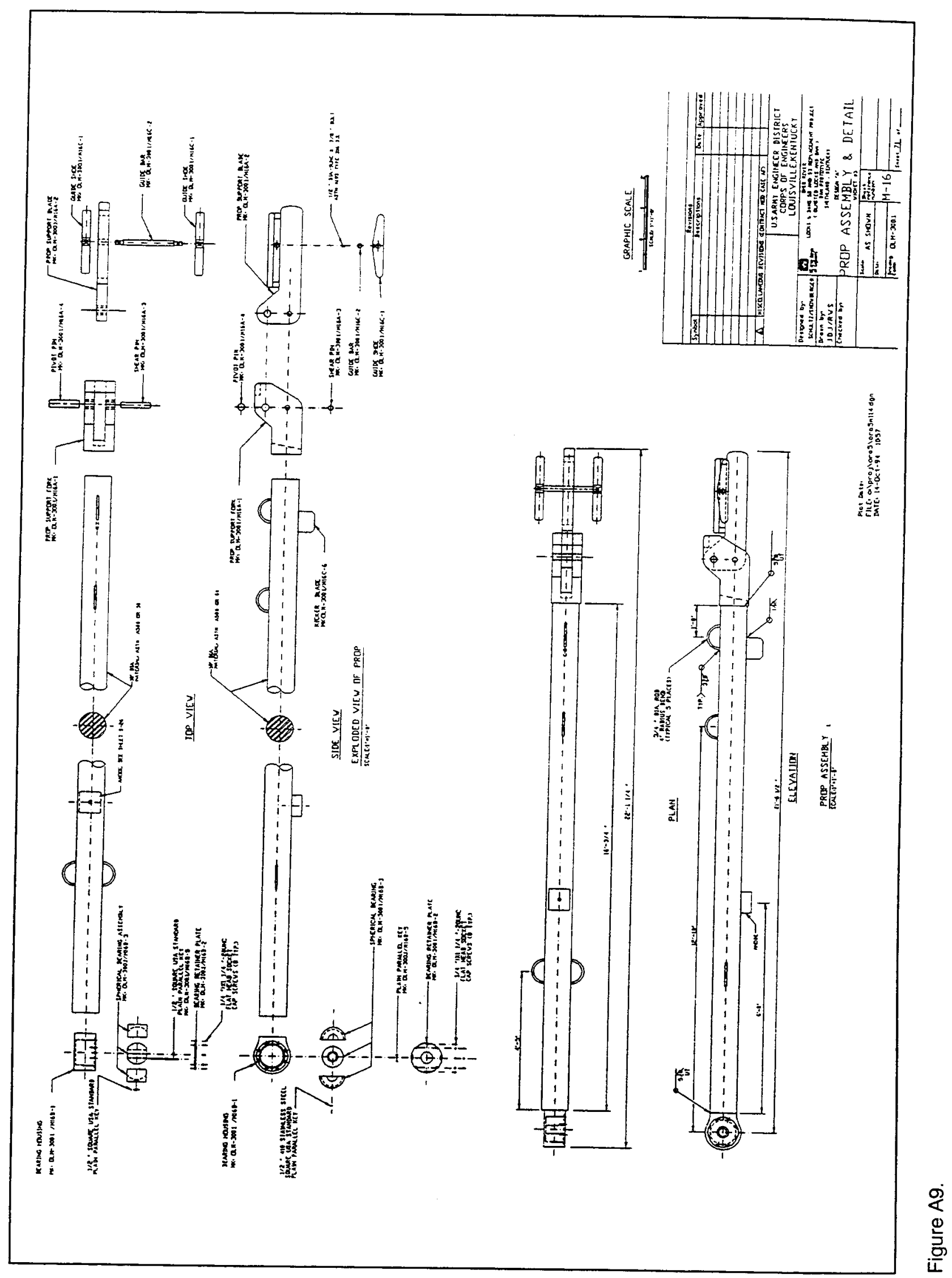




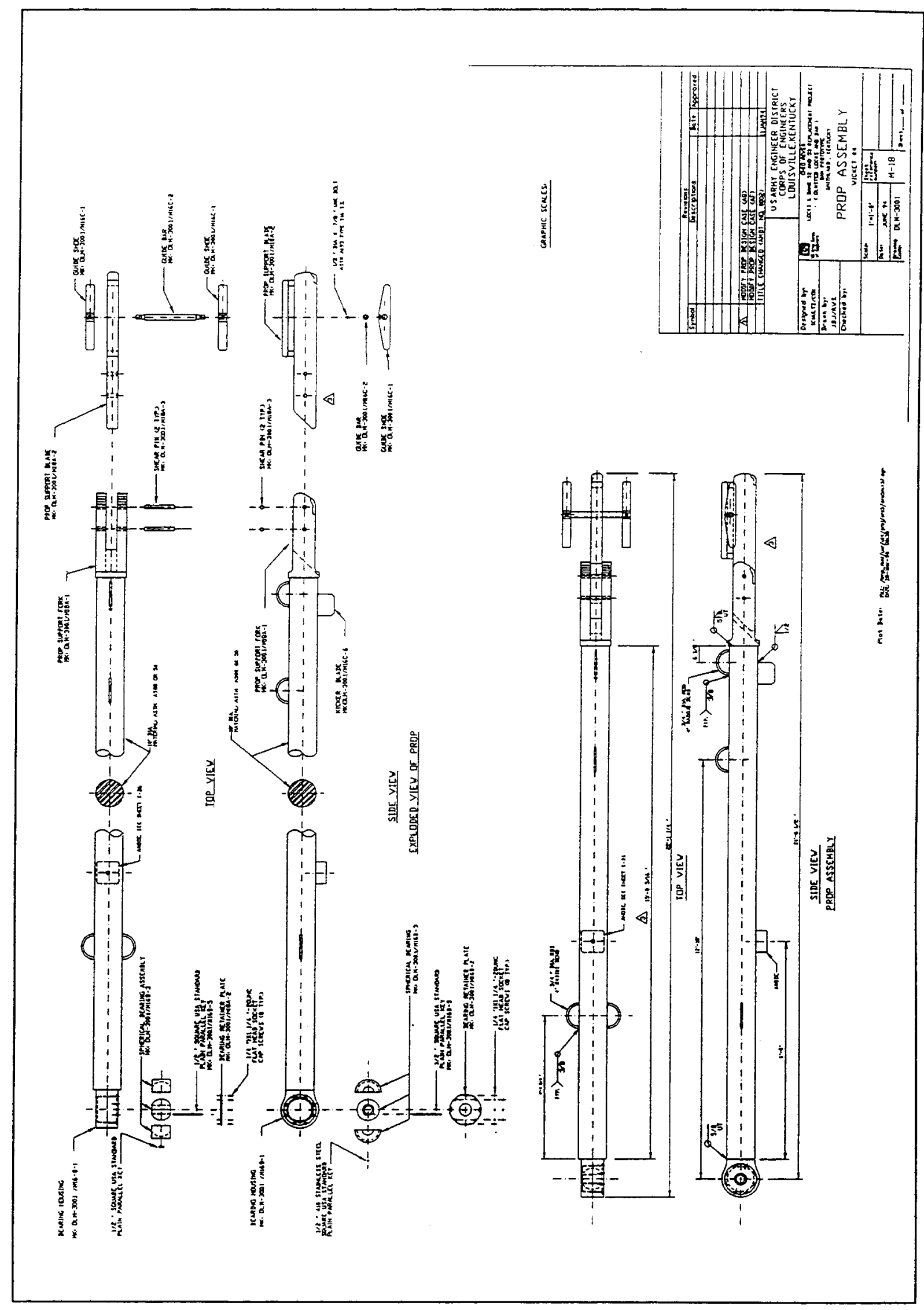

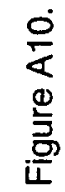




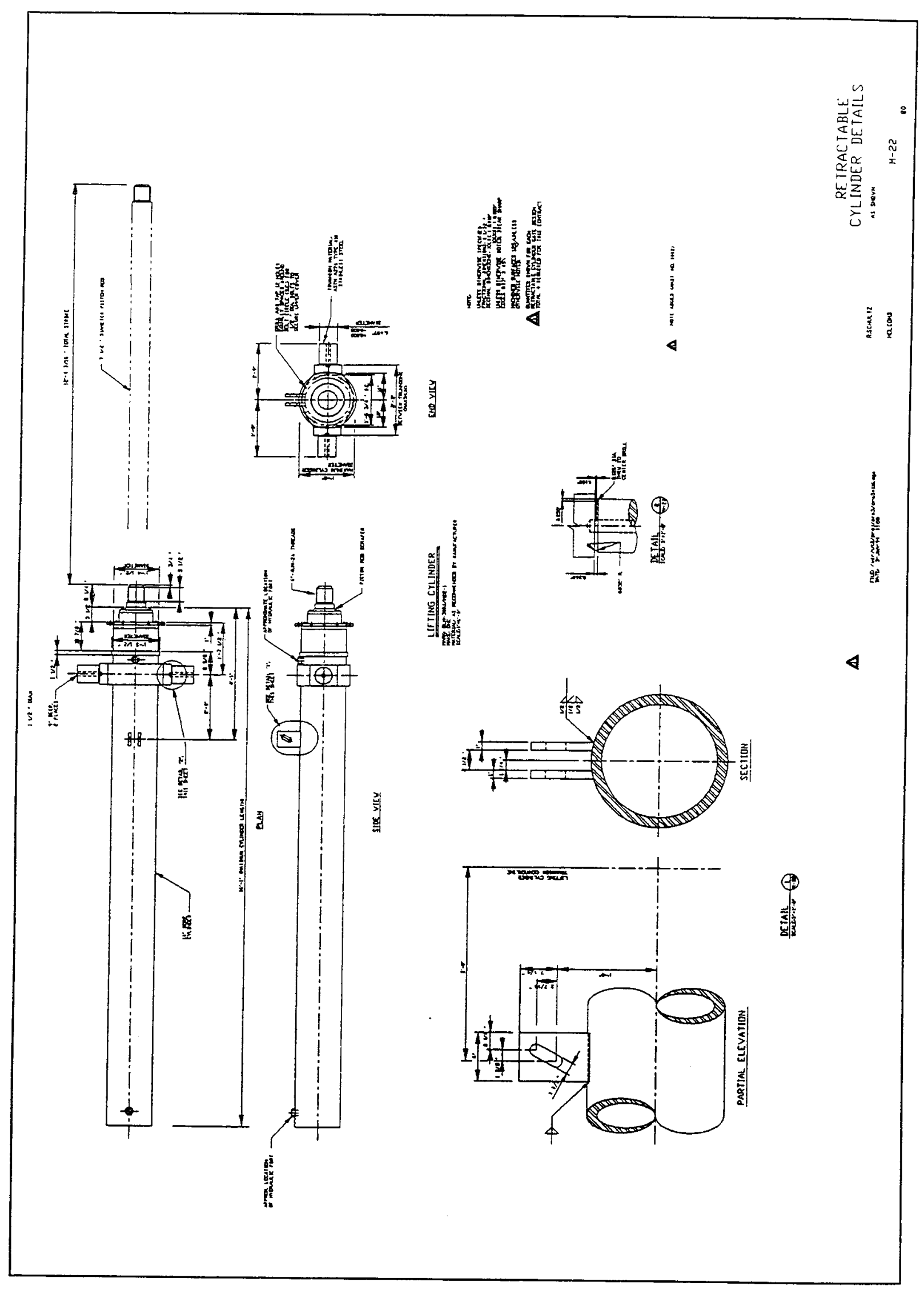

$\frac{5}{4}$
$\frac{1}{3}$
$\frac{0}{1}$ 


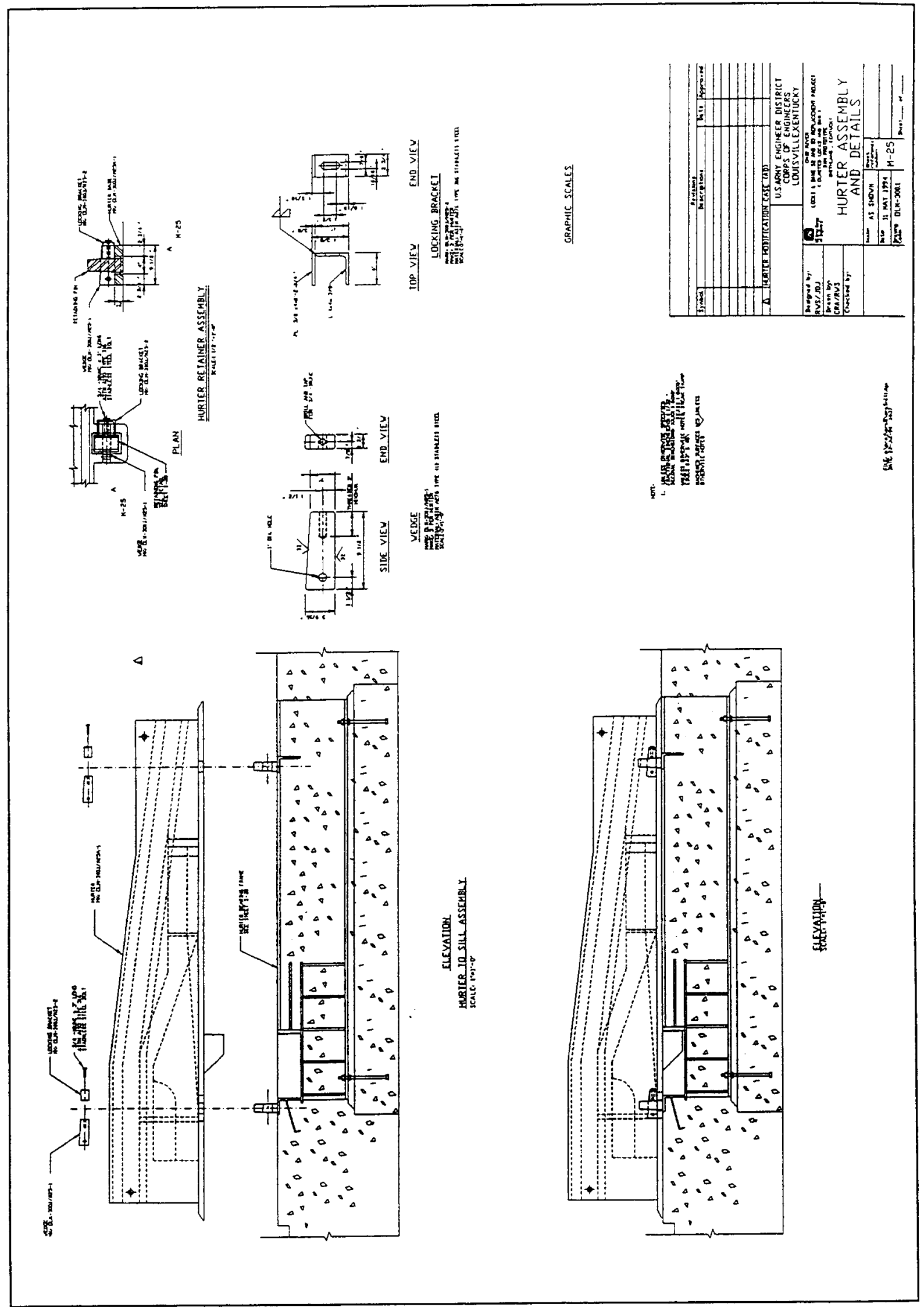

$\frac{N}{4}$
$\frac{0}{5}$
$\frac{9}{1}$ 

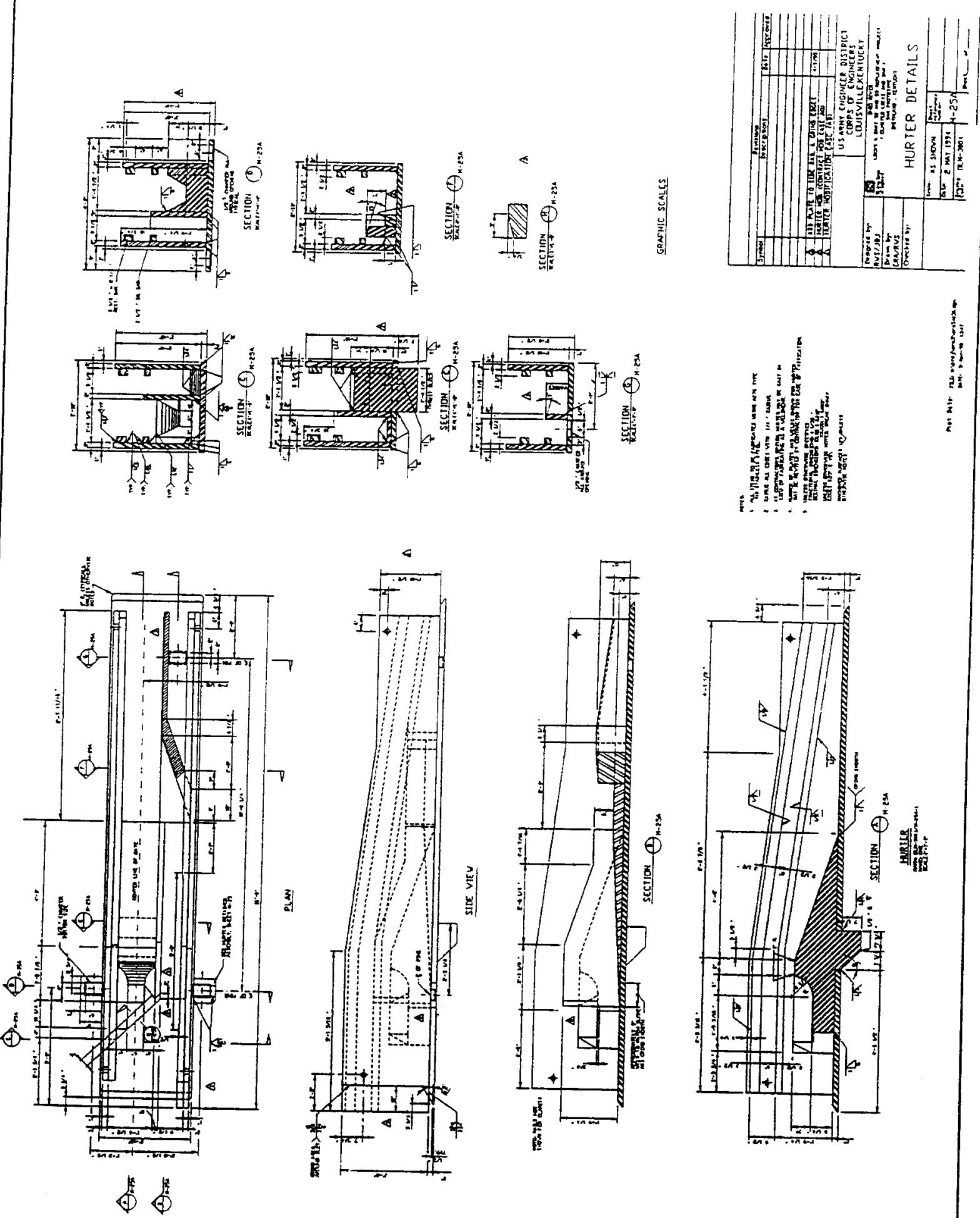

$\frac{1}{4}$
$\frac{0}{5}$
$\frac{0}{2}$ 


\section{Appendix B Lifting Cylinder Drawings}




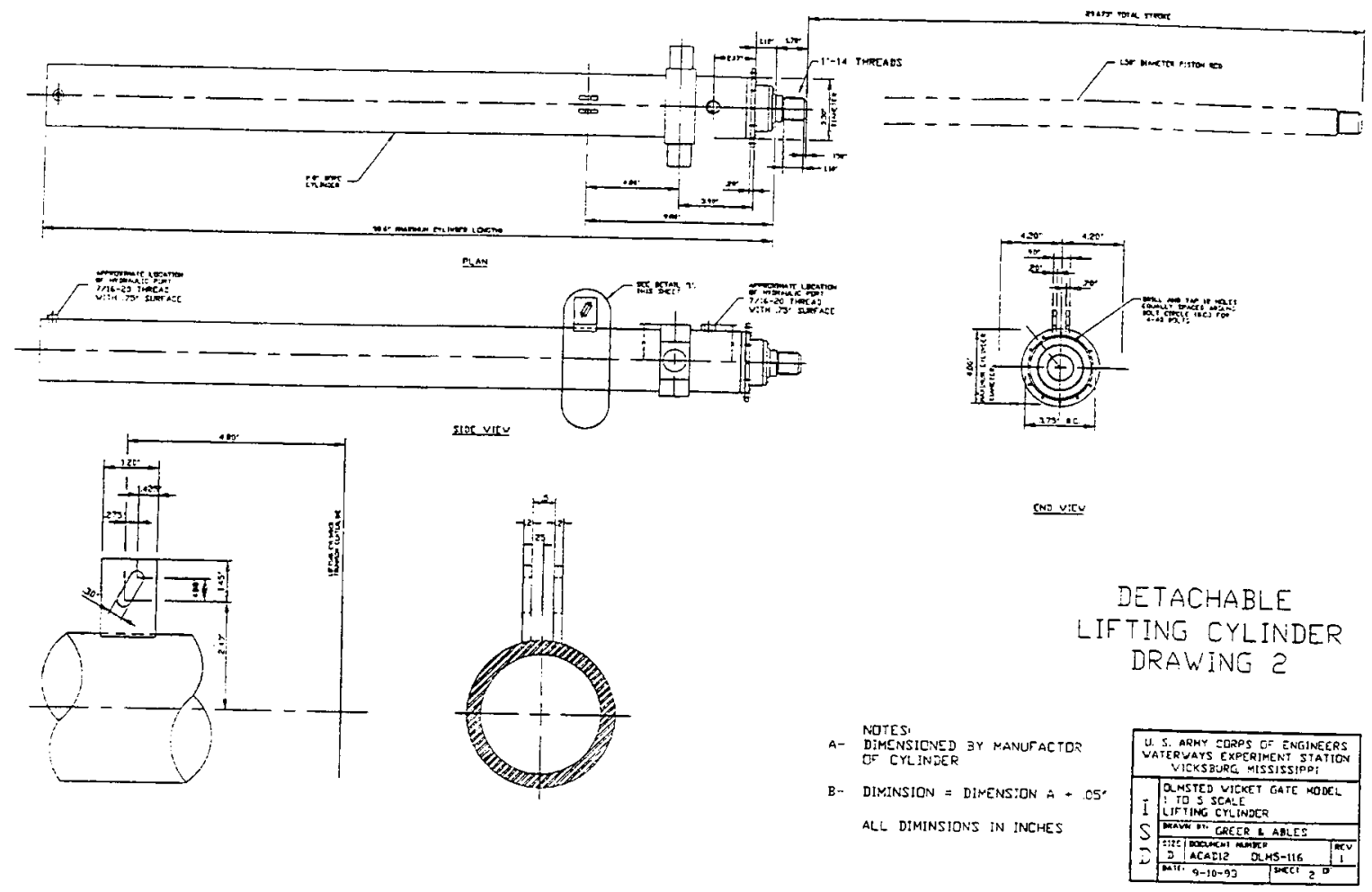

Figure B1.
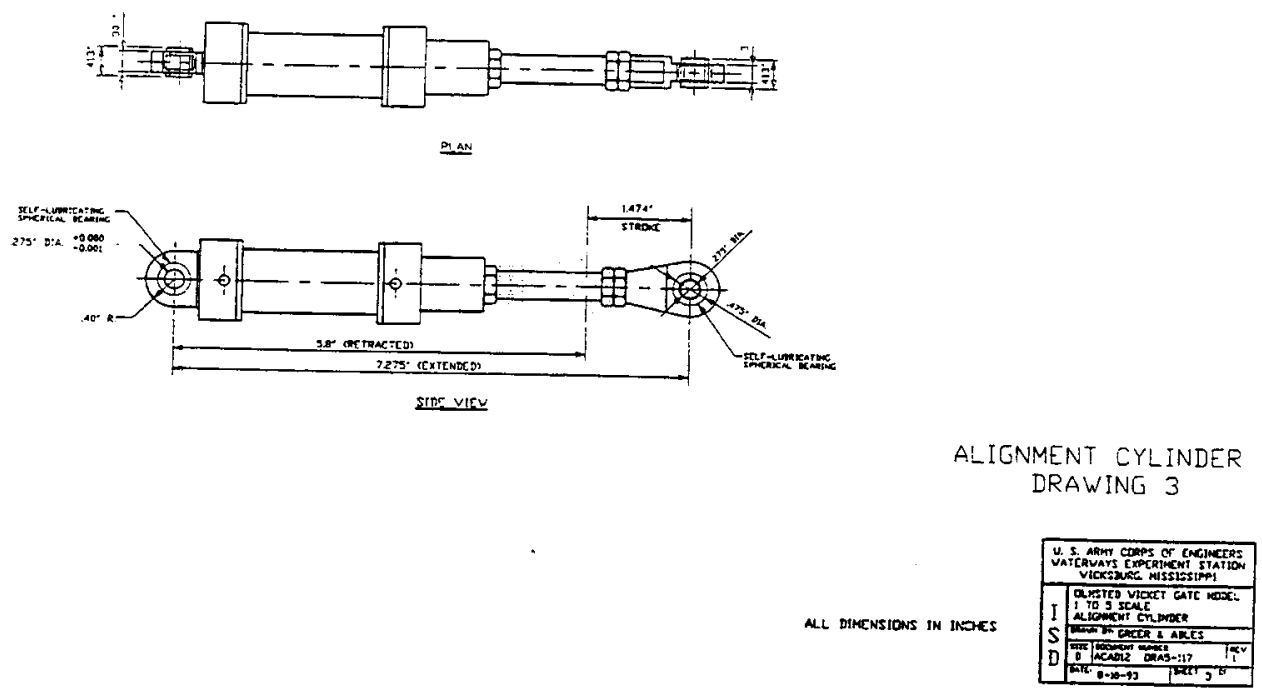

Figure B2.

B2 


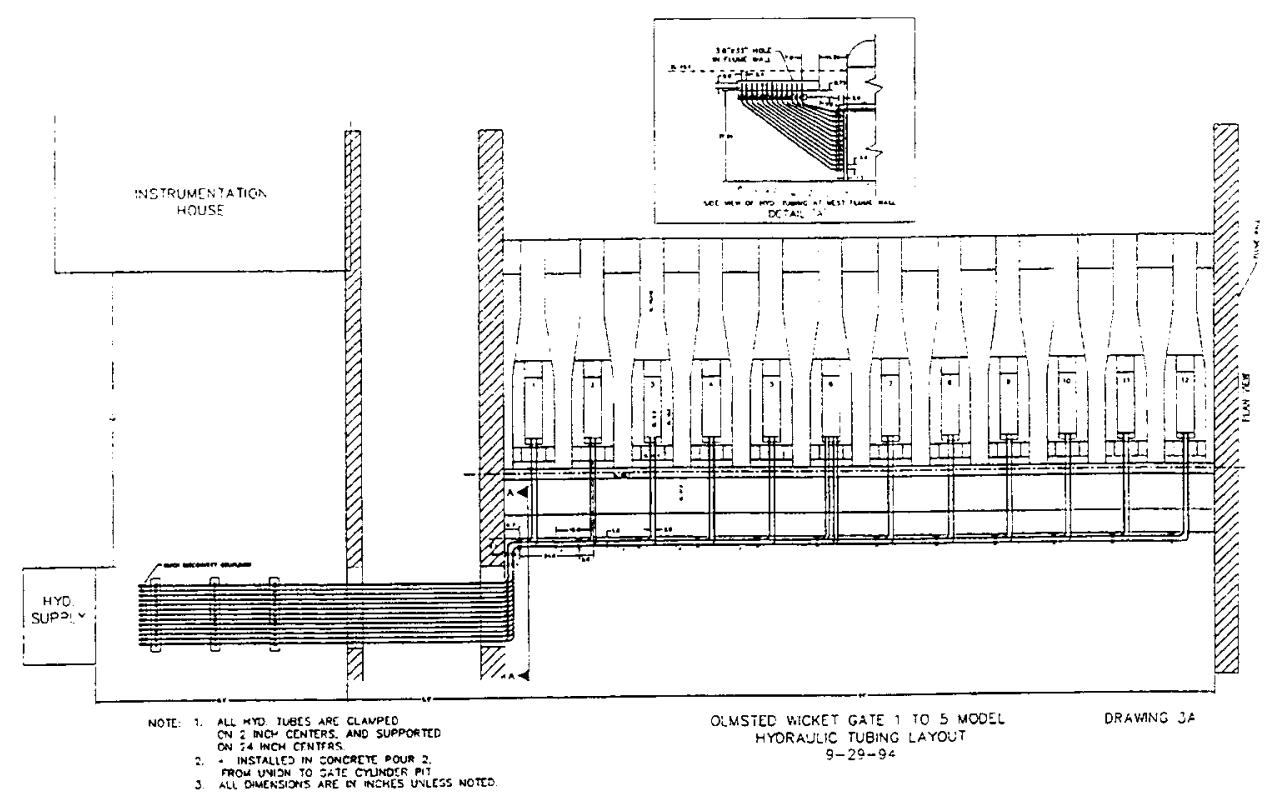

Figure B3.

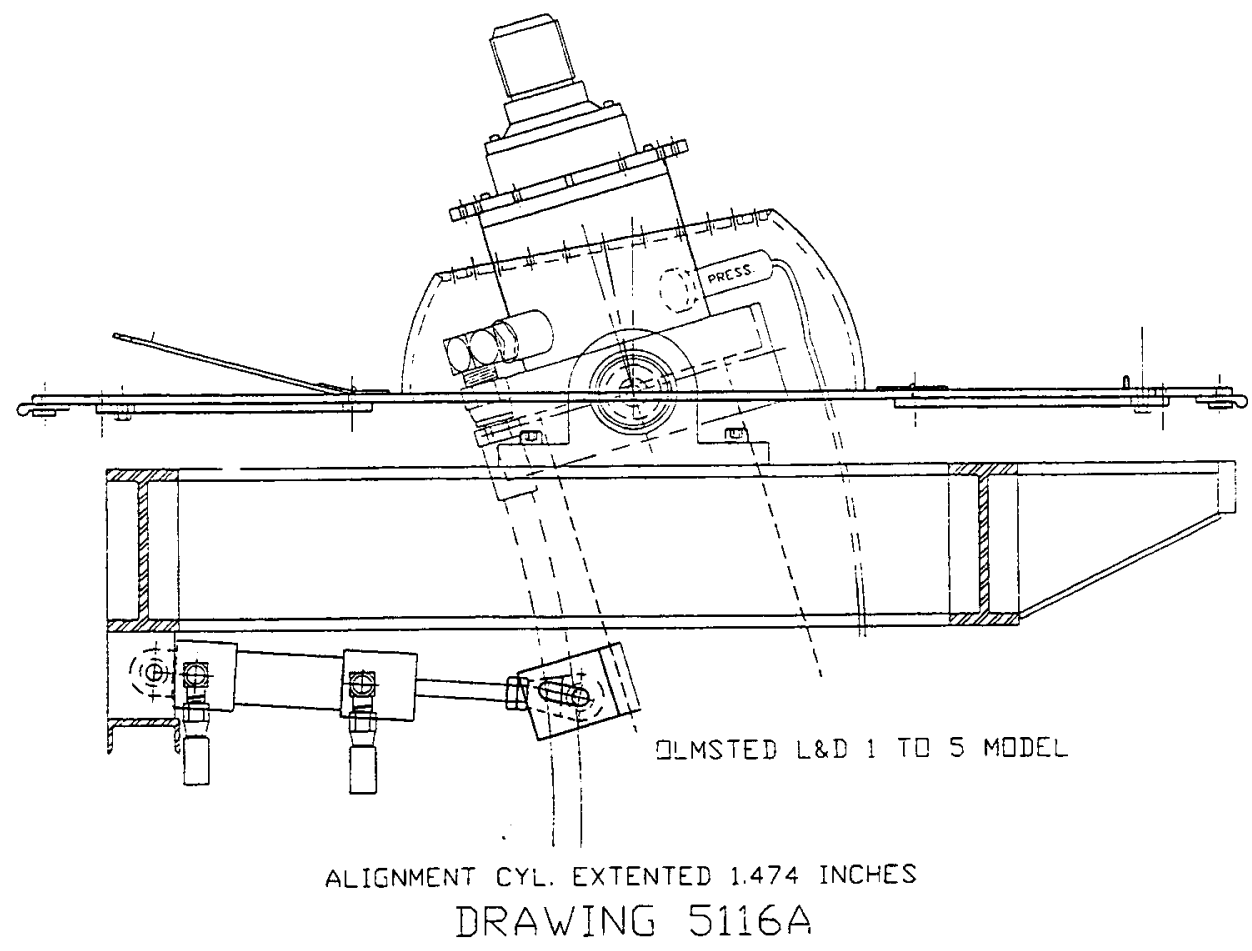

Figure B4. 

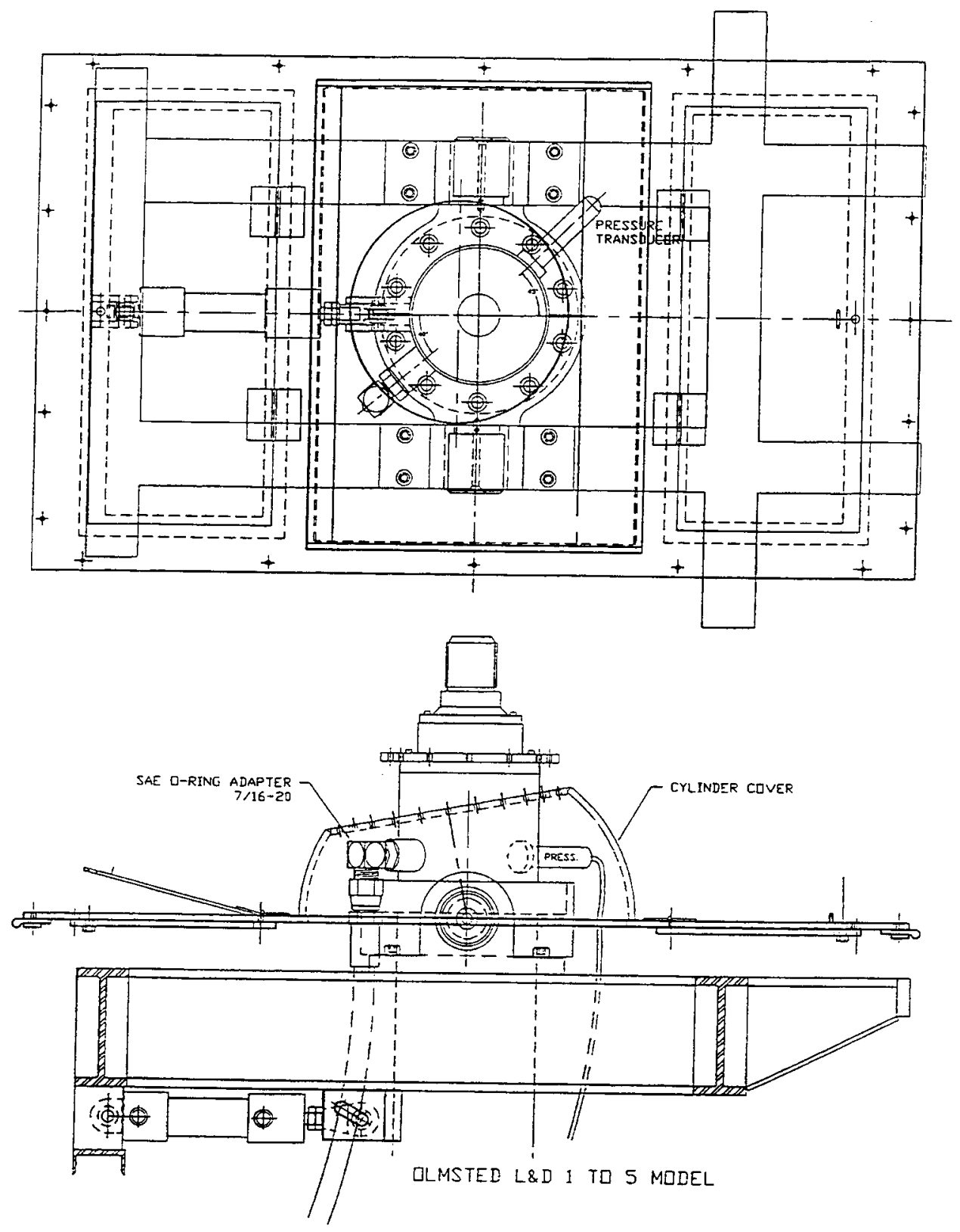

Figure B5. 


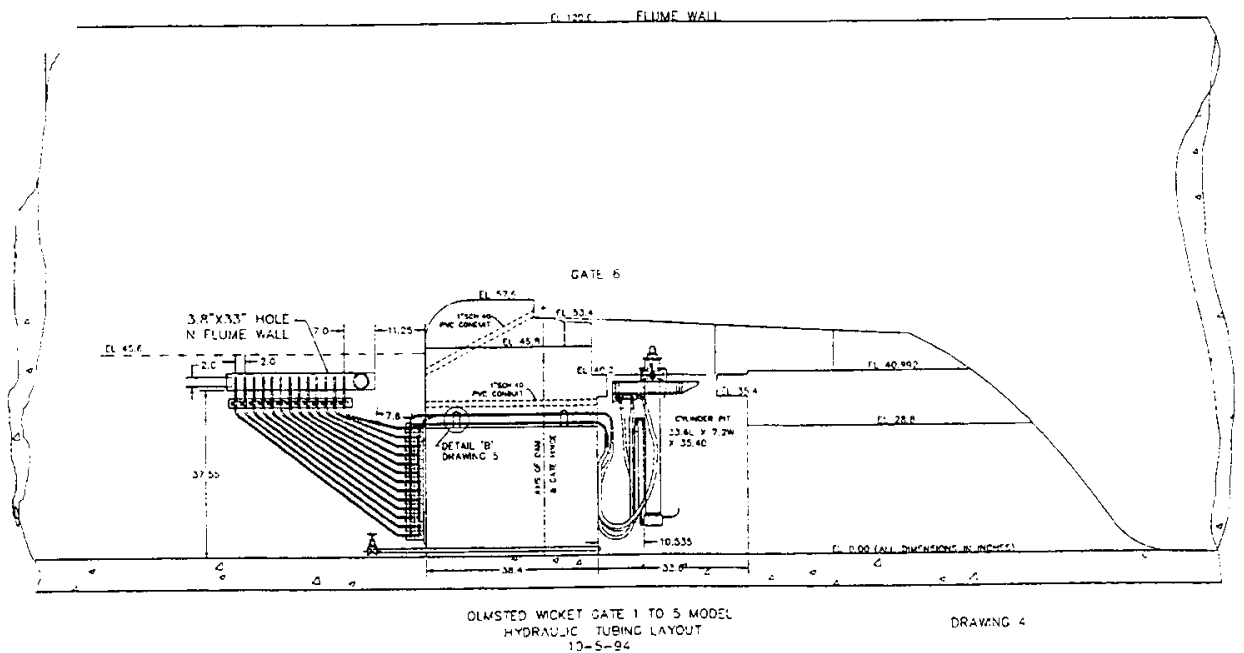

Figure B6.

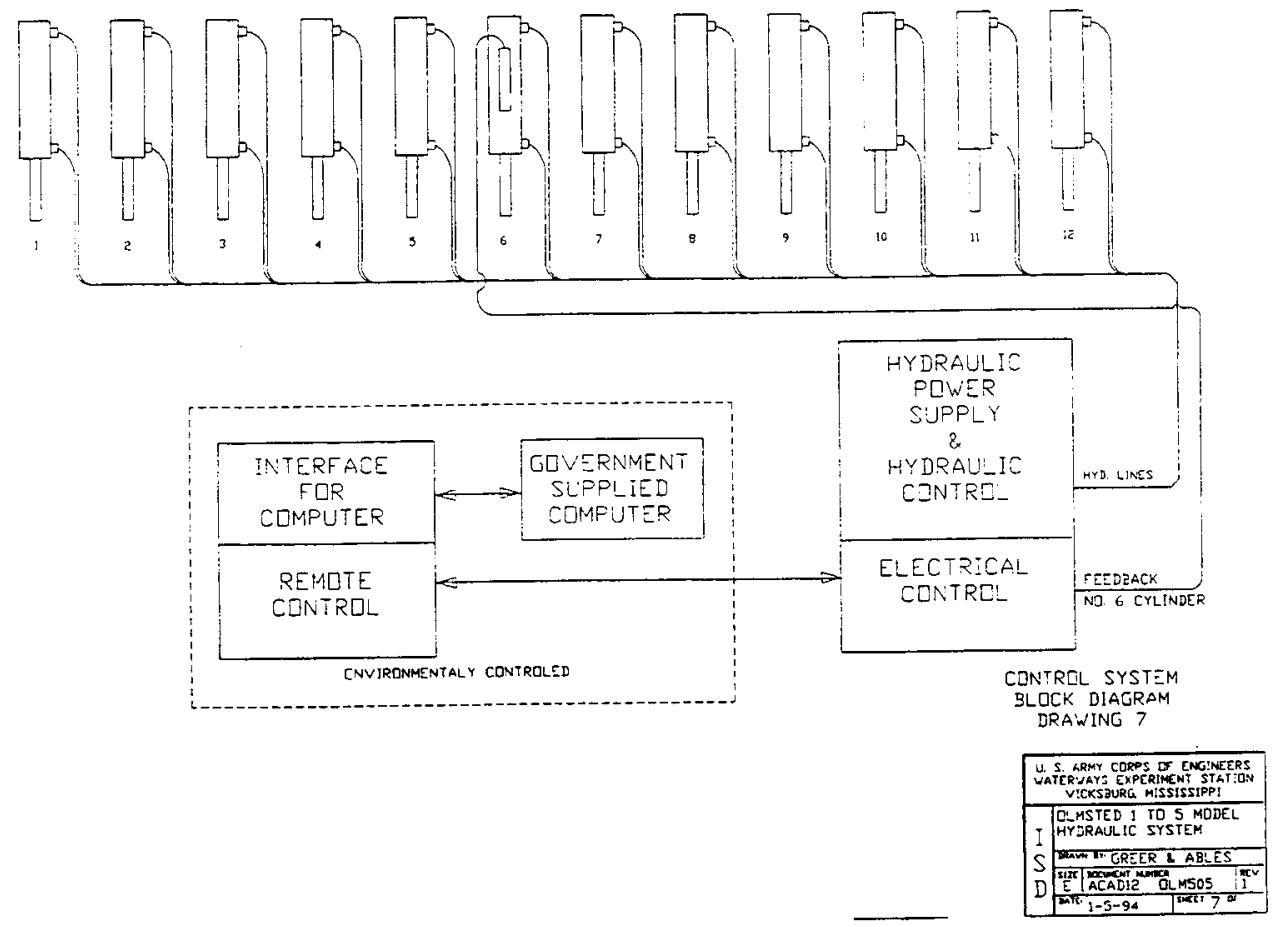

Figure B7. 
Putlic reporting burden for this collection of information is estimated to average 1 hour per response, including the time for reviewing instructions, searcting existing data sources, gathering and maintaining the data needed, and completing and revieming the collection of information. Send comments regarding this burden estimate or any other aspect of this collection of information, including suggestions

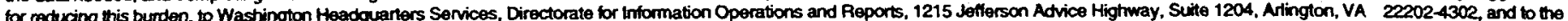
for reducing this bunden, to Washington Haadquarters Services, Directorate for thiformaton Operations and
1. AGENCY USE ONLY (Leave blank)
2. REPORT DATE
3. REPORT TYPE AND DATES COVERED
April 1998
Final report

4. TITLE AND SUBTITLE

Flow-Induced Structural Response of a 1:5-Scale Olmsted Wicket Model

5. FUNDING NUMBERS

6. AUTHOR(S)

Mostafiz R. Chowdhury, Robert L. Hall, Winston Glenn Davis

7. PERFORMING ORGANZATION NAME(S) AND ADDRESS(ES)

U.S. Army Engineer Waterways Experiment Station

3909 Halls Ferry Road

8. PERFORMING ORGANIZATION

REPORT NUMBER

Vicksburg, MS 39180-6199

Technical Report SL-98-2

9. SPONSORINGMONITORING AGENCY NAME(S) AND ADDRESS(ES)

Headquarters, U.S. Army Corps of Engineers, Washington, DC 20314-1000

10. SPONSORINGMONITORING

AGENCY REPORT NUMBER

U.S. Army Engineer District, Louisville, Louisville, KY 40201-0059

11. SUPPLEMENTARY NOTES

Available from National Technical Information Service, 5285 Port Royal Road, Springfield, VA 22161.

12a. DISTRIBUTIONAVAILABILTY STATEMENT

12b. DISTRIBUTION CODE

Approved for public release; distribution is unlimited.

13. ABSTRACT (Maximum 200 words)

This report presents the technical contributions of different physical models for studying the flow-induced vibrations of Olmsted wickets. Experimental results of two Froude-scale models (1:25 and 1:5 scales) for different flow configurations are compared to examine the effectiveness of the physical similitude models. It also indicates that the sensitivity of the size effects decreases as the model size increases. This experiment demonstrates that the global design parameters could be roughly simulated with a small-scale model (1:25) without going through elaborate reproduction of an exact replica of its prototype. Sensitivity of the sensors and the reproduction of mechanical joints in a small-scale model are the primary factors governing the effectiveness of a very small-scale model. Experimental results of a hybrid rigid body model of the wicket suggest that the higher-order frequency contributions on the predicted response are negligible. The knowledge gained from the models and prototype studies complemented each other by providing information unique to each of the systems. For the flow configurations examined in this investigation, a Froude model adequately reproduces the structural as well as hydraulic phenomena of the Olmsted wicket.

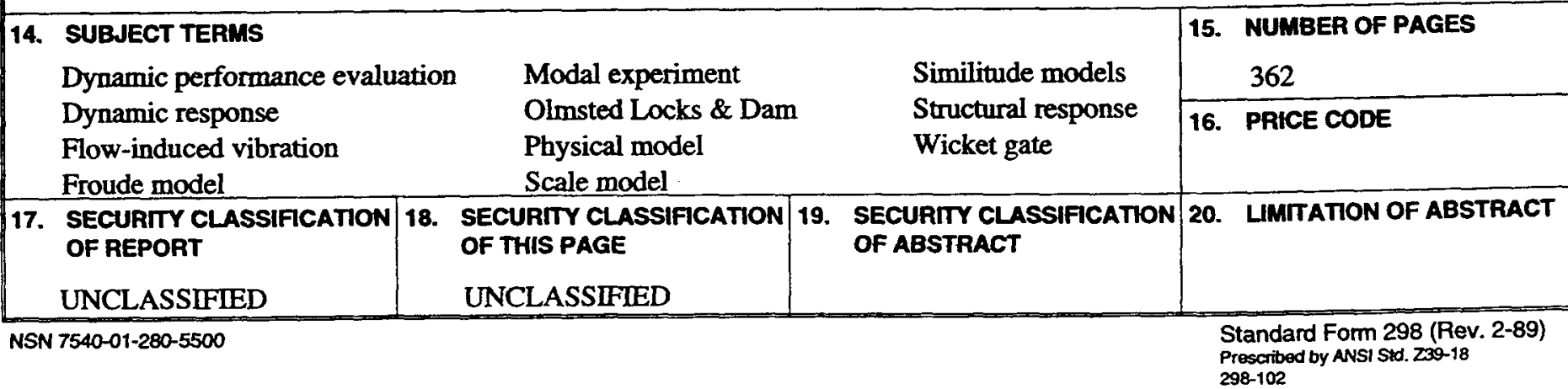

